Florida International University FIU Digital Commons

$3-12-2010$

\title{
Analytical Approximations to Predict Performance Measures of Manufacturing Systems with Job Failures and Parallel Processing
}

Maria Hulett

Florida International University, maluhulett@yahoo.com

DOI: $10.25148 /$ etd.FI10041614

Follow this and additional works at: https://digitalcommons.fiu.edu/etd

Part of the Operations Research, Systems Engineering and Industrial Engineering Commons

\section{Recommended Citation}

Hulett, Maria, "Analytical Approximations to Predict Performance Measures of Manufacturing Systems with Job Failures and Parallel Processing" (2010). FIU Electronic Theses and Dissertations. 167.

https://digitalcommons.fiu.edu/etd/167 


\section{FLORIDA INTERNATIONAL UNIVERSITY}

Miami, Florida

\section{ANALYTICAL APPROXIMATIONS TO PREDICT PERFORMANCE MEASURES OF MANUFACTURING SYSTEMS WITH JOB FAILURES AND PARALLEL PROCESSING}

A dissertation submitted in partial fulfillment of the requirements for the degree of DOCTOR OF PHILOSOPHY in

INDUSTRIAL AND SYSTEMS ENGINEERING

by

Maria de Lourdes Hulett 


\section{To: Dean Amir Mirmiran}

College of Engineering and Computing

This dissertation, written by Maria de Lourdes Hulett, and entitled Analytical Approximations to Predict Performance Measures of Manufacturing Systems with Job Failures and Parallel Processing, having been approved in respect to style and intellectual content, is referred to you for judgment.

We have read this dissertation and recommend that it be approved.

\section{Ronald Giachetti}

Sushil Gupta

Purushothaman Damodaran, Co-Major Professor

Chin-Sheng Chen, Co-Major Professor

Date of Defense: March 12, 2010

The dissertation of Maria de Lourdes Hulett is approved.

\begin{tabular}{r} 
Dean Amir Mirmiran \\
College of Engineering and Computing \\
\hline Interim Dean Kevin O’Shea \\
University Graduate School
\end{tabular}

Florida International University, 2010 
(C) Copyright 2010 by Maria de Lourdes Hulett All rights reserved. 


\section{DEDICATION}

I dedicate this dissertation to my wonderful family, particularly to my husband. There is no doubt in my mind that without his love, patience, understating, and support, the completion of this work would not have been possible. 


\section{ACKNOWLEDGMENTS}

My thanks and appreciation to my advisor, Dr. Damodaran, for all his guidance, encouragement, and support throughout the doctorate program. Also, I would like to thank my committee members for their comments and suggestions, and all of those in the Industrial and Systems Department who gave me your support and assistance during my graduate studies at FIU. 


\section{ABSTRACT OF THE DISSERTATION \\ ANALYTICAL APPROXIMATIONS TO PREDICT PERFORMANCE MEASURES \\ OF MANUFACTURING SYSTEMS WITH JOB FAILURES \\ AND PARALLEL PROCESSING \\ by}

Maria de Lourdes Hulett

Florida International University, 2009

Miami, Florida

\section{Professor Purushothaman Damodaran, Major Professor}

Parallel processing is prevalent in many manufacturing and service systems. Many manufactured products are built and assembled from several components fabricated in parallel lines. An example of this manufacturing system configuration is observed at a manufacturing facility equipped to assemble and test web servers. Characteristics of a typical web server assembly line are: multiple products, job circulation, and paralleling processing. The primary objective of this research was to develop analytical approximations to predict performance measures of manufacturing systems with job failures and parallel processing. The analytical formulations extend previous queueing models used in assembly manufacturing systems in that they can handle serial and different configurations of paralleling processing with multiple product classes, and job circulation due to random part failures. In addition, appropriate correction terms via regression analysis were added to the approximations in order to minimize the gap in the error between the analytical approximation and the simulation models. Markovian and general type manufacturing systems, with multiple product classes, job circulation due to failures, and fork and join systems to model parallel processing were studied. In the Markovian and general case, the approximations without correction terms performed quite well for one and two product problem instances. However, it was observed that the flow time error increased as the number of products 
and net traffic intensity increased. Therefore, correction terms for single and fork-join stations were developed via regression analysis to deal with more than two products. The numerical comparisons showed that the approximations perform remarkably well when the corrections factors were used in the approximations. In general, the average flow time error was reduced from $38.19 \%$ to $5.59 \%$ in the Markovian case, and from $26.39 \%$ to $7.23 \%$ in the general case. All the equations stated in the analytical formulations were implemented as a set of Matlab scripts. By using this set, operations managers of web server assembly lines, manufacturing or other service systems with similar characteristics can estimate different system performance measures, and make judicious decisions - especially setting delivery due dates, capacity planning, and bottleneck mitigation, among others. 


\section{TABLE OF CONTENTS}

CHAPTER

PAGE

1 INTRODUCTION 1

1.1 Background and Motivation . . . . . . . . . . . . . . . . . . 1

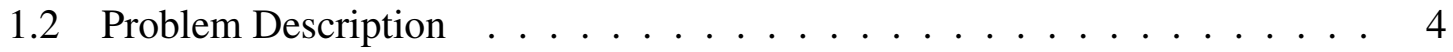

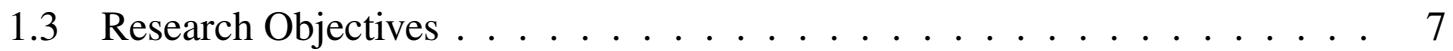

1.4 Overview of Solution Approach $\ldots \ldots \ldots$. . . . . . . . . . . . 7

1.5 Contribution and Significance . . . . . . . . . . . . . . . . . . 9

1.6 Dissertation Outline . . . . . . . . . . . . . . . . . . . . . . . . 10

$\begin{array}{lll}2 & \text { LITERATURE REVIEW } & 11\end{array}$

2.1 Performance Analysis . . . . . . . . . . . . . . . . . . . 11

2.2 Manufacturing Systems with Multiple Classes of Jobs . . . . . . . . . . . . 16

2.3 Manufacturing System with Job Circulation . . . . . . . . . . . . . . . . . . . . . . . . . . . .

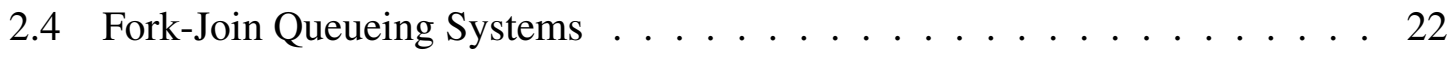

3 FORMULATION OF THE ANALYTICAL MODEL FOR THE $M / M / c$ SYSTEM 36

3.1 The Internal Flow Parameters $\ldots \ldots \ldots$. . . . . . . . . . . 38

3.2 The Performance Measures . . . . . . . . . . . . . . . . . . . . . . . 39

3.3 Pseudocode . . . . . . . . . . . . . . . . . . . . . 44

3.4 Simulation Models $\ldots \ldots \ldots$. . . . . . . . . . . . . . . 46

3.4 .1 Problem analysis and information collection . . . . . . . . . . . 46

3.4 .2 Model construction, and verification . . . . . . . . . . . . . . . 47

3.4.3 Designing and conducting simulation experiments . . . . . . . . . . 48

3.5 Computational Results . . . . . . . . . . . . . . . . . . . 49

3.6 Correction Factors $\ldots \ldots \ldots$. . . . . . . . . . . . . . . . 61

3.6 .1 The case of single stations . . . . . . . . . . . . . . . 61

3.6 .2 The case of fork-join stations . . . . . . . . . . . . . . . . . . . . . . . . . 64

3.7 Numerical Comparisons . . . . . . . . . . . . . . . . . 67

4 FORMULATION OF THE ANALYTICAL MODEL FOR THE $G / G / c$ SYSTEM 75

4.1 The Internal Flow Parameters . . . . . . . . . . . . . . 76

4.2 The Performance Measures . . . . . . . . . . . . . . . . . . . . 78

$4.2 .1 \quad$ The single stations $\ldots \ldots \ldots$. . . . . . . . . . . . . . 79

4.2 .2 The fork-Join stations . . . . . . . . . . . . . . 81

4.3 Pseudocode . . . . . . . . . . . . . . . . . . . 86

4.4 Computational Results . . . . . . . . . . . . . . . . . . . . . 88

4.5 Correction Factors $\ldots \ldots \ldots$. . . . . . . . . . . . . . . . . . . . . . . . . 104

4.5 .1 The single stations in the general case . . . . . . . . . . . . . 104

4.5.2 The case of fork-join stations for the general case . . . . . . . . . . 105

4.6 Numerical comparisons . . . . . . . . . . . . . . . . . 107 
5 CONCLUSIONS AND FUTURE WORK 123

5.1 Conclusions . . . . . . . . . . . . . . . . . . . . . 123

5.2 Future work . . . . . . . . . . . . . . . . . . . . 127

\begin{tabular}{ll}
\hline LIST OF REFERENCES & 129
\end{tabular}

\begin{tabular}{ll}
\hline APPENDICES & 138
\end{tabular}

\begin{tabular}{ll}
\hline VITA & 241
\end{tabular} 


\section{LIST OF TABLES}

TABLE

PAGE

3.1 Problem instances configuration . . . . . . . . . . . . . 51

3.2 Fork-join node configuration . . . . . . . . . . . . . . . 53

3.3 Paired-t test results per product class . . . . . . . . . . . . . 56

$3.4 \quad$ Paired-t test for problem instances where the number of subtasks was less than the number of servers in the fork-join nodes . . . . . . . . . . . . . . 61

3.5 Paired-t test results per product class when the correction factors were used in the approximations $\ldots \ldots \ldots \ldots \ldots \ldots$

3.6 Comparisons of average flow time error by product class $\ldots . . . . . . .73$

$4.1 \quad$ Problem instance configuration for single product class . . . . . . . . . . . . 89

4.2 Problem instance configuration for two product classes . . . . . . . . . . . 90

4.3 Problem instance configuration for three product classes . . . . . . . . . . . . 91

4.4 Problem instance configuration for four product classes . . . . . . . . . . . . 91

4.5 Problem instance configuration for five product classes . . . . . . . . . . . . 92

4.6 Problem instance configuration for six product classes $\ldots . . . . . . .93$

4.7 Paired-t test results per product class . . . . . . . . . . . . . . . . . 95

4.8 Paired-t test results for one product class $\ldots \ldots \ldots$. . . . . . . . . 95

4.9 Paired-t test results for two product classes . . . . . . . . . . . . . . . 95

4.10 Paired-t test results for three product classes $\ldots \ldots$. . . . . . . . . . 96

4.11 Paired-t test results for four product classes . . . . . . . . . . . . . 96

4.12 Paired-t test results for five product classes . . . . . . . . . . . . . . . . 96

4.13 Paired-t test results for six product classes . . . . . . . . . . . . . . 97

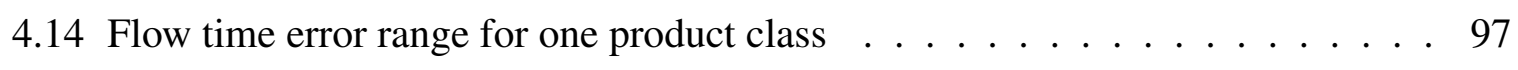


4.15 Flow time error range for two product classes . . . . . . . . . . . . . . . . 97

4.16 Flow time error range for three product classes $\ldots \ldots$. . . . . . . . . . . 99

4.17 Flow time error range for four product classes . . . . . . . . . . . . . 100

4.18 Flow time error range for five product classes $\ldots \ldots$. . . . . . . . . 101

4.19 Flow time error range for six product classes . . . . . . . . . . . . . . 103

4.20 Paired-t test results per product class using the correction factors . . . . . . . 111

4.21 Flow time error range for three product classes by case . . . . . . . . . . 113

4.22 Average flow time error for three product classes by case . . . . . . . . . . 113

4.23 Paired-t test results for three product classes using correction factors by case . . 115

4.24 Flow time error range for four product classes by case . . . . . . . . . . . 116

4.25 Average flow time error for four product classes by case . . . . . . . . . . . 116

4.26 Paired-t test results for four product classes using correction factors by case . . 117

4.27 Flow time error range for five product classes by case . . . . . . . . . . . . 117

4.28 Average flow time error for five product classes by case . . . . . . . . . . . . 118

4.29 Paired-t test results for five product classes using correction factors by case . . 118

4.30 Flow time error range for six product classes by case . . . . . . . . . . . 120

4.31 Average flow time error for six product classes by case . . . . . . . . . . 120

4.32 Paired-t test results for six product classes using correction factors by case . . . 122 


\section{LIST OF FIGURES}

FIGURE

PAGE

1.1 Flow chart of the server assembly process. . . . . . . . . . . . . . . 2

1.2 Fork-join queuing model. . . . . . . . . . . . . . . . . 4

1.3 Problem representation. . . . . . . . . . . . . . . . . . 6

$2.1 \quad$ Centralized splitting model. . . . . . . . . . . . . . . . 23

2.2 Distributed splitting model with synchronization queue. . . . . . . . . . . . . . 24

2.3 Centralized splitting model without synchronization queue. . . . . . . . . . . . 24

$3.1 \quad$ Simulation Model Example $\ldots \ldots \ldots$. . . . . . . . . . . 48

3.2 Overall flow time error distribution. . . . . . . . . . . . . 56

3.3 Simulation and analytical flow time trends. . . . . . . . . . . . 56

3.4 Flow time distribution for one product. . . . . . . . . . . . . . . . . 57

3.5 Flow time distribution for two products. . . . . . . . . . . . . . . 57

3.6 Flow time distribution for three products. . . . . . . . . . . . . 58

3.7 Flow time distribution for four products. . . . . . . . . . . . . 58

3.8 Flow time distribution for five products. . . . . . . . . . . . . . . . 59

3.9 Flow time distribution for six products. . . . . . . . . . . . . . . . . . 59

3.10 Flow time error by product. . . . . . . . . . . . . . . . . . 60

\begin{tabular}{ll}
3.11 Flow time error for problems with less subtasks than number of servers at the \\
\hline \hline fork-join nodes. $\ldots \ldots \ldots \ldots \ldots \ldots \ldots \ldots \ldots \ldots \ldots \ldots \ldots \ldots \ldots \ldots$
\end{tabular}

3.12 Service and waiting time average error $\ldots \ldots \ldots$. . . . . . . . . 63

3.13 Flow time error in the waiting time . . . . . . . . . . . . . 63

3.14 Regression equation parameters in terms of the number of products. . . . . . . 64 
3.15 Predicted and observed flow time error. . . . . . . . . . . . . . . . . 64

3.16 Flow time error of fork-join nodes against net traffic intensity. . . . . . . . . 66

3.17 Flow time error from fork-join nodes against failure rate for one product instances. ......................... 66

3.18 Flow time error from fork-join nodes against failure rate for two product in-

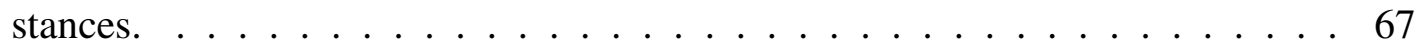

3.19 Flow time error from fork-join nodes against failure rate for three product in-

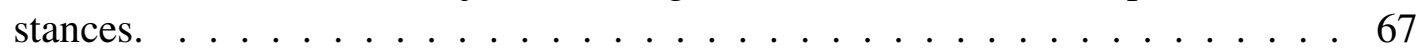

3.20 Flow time error from fork-join nodes against failure rate for four product instances. .......................... 68

3.21 Flow time error from fork-join nodes against failure rate for five product instances. .......................... 68

3.22 Flow time error from fork-join nodes against failure rate for six product instances. 69

3.23 Trend of predicted values for fork and join stations. . . . . . . . . . . . . . 69

3.24 Flow time error using the correction factors. . . . . . . . . . . . . . . . 70

3.25 Flow time error obtained using correction factors. . . . . . . . . . . . . . 70

3.26 Flow time error using correction factors for three product instances. . . . . . . 71

3.27 Flow time error using correction factors for four product instances. . . . . . . . 71

3.28 Flow time error using correction factors for five product instances. . . . . . . . 72

3.29 Flow time error using correction factors for six product instances. . . . . . . . 72

3.30 Flow time error for three product instances using the correction factors. . . . . 73

3.31 Flow time error for four product instances using the correction factors. . . . . . 73

3.32 Flow time error for five product instances using the correction factors. . . . . . 74

3.33 Flow time error for six product instances using the correction factor. . . . . . . 74

3.34 Flow time error using correction factors for problem instances where the number of subtasks is less than servers. . . . . . . . . . . . . . . . . . 74 
4.1 Overall flow time distribution for the general type system. . . . . . . . . . . . . 94

4.2 Analytical and simulated flow times for problem instances with general distri-

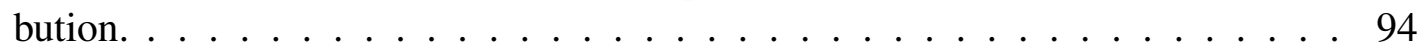

4.3 Flow time error distribution for one product class with general type distribution. 97

4.4 Flow time error distribution for one product class by type of distribution. . . . . 97

4.5 Flow time error distribution for two product classes with general type distribution. 98

$4.6 \quad$ Flow time error distribution for two product classes by type of distribution. . . 98

4.7 Flow time error distribution for three product classes with general type distri-

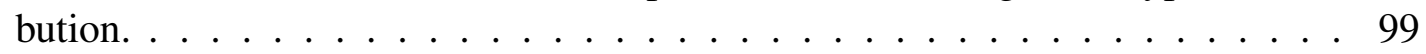

$4.8 \quad$ Flow time error distribution for three product classes by type of distribution. . . 99

4.9 Flow time error distribution for four product classes with general type distri-

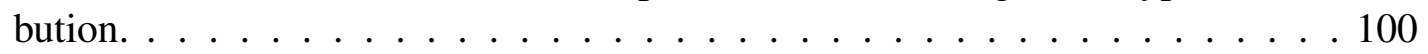

4.10 Flow time error distribution for four product classes by type of distribution. . . 100

4.11 Flow time error distribution for five product classes with general type distribution. 101

4.12 Flow time error distribution for five product classes by type of distribution. . . . 102

4.13 Flow time error distribution for six product classes with general type distribution. 102

4.14 Flow time error distribution for six product classes by type of distribution. . . . 103

4.15 Average flow time error by type of distribution. . . . . . . . . . . . 103

4.16 Flow time error vs. net traffic intensity of single stations for the general case. 105

4.17 Distribution of the flow time error when the correction factors are used in the

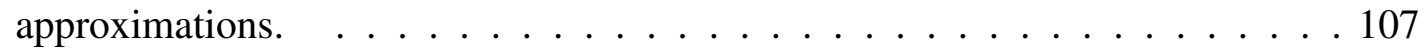

4.18 Overall trend of the flow time error using the correction factors in the approxi-

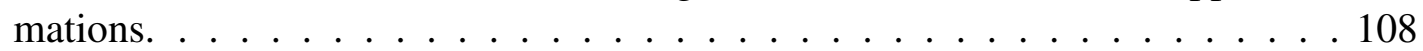

4.19 Flow time error for three product instances using correction factors in the ap-

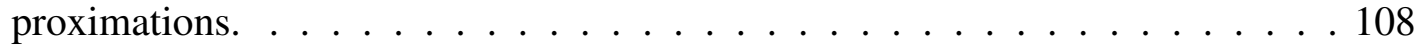

4.20 Flow time error for four product instances using correction factors in the ap-

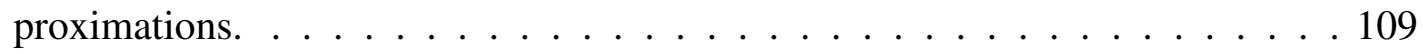


4.21 Flow time error for five product instances using correction factors in the ap-

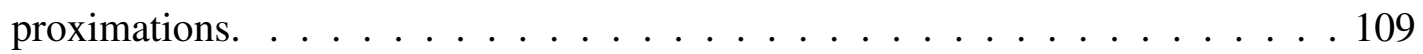

4.22 Flow time error for six product instances using correction factors in the ap-

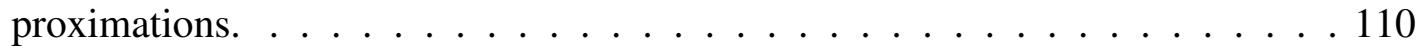

4.23 Flow time error distribution for three product classes with general type distribution using the correction factors. . . . . . . . . . . . . . . 110

4.24 Flow time error distribution for four product classes with general type distribution using the correction factors. . . . . . . . . . . . . . 111

4.25 Flow time error distribution for five product classes with general type distribution using the correction factors. . . . . . . . . . . . . . 111

4.26 Flow time error distribution for six product classes with general type distribution using the correction factors. . . . . . . . . . . . . . . . . . 112

4.27 Flow time error for three product instances using correction factors in the $E_{2}-$

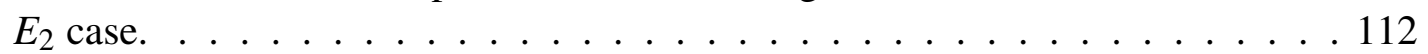

4.28 Flow time error for three product instances using correction factors in the $E_{2}-$ Exp case . . . . . . . . . . . . . . . . . . . 113

4.29 Flow time error for three product instances using correction factors in the $E x p-H_{2}$ case. . . . . . . . . . . . . . . . 113

4.30 Flow time error for three product instances using correction factors in the

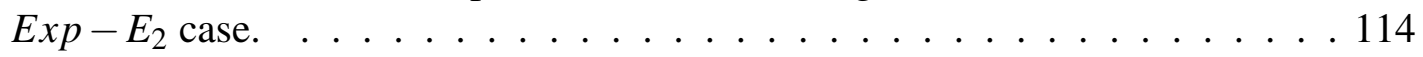

4.31 Flow time error for three product instances using correction factors in the $\mathrm{H}_{2}-$

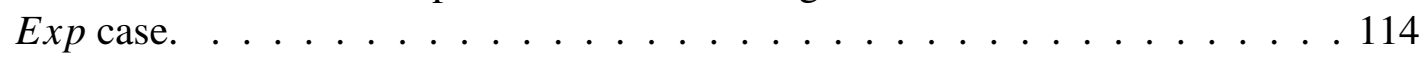

4.32 Flow time error for four product instances using correction factors in the $E_{2}-$

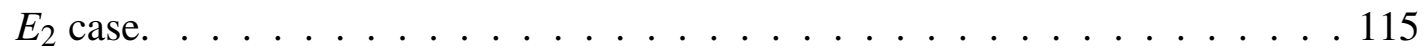

4.33 Flow time error for four product instances using correction factors in the $E_{2}-$

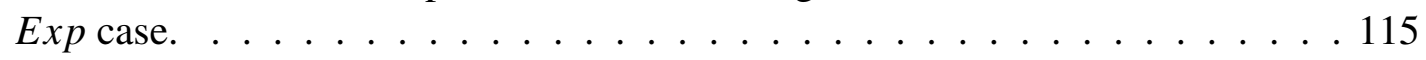

4.34 Flow time error for four product instances using correction factors in the Exp-

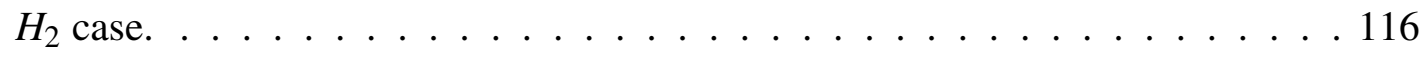

4.35 Flow time error for four product instances using correction factors in the Exp-

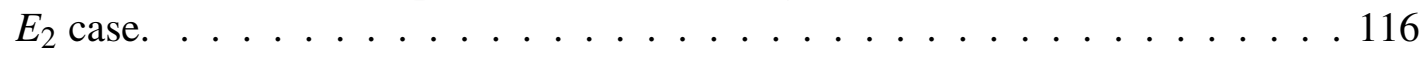


4.36 Flow time error for four product instances using correction factors in the $\mathrm{H}_{2}-$

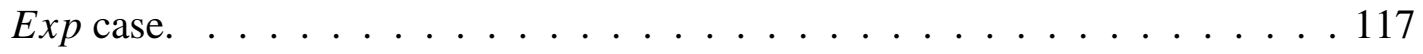

4.37 Flow time error for five product instances using correction factors in the $E_{2}-$

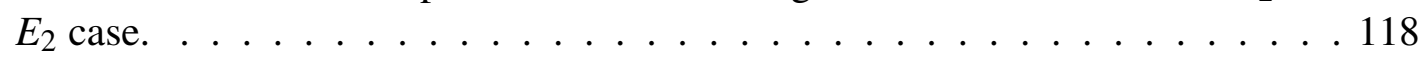

4.38 Flow time error for five product instances using correction factors in the $E_{2}-$

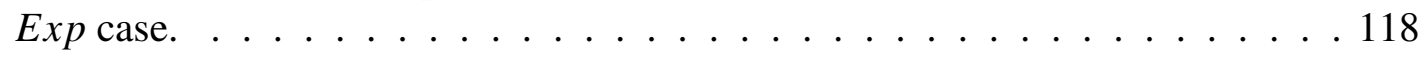

4.39 Flow time error for five product instances using correction factors in the Exp-

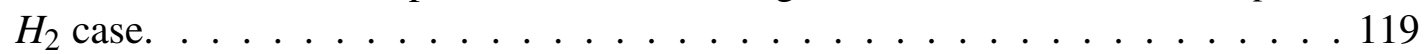

4.40 Flow time error for five product instances using correction factors in the Exp-

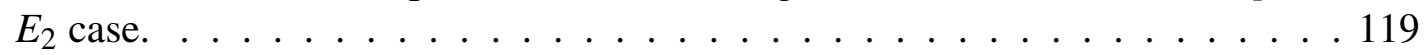

4.41 Flow time error for five product instances using correction factors in the $\mathrm{H}_{2}-$

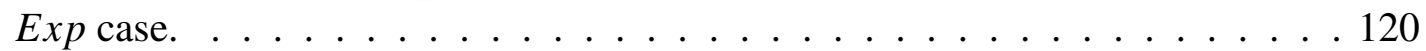

4.42 Flow time error for six product instances using correction factors in the $E_{2}-E_{2}$

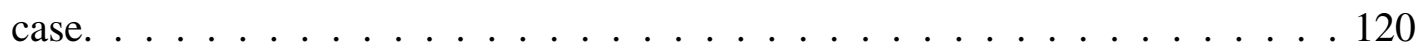

4.43 Flow time error for six product instances using correction factors in the $E_{2}-$ Exp case . . . . . . . . . . . . . . . . . . . 121

4.44 Flow time error for six product instances using correction factors in the Exp-

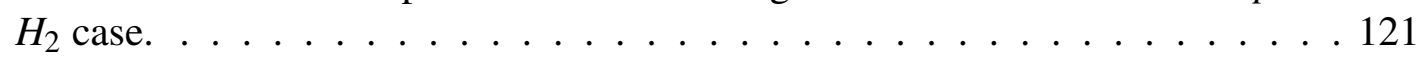

4.45 Flow time error for six product instances using correction factors in the Exp-

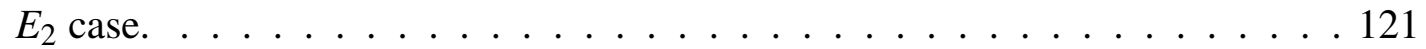

4.46 Flow time error for six product instances using correction factors in the $\mathrm{H}_{2}-$

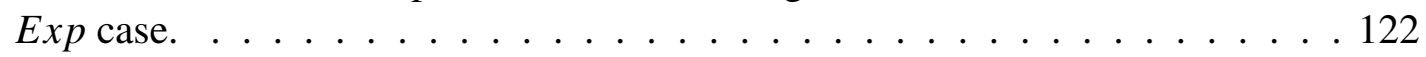

5.1 Comparisons of the average flow time error per class in the Markovian case . . 126 5.2 Comparisons of the average flow time error per class in the general case . . . . 127 


\section{CHAPTER 1}

\section{INTRODUCTION}

\subsection{Background and Motivation}

Web servers are highly customizable. Consequently, web server assembly facilities typically adopt a make-to-order operation mode. In a make-to-order environment, the assembly or production is triggered only after a firm customer order is placed. The following are the characteristics of a typical web server assembly line:

1. It is common to assemble multiple classes (or types) of products;

2. Several products may share the same resources;

3. The process route of each product class may be unique;

4. Job circulation may occur - when a server fails during testing it has to be disassembled, the failed component is replaced, and the assembly is retested;

5. Portions of the assembly can be carried out in parallel.

Figure 1.1 shows the flow diagram of a typical web server assembly process. Each order may consist of one or more servers and depending on the customer need, each server can have one or more racks. Based on the configuration of the server, some portions of the assembly process can be handled in parallel and some of them in series. The hardware components/parts that need to be assembled for each rack are grouped together to form a kit, which later are assembled to a prefabricated subassembly in the build area. The racks are then stored in a temporary storage area waiting for the remaining racks associated with 
the order to be ready. As soon as all the racks are built, they are merged together in the merge area. For customer orders with one rack, the merging process is bypassed.

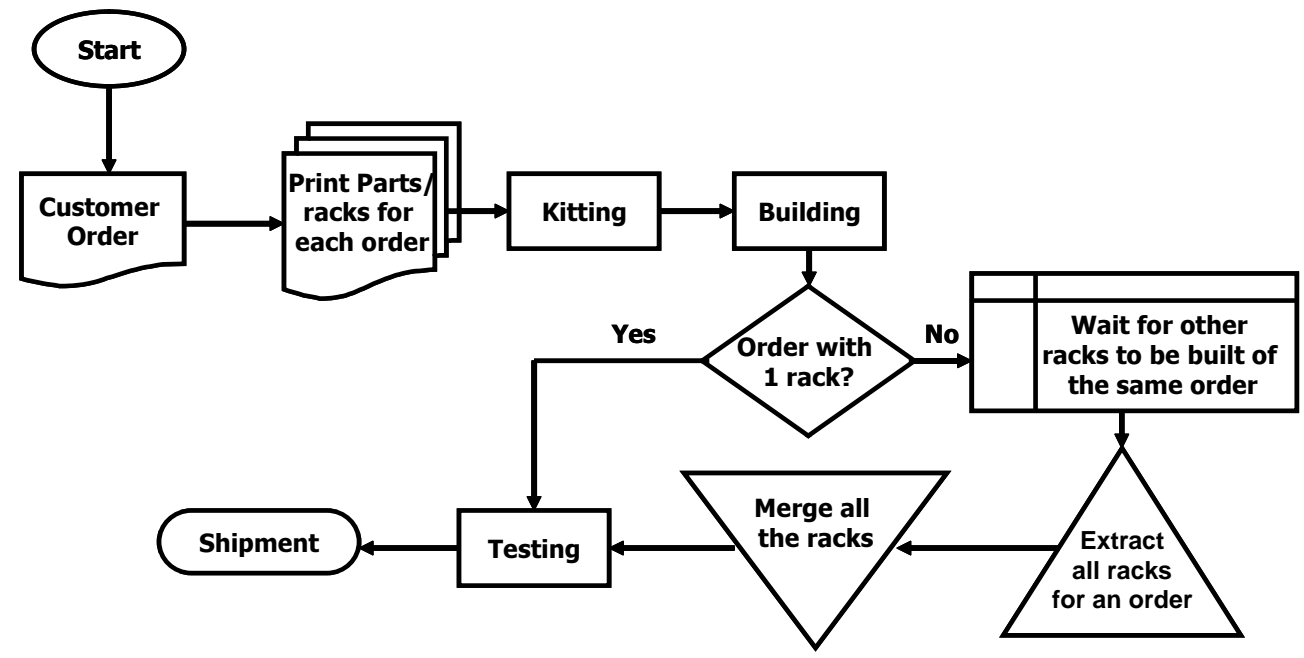

Figure 1.1: Flow chart of the server assembly process.

The merged assembly is later tested to detect early failures. When a failure is observed during the testing phase, the server has to be disassembled, reassembled (failed components are replaced), and retested. The process is repeated until the server passes all the tests. Consequently, job/server circulation is typical under this environment. When severs fail repeatedly at some stage (s), the retesting and rework can increase the time required to fulfill an order (i.e., flow time) and stress some assembly and test resources. This could lead to missed delivery dates and their respective penalties, loss of customer good will, and so on. Consequently, procedures to estimate performance measures of the server assembly system (i.e., flow time, resource utilization, etc.) accurately can help the operations managers to make more informed decisions, especially with respect to shipment dates and allocation of resources.

Parallel processing is prevalent in many manufacturing and service systems (e.g., diagnostic and treatment activities in an ambulatory medical care, warehouse order picking process, 
mask lithography deposition of multiple circuits simultaneously on a single semiconductor wafer, molecular components simultaneously processed, paralleling processing of multiple wafer, testing multiple products or subcomponents simultaneously, among others). Many manufactured products are built and assembled from several components fabricated in parallel lines. In assembly operations, components have to wait for other components before the assembly operation can begin. Hence, synchronization constraints arise between some workstations in the manufacturing process. Although assembly manufacturing systems have been studied using analytical models, these models do not incorporate all the important aspects of the system which are of practical interest. Many of the analytical models study a subsystem of an assembly line or make simplifying assumptions. Moreover, this type of assembly process considerably differs from semiconductors and electronics manufacturing (re-entrant lines), which has been widely studied in the past. In semiconductors and electronics manufacturing, the processes are represented deterministically, and jobs could return more than once to some of the workstations for repeated stages of processing [13]. Under this scenario, the number of job visits to each workstation is fixed by the original process. In contrast, the web assembly process deals with stochastic flow of jobs between different workstations due to job failures.

This research is motivated by the fact that the web server assembly facilities do not have appropriate analytical tools to estimate their performance measures. Consequently, the overall aim of this research is to develop analytical models to estimate performance measures of manufacturing systems with the characteristics of the web server assembly line such as: serial and parallel processing, multiple product classes sharing some resources, and job circulation due to random failures. 


\subsection{Problem Description}

In the server assembly manufacturing process, some portions of the process can be handled in parallel and some of them in series. The kitting, merging, and testing process steps are done sequentially, whereas the rack assembly is done in parallel. Multiple racks of a server can be assembled in tandem. Once all the racks are assembled, they are merged together before the entire server is subjected to a series of tests. The parallel processing with synchronization constraints can be modeled by using fork and join stations [103]. In the fork and join subsystems, each job is divided at the fork point into $l$ identical subtasks that are submitted to a unique server/resource/station within the fork and join subsystem. The parallel queues are independent and identically distributed (iid), and the number of subtasks can be less than or equal to the number of servers in the subsystem. On completing service, each subtask waits at the join point for its sibling subtask to complete service without preventing the server from working on another subtask. A job is ready for merge operation if all of its subtasks have been assembled. Figure 1.2 presents an example for the fork-join queuing model.

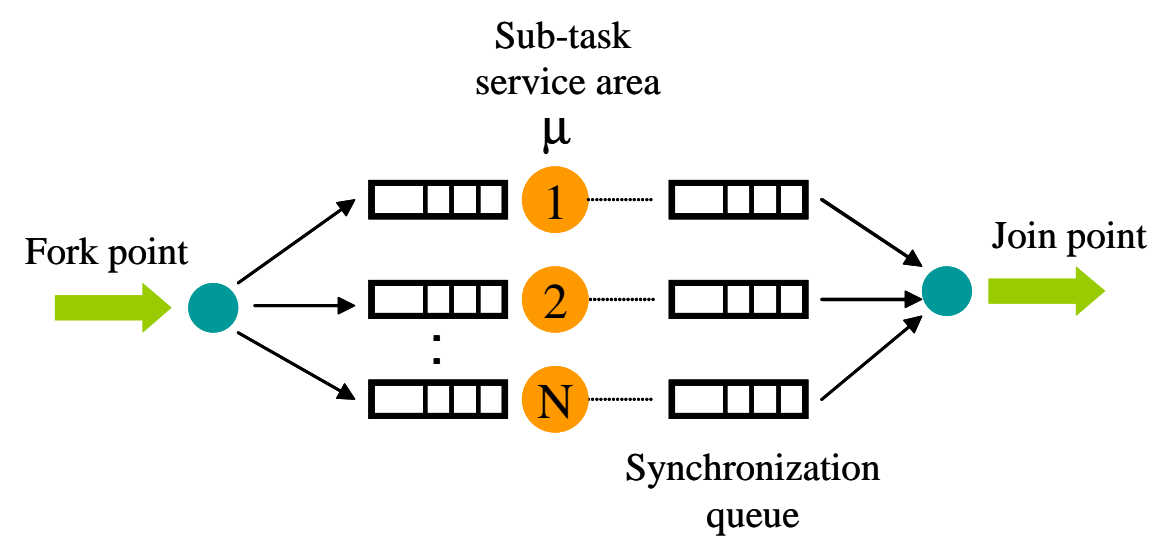

Figure 1.2: Fork-join queuing model. 
Systems such as the web server assembly process are complex to analyze mathematically [106, 66, 76] and exact analytical solutions for fork and join system have been provided only for two- server fork and join queues [77, 30, 29]. As a result, the performance measures of fork and join queues with more than two servers have been computed using approximations and bounding techniques [103, 66, 77, 61].

Different manufacturing systems have been studied analytically using the queuing network approach. For simple systems, exact solution is derived. For example, single server systems can be analytically modeled easily. However, for complex systems only approximate solutions are practical. Performance analysis of manufacturing systems with multiple product classes, and job circulation due to random part failures has not been widely studied in the context of parallel processing. Many works done with the characteristics of parallel processing has been in parallelism in computer and storage systems [105, 103, 104, 106]. Additionally, majority of the analysis of fork-join studies assume that the inputs to the individual buffers are Poisson process [77, 105, 103, 104]. Although [68, 86] consider random job failures, they do not consider a fork-join subsystem.

In this research, we are interested in predicting performance measures such as the flow time, work in progress, and waiting time for manufacturing systems with parallel processing. Specifically, we consider an open system where the products are allowed to leave the system and the external arrivals arise from an infinite population of products. It is assumed that a collection of products types, which are routed through a finite collection of workstations, where some portions of the process can be handled in parallel and some of them in series. The jobs circulate probabilistically in the system depending on the nature of the failure, thus, the job may visit one or more workstations multiple times. Job splitting or preemption is not allowed, and machine breakdowns are not considered. Arrival and service rates are assumed from a Markovian and from general distribution. Jobs are processed according 
to a first-in-first-out (FIFO) discipline at each station, and all jobs/products must leave the system.

Figure 1.3 presents a queueing network to illustrate the characteristics of the problem under study. In this example, two different products is considered. Each product has its respective arrival, service, and failure rates, and is routed through a finite collection of workstations. Additionally, one portion of the process is handled in parallel, which is modeled as a forkjoin system. On the other hand, the fork-join subsystem has three homogeneous stations, where product 1 is split into two subtasks, and product 2 into three subtasks. Product 1 must visit workstation one, the parallel system (fork-join node) with two subtasks, and workstations five, six, and eight in that order (shown by dashed lines in the figure). On the other hand, product 2 must visit workstations one, the parallel system (fork-join node) with three subtasks, and workstations five, seven and eight. Although in web server assembly lines server failures are typically noticed after the testing stage, we consider failures at any stage in our study so that our discussions are applicable to other manufacturing systems where failures can occur at any stage in the assembly.

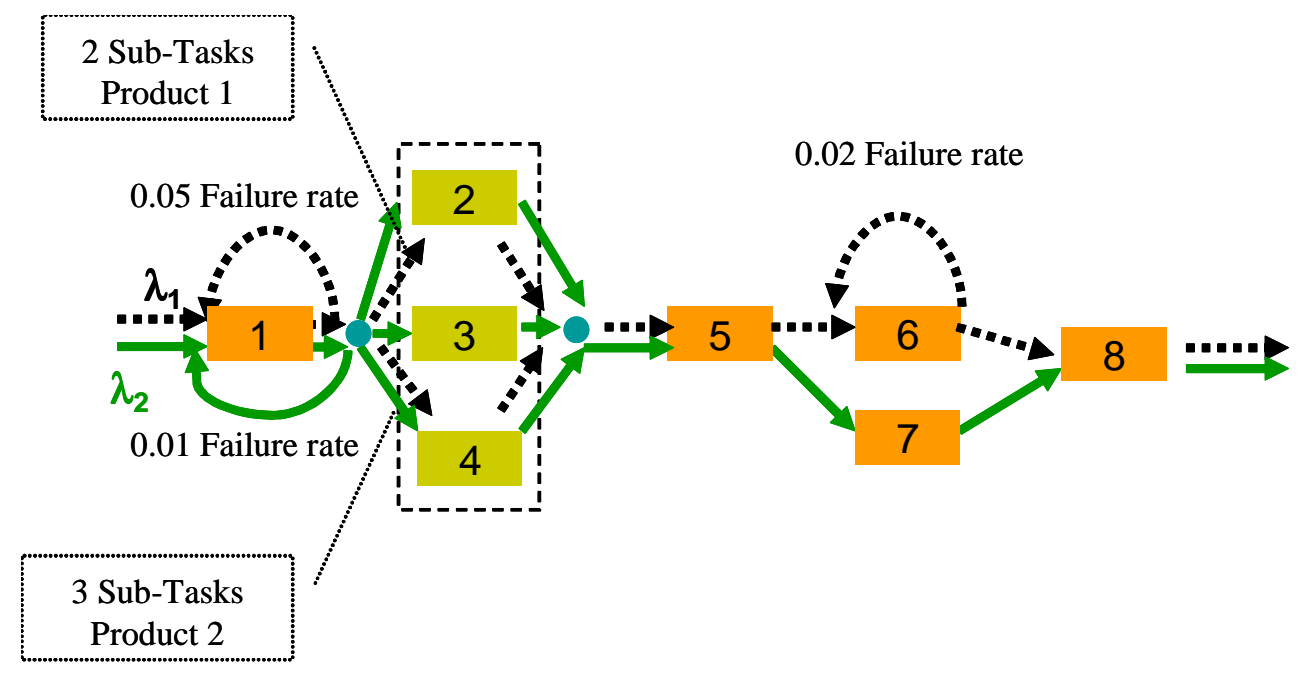

Figure 1.3: Problem representation. 


\subsection{Research Objectives}

The primary objective of this research was to develop analytical approximations to predict performance measures of manufacturing systems with random job failures and parallel processing. Specifically in this research, analytical approximations to estimate the performance measures for Markovian and general type of multiclass manufacturing systems with random job circulation, and in which is included fork and join system to model parallel processing were developed. To accomplish this goal, the following specific objectives were considered: (1) design of the analytical approximations considering Markovian type of manufacturing systems, (2) design of the analytical approximations considering Gen-

eral type of manufacturing systems, (3) develop discrete event simulation models to verify the accuracy of the results of these analytical approximations, and (4) develop appropriate correction terms via regression analysis to bridge the gap between the analytical approximations and the simulation models where the error is large $(>20 \%)$.

\subsection{Overview of Solution Approach}

Queueing theory enables mathematical analysis of several related processes in a manufacturing system. A typical manufacturing system consist of a set of resources that process a variety of products. The arrival pattern of products are dependent on the product demand, which is stochastic; and service rates of the resources show a natural variation in their performance [32]. Jobs arriving at different workstations have to wait in queues if the resources are not available. Jobs that are successfully processed leave the workstation and join the queue for service at the following workstation. Jobs that fail are routed to the same workstation or to one of the previous workstations. These characteristics of manufacturing systems make them suitable for modeling as a network of queues consisting of a set of nodes (workstations), and arcs (movement of the job between workstations) [86]. 
Exact analytical solutions have been developed for some classes of queueing networks; however, an alternative to deal with more complex systems, such as the problem under study, has been to have an approximate analytical solution. Therefore, queueing network models can be classified according to whether they yield exact results or whether they rely on approximations. The approximation models can be classified into decomposition models and diffusion models. According to Sunkyo [97] most standard decomposition methods reduce complex behavior of the queueing networks into three network operations on a point process: queueing, splitting and superposition. According to Govil and Fu [32] these approaches make two basic assumptions: 1) the stations in the queueing network can be treated as being stochastically independent, and 2) the input to each queue is a renewal process characterized by the mean and the variance of the arrival time distributions. By contrast, the diffusion approximations are based on the heavy traffic intensity theorems. These approximations are valid when the traffic intensities at the stations are close to one [32].

In this research, we use the parametric decomposition approach in order to decompose the queueing network into queueing systems with a single station. We choose this approach because according to Sunkyo [97] decomposition approximation of queueing network, based on the two-moment renewal approximation of the departure process, performs well in approximating some performance measures at stations with moderate traffic intensity.

Under this approach, individual queues are analyzed separately after approximately characterizing the aggregate arrival process. Later, the whole system performance is computed by aggregating the individual queue performances. By using this approach, we can analyze independently single and fork-join stations within the same framework.

Markovian systems (i.e., interarrival and service times are from an exponential distribution), and general type systems (i.e., interarrival and service times are from Erlang-2, hy- 
perexponential, and/or exponential distribution) are studied. Random problem instances are generated and the analytical approximations are used to estimate the performance measures. Discrete event simulation models are developed to simulate the problem instances. The results from the analytical approximations and simulation are compared to evaluate the accuracy of the analytical approximations, and correction terms via regression analysis are developed to minimize the gap between the analytical approximations and the simulation models where the error was greater than $20 \%$.

The analytical approximations are implemented in MatLab, the simulation models are developed in Arena 11.0, and the corrections terms are obtained by using Microsoft Excel and Statistical Analysis Software (SAS).

\subsection{Contribution and Significance}

In this research, new analytical formulations are proposed to estimate the performance of complex manufacturing systems where several issues of practical interest such as parallel processing with synchronization queue, multiple product classes, and job circulation due to random part failures are simultaneously considered. Although many researchers have proposed analytical formulations for manufacturing systems with some similar characteristics, most of these previous efforts have not dealt with all the issues that in this study are considered. Especially, prior work on paralleling processing with the same characteristics of the problem under study, has not been taken into account multiple product classes and failure rates at the fork point. Also, the majority of the analysis of fork-join systems assume that the inputs to the individual buffers are Poisson process. In this research, existing analytical formulations are modified to incorporate the fact that a node can be visited by different product classes, and appropriate correction terms via regression analysis are added to the 
approximations in order to minimize the gap in the error between the analytical approximations and the simulation models.

The analytical approximations can help operations managers of web server assembly lines, and manufacturing or other service systems with similar characteristics to estimate system performances. By estimating the system performances accurately, they may be able to make judicious decisions - especially setting delivery due dates, capacity planning, and bottleneck mitigation, among others.

\subsection{Dissertation Outline}

The dissertation is organized as follows: Chapter 1 presents the motivation for this research, the problem description, research objectives, and the solution approach to follow. Chapter 2 provides the literature review from four perspectives. The first section reviews previous efforts which utilized queueing theory as the principal tool for performance estimation in manufacturing systems. The second and third sections address previous research on manufacturing systems with multiple classes of jobs, and job circulation respectively, and the fourth section reviews previous research on fork and join systems (parallel queues) and tools for analyzing their performance. In Chapters 3 and 4 the analytical formulations for Markovian and general type of manufacturing systems are presented. The experimental study was conducted to determine the gap in the performance estimates between the analytical and simulation models. The details of the experimental study and the procedure followed to bridge the gap is also presented. Finally, the conclusions and directions for future work are summarized in Chapter 5. 


\section{CHAPTER 2}

\section{LITERATURE REVIEW}

This research is concerned with the development of analytical formulations for manufacturing systems that deals with parallel processing, multiple product classes, job circulation due to random part failures, and shared workstations. Several lines of research exists which are pertinent to this problem. We restrict our literature search to four lines of research: 1) performance analysis, 2) manufacturing systems with multiple classes of jobs, 3) manufacturing system with job circulation, and 4) fork and join queueing systems.

\subsection{Performance Analysis}

Performance evaluation involves the development of models to measure the effectiveness of a manufacturing organization in achieving its goals. There is considerable research that reports a number of techniques or tools for performance evaluation of manufacturing systems. Leung and Suri [67] provided an overview of performance evaluation of discrete

manufacturing systems. They described the major type of techniques indicating where and when the different tools are most applicable. Techniques such as physical experimentation, probability models, queueing models (as a subset of probability models), discrete event simulation, sample path based sensitivity analysis, Petri nets, algebraic models, untimed (qualitative) models, and hybrid models were discussed.

Several queueing network model implementations have been used in the performance evaluation of manufacturing systems. Exact results exist for Markovian systems with the seminal papers of Jackson [47] for job shops and Koenigsberg [56] for cyclic systems. Jackson showed that the equilibrium joint probability distribution for a wide class of Markovian 
models, was the product of the marginal distributions, where the mean arrival rate of customers depends almost arbitrarily on the number already present, and the mean service rate at each service center depends almost arbitrarily on the length. On the other hand, Koenigsberg studied a special case of a job shop where all jobs have to pass the machines over the same route like in the flow shop but some of the machines in the route can be met more than once.

Several generalizations of these results are derived by Baskett et al. [10] and Kelly [50, 51]. Baskett et al. showed that the product-form stationary distribution characteristic of a Jackson network in fact holds for arbitrary service-time distributions that have the same means. Kelly [50] addressed the behavior of equilibrium of networks of queues that deals with multiclass jobs, and in [51] the concept of reversibility and quasi-reversibility in stochastic process is explored. Additionally, Meyn and Down [73] studied open generalized Jackson networks with general arrival streams and general service time distribution. The stability of the network was deduced by bounding the expected waiting time for a customer entering the network.

Two other remarkable contributions to this line of research were made by Burke [17] and Little [70]. Burke showed that for a queueing system with a Poisson input and exponential service times, the equilibrium distribution of the number of service completions in an arbitrary time interval is a Poisson distribution for any number of servers. Little showed that if the mean time between arrivals of two consecutive units, the number of units in the systems, and the mean time spent by a unit in the systems are finite and are in steady state, then the number of customers in the system is proportional to the the waiting time of the customers in the system no matter what the arrival or service distribution is.

The desire of dealing with more complex systems, such as the problem under study, has led to the development of approximate analytical solutions. According to Bitran and Sarkar 
[14] the approximate methods can be classified into i) diffusion methods, ii) mean value analysis, iii) operational analysis, and iv) decomposition methods. Diffusion methods approximations are based on the heavy traffic limits theorems. Works by Iglehart and Whitt [44, 45], Harrison [36], Harrison and Reiman [37], Reiman [88], Harrison and Wein [38], Chen and Maldembaum [21], Harrison and Williams [34], Bramson and Dai [16] are representative papers of this line of research.

Mean value analysis is an algorithm that allows analyzing closed queueing networks and obtaining mean values for queue lengths and response times, as well as throughputs. It has been studied by Reiser and Lavenberg [90, 65], Bard [8], Schwitzer [92], and Akyildiz [1] among others.

Operational analysis provides a framework to study queueing networks during finite time periods. Under this approach flow balance assumption is used in the derivation of many operational formulas, and different operational assumptions are used to derive several formulas for the mean queue length and response time of an isolated service center. Representative work in this area has been done by Buzen [18], Denning and Buzen [28] and Bengtson [11].

Decomposition methods decompose the queueing network into queueing subsystems each with a single station. This approach is based on two assumptions: a) the nodes can be treated as being stochastically independent; and b) the arrival and departure streams are renewal processes, which are characterized by the first two moments, mean and variance [97]. According to [15] this approach follows three basic steps: 1) analysis of the interactions between nodes; this is analyzed by considering the network as a combination of three basic processes: a) arrivals at a station, b) departures from a station, and c) splitting and merging, which represents the products routing in the network; 2) decomposition of 
the network into individual nodes; and 3) aggregation of individual queue performances to obtain the performance measures of the whole system.

The pioneers in this field are Reiser and Kobayashi [89], who implicitly used a decomposition approach to analyze open and closed queueing networks with general service time distributions and FCFS discipline. Contributions were made by Chandy and Sauser [19], Kuehn [60], Shanthikumar and Buzacott [95], Whitt [110], and Bitran and Sarkar [14]. Chandy and Sauser discussed approximation methods for the analysis of computing system. In [60] an approximate method for the analysis of general queueing networks was proposed. The analysis is based on the method of decomposition, where the subsystems are analyzed individually by assuming renewal arrival and departure processes. All related processes are considered with respect to their first two moments only.

Shanthikumar and Buzacott [95] developed an approximate decomposition approach to analyze an open queueing network model of dynamic job shops with general service time distributions and first come first served or shortest processing time service discipline. Later a software package called the Queueing Network Analyzer (QNA) was proposed by Whitt [110] to calculate approximate performance measures for systems that can be modeled as general open queueing networks. The purpose of the first version of this computer-based package was to analyze packet communication network. It considered open networks of multiserver nodes with the first-come first-served (FCFS) discipline and no capacity constraints. The arrival processes need not be Poisson and service time distributions need not be exponential. The nodes are analyzed as standard GI/G/m queues partially characterized by the first two moments of the interarrival and service time distributions. Later, Segal and Whitt [93] contributed to the field by using this methodology to address applications in manufacturing. 
Bitran and Sarkar [14] considered an open network with multiple product classes with deterministic routes and FCFS discipline. They showed that the parametric decomposition approach does not perform well in certain cases with large number of jobs. Thus, they refined the decomposition approach by taking into account the distortion in the variability of a given product due to the presence of the other products. This is referred to as the interference effect. In contrast with other approaches, they expressed the variation of the interdeparture intervals for each product at each station as the sum of two terms. The first term that reflects the congestion and the service at the station, and the second one that captures the effect of the distribution of the arrivals of other products. The interference that the product suffers is incorporated by taking into account the second term.

In manufacturing flow lines, which consist of stations such that parts visit them exactly once in a fixed sequence, have also used decomposition methods for their analysis. Work in this field was originally introduced by Gershwin in [31]. He presented a method for evaluating performance measures for a class of tandem queueing systems with finite buffer. The work is based on an approximate decomposition method in which the flow line is treated as a set of two-machine lines, leading to a set of equations that are solved using an interactive algorithm. Later, Dallery et al. [25] extended the work done by Gershwin in [31]. They replaced the original set of equations by an equivalent one, obtaining a simpler algorithm with a lower computational complexity. Altiok [3], Yeralan and Tan [118] [100], Helber [42], among others, are representative papers of this field. Dallery and Gershwin [27] described the most important models and results of the manufacturing flow line literature, as well as the exact and approximate methods to obtain performance measures of the system. It includes the major classes of models (asynchronous, synchronous, and continuous), the major properties (conservation flow, flow rate-idle time, reversibility, among others), and the relationships among different models. 
During the last several years, numerous research publications have contributed to expanding this area. Whitt [115], Sunkyo [97], Baris [9], Dallery and Frein [26], and Krishnamurthy and Suri [59] among others, are representative of this work. The review by Govil and $\mathrm{Fu}[32]$ provides a survey of the literature on analytical queueing theory models applied to discrete parts manufacturing systems. They present exact and approximate models for job shop systems, flexible manufacturing systems, assembly/disassembly networks, and manufacturing flow lines.

\subsection{Manufacturing Systems with Multiple Classes of Jobs}

Typically manufacturing systems deal with different types of jobs whose service times and route are determined by the job type. This type of queueing networks have been approached in the past by different researchers. Baskett et al. [10], motivated by the desire to model computers systems, extended the work of Jackson to multiclass networks for a variety of system configurations. They derived the equilibrium distribution of states of a model containing four different types of service centers and different classes of products. They considered open, closed, and mixed networks of queues with multiple class customers; and scheduling disciplines including FCFS, processor sharing, no queueing, and last come first served. Kelly [50] also worked with more general queueing networks where all service and interarrival times are assumed to be independent and exponentially distributed, with each customer class served at a given station having a common exponential service time distribution.

Whitt [110] with the QNA provides the option of defining different customer classes. Each type of customer has its own deterministic flow in the network and external arrival process, which is characterized by the mean and variability parameters. Additionally, each class may have its own service time distribution, which may be different at the same node in different 
visits. These service distributions are also characterized by the same parameters. The performance measures are derived based on the individual node analysis after the parameters for the internal flows are determined. Later, Segal and Whitt [93] described a new version of the QNA software package, which was developed especially to analyze manufacturing lines. This new version still employs the parametric decomposition approach method, but it was modified to represent machine breakdown, batch services, lot sizes, product testing and rework.

Bitran and Tirupati [15] extended the work done by Whitt. They showed that the approximations by using parametric decomposition approach do not perform well in some cases with large number of products due to interference among products. Thus, they enriched the approach by taking into account the presence of other products and describing its effect on the variance of the departure streams. The authors computed this interference effect for a product at a given station, by aggregating all other products into a single product. Therefore, the determination of interference was reduced to the analysis of two product classes. An open queueing network with multiple product classes, deterministic routing and general arrival and service distributions as a two-class network was analyzed in this paper. Later, Whitt [114] extended the parametric-decomposition approximation method to treat queueing networks with several classes. In this paper, methods were developed for approximately characterizing the departure process of each customer class from a multiclass single-server queue with unlimited waiting space and the FIFO service discipline. The approximations extended the ones developed by Bitran and Tirupati by considering the effect of class-dependent service times and developing a more general formula that can be used for non Poisson class-2 arrivals.

Bhat [12] presented a new method to approximate the squared coefficient of variation of the interarrival time of a merged process, which takes into account the variations among customer classes. Additionally, he presented several methods to approximate the expected 
waiting time of each customer class in a multiclass first come first served (FCFS) single server queue, and three approaches to derive the first two moments of the busy periods in a GI/G/l queue.

Whitt [113] showed that feedback together with significant differences in class-dependent service times can lead to dramatic fluctuations in the queue-length processes at the stations in the network. These fluctuations rule out conventional heavy-traffic limit theorems. He showed how to obtain proper heavy-traffic limits for a deterministic four-class two-queue multiclass open network of single-server FIFO queues with traffic intensity of one at each queue. Sunkyo [98] presented the heavy-traffic bottleneck phenomenon under multiclass deterministic routing and discuss how it can be addressed by decomposition. They showed that decomposition methods for deterministic routing by Bitran and Tirupati [15] and Whitt [114] can be combined with the Whitt's variability functions [115] to consider the heavytraffic bottleneck phenomenon under deterministic routing.

Harten and Sleptchenko [39] derived a method for the exact analysis of multiclass, multiserver queues, based on a classical method using the stationary state equations. They provided some new approximations based on special multiplicative eigenmodes, including the dominant mode in the heavy traffic limit. In the field of flexible queueing systems, Gurumurthi and Benjaafar [33] provided a framework for the modeling and analysis of systems with multiple classes of arrivals and heterogeneous servers where customers have the flexibility of being routed to more than one server and servers possess the capability of processing more than one customer class. They extended the work in this area by including a broader class of system configurations and control policies and a less restrictive set of assumptions. 


\subsection{Manufacturing System with Job Circulation}

Job circulation can be found in different manufacturing environments. Flow of jobs between different stations where parts may return more than once to the same machine for repeated stages of processing, and where the number of visits would be fixed by the original sequence is referred to as re-entrant lines [62]. On the other hand, manufacturing systems with stochastic flow of jobs between different stations due to job failures [86] is also observed. Under this scenario, the jobs entering the system have different repeated cycle requirements, contrasting with the deterministic re-entrant lines where jobs would have fixed cycle needs [80].

Examples of re-entrant lines include semiconductor wafer fabrication facilities [23] [83], circuit card assembly, thin film lines, and systems with rework tasks [74] [91]. In [62] Kumar explains re-entrant lines in detail. He provided some bounds on attainable mean delay in a stochastic framework, under the assumption that the service and inter-arrival times are exponentially distributed. He examined the issue of stability of re-entrant lines, under a deterministic, bursty model of arrivals, and the problem of minimizing the variance of delay. Moreover, he provided a stochastic control approach to the problem of production control under random machines failures, and examined scheduling issues if set-up times are incurred whenever processing is switched from one buffer to another.

In another research work, Lu et al. [72] addressed the problem of reducing the mean and variance of cycle time in semiconductor manufacturing plants. They proposed a new approach of smoothing the fluctuations in all the flows of the network. They developed a new class of scheduling policies called Fluctuation Smoothing policies, which achieved the best mean and standard deviation of cycle time, in all the system configurations and release policies tested. 
Narahari and Khan [75] presented an approximate technique based on Mean Value Analysis (MVA) for analytical performance prediction of re-entrant lines with priority scheduling among the buffers at a work center and different processing times on different visits. Park et al. [83] extended the Narahari and Khan's methodology by including the workstations of batch machines. They proposed an approximate analytical method based on MVA for estimating the average performance of re-entrant flow shops with single-job (or discrete) machines and batch machines. It assumed a closed queueing network and a single type of job flowing through a fixed routing. The authors obtained the mean waiting time at each buffer of the workstation and developed a heuristic method to improve the result of the analytical method. Later, in [84] they extended their work by taking into account multiclass jobs. However, in these works the flow of jobs through the system have deterministic routes.

Connor et al. [23] developed an open queueing network model, with FCFS scheduling policy at all nodes, for rapid performance analysis of semiconductor manufacturing facilities. In this paper, they modeled five different types of tool groups found in semiconductor wafer fabrication, as well as events that disrupted the normal process at the tools. In addition, they conducted a detailed analysis of the scrap and rework processes to capture the effect of variable job sizes on the workload and utilization of the tool group. Thus, the manufacturing system has a stochastic flow of jobs between different stations where the cycle requirements depend on the job failures such as the problem under study. Narahari and Khan [74] extended the work on reentrant lines done by Connor et al. [23] as a closed queueing network. They considered probabilistic routing and proposed a model for reentrant manufacturing systems with inspections. They developed an analytical technique based on MVA to compute the mean steady state cycle time and throughput rate of such models under various scheduling policies. The method can determine the minimum number of inspection stations to ensure a given quality level, the optimal location to maximize 
throughput, and the lowest possible speeds of the inspection stations that will not interfere with the system performance.

More recently, Kim et al. [53] extended the model proposed by Park et al. [84] by considering re-entrant manufacturing system with production loss due to machine failures and yields. A methodology for analyzing the system approximately is proposed using the iterative procedures based on the MVA and some heuristic adjustments.

Kumar and Omar [64] presented a modified analytical model based on approximate MVA with probabilistic re-entrant line to predict the total mean waiting time and the throughput rate for the environmental stress testing operation in the semiconductor assembly industry. They analyzed a five-stage queuing system with re-entrant to the second stage under various stochastic routing. In this paper, a correction factor in order to deal with a large number of lots was introduced. Later, Kumar and Omar [63] considered the same environmental stress testing operation, and presented a modified analytical model based on MVA technique and virtual clustering method. This method allows measuring the cycle times in terms of lots clusters. Omar and Kumar [80] extended their work in [63] improving the formulation in order to avoid the correction factor that is case dependent, simplifying the computation process. The objective of this work was to develop a method to determine the total cycle time and mean throughput in the same manufacturing system for a given lot loaded into the system. In addition, they determined the maximum number of lots that can be loaded into the system and the target cycle time.

Pradhan et al. [86] presented analytical approximations to estimate the performance measures of an optoelectronic manufacturing system with multiple product classes, job circulations due to failures, and some resources being shared among different product classes. In this paper they analyzed a system with exponential interarrival and service times $(M / M / c)$ based on the modified version of the QNA developed by Whitt [110] and modified it to 
incorporate the stochastic flow of jobs between different nodes in the network. Later, in [85] Pradhan and Damodaran analyzed the same problem for $G / G / c$ type of systems. This work has some similarities with the problem under study; however, they did not consider parallel processing in the manufacturing system.

In another research work, Omar et al. [81] extended their work in [64] including a routing probability factor in the algorithm that allows dealing with the stochastic nature of the re-entrant lines in the manufacturing industry. In addition, they added a yield probability factor to the algorithm in order to take into account the defects of lots that have to be removed from the system.

\subsection{Fork-Join Queueing Systems}

Fork-join queueing systems take place in the performance analysis of parallel processing in queueing models of manufacturing, service and computer systems. In some queueing models of assembly systems, the assembly station is also modeled using a fork-join station. In such systems each job is decomposed, at the fork point, into $l$ identical subtasks that are submitted to a unique parallel station within the fork and join system. On completing service, each subtask waits at the join point for its sibling subtask. A job is completed if all of its subtasks have completed service. The time elapsed between the fork and join operation of a job is called the response time, and the time that a subtask waits for the other siblings is the synchronization delay.

Previous research in this area reports different types of fork-join queueing systems, such as the centralized splitting model, the distributed splitting model with synchronization queue, and the centralized splitting model without synchronization queue. In the centralized splitting model [78, 76], the subtasks wait in a centralized queue at the fork point, whenever a 
server becomes free, one of the waiting subtasks (if any) is scheduled on to this server. The subtasks synchronize at the join point (see Figure 2.1). In the distributed splitting model with synchronization queue [78, 76], the subtasks wait in an independent server queue. A subtask finishing service waits in its synchronization queue until all the other subtasks also finish service at their respective servers (see Figure 2.2). In the centralized splitting model without synchronization queue [76], a subtask finishing service at the server is forced to wait there until all the other subtasks also finish their service, preventing the server from working on another subtask (see Figure 2.3). Another variation is the case where the server, just before a service initiation, verifies whether or not its downstream buffers are free. If at least one of these buffers is full, the service initiation is postponed until all downstream buffers are free [82].

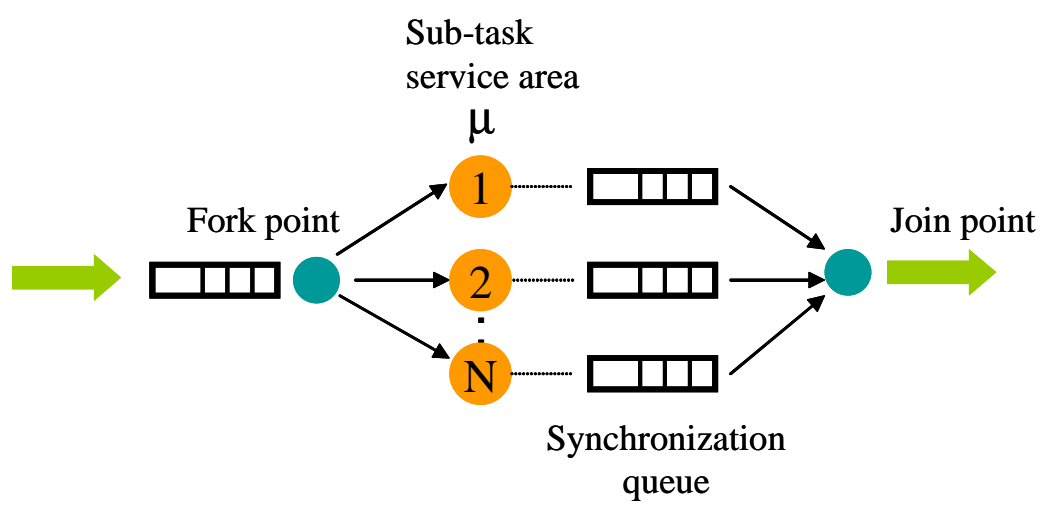

Figure 2.1: Centralized splitting model.

Fork and join systems have been studied extensively during the past several years, and in many cases the focus of the study was parallelism in computer and storage systems. The 


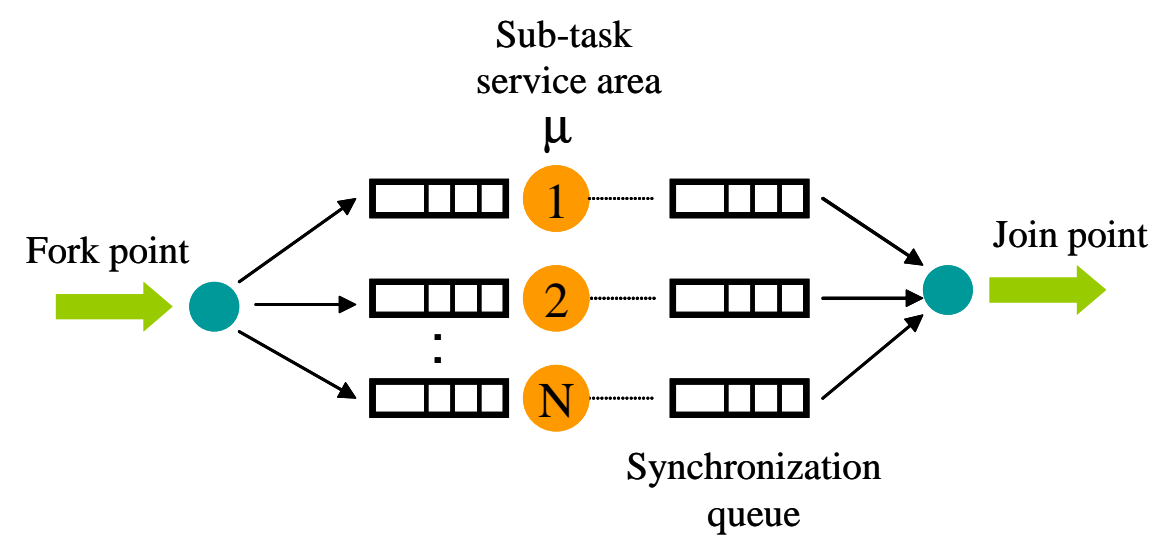

Figure 2.2: Distributed splitting model with synchronization queue.

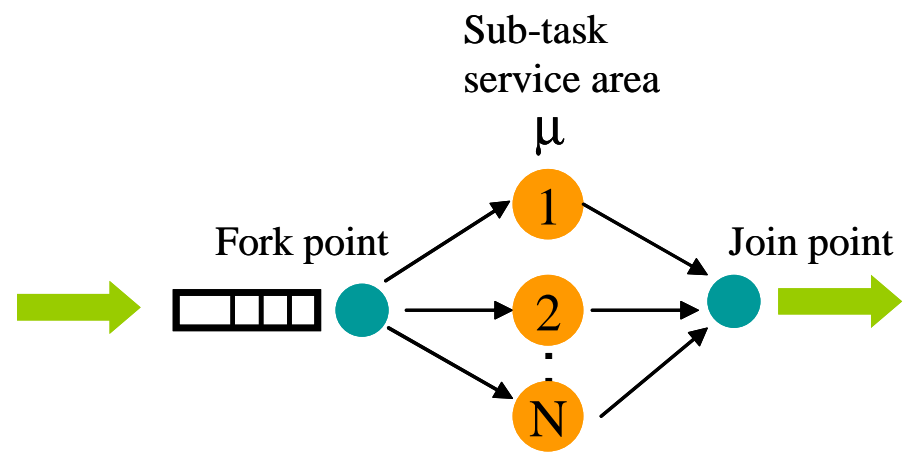

Figure 2.3: Centralized splitting model without synchronization queue.

analysis of such systems is analytically intractable due to the parallelism of the tasks [77], and the synchronization, demanded by the forks and joins, that destroys all nice properties like product form or insensitivity [4]. Thus, exact analysis of the fork and join queue is presented only for two server fork-join queues, requiring approximation and bounding techniques to compute performance measures of fork-join queues for more servers [106].

Early efforts in this field have been done by Heidelberger and Trivedi [40, 41]. In [40], computer performance models of certain types of parallel processing systems is considered, in which a job is subdivided into two or more tasks at some point during its execution. In this case the synchronization among tasks is not required. They developed an approximate method which iterates through a sequence of product-form networks. In addition, bounds on performance improvement due to overlap are derived. Later, in [41] they extended their 
work by requiring synchronization among the tasks, but analyzed closed systems. Two approximate solution methods to predict performance measures in such systems were developed. The first approximation is based on a decomposition approach, and the second approximation interactively solves a sequence of product-form networks. In this approximation the synchronization delay is modeled by an infinite server queue.

Flatto and Hahn [30] obtained asymptotic formulas for the steady state of an open queueing network with two heterogeneous servers, each having its own queue. They assumed that the arrival rate follows a Poisson distribution with mean 1 and the servers have exponential services times. Later, Flatto [29] continued his previous work using the asymptotic results to study the interdependence between the length of the server queues. He derived limit laws for the expectation and distribution for either of the queue lengths conditioned on the other.

Nelson et al. [78] obtained an expression for the mean response time in centralized parallel processing systems with job splitting into independent tasks. They modeled the system as a continuous time, discrete Markov process. In this work the response time $T$ of a random job was expressed as the sum of two terms:

$$
E[T]=E[W]+E[S]
$$

where $W$ is the job waiting time and corresponds to the time that the job waits in the queue before the first of its tasks is scheduled. This waiting time was analyzed by studying the $M^{X} / M / K$ bulk arrival queueing system that represents the system under study, and calculated by solving a set of recurrence relations. On the other hand, $S$ is the job service time and corresponds to the time required to process all the tasks associated to the job once the first task is scheduled. The value of the mean job service time for a random job was given by the following equation: 


$$
E[S]=\frac{H_{K}}{\mu}
$$

where $H_{K}$ is the harmonic series given by

$$
H_{K}=\sum_{i=1}^{K} \frac{1}{i} .
$$

Nelson and Tantawi [77] considered a distributed parallel processing system, where the jobs arrive with rate $\lambda$, Poisson distribution, and service times are independent and exponentially distributed. They developed a new approximation technique called the scaling approximation, which is applied to the analysis of two different computer systems models consisting of homogeneous fork-join queuing systems with two or more servers. The nature of the approximation arises from the observation that there exist upper and lower bounds which grow at the same rate as a function of the number of servers $(K)$ in the fork-join system. From this observation they concluded that the mean time $\left(T_{K}\right)$ for $K \geq 2$ is given by

$$
T_{K} \simeq S_{K}(\rho) T_{2}
$$

where $S_{K}(\rho)=\alpha(\rho)+\frac{1-\alpha(\rho)}{H_{2}} H_{K}$ is a scaling factor, and $T_{2}=\left(\frac{12-\rho}{8}\right)\left(\frac{1}{\mu-\lambda}\right)$ is the mean job response time for two homogeneous servers. Using simulation results, they found a good approximation for $\alpha(\rho)$, leading to the following approximate expression for $T_{K}$

$$
T_{K}=\left[\frac{H_{K}}{H_{2}}+\frac{4}{11}\left(1-\frac{H_{K}}{H_{2}}\right) \rho\right] T_{2} .
$$

Baccelli et al. [4], studied the class acyclic fork-join system that takes place in applications like parallel processing and flexible manufacturing. They derived the evolution equations that rule the behavior of such systems, and developed upper and lower bounds on the network response times. Kim and Agrawala [52] obtained the transient and steady-state solutions of the fork-join queue in terms of virtual waiting times, which can be used to calculate 
the response time. The approximate solutions in the steady state are presented for the cases where the interarrival time distributions are exponential and two-stage Erlangian.

For a fork-join network with $K$ servers, Poisson arrival and general independent services times, Thomasian and Tantawi [101] developed approximate techniques to calculate its mean response time. This was expressed as the sum of the mean response time at one of the queues and the mean synchronization delay. The mean response time for a fork-join system with a server utilization $\rho$, is given by

$$
R_{K}^{F / J}(\rho)=R_{1}(\rho)+F_{K} \alpha(\rho) \sigma_{1}(\rho)
$$

where $F_{k}$ is obtained from the previous equation at $\rho=0$ (since $\left.\alpha_{K}(0) \triangleq 1\right), R_{1}(\rho)$ and $\sigma_{1}(\rho)$ are the mean and the standard deviation of response time at any one of the queues respectively, and $\alpha_{K}(\rho)$ is a low-degree service-time distribution dependent polynomial in $\rho$, whose coefficients are determined from simulation results.

Varma and Makowski [107] proposed a family of heuristics approximations for the expected response time of $K$-dimensional symmetric Fork-Join systems in statistical equilibrium with general interarrival and service time distributions. They first developed approximations for the case $K=2$, and for arbitrary $K \geq 2$, with Poisson arrivals and exponential service times. By observing agreement between the light traffic derivative and the heavy traffic limit for this system under Markovian assumptions, they were able to developed an approximation to the heavy traffic limit for $K$-dimensional systems with general interarrival and service distributions. They generated interpolation approximations by combining heavy traffic limit with light traffic limits. They approached the non-Markovian cases for a $K$-dimensional Fork-Join queue with Erlang-2 interarrival and service time distributions, Erlang-2 arrivals and exponential services, Poisson arrivals and hyper-exponential services, Poisson arrivals and Erlang-2 services, and hyper-exponential arrivals and exponential ser- 
vices. This research is distinct from that effort in that, it considers multiclass products, and failures rates at the fork-join nodes, and the parallel processing modeled by fork-join queues is only one part of the server assembly process.

Nguyen [79] presented a heavy traffic analysis of feedforward fork-join networks with heterogeneous customers. They showed that the heavy traffic limit of certain processes associated with heterogeneous fork-join networks can be expressed as a semimartingale reflected Brownian motion with polyhedral state space. Kumar and Shorey [61] obtained upper and lower bounds to the mean job response time in a model of parallel processing system where each job, in a multicomputer system, forks into a random number of subtasks that can be processed independently on any of the computers. Upper bounds are obtained using the concept of associated random variables, and the lower bounds are obtained by neglecting and including queueing delays.

Balsamo and Mura [6] proposed a new bounded approximate method, based on Markov chain analysis, to evaluate response time distribution function in a fork-join system with $K \geq 2$ heterogeneous service centers, Poisson job arrival, and exponential service time distribution. They defined two approximate models, based on the matrix-geometric technique, which provide an upper and lower bound on the response time distribution. In [7] they extended their work to general service time distributions to obtain a bounded approximation of the moments of any order for the number of jobs in the fork-join system. They presented an algorithm to derive approximate solutions of the open fork-join queueing networks with Poisson arrivals and Coaxial service time distribution, which provide lower and upper bounds on the queue length moments. Later, Balsamo et al. in [5] extended their previous work by providing a method that allows controlling the spread of bounds to meet a given accuracy. 
Varki [105] presented an exact analysis of mean response time of two identical parallel queueing systems, consisting of a single server and an infinite capacity queue. In this paper, a formal parallel wait time analysis, similar to the wait time analysis of non-parallel systems is presented. The analysis is carried out using event/time sequence (ET-S) trees. On the other hand, Narahari and Sundarrajan [76] studied the performability with respect to average response time of three fork-join queueing models: the centralized splitting model, the distributed splitting model with synchronization queue, and the distributed splitting model without synchronization queue. They considered fork-join queueing systems with $K$ identical servers prone to fail, and followed the randomization approach to compute the cumulative distribution of performability. Paik [82] presented throughput upper and lower bounding methods for another fork-join network configuration. Under this fork-join model the join server may initiate its service only when each of its upstream buffers is occupied, and a server initiates the service until all downstream buffers are free, that is, blocking before service. He developed an approximation method for the throughput, which are based on decomposition/aggregation principles.

Chen [22] introduced a dynamic bubblesort technique that combines analysis and simulation to obtain the mean response time $\left(T_{K}\right)$ in a homogeneous fork-join queueing system of $K \geq 2$, where jobs arrive with rate $\lambda$ and a general arrival distribution, the service follows a general distribution, and the buffers have infinite capacity. This technique sorts the branches of the queues based on the number of the tasks waiting for synchronization in each branch. The response time $T_{K}$ is given by

$$
T_{K}=T_{1}+\frac{1}{\lambda} \sum_{J=2}^{K} \frac{\left.\overline{x_{J, 2}(t)}\right|_{E_{J(t)} \varepsilon A_{J}}}{J}, \quad K \geq 2
$$

where $\left.\overline{x_{J, 2}(t)}\right|_{E_{J(t)} \varepsilon A_{J}}$ is the average number of tasks waiting, in the synchronization queue at branch number two, for their siblings tasks to complete. In addition, he presented an upper bound expression for the K-queue fork-join system, which is given by 


$$
T_{k} \leq T_{1}+2\left(T_{2}-T_{1}\right)\left(H_{K}-1\right)
$$

Varki et al. [106] presented simple pessimistic and optimistic mean response time bounds and a mean response time approximation $R_{N}^{K}$ for a homogeneous fork-join queue, and exponential service times. In contrast with other works, this paper analyzed the $N$-server fork-join queues in which the jobs divide into $K$ identical subtasks, where $1 \leq K \leq N$, such as the problem object of this study. However, this research is distinct from that effort in that, it considers multiclass products, and failures rates; and the parallel processing is only one part of the whole network of queues that model the manufacturing system under study.

The authors presented the response time bounds $R_{N}^{K}$ of a $N$-server fork-join queue, where each arriving job divides into $1 \leq K \leq N$

$$
\frac{1}{\mu}\left(H_{N}+\rho * \operatorname{Sum}_{K(K-\rho)}\right) \leq R_{N}^{K} \leq \frac{H_{N}}{\mu}\left(1+\frac{\rho}{1-\rho}\right),
$$

where $\rho=\lambda * k / N * \mu$ is the utilization of a server in the fork-join queue, $H_{N}$ is the harmonic number, and $\operatorname{Sum}_{K(K-\rho)}=\frac{1}{1-\rho}+\frac{1}{2} \frac{1}{2-\rho}+\frac{1}{3} \frac{1}{3-\rho}+\ldots+\frac{1}{K} \frac{1}{K-\rho}$ is the $K^{\text {th }}$ partial sum of the sequence $\left(\frac{1}{1-\rho}+\frac{1}{2} \frac{1}{2-\rho}+\ldots+\frac{1}{K} \frac{1}{K-\rho}, \ldots\right)$. Then, the authors used those bounds to compute the response approximation

$$
R_{N}^{K}=\frac{1}{\mu}\left(H_{N}+\frac{\rho}{2(1-\rho)}\left(\operatorname{Sum}_{K-\rho}+(1-2 \rho) * \operatorname{Sum}_{K(K-\rho)}\right)\right),
$$

where $\operatorname{Sum}_{K-\rho}=\frac{1}{1-\rho}+\frac{1}{2-\rho}+\frac{1}{3-\rho}+\ldots+\frac{1}{K-\rho}$ is the $K^{\text {th }}$ partial sum of the sequence $\left(\frac{1}{1-\rho}+\frac{1}{2-\rho}+\ldots+\frac{1}{K-\rho}, \ldots\right)$.

Ko and Serfoso [55] presented a closed-form formula for approximating the distribution of the response time for a $M / M / s$ fork-join in equilibrium. The response time formula is based on a linear combination of bounds, where certain constants are obtained by auxiliary network properties and simulation results. This is given by 


$$
F_{W}(t) \approx 1-\sum_{i=1}^{m} c_{i}\left[1-F_{W^{J}}(t)\right] \prod_{j=1}^{i-1} F_{W^{J}}(t)
$$

where

$$
\begin{gathered}
F_{W^{i}}(t)=\frac{1}{s_{i}\left(1-\rho_{i}\right)}\left(\left[s_{i}\left(1-\rho_{i}\right)-\left(1-q_{i}\right)\right]\left[1-e^{-\mu_{i} t}\right]-q_{i}\left[1-e^{-\left(s_{i} \mu_{i}-\lambda\right) t}\right]\right) \\
c_{m+1}=0, c_{1}=1, c_{2}=1-q_{i} / 4, \text { and } \\
c_{i}=\left(1-\frac{q_{i-2}}{8}\right)\left(1-\frac{q_{i-1}}{4}\right), \quad 3 \leq i \leq m
\end{gathered}
$$

where

$$
q_{i}=P\left\{Q^{i} \geq s_{i}\right\}=\frac{P\left\{Q^{i}=0\right\}\left(s_{i} \rho_{i}\right)^{s_{i}}}{s_{i} !\left(1-\rho_{i}\right)}
$$

and

$$
P\left\{Q^{i}=0\right\}=\left[\sum_{n=0}^{s_{i}-1} \frac{\left(s_{i} \rho_{i}\right)}{n !}+\frac{\left(s_{i} \rho_{i}\right)^{s_{i}}}{s_{i} !\left(1-\rho_{i}\right)}\right]^{-1}
$$

For the case of single-server nodes, the authors presented the following approximation for the distribution of the response time

$$
F_{W}(t) \approx 1-e^{-\gamma_{1} t}-\sum_{i=2}^{m} c_{i} e^{-\gamma_{i} t} \prod_{j=1}^{i-1}\left(1-e^{-\gamma_{J} t}\right) .
$$

For the two-node network, this approximation is

$$
F_{w}(t) \approx 1-e^{-\gamma_{1} t}-\left(1-\frac{\rho_{1}}{4}\right)\left[e^{-\gamma_{2} t}-e^{-\left(\gamma_{1}+\gamma_{2}\right) t}\right]
$$

and its mean is

$$
E W \approx \frac{1}{\gamma_{1}}+\left(1-\frac{\rho_{1}}{4}\right)\left(\frac{1}{\gamma_{2}}-\frac{1}{\gamma_{1}+\gamma_{2}}\right)
$$


They also presented a formula for approximating the distribution of the number of jobs for the $M / M / 1$ fork-join network. This was obtained by relating the number of jobs in the network (queue length) to the response time and applying the Little's law for distributions. Their approximation proposes:

$$
F_{Q}(n) \approx 1-\rho_{1}^{n+1}-\sum_{i=2}^{m} c_{i} g_{i}(n)
$$

where $c_{i}$ is calculated by equation $2.13, \gamma_{1}=\mu_{1}-\lambda$, and

$$
g_{i}=\sum_{k_{1} \in\{0,1\}} \ldots \quad \sum_{k_{i-1} \in\{0.1\}}(-1)^{\sum_{j=1}^{t-1} k_{J}}\left(\frac{\lambda}{\mu_{i}+\sum_{j=1}^{i-1} \gamma_{J} k_{J}}\right)^{n+1} .
$$

For the two-network,

$$
F_{Q}(n) \approx 1-\rho_{1}^{n+1}-\left(1-\frac{\rho_{1}}{4}\right)\left[\rho_{2}^{n+1}-\left(\frac{\lambda}{\gamma_{1}+\gamma_{2}+\lambda}\right)^{n+1}\right]
$$

and its mean

$$
E Q \approx \lambda\left[\frac{1}{\gamma_{1}}+\left(1-\frac{\rho_{1}}{4}\right)\left(\frac{1}{\gamma_{2}}-\frac{1}{\gamma_{1}+\gamma_{2}}\right)\right]
$$

Later, Ko [54] presented formulas for approximating the distribution of the cycle time of a job in a two-stage fork-join network in equilibrium with two node server, in which jobs arrive according to a Poisson process and the server times are exponentially distributed. Basically, when the tasks for a job are completed, they are joined completing the fist stage; then, they enter to the second stage that is the same as the first stage. A job will exit the system when all tasks are completed in the last-stage. Additionally, they developed approximations for $m$-stage networks. Their approximation proposes that the mean cycle time in a $m$-stage fork-join network is calculated by

$$
E\left[W_{1}+\ldots+W_{m}\right] \approx \sum_{j=1}^{m} \frac{\left(12-\lambda / \mu_{j}\right)}{8\left(\mu_{j}-\lambda\right)}
$$


More complex fork-join systems, consisting of different types of execution stages and multiple interactions of this collection of stages, were analyzed by Squillante et al. [96]. They presented an exact matrix-analytic analysis of generalized parallel-server fork-join queueing systems, for small instances of the stochastic model, and presented an approximate matrix-analytic analysis and fixed-point solution, for larger instances of the model.

Response time for fork-join queueing networks in closed systems have also been obtained. Liu and Perros [71] presented an iterative approximation algorithm for analyzing a closed system with a $K$-sibling fork-join queue. This iterative procedure is based on a combination of nearly complete decomposability and the Gauss-Seidel method, and converge to the exact solution in the case of the closed 3-sibling fork-join queue. Varki [102] presented a technique for computing mean performance measures of closed single-class fork-join networks with exponential service time distribution. The technique is based on the mean value equation for fork-join networks which relates the response time of a network to the mean service times at the service centers and the mean queue length of the system with one customer less. This equation is an approximation and the technique computes lower performance bound values for the fork-join network. Later, Varki [103] presented another response time approximation in closed system with applications in parallel computer and storage systems. This approximation was extended to cover cases when a job splits into an arbitrary number of tasks upon arrival at the parallel system.

In the literature, fork-join synchronization stations have also been used to analyze kitting systems where, in contrast with the problem under study, input streams differ in terms of rates or predefined buffer limits. Furthermore, in this type of kitting systems most of the manufacturing setting imposes specific limits on component inventories, such as kanban control mechanisms, resulting in closed systems. Harrison [35] presented a queueing theoretic model of an assembly operation, which consist of some $K$ different input processes and a single server that assembles these input items into finished products. He provided im- 
portant conditions for the stability of synchronization stations. Lipper and Sengupta [69] obtained upper and lower bounds for the mean sojourn time of an assembly-like queue, and derived asymptotic solutions from the bounds. Sengupta [94] modeled the store of assembly shop by means of an assembly queue, and showed how to obtain the transient and steady state distribution of queue length and time spent in the system for this queue. On the other hand, Takahashi et al. [99] and Krishnamurthy [58] considered synchronization queues with two input streams different from Poisson distribution. More recently, Ramakrishnan and Krishnamurthy [87] developed closed form approximations for key performance measures of kitting systems with multiple input streams.

Fork-join stations also have been used to model paralleling processing in health care systems. Jiang and Giachetti [49] developed an open queueing network model using the twomoment parametric decomposition approximation method to predict the patient flow time in an urgent care center. They used fork-join queues without synchronization to represent the parallelization of patient flow. In this work, the service time encountered by the patient entering the fork-join subsystem was considered as the maximum of the service times that patient spends in the sibling queues.

The preceding discussion reveals that most of the research done on the fork-join, under the characteristic of the problem object of this study, has addressed parallel processing in parallel computer and storage systems environments, and rarely has been considered with multiple products. Varki et al. [106] and Varma and Makowski [107] analyzed parallel processing with similar characteristics; however, multiclass products and failures rates were not considered in those works. Although Pradhan and Damodaran [86, 85] addressed optoelectronic manufacturing system with multiple product classes and job circulations due to failure, this dissertation differs in that, the problem object of this study considers parallel processing. Furthermore, from the literature review it can be concluded that performance analysis of manufacturing systems considering simultaneously multiple product classes, 
job circulation due to random part failures have not been widely studied in the context of parallel processing. 


\section{CHAPTER 3}

\section{FORMULATION OF THE ANALYTICAL MODEL FOR THE $M / M / c$ SYSTEM}

As stated earlier the web server assembly process arises a variety and complexity of different issues such as, parallel processing with synchronization constraints, multiple product classes, and job circulation due to random part failures, that make this type of systems difficult to analyze. Although many researchers have proposed analytical formulations for manufacturing systems with some similar characteristics, most of these previous efforts have not dealt with all the issues that in this study are considered. Especially, prior work on paralleling processing, with the same characteristics of the problem under study, has not taken into account multiple products and failure rates at the fork point. In this research existing analytical formulations related to fork-join nodes are modified to incorporate the fact that a node can be visited by different product classes, and appropriate correction terms via regression analysis are added to the approximations in order to bridge the gap in the error between the analytical approximation and the simulation models.

Exact analytical solutions have only been developed for parallel processing with two servers, and for more complex systems, such as the problem under study, approximation or bounding analysis has been used to predict performance measures. Parametric decomposition approximation method, based on the Whitt's approach [110], is used to analyze the server manufacturing process. Under this method, the queueing network is decomposed into individual queues that are analyzed separately after approximately characterizing the aggregate arrival process. By using renewal approximation, the arrivals and the departures in the network are characterized by the first two moments, namely mean and variance, and are based on three network operations: flow through a queue (departure), which is the operation that transforms an arrival process to a departure process [97]; splitting, in which a job splits 
itself along different arcs to multiple arcs; and superposition (merging), in which different jobs merge along with different arcs to a node [86]. In this approach, when calculating the performance measures for the network as a whole, the nodes are treated (approximately) as being stochastically independent. However, the method seeks to capture the dependence among nodes approximately through the calculation of internal flow parameters [110].

The formulations for the $M / M / c$ system presented in this section are based on the work done by Jackson [47], Whitt [110], the modifications done by Pradhan et al. [86] to incorporate the probabilistic flow of jobs between stations in the network system, and the work done on fork-join queues with variable substasks by Varki [106]. The notation used is as follows:

\section{Notation}

$k \quad$ product class index $k \in K$

$n_{k} \quad$ total number of nodes visited by product class $k$

$c_{j} \quad$ number of servers at node $j$

$J_{k} \quad$ set of nodes visited by product class $k$

$F_{k} \quad$ set of fork-join nodes visited by product class $k$

$q_{i j k} \quad$ transition probability from node $i$ to node $j$ by product class $k$

$\Phi_{k} \quad$ transition probability matrix

$\lambda_{0 j k} \quad$ external arrival rate at node $j$ by product class $k$

$\mu_{j k} \quad$ service rate at node $j$ for product class $k$

$\tau_{j k} \quad$ mean service time at node $j$ by product class $k$

$N F J_{j} \quad$ number of nodes at the fork-join $j$

$\mu F J_{j k} \quad$ service rate for product class $k$ at the fork-join $j$

$M F J_{j k} \quad$ number of subtasks at the fork-join $j$ by product class $k$ 
Only limited information is required to analyze the queueing network. For each product class it is necessary to specify the number of nodes visited by each product class $\left(n_{k}\right)$, number of servers at each node $\left(c_{j}\right)$, external arrival rate to each node $\left(\lambda_{0 j k}\right)$, mean service time at each node $\left(\tau_{j k}\right)$, and the transition probability matrix $\left(\Phi_{k}\right)$, which specifies the sequence of operations for each product class through the network.

\subsection{The Internal Flow Parameters}

Following the Whitt's approach [110] the first step to determine the performance measures is to calculate the internal flow parameters. The traffic rates can be computed by solving a system of linear equations. The system of linear equations are those used in Jackson Networks, which has an unique solution. The traffic rate at node $j$ by product class $k$ is the summation of the input that is received from outside (external to the system), $\lambda_{0 j k}$, and any output from the other stations routed to $j$ [47].

$$
\lambda_{j k}=\lambda_{0 j k}+\sum_{i \varepsilon J_{k}} q_{i j k} \lambda_{i k} \quad \forall j \in J, k \in K
$$

Or in matrix notation

$$
\Lambda_{k}=\Lambda_{0 k}\left(I-\Phi_{k}\right)^{-1}
$$

where $\Lambda_{0 k}$ is the external arrival rate vector, $\Phi_{k}$ is the transition probability matrix, and $I$ is the identity matrix.

The problem under study deals with probabilistic flow of jobs due to random failures; thus, a job may return more than once to the same station. Similarly, the mean number of visits $\left(v_{j k}\right)$ to each node $j$ for product class $k$ can be calculated by solving a system of linear equations. The set of equations is given by 


$$
v_{j k}=q_{0 j k}+\sum_{i \varepsilon J_{k}} q_{i j k} v_{i k} \quad \forall j \in J, k \in K
$$

After the net arrival rate into each node is computed using 3.1 , the proportion of arrivals to node $j$ that came from node $i$ for product class $k$ can be computed.

$$
p_{i j k}=\frac{\lambda_{i j k}}{\lambda_{j k}}
$$

where $\lambda_{i j k}=\lambda_{i k} q_{i j k}$.

Given the arrival rates, it is possible to determine the traffic intensity $\rho_{j}$ at each node, which is defined as the ratio of the arrival rate $\lambda_{j k}$ to the maximum service rate $c_{j} \mu_{j}$ [48]. Since the problem object of this research considers different product classes, which can have different service rates at a given node, an average of the service time need to be calculated. The aggregate service time at node $j\left(\tau_{j}\right)$ can be computed using Pradhan's equation [86], which is a modified version of its deterministic equivalent given by Whitt [110]

$$
\tau_{j}=\frac{\sum_{k \in K} \lambda_{0 j k} \tau_{j k}+\sum_{k \in K} \sum_{i \in I_{k}} \lambda_{i j k} \tau_{j k} v_{j k}}{\sum_{k \in K} \lambda_{0 j k}+\sum_{k \in K} \sum_{i \in I_{k}} \lambda_{i j k} v_{j k}} \quad \forall j \in J
$$

The equation 3.5 calculates a weighted average of the service time taking into account the external arrival rate to node $j$, and the inputs from the other stations routed to that node. In the equation, the external arrival is not multiplied by the number of visits to the node $v_{j k}$, since it equals one [86]. Later, the net traffic intensity at node $j$ is calculated using

$$
\rho_{j}=\frac{\sum_{k \in K} \lambda_{j k} \tau_{j}}{c_{j}}
$$

\subsection{The Performance Measures}

All the stations with the exception of the fork-join can be modeled as $M / M / c$ queues since

all external arrivals are Poisson processes, each queue within the network has unlimited 
capacity, exponential service times, and independent transfer probabilities. Under these conditions, the steady-state results are valid. Based on the Jackson's work [46], each station in the network can be analyzed independently using the formulas for a single queue; and for the case of the fork-join subsystems, the $N$ servers can be modeled as $M / M / 1$ queues with synchronized arrivals [106]. The performance measures for the network as a whole are calculated by aggregating the individual performance measures.

One of the first measures of interest is the expected number of jobs in the system for product class $k$, denoted by $L_{k}$. This is given by Vohra [108] as the summation of the expected number of jobs at each node $\left(L_{j k}\right)$ for product class $k$. The formula is given by

$$
L_{k}=\sum_{j \in J_{k}} L_{j k} \quad \forall k \in K
$$

The expected number of jobs at each node $\left(L_{j k}\right)$ for product class $k$ is calculated by adding the expected number of jobs in service for each product class $\left(L s_{j k}\right)$ and the number of jobs in queue for each product class $\left(L q_{j k}\right)$. This is defined by

$$
L_{j k}=L s_{j k}+L q_{j k} \quad \forall j \in J_{k}, k \in K
$$

where

$$
\begin{gathered}
L s_{j k}=\frac{\lambda_{j k}}{\mu_{j k}} \quad \forall j \in J_{k}, k \in K, \\
L q_{j k}=\left[\frac{\left(\frac{\lambda_{j k}}{\mu_{j k}}\right)^{c_{j}} \rho_{j}}{c_{j} !\left(1-\rho_{j}\right)}\right] \pi_{0_{i k}} \quad \forall j \in J_{k}, k \in K, \\
\pi_{0_{j k}}=\left[\left(\sum_{n=0}^{c_{j}-1} \frac{\left(\lambda_{j k} / \mu_{j k}\right)^{n}}{n !}\right)+\frac{\left(\lambda_{j k} / \mu_{j k}\right)^{c_{j}}}{c_{j} !\left(1-\rho_{j}\right)}\right]^{-1} \forall j \in J_{k}, k \in K,
\end{gathered}
$$


and $\lambda_{j k}$ is the net traffic rate in $3.1, \rho_{j}$ is the net traffic intensity in $3.6, c_{j}$ is the number of servers, $\mu_{j k}$ is service rate at node $j$ for product class $k$, and $\pi_{0_{j k}}$ is the probability of zero jobs in the system.

Another important measure of interest is the expected flow time $\left(W_{k}\right)$ through the network of a job for product class $k$. This can be calculated by determining the mean time a job of product class $k$ spends at node $j$ per visit, denoted by $W_{j k}$, and the number of times each node is visited $\left(v_{j k}\right)$ [48]. The formula is given by

$$
W_{k}=\sum_{j \in J_{k}} W_{j k} v_{j k} \quad \forall k \in K
$$

where

$$
\begin{gathered}
W_{j k}=W s_{j k}+W q_{j} \quad \forall j \in J_{k}, k \in K, \\
W s_{j k}=\frac{1}{\mu_{j k}}
\end{gathered}
$$

and

$$
W q_{j k}=\frac{L q_{j k}}{\lambda_{j k}},
$$

where $W s_{j k}$ is the expected time of a job in service and $W q_{j k}$ is the expected time in queue.

Since the problem under study considers multiple product classes, an average waiting time in queue at node $j\left(\bar{W} q_{j}\right)$ needs to be calculated [86]. According to Pradhan et al. [86] the mean time a job for product class $k$ spends at node $j$ per visit is calculated by

$$
W_{j k}=W s_{j k}+\bar{W} q_{j} \quad \forall j \in J_{k}, k \in K,
$$

where 


$$
\bar{W} q_{j}=\frac{\sum_{k \in K} W q_{j k}}{\sum_{k \in K} Z_{j k}} \quad \forall j \in J_{k}
$$

and $Z_{j k}$ is 1 if node $j$ is visited by class $k$ and 0 otherwise.

In the server manufacturing system, the operations (e.g., building) handled in parallel can be modeled by using fork and join stations. Using parametric decomposition method we can analyze single (non fork-join stations) and fork-join stations separately after the input parameters to those subsystems are determined. The fork-join stations are modeled as homogeneous $N$ server fork-join queues with inputs to the individual buffers drawn from a Poisson process. Each arriving job of product class $k$ is divided into $M$ sub-tasks (typically $M \leq N)$, which are submitted to a unique server within the fork-join queue with service rate $\mu F J_{j k}$.

The response time $\left(R_{j k}\right)$ for product class $k$ in the fork-join subsystem $j$ for a two server fork-join queue can be computed by using the approximation developed by Nelson and Tantawi [77], and for a $N$ server fork-join queue by using the approximation developed by Varki et al. [106]. Those approximations are modified to incorporate the fact that a node can be visited by different product classes.

The response time for a two server fork-join queue is given by

$$
R_{j k}=\left(\frac{12-\rho F J_{j}}{8}\right)\left(\frac{1}{\mu F J_{j k}\left(1-\rho F J_{j}\right)}\right) \quad \forall j \in F_{k}, k \in K,
$$

and for a $N$ server fork-join queue is given by

$$
\begin{array}{r}
R_{j k}=\frac{1}{\mu F J_{j k}}\left(H_{N}+\frac{\rho F J_{j}}{2\left(1-\rho F J_{j}\right)}\left(\operatorname{Sum}_{M F J_{j k}-\rho F J_{j}}+\left(1-2 \rho F J_{j}\right) * \operatorname{Sum}_{M F J_{j k}\left(M F J_{j k}-\rho F J_{j}\right)}\right)\right) \\
\forall j \in F_{k}, k \in K,
\end{array}
$$


where $\rho F J_{j}$ is the net traffic intensity at fork-join $j$ given by

$$
\rho F J_{j}=\frac{\rho_{i}}{N F J_{j}}\left(\frac{\sum_{k \in K} M F J_{j k}}{|K|}\right),
$$

and

$\mu F J_{j k}$ is the service rate for product class $k$ at fork-join $j, H_{N}$ is the harmonic number given by 2.3. $\operatorname{Sum}_{M F J_{j k}-\rho F J_{j}}=\frac{1}{1-\rho F J_{j}}+\frac{1}{2-\rho F J_{j}}+\frac{1}{3-\rho F J_{j}}+\ldots+\frac{1}{M F J_{j k}-\rho F J_{j}}$ is the $M F J_{j k}^{t h}$ partial sum of the sequence $\left(\frac{1}{1-\rho F J_{j}}, \frac{1}{2-\rho F J_{j}}, \ldots, \frac{1}{M F J_{j}-\rho F J_{j}}\right)$, and $\operatorname{Sum}_{M F J_{j k}}\left(M F J_{j k}-\rho F J_{j}\right)=$ $\frac{1}{1-\rho F J_{j}}+\frac{1}{2}\left(\frac{1}{2-\rho F J_{j}}\right)+\frac{1}{3}\left(\frac{1}{3-\rho F J_{j}}\right)+\ldots+\frac{1}{M F J_{j k}}\left(\frac{1}{M F J_{j k}-\rho F J_{j}}\right)$ is the $M F J_{j k}^{t h}$ partial sum of the sequence $\left(\frac{1}{1-\rho F J_{j}}, \frac{1}{2}\left(\frac{1}{2-\rho F J_{j}}\right), \ldots, \frac{1}{M F J_{j k}}\left(\frac{1}{M F J_{j k}-\rho F J_{j}}\right)\right)$.

The net traffic intensity at fork-join $j$ in 3.20 is determined by the product of the net traffic intensity in 3.6, which considers different product classes, and the server access probability, which is the probability that a sub-task is submitted to a server. If the allocation policy assumed treats each server uniformly, according to Varki [106] serveraccess probability = $\frac{\text { numberof subtasks }}{\text { numberof servers }}$ (i.e., $\frac{M F J_{j k}}{N F J_{j}}$ ). Equation 3.2 differs from the original equation 2.10 with respect to the traffic intensity, and the server access probability. In the modified version, a net traffic intensity and an average of the server access probability are used to take into account the fact that the nodes within the fork-join subsystem can be visited by different products classes. By using these terms, it is possible to deal with different service times, and different number of subtasks in the fork-join stations.

To obtain the total expected flow time of a job through the network, individual expected flow times of single and fork-join stations are aggregated. The flow time for each product class $k$ is given by

$$
\text { Total Flow Time } \text { T }_{k}=\sum_{j \in J_{k}}\left(\bar{W} q_{j}+W s_{j k}\right) v_{j k}+\sum_{i \in F J_{k}} R_{j k}
$$




\subsection{Pseudocode}

The high-level description of the computer programming algorithm used to calculated the performance measures is as follows:

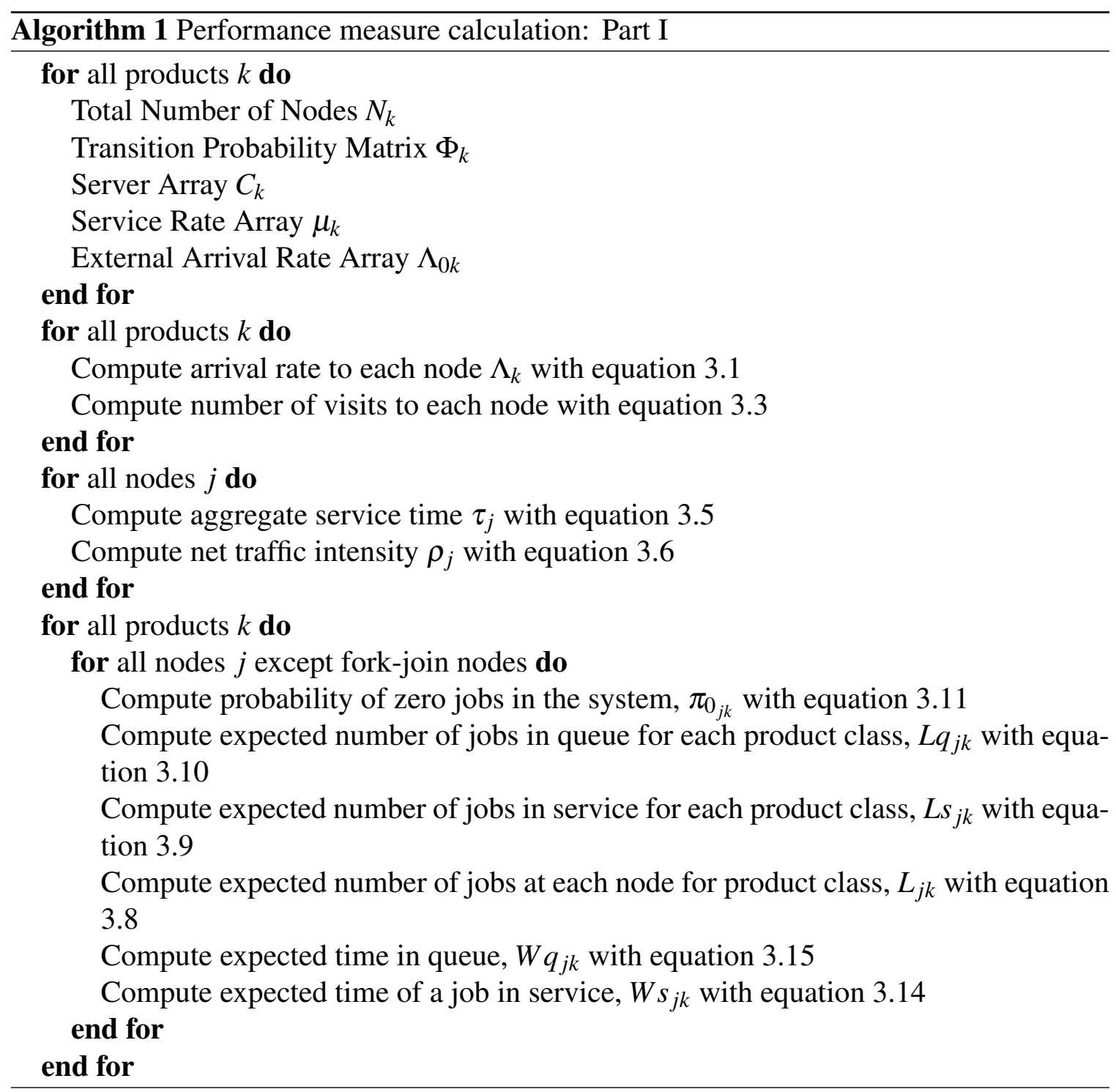




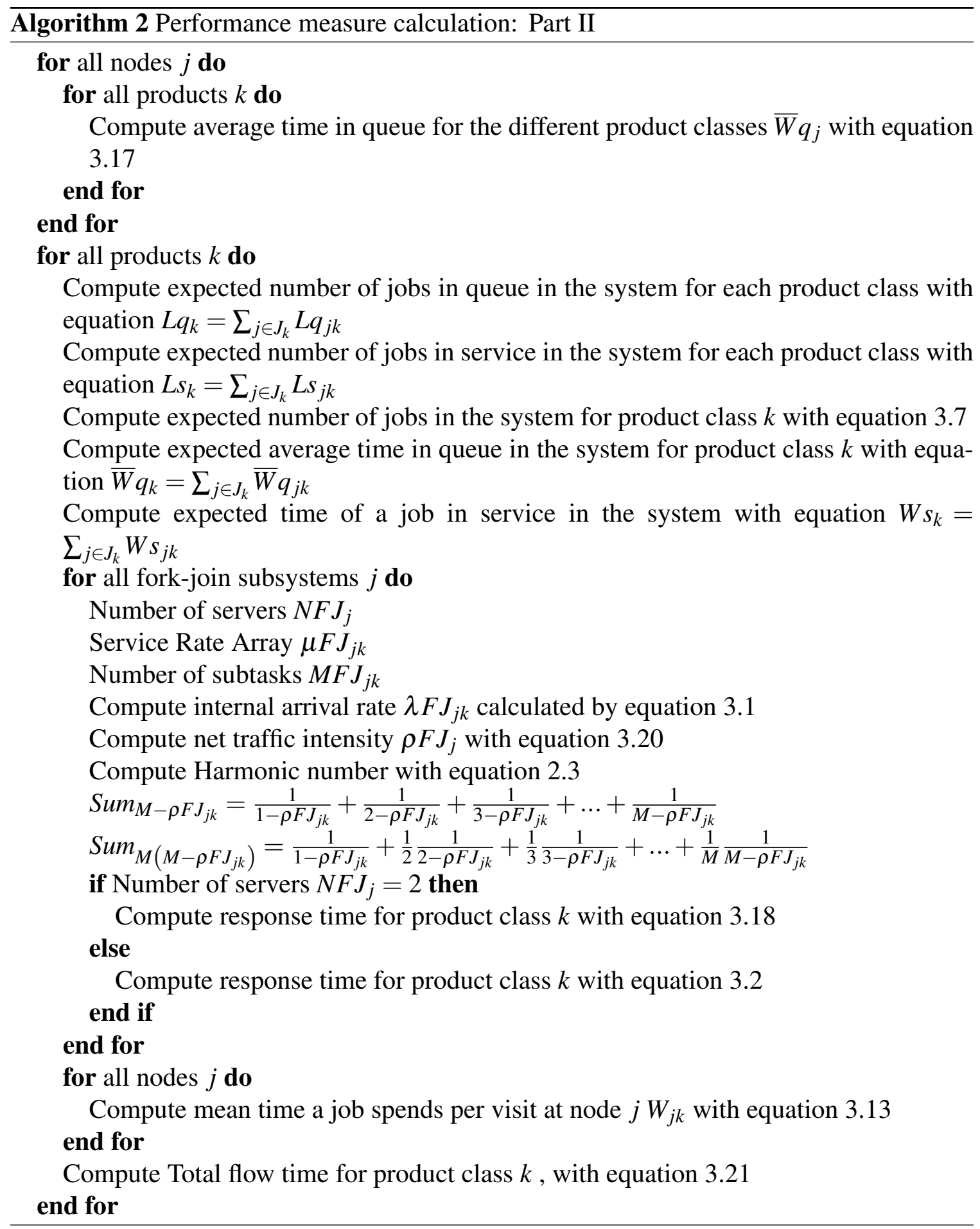




\subsection{Simulation Models}

To verify the accuracy of the results of the analytical approximations, the analytical results were compared to simulation. The process of developing discrete event simulation models for the problem under study, can be summarized by the following major steps: problem analysis and information collection, model construction and verification, designing and conducting simulation experiments, and output analysis. In the following sections, each step is discussed.

\subsubsection{Problem analysis and information collection}

The first step in developing the simulation models was to analyze each operation of the server manufacturing process in order to identify input parameters, relationships between parameters and variables, and rules governing the operation of system components. In this phase, the sequence of events that represents the behavior of the system, and the flows of jobs through the system were identified. Based on the configuration of the web server, some portions of the assembly process can be handled in parallel and some of them in series. Therefore, in order to model the problem under study it was considered as events in series: the customer order processing, kitting, assembly and testing operations. In contrast, the building operation, where each rack is built, was thought as set of events with a parallel processing structure, and a join point where all the racks wait for the remaining racks associated to the order. In addition, job/server circulation due to job failures was considered 


\subsubsection{Model construction, and verification}

In this phase a discrete event simulation model was developed to represent the nature of the problem under study. The model was developed and implemented in Arena 11 simulation package, taking into account the portions of the assembly process that are handled in series and in parallel with synchronization queues. The Figure 3.1 shows a configuration of parallel processing in the network which is modeled as a two server fork-join queue with two products. The duplicate block was used to model when the job is divided at the fork point into lidentical subtasks that are submitted to a unique server/resource/station within the fork and join subsystem, and the group block to model the activity where the subtask waits at the join point for its sibling subtask to complete service without preventing the server from working on another subtask. In addition, the distribution functions assumed in the simulation models correspond to the case analyzed in this research: Exponential (section 3), Erlang-2, and hyper geometric with two phases (section 4). Since Arena does not accommodate the hyper geometric distribution directly, the following expression was used to create the distribution: $\operatorname{DISC}\left(p_{1}, E X P O\left(m_{1}\right), p_{2}, E X P O\left(m_{2}\right)\right.$.

In order to verify and validate the model, the model code was compared to model specifications, and analyzed if the model's input and output relationships were reasonable. On the other hand, by making test runs to observe the model's animated evolution and using variable trace values it was possible to track the flow of jobs in the system, and verify that a proper flow of entities was maintained through selected model components. These methods allowed determining if the logic was correct and the model was operating according to the nature of the process. 


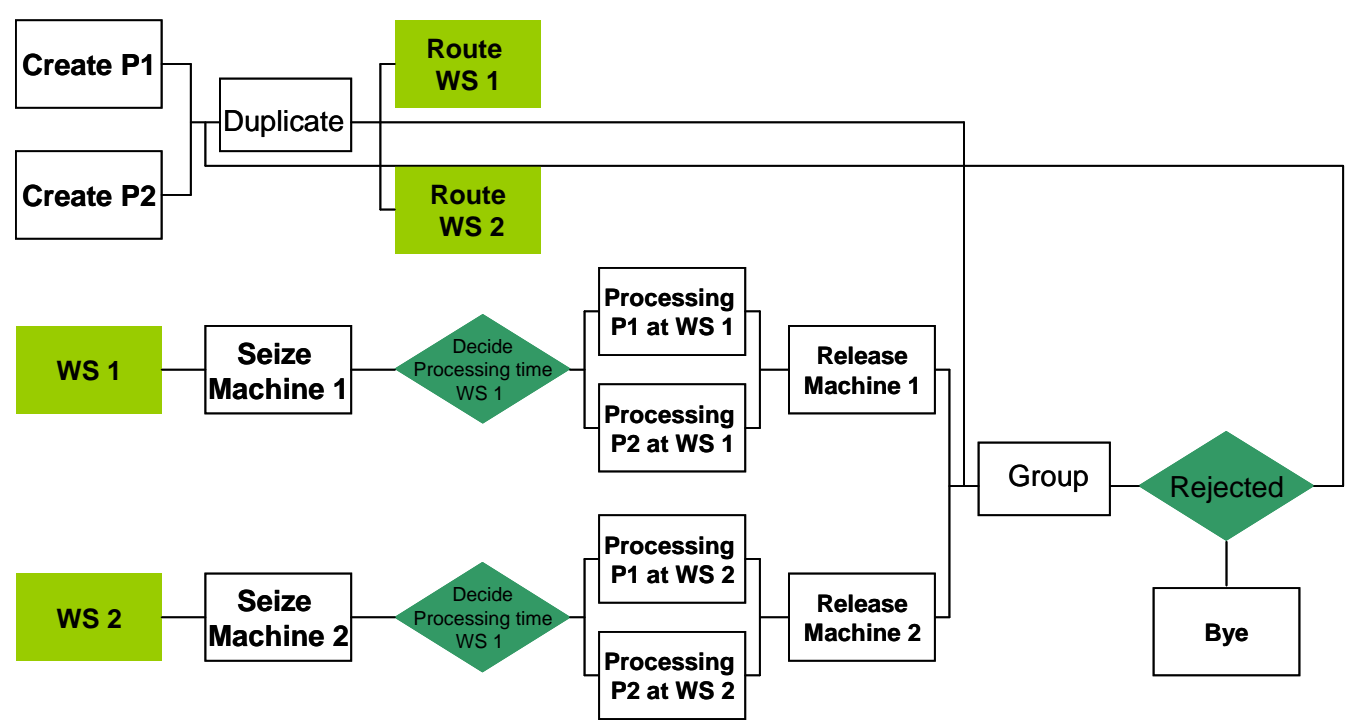

Figure 3.1: Simulation Model Example

\subsubsection{Designing and conducting simulation experiments}

Once the model was validated a set of simulation experiments was designed to estimate the performance measures of interest. The data set used for evaluating the analytical model was used in the simulation experiments. In order to determine the length of the warm-up period to attain steady-state, the graphical procedure of Welch [109] was used. By using this approach, it was possible to observe experimentally when the time variability of the statistics is largely eliminated. According to Alexopoulos [2] this graphical procedure uses $n$ replications of the simulation each with run length $m$, producing the $i^{t h}$ observation from the $j^{t h}$ replication, $X_{j i}$. The averages over the replications is calculated by $\bar{X}_{i}=\sum_{j=1}^{n} \frac{X_{j i}}{n}$, for $i=1,2 \ldots m$. Then, we define a moving average $\bar{X}_{i}(w)$ to smooth out the high frequency oscillations in $\bar{X}_{i}(w)$. By using a window $(w)$ less than or equal to $m / 4$, the moving averages are calculated as follows:

$$
\bar{X}_{i}(w)=\left\{\begin{array}{lc}
\frac{1}{2 w+1} \sum_{s=-w}^{w} \bar{X}_{i+s} & w+1 \leq i \leq m-w \\
\frac{1}{2 i-1} \sum_{s=-(i-1)}^{i-1} \bar{X}_{i+s} & 1 \leq i \leq w
\end{array}\right.
$$


Later, the moving average $\bar{X}_{i}(w)$ is plotted for $i=1,2, \ldots, m-w$ and the warm-up period is set at that value of $i$ beyond which $\bar{X}_{i}(w)$ appears to be converged (variability of the statistics is largely eliminated). Based on this, the length of the warm-up was set at 30,000 time units.

The guiding principle to set the replication length was the stabilization of the statistics of interest. The replication length was set as 500,000 time units (roughly more than 30,000 jobs) when records of the statistics collected from several successive increments were sufficiently close. In addition, the number of replications $n$ was set as 10. It was calculated by setting a specific tolerance for the average value of the measures of performance, $h^{*}$. This desired half-width interval was determined by selecting a value no greater than $10 \%$ of the numeric value of the average obtained by running the simulation model for $m$ replications. That value was then compared to $h=t_{n-1,1-\alpha / 2} \frac{\sigma}{\sqrt{n}}$, which is the half width interval using the initial number of replications $m$, if $h^{*}<h$ the number of replications is computed by using

$$
n^{*}=\text { Round }\left[n\left(\frac{h}{h^{*}}\right)^{2}\right] \text {. }
$$

Otherwise, the number of replications is $n=m$.

\subsection{Computational Results}

In order to design the problem instances that represent the problem under study, five subprocesses of the web server assembly process were considered: the customer order processing, kitting, building (parallel processing), assembly and testing operations. In web server assembly lines server failures are typically noticed after the testing stage. A queueing network with four single stations, one fork-join subsystem, and job circulation in the testing station is a typical representation of the problem under study. However, different number of 
single and fork-join stations, and failures at any stage are considered so that the analytical approximations are applicable to other manufacturing systems with different subprocesses, and where failures can occur at any stage in the assembly. Ninety problem instances were designed to test the accuracy of the analytical estimates. Each problem instance is defined by the number of nodes in the network, number of servers at each node, number of product classes, nodes that are shared by different product classes; and for each product class, its arrival and service rates and the transition probability matrix. All the elements of the data set were selected taking into account the nature and previous knowledge of the web server assembly process.

The number of nodes for each problem instance was varied from five to eight, product classes from one to six, and shared nodes from $43 \%$ to $100 \%$ of the total number of stations. The number of fork and join subsystems considered was either one or two, the number of servers in it two, three or four, and the number of subtasks two, three or four. The location of the fork-join nodes in the network was varied from node one to last node -1, keeping the nature of the server assembly process. The transition probability matrix for each product class was randomly generated, varying failure rates from 0.01 to 0.2 . Arrival and service rates were selected in such way that the service rates at all stations were greater than the arrival rates. All the equations stated in the analytical formulation were implemented in Matlab 7.0. in order to determine the performance measures of interest.

Table 3.1 describes each problem instance in terms of the number of nodes, product classes, shared nodes, and fork-join nodes along with their location in the network. Problem instances 1-9 only deal with one product class, and failure rates between 0.01 and 0.12 . Instances 10-38 are for two product classes, with failures rates for instances 10-15 between 0.01 and 0.12 and for instances 15-38 between 0.1 and 0.2. Instances 39-51 are for three product classes, 52-61 for four product classes, 62-71 for five product classes, and 72-78 for six product classes. Instances $79-90$ consider product classes from three to six varying 
the number of subtasks in the fork and join node. In problem instances 39-90, fork-join nodes were varied from one to two, and failures rates from 0.01 to 0.15 . In addition, instances $31-33,44,46,49,54-56,59,64-66,69,74-76,79,85$ consider recirculation of jobs at the fork point due to product failures. Instances 34-38, 82-90 arise the case where at the fork-join node the number of subtasks is less than the number of servers. Table 3.2 presents the configuration of the fork-join nodes for each problem instance in terms of number of servers, and number of subtasks.

Table 3.1: Problem instances configuration

\begin{tabular}{|c|c|c|c|c|c|}
\hline Instance & Nod. & Prod classes & Shared nod. & $\mathrm{F} / \mathrm{J}$ nodes & F/J location \\
\hline 1 & 7 & 1 & & 1 & node 4 \\
\hline 2 & 6 & 1 & & 1 & node 4 \\
\hline 3 & 7 & 1 & & 2 & node 2 , node 5 \\
\hline 4 & 7 & 1 & & 2 & node 2 , node 5 \\
\hline 5 & 7 & 1 & & 1 & node 2 \\
\hline 6 & 7 & 1 & & 1 & node 2 \\
\hline 7 & 6 & 1 & & 1 & node 4 \\
\hline 8 & 6 & 1 & & 1 & node 4 \\
\hline 9 & 8 & 1 & & 1 & node 3 \\
\hline 10 & 7 & 2 & 5 & 1 & node 4 \\
\hline 11 & 5 & 2 & 3 & 1 & node 4 \\
\hline 12 & 7 & 2 & 5 & 2 & node 2 , node 4 \\
\hline 13 & 7 & 2 & 7 & 2 & node 2 , node 5 \\
\hline 14 & 7 & 2 & 6 & 1 & node 2 \\
\hline 15 & 6 & 2 & 5 & 1 & node 4 \\
\hline 16 & 5 & 2 & 4 & 1 & node 4 \\
\hline 17 & 5 & 2 & 5 & 2 & node 2 , node 4 \\
\hline 18 & 6 & 2 & 6 & 2 & node 2 , node 5 \\
\hline 19 & 5 & 2 & 4 & 1 & node 1 \\
\hline 20 & 7 & 2 & 5 & 1 & node 4 \\
\hline 21 & 7 & 2 & 7 & 1 & node 2 \\
\hline 22 & 7 & 2 & 5 & 2 & node 2 , node 4 \\
\hline 23 & 7 & 2 & 7 & 2 & node 2 , node 5 \\
\hline 24 & 7 & 2 & 6 & 1 & node 2 \\
\hline 25 & 6 & 2 & 5 & 1 & node 4 \\
\hline 26 & 6 & 2 & 6 & 1 & node 4 \\
\hline 27 & 5 & 2 & 5 & 2 & node 2 , node 4 \\
\hline
\end{tabular}




\begin{tabular}{|c|c|c|c|c|c|}
\hline Instance & Nod. & Prod classes & Shared nod. & $\mathrm{F} / \mathrm{J}$ nodes & $\mathrm{F} / \mathrm{J}$ location \\
\hline 28 & 6 & 2 & 6 & 2 & node 2 , node 5 \\
\hline 29 & 7 & 2 & 7 & 1 & node 2 \\
\hline 30 & 7 & 2 & 7 & 2 & node 2 , node 5 \\
\hline 31 & 7 & 2 & 6 & 1 & node 2 \\
\hline 32 & 5 & 2 & 4 & 1 & node 4 \\
\hline 33 & 6 & 2 & 6 & 2 & node 2 , node 5 \\
\hline 34 & 5 & 2 & 4 & 1 & node 4 \\
\hline 35 & 5 & 2 & 5 & 2 & node 2 , node 4 \\
\hline 36 & 7 & 2 & 7 & 2 & node 2 , node 5 \\
\hline 37 & 6 & 2 & 6 & 2 & node 2 , node 5 \\
\hline 38 & 6 & 2 & 3 & 1 & node 2 \\
\hline 39 & 5 & 3 & 5 & 1 & node 4 \\
\hline 40 & 5 & 3 & 4 & 2 & node 2 , node 4 \\
\hline 41 & 6 & 3 & 5 & 1 & node 2 \\
\hline 42 & 6 & 3 & 3 & 1 & node 2 \\
\hline 43 & 7 & 3 & 3 & 2 & node 2 , node 5 \\
\hline 44 & 5 & 3 & 3 & 1 & node 4 \\
\hline 45 & 5 & 3 & 3 & 1 & node 2 \\
\hline 46 & 6 & 3 & 3 & 2 & node 3 , node 5 \\
\hline 47 & 7 & 3 & 4 & 1 & node 6 \\
\hline 48 & 8 & 3 & 4 & 1 & node 3 \\
\hline 49 & 6 & 3 & 3 & 1 & node 3 \\
\hline 50 & 5 & 3 & 3 & 1 & node 3 \\
\hline 51 & 7 & 3 & 3 & 1 & node 2 \\
\hline 52 & 6 & 4 & 3 & 1 & node 2 \\
\hline 53 & 7 & 4 & 3,7 & 2 & node 2 , node 5 \\
\hline 54 & 5 & 4 & 3 & 1 & node 4 \\
\hline 55 & 5 & 4 & 3 & 1 & node 2 \\
\hline 56 & 6 & 4 & 3,4 & 2 & node 3 , node 5 \\
\hline 57 & 8 & 4 & 4 & 1 & node 6 \\
\hline 58 & 8 & 4 & 4 & 1 & node 3 \\
\hline 59 & 6 & 4 & 3 & 1 & node 3 \\
\hline 60 & 5 & 4 & 3 & 1 & node 3 \\
\hline 61 & 7 & 4 & 3 & 1 & node 2 \\
\hline 62 & 6 & 5 & 3 & 1 & node 2 \\
\hline 63 & 7 & 5 & 3,7 & 2 & node 2 , node 5 \\
\hline 64 & 5 & 5 & 3 & 1 & node 4 \\
\hline 65 & 5 & 5 & 3 & 1 & node 2 \\
\hline 66 & 6 & 5 & 3 & 2 & node 3 , node 5 \\
\hline 67 & 8 & 5 & 4 & 1 & node 6 \\
\hline 68 & 8 & 5 & 4 & 1 & node 3 \\
\hline
\end{tabular}




\begin{tabular}{|c|c|c|c|c|c|}
\hline Instance & \multicolumn{1}{l}{ Nod. } & Prod classes & \multicolumn{1}{c}{ Shared nod. } & F/J nodes & F/J location \\
\hline 69 & 6 & 5 & 3 & 1 & node 3 \\
\hline 70 & 5 & 5 & 3 & 1 & node 3 \\
\hline 71 & 7 & 5 & 3 & 1 & node 2 \\
\hline 72 & 6 & 6 & 3 & 1 & node 2 \\
\hline 73 & 7 & 6 & 3,4 & 2 & node 2, node 5 \\
\hline 74 & 5 & 6 & 3 & 1 & node 4 \\
\hline 75 & 7 & 6 & $3,4,7$ & 1 & node 2 \\
\hline 76 & 6 & 6 & 3 & 2 & node 3, node 5 \\
\hline 77 & 7 & 6 & 4 & 1 & node 6 \\
\hline 78 & 8 & 6 & 4 & 1 & node 3 \\
\hline 79 & 6 & 4 & 3 & 1 & node 3 \\
\hline 80 & 5 & 4 & 3 & 1 & node 3 \\
\hline 81 & 7 & 4 & 3 & 1 & node 2 \\
\hline 82 & 6 & 3 & 3 & 1 & node 2 \\
\hline 83 & 6 & 3 & 3 & 2 & node 3, node 5 \\
\hline 84 & 5 & 4 & 3 & 1 & node 4 \\
\hline 85 & 8 & 4 & 4 & 1 & node 3 \\
\hline 86 & 5 & 5 & 3 & 1 & node 4 \\
\hline 87 & 8 & 4 & 4 & 1 & node 3 \\
\hline 88 & 5 & 5 & 3 & 1 & node 3 \\
\hline 89 & 5 & 6 & 3 & 1 & node 4 \\
\hline 90 & 8 & 6 & 4 & 1 & node 3 \\
\hline
\end{tabular}

Table 3.2: Fork-join node configuration

\begin{tabular}{|c|c|c|c|c|c|c|c|c|c|}
\hline \multirow[t]{2}{*}{ Instance } & \multicolumn{2}{|c|}{ Fork-join_1 } & \multicolumn{2}{|c|}{ Fork-Join_2 } & \multirow[t]{2}{*}{ Instance } & \multicolumn{2}{|c|}{ Fork-join_1 } & \multicolumn{2}{|c|}{ Fork-Join_2 } \\
\hline & Servers & Subt & Servers & Subt & & Servers & Subt & Servers & Subt \\
\hline 1 & 3 & 3 & - & - & 39 & 3 & 3 & - & - \\
\hline 2 & 3 & 3 & - & - & 40 & 3 & 3 & 2 & 2 \\
\hline 3 & 3 & 3 & 2 & 2 & 41 & 2 & 2 & - & - \\
\hline 4 & 3 & 3 & 2 & 2 & 42 & 3 & 3 & - & - \\
\hline 5 & 2 & 2 & - & - & 43 & 2 & 2 & - & - \\
\hline 6 & 2 & 2 & - & - & 44 & 3 & 3 & - & - \\
\hline 7 & 3 & 3 & - & - & 45 & 3 & 3 & - & - \\
\hline 8 & 3 & 3 & 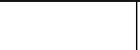 & & 46 & 3 & 3 & 3 & 3 \\
\hline 9 & 4 & 4 & 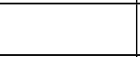 & . & 47 & 3 & 3 & - & - \\
\hline 10 & 3 & 3 & - & - & 48 & 4 & 4 & - & - \\
\hline 11 & 2 & 2 & - & - & 49 & 3 & 3 & - & - \\
\hline 12 & 3 & 3 & 3 & 3 & 50 & 3 & 3 & - & - \\
\hline
\end{tabular}




\begin{tabular}{|c|c|c|c|c|c|c|c|c|c|}
\hline \multirow[t]{2}{*}{ Instance } & \multicolumn{2}{|c|}{ Fork-join_1 } & \multicolumn{2}{|c|}{ Fork-Join_2 } & \multirow[t]{2}{*}{ Instance } & \multicolumn{2}{|c|}{ Fork-join_1 } & \multicolumn{2}{|c|}{ Fork-Join_2 } \\
\hline & Servers & Subt & Servers & Subt & & Servers & Subt & Servers & Subt \\
\hline 13 & 3 & 3 & 2 & 2 & 51 & 3 & 3 & - & - \\
\hline 14 & 2 & 2 & - & - & 52 & 3 & 3 & - & - \\
\hline 15 & 3 & 3 & - & - & 50 & 3 & 3 & - & - \\
\hline 16 & 3 & 3 & - & - & 50 & 3 & 3 & - & - \\
\hline 17 & 3 & 3 & 3 & 3 & 51 & 3 & 3 & - & - \\
\hline 18 & 3 & 3 & 2 & 2 & 52 & 3 & 3 & - & - \\
\hline 19 & 3 & 3 & - & - & 53 & 2 & 2 & - & - \\
\hline 20 & 3 & 3 & - & - & 54 & 3 & 3 & - & - \\
\hline 21 & 2 & 2 & - & - & 55 & 3 & 3 & - & - \\
\hline 22 & 3 & 3 & 3 & 3 & 56 & 3 & 3 & 3 & 3 \\
\hline 23 & 3 & 3 & 2 & 2 & 57 & 3 & 3 & - & - \\
\hline 24 & 2 & 2 & - & - & 58 & 4 & 4 & - & - \\
\hline 25 & 3 & 3 & - & - & 59 & 3 & 3 & - & - \\
\hline 26 & 3 & 3 & - & - & 60 & 3 & 3 & - & - \\
\hline 27 & 3 & 3 & 3 & 3 & 61 & 3 & 3 & - & - \\
\hline 28 & 3 & 3 & 2 & 2 & 62 & 3 & 3 & - & - \\
\hline 29 & 2 & 2 & - & - & 63 & 2 & 2 & - & - \\
\hline 30 & 3 & 3 & 2 & 2 & 64 & 3 & 3 & - & - \\
\hline 31 & 2 & 2 & - & - & 65 & 3 & 3 & - & - \\
\hline 32 & 3 & 3 & - & - & 65 & 3 & 3 & - & - \\
\hline 33 & 3 & 3 & 2 & 2 & 66 & 3 & 3 & 3 & 3 \\
\hline 34 & 3 & 2 & - & - & 67 & 3 & 3 & - & - \\
\hline 35 & 3 & 2 & 3 & 2 & 68 & 4 & 4 & - & - \\
\hline 36 & 3 & 2 & 2 & 2 & 69 & 3 & 3 & - & - \\
\hline 37 & 3 & 2 & 2 & 2 & 70 & 3 & 3 & - & - \\
\hline 38 & 3 & 2 & - & - & 71 & 3 & 3 & - & - \\
\hline 72 & 3 & 3 & - & - & 82 & 3 & 2 & - & - \\
\hline 73 & 2 & 2 & - & - & 83 & 3 & 2 & 3 & 2 \\
\hline 74 & 3 & 3 & - & - & 84 & 3 & 2 & - & - \\
\hline 75 & 3 & 3 & - & - & 85 & 4 & 2 & - & - \\
\hline 76 & 3 & 3 & 3 & 3 & 86 & 3 & 2 & - & - \\
\hline 77 & 3 & 3 & - & - & 87 & 4 & 3 & - & - \\
\hline 78 & 4 & 4 & - & - & 88 & 3 & 2 & - & - \\
\hline 79 & 3 & 3 & - & - & 89 & 3 & 2 & - & - \\
\hline 80 & 3 & 3 & - & - & 90 & 4 & 3 & - & - \\
\hline 81 & 3 & 3 & - & - & & & & & \\
\hline
\end{tabular}


One of the first measures of interest is the product flow time in the system. The tightness of the flow time approximations is verified by comparing with simulation results. The error in the flow time approximation is calculated by using

$$
\text { Relative approx error }=\frac{\text { Flow Time }_{\text {approx }}-\text { Flow Time }_{\text {simulation }}}{\text { Flow Time }_{\text {simulation }}} * 100 .
$$

In Appendix $\mathrm{A}$ is presented the approximate and simulated mean flow time values along with the error in the approximation for one, two, three, four, five, and six product instances respectively. Likewise, comparisons between results for instances where the number of subtasks is less than the number of servers in the fork-join node, and where the number of subtasks equals the number of servers in the fork-join node are also presented in the appendix.

In order to show the nature of the distribution of the error for the flow time, and identify what proportion of data points fall into each interval, a histogram of the errors was plotted for all problem instances (see Figure 3.2). The graph shows that the error seems to be distributed between zero and $85 \%$. Figure 3.3 presents the trend of the simulation and analytical flow times for all problem instances.

Detailed analysis was done by plotting the error for the different types of problem instances, and a paired-t test was conducted to determine whether there was significant difference between the flow time from the simulation models and the analytical formulations (see Table 3.3). Figure 3.4 displays that the error for most of the one product problem instances was between zero and 5\%, and the maximum error was $8 \%$. The data strongly suggests that the means are the same at 0.05 level of significance. Figure 3.5 shows that the error 


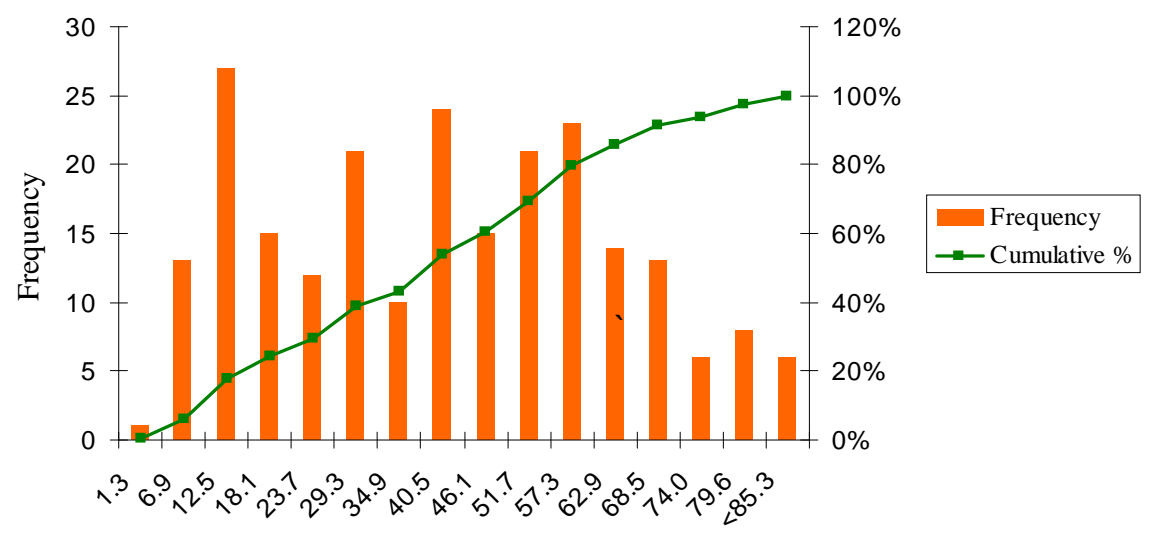

Flow Time Error (\%)

Figure 3.2: Overall flow time error distribution.

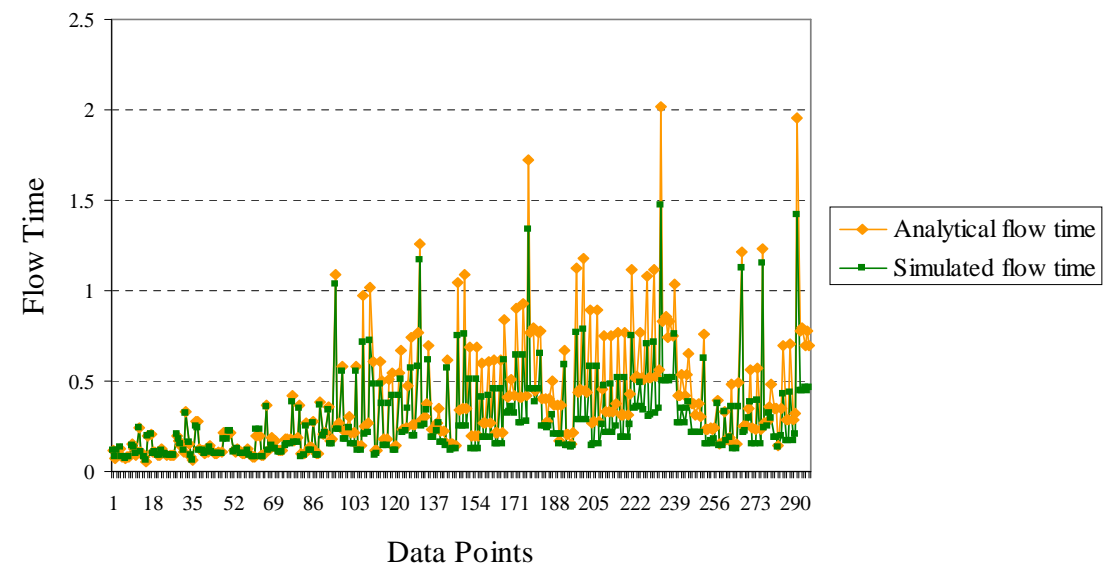

Figure 3.3: Simulation and analytical flow time trends.

for $86 \%$ of two product instances was between zero and $10 \%$, and the maximum error was $17 \%$. The data strongly suggests that at 0.05 level of significance the means are the same.

Table 3.3: Paired-t test results per product class

\begin{tabular}{|c|c|c|c|c|c|c|}
\hline \multicolumn{7}{|c|}{ Product Class } \\
\hline \hline & 1 & 2 & 3 & 4 & 5 & 6 \\
\hline t Stat & -2.09 & 0.94 & 16.33 & -8.43 & -12.73 & -16.82 \\
\hline t Critical two-tail & 2.31 & 2.00 & 1.97 & 2.01 & 2.00 & 1.99 \\
\hline $\mathrm{P}(\mathrm{T}<=\mathrm{t})$ two-tail & 0.07 & 0.35 & 0.00 & 0.00 & 0.00 & 0.00 \\
\hline
\end{tabular}




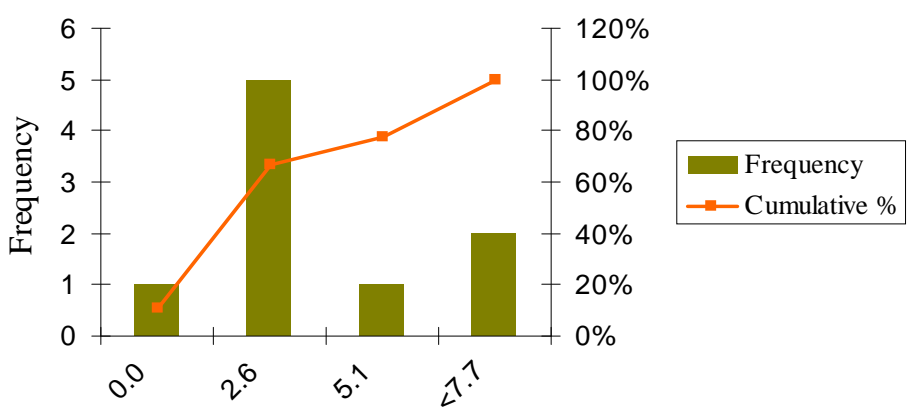

Flow Time Error $(\%)$

Figure 3.4: Flow time distribution for one product.

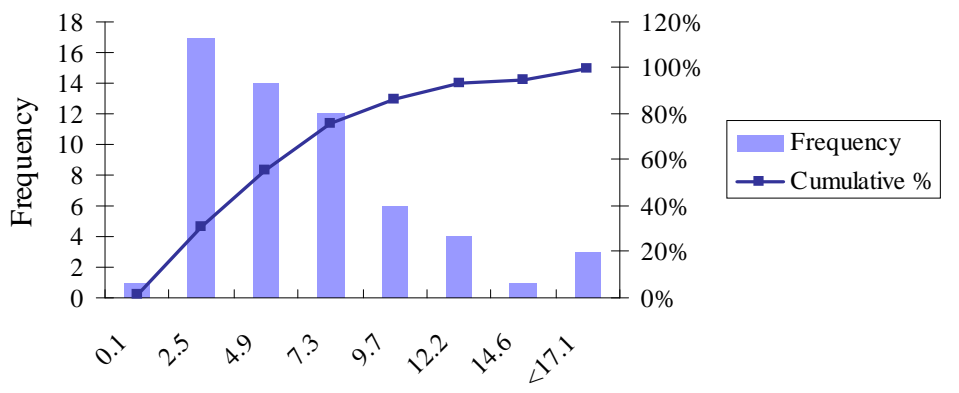

Flow Time Error $(\%)$

Figure 3.5: Flow time distribution for two products.

In the case of three product classes, Figure 3.6 displays that the error for $87 \%$ of the instances was between $1 \%$ and $26 \%$, and the maximum error was $39.43 \%$. The data strongly suggest that the means are different at 0.01 level of significance. Figure 3.7 shows that the error was between $7 \%$ and $35 \%$ for $81 \%$ of the four product instances. The data strongly suggest that the means are different at 0.01 level of significance. A maximum error of $40.88 \%$ was observed. In Figure 3.8, the error is shown for five product classes. The error for $91 \%$ of the instances appears to be distributed between $7 \%$ and $73 \%$, and the maximum error was $82.36 \%$. The data strongly suggest that the means are different at 0.01 level of 


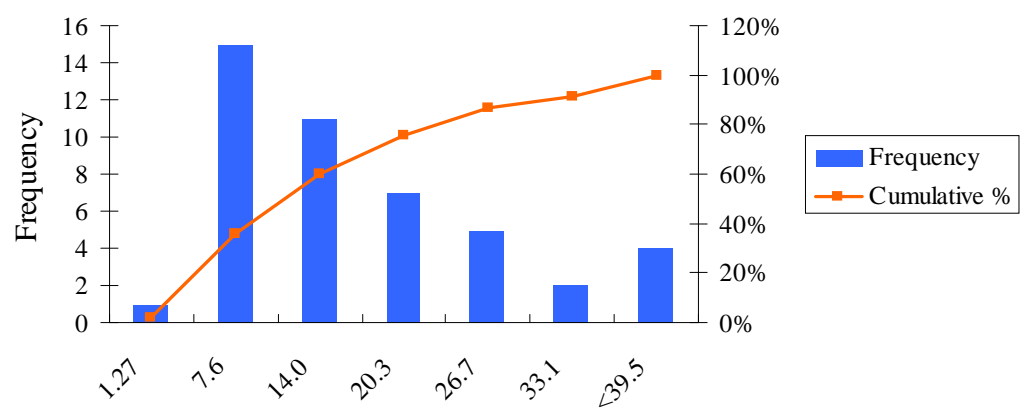

Flow Time Error $(\%)$

Figure 3.6: Flow time distribution for three products.

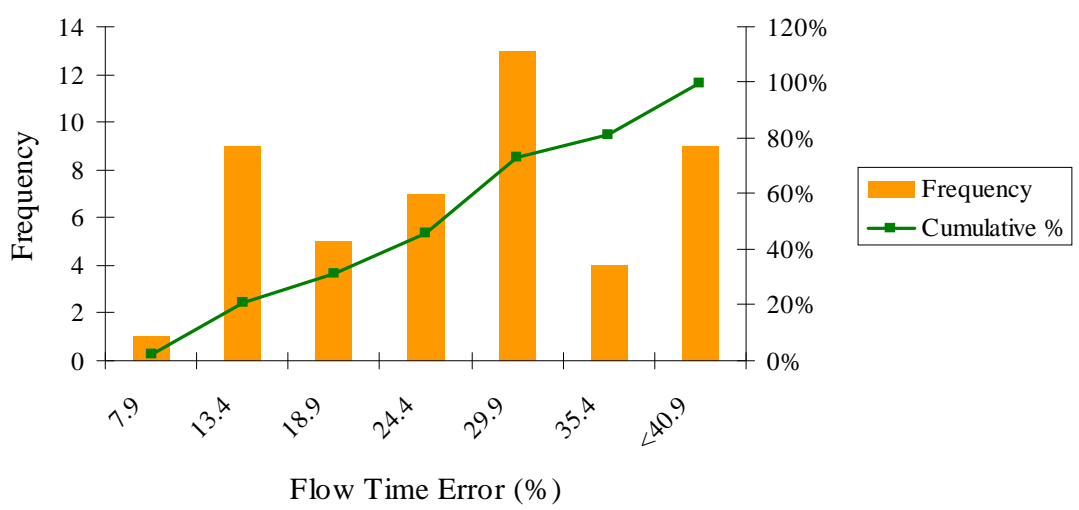

Figure 3.7: Flow time distribution for four products.

significance. Figure 3.9 displays that the flow time error for $94 \%$ of six product instances was between $21 \%$ and $77 \%$. The data strongly suggest that the means are different at 0.01 level of significance. 


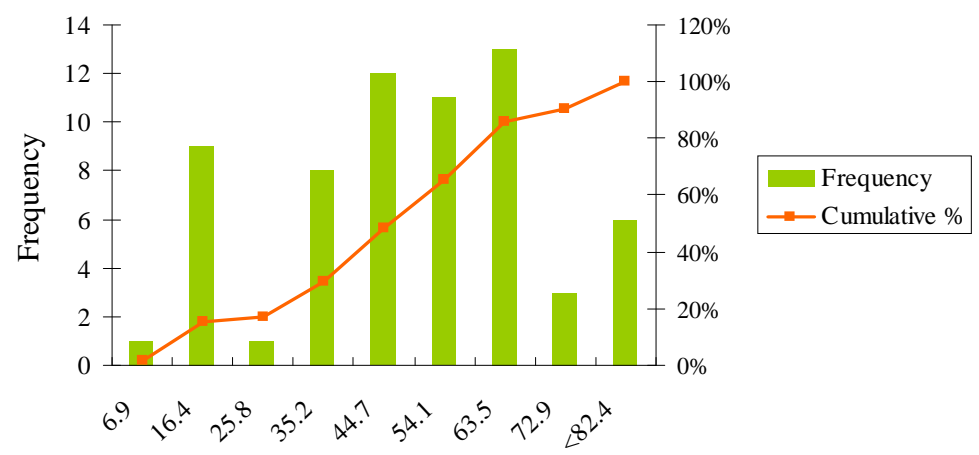

Flow Time Error $(\%)$

Figure 3.8: Flow time distribution for five products.

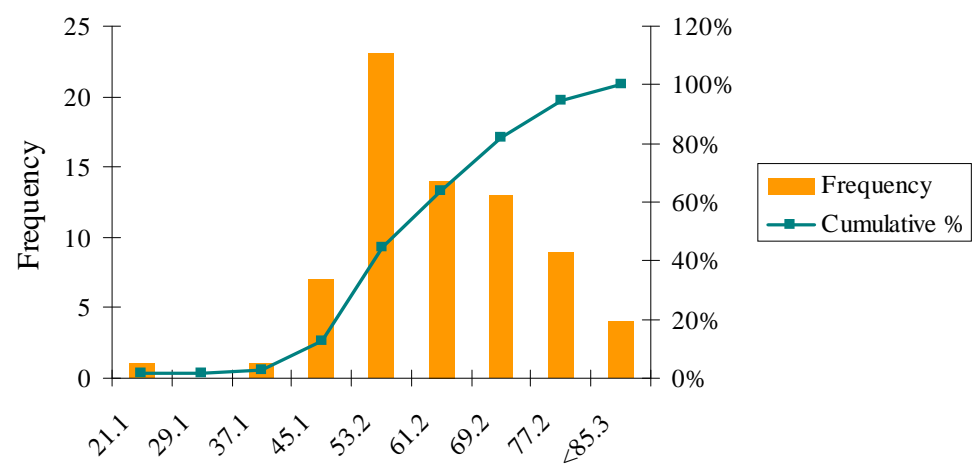

Flow Time Error $(\%)$

Figure 3.9: Flow time distribution for six products.

Figure 3.10 presents patterns of the flow time error for one, two, three, four, five, and six product instances. In this graph, it is possible to identify that flow time error values for five and six product instances are concentrated at the upper level of the graph, and for the case of one and two products are concentrated at the lower end of the graph. Therefore, it is apparent from the figure that the flow time error increased as the number of products increased.

Figure 3.11 presents the error in flow time for problems where the number of subtasks was less than the number of servers in the fork-join nodes. The flow time error was compared 


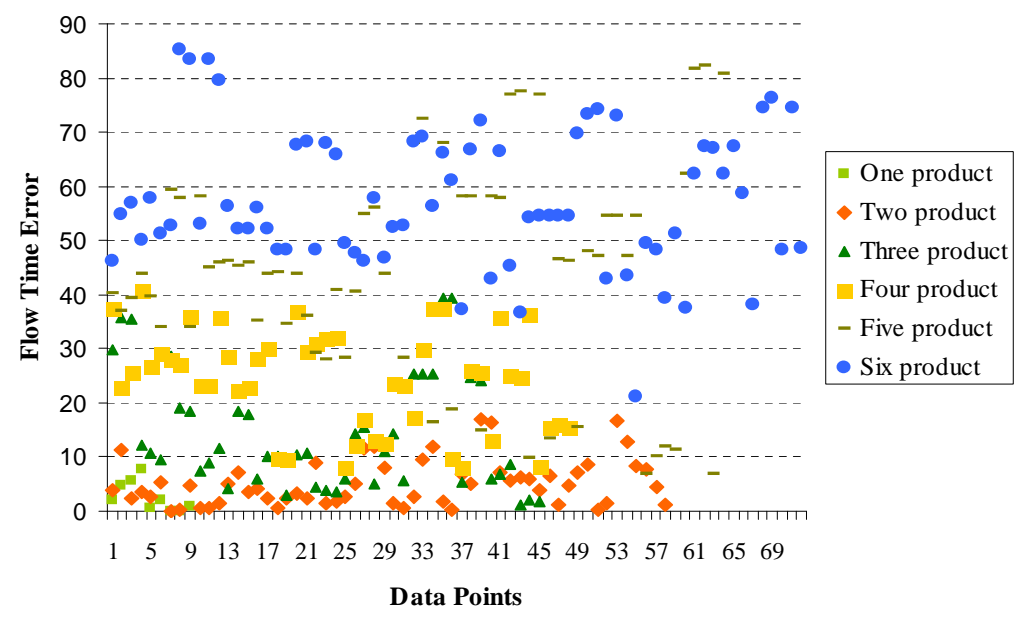

Figure 3.10: Flow time error by product.

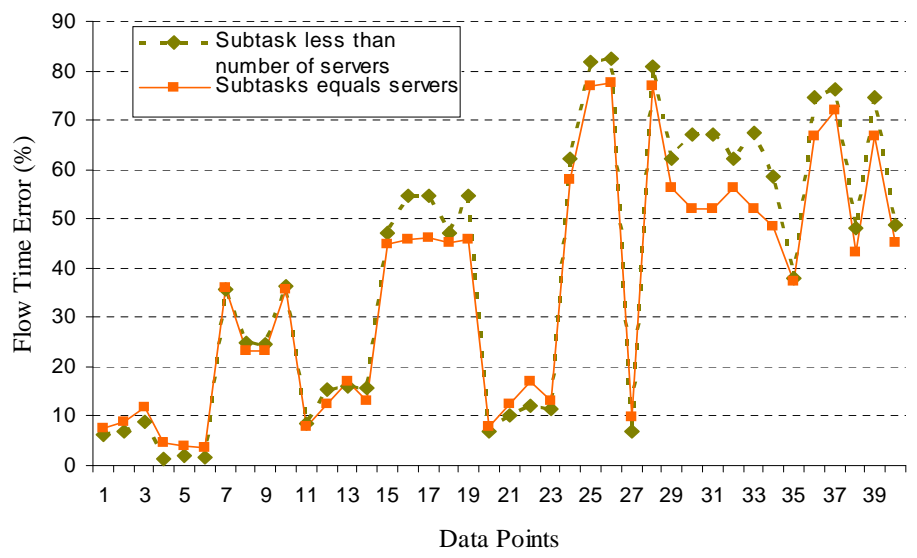

Figure 3.11: Flow time error for problems with less subtasks than number of servers at the fork-join nodes.

with the results from the same examples with equal number of subtasks and servers. The flow time error for $67.5 \%$ of the data points was greater in the cases where the number of subtasks was less than the number of servers in the fork-join nodes. A paired-t test was conducted to determine whether there was significant difference between the flow time errors (see Table 3.4). The data strongly suggests that the flow time errors are not the same at 0.01 level of significance. Based on these results, it can be inferred that the number of subtasks in the fork-join nodes are affecting the error in flow time. 
Table 3.4: Paired-t test for problem instances where the number of subtasks was less than the number of servers in the fork-join nodes

\begin{tabular}{|c|c|}
\hline $\mathrm{t}$ Stat & 3.89 \\
\hline $\mathrm{t}$ Critical two-tail & 2.02 \\
\hline $\mathrm{P}(\mathrm{T}<=\mathrm{t})$ two-tail & 0.00 \\
\hline
\end{tabular}

\subsection{Correction Factors}

The flow time was obtained from the analytical and simulation results, and the relative percentage error was calculated between them. Based on the data set, the flow time error from three to six products was roughly between $1 \%$ and $85 \%$, and the data strongly suggest that the means for those problem classes are different at 0.01 level of significance. In order to improve the results for Markovian system instances with more than two products, efforts were made to develop regression equations to predict the error between the analytical and simulation results for single (no fork-join stations) and fork-join stations. Since the flow time in the network is the aggregation of the flow time at each station, for simplicity one station instances were considered to develop the correction factors. Therefore, the error in the flow time was adjusted at each station with the correction factor.

\subsubsection{The case of single stations}

Thirty two problem instances (144 data points) for single stations were generated in order to predict the flow time. The number of product classes were varied from three to six, and failure rates from zero to $35 \%$. After analyzing the data, it was identified that the main error of the flow time came from the waiting time (see Figure 3.12 ; the variables affecting the results most were the number of products in the network, and the net traffic intensity at node $i$, and there was a nonlinearity in the parameters (see Figure 3.13). The correction term was developed by analyzing the behavior of the error in the waiting time 
and using Microsoft Excel to determine the best regression equation. Figure 3.13 shows the regression equation by products as a function of the net traffic intensity. Later, by using those equations it was possible to determine the regression model in terms of the net traffic intensity and the number of products (see Figure 3.14). The final regression equation is given by

$$
\hat{E}_{j}=\left(-0.8329 * p^{2}+8.2267 * p-8.3643\right) \exp ^{\left(0.0628 * p^{2}-0.5157 * p+4.258\right) \rho_{j}}
$$

where $\hat{E}_{j}$ is the percentage of predicted error in the waiting time at node $j, p$ is the number of products in the network and $\rho_{j}$ is the the net traffic intensity at node $j$, which is calculated by equation 3.6 .

The approximation to determine the adjusted expected waiting time using the correction factor is of the form

$$
W q A d_{j k}=\frac{\left(\frac{L q_{j k}}{\lambda_{j k}}\right)}{\left(1+\frac{\hat{E}_{j}}{100}\right)}
$$

and the adjusted mean flow time $\left(W A d j_{j k}\right)$ of a job for product class $k$ at node $j$ per visit is calculated by

$$
W A d_{j k}=W s_{j k}+\bar{W} q A d_{j k} \quad \forall j \in J_{k}, k \in K
$$

where

$$
\bar{W} q A d_{j}=\frac{\sum_{k \in K} W q A d_{j k}}{\sum_{k \in K} Z_{j k}} \quad \forall j \in J_{k},
$$

and $W s_{j k}$ is computed using equation 3.14. Later the adjusted expected flow time through the network of a job for product class $k\left(W A d j_{k}\right)$ is given by 


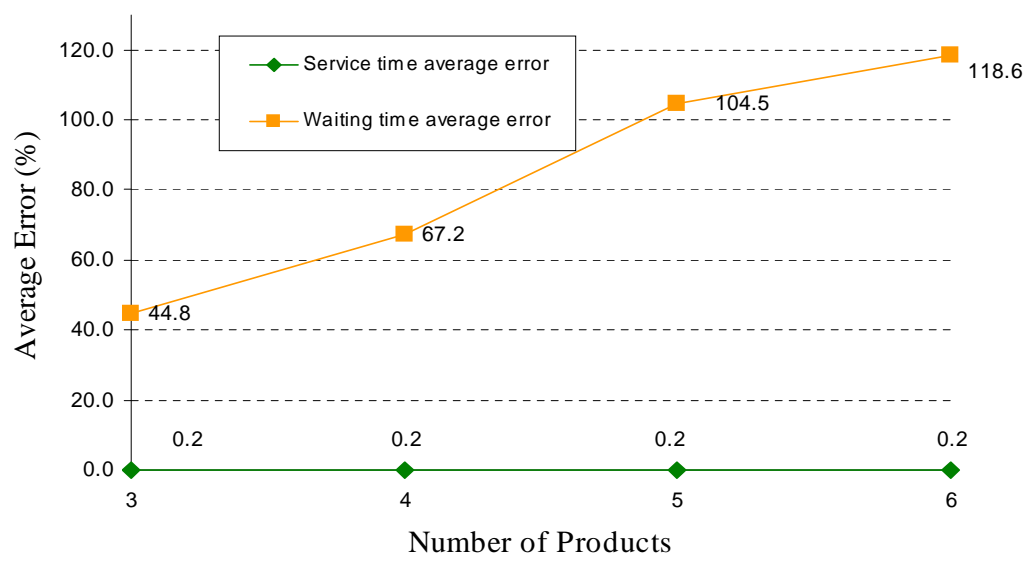

Figure 3.12: Service and waiting time average error

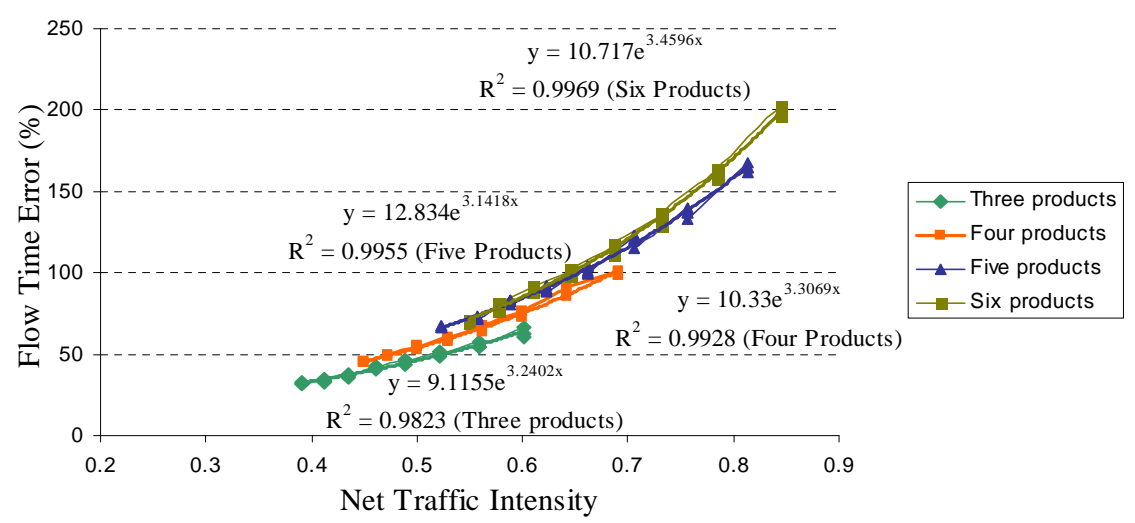

Figure 3.13: Flow time error in the waiting time.

$$
W A d_{k}=\sum_{j \in J_{k}} W A d_{j k} v_{j k} \quad \forall k \in K
$$

Appendix B presents the predicted flow time error using the regression equation for the thirty two problem instances. Figure 3.15 displays trends of the predicted and observed errors. It is evident from this figure that the predicted values from the regression equation follow the same trend as the observed values. 


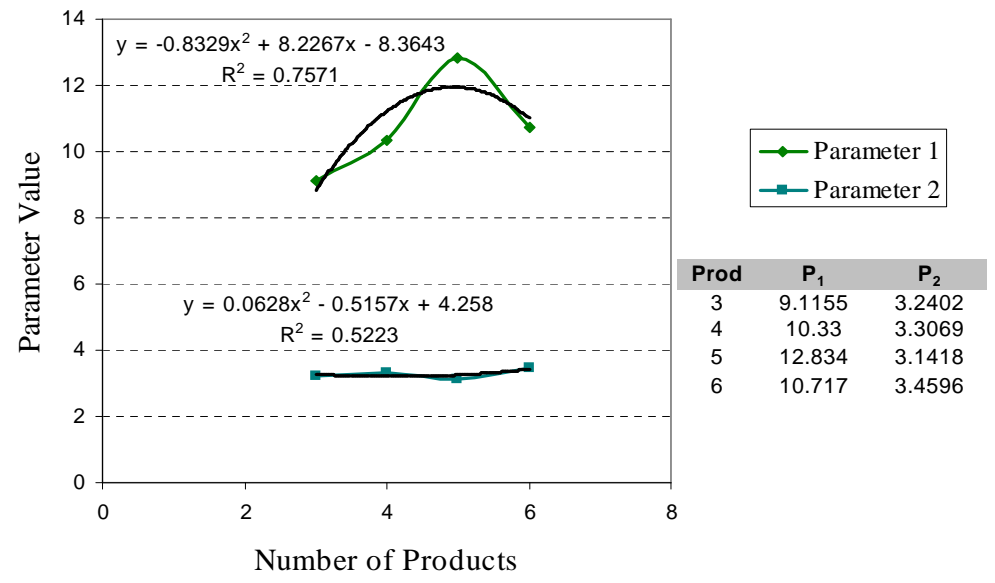

Figure 3.14: Regression equation parameters in terms of the number of products.

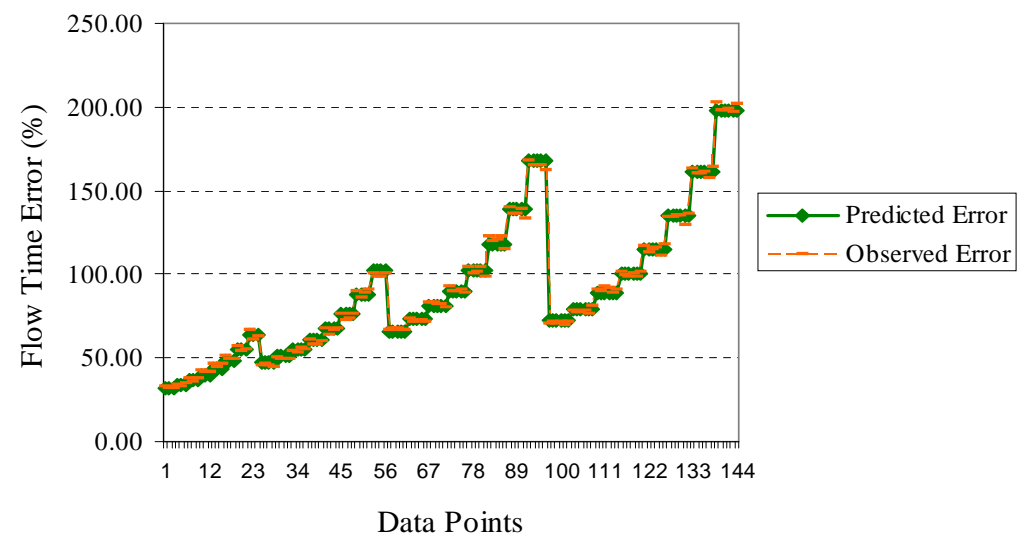

Figure 3.15: Predicted and observed flow time error.

\subsubsection{The case of fork-join stations}

For fork and join stations, seven hundred thirty five problem instances (2,547 data points) were generated. In order to develop the regression equation, numerical examples with interarrival and service times from exponential distributions and from general type distributions were considered. The cases of interarrival times and service times with general distributions are analyzed in section 4 . The objective was to try to have a single correction factor that could be used in any case analyzed in this research. 
One hundred ninety five problem instances (657 data points) were generated for the Markovian case. The number of product classes in the problem instances was varied from one to six, failures rates from zero to $30 \%$, and the number of servers and subtasks from two to four.

Figure 3.16 presents the flow time error of fork-join nodes against net traffic intensity for the problem instances generated. It is possible to identify a straight line relationship between both variables. The same relationship was observed with the variable failure rate (see Figures 3.17, 3.18, 3.19, 3.20, 3.21, and 3.22). Since it was observed a straight relationship between the variables, the Statistical Analysis Software (SAS) was used to determine the parameters of the model, and the stepwise procedure was used to identify the significant variables in the regression model. The regression equation with a R-squared of 0.5 for the Markovian case is given by

$$
E \hat{F} J_{j}=7.0274-2.85 * p-4.4 * N F J_{j}+2.62 * \sum_{k \varepsilon K} \frac{M F J_{j k}}{K}-1.08 * f+10.95 * \rho F J_{j}
$$

where $E \hat{F} J_{j}$ is the predicted flow time error at the fork-join $j, p$ the number of products at the fork-join node $j, N F J_{j}$ the number of nodes at the fork-join $j, \sum_{k \varepsilon K} \frac{M F J_{j k}}{K}$ an average of the number of subtasks at the fork-join $j, f$ is the failure rate at the fork point, and $\rho F J_{j}$ is the net traffic intensity at the fork-join $j$. Based on this regression equation, the number of products, the number of subtasks, the failure rate at the fork point, and the net traffic intensity at the fork-join nodes are significantly affecting the flow time in the node.

The approximation to determine the adjusted flow time at the fork-join $j$ of product class $k$ using the correction factor is given by 


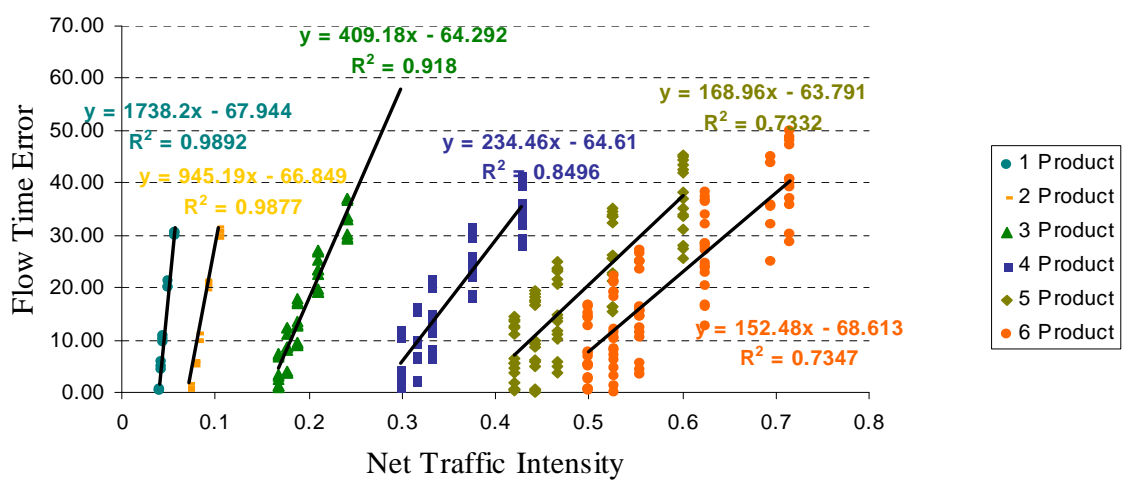

Figure 3.16: Flow time error of fork-join nodes against net traffic intensity.

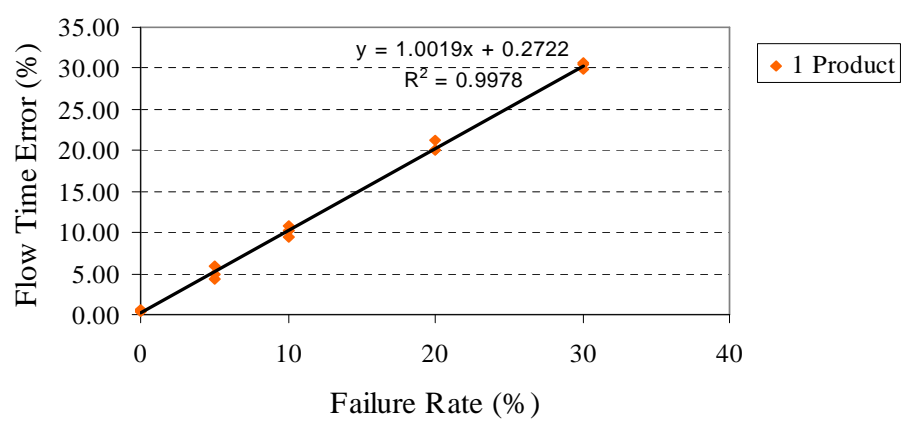

Figure 3.17: Flow time error from fork-join nodes against failure rate for one product instances.

$$
R A d_{j k}=\frac{R_{j k}}{\left(1+\frac{E \hat{F} J_{i}}{100}\right)}
$$

where $R_{j k}$ is computed either using equation 3.18 for two-serverfork-join queue, or using equation 3.2 for $N$ server fork-join queue.

Appendix $\mathrm{C}$ presents the predicted error in flow time along with the observed values for one hundred and fifty fork-join problem instances with Poisson arrivals and exponential service times. Figure 3.23 displays the trend of the predicted error and the observed values. The graph shows that the predicted values follow pretty much the same trend as the observed values. 


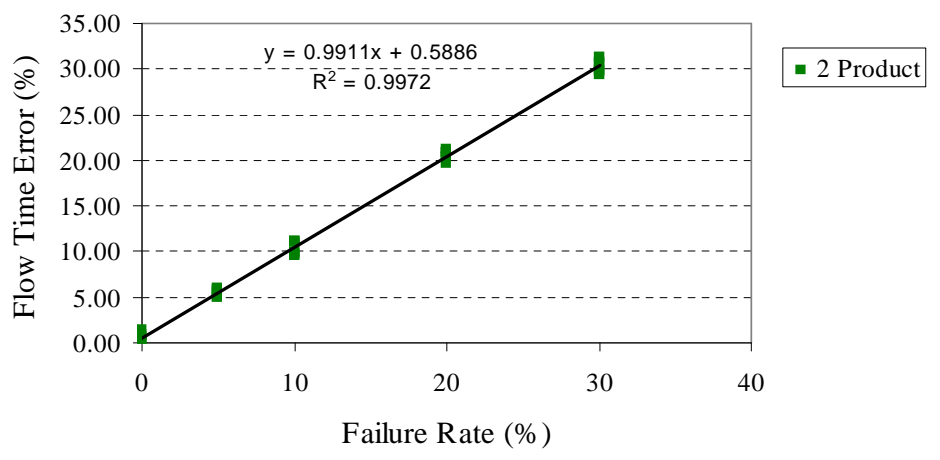

Figure 3.18: Flow time error from fork-join nodes against failure rate for two product instances.

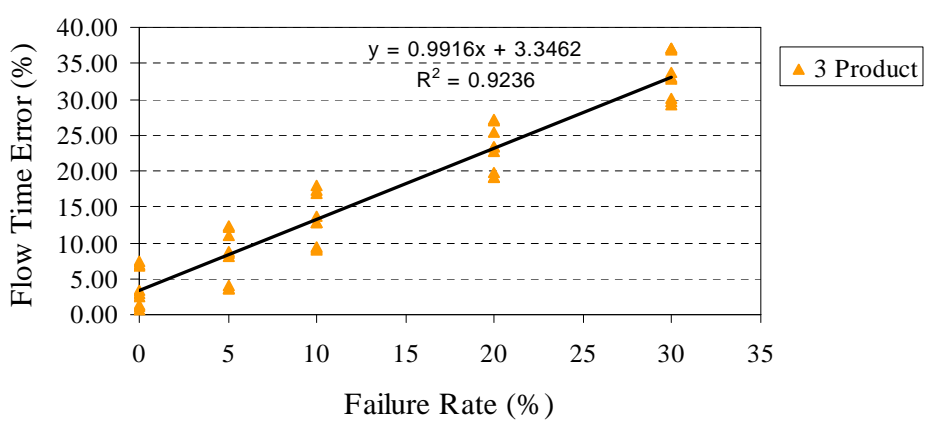

Figure 3.19: Flow time error from fork-join nodes against failure rate for three product instances.

\subsection{Numerical Comparisons}

Numerical comparisons were conducted in order to evaluate the performance of the correction factors in the approximations. Appendix D presents the error in flow time using the corrections factors for three, four, five and six product instances; and for problem instances where the number of subtasks was less than the number of servers in the fork-join stations. These values were compared with the results obtained using the analytical formulations without the correction factors. 


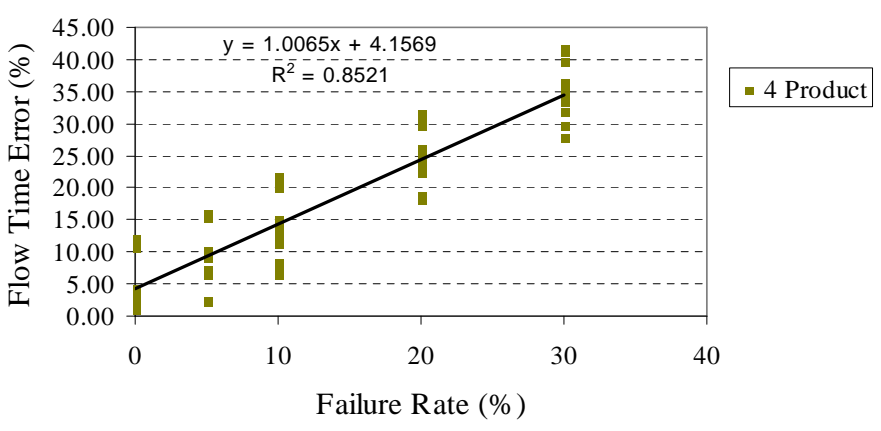

Figure 3.20: Flow time error from fork-join nodes against failure rate for four product instances.

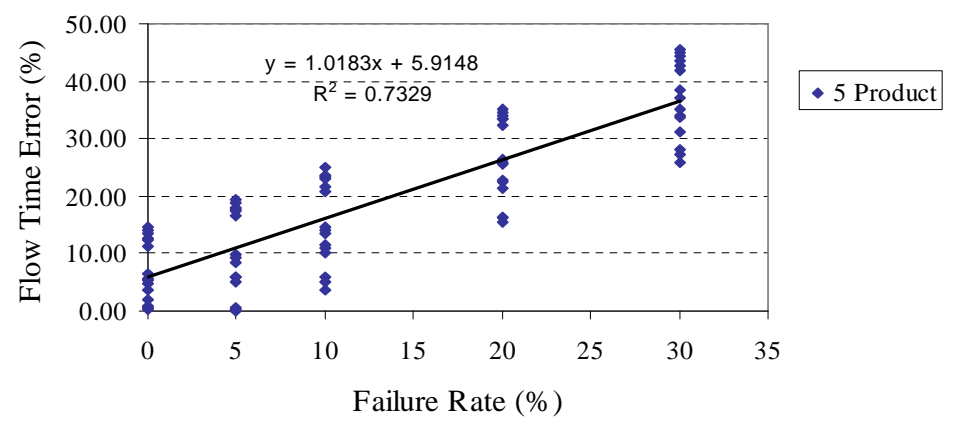

Figure 3.21: Flow time error from fork-join nodes against failure rate for five product instances.

Figure 3.24 shows the distribution of the flow time error for the ninety problem instances when the correction factors were used in the approximations. The histogram indicates that the error seems to be distributed now between zero and $20 \%$, and the error in $90 \%$ of the problem instances (207 out of 229 data points) was between zero and 13\%. Additionally, in Figure 3.25 is shown the performance of the approximations by comparing the flow time error with previous results where the correction terms are not used.

Detailed analysis by product is presented in Figures 3.26, 3.27, 3.28, and 3.29. These figures show the trend of the flow time error when the correction terms are used in the approximations for three, four, five and six product instances respectively. In addition, these results are compared with the flow time error when the correction terms are not used in the 


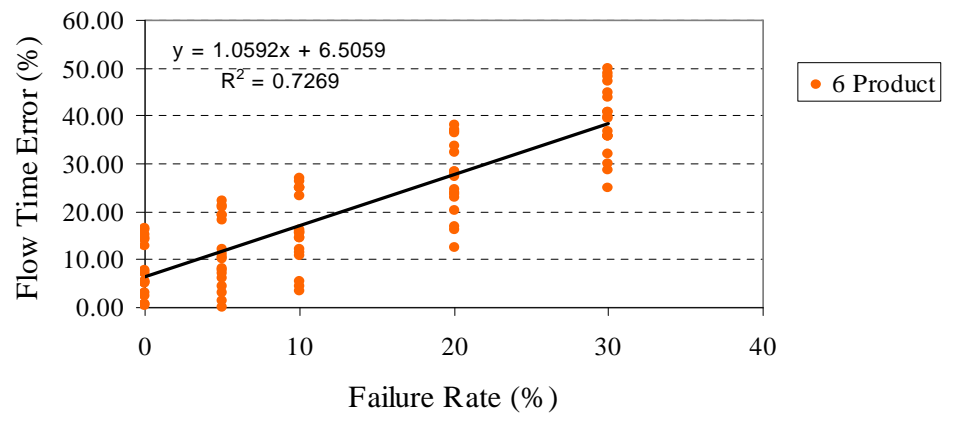

Figure 3.22: Flow time error from fork-join nodes against failure rate for six product instances.

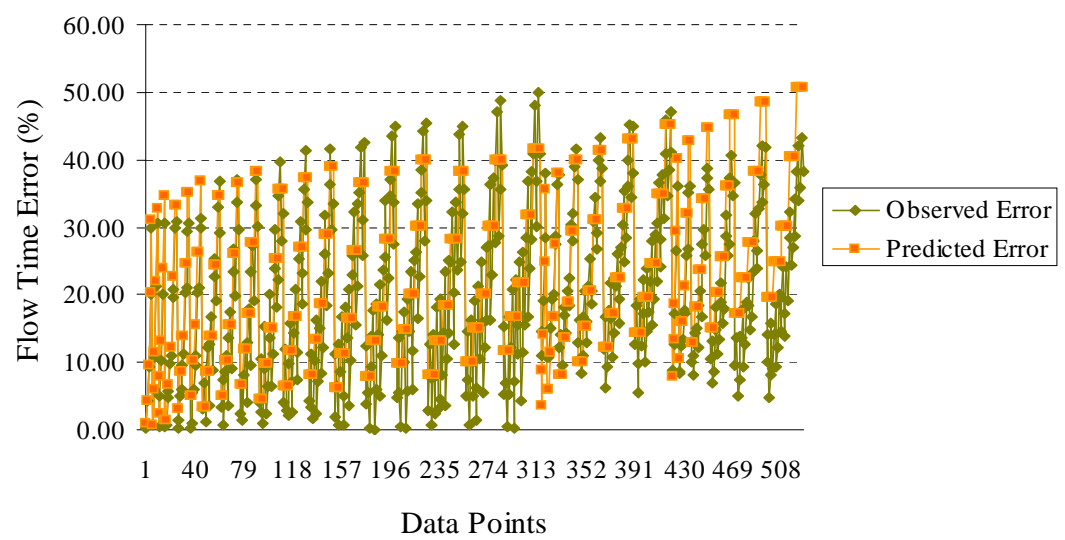

Figure 3.23: Trend of predicted values for fork and join stations.

approximations. The graphs clearly indicate that the flow time error for most of the problem instances was reduced when the correction terms were added to the approximations. Additionally, in Table 3.5 is presented the results of the paired-t test by product, and in Table 3.6 is presented comparisons of the average error by product class.

In the case of three product instances, the flow time error in $93 \%$ of the data points (42 out of 45 data points) was between zero and 16\%, and the maximum error was 19\% (see Figure 3.30). The data strongly suggests that the means are the same at 0.01 level of significance. On the other hand, for four product instances the error in $92 \%$ of the data points (44 out of 48 data points) was between zero and 17\% (see Figure 3.31), and for five product instances 


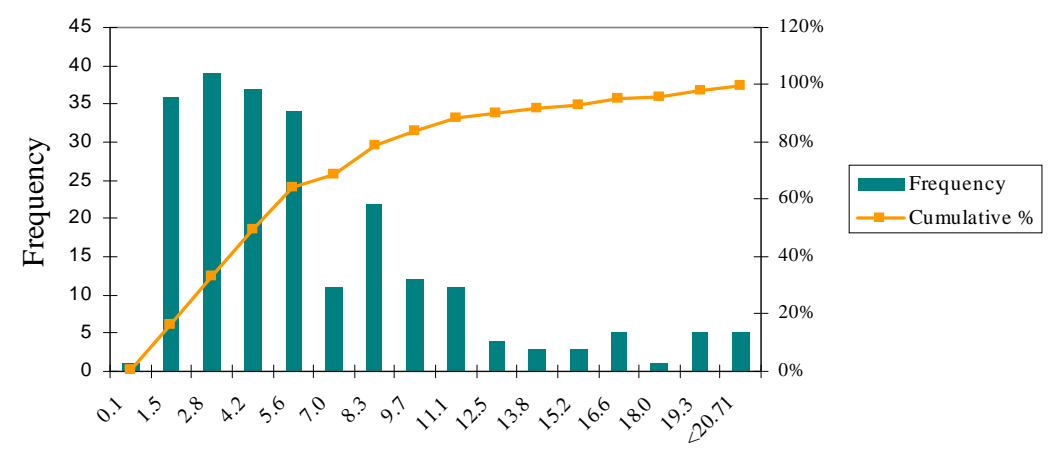

Flow Time Error $(\%)$

Figure 3.24: Flow time error using the correction factors.

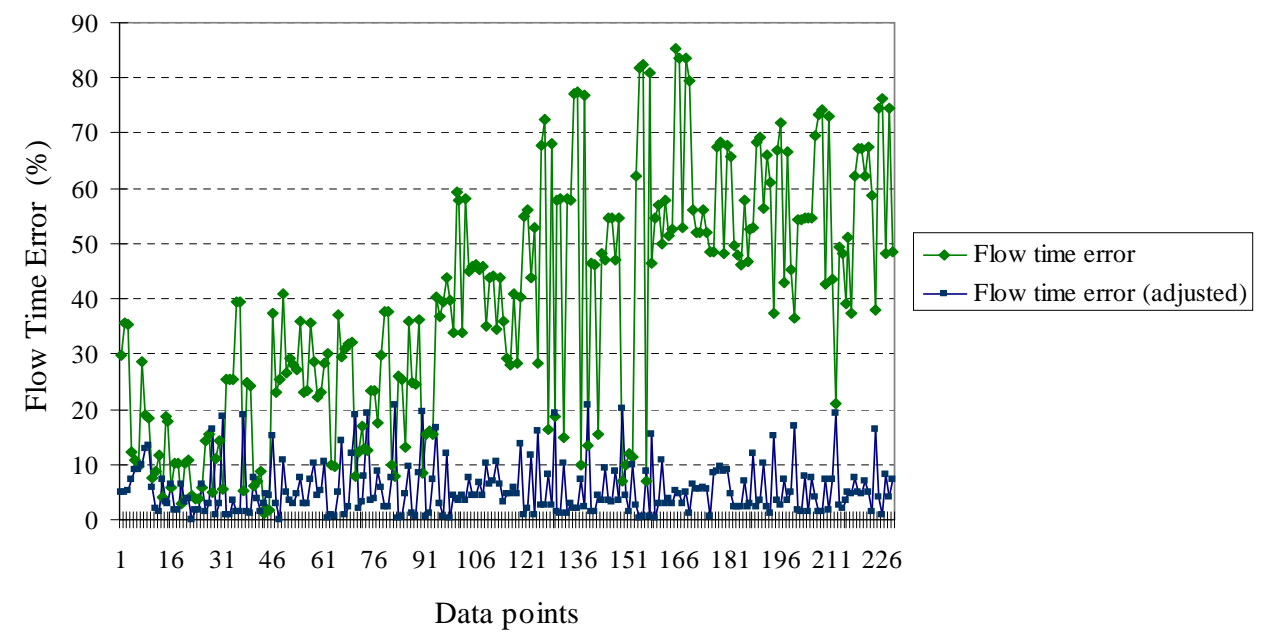

Figure 3.25: Flow time error obtained using correction factors.

the error in $95 \%$ of the data points (61 out of 64 data points) was between zero and $18 \%$ (see Figure 3.32). In the case of six products the error in $97 \%$ of the data points (70 out of 72) was between zero and 17\%, and the maximum error was 19\% (see Figure 3.33). The data strongly suggests that at 0.01 level of significance the means are not the same for four five, and six product problem instances.

Additional analysis was conducted to study the case when the number of subtasks was less than the number of servers. Figure 3.34 shows the trend of the flow time error when the correction terms are applied in the approximations. In addition, these results are compared 


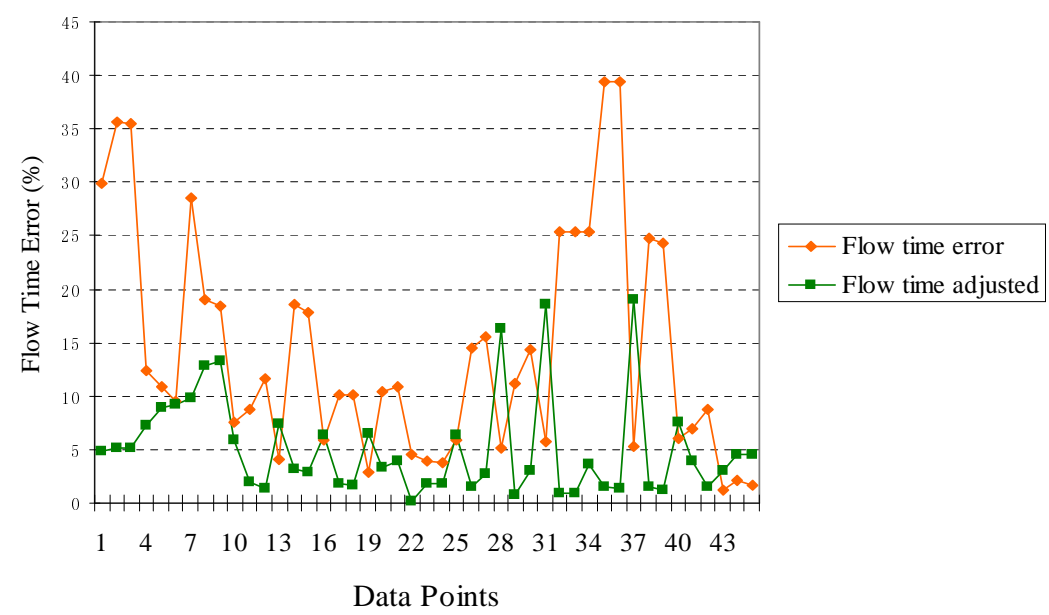

Figure 3.26: Flow time error using correction factors for three product instances.

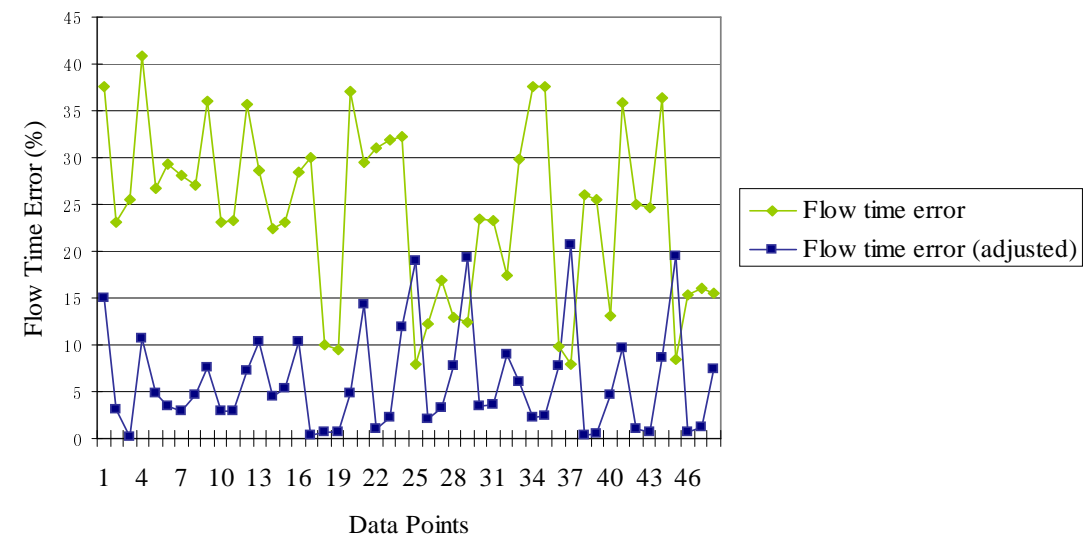

Figure 3.27: Flow time error using correction factors for four product instances.

with the flow time error when the correction terms are not used in the approximations. The trends indicate that the flow time error considerably reduced when the corrections term were used, and for most of the problems the error was between zero and $10 \%$. The numerical comparisons show that the average flow time error was reduced about $85.8 \%$. The average error decreased from $39.03 \%$ to $5.54 \%$.

In summary, the numerical comparisons show that the approximations perform remarkably well when the corrections factors are used. The average flow time error for three product instances reduced from $14.20 \%$ to $5.13 \%$, for four product instances from $24.19 \%$ to 


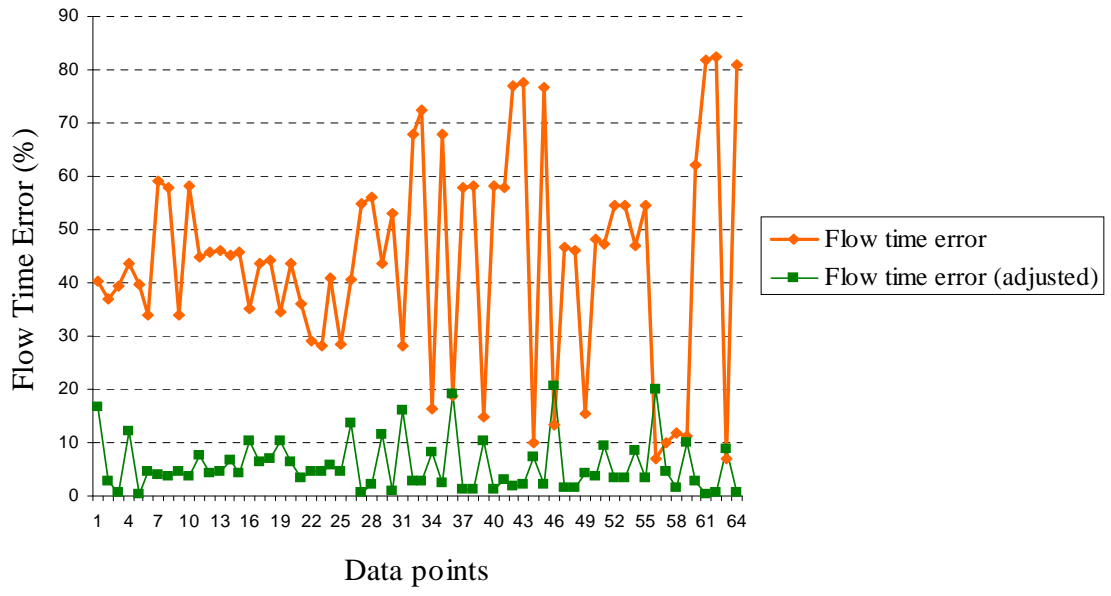

Figure 3.28: Flow time error using correction factors for five product instances.

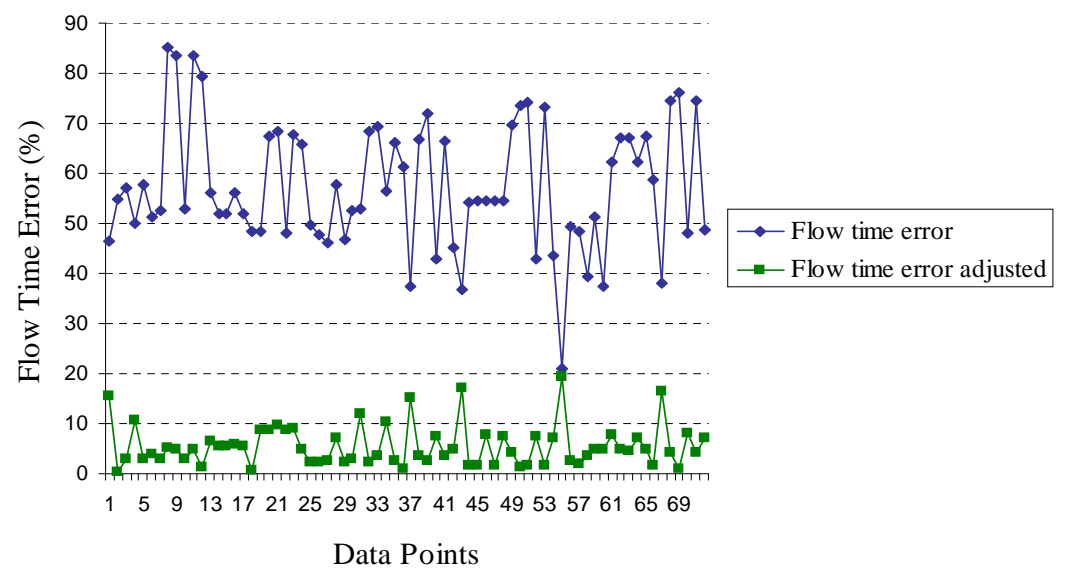

Figure 3.29: Flow time error using correction factors for six product instances.

$6.10 \%$, for five product instances from $44.13 \%$ to $5.66 \%$, and for six product instances from $57.23 \%$ to $5.47 \%$. In general, the average flow time error was reduced about $85.36 \%$ from $38.19 \%$ to $5.59 \%$. 
Table 3.5: Paired-t test results per product class when the correction factors were used in the approximations

\begin{tabular}{|c|c|c|c|c|}
\hline \multicolumn{5}{|c|}{ Product Class } \\
\hline \hline & 3 & 4 & 5 & 6 \\
\hline t Stat & 2.88 & 3.85 & 3.81 & 3.84 \\
\hline t Critical two-tail & 2.69 & 2.01 & 2.00 & 1.99 \\
\hline $\mathrm{P}(\mathrm{T}<=\mathrm{t})$ two-tail & 0.01 & 0.00 & 0.00 & 0.00 \\
\hline
\end{tabular}

Table 3.6: Comparisons of average flow time error by product class

\begin{tabular}{|c|c|c|c|c|}
\hline \multicolumn{5}{|c|}{ Product Class } \\
\hline \hline$(\%)$ & 3 & 4 & 5 & 6 \\
\hline Average Error (no corr. Fact.) & 14.2 & 24.2 & 44.1 & 57.2 \\
\hline Average Error (using corr. Fact.) & 5.1 & 6.1 & 5.7 & 5.5 \\
\hline
\end{tabular}

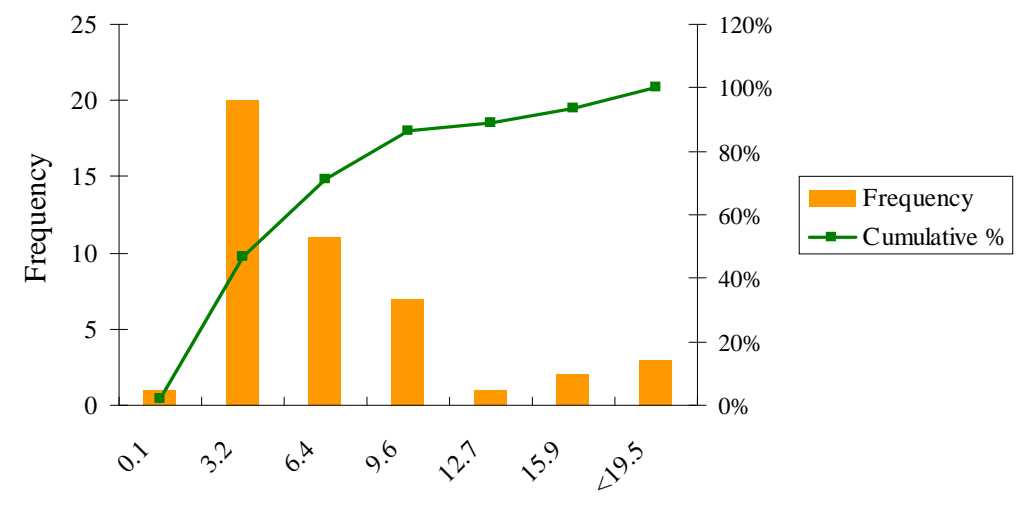

Flow Time Error $(\%)$

Figure 3.30: Flow time error for three product instances using the correction factors.

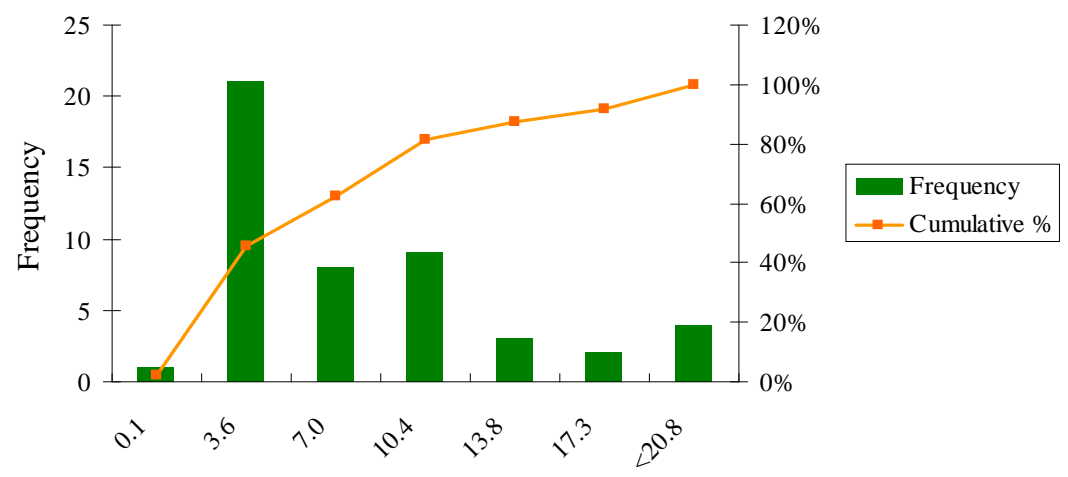

Flow Time Error (\%)

Figure 3.31: Flow time error for four product instances using the correction factors. 


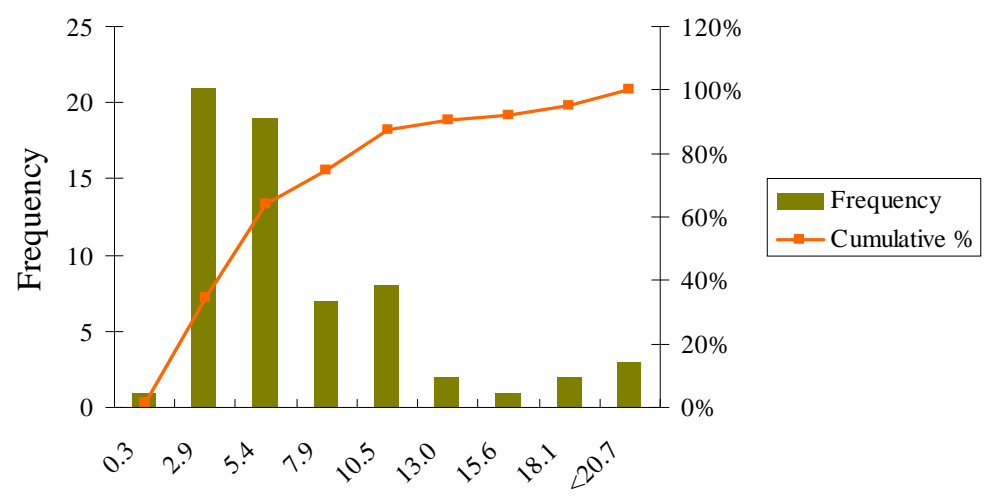

Flow Time Error (\%)

Figure 3.32: Flow time error for five product instances using the correction factors.

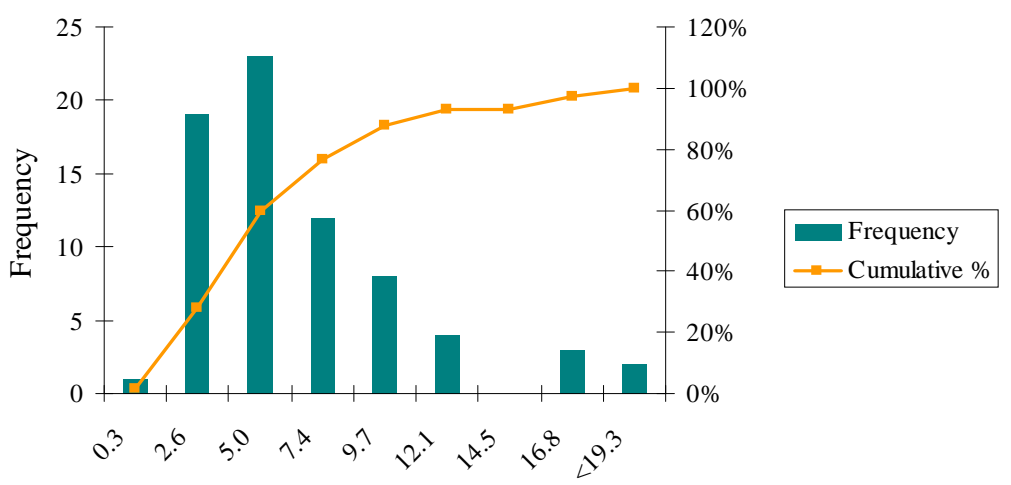

Flow Time Error $(\%)$

Figure 3.33: Flow time error for six product instances using the correction factor.

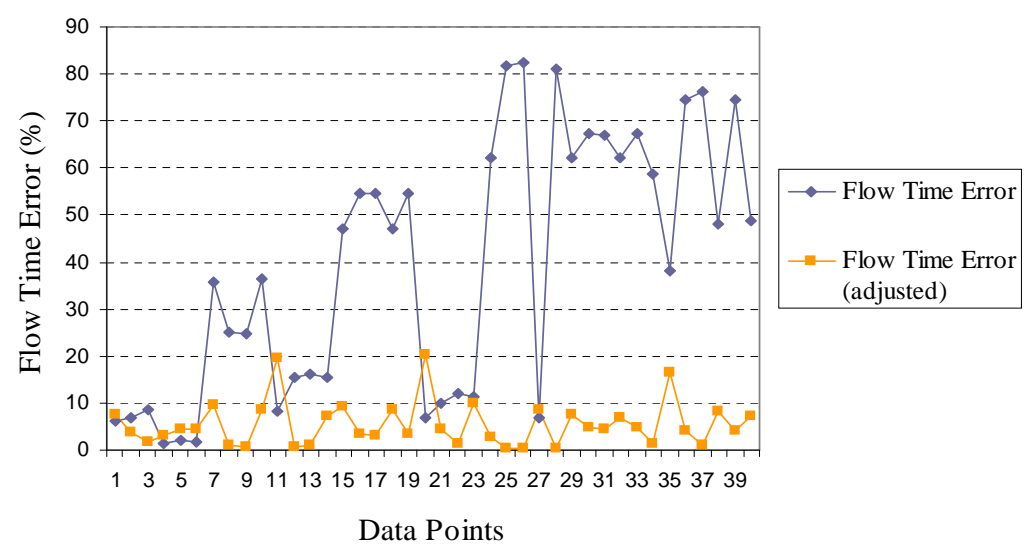

Figure 3.34: Flow time error using correction factors for problem instances where the number of subtasks is less than servers. 


\section{CHAPTER 4}

\section{FORMULATION OF THE ANALYTICAL MODEL FOR THE G/G/c SYSTEM}

This chapter analyzes the case of the web server manufacturing process as an open queueing network with independent $G / G / c$ queues. The standard $G / G / c$ system has a single service facility with $c$ identical servers, unlimited waiting room and the FCFS discipline. The interarrival times are independent of the service times, and the service times are independent and identically distributed with a general distribution.

The approximations presented in this chapter are similar to the Markovian case discussed in chapter 3. The parametric decomposition approximation method, based on the Whitt's approach [110], is used to analyze the server manufacturing process. Under this method, the queueing network is decomposed into individual $G / G / c$ queues that are analyzed separately, after characterizing the interarrival and service times by their first two moments $\left(\lambda, C_{a}, \tau, C_{s}\right)$.

The formulations for the $G / G / c$ system, presented in this chapter, are based on the work done by Jackson [47], Whitt [110] [112], the modifications done by Pradhan et al. [86] to incorporate the probabilistic flow of jobs between stations in the network system, and the work done on fork-join queues with general distributions by Varma and Makowski [107]. The new notation used in this section is as follows (the remaining notation was presented in chapter 3): 


\section{Notation}

$C a_{0 j} \quad$ squared coefficient of variation of the external arrival process to node $j$.

$C a_{j k} \quad$ squared coefficient of variation of the arrival process to node $j$ for class $k$.

$C s_{j k} \quad$ squared coefficient of variation of the service time distribution at node $j$ for class $k$.

\subsection{The Internal Flow Parameters}

In the case of $G / G / c$ systems additional information is required to analyze the queueing network for the web server manufacturing process. For each product class, it is necessary to specify the squared coefficient of variation of the external arrival process $C a_{0 j k}$, and the service time distribution $C s_{j k}$. The dependence among successive interarrival times, and the actual properties of the arrival process at each queue is captured by the two arrival parameters $\lambda_{j k}$, and $C a_{j k}[112]$.

The parameters for the internal flows such as traffic rates and coefficients of variation for the arrival processes, are calculated by solving systems of linear equations. As stated in chapter 3 the traffic rate $\left(\lambda_{j k}\right)$ at node $j$ by product class $k$ is calculated by equation 3.1 , the mean number of visits $\left(v_{k}\right)$ to each node for product class $k$ by equation 3.3 , the aggregate service time $\left(\tau_{j}\right)$ at node $j$ by equation 3.5 , and the traffic intensity at each node by equation 3.6 .

The variability parameter of the service time distribution at the node $j$ can be calculated using Pradhan's equation [86], which incorporates the stochastic flow of jobs between nodes. The formula is based on the work done by Whitt [110], in which the node variability parameters, $C s_{j}$ is calculated using the property that "the second moment of a mixture of 
distributions is the mixture of the second moments". The aggregate value of $C s_{j}$ is calculated by using

$$
\tau_{j}^{2}\left(C s_{j}+1\right)=\frac{\sum_{k \in K} \lambda_{0 j k} \tau_{j k}^{2}\left(C s_{j k}+1\right)+\sum_{k \in K} \sum_{i \in I_{k}} \lambda_{i j k} \tau_{j k}^{2}\left(C s_{j k}+1\right) v_{j k}}{\sum_{k \in K} \lambda_{0 j k}+\sum_{k \in K} \sum_{i \in I_{k}} \lambda_{i j k} v_{j k}} \quad \forall j \in J .
$$

In order to calculate the coefficients of variation for the arrival process for each product class, the approximations developed by Whitt [110] were used. This set of simultaneous equations are based on the asymptotic and the stationary interval methods, as well as hybrid approximations for superposition of arrival processes. The equations are linear, of the form

$$
C a_{j k}=a_{j k}+\sum_{i \in J_{k}} C a_{i k} b_{i j k} \quad \forall j \in J_{k}, k \in K
$$

where $a_{j k}$ and $b_{i j k}$ are constants, depending on the input data

$$
a_{j k}=1+w_{j k}\left\{\left(p_{0 j k} C a_{0 j}-1\right)+\sum_{i \in J_{k}} p_{i j k}\left[\left(1-q_{i j k}\right)+\left(1-v_{i j k}\right) q_{i j k} \rho_{i}^{2} x_{i}\right]\right\}
$$

and

$$
b_{i j k}=w_{j k} p_{i j k} q_{i j k}\left[v_{i j k}+\left(1-v_{i j k}\right)\left(1-\rho_{i}^{2}\right)\right]
$$

The variables $x_{i}$ and $v_{i j k}$ are used to specify the departure operation, and the variable $w_{j k}$ is used to specify the superposition operation. The specific values of this variables according to Whitt [110] are

$$
\begin{gathered}
x_{i}=1+\frac{\max \left(C s_{i}, 0.2\right)-1}{\sqrt{c_{i}}}, \\
v_{i j k}=0, \\
w_{j k}=\frac{1}{\left[1+4\left(1-\rho_{j}\right)^{2}\left(\bar{v}_{j k}-1\right)\right]},
\end{gathered}
$$




$$
\bar{v}_{j k}=\left[\sum_{i=0}^{I_{k}} p_{i j k}^{2}\right]^{-1}
$$

The value of $v_{i j k}$ is chosen to satisfy $0 \leq v_{i j k} \leq 1$ and increasing in $\rho_{j}$. However, according to previous work done by Whitt [111] it has not yet been found that positive value of $v_{i j k}$ helps; thus, it was set to zero. The variables $x_{i}, v_{i j k}$, and $w_{j k}$ depend on the data calculated previously, e.g., $\rho_{j}$ is the net traffic intensity in $3.6, c_{i}$ is the number of servers, and $C s_{j}$ is the variability parameter of the service time distribution at the node $j$ in 4.1 .

The variability parameters $C a_{0 j}$ of the external arrival process is calculated by using a hybrid approximation for superposition arrival processes [110] . If $\lambda_{0 j}=0$, then $C a_{0 j}=1$. Otherwise,

$$
C a_{0 j}=\left(1-\bar{w}_{j}\right)+\bar{w}_{j}\left[\sum_{k \in K} C a_{0 j k}\left(\frac{\lambda_{0 j k}}{\sum_{k \in K} \lambda_{0 j k}}\right)\right],
$$

where

$$
\bar{w}_{j}=\frac{1}{\left[1+4\left(1-\rho_{j}\right)^{2}\left(\bar{v}_{j}-1\right)\right]},
$$

and

$$
\bar{v}_{j}=\left[\sum_{k \in K}\left(\frac{\lambda_{0 j k}}{\sum_{k \in K} \lambda_{0 j k}}\right)^{2}\right]^{-1}
$$

\subsection{The Performance Measures}

As for the Markovian case each station in the network can be analyzed independently, and the performance measures for the network as a whole are calculated by aggregating the individual performance measures. In the case of single stations, the formulations for the $M / M / c$ system are used to construct the approximations for the $G / G / c$ system [112] and 
for the fork-join stations the interpolations between heavy and light traffic limits are used to predict the response time in those nodes [107].

\subsubsection{The single stations}

In the case of general distributions, the expected number of jobs at each node for product class $L(G / G / c)_{j k}$ is calculated by adding the expected number of jobs in service for each product class $L s(G / G / c)_{j k}$ (see equation 3.9, and the number of jobs in queue for each product class $L q(G / G / c)_{j k}$. The number of jobs in queue for each product class $L q(G / G / c)_{j k}$ is calculated by using the Little's formula

$$
L q(G / G / c)_{j k}=\lambda_{j k} W_{q}(G / G / c)_{j k}
$$

where $\lambda_{j k}$ is calculated using equation 3.1 , and $W_{q}(G / G / c)_{j k}$ is the waiting time at node $j$ for product class $k$ for general distributions. The waiting time at node $j$ for product class $k$ is computed using the generic equations for a $G / G / c$ system developed by Whitt [112], based on previous work done by Cosmetatos [24], and Kraemer and Langenbach-Belz [57]. The approximation is of the form

$$
W_{q}(G / G / c)_{j k}=\phi\left(\rho_{j}, C a_{j k}, C s_{j}, c_{j}\right)\left(\frac{C a_{j k}+C s_{j}}{2}\right) W_{q}(M / M / c)_{j k},
$$

where

$$
\begin{aligned}
\phi & \left(\rho_{j}, C a_{j k}, C s_{j}, c_{j}\right) \\
= & \begin{cases}\left(\frac{4\left(C a_{j k}-C s_{j}\right)}{4 C a_{j k}-3 C s_{j}}\right) \phi_{1}\left(c_{j}, \rho_{j}\right)+\left(\frac{C s_{j}}{4 C a_{j k}-3 C s_{j}}\right) \psi\left(\frac{C a_{j k}+C s_{j}}{2}, c_{j}, \rho_{j}\right), & C a_{j k} \geq C s_{j} \\
\left(\frac{C s_{j k}-C a_{j k}}{2 C a_{j k}-2 C s_{j}}\right) \phi_{3}\left(c_{j}, \rho_{j}\right)+\left(\frac{C s_{j k}+3 C a_{j k}}{2 C a_{j k}-2 C s_{j}}\right) \psi\left(\frac{C a_{j k}+C s_{j}}{2}, c_{j}, \rho_{j}\right), & C a_{j k} \leq C s_{j}\end{cases}
\end{aligned}
$$




$$
\begin{gathered}
\phi_{1}\left(c_{j}, \rho_{j}\right)=1+\min \left\{0.24, \frac{\left(1-\rho_{j}\right)\left(c_{j}-1\right)\left(\left(4+5 c_{j}\right)^{1 / 2}-2\right)}{16 c_{j} \rho_{j}}\right\}, \\
\phi_{3}\left(c_{j}, \rho_{j}\right)=\left(1-4 \min \left\{0.24, \frac{\left(1-\rho_{j}\right)(c-1)\left(\left(4+5 c_{j}\right)^{1 / 2}-2\right)}{16 c_{j} \rho_{j}}\right\}\right) \exp \left(\frac{-2\left(1-\rho_{j}\right)}{3 \rho_{j}}\right), \\
\psi\left(\frac{C a_{j k}+C s_{j}}{2}, c_{j}, \rho_{j}\right)=\left\{\begin{array}{l}
1, \\
\phi_{4}\left(c_{j}, \rho_{j}\right)^{2\left(1-\frac{C a_{j k}+C s_{j k}}{2}\right)} \quad \frac{C a_{j k}+C s_{j}}{2} \geq 1
\end{array}\right] \\
\phi_{4}\left(c_{j}, \rho_{j}\right)=\min \left\{1, \frac{C a_{j k}+C s_{j}}{2} \leq 1\right.
\end{gathered}
$$

and the waiting time for the Markovian case $W_{q}(M / M / c)_{j k}$ is calculated by equation 3.26 However, since the problem under study considers multiple products, the average of the waiting time at a given node need to be calculated. The equation is given by

$$
\bar{W}_{q}(G / G / c)_{j k}=\frac{\sum_{k \in K} W_{q}(G / G / c)_{j k}}{\sum_{k \in K} Z_{j k}} \quad \forall j \in J_{k} .
$$

Another congestion measure is the expected flow time of a job through the network. In the case of singles stations, this is calculated by

$$
W(G / G / c)_{k}=\sum_{j \in J_{k}} W(G / G / c)_{j k} v_{j k} \quad \forall k \in K,
$$

where 


$$
W(G / G / c)_{j k}=W s_{j k}+\bar{W}_{q}(G / G / c)_{j k} \quad \forall j \in J_{k}, k \in K,
$$

the expected time of a job in service $W s_{j k}$ is calculated by equation 3.14 and $\bar{W}_{q}(G / G / c)_{j k}$ is computed with equation 4.13 .

\subsubsection{The fork-Join stations}

The formulations to predict the response time at $N$ homogeneous server fork-join queues for the general case are based on the work done by Varma and Makowski [107]. They obtained interpolation approximations for fork-join queues for general arrival and service times by using an estimate of the heavy traffic limit in conjunction with light traffic information. In contrast with the original formulas, in this research the net traffic intensity at fork-join $j$ is calculated by equation 3.20 . This modified formula takes into account that a node can be visited by different product classes, and the job at the fork point is divided into a number of subtasks that could be less than the number of servers in the fork-join node. The following cases were analyzed:

1. Erlang-2 arrivals and Erlang-2 service times.

2. Erlang-2 arrivals and exponential service times.

3. Poisson arrivals and hyper-exponential service times.

4. Poisson arrivals and Erlang-2 service times.

5. Hyper-exponential arrivals and exponential service times. 


\subsubsection{The Erlang-2 arrivals and Erlang-2 service times case}

The response time for product class $k$ in the homogeneous $N$ server fork-join subsystem $j$ with Erlang-2 interarrival and service time distributions is calculated as follows :

$$
R_{j k}=\left[F_{N}+\left(\frac{V_{N}}{2}-F_{N}\right) \rho F J_{j}\right] \frac{1}{\mu F J_{j k}\left(1-\rho F J_{j}\right)},
$$

where

$$
\begin{gathered}
F_{N}=\sum_{r=1}^{N}\left(\begin{array}{c}
N \\
r
\end{array}\right)(-1)^{r-1} \sum_{m=0}^{r}\left(\begin{array}{c}
r \\
m
\end{array}\right) \frac{m !}{2 r^{m+1}}, \\
V_{N}=\sum_{r=1}^{N}\left(\begin{array}{c}
N \\
r
\end{array}\right)(-1)^{r-1} \sum_{m=1}^{r}\left(\begin{array}{c}
r \\
m
\end{array}\right) \frac{(m-1) !}{r^{m+1}},
\end{gathered}
$$

and $\rho F J_{j}$ is the net traffic intensity at fork-join $j$ calculated by equation 3.20 [107].

\subsubsection{The Erlang-2 arrivals and exponential service times case}

The response time for product class $k$ in the homogeneous $N$ server fork-join subsystem $j$ with Erlang-2 interarrival and exponential service time distributions is calculated by the equation

$$
R_{j k}=\left[H_{N}+\left(\frac{2}{3} V_{N}-\frac{5}{6} H_{N}-\frac{1}{12}\right) \rho F J_{j k}\right] \frac{1}{\mu F J_{j k}\left(1-\rho F J_{j}\right)},
$$

where $H_{N}$ is calculated by equation 2.3. $F_{N}$ by equation 4.23, and $V_{N}$ by equation 4.24 [107]. 


\subsubsection{The Poisson arrivals and hyper-exponential service times case}

In the case of Poisson arrivals and hyper-exponential service times, a $\mathrm{H}_{2}$ service time distribution is assumed with mean $\mu F J_{j k}=p_{1} / \mu_{1} F J_{j k}+p_{2} / \mu_{2} F J_{j k}, 0<p_{1}<1, p_{2}=1-p_{1}$, and $\mu_{1} F J_{j k}>\mu_{2} F J_{j k}$. In addition, it was considered balanced means, which produces an $H_{2}$ distribution approximately in the middle of the range for given first two moments [112]. Balanced means considers, $p_{1} / \mu_{1} F J_{j k}=p_{2} / \mu_{2} F J_{j k}=\frac{1}{2}$ so that $\mu_{1} F J_{j k}+\mu_{2} F J_{j k}=2$.

The response time for product class $k$ in the homogeneous $N$ server fork-join subsystem $j$ with Poisson arrivals and $\mathrm{H}_{2}$ service time distributions is calculated by the equation

$$
\begin{array}{r}
R_{j k}=\frac{\left(\mu F J_{j k}\right) D_{N}}{\mu F J_{j k}\left(1-\rho F J_{j}\right)}+\left[\left(\mu F J_{j k}\right) E_{N}-D_{N}\right] \frac{\lambda F J_{j k}}{\mu F J_{j k}\left(1-\rho F J_{j}\right)}+ \\
{\left[h T r a f f i c-\left(\mu F J_{j k}\right)^{2} E_{N}\right] \frac{\left(\rho F J_{j}\right)^{2}}{\mu F J_{j k}\left(1-\rho F J_{j}\right)},}
\end{array}
$$

where

$$
D_{N}=\sum_{r=1}^{N}\left(\begin{array}{c}
N \\
r
\end{array}\right)(-1)^{r+1} \sum_{m=0}^{r}\left(\begin{array}{c}
r \\
m
\end{array}\right) \frac{p_{1}^{m} p_{2}^{r-m}}{m\left(\mu_{1} F J_{j k}\right)+(r-m)\left(\mu_{2} F J_{j k}\right)}
$$




$$
\begin{aligned}
& E_{N}=\sum_{r=1}^{N}\left(\begin{array}{l}
N \\
r
\end{array}\right)(-1)^{r+1} \sum_{m_{1}=0}^{N-r}(-1)^{m_{1}} \sum_{m_{2}=0}^{m_{1}}\left(\begin{array}{l}
m_{1} \\
m_{2}
\end{array}\right) p_{1}^{m_{2}} p_{2}^{m_{1}-m_{2}} \\
& \times \sum_{k_{1}=0}^{r}\left(\begin{array}{c}
r \\
k_{1}
\end{array}\right)\left(\frac{p_{1} p_{2}}{\mu_{1} F J_{j k}-\mu_{2} F J_{j k}}\right)^{r-k_{1}} \sum_{k_{2}=0}^{k_{1}}\left(\begin{array}{c}
k_{1} \\
k_{2}
\end{array}\right)\left(p_{1}^{2} \mu_{1} F J_{j k}\right)^{k_{2}}\left(p_{2}^{2} \mu_{2} F J_{j k}\right)^{k_{1}-k_{2}} \\
& \times \sum_{k_{3}=0}^{r-k_{1}}\left(\begin{array}{c}
r-k_{1} \\
k_{3}
\end{array}\right)(-1)^{r-k_{1}-k_{3}} \sum_{k_{4}=0}^{r-k_{1}}\left(\begin{array}{c}
r-k_{1} \\
k_{4}
\end{array}\right)\left(\mu_{2} F J_{j k}\right)^{k_{4}}\left(\mu_{1} F J_{j k}\right)^{r-k_{1}-k_{4}} \\
& \times \frac{k_{1} \text { ! }}{\left(\mu_{1} F J_{j k}\left(m_{2}+k_{2}+k_{3}\right)+\mu_{2} F J_{j k}\left(m_{1}-m_{2}-k_{2}+r-k_{3}\right)\right)^{k_{1}+1}} \\
& \times \frac{1}{\mu_{1} F J_{j k}\left(k_{2}+k_{4}\right)+\mu_{2} F J_{j k}\left(r-k_{2}-k_{4}\right)}, \\
& \text { hTraffic }=\left[H_{N}+\left(4 V_{N}-3 H_{N}-1\right) \beta+2\left(1+H_{N}-2 V_{N}\right) \beta^{2}\right] \frac{\sigma^{2}+\sigma_{0}^{2}}{2} \mu F J_{j k}^{2}, \\
& \sigma^{2}=2\left[\frac{p_{1}}{\left(\mu_{1} F J_{j k}\right)^{2}}+\frac{p_{2}}{\left(\mu_{2} F J_{j k}\right)^{2}}\right]-\left[\frac{p_{1}}{\mu_{1} F J_{j k}}+\frac{p_{2}}{\mu_{2} F J_{j k}}\right]^{2}, \\
& \sigma_{0}^{2}=\frac{1}{\left(\mu F J_{j k}\right)^{2}}
\end{aligned}
$$

and

$$
\beta=\frac{\sigma_{0}^{2}}{\sigma_{0}^{2}+\sigma^{2}},
$$

where $H_{N}$ is calculated by equation 2.3, $V_{N}$ by equation $4.24, \sigma^{2}$ and $\sigma_{0}^{2}$ are the variances of the service time and interarrival distributions respectively, and $\beta$ is a valued parameter in the heavy traffic limit [107]. 


\subsubsection{The Poisson arrivals and Erlang-2 service times case}

The response time approximation for product class $k$ in the homogeneous $N$ server fork-join subsystem $j$ with Poisson arrivals and Erlang-2 service times is given by

$$
R_{j k}=\left[F_{N}+\left(\frac{1}{6}-\frac{H_{N}}{12}+\frac{2}{3} V_{N}-F_{N}\right) \rho F J_{j}\right] \frac{1}{\mu F J_{j k}\left(1-\rho F J_{j}\right)},
$$

where $H_{N}$ is calculated by equation 2.3, $F_{N}$ by equation $4.23, V_{N}$ by equation 4.24 , and $\rho F J_{j}$ is the net traffic intensity at fork-join $j[107$.

\subsubsection{The hyper-exponential arrivals and exponential service times case}

In this case, it is assumed a $H_{2}$ arrival distribution with mean $\lambda F J_{j k}=p_{1} / \lambda_{1} F J_{j k}+p_{2} / \lambda_{2} F J_{j k}$,

$0<p_{1}<1, p_{2}=1-p_{1}, \lambda_{1} F J_{j k}>\lambda_{2} F J_{j k}$, and variance calculated by equation 4.30 . In addition, the variance of the service time is calculated by equation 4.31 . The response time approximation is of the form

$$
R_{j k}=\left[H_{N}+\left(h \text { Traffic }-H_{N}\right) \rho F J_{j}\right] \frac{1}{\mu F J_{j k}\left(1-\rho F J_{j}\right)},
$$

where $H_{N}$ is calculated by equation 2.3, hTraffic by 4.29, and $\beta$ by equation 4.32 [107].

To obtain the total expected flow time of a job through the network, individual expected flow times of single and fork-join stations are aggregated. This calculated using formula 3.21 stated in section 3 . 


\subsection{Pseudocode}

The high-level description of the computer programming algorithm used to calculated the performance measures for the general case is as follows:

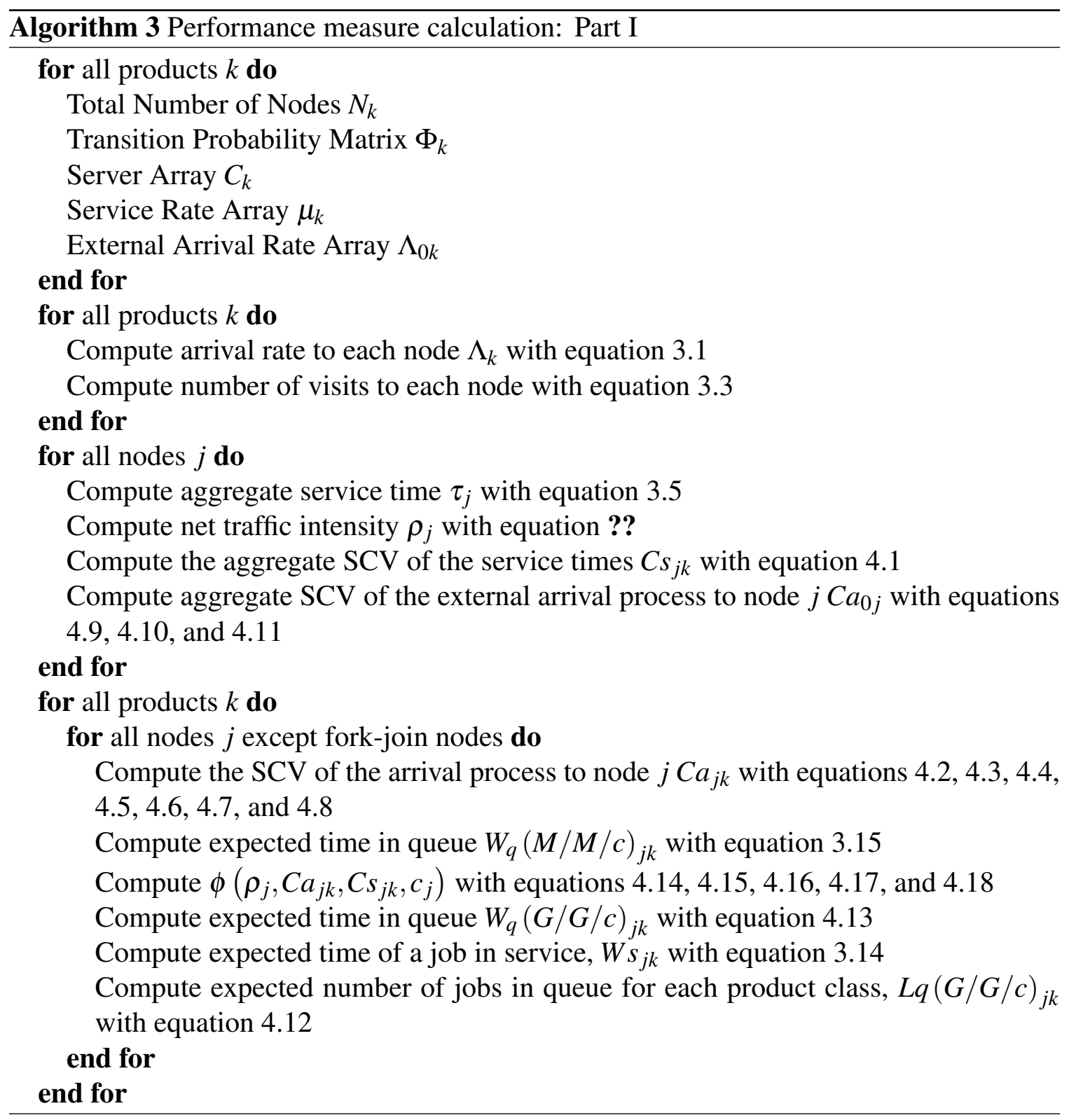




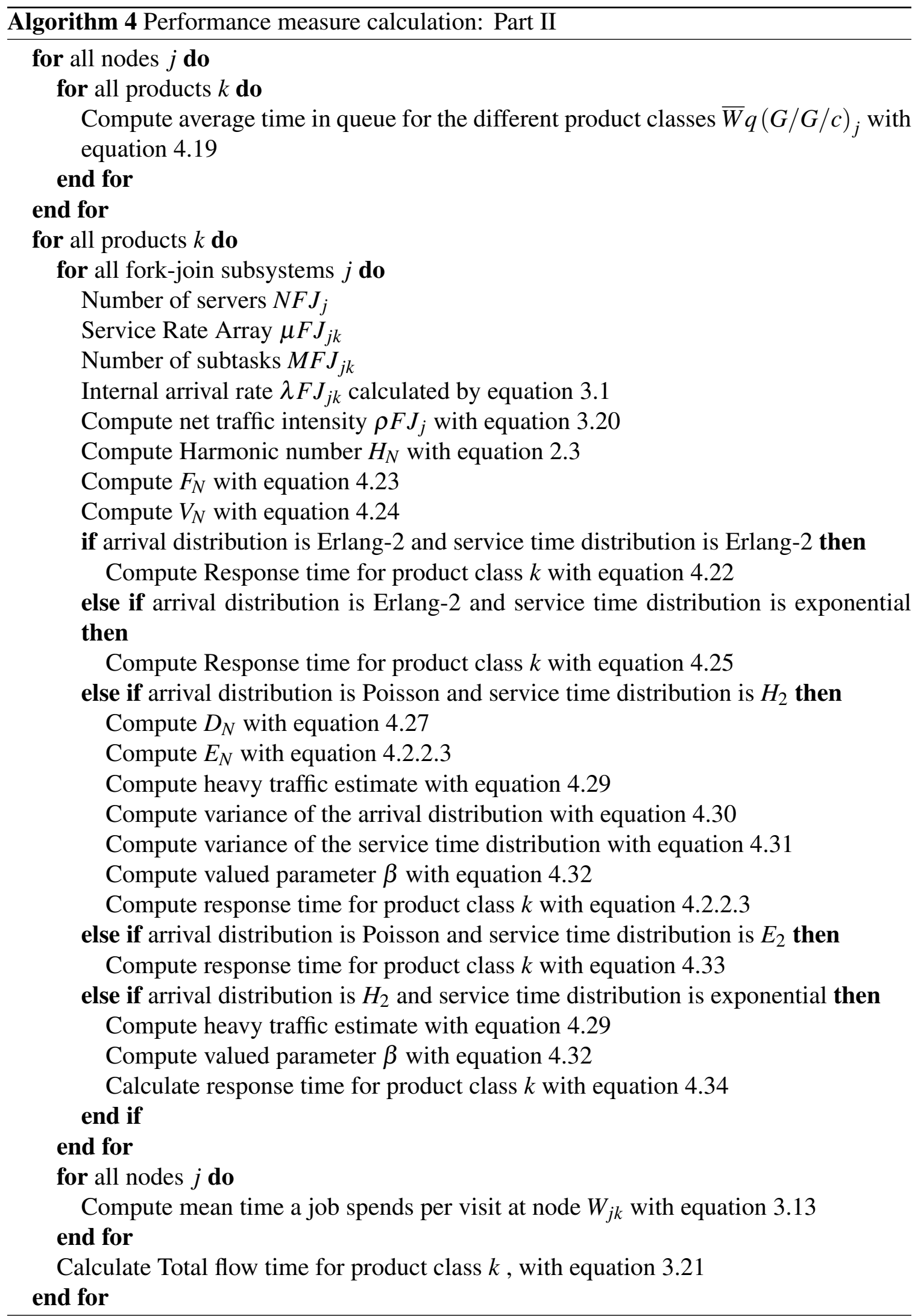




\subsection{Computational Results}

One hundred twenty problem instances were designed to test the accuracy of the analytical estimates, and discrete event simulation models were developed to simulate the problem instances. Based on previous formulations of fork-join nodes with general distributions [107], interarrival and service times were assumed to be Erlang with two phases (Erlang-2), hyperexponential with two phases $\left(H_{2}\right)$, and/or exponential distribution. Five different scenarios were analyzed:

1. Erlang-2 arrivals and Erlang-2 service times $\left(E_{2}-E_{2}\right)$;

$$
C a_{0 j k}=0.5, C s_{j k}=0.5 \text {. }
$$

2. Erlang-2 arrivals and exponential service times $\left(E_{2}-E x p\right)$;

$$
C a_{0 j k}=0.5, C s_{j k}=1 \text {. }
$$

3. Poisson arrivals and hyper-exponential service times $\left(\operatorname{Exp}-H_{2}\right)$;

$$
C a_{0 j k}=1, C s_{j k}=1.5 \text { and } 2 .
$$

4. Poisson arrivals and Erlang-2 service times $\left(\operatorname{Exp}-E_{2}\right)$;

$$
C a_{0 j k}=1, C s_{j k}=0.5 \text {. }
$$

5. Hyper-exponential arrivals and exponential services $\left(H_{2}-E x p\right)$;

$$
C a_{0 j k}=1.5, \text { and } 2, C s_{j k}=1 \text {. }
$$

Each problem instance is defined by the number of nodes in the network, number of servers at each node, number of product classes, nodes that are shared by different product classes; and for each product class, its arrival and service time distributions and the transition probability matrix. The number of nodes for each problem instance was varied from five to eight, 
product classes from one to six, and shared nodes from $50 \%$ to $67 \%$ of the total number of nodes in the network. The number of fork and join subsystems considered was either one or two, and the number of servers in it two, three or four. The location of the fork-join nodes in the network was varied from node two to last node -1, keeping the nature of the server assembly process. The transition probability matrix for each product class was randomly generated, varying failure rates from 0.02 to 0.15 . Arrival and service rates were selected in such way that the service rates at all stations were greater than the arrival rates. All the equations stated in the analytical formulation were implemented in Matlab 7.0 as for the Markovian case, and the simulations models were developed following the same procedure as described in section 3.4.

Tables 4.1, 4.2, 4.3, 4.4, 4.5, and 4.6 describe the problem instances for one, two, three, four, five and six product classes respectively in terms of the type of arrival and service time distribution (I) along with its squared coefficient of variation (II), number of nodes (III), shared nodes (IV) and number of fork-join nodes (V) along with their location in the network (VI).

Table 4.1: Problem instance configuration for single product class

\begin{tabular}{cccccc|c|}
\multicolumn{1}{c}{$(\mathrm{I})$} & $(\mathrm{II})$ & $(\mathrm{III})$ & $(\mathrm{IV})$ & $(\mathrm{V})$ & $(\mathrm{VI})$ \\
\hline Inst. & $\begin{array}{c}\text { Arr. and } \\
\text { Serv. Distr. }\end{array}$ & SCV & Nod. & SN & F/J nodes & $\begin{array}{c}\text { F/J } \\
\text { location }\end{array}$ \\
\hline 1 & E2_E2 & $0.5-0.5$ & 6 & 0 & 2 & node 3, node 5 \\
\hline 7 & E2_E2 & $0.5-0.5$ & 8 & 0 & 1 & node 6 \\
\hline 13 & E2_E2 & $0.5-0.5$ & 8 & 0 & 1 & node 3 \\
\hline 19 & E2_E2 & $0.5-0.5$ & 5 & 0 & 1 & node 3 \\
\hline 25 & E2_Exp & $0.5-1.0$ & 6 & 0 & 2 & node 3, node 5 \\
\hline 31 & E2_Exp & $0.5-1.0$ & 8 & 0 & 1 & node 6 \\
\hline 37 & E2_Exp & $0.5-1.0$ & 8 & 0 & 1 & node 3 \\
\hline 43 & E2_Exp & $0.5-1.0$ & 5 & 0 & 1 & node 3 \\
\hline 49 & Exp_H2 & $0.5-1.5$ & 6 & 0 & 2 & node 3, node 5 \\
\hline 55 & Exp_H2 & $0.5-2.0$ & 8 & 0 & 1 & node 6 \\
\hline 61 & Exp_H2 & $0.5-2.0$ & 8 & 0 & 1 & node 3 \\
\hline
\end{tabular}


(I)

(II) (III) (IV)

(V)

(VI)

\begin{tabular}{llllll}
\hline Inst. Arr. and & SCV & Nod. & SN & F/J nodes & F/J
\end{tabular}

Serv. Distr.

location

\begin{tabular}{|c|c|c|c|c|c|c|}
\hline 67 & Exp_H2 & $0.5-1.5$ & 5 & 0 & 1 & node 3 \\
\hline 73 & Exp_E2 & $1.0-0.5$ & 6 & 0 & 2 & node 3, node 5 \\
\hline 79 & Exp_E2 & $1.0-0.5$ & 8 & 0 & 1 & node 6 \\
\hline 85 & Exp_E2 & $1.0-0.5$ & 8 & 0 & 1 & node 3 \\
\hline 91 & Exp_E2 & $1.0-0.5$ & 5 & 0 & 1 & node 3 \\
\hline 97 & H2_Exp & $1.5-1.0$ & 6 & 0 & 2 & node 3, node 5 \\
\hline 103 & H2_Exp & $2.0-1.0$ & 8 & 0 & 1 & node 6 \\
\hline 109 & H2_Exp & $1.5-1.0$ & 8 & 0 & 1 & node 3 \\
\hline 115 & H2_Exp & $1.5-1.0$ & 5 & 0 & 1 & node 3 \\
\hline
\end{tabular}

Table 4.2: Problem instance configuration for two product classes

(I)

(II)

(III) (IV)

(V)

(VI)

\begin{tabular}{lcccccc}
\hline Inst. & $\begin{array}{c}\text { Arr. and } \\
\text { Serv. Distr. }\end{array}$ & SCV & Nod. & SN & F/J nodes & $\begin{array}{c}\text { F/J } \\
\text { location }\end{array}$ \\
\hline
\end{tabular}

\begin{tabular}{|c|c|c|c|c|c|c|}
\hline 2 & E2_E2 & $0.5-0.5$ & 6 & 3 & 2 & node 3, node 5 \\
\hline 8 & E2_E2 & $0.5-0.5$ & 8 & 4 & 1 & node 6 \\
\hline 14 & E2_E2 & $0.5-0.5$ & 8 & 4 & 1 & node 3 \\
\hline 20 & E2_E2 & $0.5-0.5$ & 5 & 3 & 1 & node 3 \\
\hline 26 & E2_Exp & $0.5-1.0$ & 6 & 3 & 2 & node 3, node 5 \\
\hline 32 & E2_Exp & $0.5-1.0$ & 8 & 4 & 1 & node 6 \\
\hline 38 & E2_Exp & $0.5-1.0$ & 8 & 4 & 1 & node 3 \\
\hline 44 & E2_Exp & $0.5-1.0$ & 5 & 3 & 1 & node 3 \\
\hline 50 & Exp_H2 & $0.5-1.5$ & 6 & 3 & 2 & node 3, node 5 \\
\hline 56 & Exp_H2 & $0.5-2.0$ & 8 & 4 & 1 & node 6 \\
\hline 62 & Exp_H2 & $0.5-2.0$ & 8 & 4 & 1 & node 3 \\
\hline 68 & Exp_H2 & $0.5-1.5$ & 5 & 3 & 1 & node 3 \\
\hline 74 & Exp_E2 & $1.0-0.5$ & 6 & 3 & 2 & node 3, node 5 \\
\hline 80 & Exp_E2 & $1.0-0.5$ & 8 & 4 & 1 & node 6 \\
\hline 86 & Exp_E2 & $1.0-0.5$ & 8 & 4 & 1 & node 3 \\
\hline 92 & Exp_E2 & $1.0-0.5$ & 5 & 3 & 1 & node 3 \\
\hline 98 & H2_Exp & $1.5-1.0$ & 6 & 3 & 2 & node 3, node 5 \\
\hline 104 & H2_Exp & $2.0-1.0$ & 8 & 4 & 1 & node 6 \\
\hline 110 & H2_Exp & $1.5-1.0$ & 8 & 4 & 1 & node 3 \\
\hline 116 & H2_Exp & $1.5-1.0$ & 5 & 3 & 1 & node 3 \\
\hline
\end{tabular}


Table 4.3: Problem instance configuration for three product classes

(I)

(II) (III) (IV) (V)

(VI)

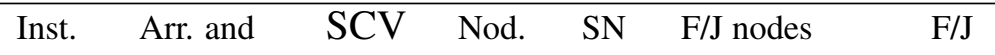

Serv. Distr.

location

\begin{tabular}{|c|c|c|c|c|c|c|}
\hline 3 & E2_E2 & $0.5-0.5$ & 6 & 3 & 2 & node 3, node 5 \\
\hline 9 & E2_E2 & $0.5-0.5$ & 8 & 4 & 1 & node 6 \\
\hline 15 & E2_E2 & $0.5-0.5$ & 8 & 4 & 1 & node 3 \\
\hline 21 & E2_E2 & $0.5-0.5$ & 5 & 3 & 1 & node 3 \\
\hline 27 & E2_Exp & $0.5-1.0$ & 6 & 3 & 2 & node 3, node 5 \\
\hline 33 & E2_Exp & $0.5-1.0$ & 8 & 4 & 1 & node 6 \\
\hline 39 & E2_Exp & $0.5-1.0$ & 8 & 4 & 1 & node 3 \\
\hline 45 & E2_Exp & $0.5-1.0$ & 5 & 3 & 1 & node 3 \\
\hline 51 & Exp_H2 & $0.5-1.5$ & 6 & 3 & 2 & node 3, node 5 \\
\hline 57 & Exp_H2 & $0.5-2.0$ & 8 & 4 & 1 & node 6 \\
\hline 63 & Exp_H2 & $0.5-2.0$ & 8 & 4 & 1 & node 3 \\
\hline 69 & Exp_H2 & $0.5-1.5$ & 5 & 3 & 1 & node 3 \\
\hline 75 & Exp_E2 & $1.0-0.5$ & 6 & 3 & 2 & node 3, node 5 \\
\hline 81 & Exp_E2 & $1.0-0.5$ & 8 & 4 & 1 & node 6 \\
\hline 87 & Exp_E2 & $1.0-0.5$ & 8 & 4 & 1 & node 3 \\
\hline 93 & Exp_E2 & $1.0-0.5$ & 5 & 3 & 1 & node 3 \\
\hline 99 & H2_Exp & $1.5-1.0$ & 6 & 3 & 2 & node 3, node 5 \\
\hline 105 & H2_Exp & $2.0-1.0$ & 8 & 4 & 1 & node 6 \\
\hline 111 & H2_Exp & $1.5-1.0$ & 8 & 4 & 1 & node 3 \\
\hline 117 & H2_Exp & $1.5-1.0$ & 5 & 3 & 1 & node 3 \\
\hline
\end{tabular}

Table 4.4: Problem instance configuration for four product classes

$\begin{array}{llllll}\text { (I) } & \text { (II) } & \text { (III) } & \text { (IV) } & \text { (V) } & \text { (VI) }\end{array}$

\begin{tabular}{|c|c|c|c|c|c|c|}
\hline Inst. & $\begin{array}{c}\text { Arr. and } \\
\text { Serv. Distr. }\end{array}$ & $\mathrm{SCV}$ & Nod. & $\mathrm{SN}$ & $\mathrm{F} / \mathrm{J}$ nodes & $\begin{array}{c}\mathrm{F} / \mathrm{J} \\
\text { location }\end{array}$ \\
\hline 4 & E2_E2 & $0.5-0.5$ & 6 & 3,4 & 2 & node 3 , node 5 \\
\hline 10 & E2_E2 & $0.5-0.5$ & 8 & 4,8 & 1 & node 6 \\
\hline 16 & E2_E2 & $0.5-0.5$ & 8 & 4 & 1 & node 3 \\
\hline 22 & E2_E2 & $0.5-0.5$ & 5 & 3 & 1 & node 3 \\
\hline 28 & E2_Exp & $0.5-1.0$ & 6 & 3,4 & 2 & node 3 , node 5 \\
\hline 34 & E2_Exp & $0.5-1.0$ & 8 & 4,8 & 1 & node 6 \\
\hline 40 & E2_Exp & $0.5-1.0$ & 8 & 4 & 1 & node 3 \\
\hline 46 & E2_Exp & $0.5-1.0$ & 5 & 3 & 1 & node 3 \\
\hline 52 & Exp_H2 & $0.5-1.5$ & 6 & 3,4 & 2 & node 3 , node 5 \\
\hline 58 & Exp_H2 & $0.5-2.0$ & 8 & 4,8 & 1 & node 6 \\
\hline 64 & Exp_H2 & $0.5-2.0$ & 8 & 4 & 1 & node 3 \\
\hline
\end{tabular}


(I)

(II) (III) (IV) (V)

(VI)

\begin{tabular}{c|c|c|c|c|c|c|}
\hline Inst. & $\begin{array}{c}\text { Arr. and } \\
\text { Serv. Distr. }\end{array}$ & SCV & Nod. & SN & F/J nodes & $\begin{array}{c}\text { F/J } \\
\text { location }\end{array}$ \\
\hline 70 & Exp_H2 & $0.5-1.5$ & 5 & 3 & 1 & node 3 \\
\hline 76 & Exp_E2 & $1.0-0.5$ & 6 & 3,4 & 2 & node 3, node 5 \\
\hline 82 & Exp_E2 & $1.0-0.5$ & 8 & 4,8 & 1 & node 6 \\
\hline 88 & Exp_E2 & $1.0-0.5$ & 8 & 4 & 1 & node 3 \\
\hline 94 & Exp_E2 & $1.0-0.5$ & 5 & 3 & 1 & node 3 \\
\hline 100 & H2_Exp & $1.5-1.0$ & 6 & 3,4 & 2 & node 3, node 5 \\
\hline 106 & H2_Exp & $2.0-1.0$ & 8 & 4,8 & 1 & node 6 \\
\hline 112 & H2_Exp & $1.5-1.0$ & 8 & 4 & 1 & node 3 \\
\hline 118 & H2_Exp & $1.5-1.0$ & 5 & 3 & 1 & node 3 \\
\hline
\end{tabular}

Table 4.5: Problem instance configuration for five product classes

$\begin{array}{lllll}\text { (I) } & \text { (II) } & \text { (III) } & \text { (IV) } & \text { (V) }\end{array}$

\begin{tabular}{ccccccc}
\hline Inst. & $\begin{array}{c}\text { Arr. and } \\
\text { Serv. Distr. }\end{array}$ & SCV & Nod. & SN & F/J nodes & $\begin{array}{c}\text { F/J } \\
\text { location }\end{array}$ \\
\hline
\end{tabular}

\begin{tabular}{|c|c|c|c|c|c|c|}
\hline 5 & E2_E2 & $0.5-0.5$ & 6 & 3,4 & 2 & node 3, node 5 \\
\hline 11 & E2_E2 & $0.5-0.5$ & 8 & 4,8 & 1 & node 6 \\
\hline 17 & E2_E2 & $0.5-0.5$ & 8 & 4 & 1 & node 3 \\
\hline 23 & E2_E2 & $0.5-0.5$ & 5 & 3 & 1 & node 3 \\
\hline 29 & E2_Exp & $0.5-1.0$ & 6 & 3,4 & 2 & node 3, node 5 \\
\hline 35 & E2_Exp & $0.5-1.0$ & 8 & 4,8 & 1 & node 6 \\
\hline 41 & E2_Exp & $0.5-1.0$ & 8 & 4 & 1 & node 3 \\
\hline 47 & E2_Exp & $0.5-1.0$ & 5 & 3 & 1 & node 3 \\
\hline 53 & Exp_H2 & $0.5-1.5$ & 6 & 3,4 & 2 & node 3, node 5 \\
\hline 59 & Exp_H2 & $0.5-2.0$ & 8 & 4,8 & 1 & node 6 \\
\hline 65 & Exp_H2 & $0.5-2.0$ & 8 & 4 & 1 & node 3 \\
\hline 71 & Exp_H2 & $0.5-1.5$ & 5 & 3 & 1 & node 3 \\
\hline 77 & Exp_E2 & $1.0-0.5$ & 6 & 3,4 & 2 & node 3, node 5 \\
\hline 83 & Exp_E2 & $1.0-0.5$ & 8 & 4,8 & 1 & node 6 \\
\hline 89 & Exp_E2 & $1.0-0.5$ & 8 & 4 & 1 & node 3 \\
\hline 95 & Exp_E2 & $1.0-0.5$ & 5 & 3 & 1 & node 3 \\
\hline 101 & H2_Exp & $1.5-1.0$ & 6 & 3,4 & 2 & node 3, node 5 \\
\hline 107 & H2_Exp & $2.0-1.0$ & 8 & 4,8 & 1 & node 6 \\
\hline 113 & H2_Exp & $1.5-1.0$ & 8 & 4 & 1 & node 3 \\
\hline 119 & H2_Exp & $1.5-1.0$ & 5 & 3 & 1 & node 3 \\
\hline
\end{tabular}


Table 4.6: Problem instance configuration for six product classes

\begin{tabular}{|c|c|c|c|c|c|c|}
\hline & (I) & (II) & (III) & (IV) & $(\mathrm{V})$ & (VI) \\
\hline Inst. & $\begin{array}{c}\text { Arr. and } \\
\text { Serv. Distr. }\end{array}$ & SCV & Nod. & SN & $\mathrm{F} / \mathrm{J}$ nodes & $\begin{array}{c}\mathrm{F} / \mathrm{J} \\
\text { location }\end{array}$ \\
\hline 6 & E2_E2 & $0.5-0.5$ & 6 & 3,4 & 2 & node 3 , node 5 \\
\hline 12 & E2_E2 & $0.5-0.5$ & 8 & 4,8 & 1 & node 6 \\
\hline 18 & E2_E2 & $0.5-0.5$ & 8 & 4 & 1 & node 3 \\
\hline 24 & E2_E2 & $0.5-0.5$ & 5 & 3 & 1 & node 3 \\
\hline 30 & E2_Exp & $0.5-1.0$ & 6 & 3,4 & 2 & node 3 , node 5 \\
\hline 36 & E2_Exp & $0.5-1.0$ & 8 & 4,8 & 1 & node 6 \\
\hline 42 & E2_Exp & $0.5-1.0$ & 8 & 4 & 1 & node 3 \\
\hline 48 & E2_Exp & $0.5-1.0$ & 5 & 3 & 1 & node 3 \\
\hline 54 & Exp_H2 & $0.5-1.5$ & 6 & 3,4 & 2 & node 3 , node 5 \\
\hline 60 & Exp_H2 & $0.5-2.0$ & 8 & 4,8 & 1 & node 6 \\
\hline 66 & Exp_H2 & $0.5-2.0$ & 8 & 4 & 1 & node 3 \\
\hline 72 & Exp_H2 & $0.5-1.5$ & 5 & 3 & 1 & node 3 \\
\hline 78 & Exp_E2 & $1.0-0.5$ & 6 & 3,4 & 2 & node 3 , node 5 \\
\hline 84 & Exp_E2 & $1.0-0.5$ & 8 & 4,8 & 1 & node 6 \\
\hline 90 & Exp_E2 & $1.0-0.5$ & 8 & 4 & 1 & node 3 \\
\hline 96 & Exp_E2 & $1.0-0.5$ & 5 & 3 & 1 & node 3 \\
\hline 102 & H2_Exp & $1.5-1.0$ & 6 & 3,4 & 2 & node 3 , node 5 \\
\hline 108 & H2_Exp & $2.0-1.0$ & 8 & 4,8 & 1 & node 6 \\
\hline 114 & H2_Exp & $1.5-1.0$ & 8 & 4 & 1 & node 3 \\
\hline 120 & H2_Exp & $1.5-1.0$ & 5 & 3 & 1 & node 3 \\
\hline
\end{tabular}

Results for flow times were obtained using the analytical formulations and the simulation models. The tightness of the flow time approximations was computed using equation 3.24 In Appendix E is presented the approximate and simulated mean flow time values along with the error in the approximation for one, two, three, four, five, and six product instances respectively.

The nature of the distribution of the error for the flow time in the case of general distributions is shown in Figure 4.1. The graph shows that the error seems to be distributed between zero and 63\%, and the average error was $23.19 \%$. Figure 4.2 presents the trend of the simulation and analytical flow times for all problem instances. It is apparent from the graph that the analytical values follow the same trend that the simulated values. 


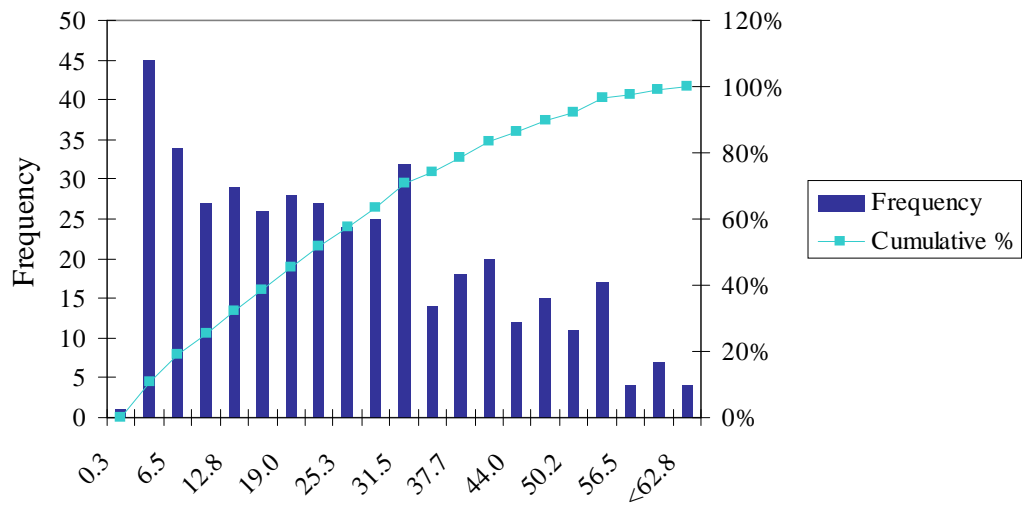

Flow Time Error $(\%)$

Figure 4.1: Overall flow time distribution for the general type system.

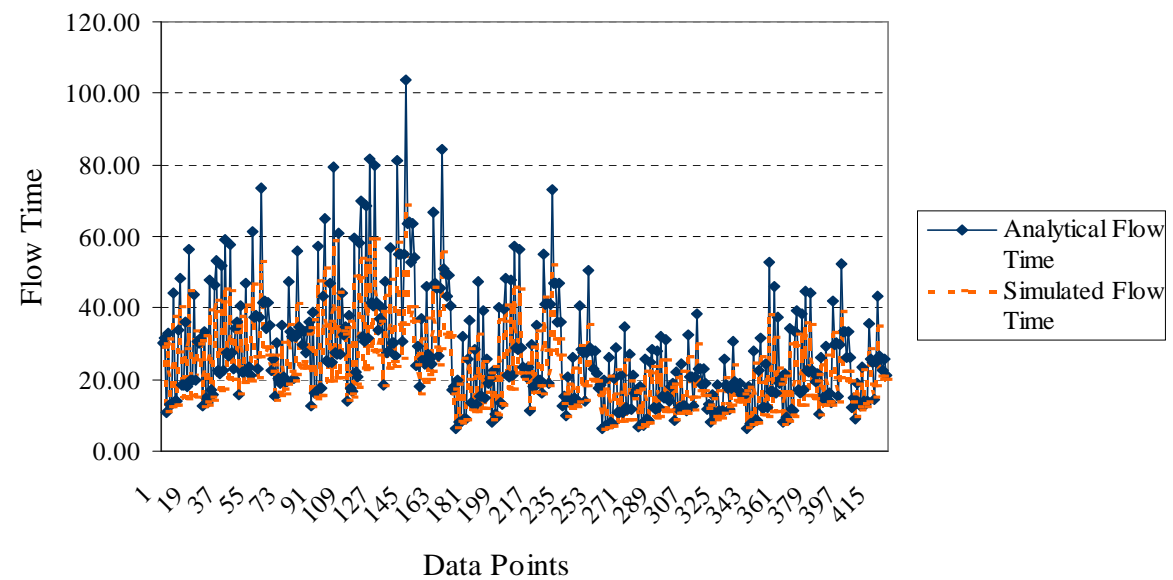

Figure 4.2: Analytical and simulated flow times for problem instances with general distribution.

Further analysis was done to analyze the error for the different problem instances. The error in flow time was plotted by product class and later by product class and arrival and service time distribution. In addition, a paired-t test was conducted to determine whether there was significant difference between the flow time from the simulation models and the analytical formulations (see Tables 4.7, 4.8, 4.9, 4.10, 4.11, 4.12, and 4.13).

Figure 4.3 displays the error distribution for one product class instances for all type of distributions. The error for most of the problem instances was between zero and 10\%, and 
Table 4.7: Paired-t test results per product class

\begin{tabular}{|c|c|c|c|c|c|c|}
\hline \multicolumn{7}{|c|}{ Product Class } \\
\hline \hline & 1 & 2 & 3 & 4 & 5 & 6 \\
\hline t Stat & -1.48 & -3.44 & -9.29 & -11.16 & -14.78 & -17.64 \\
\hline t Critical two-tail & 1.73 & 2.02 & 2.00 & 1.99 & 1.98 & 1.98 \\
\hline $\mathrm{P}(\mathrm{T}<=\mathrm{t})$ two-tail & $1.6 \mathrm{E}-01$ & $1.4 \mathrm{E}-03$ & $3.8 \mathrm{E}-13$ & $6.6 \mathrm{E}-18$ & $8.4 \mathrm{E}-27$ & $5.3 \mathrm{E}-35$ \\
\hline
\end{tabular}

Table 4.8: Paired-t test results for one product class

\begin{tabular}{|c|c|c|c|c|c|}
\hline & $E_{2}-E_{2}$ & $E_{2}-\operatorname{Exp}$ & $\operatorname{Exp}-H_{2}$ & $\operatorname{Exp}-E_{2}$ & $H_{2}-\operatorname{Exp}$ \\
\hline \hline $\mathrm{t}$ Stat & 3.32 & 1.95 & -0.66 & 1.43 & -0.96 \\
\hline $\mathrm{t}$ Critical two-tail & 3.18 & 3.18 & 3.18 & 3.18 & 3.18 \\
\hline $\mathrm{P}(\mathrm{T}<=\mathrm{t})$ two-tail & 0.05 & 0.15 & 0.55 & 0.25 & 0.41 \\
\hline
\end{tabular}

the maximum error was $13.4 \%$. The data strongly suggests that the means are the same at 0.05 level of significance (see Table 4.7). Figure 4.4 shows the flow time error distribution by each scenario analyzed. From the graph it is possible to identify that the maximum error comes from the case $E_{2}-E_{2}$. On average the error for the $E_{2}-E_{2}$ case was $3 \%$, for $E_{2}-\operatorname{Exp}$ was $1.8 \%$, for $\operatorname{Exp}-\mathrm{H}_{2}$ was $5.5 \%$, for $\operatorname{Exp}-E_{2}$ was $4.3 \%$, and for $\mathrm{H}_{2}-\operatorname{Exp}$ was $3.1 \%$. Table 4.14 presents the error distribution for each case. Based on the results of the paired-t test (see Table 4.8), the data strongly suggests that at 0.04 level of significance the means are the same for the $E_{2}-E_{2}$ case, and at 0.05 level of significance for the cases $E_{2}-\operatorname{Exp}, \operatorname{Exp}-H_{2}, \operatorname{Exp}-E_{2}$, and $H_{2}-\operatorname{Exp}$.

Figure 4.5 displays the error distribution for two product classes instances for all type of distributions. The error for $95 \%$ of the instances was between zero and $12.4 \%$, and the maximum error was $14.8 \%$. The data strongly suggests that the means, taking into account

Table 4.9: Paired-t test results for two product classes

\begin{tabular}{|c|c|c|c|c|c|}
\hline & $E_{2}-E_{2}$ & $E_{2}-\operatorname{Exp}$ & $\operatorname{Exp}-H_{2}$ & $\operatorname{Exp}-E_{2}$ & $H_{2}-\operatorname{Exp}$ \\
\hline \hline $\mathrm{t}$ Stat & -3.34 & -3.50 & 0.82 & -2.52 & -0.16 \\
\hline $\mathrm{t}$ Critical two-tail & 2.36 & 2.36 & 2.36 & 2.36 & 2.36 \\
\hline $\mathrm{P}(\mathrm{T}<=\mathrm{t})$ two-tail & 0.01 & 0.01 & 0.44 & 0.04 & 0.88 \\
\hline
\end{tabular}


Table 4.10: Paired-t test results for three product classes

\begin{tabular}{|c|c|c|c|c|c|}
\hline & $E_{2}-E_{2}$ & $E_{2}-\operatorname{Exp}$ & $\operatorname{Exp}-H_{2}$ & $\operatorname{Exp}-E_{2}$ & $H_{2}-\operatorname{Exp}$ \\
\hline \hline $\mathrm{t}$ Stat & -6.64 & -6.55 & -2.71 & -6.66 & -4.67 \\
\hline $\mathrm{t}$ Critical two-tail & 2.20 & 2.20 & 2.20 & 2.20 & 2.20 \\
\hline $\mathrm{P}(\mathrm{T}<=\mathrm{t})$ two-tail & 0.00 & 0.00 & 0.02 & 0.00 & 0.00 \\
\hline
\end{tabular}

Table 4.11: Paired-t test results for four product classes

\begin{tabular}{|c|c|c|c|c|c|}
\hline & $E_{2}-E_{2}$ & $E_{2}-\operatorname{Exp}$ & $\operatorname{Exp}-H_{2}$ & $\operatorname{Exp}-E_{2}$ & $H_{2}-\operatorname{Exp}$ \\
\hline \hline $\mathrm{t}$ Stat & -6.62 & -7.56 & -4.66 & -6.03 & -5.95 \\
\hline $\mathrm{t}$ Critical two-tail & 2.13 & 2.13 & 2.13 & 2.13 & 2.13 \\
\hline $\mathrm{P}(\mathrm{T}<=\mathrm{t})$ two-tail & 0.00 & 0.00 & 0.00 & 0.00 & 0.00 \\
\hline
\end{tabular}

all the distributions, are not the same at 0.01 level of significance (see Table 4.7). Figure 4.6 shows the flow time error distribution by each scenario analyzed. It is possible to identify that the maximum error comes from the case $\operatorname{Exp}-E_{2}$. In Table 4.15 is presented the error range of each case analyzed. On average the error was $3.79 \%, 4.33 \%, 4.14 \%$, $5.35 \%$, and $3.65 \%$ for the $E_{2}-E_{2}, E_{2}-E x p, E x p-H_{2}, E x p-E_{2}$, and $H_{2}-E x p$ cases, respectively. Based on the results of the paired-t test (see Table 4.9), the data strongly suggests that the means are the same for the $\operatorname{Exp}-\mathrm{H}_{2}$, and $\mathrm{H}_{2}-\operatorname{Exp}$ configurations at 0.05 level of significance, for $E x p-E_{2}$ at 0.03 level of significance, and for $E_{2}-E_{2}$ at 0.01 level of significance. The means are not the same for the $E_{2}-\operatorname{Exp}$ case at 0.01 level of significance.

In the case of three product classes, Figure 4.7 displays that the error, taking into account all the configurations, for $93 \%$ of the instances was between zero and $21 \%$, and the maximum error was $29.44 \%$. The data strongly suggest that the means are different at 0.01 level of

Table 4.12: Paired-t test results for five product classes

\begin{tabular}{|c|c|c|c|c|c|}
\hline & $E_{2}-E_{2}$ & $E_{2}-\operatorname{Exp}$ & $\operatorname{Exp}-H_{2}$ & $\operatorname{Exp}-E_{2}$ & $H_{2}-\operatorname{Exp}$ \\
\hline \hline $\mathrm{t}$ Stat & -8.91 & -9.09 & -7.17 & -10.43 & -8.76 \\
\hline $\mathrm{t}$ Critical two-tail & 2.09 & 2.09 & 2.09 & 2.09 & 2.09 \\
\hline $\mathrm{P}(\mathrm{T}<=\mathrm{t})$ two-tail & 0.00 & 0.00 & 0.00 & 0.00 & 0.00 \\
\hline
\end{tabular}


Table 4.13: Paired-t test results for six product classes

\begin{tabular}{|c|c|c|c|c|c|}
\hline & $E_{2}-E_{2}$ & $E_{2}-\operatorname{Exp}$ & $\operatorname{Exp}-H_{2}$ & $\operatorname{Exp}-E_{2}$ & $H_{2}-\operatorname{Exp}$ \\
\hline \hline $\mathrm{t}$ Stat & -10.65 & -11.10 & -9.26 & -13.36 & -10.88 \\
\hline $\mathrm{t}$ Critical two-tail & 2.07 & 2.07 & 2.07 & 2.07 & 2.07 \\
\hline $\mathrm{P}(\mathrm{T}<=\mathrm{t})$ two-tail & 0.00 & 0.00 & 0.00 & 0.00 & 0.00 \\
\hline
\end{tabular}

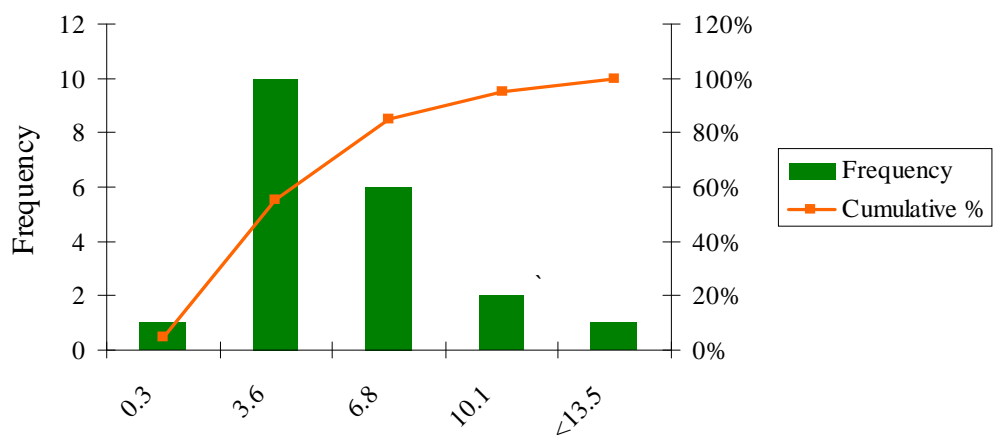

Flow Time Error (\%)

Figure 4.3: Flow time error distribution for one product class with general type distribution.

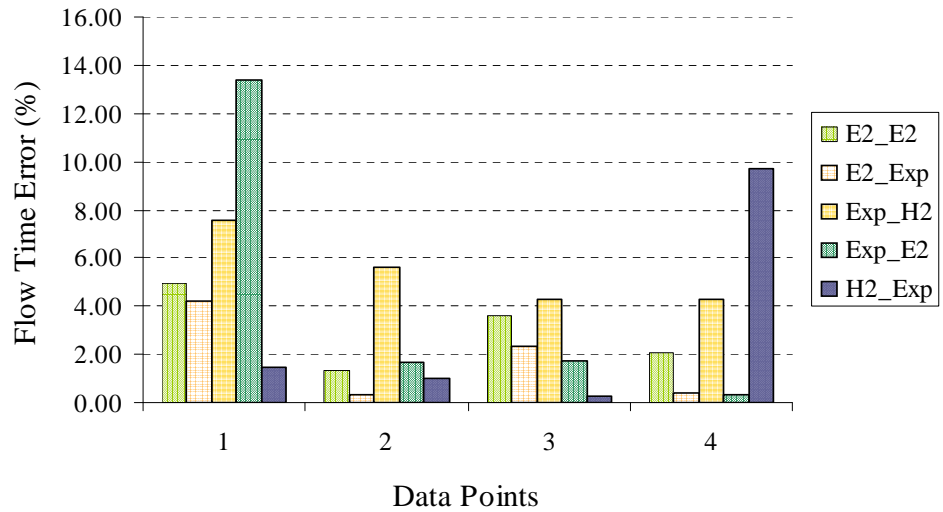

Figure 4.4: Flow time error distribution for one product class by type of distribution.

Table 4.14: Flow time error range for one product class

\begin{tabular}{|c|c|c|c|c|c|}
\hline & $E_{2}-E_{2}$ & $E_{2}-\operatorname{Exp}$ & $\operatorname{Exp}-H_{2}$ & $\operatorname{Exp}-E_{2}$ & $H_{2}-\operatorname{Exp}$ \\
\hline \hline Error (\%) & $1.4-5.0$ & $0.3-4.3$ & $4.2-7.7$ & $0.3-13.4$ & $0.2-9.7$ \\
\hline
\end{tabular}

Table 4.15: Flow time error range for two product classes

\begin{tabular}{|c|c|c|c|c|c|}
\hline & $E_{2}-E_{2}$ & $E_{2}-\operatorname{Exp}$ & $\operatorname{Exp}-H_{2}$ & $\operatorname{Exp}-E_{2}$ & $H_{2}-\operatorname{Exp}$ \\
\hline \hline Error (\%) & $0.3-10.6$ & $0.8-13.2$ & $1.2-7.3$ & $2.3-14.9$ & $0.5-8.1$ \\
\hline
\end{tabular}




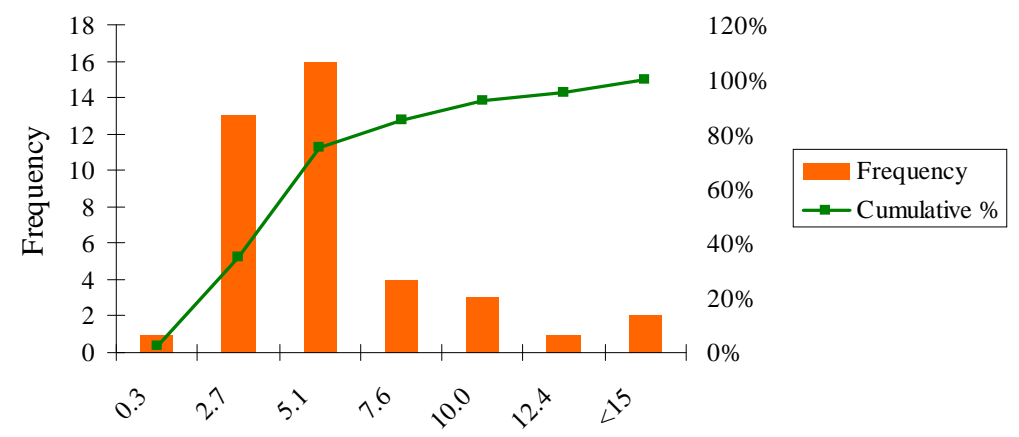

Flow Time Error (\%)

Figure 4.5: Flow time error distribution for two product classes with general type distribution.

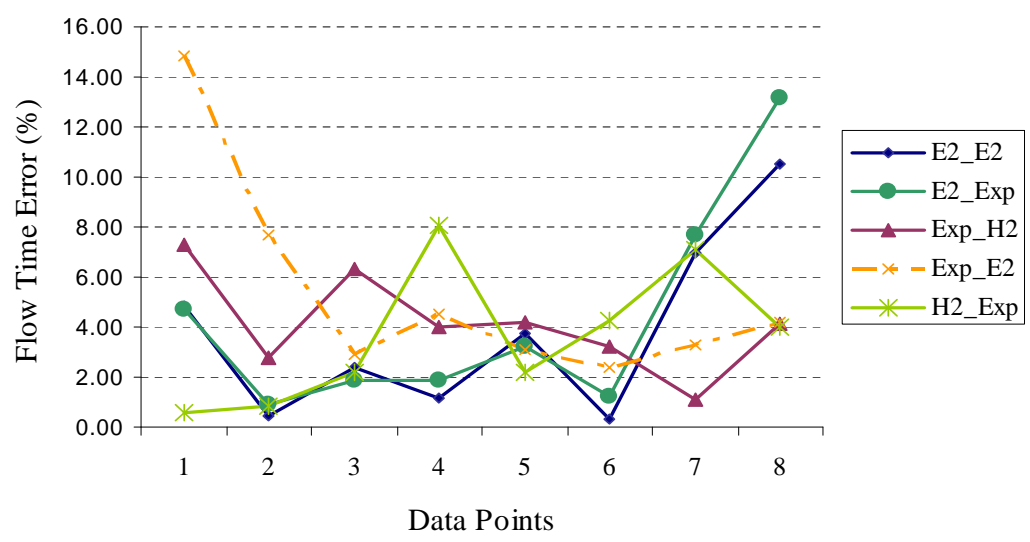

Figure 4.6: Flow time error distribution for two product classes by type of distribution.

significance (see Table 4.7). Figure 4.8 shows the trend error for each scenario analyzed. The graph displays that the maximum error comes from the case $E_{2}-\operatorname{Exp}$. On average the error for the $E_{2}-E_{2}$ case was $12.59 \%$, for $E_{2}-\operatorname{Exp}$ was $15.13 \%$, for $\operatorname{Exp}-H_{2}$ was $7.75 \%$, for $\operatorname{Exp}-E_{2}$ was $11.76 \%$, and for $H_{2}-\operatorname{Exp}$ was $7.97 \%$. Table 4.16 presents the error distribution for each case. Based on the results from the paired-t test (see Table 4.10), the data strongly suggests that at 0.01 level of significance the means are not the same for the $E_{2}-E_{2}, E_{2}-\operatorname{Exp}, \operatorname{Exp}-E_{2}$, and $H_{2}-\operatorname{Exp}$ cases. In the case of $\operatorname{Exp}-H_{2}$, the data strongly suggests that at 0.02 level of significance the means are the same. 


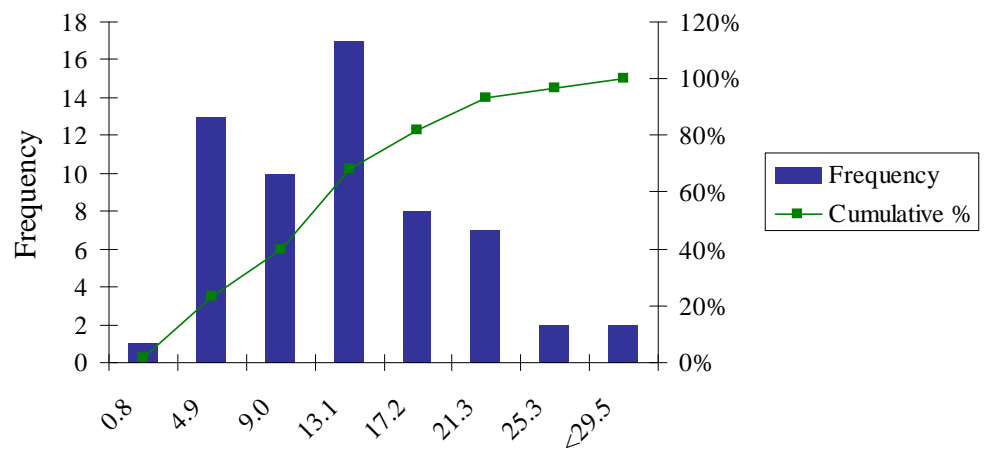

Flow Time Error (\%)

Figure 4.7: Flow time error distribution for three product classes with general type distribution.

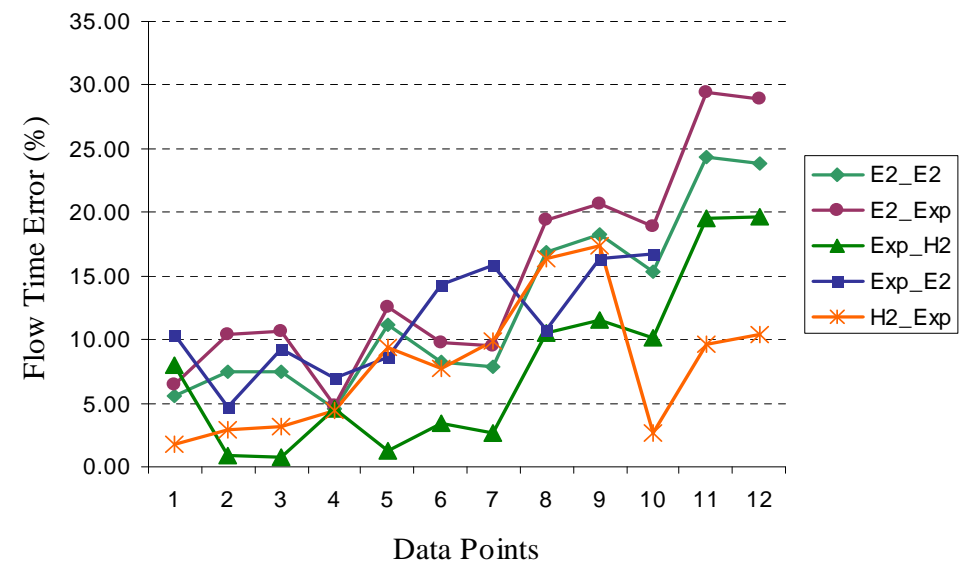

Figure 4.8: Flow time error distribution for three product classes by type of distribution.

Figure 4.9 displays the error distribution for four product instances for all type of distributions. The error for $95 \%$ of the instances was between $1 \%$ and $25.7 \%$, and the maximum error was $29.15 \%$. The data strongly suggests that the means, taking into account all the cases, are not the same at 0.01 level of significance (see Table 4.7). Figure 4.10 shows the flow time error distribution for each scenario analyzed, and in Table 4.17 is presented the

Table 4.16: Flow time error range for three product classes

\begin{tabular}{|c|c|c|c|c|c|}
\hline & $E_{2}-E_{2}$ & $E_{2}-\operatorname{Exp}$ & $\operatorname{Exp}-H_{2}$ & $\operatorname{Exp}-E_{2}$ & $H_{2}-\operatorname{Exp}$ \\
\hline \hline Error (\%) & $4.5-24.4$ & $4.8-29.5$ & $0.7-19.7$ & $4.6-16.8$ & $1.7-17.5$ \\
\hline
\end{tabular}




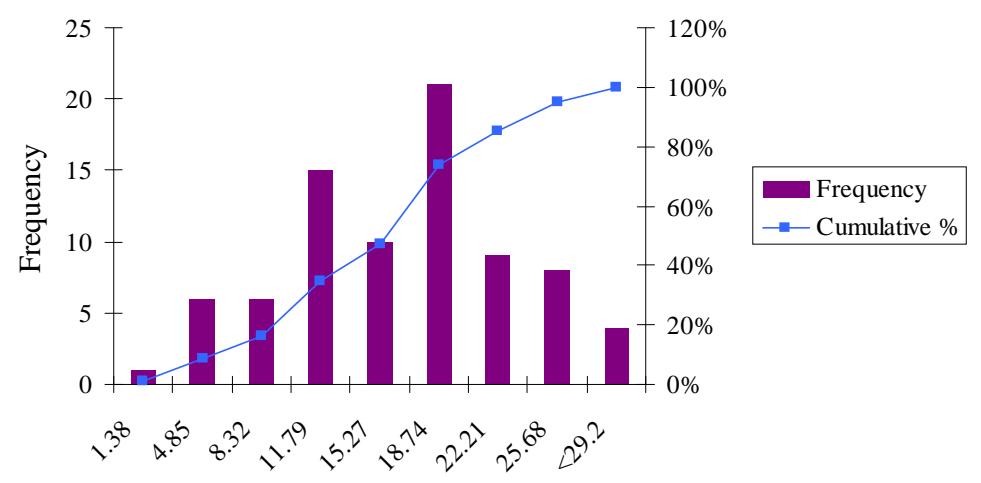

Flow Time Error $(\%)$

Figure 4.9: Flow time error distribution for four product classes with general type distribution.

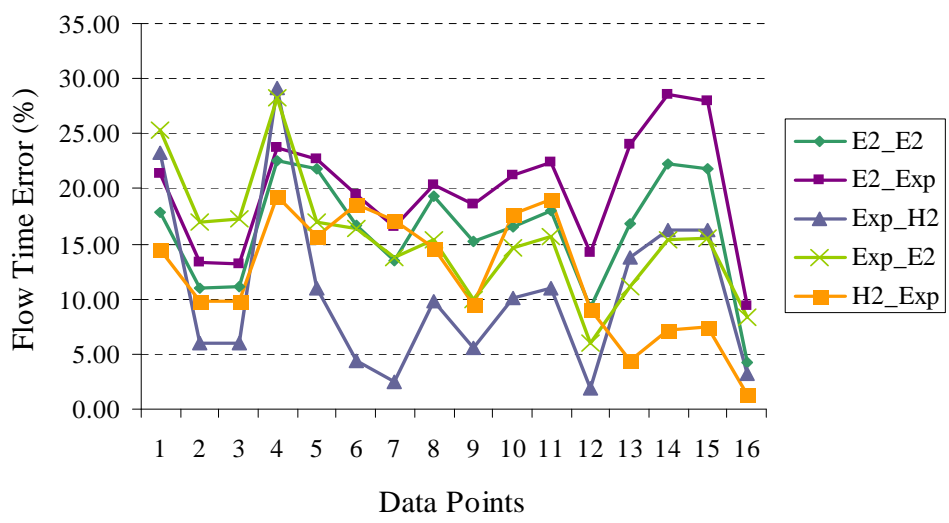

Figure 4.10: Flow time error distribution for four product classes by type of distribution.

range of the error. It is possible to identify that the maximum error comes from the case $\operatorname{Exp}-\mathrm{H}_{2}$. On average the flow time error was $16.13 \%, 19.81 \%, 10.63 \%, 15.44 \%$, and $12.21 \%$ for the $E_{2}-E_{2}, E_{2}-E x p, E x p-H_{2}, E x p-E_{2}$, and $H_{2}-E x p$ cases, respectively. The data strongly suggests that the means are not the same for any case at 0.01 level of significance (see Table 4.11).

Table 4.17: Flow time error range for four product classes

\begin{tabular}{|c|c|c|c|c|c|}
\hline & $E_{2}-E_{2}$ & $E_{2}-\operatorname{Exp}$ & $\operatorname{Exp}-H_{2}$ & $\operatorname{Exp}-E_{2}$ & $H_{2}-\operatorname{Exp}$ \\
\hline \hline Error (\%) & $4.0-22.7$ & $9.3-28.5$ & $1.9-29.2$ & $6.0-28.4$ & $1.3-19.4$ \\
\hline
\end{tabular}




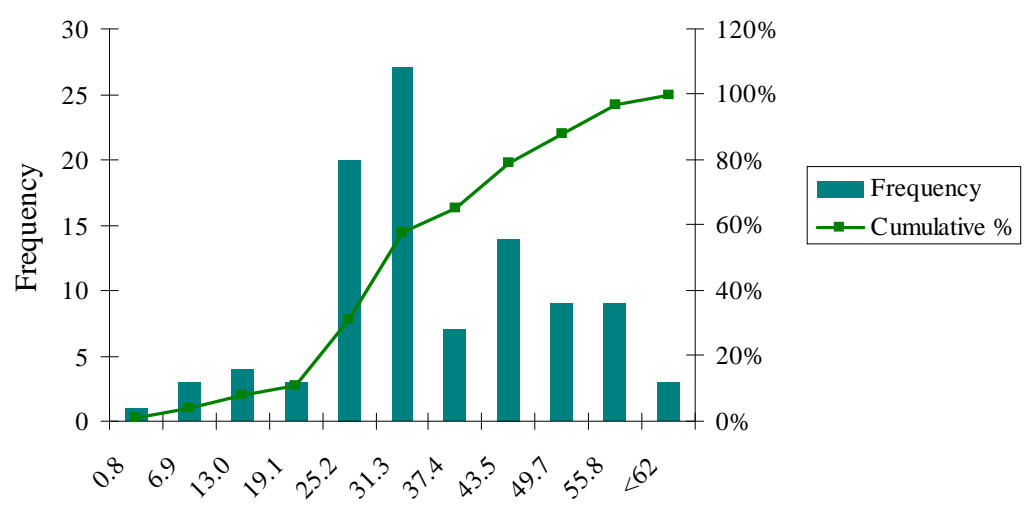

Flow Time Error (\%)

Figure 4.11: Flow time error distribution for five product classes with general type distribution.

Table 4.18: Flow time error range for five product classes

\begin{tabular}{|c|c|c|c|c|c|}
\hline & $E_{2}-E_{2}$ & $E_{2}-E x p$ & $E x p-H_{2}$ & $E x p-E_{2}$ & $H_{2}-\operatorname{Exp}$ \\
\hline \hline Error (\%) & $4.1-52.0$ & $10.9-61.9$ & $0.7-52.3$ & $7.7-43.7$ & $1.4-48.1$ \\
\hline
\end{tabular}

The error distribution for five product instances for all distribution types is shown in Figure 4.11. The error for $88 \%$ of the problem instances was between zero and $50 \%$, and the maximum error was $61.9 \%$. The data strongly suggest that the means are different at 0.01 level of significance (see Table 4.7). Figure 4.12 shows the trend error for all distribution types, and in Table 4.18 is presented the range of the error. The graph displays that the maximum error comes from the case $E_{2}-E x p$. The average error for the $E_{2}-E_{2}$ case was $31.27 \%$, for $E_{2}-\operatorname{Exp}$ was $39.02 \%$, for $\operatorname{Exp}-H_{2}$ was $29.85 \%$, for $\operatorname{Exp}-E_{2}$ was $29.59 \%$, and for $H_{2}-\operatorname{Exp}$ was $29.4 \%$. The data strongly suggests that at 0.01 level of significance the means are not the same for any type of configuration (see Table 4.12).

For six product classes Figure 4.13 displays the error distribution for all configuration types. The error for $88 \%$ of the instances was between $6 \%$ and $51 \%$, and the maximum error was 62.71\%. The data strongly suggest that the means are different at 0.01 level of significance (see Table 4.7). Figure 4.14 shows the trend error for each scenario analyzed. The graph displays that the maximum error comes from the case $H_{2}-$ Exp. In Table 4.19 is presented 


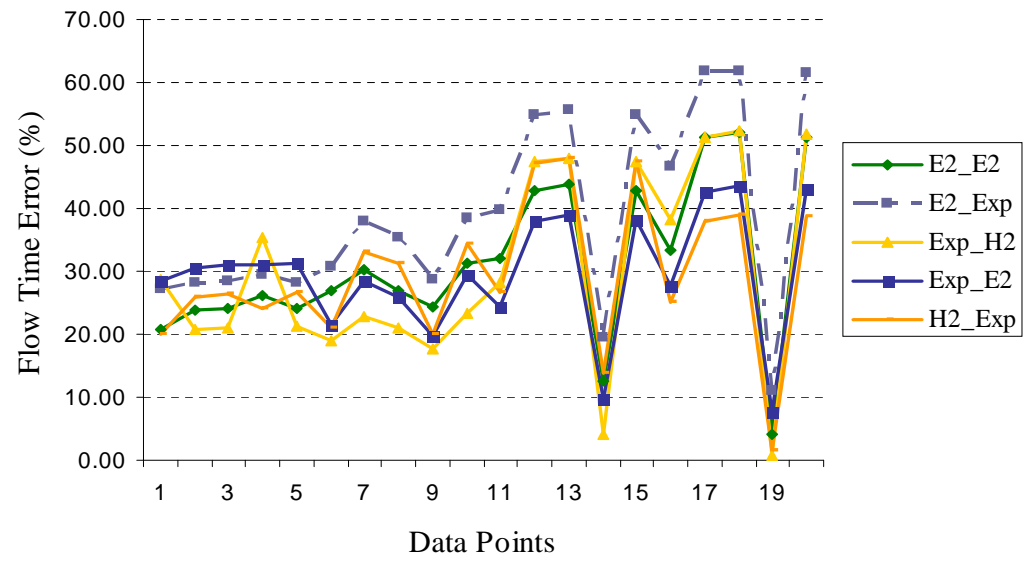

Figure 4.12: Flow time error distribution for five product classes by type of distribution.

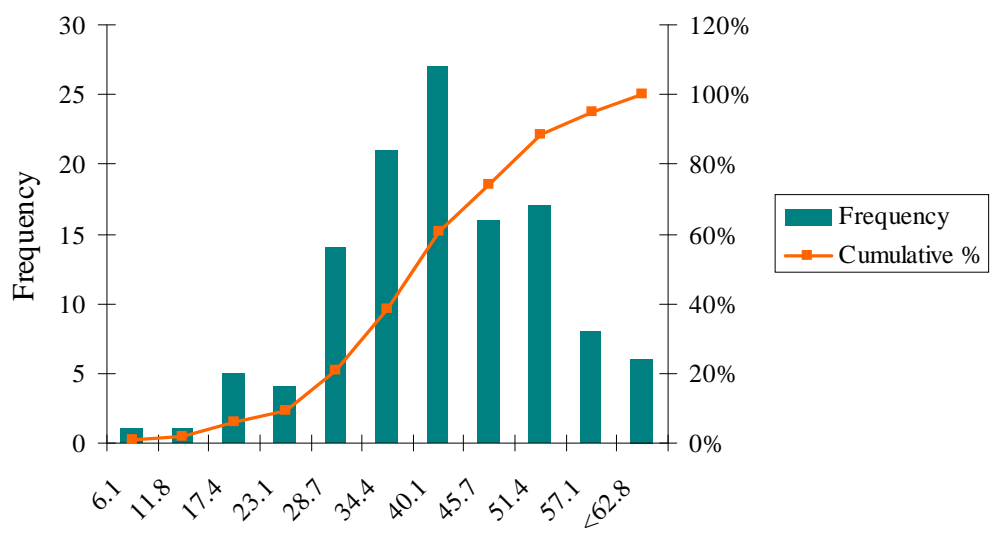

Flow Time Error (\%)

Figure 4.13: Flow time error distribution for six product classes with general type distribution.

the error distribution for each case. The average flow time error was 33.22\%, 44\%, 37.08\%, $33.4 \%$, and $38.34 \%$ for the $E_{2}-E_{2}, E_{2}-\operatorname{Exp}, \operatorname{Exp}-H_{2}, E x p-E_{2}$, and $H_{2}-E x p$ cases, respectively. Based on the results from the paired-t test, the data strongly suggests that at 0.01 level of significance the means are not the same for any configuration analyzed (see Table 4.13).

Figure 4.15 presents the average flow time error by type of distribution. The average error for one and two product instances was between $1 \%$ and $5.3 \%$, and for three and four prod- 


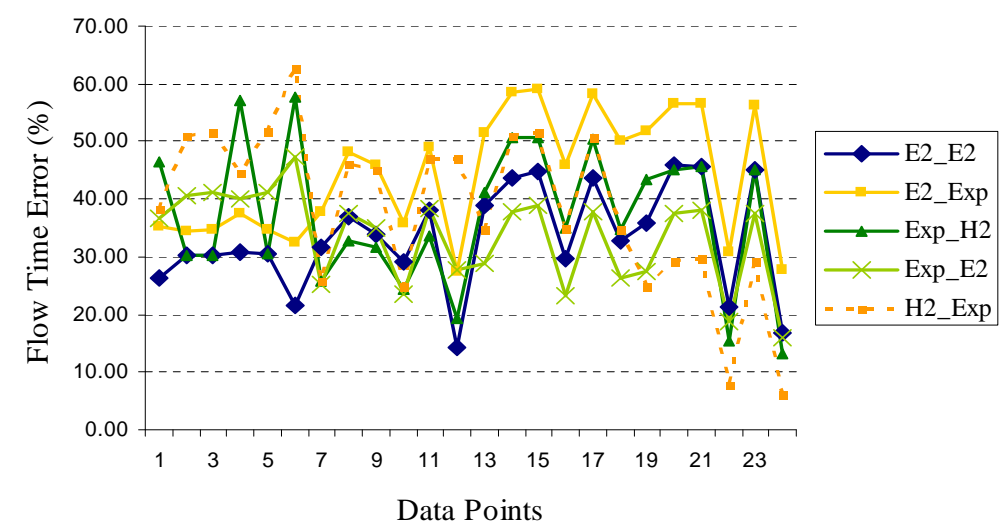

Figure 4.14: Flow time error distribution for six product classes by type of distribution.

Table 4.19: Flow time error range for six product classes

\begin{tabular}{|c|c|c|c|c|c|}
\hline & $E_{2}-E_{2}$ & $E_{2}-\operatorname{Exp}$ & $\operatorname{Exp}-H_{2}$ & $\operatorname{Exp}-E_{2}$ & $H_{2}-\operatorname{Exp}$ \\
\hline \hline Error $(\%)$ & $14.2-45.9$ & $27.4-59.2$ & $13.1-57.7$ & $15.8-47.3$ & $6.1-62.8$ \\
\hline
\end{tabular}

ucts was between $7.7 \%$ and $20 \%$. In the case of five and six product classes the average error in flow time was between $31.2 \%$ and $44 \%$. It is evident from this graph that the flow time error increased as the number of products increased. In addition, it is possible to identify that the average flow time error for three, four, five, and six product classes was greater for the case $E_{2}-\operatorname{Exp}$.

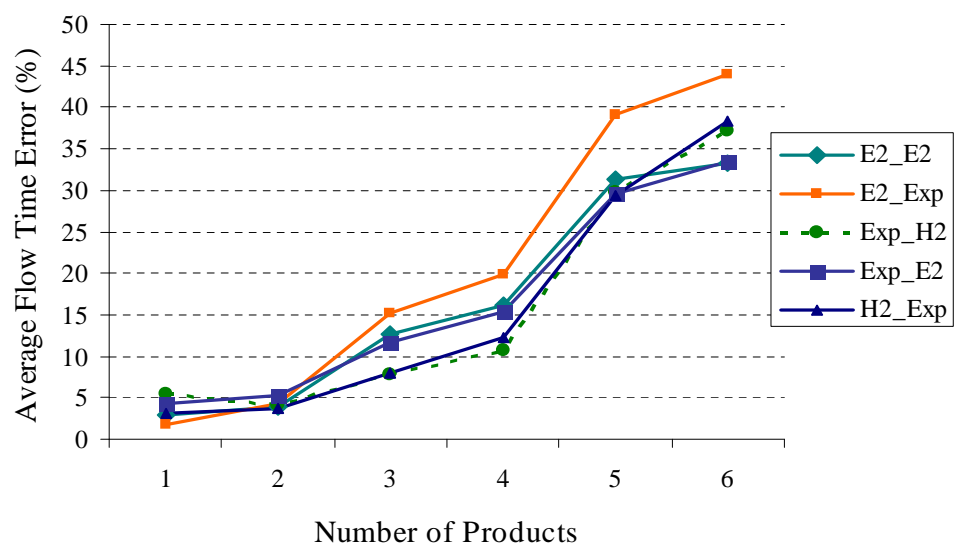

Figure 4.15: Average flow time error by type of distribution. 


\subsection{Correction Factors}

Based on the results, the flow time error for one and two product instances was between zero and $12.4 \%$, and from three to six product classes was roughly between zero and $62.71 \%$. The data strongly suggest that the means for instances with three to six classes are different at 0.01 level of significance. In order to develop regression equations to bridge the gap between the analytical approximations and the simulation models for the general case, the corrections factors for the Markovian case were used. The idea was to have correction terms that could work for both the Markovian and general type system under the five scenarios analyzed.

\subsubsection{The single stations in the general case}

The single stations in the general case were analyzed following the same Markovian case approach. It was identified that the main error of the flow time came from the waiting time, and variables affecting the flow time were the number of products in the network, and the net traffic intensity at node $i$ (see Figure 4.16). Formula 4.13 in section 4.2.1 presents the approximation of the expected waiting time at a given node for the general $G / G / c$ model developed by Whitt [112]. In this approximation, the waiting time for a $M / M / c$ type of system, the coefficients of variation of the arrival and service time, and some correction factors to determine the expected waiting time are used. The regression equation (or the correction term) developed for the Markovian case (see section 3.6.1) is used to determine the waiting time. The adjusted expected waiting time for a $M / M / c$ type of system is then used in the Whitt's formula to determine the adjusted expected waiting time for the general case. The approximation is of the form

$$
W_{q} A d j(G / G / c)_{j k}=\phi\left(\rho, C a_{j k}, C s_{j k}, c\right)\left(\frac{C a_{j k}+C s_{j k}}{2}\right) W_{q} A d j(M / M / c)_{j k},
$$




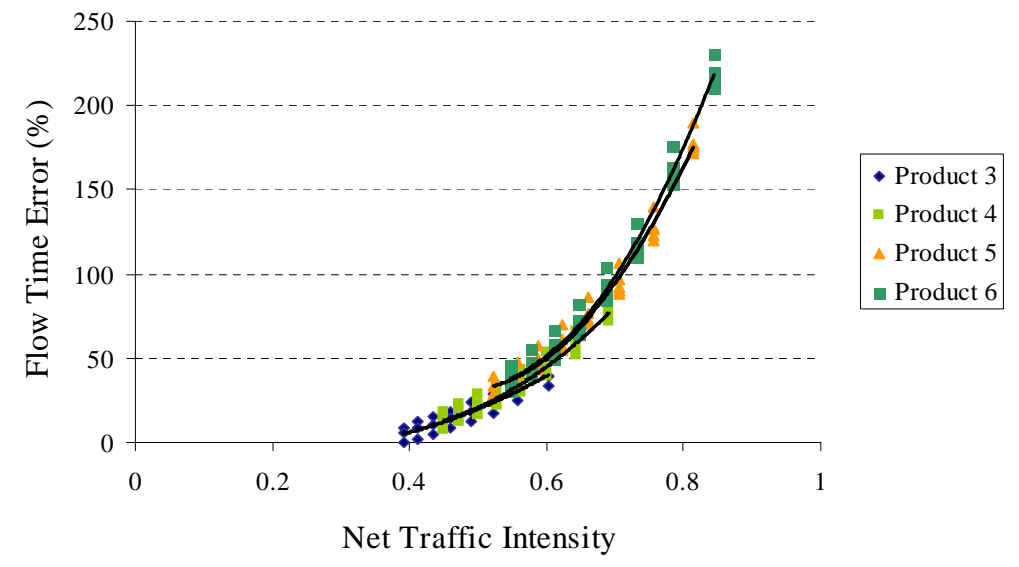

Figure 4.16: Flow time error vs. net traffic intensity of single stations for the general case.

where $W_{q} A d j(M / M / c)_{j k}$ is calculated by equation 3.26 .

\subsubsection{The case of fork-join stations for the general case}

As stated in section 3.6.2 the regression equation to predict the flow time error at the the fork-join stations was developed using seven hundred thirty five problem instances $(2,547$ data points) with interarrival and service times from exponential distributions and from general type distributions. In the general case, five hundred forty problem instances $(1,890$ data points) were generated. The arrivals and service times were from Erlang with two phases (Erlang-2), hyperexponential with two phases $\left(\mathrm{H}_{2}\right)$, and/or exponential distributions. For each scenario analyzed one hundred eight fork-join problem instances were generated. The number of product classes in the problem instances varied from one to six, failures rates from zero to $15 \%$, and the number of servers from two to four. The regression equation with a R-squared of 0.5 for the general case is given by 


$$
\begin{array}{r}
E \hat{F} J_{j}=0.406-2.85 * p-4.4 * N F J_{j}+2.62 * \sum_{k \varepsilon K} \frac{M F J_{j k}}{K}-1.08 * f+ \\
10.95 * \rho F J_{j}+5.02 * C a_{j k}+1.6 * C s_{j}
\end{array}
$$

where $E \hat{F} J_{j}$ is the percentage of predicted flow time error at the fork-join $j, p$ the number of products at the fork-join node $j, N F J_{j}$ the number of nodes at the fork-join $j, \sum_{k \varepsilon K} \frac{M F J_{j k}}{K}$ an average of the number of subtasks at the fork-join $j, f$ is the failure rate at the fork point, $\rho F J_{j}$ is the net traffic intensity at the fork-join $j, C a_{j k}$ is the coefficient of variation of the arrival process for product class $k$, and $C s_{j}$ is the aggregate coefficient of variation for the service time distribution at node $j$. Based on this regression equation, the number of products, the number of subtasks, the failure rate at the fork point, the net traffic intensity at the fork-join nodes, the coefficient of variation of the arrival process, and the aggregate coefficient of variation for the service time are significantly affecting the flow time in the node. Note that when $C a_{j k}$ and $C s_{j}$ equal to 1 (Markovian case) equation 4.5.2 agrees with equation 3.30 .

The approximation to determine the adjusted flow time at the fork-join $j$ of product class $k$ using the correction factor is calculated using equation 3.31, where $R_{j k}$ is computed according to the case analyzed. In Appendix $\mathrm{F}$ is presented the predicted error in flow time along with the observed values for the five scenarios analyzed. It should be noted that the correction terms hold good for the Markovian case and for the general case under the configurations analyzed in this research. 


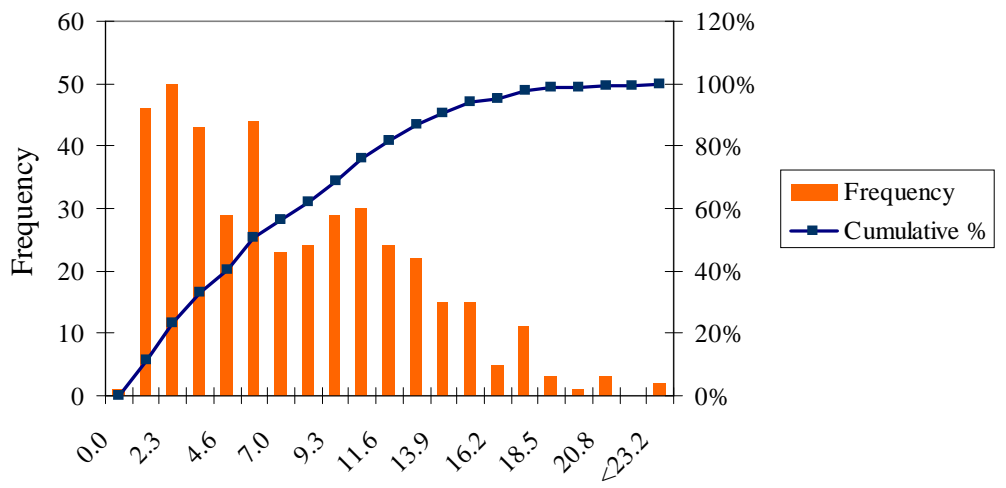

Flow Time Error $(\%)$

Figure 4.17: Distribution of the flow time error when the correction factors are used in the approximations.

\subsection{Numerical comparisons}

The analytical approximations using the correction factors were used to predict the flow time of the problem instances of section 4.4. In order to evaluate the performance of the correction factors in the approximations, flow time results of section 4.4 were compared with the values obtained by using the correction factors in the approximations. Appendix $\mathrm{G}$ presents the results for three, four, five and six product instances. These numerical comparisons show that using the correction factors in the approximation help to predict the flow time more accurately.

Figure 4.17 shows the distribution of the flow time error for the one hundred twenty problem instances when the correction factors were used in the approximations. The histogram indicates that the error seems to be distributed now between zero and $23 \%$, and the error in $95 \%$ of the problem instances (400 out of 420 data points) was between zero and $16 \%$. Figure 4.18 displays the performance of the approximations using the correction terms.

Detailed analysis by product class is presented in Figures 4.19, 4.20, 4.21, and 4.22, In these figures is shown the trend of the flow time error when the correction factors are added 


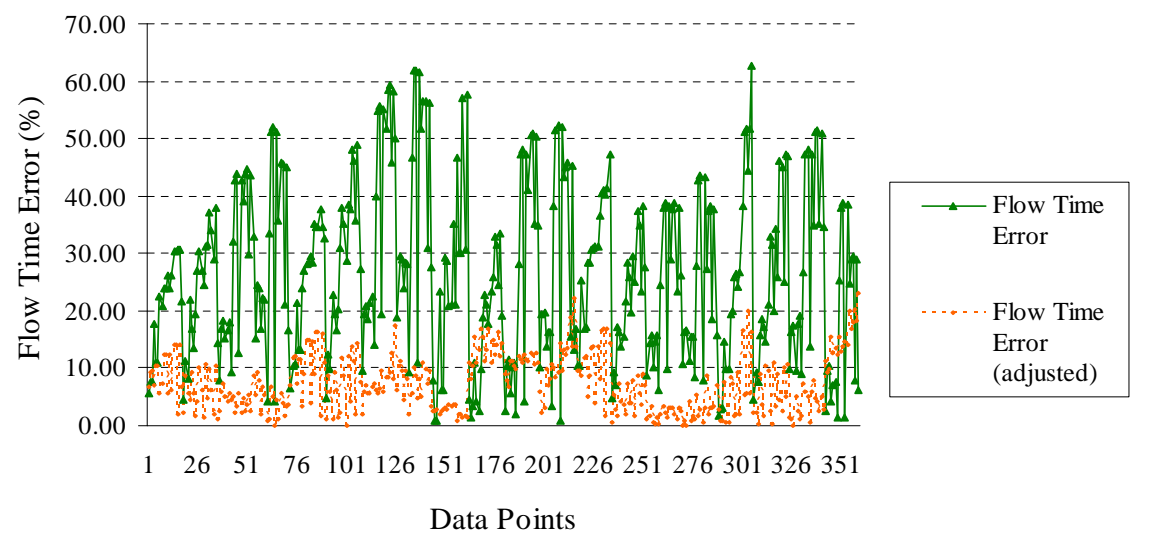

Figure 4.18: Overall trend of the flow time error using the correction factors in the approximations.

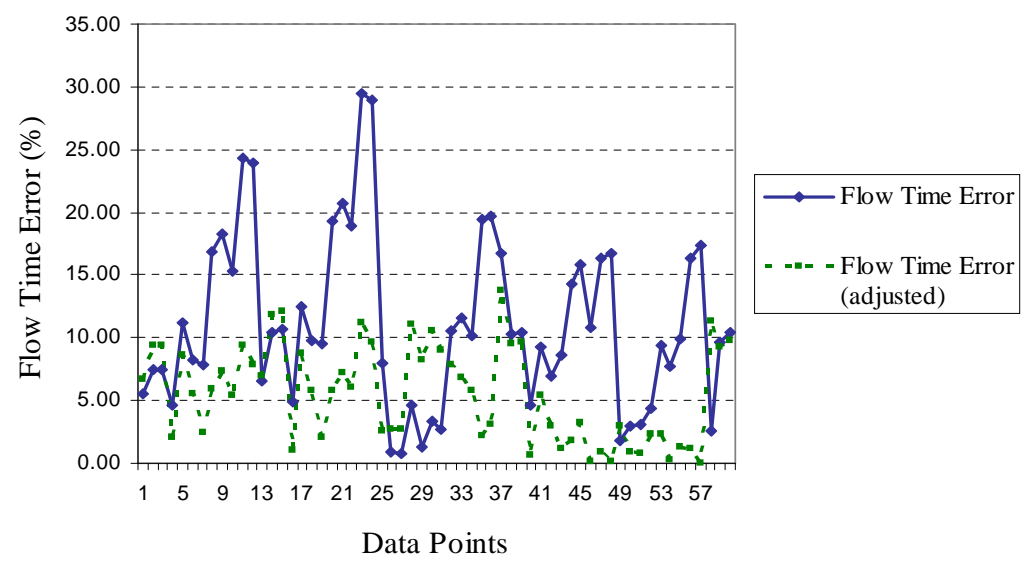

Figure 4.19: Flow time error for three product instances using correction factors in the approximations.

to the approximations for three, four, five and six product instances respectively. The results are compared with previous results obtained in section 4.4. These graphs clearly indicate that the error for most of the problem instances was reduced when the correction terms were used .

Histograms for the flow time error were plotted in order to obtain the overall distribution of the error by product classes. In Figure 4.23 is presented the flow time error distribution for three product classes. The error in $95 \%$ of the data points (57 out of 60 data points) was between zero and $11.8 \%$, and the maximum error was $13.81 \%$. Figure 4.24 shows the 


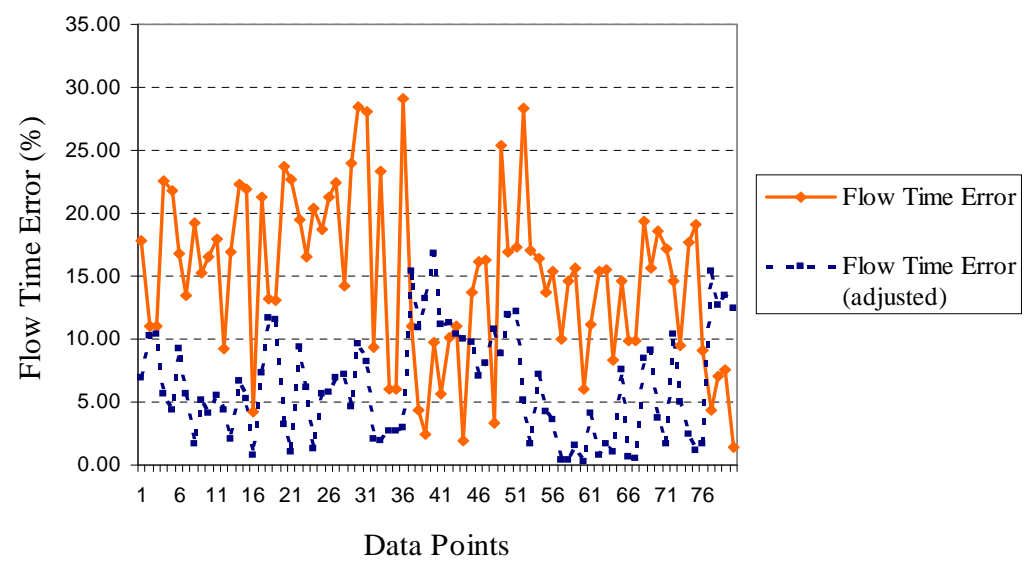

Figure 4.20: Flow time error for four product instances using correction factors in the approximations.

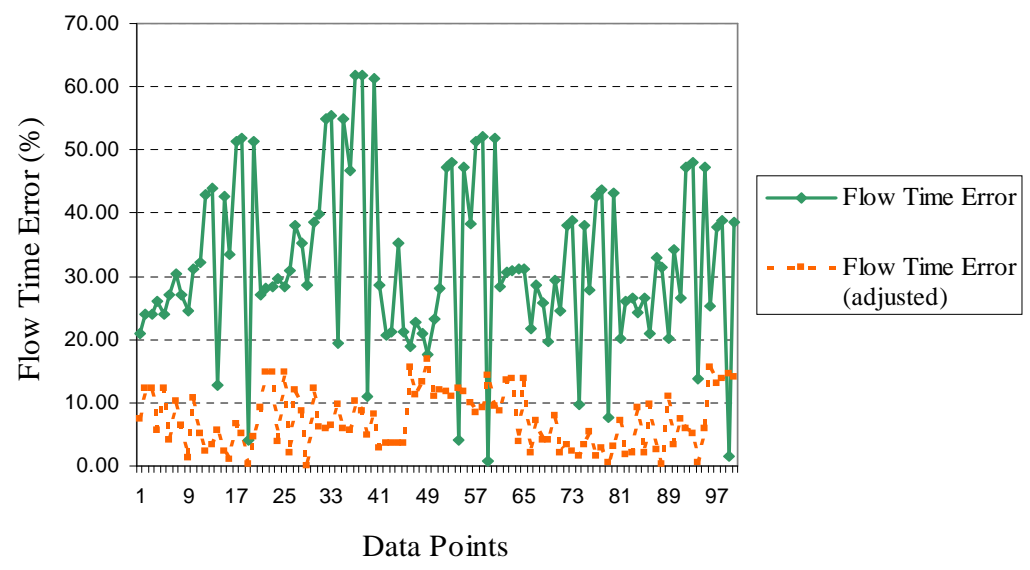

Figure 4.21: Flow time error for five product instances using correction factors in the approximations.

flow time error distribution for four product classes. The error in $96 \%$ of the data points (77 out of 80 data points) ranged between zero and 14.7\%, and the maximum error was $16.8 \%$. In the case of five product instances the error in $97 \%$ of the data points (97 out of 100 data points) was between zero and $15.3 \%$, and the maximum error was $16.97 \%$ (see Figure 4.25). In Figure 4.26 is presented the flow time error distribution for six product classes. The error in $95 \%$ of the data points (114 out of 120 data points) was between zero and $18.7 \%$, and the maximum error was $23.15 \%$ 


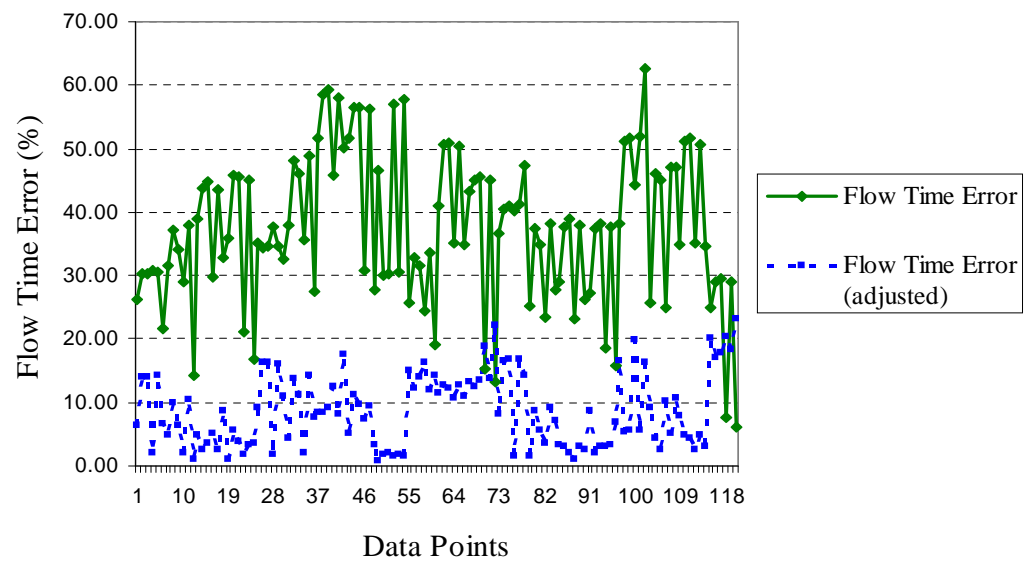

Figure 4.22: Flow time error for six product instances using correction factors in the approximations.

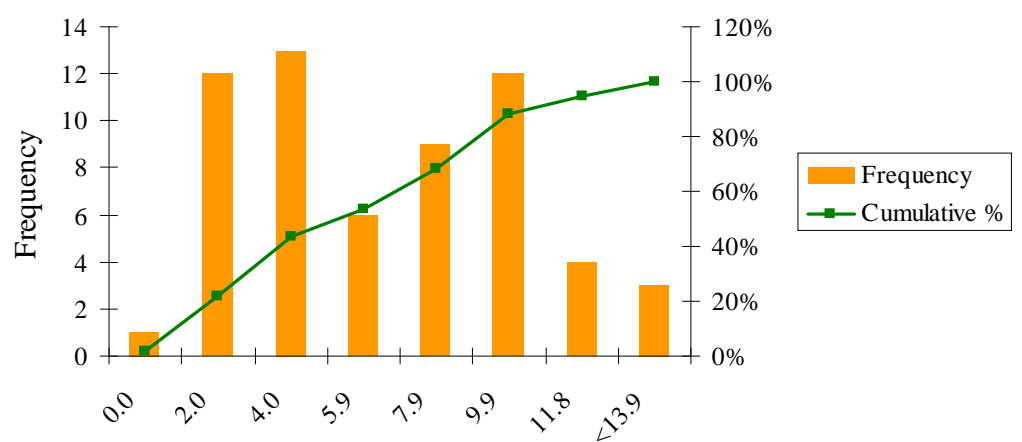

Flow Time Error $(\%)$

Figure 4.23: Flow time error distribution for three product classes with general type distribution using the correction factors.

Additional analysis was done by conducting a paired-t test for three, four, five and six product classes to determine whether there was significant difference between the flow time from the simulation models and the analytical formulations using the correction factors (see Table 4.20). Based on the paired-t test, the data strongly suggests that the means are the same for four, five and six product classes at 0.05 level of significance, and for three product classes at 0.02 level of significance taking into account all distribution types.

Further analysis were conducted by product class and arrival and service time distribution. Figures 4.27, 4.28, 4.34, 4.30, and 4.31 present, for each case, comparisons of the flow time 


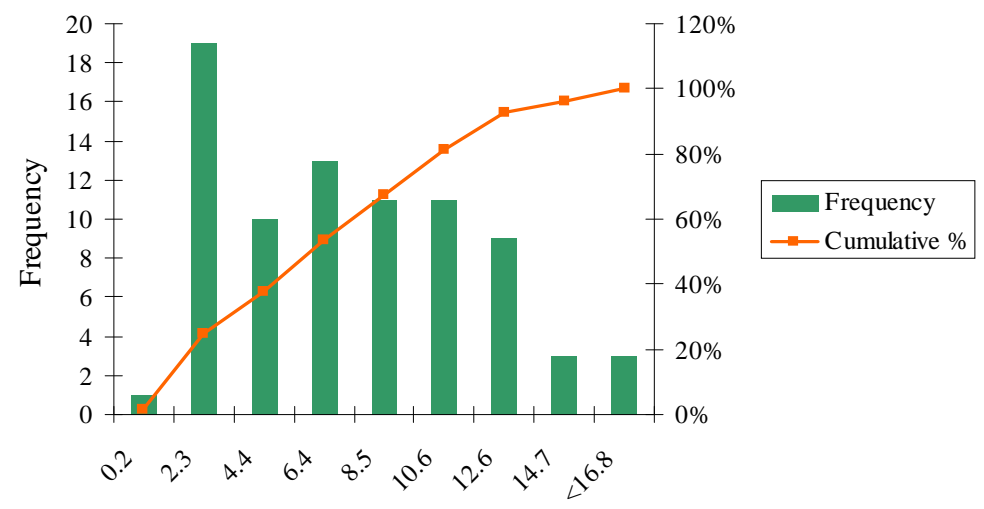

Flow Time Error (\%)

Figure 4.24: Flow time error distribution for four product classes with general type distribution using the correction factors.

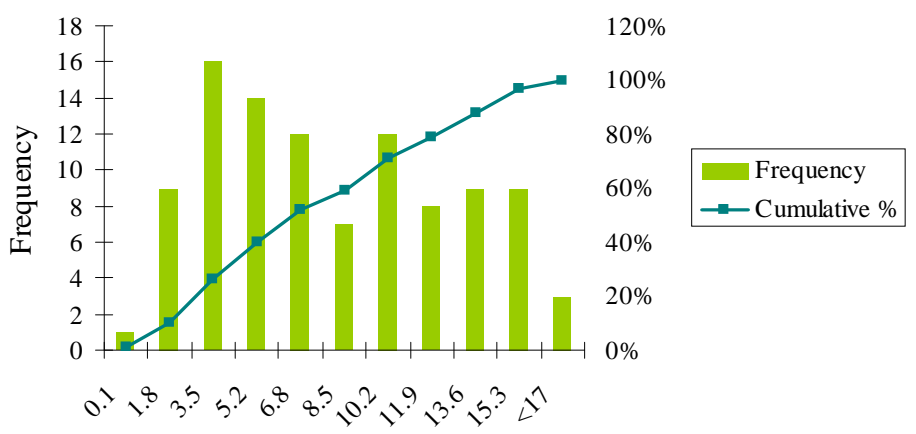

Flow Time Error (\%)

Figure 4.25: Flow time error distribution for five product classes with general type distribution using the correction factors.

error trend for three product instances. These figures clearly indicate that the error for most of the problem instances was reduced by adding the correction terms in the approximations. In Tables 4.21 and 4.22 are presented respectively the flow time error range, and the average

Table 4.20: Paired-t test results per product class using the correction factors

\begin{tabular}{|c|c|c|c|c|}
\hline \multicolumn{5}{|c|}{ Product Class } \\
\hline \hline & 3 & 4 & 5 & 6 \\
\hline t Stat & -2.37 & 0.27 & 0.08 & 0.26 \\
\hline t Critical two-tail & 2.00 & 1.99 & 1.98 & 1.98 \\
\hline $\mathrm{P}(\mathrm{T}<=\mathrm{t})$ two-tail & 0.02 & 0.79 & 0.94 & 0.79 \\
\hline
\end{tabular}




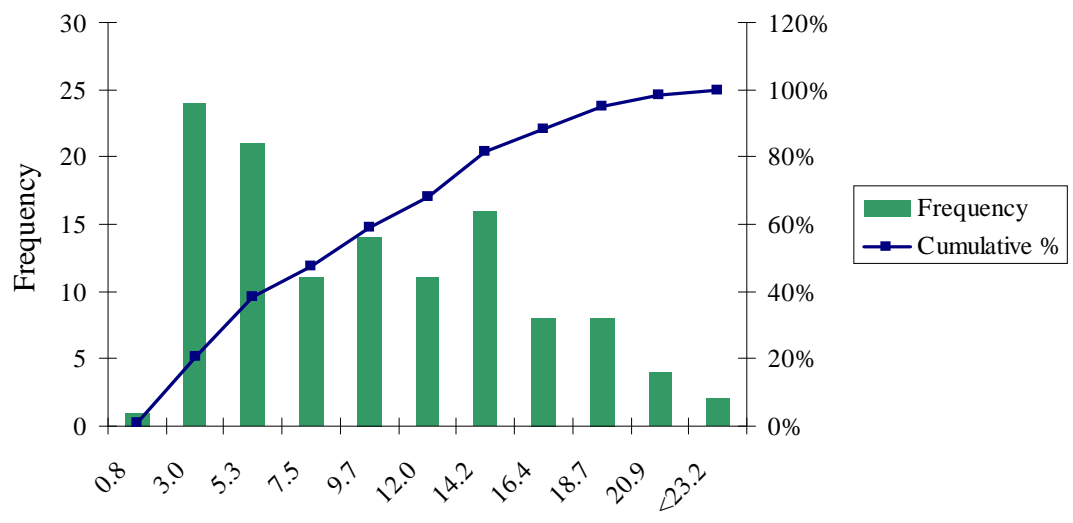

Flow Time Error (\%)

Figure 4.26: Flow time error distribution for six product classes with general type distribution using the correction factors.

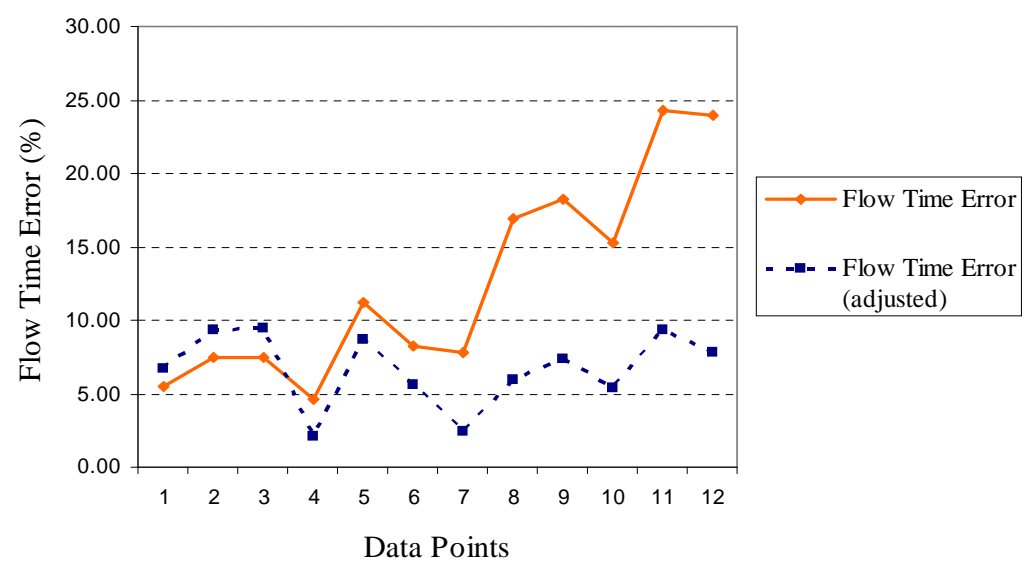

Figure 4.27: Flow time error for three product instances using correction factors in the $E_{2}-E_{2}$ case.

error for each case. On average, the flow time error was reduced by 50\%, from $11.04 \%$ to $5.55 \%$. Based on the results of the paired-t test, the data strongly suggests that the means are the same for the $\operatorname{Exp}-\mathrm{H}_{2}, \operatorname{Exp}-E_{2}$, and $H_{2}-\operatorname{Exp}$ configurations at $0.01,0.04$, and 0.03 level of significance respectively, and they are not the same for the $E_{2}-E_{2}$ and $E_{2}-E x p$ cases at 0.01 level of significance (see Table 4.23).

In the case of four product instances, Figures 4.32, 4.33, 4.34, 4.35, and 4.36 present for each configuration, comparisons of the trend of the flow time error when the correction 


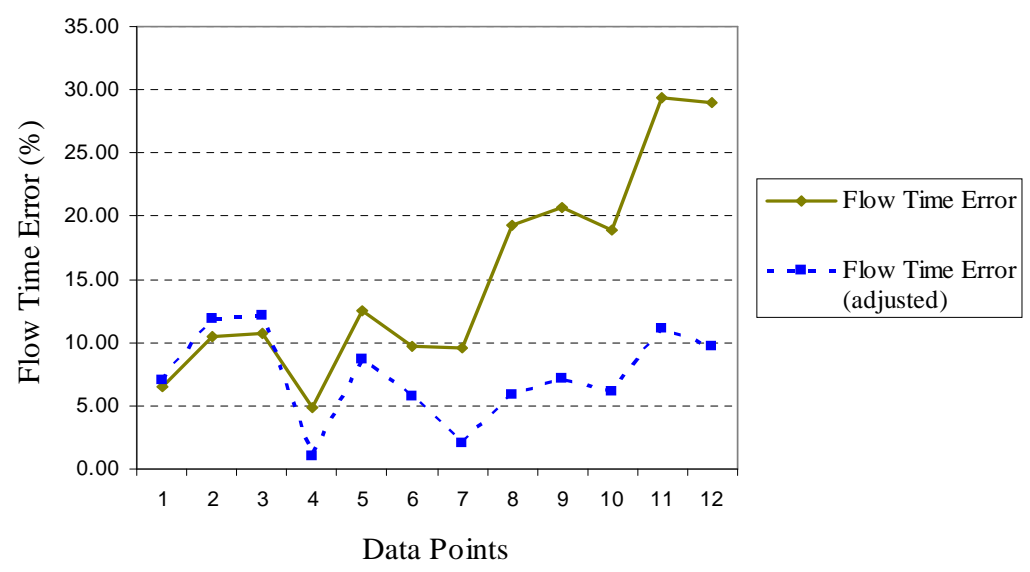

Figure 4.28: Flow time error for three product instances using correction factors in the $E_{2}-E x p$ case.

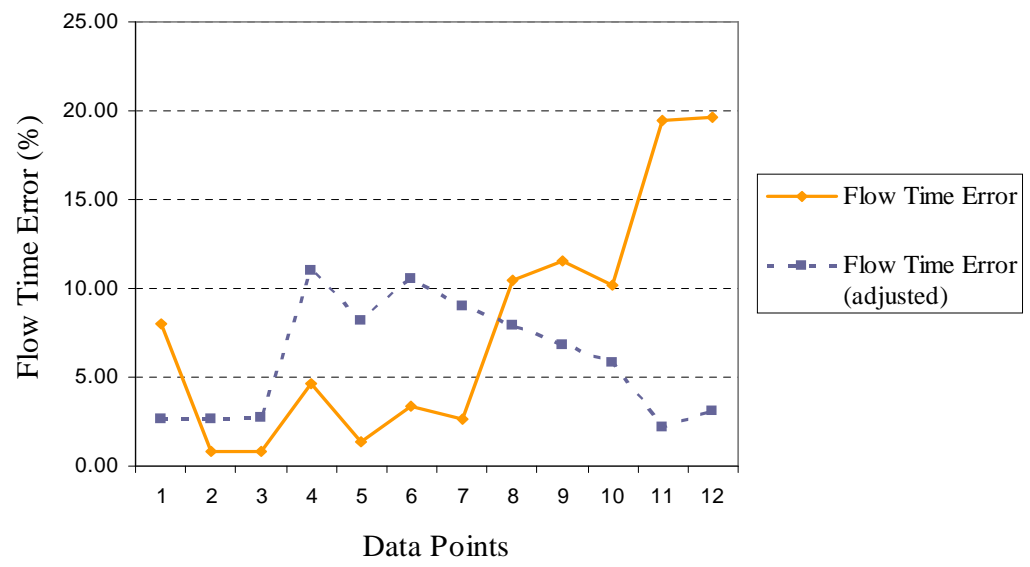

Figure 4.29: Flow time error for three product instances using correction factors in the $\operatorname{Exp}-\mathrm{H}_{2}$ case.

Table 4.21: Flow time error range for three product classes by case

\begin{tabular}{|c|c|c|c|c|c|}
\hline & $E_{2}-E_{2}$ & $E_{2}-\operatorname{Exp}$ & $\operatorname{Exp}-H_{2}$ & $\operatorname{Exp}-E_{2}$ & $H_{2}-\operatorname{Exp}$ \\
\hline \hline Error (\%) & $4.5-24.4$ & $4.8-29.5$ & $0.7-19.7$ & $4.6-16.8$ & $1.7-17.5$ \\
\hline Error-Correction Factor (\%) & $2.1-9.5$ & $1.0-12.2$ & $2.1-11.1$ & $0.0-13.9$ & $0.0-11.4$ \\
\hline
\end{tabular}

Table 4.22: Average flow time error for three product classes by case

\begin{tabular}{|c|c|c|c|c|c|}
\hline & $E_{2}-E_{2}$ & $E_{2}-\operatorname{Exp}$ & $\operatorname{Exp}-H_{2}$ & $\operatorname{Exp}-E_{2}$ & $H_{2}-\operatorname{Exp}$ \\
\hline \hline Average Error (\%) & 12.59 & 15.13 & 7.75 & 11.76 & 7.97 \\
\hline Average Error-Correction Factor (\%) & 6.68 & 7.38 & 6.05 & 4.1 & 3.53 \\
\hline
\end{tabular}




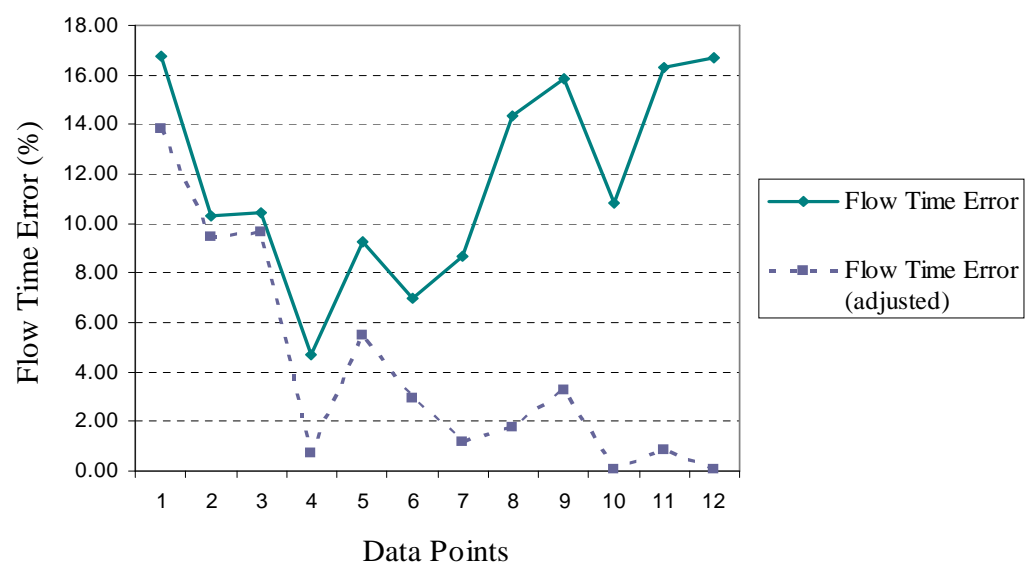

Figure 4.30: Flow time error for three product instances using correction factors in the $\operatorname{Exp}-E_{2}$ case.

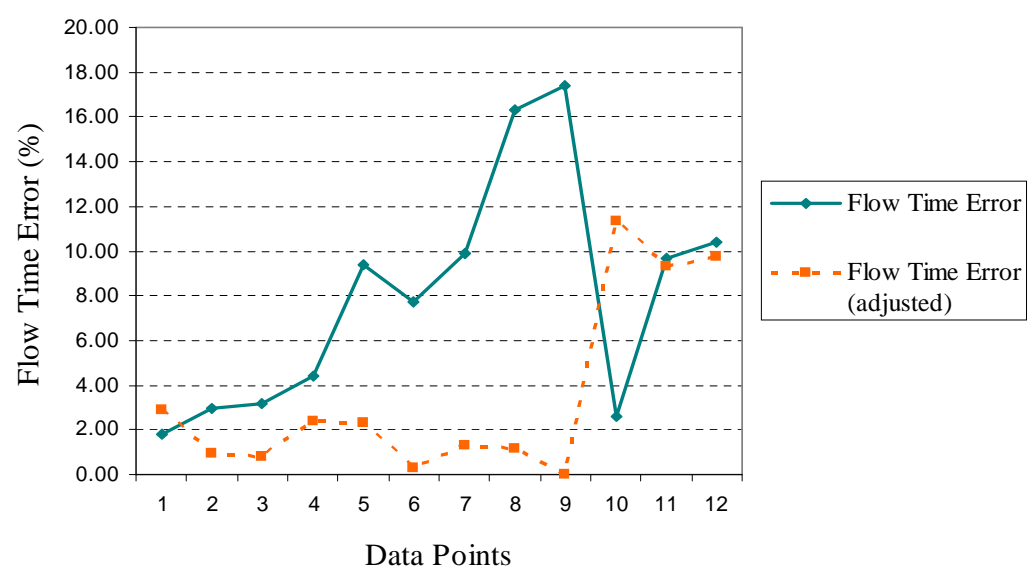

Figure 4.31: Flow time error for three product instances using correction factors in the $H_{2}-\operatorname{Exp}$ case.

terms are used in the approximations. It is apparent from the graphs that the correction factors help to reduce the flow time error in the approximations. In addition, Tables 4.24 and 4.25 contain comparisons of the flow time error range, and the average error for each case analyzed. Taking into account all data points for four product instances, the flow time error was reduced by $57.4 \%$ (from $14.85 \%$ to $6.33 \%$ ). From this perspective, the approximations are quite satisfactory, even thought the paired-t test suggests that the means 
Table 4.23: Paired-t test results for three product classes using correction factors by case

\begin{tabular}{|c|c|c|c|c|c|}
\hline & $E_{2}-E_{2}$ & $E_{2}-\operatorname{Exp}$ & $\operatorname{Exp}-H_{2}$ & $\operatorname{Exp}-E_{2}$ & $H_{2}-\operatorname{Exp}$ \\
\hline \hline t Stat & -10.89 & -8.78 & 3.45 & -2.33 & 2.48 \\
\hline $\mathrm{t}$ Critical two-tail & 2.20 & 2.20 & 2.20 & 2.20 & 2.20 \\
\hline $\mathrm{P}(\mathrm{T}<=\mathrm{t})$ two-tail & 0.00 & 0.00 & 0.01 & 0.04 & 0.03 \\
\hline
\end{tabular}

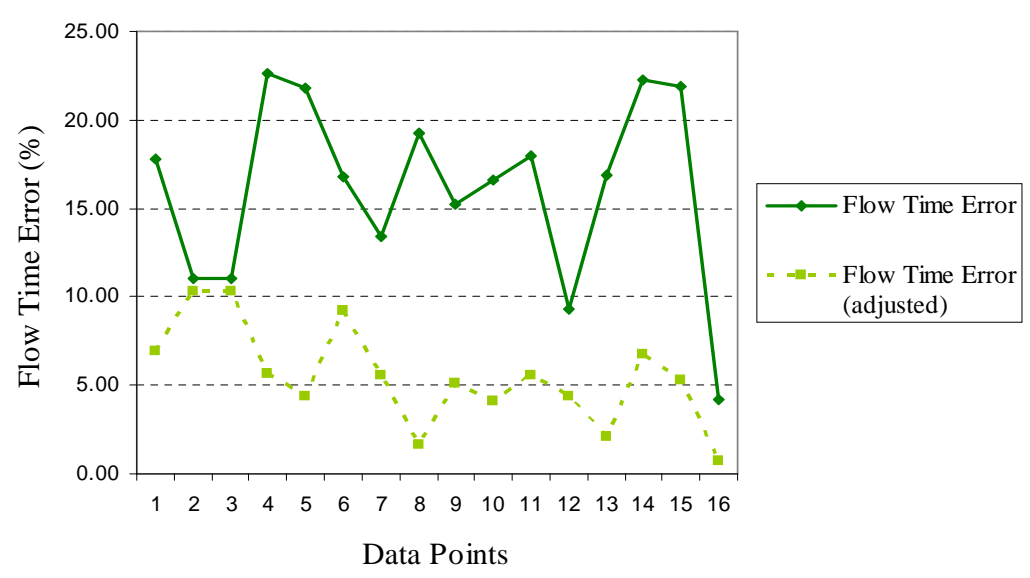

Figure 4.32: Flow time error for four product instances using correction factors in the $E_{2}-E_{2}$ case.

are not the same for the $E_{2}-E_{2}, E_{2}-E x p, E x p-H_{2}$, and $H_{2}-E x p$ configurations at 0.01 level of significance respectively (see Table 4.26).

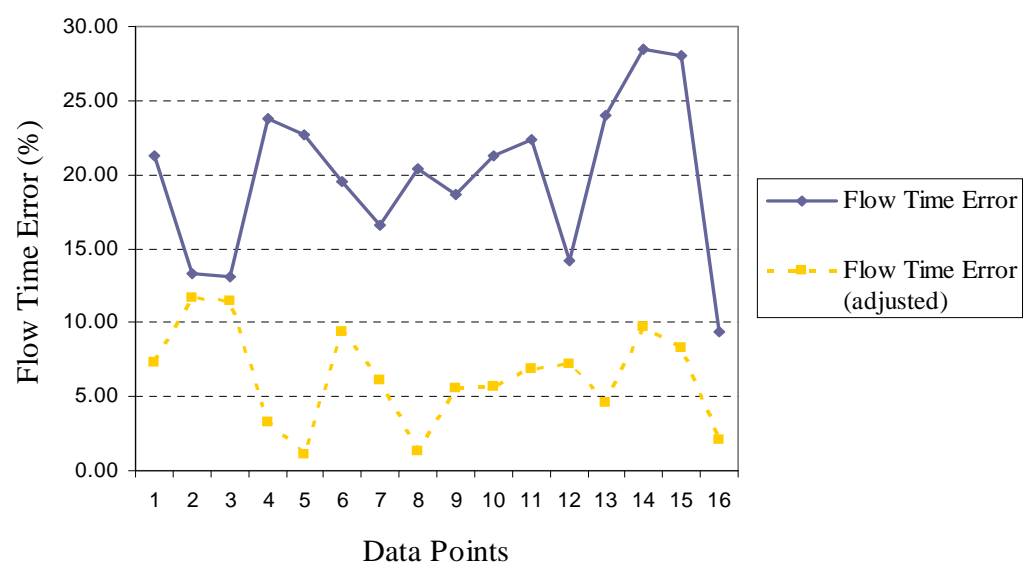

Figure 4.33: Flow time error for four product instances using correction factors in the $E_{2}-\operatorname{Exp}$ case. 


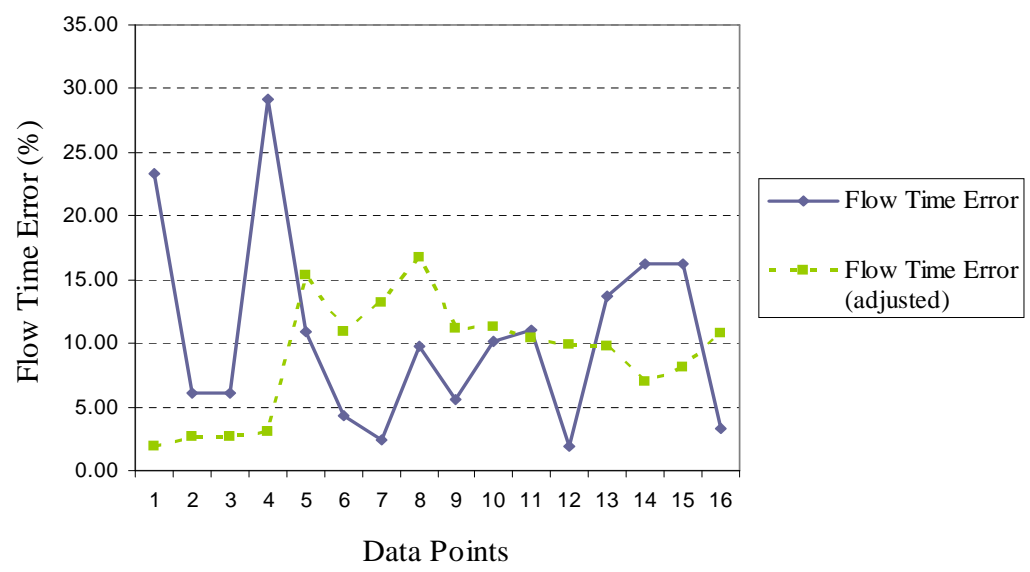

Figure 4.34: Flow time error for four product instances using correction factors in the $\operatorname{Exp}-\mathrm{H}_{2}$ case.

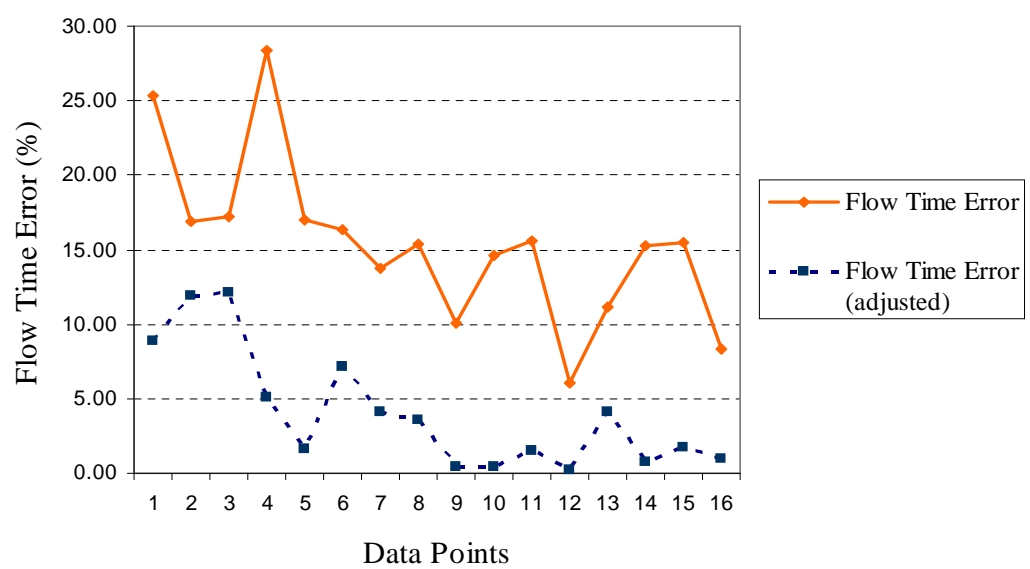

Figure 4.35: Flow time error for four product instances using correction factors in the $\operatorname{Exp}-E_{2}$ case.

Table 4.24: Flow time error range for four product classes by case

\begin{tabular}{|c|c|c|c|c|c|}
\hline & $E_{2}-E_{2}$ & $E_{2}-\operatorname{Exp}$ & $\operatorname{Exp}-H_{2}$ & $\operatorname{Exp}-E_{2}$ & $H_{2}-\operatorname{Exp}$ \\
\hline \hline Error (\%) & $4.0-22.7$ & $9.3-28.5$ & $1.9-29.2$ & $6.0-28.4$ & $1.3-19.4$ \\
\hline Adjusted Error (\%) & $0.7-10.4$ & $1.1-11.7$ & $1.9-16.8$ & $0.2-12.2$ & $0.5-15.4$ \\
\hline
\end{tabular}

Table 4.25: Average flow time error for four product classes by case

\begin{tabular}{|c|c|c|c|c|c|}
\hline & $E_{2}-E_{2}$ & $E_{2}-\operatorname{Exp}$ & $\operatorname{Exp}-H_{2}$ & $\operatorname{Exp}-E_{2}$ & $H_{2}-\operatorname{Exp}$ \\
\hline \hline Error (\%) & 16.13 & 19.81 & 10.63 & 15.44 & 12.21 \\
\hline Adjusted Error (\%) & 5.51 & 6.35 & 9.07 & 4.05 & 6.65 \\
\hline
\end{tabular}




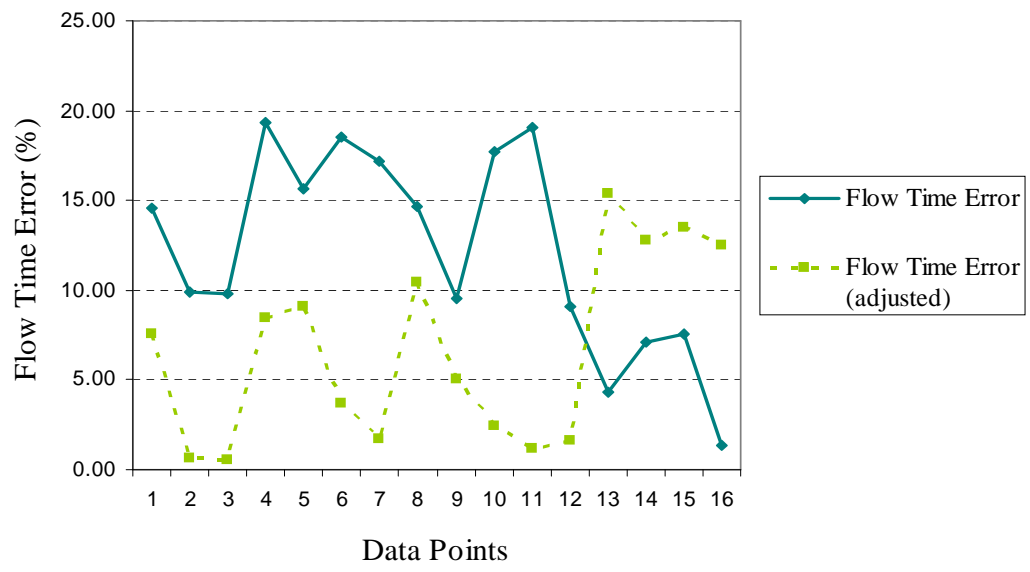

Figure 4.36: Flow time error for four product instances using correction factors in the $H_{2}-E x p$ case.

Table 4.26: Paired-t test results for four product classes using correction factors by case

\begin{tabular}{|c|c|c|c|c|c|}
\hline & $E_{2}-E_{2}$ & $E_{2}-\operatorname{Exp}$ & $\operatorname{Exp}-H_{2}$ & $\operatorname{Exp}-E_{2}$ & $H_{2}-\operatorname{Exp}$ \\
\hline \hline $\mathrm{t}$ Stat & -7.21 & -6.56 & 4.44 & -1.17 & 3.91 \\
\hline $\mathrm{t}$ Critical two-tail & 2.13 & 2.13 & 2.13 & 2.13 & 2.13 \\
\hline $\mathrm{P}(\mathrm{T}<=\mathrm{t})$ two-tail & 0.00 & 0.00 & 0.00 & 0.26 & 0.00 \\
\hline
\end{tabular}

Figures 4.37, 4.38, 4.39, 4.40, and 4.41, show that the correction terms help to reduce remarkably well the flow time error in the approximations for five product classes. Additional analysis is presented in Tables 4.27 and 4.28, which contain comparisons of the flow time error range, and the average error for each case analyzed. Although, the flow time error was reduced by $77.19 \%$ (from $31.86 \%$ to $7.27 \%$ ), the paired-t test suggests that the means are the same for only the $\operatorname{Exp}-E_{2}$ case at 0.05 level of significance (see Table 4.29).

Comparisons of the trend of the flow time error for six product classes are displayed in Figures 4.42, 4.43, 4.44, 4.45, 4.46. These graphs show that the approximations perform

Table 4.27: Flow time error range for five product classes by case

\begin{tabular}{|c|c|c|c|c|c|}
\hline & $E_{2}-E_{2}$ & $E_{2}-\operatorname{Exp}$ & $\operatorname{Exp}-H_{2}$ & $\operatorname{Exp}-E_{2}$ & $H_{2}-\operatorname{Exp}$ \\
\hline \hline Error (\%) & $4.1-52.0$ & $10.9-61.9$ & $0.7-52.3$ & $7.7-43.7$ & $1.4-48.1$ \\
\hline Adjusted Error (\%) & $0.1-12.4$ & $0.0-14.9$ & $2.7-17.0$ & $0.5-13.8$ & $0.2-15.6$ \\
\hline
\end{tabular}




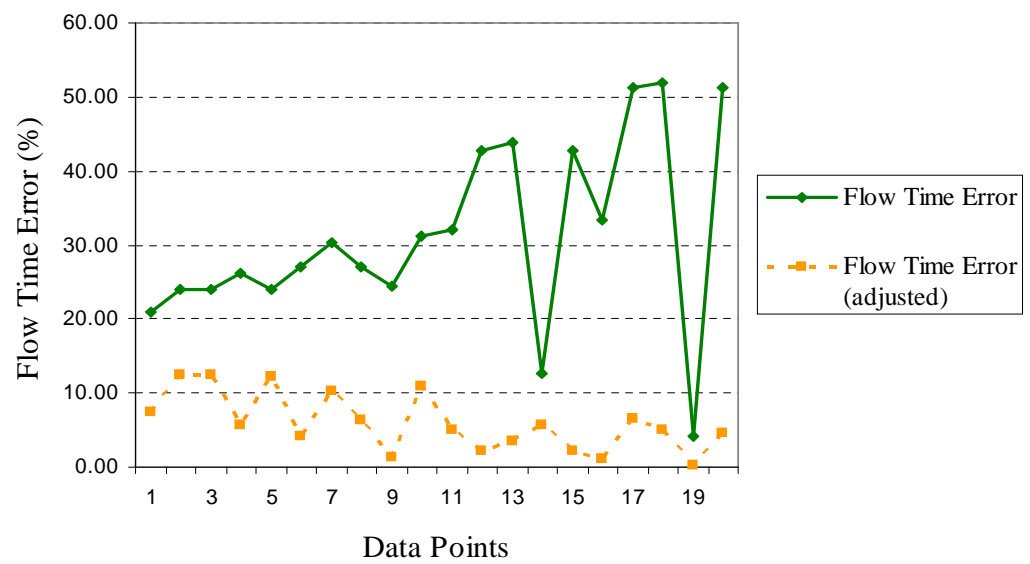

Figure 4.37: Flow time error for five product instances using correction factors in the $E_{2}-E_{2}$ case.

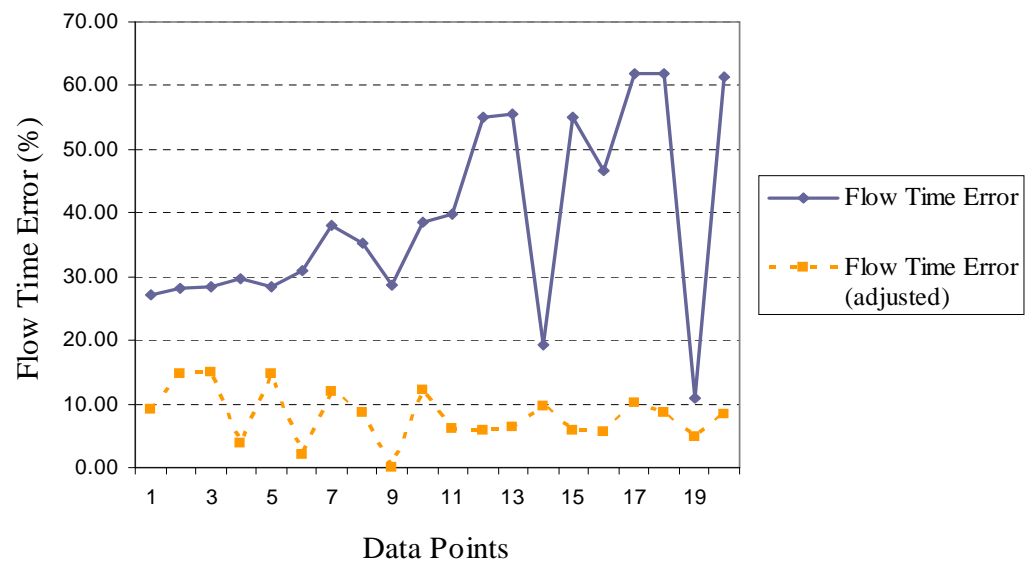

Figure 4.38: Flow time error for five product instances using correction factors in the $E_{2}-E x p$ case.

Table 4.28: Average flow time error for five product classes by case

\begin{tabular}{|c|c|c|c|c|c|}
\hline & $E_{2}-E_{2}$ & $E_{2}-\operatorname{Exp}$ & $\operatorname{Exp}-H_{2}$ & $\operatorname{Exp}-E_{2}$ & $H_{2}-\operatorname{Exp}$ \\
\hline \hline Error (\%) & 31.27 & 39.02 & 29.85 & 29.59 & 29.40 \\
\hline Adjusted Error (\%) & 5.93 & 8.20 & 9.76 & 3.90 & 7.22 \\
\hline
\end{tabular}

Table 4.29: Paired-t test results for five product classes using correction factors by case

\begin{tabular}{|c|c|c|c|c|c|}
\hline & $E_{2}-E_{2}$ & $E_{2}-\operatorname{Exp}$ & $E x p-H_{2}$ & $E x p-E_{2}$ & $H_{2}-E x p$ \\
\hline \hline t Stat & -8.20 & -10.78 & 5.92 & -0.72 & 4.47 \\
\hline t Critical two-tail & 2.09 & 2.09 & 2.09 & 2.09 & 2.09 \\
\hline $\mathrm{P}(\mathrm{T}<=\mathrm{t})$ two-tail & 0.00 & 0.00 & 0.00 & 0.48 & 0.00 \\
\hline
\end{tabular}




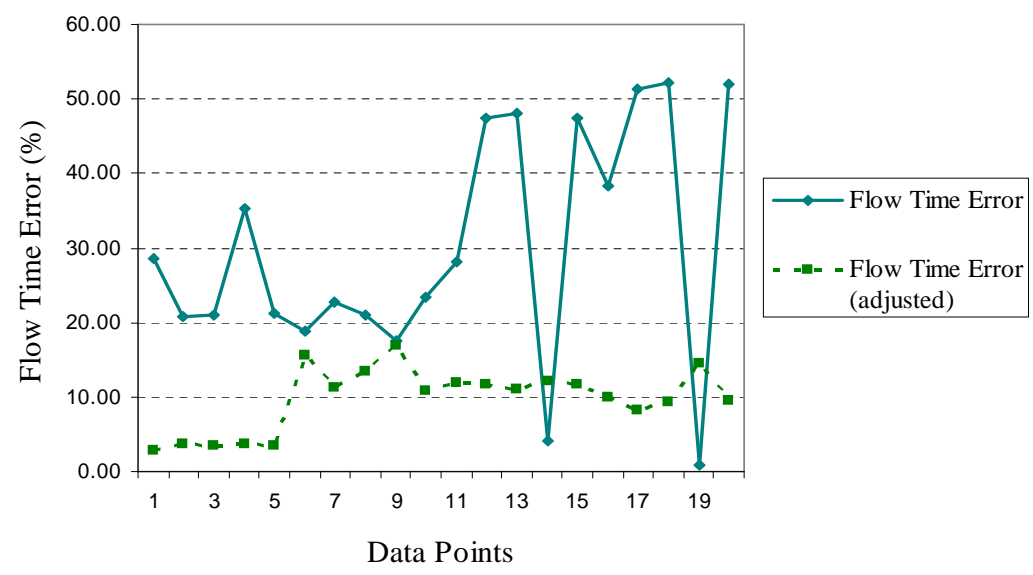

Figure 4.39: Flow time error for five product instances using correction factors in the $\operatorname{Exp}-\mathrm{H}_{2}$ case.

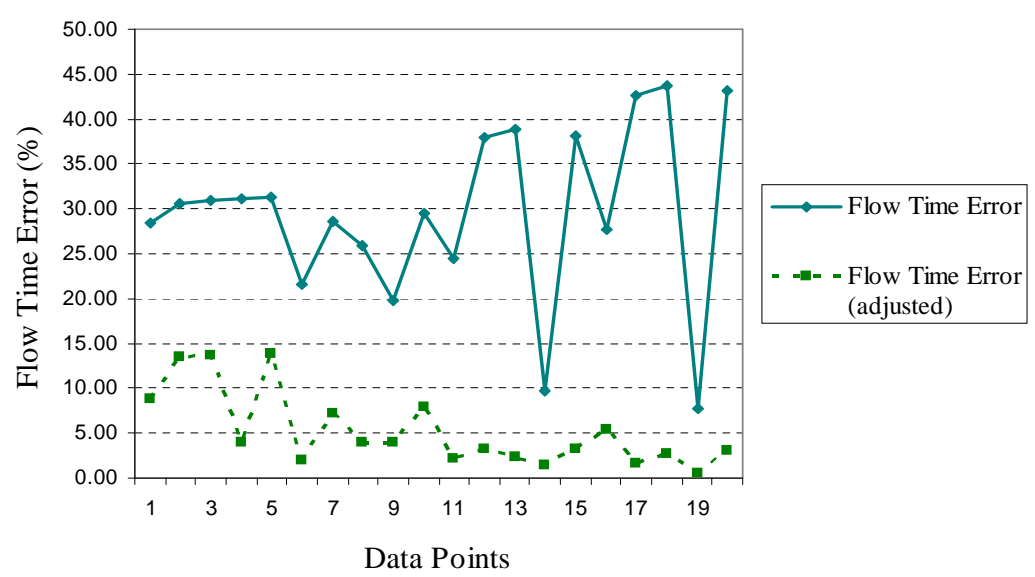

Figure 4.40: Flow time error for five product instances using correction factors in the $E x p-E_{2}$ case.

quite well when correction factors are added. In Tables 4.30 and 4.31 is presented the flow time error range, and the average error for each case analyzed. The average flow time error was reduced from $37.21 \%$ (approximations without correction terms) to $8.65 \%$. From this perspective, the approximations are quite satisfactory, even thought the paired-t test suggests that the means are same only for the $\operatorname{Exp}-E_{2}$ case at 0.05 level of significance (see Table 4.32). 


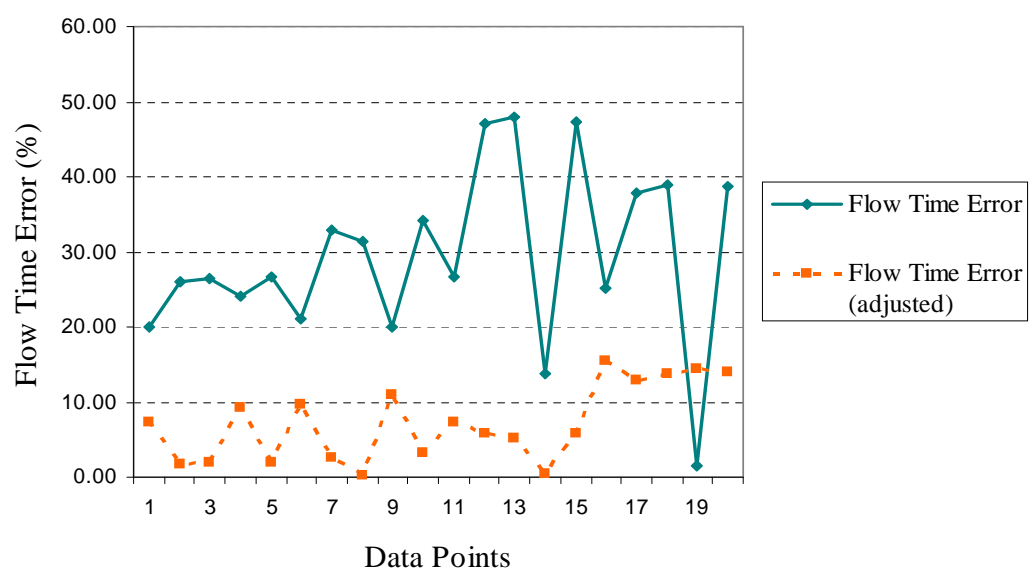

Figure 4.41: Flow time error for five product instances using correction factors in the $H_{2}-E x p$ case.

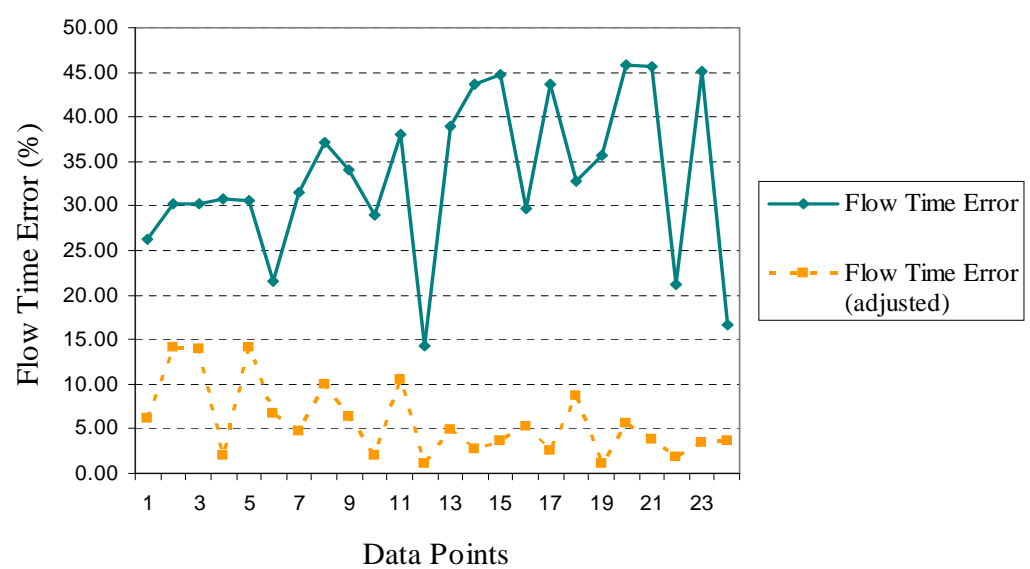

Figure 4.42: Flow time error for six product instances using correction factors in the $E_{2}-E_{2}$ case.

Table 4.30: Flow time error range for six product classes by case

\begin{tabular}{|c|c|c|c|c|c|}
\hline & $E_{2}-E_{2}$ & $E_{2}-\operatorname{Exp}$ & $\operatorname{Exp}-H_{2}$ & $\operatorname{Exp}-E_{2}$ & $H_{2}-\operatorname{Exp}$ \\
\hline \hline Error (\%) & $14.2-45.9$ & $27.4-59.2$ & $13.1-57.7$ & $15.8-47.3$ & $6.1-62.8$ \\
\hline Adjusted Error (\%) & $1.0-14.2$ & $1.7-17.6$ & $0.7-22.3$ & $0.9-16.9$ & $2.3-23.2$ \\
\hline
\end{tabular}

Table 4.31: Average flow time error for six product classes by case

\begin{tabular}{|c|c|c|c|c|c|}
\hline & $E_{2}-E_{2}$ & $E_{2}-\operatorname{Exp}$ & $\operatorname{Exp}-H_{2}$ & $\operatorname{Exp}-E_{2}$ & $H_{2}-\operatorname{Exp}$ \\
\hline \hline Error (\%) & 33.22 & 44.00 & 37.08 & 33.40 & 38.34 \\
\hline Adjusted Error (\%) & 5.77 & 9.75 & 10.76 & 6.31 & 10.65 \\
\hline
\end{tabular}




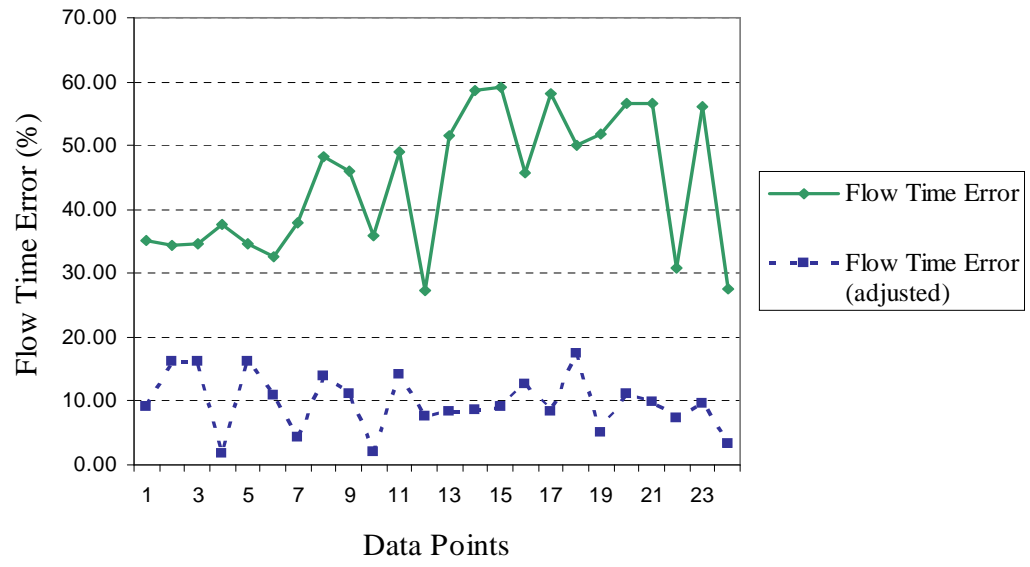

Figure 4.43: Flow time error for six product instances using correction factors in the $E_{2}-\operatorname{Exp}$ case.

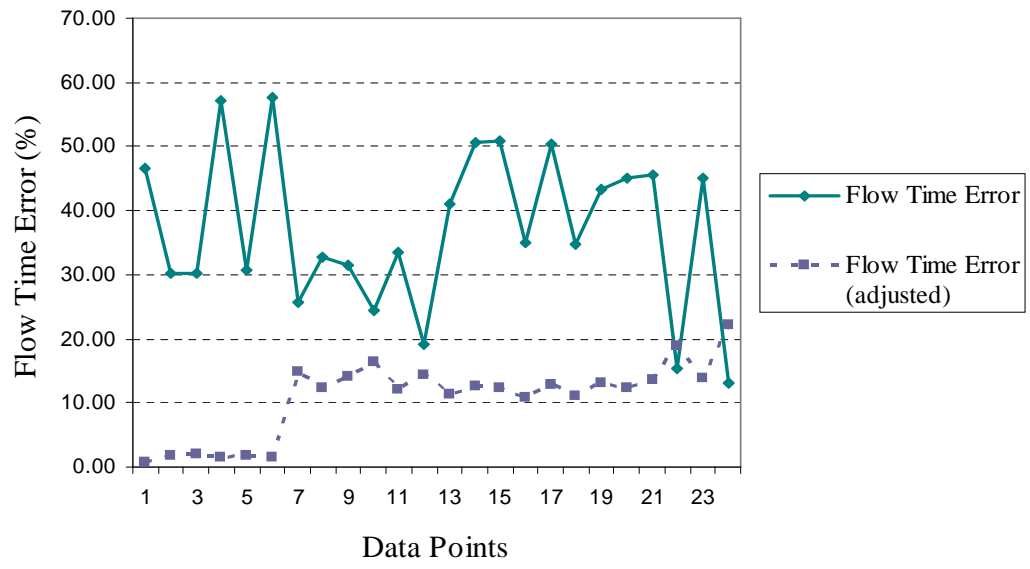

Figure 4.44: Flow time error for six product instances using correction factors in the $\operatorname{Exp}-\mathrm{H}_{2}$ case.

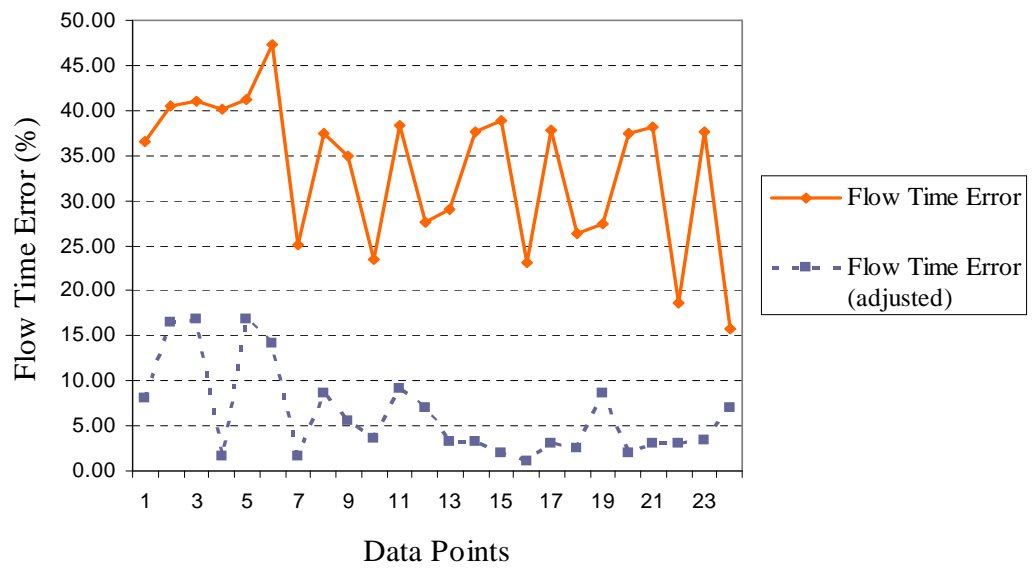

Figure 4.45: Flow time error for six product instances using correction factors in the $\operatorname{Exp}-E_{2}$ case. 


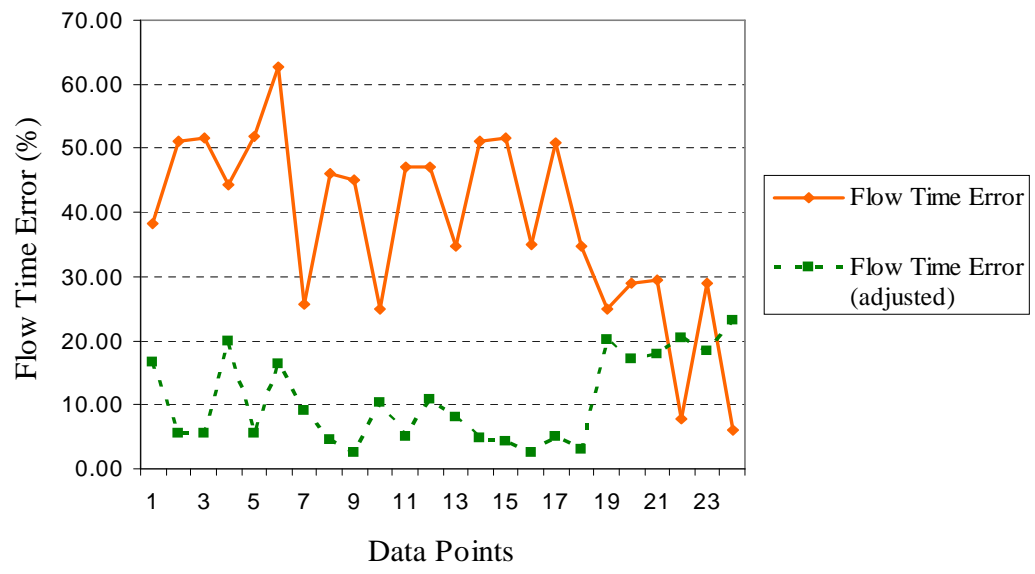

Figure 4.46: Flow time error for six product instances using correction factors in the $H_{2}-E x p$ case.

Table 4.32: Paired-t test results for six product classes using correction factors by case

\begin{tabular}{|c|c|c|c|c|c|}
\hline & $E_{2}-E_{2}$ & $E_{2}-\operatorname{Exp}$ & $\operatorname{Exp}-H_{2}$ & $\operatorname{Exp}-E_{2}$ & $H_{2}-\operatorname{Exp}$ \\
\hline \hline $\mathrm{t}$ Stat & -6.80 & -12.17 & 7.08 & -0.76 & 4.13 \\
\hline $\mathrm{t}$ Critical two-tail & 2.07 & 2.07 & 2.07 & 2.07 & 2.07 \\
\hline $\mathrm{P}(\mathrm{T}<=\mathrm{t})$ two-tail & 0.00 & 0.00 & 0.00 & 0.45 & 0.00 \\
\hline
\end{tabular}

In summary, when the analysis is conducted by product class (including all type of distributions) the data strongly suggests that the means are the same for four, five and six product classes at 0.05 level of significance, and for three product classes at 0.02 level of significance. The total average flow time error decreased from $26.39 \%$ (approximations without correction terms) to $7.23 \%$, and the maximum error found was $23.14 \%$. Based on this results, in the general type system the approximations perform much better when the corrections factors were added to the approximations. 


\section{CHAPTER 5}

\section{CONCLUSIONS AND FUTURE WORK}

In this research, new analytical formulations are proposed to deal with manufacturing systems where it simultaneously considers several issues of practical interest such as parallel processing with synchronization queue, multiple product classes, and job circulation due to random part failures. Section 5.1 summarizes the conclusions of this research and in section 5.2 suggestions for future work are presented.

\subsection{Conclusions}

The nature of the web server assembly process is complex, which includes serial and parallel processing, multiple product classes and job circulation due to random failures. Although manufacturing systems have been studied using analytical approaches, performance analysis of manufacturing systems considering simultaneously multiple product classes, and job circulation due to random part failures have not been widely studied in the context of parallel processing. Pradhan et al. [86], and Pradhan and Damodaran [85] addressed a similar problem in which was presented analytical approximations to estimate the performance measures of an optoelectronic manufacturing system with multiple product classes, job circulations due to failures, and some resources being shared among different products classes. However, this dissertation differs in that, the problem object of this study considers parallel processing.

The analytical formulations presented in this research, extend previous queueing models used in assembly manufacturing systems. Existing analytical formulations related to forkjoin queues were modified to incorporate the fact that a node can be visited by different 
product classes, and that job can return more than once to the fork point due to job failures. In addition, the methodology applied to develop the regression equation (correction factors) could be generalized to other queueing network systems.

The queueing network model proposed improves previous formulations in two main ways. First, it can handle serial and different configurations of paralleling processing in the same framework, with multiple product classes, and job circulation due to random part failures. Second, the analytical formulations have appropriate correction terms that minimize the gap in the error between the analytical approximation and the simulation models. The correction terms may have to be revised if the manufacturing system to be analyzed has parameters that are very different from what was considered in this research.

In order to to test the accuracy of the analytical estimates, random problem instances were generated to estimate the performance measures of the system. Discrete event simulation models were developed to simulate the problem instances, and the results from the analytical approximations and simulation were compared to evaluate the accuracy of the analytical approximations.

In the Markovian case, the approximations without correction terms performed quite well for one and two product problem instances. The flow time error for one product problem instances was between zero and 5\%, and for two product instances was between zero and $10 \%$. The data strongly suggested that at 0.05 level of significance the means are the same. However, it was observed that the flow time error increased as the number of products and net traffic intensity increased. Therefore, correction terms for single and fork-join stations were developed via regression analysis to deal with three, four, five, and six product instances.

Since the flow time in the network is the aggregation of the flow time at each station, for simplicity one station instances were considered to develop the correction factors. For 
the case of non fork-join stations, thirty two problem instances (144 data points) were generated in order to predict the flow time. The data analysis of single stations revealed that the main error of the flow time was coming from the waiting time, the variables affecting the results were the number of products in the network and the net traffic intensity, and there was a nonlinear relationship in the parameters. The correction term for this case was developed analyzing the behavior of the error in the waiting time and by using Microsoft Excel the best regression equation was developed. The regression model was stated in terms of the net traffic intensity and the number of products in the network.

In the case of fork-join stations, seven hundred thirty five problem instances (2547 data points) were generated to develop the correction factor. A straight line relationship between the flow time error and the net traffic intensity, and between the flow time error and failure rate was observed. Statistical Analysis Software (SAS) was used to determine the parameters of the model, and the stepwise procedure was used to identify the significant variables in the regression model. Based on the regression equation, the number of products, the number of subtasks, the failure rate at the fork point, the net traffic intensity at the fork-join nodes, the coefficient of variation of the arrival process, and the aggregate coefficient of variation for the service time were significantly affecting the flow time in the node

The numerical comparisons in the Markovian case showed that the approximations perform remarkably well when the corrections factors were used. The average flow time error for three product instances was reduced from $14.20 \%$ to $5.13 \%$, for four product instances from $24.19 \%$ to $6.10 \%$, for five product instances from $44.13 \%$ to $5.66 \%$, and for six product instances from $57.23 \%$ to $5.47 \%$ (see Figure 5.1). In general, the average flow time error was reduced from $38.12 \%$ to $5.59 \%$, and for the problem instances where the number of subtasks was less than the number of servers, the average error decreased from $39.03 \%$ to $5.54 \%$. 


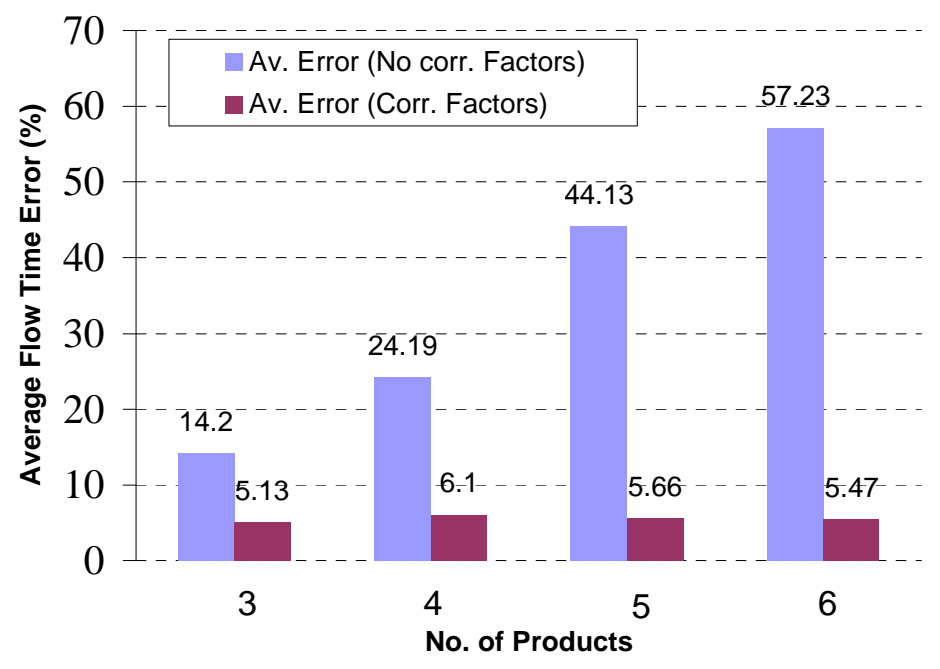

Figure 5.1: Comparisons of the average flow time error per class in the Markovian case

In the general case, the computational experiments showed that the flow time error increased as the number of products and net traffic intensity increased coinciding with the Markovian case. The approximations without correction terms performed quite well for one and two product instances, where the error was between zero and $12.4 \%$. However, from three to six product classes the flow time error was roughly between zero and $62.71 \%$, and the data strongly suggest that the means for those problem classes are different at 0.01 level of significance.

When the correction terms via regression analysis were added to the approximations, the flow time error was reduced by $50 \%$ (from $11.04 \%$ to $5.55 \%$ ) for three product classes, $57.4 \%$ (from $14.85 \%$ to $6.33 \%$ ) for four product classes, $77.19 \%$ (from $31.86 \%$ to $7.27 \%$ ) for five product classes, and $76.75 \%$ (from $37.21 \%$ to $8.65 \%$ ) for six product classes. The results indicate that the error seems to be distributed now between zero and $23 \%$, and the error in $95 \%$ of the problem instances (400 out of 420 data points) was between zero and 16\%. In summary, the average flow time error decreased from $26.39 \%$ (approximations without correction terms) to $7.23 \%$, and the maximum error was $23.14 \%$. Based on these 


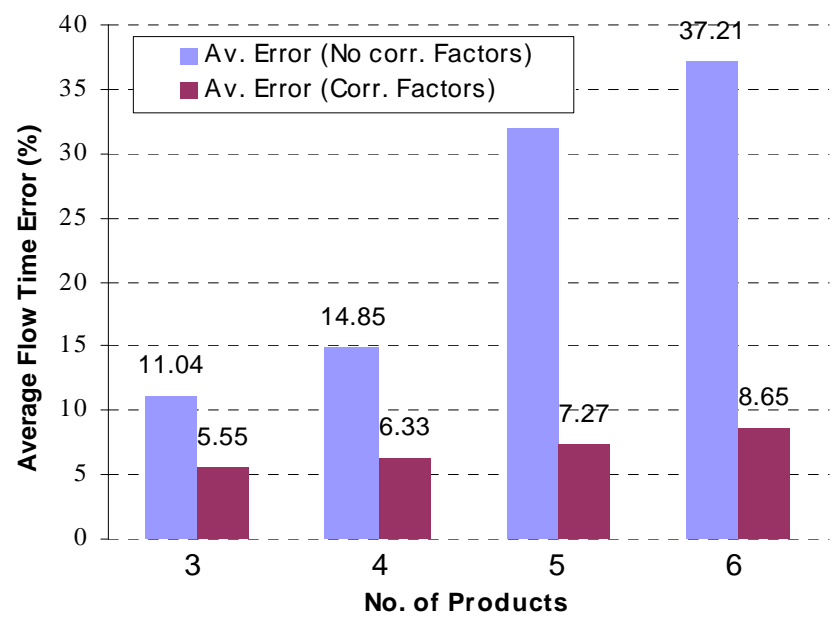

Figure 5.2: Comparisons of the average flow time error per class in the general case

results, in the general type system the approximations perform much better when the corrections factors were added to the approximations (see Figure 5.2).

All the equations stated in the analytical formulations were implemented as a set of Matlab scripts. By using this set, operations managers of web server assembly lines, or other service systems with similar characteristics can estimate different system performance measures. This can help managers to make judicious decisions - especially setting delivery due dates, capacity planning, and bottleneck mitigation, among others.

\subsection{Future work}

Performance analysis of manufacturing systems with multiple product classes, probabilistic job circulation, and parallel processing can be extended in different ways. In this study the parallel processing with synchronization constraints was modeled by using fork-join stations with homogeneous server fork-join queues. Future studies may use analytical approximations that assume heterogeneous server fork-join queues. In addition, in this research it was assumed some types of arrival and service time distributions in the fork-join 
stations; therefore, future work with the characteristic of the problem object of this study can approach other general distribution configurations.

For single stations, the correction factor developed is stated in terms of the number of products in the network, and the net traffic intensity at a given node. Another potential area for research is to analyze the impact of the coefficient of variation of the arrival process and the aggregate coefficient of variation of the service time on the flow time error in single stations.

In this research an open system with jobs being processed according to a first-in-first-out (FIFO) discipline at each station was considered. Different contributions on queueing networks with priority service disciplines have been made [20, 119, 117, 43, 116]. However, future studies may analyze this research problem under priority service disciplines at each station. Another line of research is to use these analytical models in a capacity planning model to optimize the resource allocation at various stages of production. 


\section{LIST OF REFERENCES}

[1] I. Akyildiz. Mean value analysis for blocking queueing networks. IEE Transactions on Software Engineering, 14(4):418-428, 1988.

[2] Ch. Alexopoulos. A comprehensive review of methods for simulation output analysis. In L.F. Perrone, F.P Wieland, J. Liu, B.G. Lawson, D.M. Nicol Nicol, and R.M. Fujimoto, editors, Proceedings of the 2006 Winter Simulation Conference, 2006.

[3] T. Altiok. Performance analysis of manufacturing systems. Springer, New York, 1997.

[4] F. Baccelli, W. Massey, and D. Towsley. Acyclic fork-join queueing networks. Journal of the ACM, 36(3):615-642, 1989.

[5] S. Balsamo, L. Donatiello, and N. M. Van Dijk. Bound performance models of heterogeneous parallel processing systems. IEEE Transactions on Parallel and Distributed Systems, 9(10):1041-1056, 1998.

[6] S. Balsamo and I. Mura. Approximate response time distributions in fork and join systems. In Proceedings of the 1995 ACM SIGMETRICS Joint International Conference on Measurement and Modeling of Computer Systems, pages 305-306. ACM, 1995.

[7] S. Balsamo and I. Mura. On queue length moments in fork and join queuing networks with general services times, volume 1245 of Computer Performance Evaluation Modelling Techniques and Tools, pages 218-231. Springer, Berlin, 1997.

[8] Y. Bard. Some extensions to multiclass queueing network analysis. Performance of Computer Systems, 1979.

[9] T. Baris. A decomposition method for multistation production systems. PhD thesis, 1994.

[10] F. Baskett, K. Chandy, and R. Muntz. Open, closed, and mixed networks of queues with different classes of customers. Journal of the ACM, 22(2):248-260, 1975.

[11] N. Bengtson. Measuring errors in operational analysis assumptions. IEE Transactions on Software Engineering, 13(7):767-776, 1987.

[12] V. Bhat. Approximations for multiclass single server queues with applications to the parametric decomposition approach for solving queuieng networks. 1988.

[13] C. F. Bispo and S. Tayur. Managing simple re-entrant flow lines. Theoretical foundation and experimental results, 2000.

[14] G. Bitran and D. Sarkar. Throughput analysis in manufacturing networks. European Journal of Operational Research, 74:448-465, 1994. 
[15] G. Bitran and D. Tirupati. Multiproduct queueing networks with deterministic routing: Decomposition approach and the notion of interference. Management Science, 34(1):75, 1988.

[16] Maury Bramson and J. G. Dai. Heavy traffic limits for some queueing networks. The Annals of Applied Probability, 11(1):49-90, Feb. 2001.

[17] P. Burke. The output of a queueing system. Operations Research, 4:699-704, 1956.

[18] J. Buzen. Fundamental laws of computer system performance. Technical report, Center for Research in Computer Technology, Harvard University, Cambridge, 1976.

[19] K. Chandy and Ch Sauser. Approximate methods for analyzing queueing network models of computing systems. ACM Computing Surveys, 10(3):281-317, 1978.

[20] H. Chen, X. Shen, and D. Yao. Brownian approximations of multicalss openqueueing networks. Operations Research, 50(6):1032, 2002.

[21] Hong Chen and Avi Mandelbaum. Stochastic discrete flow networks: Diffusion approximations and bottlenecks. The Annals of Probability, 19(4):1463-1519, Oct. 1991.

[22] R. Chen. A hybrid solution of fork/join synchronization in parallel queues. IEEE Transactions on Parallel and Distributed Systems, 12(829), 2001.

[23] D. Connors, G. Feigin, and D. Yao. A queueing network model for semiconductor manufacturing. IEEE Transactions on Semiconductor Manufacturing, 9(3), 1996.

[24] G. P. Cosmetatos. Approximate explicit formulae for the average queueing time in the process $(\mathrm{M} / \mathrm{D} / \mathrm{r})$ and $(\mathrm{D} / \mathrm{M} / \mathrm{r})$. Information Systems and Operational Research, 13:328-331, 1975.

[25] Y. Dallery, R. David, and X. Xie. An efficient algorithm for analysis of transfer lines with unreliable machines and finite buffers. IIE Transactions, 20(3):280-283, 1988.

[26] Y. Dallery and Y. Frein. On decomposition methods for tandem queueing networks with blocking. Operations Research, 41(2), 1993.

[27] Y. Dallery and S. Gershwin. Manufacturing flow lines systems: a review of models and analytical results. Queueing System, 12(1-2):3-94, 1992.

[28] P. Denning and J. Buzen. The operational analysis of queueing networks models. ACM Computing Surveys, 10(3):225-261, 1978.

[29] L. Flatto. Two parallel queues created by arrivals with two demands II. SIAM Journal on Applied Mathematics, 45(5):861-878, Oct. 1985.

[30] L. Flatto and S. Hahn. Two parallel queues created by arrivals with two demands I. SIAM Journal on Applied Mathematics, 44(5):1041-1053, Oct. 1984. 
[31] S. Gershwin. An efficient decomposition method for the approximate evaluation of tandem queues with finite storage space and blocking. Operations Research, 35(2):291-305, 1987.

[32] Manish K. Govil and Michael C. Fu. Queueing theory in manufacturing: A survey. Journal of Manufacturing Systems, 18(3):214, 1999.

[33] S. Gurumurthi and S. Benjaafar. Modeling and analysis of flexible queueing systems. Naval Research Logistics, 51(5):755-782, 2004.

[34] J. M. Harrison and R. J. Williams. A multiclass closed queueing network with unconventional heavy traffic behavior. The Annals of Applied Probability, 6(1):1-47, Feb. 1996.

[35] J. Michael Harrison. Assembly-like queues. Journal of Applied Probability, 10:354367, 1973.

[36] J. Michael Harrison. The heavy traffic approximation for single server queues in series. Journal of Applied Probability, 10(3):613-629, Sep. 1973.

[37] J. Michael Harrison and Martin I. Reiman. On the distribution of multidimensional reflected Brownian motion. SIAM Journal on Applied Mathematics, 41(2):345-361, Oct. 1981.

[38] J. Michael Harrison and Lawrence M. Wein. Scheduling networks of queues: Heavy traffic analysis of a two-station closed network. Operations Research, 38(6):10521064, Nov. - Dec. 1990.

[39] A. V. Harten and A. Sleptchenko. On Markovian multi-class, multi-server queueing. Queueing System, 43:307-328, 2003.

[40] P. Heidelberger and K. S. Trivedi. Queueing network models for parallel processing with asynchronous tasks. IEEE Transactions on Computers, C-31(11):1099-1109, 1982.

[41] P. Heidelberger and K. S. Trivedi. Analytic queueing models for programs with internal concurrency. IEEE Transactions on Computers, C-32(1):73-82, 1983.

[42] S. Helber. Performance analysis of flow-lines with nonlinear flow of materials, volume 473. Springer, Berlin, 1999.

[43] Horvath G. \& Telek M. Horvath, A. A traffic based decomposition of two-class queueing networks with priority service. Computer Networks, 53(8):1235, 2009.

[44] D. Iglehart and W. Whitt. Multiple channel queues in heavy traffic. I. Advances in Applied Probability, 2(1):150-177, Spring 1970. 
[45] D. Iglehart and W. Whitt. Multiple channel queues in heavy traffic. II: Sequences, networks, and batches. Advances in Applied Probability, 2(2):355-369, Autumn 1970.

[46] James R. Jackson. Networks of waiting lines. Operations Research, 5(4):518-521, 1957.

[47] R. Jackson. Queueing systems with phase type service. Operations Research Quartely, 5(2):109-120, 1954.

[48] P. Jensen and J. Bard. Operations Research Models and Methods. John Wiley \& Sons, Inc., 2003.

[49] L. Jiang and R. Giachetti. A queueing network model to analize the impact of parallelization of care on patient cycle time. Health Care Management Science, 11:248$261,2008$.

[50] F. Kelly. Networks of queues with customers of different types. Journal of Applied Probability, 12(3):542-554, 1975.

[51] F. Kelly. Networks of queues. Advances in Applied Probability, 8(2):416-432, 1976.

[52] Ch. Kim and A. Agrawala. Analysis of the fork-join queue. IEE Transactions on Computers, 38(2):250-255, 1989.

[53] S. Kim, Y. Park, and C. H Jun. Performance evaluation of re-entrant manufacturing system with production loss using mean value analysis. Computers \& Operations Research, 33(5):1308-1325, 2006.

[54] S. S Ko. Cycle times in a Serial Fork-Join Network, volume 4705 of Computational Science and Its applications, pages 758-766. Springer, Berlin, 2007.

[55] S. S Ko and R. Serfozo. Response times in M/M/s fork-join networks. Advances in Applied Probability, 36:854-871, 2004.

[56] E. Koenigsberg. Cyclic queues. Operations Research Quartely, 9(1):22-35, 1958.

[57] W. Kraemer and M. Langenbach-Belz. Approximate formulae for the delay in the queueing system GI/GI. Proccedings of Eighth International Telegraffic Congress, 235:1-8, 1976.

[58] A. Krishnamurthy, Suri R., and M. Vernon. Analysis of a fork/join synchronization station with inputs from coxian servers in a closed queuing network. Annals of Operations Research, 125:69-94, 2004.

[59] A. Krishnamurthy and R. Suri. Performance analysis of single stage kanban controlled production systems using parametric decomposition. Queueing System, 54:141-162, 2006. 
[60] P. R. Kuehn. Approximate analysis of general queuing networks by demposition. IEEE on Communications, 27(1), 1979.

[61] A. Kumar and R. Shorey. Performance analysis and scheduling of stochastic forkjoin jobs in a multicomputer system. IEEE Transactions on Parallel and Distributed Systems, 4(10):1147-1164, 1993.

[62] P. R. Kumar. Re-entrant lines. Queueing System, 13:87-110, 1993.

[63] S. Kumar and M. K. Omar. Performance measure in a probabilistic reflow screening line using mean value analysis. The AIUB Journal of Science and Engineering, 4(1):53-58, 2006.

[64] S. Kumar and M. K. Omar. Stochastic re-entrant line modeling for an environment stress testing in a semiconductor assembly industry. Applied Mathematics and Computation, 173:603-615, 2006.

[65] S. S. Lavenberg and M. Reiser. Stationary state probabilities at arrival instants for closed queueing networks with multiple types of customers. Journal of Applied Probability, 17(4):1048-1061, Dec. 1980.

[66] A. Lebrecht and W. Knottenbelt. Response time approximations in fork-join queues.

[67] Y. Leung and R. Suri. Performance evaluation of discrete manufacturing systems. Control Systems Magazine, IEEE, 10(4):77-86, 1990.

[68] J. Li. Performance analysis of production systems with rework loops. IIE Transactions, 36:755-765, 2004.

[69] E.H. Lipper and Sengupta B. Assembly-like queues with finite capacity: Bounds, asymptotics and approximations. Queueing System, 1(1):67-83, 1986.

[70] I. D. C. Little. A proof for the queueing formula $\mathrm{L}=$ lambda*W. Operations Research, 9:383-387, 1961.

[71] Y. C. Liu and H. G. Perros. A decomposition procedure for the analysis of a closed fork/join queueing system. IEEE Transactions on Computers, 40(3):365-370, 1991.

[72] S. Lu, D. Ramaswamy, and P. R. Kumar. Efficient scheduling policies to reduce mean and variance of cycle-time in semiconductor manufacturing plant. IEEE Transactions Semiconductor Manufacturing, 7(3):374-388, 1994.

[73] S. Meyn and D. Down. Stability of generalized jackson networks. The Annals of Applied Probability, 4(1):124-148, 1994.

[74] Y. Narahari and L. M. Khan. Modeling reentrant manufacturing systems with inspection stations. Journal of Manufacturing Systems, 15(6):367-378, 1996. 
[75] Y. Narahari and L. M. Khan. Performance analysis of scheduling policies in reentrant manufacturing systems. Computers \& Operations Research, 23(1):37-51, 1996.

[76] Y. Narahari and P. Sundarrajan. Performability analysis of fork-join queueing systems. Journal of the Operational Research Society, 46:1237-1249, 1995.

[77] R. Nelson and N. Tantawi. Approximate analysis of fork/join synchronization in parallel queues. IEEE Transactions on Computers, 37(6):739, 1988.

[78] R. Nelson, D. Towsley, and A. Tantawi. Performance analysis of parallel processing systems. IEEE Transactions on Software Engineering, 14(4):532-540, 1988.

[79] V. Nguyen. The trouble with diversity:fork-join networks with heterogeneous customer population. Working paper, Massachusetts Institute of Technology, Operations Research Center, 1992.

[80] M. K. Omar and S. Kumar. Performance analysis in a probabilistic re-entrant for an environmental stress testing operation. Computers \& Industrial Engineering, 54:932-944, 2008.

[81] M. K. Omar, S. Kumar, and Y. Suppiah. Performance analysis in a re-entrant operation with combinational routing and yield probabilities. Applied Mathematical Modeling, 33:1601-1612, 2009.

[82] C. H Paik. Throughput bounding and simple approximation methods for exponential fork/join queueing networks with blocking. Computers \& Industrial Engineering, 35(3,4):563-566, 1998.

[83] Y. Park, S. Kim, and C. H Jun. Performance analysis of re-entrant flow shop with single-job and batch machines using mean value analysis. Production Planning \& Control, 11(6):537-546, 2000.

[84] Y. Park, S. Kim, and C. H Jun. Mean value analysis of re-entrant line with batch machines and multi-class jobs. Computers \& Operations Research, 29:1009-1024, 2002.

[85] S. Pradhan and P. Damodaran. Performance characterization of complex manufacturing systems with general distributions and job failures. European Journal of Operational Research, 197:588-598, 2009.

[86] S. Pradhan, P. Damodaran, and K. Srihari. Predicting performance measures for markovian type of manufacturing systems with product failures. European Journal of Operational Research, 184:725-744, 2008.

[87] R. Ramakrishnan and A. Krishnamurthy. Analytical approximations for kitting systems with multiple inputs. Asia-Pacific Journal of Operational Research, 25(2):187216, 2008. 
[88] Martin I. Reiman. Open queueing networks in heavy traffic. Mathematics of Operations Research, 9(3), 1984.

[89] M. Reiser and H. Kobayashi. The effects of service time distributions on system performance. Informs Process, 74, 1974.

[90] M. Reiser and S. S. Lavenberg. Mean-value analysis of closed multichain queueing networks. Journal of the ACM, 27(2):313-322, 1980.

[91] S. Saboo, L. Wang, and W. E. Wihelm. Recursion models for describing and managing the transient flow of materials in generalized flowlines. Management Science, 35(6):722-742, 1989.

[92] P. Schwitzer. Approximate analysis of muticlass closed networks of queues. In International Conference on Stochastic Control and Optimization, (Amsterdam), 1979.

[93] M. Segal and W. Whitt. A queueing network analyzer for manufacturing. Proceedings of the 12th International Teletraffic Conference, 2:1146-1152, 1989.

[94] B. Sengupta. On modeling the store of an assembly shop by due date processes. Operations Research, 37(3):437-446, 1989.

[95] J. Shanthikumar and J. Buzacott. Open queueing network models of dynamic job shops. International Journal of production Research, 19(3):255-266, 1981.

[96] M. Squillante, Y. Zhang, A. Sivasubramaniam, and G. Natarajan. Generalized parallel-server frok-join queues with dynamic task scheduling. Annals of Operations Research, 160:227-255, 2008.

[97] K. Sunkyo. Parametric Decomposition Approximation of Queueing Networks. PhD thesis, Purdue University, 2000.

[98] K. Sunkyo. Approximations of multiclass queueing networks with highly variable arrivals under deterministic routing. Naval Research Logistics, 52(5):399-408, 2005.

[99] M. Takahashi, H. Osawa, and T. Fujisawa. On a synchronization queue with two finite buffers. Queueing System, 36:107-123, 2000.

[100] B. Tan and S. Yeralan. Analysis of multistation production systems with limited buffer capacity, part ii. Mathematical and Computer Modeling, 25(11):109-123, 1997.

[101] A. Thomasian and A. Tantawi. Approximate solutions for $\mathrm{m} / \mathrm{g} / 1$ for/join synchronization. In Proceedings of the 26th conference on Winter simulation, pages 361368, San Diego, CA, USA, 1994. Society for Computer Simulation International. 
[102] E. Varki. Mean value technique for closed fork-join networks. In Proceedings of ACM SIGMETRICS Conference on Measurement and Modeling of Computer Systems, 1999.

[103] E. Varki. Response time analysis of parallel computer and storage systems. IEE Transactions on Parallel and Distributed Systems, 12(11):1146-1161, 2001.

[104] E. Varki and L. Dowdy. Analysis of balanced fork-join queueing networks.

[105] E. Varki and L. Dowdy. Response time analysis of two server fork-join systems. 1995.

[106] E. Varki, A. Merchant, and H. Chen. The $\mathrm{m} / \mathrm{m} / 1$ fork-join queue with variable subtasks. 2008.

[107] S. Varma and A. Makowski. Interpolation approximations for symmetric fork-join queues. Performance Evaluation, 20:245-265, 2004.

[108] N. Vohra. Quantitative Techniques in Management. Tata McGraw Hill, New Delphi, India, 1997.

[109] P. Welch. The computer modeling handbook, chapter The statistical analysis of simulation results, pages 268-328. New York: Academic Press, 1983.

[110] W. Whitt. The queueing network analizer. The Bell System Techinical Journal, 62(9):2779-2815, 1983.

[111] W. Whitt. Approximations for departure processes and queues in series. Naval Research Logistics Quarterly, 31:499-521, 1984.

[112] W. Whitt. Approximations for the gi/g/m queue. Production and Operations Management, 2(2):114-161, 1993.

[113] W. Whitt. Large fluctuations in a deterministic multiclass network of queues. Management Science, 39(8):1020-1028, 1993.

[114] W. Whitt. Towards better multi-class parametric decomposition approximations for open queueing networks. Annals of Operations Research, 48:221-248, 1994.

[115] W. Whitt. Variability functions for parametric-decomposition approximations of queueing networks. Management Science, 41(10):1704, 1995.

[116] Jiankui Yang. Stability of the multi-type reentrant lines with type pririty service discipline. Asia-Pacific Journal of Operational Research, 26(3):341-349, 2009.

[117] Heng-Qing Ye. A paradox for admission control of multiclass queueing network with differentiated service. Journal of Applied Probability, 44(2):321, 2007. 
[118] S. Yeralan and B. Tan. Analysis of multistation production systems with limited buffer capacity, part i. Mathematical and Computer Modeling, 25(7):109-122, 1997.

[119] T. Zheng. Optimization of distributed real-time systems with scenario deadlines. $\mathrm{PhD}$ thesis, Carleton University (Canada), 2002. 


\section{APPENDIX A}

Approximate and simulated flow times for one product instances

\begin{tabular}{|c|c|c|c|}
\hline \multicolumn{1}{c}{ Inst. } & \multicolumn{1}{c}{ Appr. } & \multicolumn{1}{c}{ Sim. } & Error (\%) \\
\hline 1 & 0.1125 & 0.1148 & 2.00 \\
\hline 2 & 0.0748 & 0.07854 & 4.76 \\
\hline 3 & 0.1076 & 0.1141 & 5.77 \\
\hline 4 & 0.1219 & 0.13202 & 7.67 \\
\hline 5 & 0.0796 & 0.08006 & 0.57 \\
\hline 6 & 0.0721 & 0.07354 & 1.96 \\
\hline 7 & 0.0829 & 0.08281 & 0.11 \\
\hline 8 & 0.0829 & 0.08292 & 0.02 \\
\hline 9 & 0.1484 & 0.14704 & 0.92 \\
\hline
\end{tabular}

Approximate and simulated flow times for two product instances

\begin{tabular}{|c|c|c|c|c|c|c|}
\hline \multirow[t]{2}{*}{ Inst. } & \multicolumn{2}{|c|}{ Appr. Flow time } & \multicolumn{2}{|c|}{ Sim. Flow time } & \multicolumn{2}{|c|}{ Error $(\%)$} \\
\hline & Prod_1 & Prod_2 & Prod_1 & Prod_2 & Prod_1 & Prod_2 \\
\hline 10 & 0.1291 & 0.0900 & 0.1345 & 0.1015 & 4.01 & 11.32 \\
\hline 11 & 0.2454 & 0.1097 & 0.2394 & 0.1060 & 23.64 & 22.55 \\
\hline 12 & 0.0796 & 0.0559 & 0.0819 & 0.0590 & 2.82 & 5.29 \\
\hline 13 & 0.2003 & 0.2026 & 0.2004 & 0.2030 & 0.60 & 0.78 \\
\hline 14 & 0.1048 & 0.1090 & 0.1001 & 0.1085 & 9.71 & 1.46 \\
\hline 15 & 0.0897 & 0.0916 & 0.0904 & 0.0930 & 0.71 & 1.55 \\
\hline 16 & 0.1244 & 0.1024 & 0.1185 & 0.0957 & 4.95 & 7.03 \\
\hline 17 & 0.0873 & 0.0864 & 0.0904 & 0.0903 & 3.43 & 4.32 \\
\hline 18 & 0.0933 & 0.0903 & 0.0911 & 0.0897 & 2.46 & 0.72 \\
\hline 19 & 0.1982 & 0.1713 & 0.2032 & 0.1772 & 2.46 & 3.33 \\
\hline 20 & 0.1442 & 0.1058 & 0.1478 & 0.1161 & 2.48 & 8.90 \\
\hline 21 & 0.3274 & 0.1535 & 0.3226 & 0.1565 & 23.09 & 22.73 \\
\hline 22 & 0.0834 & 0.0581 & 0.0857 & 0.0612 & 2.69 & 5.01 \\
\hline 23 & 0.2749 & 0.2733 & 0.2466 & 0.2439 & 11.48 & 12.05 \\
\hline 24 & 0.1275 & 0.1145 & 0.1180 & 0.1163 & 8.06 & 1.57 \\
\hline 25 & 0.1018 & 0.1056 & 0.1024 & 0.1084 & 0.60 & 2.62 \\
\hline 26 & 0.1457 & 0.1190 & 0.1330 & 0.1063 & 9.52 & 11.92 \\
\hline 27 & 0.1024 & 0.0986 & 0.1005 & 0.0984 & 1.81 & 0.26 \\
\hline 28 & 0.1073 & 0.1040 & 0.1005 & 0.0991 & 6.77 & 4.96 \\
\hline 29 & 0.2132 & 0.2123 & 0.1822 & 0.1824 & 17.01 & 16.41 \\
\hline 30 & 0.2084 & 0.2123 & 0.2246 & 0.2249 & 7.22 & 5.59 \\
\hline
\end{tabular}




\begin{tabular}{|c|c|c|c|c|c|c|}
\hline Inst. & \multicolumn{2}{|c|}{ Appr. Flow time } & \multicolumn{2}{c|}{ Sim. Flow time } & \multicolumn{2}{c|}{ Error (\%) } \\
& Prod_1 & Prod_2 & \multicolumn{2}{c|}{ Prod_1 } & Prod_2 & \multicolumn{2}{c|}{ Prod_1 } & \multicolumn{1}{c|}{ Prod_2 } \\
\hline 31 & 0.1122 & 0.1111 & 0.1056 & 0.1181 & 6.29 & 5.96 \\
\hline 32 & 0.1262 & 0.1027 & 0.1214 & 0.0965 & 3.90 & 6.48 \\
\hline 33 & 0.0963 & 0.0947 & 0.0973 & 0.0994 & 1.05 & 4.69 \\
\hline 34 & 0.1222 & 0.0992 & 0.1141 & 0.0914 & 7.09 & 8.57 \\
\hline 35 & 0.0838 & 0.0828 & 0.0840 & 0.0840 & 0.28 & 1.37 \\
\hline 36 & 0.1928 & 0.1989 & 0.2315 & 0.2284 & 16.73 & 12.89 \\
\hline 37 & 0.0891 & 0.0865 & 0.0822 & 0.0802 & 8.44 & 7.85 \\
\hline 38 & 0.3688 & 0.1142 & 0.3535 & 0.1129 & 15.93 & 19.17 \\
\hline
\end{tabular}

Approximate and simulated flow times for three product instances

\begin{tabular}{|c|c|c|c|c|c|c|c|c|c|}
\hline Inst. & \multicolumn{3}{|c|}{ Approximate } & \multicolumn{3}{c|}{ Simulated } & \multicolumn{3}{c}{ Error (\%) } \\
& Prod_1 & Prod_2 & Prod_3 & Prod_1 & \multicolumn{1}{c|}{ Prod_2 } & Prod_3 & \multicolumn{1}{c|}{ Prod_1 } & Prod_2 & Prod_3 \\
\hline 39 & 0.1899 & 0.1681 & 0.1681 & 0.1463 & 0.1239 & 0.1241 & 29.85 & 35.67 & 35.50 \\
\hline 40 & 0.1175 & 0.1145 & 0.1135 & 0.1046 & 0.1033 & 0.1036 & 12.32 & 10.82 & 9.57 \\
\hline 41 & 0.1816 & 0.1809 & 0.1809 & 0.1412 & 0.1519 & 0.1528 & 28.58 & 19.08 & 18.38 \\
\hline 42 & 0.4171 & 0.1724 & 0.1833 & 0.38791 & 0.15846 & 0.16419 & 7.53 & 8.8 & 11.64 \\
\hline 43 & 0.3636 & 0.0978 & 0.1 & 0.34926 & 0.08249 & 0.08492 & 4.11 & 18.57 & 17.76 \\
\hline 44 & 0.2669 & 0.1312 & 0.1305 & 0.25188 & 0.11912 & 0.11849 & 5.95 & 10.17 & 10.12 \\
\hline 45 & 0.2769 & 0.0992 & 0.1010 & 0.26922 & 0.08992 & 0.09106 & 2.84 & 10.35 & 10.88 \\
\hline 46 & 0.3805 & 0.2077 & 0.2181 & 0.36413 & 0.19990 & 0.21028 & 4.50 & 3.90 & 3.72 \\
\hline 47 & 0.3586 & 0.1702 & 0.1743 & 0.33878 & 0.14869 & 0.15081 & 5.85 & 14.44 & 15.59 \\
\hline 48 & 1.0918 & 0.2544 & 0.2673 & 1.03883 & 0.22889 & 0.23372 & 5.10 & 11.14 & 14.38 \\
\hline 49 & 0.5834 & 0.2204 & 0.2204 & 0.55213 & 0.17586 & 0.17583 & 5.67 & 25.35 & 25.38 \\
\hline 50 & 0.2991 & 0.2177 & 0.2165 & 0.23848 & 0.15611 & 0.15529 & 25.44 & 39.43 & 39.43 \\
\hline 51 & 0.5793 & 0.1398 & 0.1414 & 0.54999 & 0.11203 & 0.11379 & 5.34 & 24.79 & 24.26 \\
\hline
\end{tabular}


Approximate and simulated flow times for four product instances

\begin{tabular}{|c|c|c|c|c|}
\hline Inst. & \multicolumn{1}{l}{ Prod } & \multicolumn{1}{l}{ Approx. } & \multicolumn{1}{l}{ Simulated } & Error $(\%)$ \\
\hline 52 & 1 & 0.9773 & 0.71068 & 37.52 \\
\hline 52 & 2 & 0.2538 & 0.20629 & 23.03 \\
\hline 52 & 3 & 0.2652 & 0.21131 & 25.51 \\
\hline 52 & 4 & 1.0202 & 0.72416 & 40.88 \\
\hline 53 & 1 & 0.609 & 0.48065 & 26.70 \\
\hline 53 & 2 & 0.1183 & 0.09152 & 29.26 \\
\hline 53 & 3 & 0.1204 & 0.09405 & 28.02 \\
\hline 53 & 4 & 0.6091 & 0.47942 & 27.05 \\
\hline 54 & 1 & 0.5042 & 0.37080 & 35.98 \\
\hline 54 & 2 & 0.1754 & 0.14239 & 23.19 \\
\hline 54 & 3 & 0.1746 & 0.14171 & 23.24 \\
\hline 54 & 4 & 0.5113 & 0.37667 & 35.73 \\
\hline 55 & 1 & 0.5410 & 0.42036 & 28.70 \\
\hline 55 & 2 & 0.1372 & 0.11216 & 22.33 \\
\hline 55 & 3 & 0.1389 & 0.11291 & 23.05 \\
\hline 55 & 4 & 0.5416 & 0.42160 & 28.46 \\
\hline 56 & 1 & 0.6660 & 0.51229 & 30.01 \\
\hline 56 & 2 & 0.2326 & 0.21162 & 9.91 \\
\hline 56 & 3 & 0.2430 & 0.22188 & 9.52 \\
\hline 56 & 4 & 0.4765 & 0.34767 & 37.06 \\
\hline 57 & 1 & 0.7440 & 0.57431 & 29.55 \\
\hline 57 & 2 & 0.2577 & 0.19680 & 30.95 \\
\hline 57 & 3 & 0.2619 & 0.19845 & 31.96 \\
\hline 57 & 4 & 0.7653 & 0.57871 & 32.24 \\
\hline 58 & 1 & 1.2592 & 1.16645 & 7.95 \\
\hline 58 & 2 & 0.2830 & 0.25201 & 12.28 \\
\hline 58 & 3 & 0.2993 & 0.25589 & 16.97 \\
\hline 58 & 4 & 0.3791 & 0.33553 & 12.99 \\
\hline 59 & 1 & 0.6924 & 0.61570 & 12.46 \\
\hline 59 & 2 & 0.2302 & 0.18642 & 23.51 \\
\hline 59 & 3 & 0.2302 & 0.18683 & 23.24 \\
\hline 59 & 4 & 0.2667 & 0.22721 & 17.40 \\
\hline 60 & 1 & 0.3496 & 0.26947 & 29.74 \\
\hline 60 & 2 & 0.2229 & 0.16198 & 37.60 \\
\hline 60 & 3 & 0.2217 & 0.16108 & 37.66 \\
\hline 60 & 4 & 0.1607 & 0.14634 & 9.82 \\
\hline 61 & 1 & 0.6165 & 0.57129 & 7.92 \\
\hline 61 & 2 & 0.1518 & 0.12051 & 25.95 \\
\hline 61 & 3 & 0.1532 & 0.12200 & 25.54 \\
\hline & & & & \\
\hline 5
\end{tabular}




\begin{tabular}{|c|c|c|c|c|}
\hline Inst. & Prod & Approx. & Simulated & Error $(\%)$ \\
\hline 61 & 4 & 0.1426 & 0.12600 & 13.15 \\
\hline
\end{tabular}

Approximate and simulated flow times for five product instances

\begin{tabular}{|c|c|c|c|c|}
\hline \multicolumn{1}{l}{ Inst. } & \multicolumn{1}{l}{ Prod } & \multicolumn{1}{l}{ Approximate } & \multicolumn{1}{l|}{ Simulated } & Error $(\%)$ \\
\hline 62 & 1 & 1.0486 & 0.74751 & 40.28 \\
\hline 62 & 2 & 0.3405 & 0.24887 & 36.82 \\
\hline 62 & 3 & 0.3525 & 0.25295 & 39.36 \\
\hline 62 & 4 & 1.0934 & 0.76084 & 43.71 \\
\hline 62 & 5 & 0.349 & 0.24996 & 39.62 \\
\hline 63 & 1 & 0.6851 & 0.51155 & 33.93 \\
\hline 63 & 2 & 0.1954 & 0.12274 & 59.20 \\
\hline 63 & 3 & 0.1973 & 0.12491 & 57.95 \\
\hline 63 & 4 & 0.6852 & 0.51131 & 34.01 \\
\hline 63 & 5 & 0.1974 & 0.12488 & 58.07 \\
\hline 64 & 1 & 0.5976 & 0.41223 & 44.97 \\
\hline 64 & 2 & 0.2689 & 0.18447 & 45.76 \\
\hline 64 & 3 & 0.2681 & 0.18353 & 46.11 \\
\hline 64 & 4 & 0.6047 & 0.41631 & 45.24 \\
\hline 64 & 5 & 0.2684 & 0.18406 & 45.85 \\
\hline 65 & 1 & 0.6142 & 0.45473 & 35.06 \\
\hline 65 & 2 & 0.2121 & 0.14756 & 43.73 \\
\hline 65 & 3 & 0.2138 & 0.14835 & 44.13 \\
\hline 65 & 4 & 0.6148 & 0.45676 & 34.59 \\
\hline 65 & 5 & 0.2125 & 0.14783 & 43.74 \\
\hline 66 & 1 & 0.8420 & 0.61882 & 36.06 \\
\hline 66 & 2 & 0.4122 & 0.31910 & 29.18 \\
\hline 66 & 3 & 0.4227 & 0.32985 & 28.15 \\
\hline 66 & 4 & 0.5086 & 0.36083 & 40.95 \\
\hline 66 & 5 & 0.4180 & 0.32542 & 28.45 \\
\hline 67 & 1 & 0.8975 & 0.63897 & 40.46 \\
\hline 67 & 2 & 0.4097 & 0.26455 & 54.85 \\
\hline 67 & 3 & 0.4138 & 0.26532 & 55.97 \\
\hline 67 & 4 & 0.9247 & 0.64335 & 43.73 \\
\hline 67 & 5 & 0.4204 & 0.27488 & 52.92 \\
\hline 68 & 1 & 1.7226 & 1.34337 & 28.23 \\
\hline 68 & 2 & 0.7672 & 0.45698 & 67.88 \\
\hline 68 & 3 & 0.7906 & 0.45866 & 72.38 \\
\hline 68 & 4 & 0.4502 & 0.38717 & 16.28 \\
\hline 68 & 5 & 0.7672 & 0.45665 & 68.00 \\
\hline 69 & 1 & 0.7761 & 0.65363 & 18.74 \\
\hline
\end{tabular}




\begin{tabular}{|c|c|c|c|c|}
\hline \multicolumn{1}{c}{ Inst. } & \multicolumn{1}{c}{ Prod } & \multicolumn{1}{c}{ Approximate } & Simulated & Error $(\%)$ \\
\hline 69 & 2 & 0.4017 & 0.25426 & 57.99 \\
\hline 69 & 3 & 0.4017 & 0.25405 & 58.12 \\
\hline 69 & 4 & 0.2789 & 0.24292 & 14.82 \\
\hline 69 & 5 & 0.4017 & 0.25417 & 58.05 \\
\hline 70 & 1 & 0.4983 & 0.31558 & 57.90 \\
\hline 70 & 2 & 0.3659 & 0.20669 & 77.01 \\
\hline 70 & 3 & 0.3647 & 0.20539 & 77.58 \\
\hline 70 & 4 & 0.1652 & 0.15032 & 9.89 \\
\hline 70 & 5 & 0.3679 & 0.20812 & 76.79 \\
\hline 71 & 1 & 0.6732 & 0.59350 & 13.43 \\
\hline 71 & 2 & 0.2085 & 0.14226 & 46.54 \\
\hline 71 & 3 & 0.2096 & 0.14353 & 46.07 \\
\hline 71 & 4 & 0.1549 & 0.13407 & 15.55 \\
\hline 71 & 5 & 0.2124 & 0.14341 & 48.10 \\
\hline
\end{tabular}

Approximate and simulated flow times for six product instances

\begin{tabular}{|c|c|c|c|c|}
\hline \multicolumn{1}{c}{ Inst. } & \multicolumn{1}{c}{ Prod } & \multicolumn{1}{c}{ Approximate } & \multicolumn{1}{c}{ Simulated } & Error $(\%)$ \\
\hline 72 & 1 & 1.1287 & 0.77136 & 46.33 \\
\hline 72 & 2 & 0.4418 & 0.28561 & 54.69 \\
\hline 72 & 3 & 0.4543 & 0.28938 & 56.99 \\
\hline 72 & 4 & 1.1749 & 0.78331 & 49.10 \\
\hline 72 & 5 & 0.4508 & 0.28556 & 57.86 \\
\hline 72 & 6 & 0.4338 & 0.28673 & 51.29 \\
\hline 73 & 1 & 0.8909 & 0.58338 & 52.71 \\
\hline 73 & 2 & 0.2721 & 0.14689 & 85.25 \\
\hline 73 & 3 & 0.2736 & 0.14906 & 83.55 \\
\hline 73 & 4 & 0.8909 & 0.58254 & 52.93 \\
\hline 73 & 5 & 0.2740 & 0.14936 & 83.45 \\
\hline 73 & 6 & 0.4574 & 0.25482 & 79.50 \\
\hline 74 & 1 & 0.7457 & 0.47743 & 56.20 \\
\hline 74 & 2 & 0.3305 & 0.21729 & 52.09 \\
\hline 74 & 3 & 0.3297 & 0.21684 & 52.06 \\
\hline 74 & 4 & 0.7528 & 0.48213 & 56.14 \\
\hline 74 & 5 & 0.3300 & 0.21708 & 52.03 \\
\hline 74 & 6 & 0.3715 & 0.25040 & 48.36 \\
\hline 75 & 1 & 0.7645 & 0.51519 & 48.39 \\
\hline 75 & 2 & 0.3144 & 0.18765 & 67.53 \\
\hline 75 & 3 & 0.3161 & 0.18772 & 68.39 \\
\hline 75 & 4 & 0.7651 & 0.51633 & 48.18 \\
\hline 75 & 5 & 0.3148 & 0.18756 & 67.82 \\
\hline & & & & \\
\hline
\end{tabular}




\begin{tabular}{|c|c|c|c|c|}
\hline Inst. & Prod & Approximate & Simulated & Error $(\%)$ \\
\hline 75 & 6 & 0.4325 & 0.26089 & 65.78 \\
\hline 76 & 1 & 1.1162 & 0.74627 & 49.57 \\
\hline 76 & 2 & 0.5144 & 0.34808 & 47.78 \\
\hline 76 & 3 & 0.5248 & 0.35930 & 46.06 \\
\hline 76 & 4 & 0.7705 & 0.48809 & 57.86 \\
\hline 76 & 5 & 0.5202 & 0.35414 & 46.89 \\
\hline 76 & 6 & 0.5117 & 0.33537 & 52.58 \\
\hline 77 & 1 & 1.0802 & 0.70681 & 52.83 \\
\hline 77 & 2 & 0.5181 & 0.30783 & 68.29 \\
\hline 77 & 3 & 0.5222 & 0.30858 & 69.23 \\
\hline 77 & 4 & 1.1124 & 0.71139 & 56.38 \\
\hline 77 & 5 & 0.5288 & 0.31819 & 66.18 \\
\hline 77 & 6 & 0.5660 & 0.35128 & 61.14 \\
\hline 78 & 1 & 2.0207 & 1.47202 & 37.27 \\
\hline 78 & 2 & 0.8265 & 0.49554 & 66.79 \\
\hline 78 & 3 & 0.8552 & 0.49721 & 71.20 \\
\hline 78 & 4 & 0.7431 & 0.51951 & 43.04 \\
\hline 78 & 5 & 0.8265 & 0.49611 & 66.60 \\
\hline 78 & 6 & 0.7533 & 0.51889 & 45.18 \\
\hline 79 & 1 & 1.0339 & 0.75650 & 36.67 \\
\hline 79 & 2 & 0.4161 & 0.26964 & 54.30 \\
\hline 79 & 3 & 0.4161 & 0.26941 & 54.43 \\
\hline 79 & 4 & 0.5345 & 0.34570 & 54.62 \\
\hline 79 & 5 & 0.4161 & 0.26921 & 54.55 \\
\hline 79 & 6 & 0.5378 & 0.34800 & 54.55 \\
\hline 80 & 1 & 0.6520 & 0.38443 & 69.59 \\
\hline 80 & 2 & 0.3766 & 0.21719 & 73.41 \\
\hline 80 & 3 & 0.3755 & 0.21569 & 74.08 \\
\hline 80 & 4 & 0.3094 & 0.21668 & 42.80 \\
\hline 80 & 5 & 0.3787 & 0.21873 & 73.13 \\
\hline 80 & 6 & 0.3077 & 0.21455 & 43.42 \\
\hline 81 & 1 & 0.7614 & 0.62892 & 21.07 \\
\hline 81 & 2 & 0.2281 & 0.15262 & 49.47 \\
\hline 81 & 3 & 0.2290 & 0.15437 & 48.35 \\
\hline 81 & 4 & 0.2417 & 0.17355 & 39.27 \\
\hline 81 & 5 & 0.2324 & 0.15368 & 51.20 \\
\hline 81 & 6 & 0.2428 & 0.17665 & 37.43 \\
\hline
\end{tabular}


Approximate and simulated flow times for instances with less number of subtasks than servers at fork-join nodes Inst. Prod. Prod Approximate Simulated Error (\%) Classes

\begin{tabular}{|c|c|c|c|c|c|}
\hline 82 & 3 & 1 & 0.3965 & 0.3737 & 6.09 \\
\hline 82 & 3 & 2 & 0.1494 & 0.1397 & 6.94 \\
\hline 82 & 3 & 3 & 0.1593 & 0.1465 & 8.74 \\
\hline 83 & 3 & 1 & 0.3314 & 0.3272 & 1.27 \\
\hline 83 & 3 & 2 & 0.1759 & 0.1724 & 2.05 \\
\hline 83 & 3 & 3 & 0.1863 & 0.1832 & 1.68 \\
\hline 84 & 4 & 1 & 0.4825 & 0.3553 & 35.81 \\
\hline 84 & 4 & 2 & 0.1537 & 0.1230 & 24.97 \\
\hline 84 & 4 & 3 & 0.1530 & 0.1228 & 24.63 \\
\hline 84 & 4 & 4 & 0.4896 & 0.3592 & 36.29 \\
\hline 85 & 4 & 1 & 1.2154 & 1.1213 & 8.39 \\
\hline 85 & 4 & 2 & 0.2480 & 0.2149 & 15.41 \\
\hline 85 & 4 & 3 & 0.2556 & 0.2201 & 16.10 \\
\hline 85 & 4 & 4 & 0.3441 & 0.2977 & 15.58 \\
\hline 86 & 5 & 1 & 0.5656 & 0.3844 & 47.15 \\
\hline 86 & 5 & 2 & 0.2369 & 0.1533 & 54.52 \\
\hline 86 & 5 & 3 & 0.2361 & 0.1528 & 54.57 \\
\hline 86 & 5 & 4 & 0.5726 & 0.3895 & 47.02 \\
\hline 86 & 5 & 5 & 0.2364 & 0.1529 & 54.65 \\
\hline 87 & 5 & 1 & 1.2353 & 1.1550 & 6.95 \\
\hline 87 & 5 & 2 & 0.2638 & 0.2398 & 10.02 \\
\hline 87 & 5 & 3 & 0.2754 & 0.2460 & 11.95 \\
\hline 87 & 5 & 4 & 0.3600 & 0.3234 & 11.31 \\
\hline 88 & 5 & 1 & 0.4840 & 0.2985 & 62.16 \\
\hline 88 & 5 & 2 & 0.3459 & 0.1903 & 81.78 \\
\hline 88 & 5 & 3 & 0.3447 & 0.1890 & 82.35 \\
\hline 88 & 5 & 4 & 0.1416 & 0.1323 & 6.99 \\
\hline 88 & 5 & 5 & 0.3479 & 0.1923 & 80.91 \\
\hline 89 & 6 & 1 & 0.6995 & 0.4311 & 62.27 \\
\hline 89 & 6 & 2 & 0.2843 & 0.1700 & 67.25 \\
\hline 89 & 6 & 3 & 0.2835 & 0.1697 & 67.08 \\
\hline 89 & 6 & 4 & 0.7066 & 0.4358 & 62.14 \\
\hline 89 & 6 & 5 & 0.2838 & 0.1696 & 67.38 \\
\hline 89 & 6 & 6 & 0.3253 & 0.2050 & 58.65 \\
\hline 90 & 6 & 1 & 1.9568 & 1.4177 & 38.02 \\
\hline 90 & 6 & 2 & 0.7754 & 0.4440 & 74.62 \\
\hline 90 & 6 & 3 & 0.7913 & 0.4490 & 76.24 \\
\hline 90 & 6 & 4 & 0.6920 & 0.4670 & 48.16 \\
\hline
\end{tabular}




Inst. $\begin{gathered}\text { Prod. } \\
\text { Classes }\end{gathered}$
\begin{tabular}{|c|c|c|c|c|c|}
\hline 90 & 6 & 5 & 0.7754 & 0.4441 & 74.61 \\
\hline 90 & 6 & 6 & 0.6965 & 0.4686 & 48.63 \\
\hline
\end{tabular}

Comparison of flow time errors at fork-join nodes

\begin{tabular}{|c|c|c|c|c|}
\hline Inst. & $\begin{array}{c}\text { Prod. } \\
\text { Classes }\end{array}$ & Prod & $\begin{array}{c}\text { Error }(\%) \\
\text { subtasks }<\text { servers }\end{array}$ & $\begin{array}{c}\text { Error }(\%) \\
\text { subtasks }=\text { servers }\end{array}$ \\
\hline 82 & 3 & 1 & 6.09 & 7.53 \\
\hline 82 & 3 & 2 & 6.94 & 8.79 \\
\hline 82 & 3 & 3 & 8.74 & 11.64 \\
\hline 83 & 3 & 1 & 1.27 & 4.49 \\
\hline 83 & 3 & 2 & 2.05 & 3.90 \\
\hline 83 & 3 & 3 & 1.68 & 3.72 \\
\hline 84 & 4 & 1 & 35.81 & 35.98 \\
\hline 84 & 4 & 2 & 24.97 & 23.19 \\
\hline 84 & 4 & 3 & 24.63 & 23.24 \\
\hline 84 & 4 & 4 & 36.29 & 35.73 \\
\hline 85 & 4 & 1 & 8.39 & 7.95 \\
\hline 85 & 4 & 2 & 15.41 & 12.28 \\
\hline 85 & 4 & 3 & 16.10 & 16.97 \\
\hline 85 & 4 & 4 & 15.58 & 12.99 \\
\hline 86 & 5 & 1 & 47.15 & 44.97 \\
\hline 86 & 5 & 2 & 54.52 & 45.76 \\
\hline 86 & 5 & 3 & 54.57 & 46.11 \\
\hline 86 & 5 & 4 & 47.02 & 45.24 \\
\hline 86 & 5 & 5 & 54.65 & 45.85 \\
\hline 87 & 5 & 1 & 6.95 & 7.95 \\
\hline 87 & 5 & 2 & 10.02 & 12.28 \\
\hline 87 & 5 & 3 & 11.95 & 16.97 \\
\hline 87 & 5 & 4 & 11.31 & 12.99 \\
\hline 88 & 5 & 1 & 62.16 & 57.90 \\
\hline 88 & 5 & 2 & 81.78 & 77.01 \\
\hline 88 & 5 & 3 & 82.35 & 77.58 \\
\hline 88 & 5 & 4 & 6.99 & 9.89 \\
\hline 88 & 5 & 5 & 80.91 & 76.79 \\
\hline 89 & 6 & 1 & 62.27 & 56.20 \\
\hline 89 & 6 & 2 & 67.25 & 52.09 \\
\hline 89 & 6 & 3 & 67.08 & 52.06 \\
\hline 89 & 6 & 4 & 62.14 & 56.14 \\
\hline 89 & 6 & 5 & 67.38 & 52.03 \\
\hline 89 & 6 & 6 & 58.65 & 48.36 \\
\hline
\end{tabular}




\begin{tabular}{c|c|c|c|c|}
\hline Inst. & $\begin{array}{c}\text { Prod. } \\
\text { Classes }\end{array}$ & \multicolumn{1}{c}{ Prod } & $\begin{array}{c}\text { Error (\%) } \\
\text { subtasks < servers }\end{array}$ & $\begin{array}{c}\text { Error (\%) } \\
\text { subtasks = servers }\end{array}$ \\
\hline 90 & 6 & 1 & 38.02 & 37.27 \\
\hline 90 & 6 & 2 & 74.62 & 66.79 \\
\hline 90 & 6 & 3 & 76.24 & 72.00 \\
\hline 90 & 6 & 4 & 48.16 & 43.04 \\
\hline 90 & 6 & 5 & 74.61 & 66.60 \\
\hline 90 & 6 & 6 & 48.63 & 45.18 \\
\hline
\end{tabular}




\section{APPENDIX B}

Approximate and simulated mean flow time values and error in the approximation

\begin{tabular}{|c|c|c|c|c|c|}
\hline Instance & Products & Failure & Net Rho & Error & Pred. Value \\
\hline & & Rate & & sim-anal $(\%)$ & (\%) \\
\hline 1 & 3 & 0 & 0.3913 & 32.66 & 31.78 \\
\hline 1 & 3 & 0 & 0.3913 & 32.17 & 31.78 \\
\hline 1 & 3 & 0 & 0.3913 & 31.62 & 31.78 \\
\hline 2 & 3 & 5 & 0.4119 & 33.75 & 34.00 \\
\hline 2 & 3 & 5 & 0.4119 & 33.11 & 34.00 \\
\hline 2 & 3 & 5 & 0.4119 & 35.06 & 34.00 \\
\hline 3 & 3 & 10 & 0.43478 & 37.76 & 36.65 \\
\hline 3 & 3 & 10 & 0.43478 & 35.77 & 36.65 \\
\hline 3 & 3 & 10 & 0.43478 & 37.18 & 36.65 \\
\hline 4 & 3 & 15 & 0.46036 & 42.72 & 39.85 \\
\hline 4 & 3 & 15 & 0.46036 & 41.04 & 39.85 \\
\hline 4 & 3 & 15 & 0.46036 & 41.27 & 39.85 \\
\hline 5 & 3 & 20 & 0.48913 & 46.76 & 43.79 \\
\hline 5 & 3 & 20 & 0.48913 & 43.98 & 43.79 \\
\hline 5 & 3 & 20 & 0.48913 & 45.95 & 43.79 \\
\hline 6 & 3 & 25 & 0.52174 & 51.63 & 48.73 \\
\hline 6 & 3 & 25 & 0.52174 & 48.84 & 48.73 \\
\hline 6 & 3 & 25 & 0.52174 & 49.26 & 48.73 \\
\hline 7 & 3 & 30 & 0.55901 & 57.08 & 55.06 \\
\hline 7 & 3 & 30 & 0.55901 & 54.24 & 55.06 \\
\hline 7 & 3 & 30 & 0.55901 & 54.59 & 55.06 \\
\hline 8 & 3 & 35 & 0.60201 & 66.44 & 63.39 \\
\hline 8 & 3 & 35 & 0.60201 & 60.54 & 63.39 \\
\hline 8 & 3 & 35 & 0.60201 & 62.62 & 63.39 \\
\hline 9 & 4 & 0 & 0.44954 & 45.05 & 47.27 \\
\hline 9 & 4 & 0 & 0.44954 & 45.97 & 47.27 \\
\hline 9 & 4 & 0 & 0.44954 & 45.67 & 47.27 \\
\hline 9 & 4 & 0 & 0.44954 & 44.61 & 47.27 \\
\hline 10 & 4 & 5 & 0.4732 & 49.75 & 50.99 \\
\hline 10 & 4 & 5 & 0.4732 & 48.80 & 50.99 \\
\hline 10 & 4 & 5 & 0.4732 & 49.07 & 50.99 \\
\hline 10 & 4 & 5 & 0.4732 & 49.64 & 50.99 \\
\hline 11 & 4 & 10 & 0.49949 & 54.21 & 55.46 \\
\hline 11 & 4 & 10 & 0.49949 & 52.85 & 55.46 \\
\hline 11 & 4 & 10 & 0.49949 & 55.57 & 55.46 \\
\hline 11 & 4 & 10 & 0.49949 & 54.62 & 55.46 \\
\hline 12 & 4 & 15 & 0.52887 & 60.99 & 60.93 \\
\hline
\end{tabular}




\begin{tabular}{|c|c|c|c|c|c|}
\hline Instance & Products & Failure & Net Rho & Error & Pred. Value \\
\hline & & Rate & & sim-anal (\%) & (\%) \\
\hline 12 & 4 & 15 & 0.52887 & 57.95 & 60.93 \\
\hline 12 & 4 & 15 & 0.52887 & 58.34 & 60.93 \\
\hline 12 & 4 & 15 & 0.52887 & 59.93 & 60.93 \\
\hline 13 & 4 & 20 & 0.56193 & 67.38 & 67.73 \\
\hline 13 & 4 & 20 & 0.56193 & 63.90 & 67.73 \\
\hline 13 & 4 & 20 & 0.56193 & 66.49 & 67.73 \\
\hline 13 & 4 & 20 & 0.56193 & 67.31 & 67.73 \\
\hline 14 & 4 & 25 & 0.59939 & 76.19 & 76.36 \\
\hline 14 & 4 & 25 & 0.59939 & 72.60 & 76.36 \\
\hline 14 & 4 & 25 & 0.59939 & 73.51 & 76.36 \\
\hline 14 & 4 & 25 & 0.59939 & 76.34 & 76.36 \\
\hline 15 & 4 & 30 & 0.6422 & 90.20 & 87.57 \\
\hline 15 & 4 & 30 & 0.6422 & 85.66 & 87.57 \\
\hline 15 & 4 & 30 & 0.6422 & 88.49 & 87.57 \\
\hline 15 & 4 & 30 & 0.6422 & 91.18 & 87.57 \\
\hline 16 & 4 & 35 & 0.6916 & 100.80 & 102.56 \\
\hline 16 & 4 & 35 & 0.6916 & 98.57 & 102.56 \\
\hline 16 & 4 & 35 & 0.6916 & 98.57 & 102.56 \\
\hline 16 & 4 & 35 & 0.6916 & 100.55 & 102.56 \\
\hline 17 & 5 & 0 & 0.522893 & 66.37 & 65.34 \\
\hline 17 & 5 & 0 & 0.522893 & 67.42 & 65.34 \\
\hline 17 & 5 & 0 & 0.522893 & 67.67 & 65.34 \\
\hline 17 & 5 & 0 & 0.522893 & 66.82 & 65.34 \\
\hline 17 & 5 & 0 & 0.522893 & 66.64 & 65.34 \\
\hline 18 & 5 & 5 & 0.55676 & 73.72 & 72.94 \\
\hline 18 & 5 & 5 & 0.55676 & 71.33 & 72.94 \\
\hline 18 & 5 & 5 & 0.55676 & 72.85 & 72.94 \\
\hline 18 & 5 & 5 & 0.55676 & 72.10 & 72.94 \\
\hline 18 & 5 & 5 & 0.55676 & 71.66 & 72.94 \\
\hline 19 & 5 & 10 & 0.5877 & 82.97 & 80.66 \\
\hline 19 & 5 & 10 & 0.5877 & 83.00 & 80.66 \\
\hline 19 & 5 & 10 & 0.5877 & 81.88 & 80.66 \\
\hline 19 & 5 & 10 & 0.5877 & 82.36 & 80.66 \\
\hline 19 & 5 & 10 & 0.5877 & 80.50 & 80.66 \\
\hline 20 & 5 & 15 & 0.62227 & 92.43 & 90.24 \\
\hline 20 & 5 & 15 & 0.62227 & 89.58 & 90.24 \\
\hline 20 & 5 & 15 & 0.62227 & 90.18 & 90.24 \\
\hline 20 & 5 & 15 & 0.62227 & 90.71 & 90.24 \\
\hline 20 & 5 & 15 & 0.62227 & 88.68 & 90.24 \\
\hline 21 & 5 & 20 & 0.66116 & 103.85 & 102.40 \\
\hline
\end{tabular}




\begin{tabular}{|c|c|c|c|c|c|}
\hline Instance & Products & Failure & Net Rho & Error & Pred. Value \\
\hline & & Rate & & sim-anal (\%) & (\%) \\
\hline 21 & 5 & 20 & 0.66116 & 100.56 & 102.40 \\
\hline 21 & 5 & 20 & 0.66116 & 101.18 & 102.40 \\
\hline 21 & 5 & 20 & 0.66116 & 104.35 & 102.40 \\
\hline 21 & 5 & 20 & 0.66116 & 98.78 & 102.40 \\
\hline 22 & 5 & 25 & 0.70523 & 122.78 & 118.17 \\
\hline 22 & 5 & 25 & 0.70523 & 119.85 & 118.17 \\
\hline 22 & 5 & 25 & 0.70523 & 121.09 & 118.17 \\
\hline 22 & 5 & 25 & 0.70523 & 122.52 & 118.17 \\
\hline 22 & 5 & 25 & 0.70523 & 115.33 & 118.17 \\
\hline 23 & 5 & 30 & 0.75561 & 140.27 & 139.19 \\
\hline 23 & 5 & 30 & 0.75561 & 136.48 & 139.19 \\
\hline 23 & 5 & 30 & 0.75561 & 136.50 & 139.19 \\
\hline 23 & 5 & 30 & 0.75561 & 138.58 & 139.19 \\
\hline 23 & 5 & 30 & 0.75561 & 132.80 & 139.19 \\
\hline 24 & 5 & 35 & 0.81373 & 168.01 & 168.12 \\
\hline 24 & 5 & 35 & 0.81373 & 165.02 & 168.12 \\
\hline 24 & 5 & 35 & 0.81373 & 164.63 & 168.12 \\
\hline 24 & 5 & 35 & 0.81373 & 165.01 & 168.12 \\
\hline 24 & 5 & 35 & 0.81373 & 161.70 & 168.12 \\
\hline 25 & 6 & 0 & 0.54868 & 70.77 & 72.09 \\
\hline 25 & 6 & 0 & 0.54868 & 71.69 & 72.09 \\
\hline 25 & 6 & 0 & 0.54868 & 70.86 & 72.09 \\
\hline 25 & 6 & 0 & 0.54868 & 71.72 & 72.09 \\
\hline 25 & 6 & 0 & 0.54868 & 69.29 & 72.09 \\
\hline 25 & 6 & 0 & 0.54868 & 71.87 & 72.09 \\
\hline 26 & 6 & 5 & 0.57756 & 78.09 & 79.59 \\
\hline 26 & 6 & 5 & 0.57756 & 76.85 & 79.59 \\
\hline 26 & 6 & 5 & 0.57756 & 77.61 & 79.59 \\
\hline 26 & 6 & 5 & 0.57756 & 78.34 & 79.59 \\
\hline 26 & 6 & 5 & 0.57756 & 76.60 & 79.59 \\
\hline 26 & 6 & 5 & 0.57756 & 80.99 & 79.59 \\
\hline 27 & 6 & 10 & 0.60964 & 91.10 & 88.83 \\
\hline 27 & 6 & 10 & 0.60964 & 89.56 & 88.83 \\
\hline 27 & 6 & 10 & 0.60964 & 92.61 & 88.83 \\
\hline 27 & 6 & 10 & 0.60964 & 91.66 & 88.83 \\
\hline 27 & 6 & 10 & 0.60964 & 88.54 & 88.83 \\
\hline 27 & 6 & 10 & 0.60964 & 90.87 & 88.83 \\
\hline 28 & 6 & 15 & 0.6455 & 101.17 & 100.44 \\
\hline 28 & 6 & 15 & 0.6455 & 99.49 & 100.44 \\
\hline 28 & 6 & 15 & 0.6455 & 98.48 & 100.44 \\
\hline
\end{tabular}




\begin{tabular}{|c|c|c|c|c|c|}
\hline Instance & Products & Failure & Net Rho & Error & Pred. Value \\
\hline & & Rate & & sim-anal (\%) & $(\%)$ \\
\hline 28 & 6 & 15 & 0.6455 & 100.43 & 100.44 \\
\hline 28 & 6 & 15 & 0.6455 & 98.20 & 100.44 \\
\hline 28 & 6 & 15 & 0.6455 & 101.80 & 100.44 \\
\hline 29 & 6 & 20 & 0.68585 & 116.37 & 115.32 \\
\hline 29 & 6 & 20 & 0.68585 & 113.40 & 115.32 \\
\hline 29 & 6 & 20 & 0.68585 & 114.66 & 115.32 \\
\hline 29 & 6 & 20 & 0.68585 & 115.76 & 115.32 \\
\hline 29 & 6 & 20 & 0.68585 & 111.38 & 115.32 \\
\hline 30 & 6 & 25 & 0.73157 & 118.01 & 115.32 \\
\hline 30 & 6 & 25 & 0.73157 & 134.47 & 134.87 \\
\hline 30 & 6 & 25 & 0.73157 & 134.35 & 134.87 \\
\hline 30 & 6 & 25 & 0.73157 & 134.75 & 134.87 \\
\hline 30 & 6 & 25 & 0.73157 & 134.94 & 134.87 \\
\hline 30 & 6 & 25 & 0.73157 & 129.34 & 134.87 \\
\hline 31 & 6 & 30 & 0.78383 & 136.42 & 134.87 \\
\hline 31 & 6 & 30 & 0.78383 & 163.11 & 161.30 \\
\hline 31 & 6 & 30 & 0.78383 & 159.92 & 161.30 \\
\hline 31 & 6 & 30 & 0.78383 & 159.93 & 161.30 \\
\hline 31 & 6 & 30 & 0.78383 & 161.49 & 161.30 \\
\hline 31 & 6 & 30 & 0.78383 & 157.58 & 161.30 \\
\hline 32 & 6 & 35 & 0.84412 & 163.86 & 161.30 \\
\hline 32 & 6 & 35 & 0.84412 & 202.33 & 198.29 \\
\hline 32 & 6 & 35 & 0.84412 & 197.69 & 198.29 \\
\hline 32 & 6 & 35 & 0.84412 & 197.49 & 198.29 \\
\hline 32 & 6 & 35 & 0.84412 & 198.45 & 198.29 \\
\hline 32 & 6 & 35 & 0.84412 & 201.32 & 198.29 \\
\hline
\end{tabular}




\section{APPENDIX C}

Predicted flow time error at fork-join stations

\begin{tabular}{|c|c|c|c|c|c|c|c|}
\hline Inst. & Products & Servers & Subtasks & $\begin{array}{l}\text { Failure } \\
\text { Rate }\end{array}$ & $\begin{array}{l}\text { Net Traffic } \\
\text { Intensity }\end{array}$ & $\begin{array}{c}\text { Error } \\
\text { sim-anal }(\%)\end{array}$ & $\begin{array}{l}\text { Predicted } \\
\text { Error }(\%)\end{array}$ \\
\hline 1 & 1 & 2 & 2 & 0 & 0.04 & 0.35 & 1.07 \\
\hline 2 & 1 & 2 & 2 & 5 & 0.04 & 4.34 & 4.29 \\
\hline 3 & 1 & 2 & 2 & 10 & 0.04 & 9.41 & 9.64 \\
\hline 4 & 1 & 2 & 2 & 20 & 0.05 & 20.06 & 20.33 \\
\hline 5 & 1 & 2 & 2 & 30 & 0.06 & 29.89 & 31.00 \\
\hline 6 & 1 & 3 & 3 & 0 & 0.04 & 0.65 & 0.70 \\
\hline 7 & 1 & 3 & 3 & 5 & 0.04 & 5.98 & 6.06 \\
\hline 8 & 1 & 3 & 3 & 10 & 0.04 & 10.76 & 11.41 \\
\hline 9 & 1 & 3 & 3 & 20 & 0.05 & 21.17 & 22.10 \\
\hline 10 & 1 & 3 & 3 & 30 & 0.06 & 30.54 & 32.78 \\
\hline 11 & 1 & 4 & 4 & 0 & 0.04 & 0.42 & 2.48 \\
\hline 12 & 1 & 4 & 4 & 5 & 0.04 & 5.01 & 7.83 \\
\hline 13 & 1 & 4 & 4 & 10 & 0.04 & 10.31 & 13.18 \\
\hline 14 & 1 & 4 & 4 & 20 & 0.05 & 19.96 & 23.87 \\
\hline 15 & 1 & 4 & 4 & 30 & 0.06 & 30.60 & 34.55 \\
\hline 16 & 2 & 2 & 2 & 0 & 0.07 & 0.39 & 1.43 \\
\hline 16 & 2 & 2 & 2 & 0 & 0.07 & 0.69 & 1.43 \\
\hline 17 & 2 & 2 & 2 & 5 & 0.08 & 4.87 & 6.76 \\
\hline 17 & 2 & 2 & 2 & 5 & 0.08 & 5.63 & 6.76 \\
\hline 18 & 2 & 2 & 2 & 10 & 0.08 & 9.86 & 12.09 \\
\hline 18 & 2 & 2 & 2 & 10 & 0.08 & 11.05 & 12.09 \\
\hline 19 & 2 & 2 & 2 & 20 & 0.09 & 19.55 & 22.73 \\
\hline 19 & 2 & 2 & 2 & 20 & 0.09 & 20.90 & 22.73 \\
\hline 20 & 2 & 2 & 2 & 30 & 0.10 & 29.80 & 33.34 \\
\hline 20 & 2 & 2 & 2 & 30 & 0.10 & 30.80 & 33.34 \\
\hline 21 & 2 & 3 & 3 & 0 & 0.07 & 0.34 & 3.20 \\
\hline 21 & 2 & 3 & 3 & 0 & 0.07 & 1.49 & 3.20 \\
\hline 22 & 2 & 3 & 3 & 5 & 0.08 & 4.98 & 8.53 \\
\hline 22 & 2 & 3 & 3 & 5 & 0.08 & 5.98 & 8.53 \\
\hline 23 & 2 & 3 & 3 & 10 & 0.08 & 9.58 & 13.86 \\
\hline 23 & 2 & 3 & 3 & 10 & 0.08 & 11.15 & 13.86 \\
\hline 24 & 2 & 3 & 3 & 20 & 0.09 & 20.35 & 24.51 \\
\hline 24 & 2 & 3 & 3 & 20 & 0.09 & 21.13 & 24.51 \\
\hline 25 & 2 & 3 & 3 & 30 & 0.10 & 29.39 & 35.12 \\
\hline 25 & 2 & 3 & 3 & 30 & 0.10 & 30.60 & 35.12 \\
\hline 26 & 2 & 4 & 4 & 0 & 0.07 & 0.35 & 4.97 \\
\hline 26 & 2 & 4 & 4 & 0 & 0.07 & 0.85 & 4.97 \\
\hline
\end{tabular}




\begin{tabular}{|c|c|c|c|c|c|c|c|}
\hline Inst. & Products & Servers & Subtasks & $\begin{array}{c}\text { Failure } \\
\text { Rate }\end{array}$ & $\begin{array}{l}\text { Net Traffic } \\
\text { Intensity }\end{array}$ & $\begin{array}{c}\text { Error } \\
\text { sim-anal }(\%)\end{array}$ & $\begin{array}{l}\text { Predicted } \\
\text { Error }(\%)\end{array}$ \\
\hline 27 & 2 & 4 & 4 & 5 & 0.08 & 5.45 & 10.31 \\
\hline 27 & 2 & 4 & 4 & 5 & 0.08 & 5.89 & 10.31 \\
\hline 28 & 2 & 4 & 4 & 10 & 0.08 & 9.65 & 15.64 \\
\hline 28 & 2 & 4 & 4 & 10 & 0.08 & 11.03 & 15.64 \\
\hline 29 & 2 & 4 & 4 & 20 & 0.09 & 20.35 & 26.28 \\
\hline 29 & 2 & 4 & 4 & 20 & 0.09 & 20.92 & 26.28 \\
\hline 30 & 2 & 4 & 4 & 30 & 0.10 & 29.78 & 36.89 \\
\hline 30 & 2 & 4 & 4 & 30 & 0.10 & 31.36 & 36.89 \\
\hline 31 & 3 & 2 & 2 & 0 & 0.17 & 3.00 & 3.23 \\
\hline 31 & 3 & 2 & 2 & 0 & 0.17 & 6.94 & 3.23 \\
\hline 31 & 3 & 2 & 2 & 0 & 0.17 & 1.11 & 3.23 \\
\hline 32 & 3 & 2 & 2 & 5 & 0.18 & 8.73 & 8.51 \\
\hline 32 & 3 & 2 & 2 & 5 & 0.18 & 12.20 & 8.51 \\
\hline 32 & 3 & 2 & 2 & 5 & 0.18 & 3.70 & 8.51 \\
\hline 33 & 3 & 2 & 2 & 10 & 0.19 & 12.74 & 13.78 \\
\hline 33 & 3 & 2 & 2 & 10 & 0.19 & 16.85 & 13.78 \\
\hline 33 & 3 & 2 & 2 & 10 & 0.19 & 8.95 & 13.78 \\
\hline 34 & 3 & 2 & 2 & 20 & 0.21 & 22.60 & 24.28 \\
\hline 34 & 3 & 2 & 2 & 20 & 0.21 & 25.31 & 24.28 \\
\hline 34 & 3 & 2 & 2 & 20 & 0.21 & 19.02 & 24.28 \\
\hline 35 & 3 & 2 & 2 & 30 & 0.24 & 32.91 & 34.70 \\
\hline 35 & 3 & 2 & 2 & 30 & 0.24 & 36.77 & 34.70 \\
\hline 35 & 3 & 2 & 2 & 30 & 0.24 & 29.23 & 34.70 \\
\hline 36 & 3 & 3 & 3 & 0 & 0.17 & 3.27 & 5.00 \\
\hline 36 & 3 & 3 & 3 & 0 & 0.17 & 7.53 & 5.00 \\
\hline 36 & 3 & 3 & 3 & 0 & 0.17 & 0.78 & 5.00 \\
\hline 37 & 3 & 3 & 3 & 5 & 0.18 & 8.53 & 10.28 \\
\hline 37 & 3 & 3 & 3 & 5 & 0.18 & 10.97 & 10.28 \\
\hline 37 & 3 & 3 & 3 & 5 & 0.18 & 3.59 & 10.28 \\
\hline 38 & 3 & 3 & 3 & 10 & 0.19 & 13.64 & 15.55 \\
\hline 38 & 3 & 3 & 3 & 10 & 0.19 & 17.38 & 15.55 \\
\hline 38 & 3 & 3 & 3 & 10 & 0.19 & 9.14 & 15.55 \\
\hline 39 & 3 & 3 & 3 & 20 & 0.21 & 23.35 & 26.05 \\
\hline 39 & 3 & 3 & 3 & 20 & 0.21 & 26.88 & 26.05 \\
\hline 39 & 3 & 3 & 3 & 20 & 0.21 & 19.73 & 26.05 \\
\hline 40 & 3 & 3 & 3 & 30 & 0.24 & 33.73 & 36.47 \\
\hline 40 & 3 & 3 & 3 & 30 & 0.24 & 37.00 & 36.47 \\
\hline 40 & 3 & 3 & 3 & 30 & 0.24 & 29.73 & 36.47 \\
\hline 41 & 3 & 4 & 4 & 0 & 0.17 & 2.41 & 6.78 \\
\hline 41 & 3 & 4 & 4 & 0 & 0.17 & 6.71 & 6.78 \\
\hline
\end{tabular}




\begin{tabular}{|c|c|c|c|c|c|c|c|}
\hline Inst. & Products & Servers & Subtasks & $\begin{array}{c}\text { Failure } \\
\text { Rate }\end{array}$ & $\begin{array}{l}\text { Net Traffic } \\
\text { Intensity }\end{array}$ & $\begin{array}{c}\text { Error } \\
\text { sim-anal }(\%)\end{array}$ & $\begin{array}{l}\text { Predicted } \\
\text { Error }(\%)\end{array}$ \\
\hline 41 & 3 & 4 & 4 & 0 & 0.17 & 1.38 & 6.78 \\
\hline 42 & 3 & 4 & 4 & 5 & 0.18 & 8.04 & 12.05 \\
\hline 42 & 3 & 4 & 4 & 5 & 0.18 & 12.41 & 12.05 \\
\hline 42 & 3 & 4 & 4 & 5 & 0.18 & 4.02 & 12.05 \\
\hline 43 & 3 & 4 & 4 & 10 & 0.19 & 12.86 & 17.32 \\
\hline 43 & 3 & 4 & 4 & 10 & 0.19 & 17.98 & 17.32 \\
\hline 43 & 3 & 4 & 4 & 10 & 0.19 & 9.50 & 17.32 \\
\hline 44 & 3 & 4 & 4 & 20 & 0.21 & 23.45 & 27.82 \\
\hline 44 & 3 & 4 & 4 & 20 & 0.21 & 27.28 & 27.82 \\
\hline 44 & 3 & 4 & 4 & 20 & 0.21 & 19.00 & 27.82 \\
\hline 45 & 3 & 4 & 4 & 30 & 0.24 & 33.15 & 38.24 \\
\hline 45 & 3 & 4 & 4 & 30 & 0.24 & 37.09 & 38.24 \\
\hline 45 & 3 & 4 & 4 & 30 & 0.24 & 30.11 & 38.24 \\
\hline 46 & 4 & 2 & 2 & 0 & 0.30 & 3.96 & 4.66 \\
\hline 46 & 4 & 2 & 2 & 0 & 0.30 & 10.51 & 4.66 \\
\hline 46 & 4 & 2 & 2 & 0 & 0.30 & 2.69 & 4.66 \\
\hline 46 & 4 & 2 & 2 & 0 & 0.30 & 0.96 & 4.66 \\
\hline 47 & 4 & 2 & 2 & 5 & 0.31 & 9.16 & 9.86 \\
\hline 47 & 4 & 2 & 2 & 5 & 0.31 & 15.35 & 9.86 \\
\hline 47 & 4 & 2 & 2 & 5 & 0.31 & 2.29 & 9.86 \\
\hline 47 & 4 & 2 & 2 & 5 & 0.31 & 6.52 & 9.86 \\
\hline 48 & 4 & 2 & 2 & 10 & 0.33 & 13.60 & 15.05 \\
\hline 48 & 4 & 2 & 2 & 10 & 0.33 & 20.01 & 15.05 \\
\hline 48 & 4 & 2 & 2 & 10 & 0.33 & 6.56 & 15.05 \\
\hline 48 & 4 & 2 & 2 & 10 & 0.33 & 11.29 & 15.05 \\
\hline 49 & 4 & 2 & 2 & 20 & 0.37 & 23.90 & 25.34 \\
\hline 49 & 4 & 2 & 2 & 20 & 0.37 & 29.75 & 25.34 \\
\hline 49 & 4 & 2 & 2 & 20 & 0.37 & 18.22 & 25.34 \\
\hline 49 & 4 & 2 & 2 & 20 & 0.37 & 22.32 & 25.34 \\
\hline 50 & 4 & 2 & 2 & 30 & 0.43 & 34.64 & 35.51 \\
\hline 50 & 4 & 2 & 2 & 30 & 0.43 & 39.62 & 35.51 \\
\hline 50 & 4 & 2 & 2 & 30 & 0.43 & 27.98 & 35.51 \\
\hline 50 & 4 & 2 & 2 & 30 & 0.43 & 31.92 & 35.51 \\
\hline 51 & 4 & 3 & 3 & 0 & 0.30 & 3.96 & 6.43 \\
\hline 51 & 4 & 3 & 3 & 0 & 0.30 & 11.97 & 6.43 \\
\hline 51 & 4 & 3 & 3 & 0 & 0.30 & 2.77 & 6.43 \\
\hline 51 & 4 & 3 & 3 & 0 & 0.30 & 2.25 & 6.43 \\
\hline 52 & 4 & 3 & 3 & 5 & 0.31 & 9.78 & 11.63 \\
\hline 52 & 4 & 3 & 3 & 5 & 0.31 & 15.67 & 11.63 \\
\hline 52 & 4 & 3 & 3 & 5 & 0.31 & 2.53 & 11.63 \\
\hline
\end{tabular}




\begin{tabular}{|c|c|c|c|c|c|c|c|}
\hline Inst. & Products & Servers & Subtasks & $\begin{array}{l}\text { Failure } \\
\text { Rate }\end{array}$ & $\begin{array}{l}\text { Net Traffic } \\
\text { Intensity }\end{array}$ & $\begin{array}{c}\text { Error } \\
\text { sim-anal }(\%)\end{array}$ & $\begin{array}{l}\text { Predicted } \\
\text { Error }(\%)\end{array}$ \\
\hline 52 & 4 & 3 & 3 & 5 & 0.31 & 7.22 & 11.63 \\
\hline 53 & 4 & 3 & 3 & 10 & 0.33 & 14.34 & 16.82 \\
\hline 53 & 4 & 3 & 3 & 10 & 0.33 & 20.84 & 16.82 \\
\hline 53 & 4 & 3 & 3 & 10 & 0.33 & 7.51 & 16.82 \\
\hline 53 & 4 & 3 & 3 & 10 & 0.33 & 11.59 & 16.82 \\
\hline 54 & 4 & 3 & 3 & 20 & 0.37 & 25.33 & 27.12 \\
\hline 54 & 4 & 3 & 3 & 20 & 0.37 & 30.82 & 27.12 \\
\hline 54 & 4 & 3 & 3 & 20 & 0.37 & 18.74 & 27.12 \\
\hline 54 & 4 & 3 & 3 & 20 & 0.37 & 23.21 & 27.12 \\
\hline 55 & 4 & 3 & 3 & 30 & 0.43 & 35.58 & 37.29 \\
\hline 55 & 4 & 3 & 3 & 30 & 0.43 & 41.34 & 37.29 \\
\hline 55 & 4 & 3 & 3 & 30 & 0.43 & 29.62 & 37.29 \\
\hline 55 & 4 & 3 & 3 & 30 & 0.43 & 33.66 & 37.29 \\
\hline 56 & 4 & 4 & 4 & 0 & 0.30 & 4.25 & 8.20 \\
\hline 56 & 4 & 4 & 4 & 0 & 0.30 & 11.20 & 8.20 \\
\hline 56 & 4 & 4 & 4 & 0 & 0.30 & 3.41 & 8.20 \\
\hline 56 & 4 & 4 & 4 & 0 & 0.30 & 1.62 & 8.20 \\
\hline 57 & 4 & 4 & 4 & 5 & 0.31 & 10.21 & 13.40 \\
\hline 57 & 4 & 4 & 4 & 5 & 0.31 & 16.19 & 13.40 \\
\hline 57 & 4 & 4 & 4 & 5 & 0.31 & 2.42 & 13.40 \\
\hline 57 & 4 & 4 & 4 & 5 & 0.31 & 7.23 & 13.40 \\
\hline 58 & 4 & 4 & 4 & 10 & 0.33 & 14.98 & 18.59 \\
\hline 58 & 4 & 4 & 4 & 10 & 0.33 & 21.95 & 18.59 \\
\hline 58 & 4 & 4 & 4 & 10 & 0.33 & 8.32 & 18.59 \\
\hline 58 & 4 & 4 & 4 & 10 & 0.33 & 12.21 & 18.59 \\
\hline 59 & 4 & 4 & 4 & 20 & 0.37 & 26.08 & 28.89 \\
\hline 59 & 4 & 4 & 4 & 20 & 0.37 & 31.72 & 28.89 \\
\hline 59 & 4 & 4 & 4 & 20 & 0.37 & 18.45 & 28.89 \\
\hline 59 & 4 & 4 & 4 & 20 & 0.37 & 23.12 & 28.89 \\
\hline 60 & 4 & 4 & 4 & 30 & 0.43 & 36.41 & 39.06 \\
\hline 60 & 4 & 4 & 4 & 30 & 0.43 & 41.61 & 39.06 \\
\hline 60 & 4 & 4 & 4 & 30 & 0.43 & 29.59 & 39.06 \\
\hline 60 & 4 & 4 & 4 & 30 & 0.43 & 33.54 & 39.06 \\
\hline 61 & 5 & 2 & 2 & 0 & 0.42 & 1.89 & 6.18 \\
\hline 61 & 5 & 2 & 2 & 0 & 0.42 & 11.17 & 6.18 \\
\hline 61 & 5 & 2 & 2 & 0 & 0.42 & 6.56 & 6.18 \\
\hline 61 & 5 & 2 & 2 & 0 & 0.42 & 0.72 & 6.18 \\
\hline 61 & 5 & 2 & 2 & 0 & 0.42 & 12.67 & 6.18 \\
\hline 62 & 5 & 2 & 2 & 5 & 0.44 & 8.54 & 11.31 \\
\hline 62 & 5 & 2 & 2 & 5 & 0.44 & 16.62 & 11.31 \\
\hline
\end{tabular}




\begin{tabular}{|c|c|c|c|c|c|c|c|}
\hline Inst. & Products & Servers & Subtasks & $\begin{array}{c}\text { Failure } \\
\text { Rate }\end{array}$ & $\begin{array}{l}\text { Net Traffic } \\
\text { Intensity }\end{array}$ & $\begin{array}{c}\text { Error } \\
\text { sim-anal }(\%)\end{array}$ & $\begin{array}{l}\text { Predicted } \\
\text { Error }(\%)\end{array}$ \\
\hline 62 & 5 & 2 & 2 & 5 & 0.44 & 0.65 & 11.31 \\
\hline 62 & 5 & 2 & 2 & 5 & 0.44 & 4.95 & 11.31 \\
\hline 62 & 5 & 2 & 2 & 5 & 0.44 & 18.06 & 11.31 \\
\hline 63 & 5 & 2 & 2 & 10 & 0.47 & 13.53 & 16.42 \\
\hline 63 & 5 & 2 & 2 & 10 & 0.47 & 20.81 & 16.42 \\
\hline 63 & 5 & 2 & 2 & 10 & 0.47 & 3.64 & 16.42 \\
\hline 63 & 5 & 2 & 2 & 10 & 0.47 & 10.20 & 16.42 \\
\hline 63 & 5 & 2 & 2 & 10 & 0.47 & 23.03 & 16.42 \\
\hline 64 & 5 & 2 & 2 & 20 & 0.53 & 25.70 & 26.54 \\
\hline 64 & 5 & 2 & 2 & 20 & 0.53 & 32.34 & 26.54 \\
\hline 64 & 5 & 2 & 2 & 20 & 0.53 & 15.56 & 26.54 \\
\hline 64 & 5 & 2 & 2 & 20 & 0.53 & 21.22 & 26.54 \\
\hline 64 & 5 & 2 & 2 & 20 & 0.53 & 33.51 & 26.54 \\
\hline 65 & 5 & 2 & 2 & 30 & 0.60 & 35.05 & 36.47 \\
\hline 65 & 5 & 2 & 2 & 30 & 0.60 & 41.78 & 36.47 \\
\hline 65 & 5 & 2 & 2 & 30 & 0.60 & 25.73 & 36.47 \\
\hline 65 & 5 & 2 & 2 & 30 & 0.60 & 31.12 & 36.47 \\
\hline 65 & 5 & 2 & 2 & 30 & 0.60 & 42.67 & 36.47 \\
\hline 66 & 5 & 3 & 3 & 0 & 0.42 & 3.72 & 7.95 \\
\hline 66 & 5 & 3 & 3 & 0 & 0.42 & 12.45 & 7.95 \\
\hline 66 & 5 & 3 & 3 & 0 & 0.42 & 5.59 & 7.95 \\
\hline 66 & 5 & 3 & 3 & 0 & 0.42 & 0.26 & 7.95 \\
\hline 66 & 5 & 3 & 3 & 0 & 0.42 & 13.93 & 7.95 \\
\hline 67 & 5 & 3 & 3 & 5 & 0.44 & 9.25 & 13.09 \\
\hline 67 & 5 & 3 & 3 & 5 & 0.44 & 17.57 & 13.09 \\
\hline 67 & 5 & 3 & 3 & 5 & 0.44 & 0.02 & 13.09 \\
\hline 67 & 5 & 3 & 3 & 5 & 0.44 & 6.04 & 13.09 \\
\hline 67 & 5 & 3 & 3 & 5 & 0.44 & 18.76 & 13.09 \\
\hline 68 & 5 & 3 & 3 & 10 & 0.47 & 14.13 & 18.19 \\
\hline 68 & 5 & 3 & 3 & 10 & 0.47 & 21.58 & 18.19 \\
\hline 68 & 5 & 3 & 3 & 10 & 0.47 & 5.01 & 18.19 \\
\hline 68 & 5 & 3 & 3 & 10 & 0.47 & 11.03 & 18.19 \\
\hline 68 & 5 & 3 & 3 & 10 & 0.47 & 23.65 & 18.19 \\
\hline 69 & 5 & 3 & 3 & 20 & 0.53 & 25.68 & 28.31 \\
\hline 69 & 5 & 3 & 3 & 20 & 0.53 & 33.86 & 28.31 \\
\hline 69 & 5 & 3 & 3 & 20 & 0.53 & 16.29 & 28.31 \\
\hline 69 & 5 & 3 & 3 & 20 & 0.53 & 22.36 & 28.31 \\
\hline 69 & 5 & 3 & 3 & 20 & 0.53 & 34.68 & 28.31 \\
\hline 70 & 5 & 3 & 3 & 30 & 0.60 & 37.05 & 38.24 \\
\hline 70 & 5 & 3 & 3 & 30 & 0.60 & 43.50 & 38.24 \\
\hline
\end{tabular}




\begin{tabular}{|c|c|c|c|c|c|c|c|}
\hline Inst. & Products & Servers & Subtasks & $\begin{array}{c}\text { Failure } \\
\text { Rate }\end{array}$ & $\begin{array}{l}\text { Net Traffic } \\
\text { Intensity }\end{array}$ & $\begin{array}{c}\text { Error } \\
\text { sim-anal }(\%)\end{array}$ & $\begin{array}{l}\text { Predicted } \\
\text { Error }(\%)\end{array}$ \\
\hline 70 & 5 & 3 & 3 & 30 & 0.60 & 27.38 & 38.24 \\
\hline 70 & 5 & 3 & 3 & 30 & 0.60 & 33.68 & 38.24 \\
\hline 70 & 5 & 3 & 3 & 30 & 0.60 & 44.95 & 38.24 \\
\hline 71 & 5 & 4 & 4 & 0 & 0.42 & 4.75 & 9.72 \\
\hline 71 & 5 & 4 & 4 & 0 & 0.42 & 13.54 & 9.72 \\
\hline 71 & 5 & 4 & 4 & 0 & 0.42 & 5.43 & 9.72 \\
\hline 71 & 5 & 4 & 4 & 0 & 0.42 & 0.55 & 9.72 \\
\hline 71 & 5 & 4 & 4 & 0 & 0.42 & 14.53 & 9.72 \\
\hline 72 & 5 & 4 & 4 & 5 & 0.44 & 9.95 & 14.86 \\
\hline 72 & 5 & 4 & 4 & 5 & 0.44 & 17.34 & 14.86 \\
\hline 72 & 5 & 4 & 4 & 5 & 0.44 & 0.28 & 14.86 \\
\hline 72 & 5 & 4 & 4 & 5 & 0.44 & 5.85 & 14.86 \\
\hline 72 & 5 & 4 & 4 & 5 & 0.44 & 19.33 & 14.86 \\
\hline 73 & 5 & 4 & 4 & 10 & 0.47 & 14.69 & 19.97 \\
\hline 73 & 5 & 4 & 4 & 10 & 0.47 & 23.31 & 19.97 \\
\hline 73 & 5 & 4 & 4 & 10 & 0.47 & 5.88 & 19.97 \\
\hline 73 & 5 & 4 & 4 & 10 & 0.47 & 11.62 & 19.97 \\
\hline 73 & 5 & 4 & 4 & 10 & 0.47 & 25.05 & 19.97 \\
\hline 74 & 5 & 4 & 4 & 20 & 0.53 & 26.35 & 30.08 \\
\hline 74 & 5 & 4 & 4 & 20 & 0.53 & 33.53 & 30.08 \\
\hline 74 & 5 & 4 & 4 & 20 & 0.53 & 16.42 & 30.08 \\
\hline 74 & 5 & 4 & 4 & 20 & 0.53 & 22.69 & 30.08 \\
\hline 74 & 5 & 4 & 4 & 20 & 0.53 & 35.15 & 30.08 \\
\hline 75 & 5 & 4 & 4 & 30 & 0.60 & 38.38 & 40.01 \\
\hline 75 & 5 & 4 & 4 & 30 & 0.60 & 44.30 & 40.01 \\
\hline 75 & 5 & 4 & 4 & 30 & 0.60 & 27.97 & 40.01 \\
\hline 75 & 5 & 4 & 4 & 30 & 0.60 & 33.93 & 40.01 \\
\hline 75 & 5 & 4 & 4 & 30 & 0.60 & 45.44 & 40.01 \\
\hline 76 & 6 & 2 & 2 & 0 & 0.50 & 2.89 & 8.16 \\
\hline 76 & 6 & 2 & 2 & 0 & 0.50 & 12.65 & 8.16 \\
\hline 76 & 6 & 2 & 2 & 0 & 0.50 & 7.61 & 8.16 \\
\hline 76 & 6 & 2 & 2 & 0 & 0.50 & 0.77 & 8.16 \\
\hline 76 & 6 & 2 & 2 & 0 & 0.50 & 14.18 & 8.16 \\
\hline 76 & 6 & 2 & 2 & 0 & 0.50 & 2.51 & 8.16 \\
\hline 77 & 6 & 2 & 2 & 5 & 0.53 & 7.89 & 13.25 \\
\hline 77 & 6 & 2 & 2 & 5 & 0.53 & 18.09 & 13.25 \\
\hline 77 & 6 & 2 & 2 & 5 & 0.53 & 2.99 & 13.25 \\
\hline 77 & 6 & 2 & 2 & 5 & 0.53 & 4.51 & 13.25 \\
\hline 77 & 6 & 2 & 2 & 5 & 0.53 & 19.27 & 13.25 \\
\hline 77 & 6 & 2 & 2 & 5 & 0.53 & 8.20 & 13.25 \\
\hline
\end{tabular}




\begin{tabular}{|c|c|c|c|c|c|c|c|}
\hline Inst. & Products & Servers & Subtasks & $\begin{array}{l}\text { Failure } \\
\text { Rate }\end{array}$ & $\begin{array}{l}\text { Net Traffic } \\
\text { Intensity }\end{array}$ & $\begin{array}{c}\text { Error } \\
\text { sim-anal }(\%)\end{array}$ & $\begin{array}{l}\text { Predicted } \\
\text { Error (\%) }\end{array}$ \\
\hline 78 & 6 & 2 & 2 & 10 & 0.56 & 14.43 & 18.30 \\
\hline 78 & 6 & 2 & 2 & 10 & 0.56 & 23.32 & 18.30 \\
\hline 78 & 6 & 2 & 2 & 10 & 0.56 & 3.49 & 18.30 \\
\hline 78 & 6 & 2 & 2 & 10 & 0.56 & 10.63 & 18.30 \\
\hline 78 & 6 & 2 & 2 & 10 & 0.56 & 25.09 & 18.30 \\
\hline 78 & 6 & 2 & 2 & 10 & 0.56 & 14.41 & 18.30 \\
\hline 79 & 6 & 2 & 2 & 20 & 0.63 & 24.77 & 28.30 \\
\hline 79 & 6 & 2 & 2 & 20 & 0.63 & 32.21 & 28.30 \\
\hline 79 & 6 & 2 & 2 & 20 & 0.63 & 12.59 & 28.30 \\
\hline 79 & 6 & 2 & 2 & 20 & 0.63 & 20.34 & 28.30 \\
\hline 79 & 6 & 2 & 2 & 20 & 0.63 & 33.82 & 28.30 \\
\hline 79 & 6 & 2 & 2 & 20 & 0.63 & 23.63 & 28.30 \\
\hline 80 & 6 & 2 & 2 & 30 & 0.69 & 35.65 & 38.28 \\
\hline 80 & 6 & 2 & 2 & 30 & 0.69 & 43.85 & 38.28 \\
\hline 80 & 6 & 2 & 2 & 30 & 0.69 & 24.92 & 38.28 \\
\hline 80 & 6 & 2 & 2 & 30 & 0.69 & 31.96 & 38.28 \\
\hline 80 & 6 & 2 & 2 & 30 & 0.69 & 44.93 & 38.28 \\
\hline 80 & 6 & 2 & 2 & 30 & 0.69 & 35.58 & 38.28 \\
\hline 81 & 6 & 3 & 3 & 0 & 0.50 & 5.11 & 9.93 \\
\hline 81 & 6 & 3 & 3 & 0 & 0.50 & 14.60 & 9.93 \\
\hline 81 & 6 & 3 & 3 & 0 & 0.50 & 7.38 & 9.93 \\
\hline 81 & 6 & 3 & 3 & 0 & 0.50 & 0.60 & 9.93 \\
\hline 81 & 6 & 3 & 3 & 0 & 0.50 & 16.20 & 9.93 \\
\hline 81 & 6 & 3 & 3 & 0 & 0.50 & 5.07 & 9.93 \\
\hline 82 & 6 & 3 & 3 & 5 & 0.53 & 10.10 & 15.02 \\
\hline 82 & 6 & 3 & 3 & 5 & 0.53 & 19.08 & 15.02 \\
\hline 82 & 6 & 3 & 3 & 5 & 0.53 & 1.38 & 15.02 \\
\hline 82 & 6 & 3 & 3 & 5 & 0.53 & 6.11 & 15.02 \\
\hline 82 & 6 & 3 & 3 & 5 & 0.53 & 21.19 & 15.02 \\
\hline 82 & 6 & 3 & 3 & 5 & 0.53 & 10.41 & 15.02 \\
\hline 83 & 6 & 3 & 3 & 10 & 0.56 & 15.83 & 20.08 \\
\hline 83 & 6 & 3 & 3 & 10 & 0.56 & 24.87 & 20.08 \\
\hline 83 & 6 & 3 & 3 & 10 & 0.56 & 5.52 & 20.08 \\
\hline 83 & 6 & 3 & 3 & 10 & 0.56 & 12.28 & 20.08 \\
\hline 83 & 6 & 3 & 3 & 10 & 0.56 & 27.03 & 20.08 \\
\hline 83 & 6 & 3 & 3 & 10 & 0.56 & 16.34 & 20.08 \\
\hline 84 & 6 & 3 & 3 & 20 & 0.63 & 27.28 & 30.07 \\
\hline 84 & 6 & 3 & 3 & 20 & 0.63 & 36.37 & 30.07 \\
\hline 84 & 6 & 3 & 3 & 20 & 0.63 & 16.34 & 30.07 \\
\hline 84 & 6 & 3 & 3 & 20 & 0.63 & 22.97 & 30.07 \\
\hline
\end{tabular}




\begin{tabular}{|c|c|c|c|c|c|c|c|}
\hline Inst. & Products & Servers & Subtasks & $\begin{array}{c}\text { Failure } \\
\text { Rate }\end{array}$ & $\begin{array}{l}\text { Net Traffic } \\
\text { Intensity }\end{array}$ & $\begin{array}{c}\text { Error } \\
\text { sim-anal }(\%)\end{array}$ & $\begin{array}{l}\text { Predicted } \\
\text { Error }(\%)\end{array}$ \\
\hline 84 & 6 & 3 & 3 & 20 & 0.63 & 37.20 & 30.07 \\
\hline 84 & 6 & 3 & 3 & 20 & 0.63 & 27.79 & 30.07 \\
\hline 85 & 6 & 3 & 3 & 30 & 0.71 & 39.86 & 39.84 \\
\hline 85 & 6 & 3 & 3 & 30 & 0.71 & 47.18 & 39.84 \\
\hline 85 & 6 & 3 & 3 & 30 & 0.71 & 28.65 & 39.84 \\
\hline 85 & 6 & 3 & 3 & 30 & 0.71 & 35.73 & 39.84 \\
\hline 85 & 6 & 3 & 3 & 30 & 0.71 & 48.77 & 39.84 \\
\hline 85 & 6 & 3 & 3 & 30 & 0.71 & 39.30 & 39.84 \\
\hline 86 & 6 & 4 & 4 & 0 & 0.50 & 5.33 & 11.70 \\
\hline 86 & 6 & 4 & 4 & 0 & 0.50 & 15.21 & 11.70 \\
\hline 86 & 6 & 4 & 4 & 0 & 0.50 & 6.93 & 11.70 \\
\hline 86 & 6 & 4 & 4 & 0 & 0.50 & 0.39 & 11.70 \\
\hline 86 & 6 & 4 & 4 & 0 & 0.50 & 16.63 & 11.70 \\
\hline 86 & 6 & 4 & 4 & 0 & 0.50 & 5.37 & 11.70 \\
\hline 87 & 6 & 4 & 4 & 5 & 0.53 & 12.09 & 16.79 \\
\hline 87 & 6 & 4 & 4 & 5 & 0.53 & 20.83 & 16.79 \\
\hline 87 & 6 & 4 & 4 & 5 & 0.53 & 0.12 & 16.79 \\
\hline 87 & 6 & 4 & 4 & 5 & 0.53 & 7.05 & 16.79 \\
\hline 87 & 6 & 4 & 4 & 5 & 0.53 & 22.29 & 16.79 \\
\hline 87 & 6 & 4 & 4 & 5 & 0.53 & 11.26 & 16.79 \\
\hline 88 & 6 & 4 & 4 & 10 & 0.56 & 15.51 & 21.85 \\
\hline 88 & 6 & 4 & 4 & 10 & 0.56 & 24.94 & 21.85 \\
\hline 88 & 6 & 4 & 4 & 10 & 0.56 & 4.39 & 21.85 \\
\hline 88 & 6 & 4 & 4 & 10 & 0.56 & 11.51 & 21.85 \\
\hline 88 & 6 & 4 & 4 & 10 & 0.56 & 26.39 & 21.85 \\
\hline 88 & 6 & 4 & 4 & 10 & 0.56 & 15.56 & 21.85 \\
\hline 89 & 6 & 4 & 4 & 20 & 0.63 & 28.48 & 31.84 \\
\hline 89 & 6 & 4 & 4 & 20 & 0.63 & 36.83 & 31.84 \\
\hline 89 & 6 & 4 & 4 & 20 & 0.63 & 16.77 & 31.84 \\
\hline 89 & 6 & 4 & 4 & 20 & 0.63 & 23.97 & 31.84 \\
\hline 89 & 6 & 4 & 4 & 20 & 0.63 & 38.17 & 31.84 \\
\hline 89 & 6 & 4 & 4 & 20 & 0.63 & 28.32 & 31.84 \\
\hline 90 & 6 & 4 & 4 & 30 & 0.71 & 40.82 & 41.62 \\
\hline 90 & 6 & 4 & 4 & 30 & 0.71 & 48.11 & 41.62 \\
\hline 90 & 6 & 4 & 4 & 30 & 0.71 & 30.16 & 41.62 \\
\hline 90 & 6 & 4 & 4 & 30 & 0.71 & 36.87 & 41.62 \\
\hline 90 & 6 & 4 & 4 & 30 & 0.71 & 49.99 & 41.62 \\
\hline 90 & 6 & 4 & 4 & 30 & 0.71 & 40.77 & 41.62 \\
\hline 91 & 1 & 3 & 2 & 0 & 0.03 & 10.98 & 3.47 \\
\hline 92 & 1 & 3 & 2 & 5 & 0.03 & 14.63 & 8.84 \\
\hline
\end{tabular}




\begin{tabular}{|c|c|c|c|c|c|c|c|}
\hline Inst. & Products & Servers & Subtasks & $\begin{array}{l}\text { Failure } \\
\text { Rate }\end{array}$ & $\begin{array}{l}\text { Net Traffic } \\
\text { Intensity }\end{array}$ & $\begin{array}{c}\text { Error } \\
\text { sim-anal }(\%)\end{array}$ & $\begin{array}{l}\text { Predicted } \\
\text { Error }(\%)\end{array}$ \\
\hline 93 & 1 & 3 & 2 & 10 & 0.03 & 19.11 & 14.20 \\
\hline 94 & 1 & 3 & 2 & 20 & 0.03 & 28.51 & 24.91 \\
\hline 95 & 1 & 3 & 2 & 30 & 0.04 & 37.95 & 35.61 \\
\hline 96 & 2 & 3 & 2 & 0 & 0.05 & 10.77 & 6.09 \\
\hline 96 & 2 & 3 & 2 & 0 & 0.05 & 10.65 & 6.09 \\
\hline 97 & 2 & 3 & 2 & 5 & 0.05 & 15.18 & 11.44 \\
\hline 97 & 2 & 3 & 2 & 5 & 0.05 & 15.04 & 11.44 \\
\hline 98 & 2 & 3 & 2 & 10 & 0.05 & 19.40 & 16.78 \\
\hline 98 & 2 & 3 & 2 & 10 & 0.05 & 19.99 & 16.78 \\
\hline 99 & 2 & 3 & 2 & 20 & 0.06 & 27.53 & 27.46 \\
\hline 99 & 2 & 3 & 2 & 20 & 0.06 & 28.63 & 27.46 \\
\hline 100 & 2 & 3 & 2 & 30 & 0.07 & 36.23 & 38.12 \\
\hline 100 & 2 & 3 & 2 & 30 & 0.07 & 37.83 & 38.12 \\
\hline 101 & 3 & 3 & 2 & 0 & 0.11 & 12.30 & 8.24 \\
\hline 101 & 3 & 3 & 2 & 0 & 0.11 & 14.17 & 8.24 \\
\hline 101 & 3 & 3 & 2 & 0 & 0.11 & 9.53 & 8.24 \\
\hline 102 & 3 & 3 & 2 & 5 & 0.12 & 16.95 & 13.55 \\
\hline 102 & 3 & 3 & 2 & 5 & 0.12 & 18.10 & 13.55 \\
\hline 102 & 3 & 3 & 2 & 5 & 0.12 & 14.25 & 13.55 \\
\hline 103 & 3 & 3 & 2 & 10 & 0.12 & 20.55 & 18.86 \\
\hline 103 & 3 & 3 & 2 & 10 & 0.12 & 22.59 & 18.86 \\
\hline 103 & 3 & 3 & 2 & 10 & 0.12 & 18.49 & 18.86 \\
\hline 104 & 3 & 3 & 2 & 20 & 0.14 & 30.29 & 29.44 \\
\hline 104 & 3 & 3 & 2 & 20 & 0.14 & 31.97 & 29.44 \\
\hline 104 & 3 & 3 & 2 & 20 & 0.14 & 27.89 & 29.44 \\
\hline 105 & 3 & 3 & 2 & 30 & 0.16 & 38.90 & 39.98 \\
\hline 105 & 3 & 3 & 2 & 30 & 0.16 & 41.53 & 39.98 \\
\hline 105 & 3 & 3 & 2 & 30 & 0.16 & 37.14 & 39.98 \\
\hline 106 & 4 & 3 & 2 & 0 & 0.20 & 12.80 & 10.14 \\
\hline 106 & 4 & 3 & 2 & 0 & 0.20 & 16.22 & 10.14 \\
\hline 106 & 4 & 3 & 2 & 0 & 0.20 & 8.47 & 10.14 \\
\hline 106 & 4 & 3 & 2 & 0 & 0.20 & 11.04 & 10.14 \\
\hline 107 & 4 & 3 & 2 & 5 & 0.21 & 16.64 & 15.41 \\
\hline 107 & 4 & 3 & 2 & 5 & 0.21 & 20.47 & 15.41 \\
\hline 107 & 4 & 3 & 2 & 5 & 0.21 & 12.97 & 15.41 \\
\hline 107 & 4 & 3 & 2 & 5 & 0.21 & 16.12 & 15.41 \\
\hline 108 & 4 & 3 & 2 & 10 & 0.22 & 21.24 & 20.65 \\
\hline 108 & 4 & 3 & 2 & 10 & 0.22 & 25.30 & 20.65 \\
\hline 108 & 4 & 3 & 2 & 10 & 0.22 & 18.53 & 20.65 \\
\hline 108 & 4 & 3 & 2 & 10 & 0.22 & 20.52 & 20.65 \\
\hline
\end{tabular}




\begin{tabular}{|c|c|c|c|c|c|c|c|}
\hline Inst. & Products & Servers & Subtasks & $\begin{array}{l}\text { Failure } \\
\text { Rate }\end{array}$ & $\begin{array}{l}\text { Net Traffic } \\
\text { Intensity }\end{array}$ & $\begin{array}{c}\text { Error } \\
\text { sim-anal }(\%)\end{array}$ & $\begin{array}{l}\text { Predicted } \\
\text { Error }(\%)\end{array}$ \\
\hline 109 & 4 & 3 & 2 & 20 & 0.25 & 30.85 & 31.11 \\
\hline 109 & 4 & 3 & 2 & 20 & 0.25 & 34.45 & 31.11 \\
\hline 109 & 4 & 3 & 2 & 20 & 0.25 & 26.66 & 31.11 \\
\hline 109 & 4 & 3 & 2 & 20 & 0.25 & 29.18 & 31.11 \\
\hline 110 & 4 & 3 & 2 & 30 & 0.28 & 39.85 & 41.47 \\
\hline 110 & 4 & 3 & 2 & 30 & 0.28 & 43.37 & 41.47 \\
\hline 110 & 4 & 3 & 2 & 30 & 0.28 & 36.78 & 41.47 \\
\hline 110 & 4 & 3 & 2 & 30 & 0.28 & 38.62 & 41.47 \\
\hline 111 & 5 & 3 & 2 & 0 & 0.28 & 11.89 & 12.11 \\
\hline 111 & 5 & 3 & 2 & 0 & 0.28 & 16.81 & 12.11 \\
\hline 111 & 5 & 3 & 2 & 0 & 0.28 & 6.25 & 12.11 \\
\hline 111 & 5 & 3 & 2 & 0 & 0.28 & 9.41 & 12.11 \\
\hline 111 & 5 & 3 & 2 & 0 & 0.28 & 17.75 & 12.11 \\
\hline 112 & 5 & 3 & 2 & 5 & 0.29 & 16.54 & 17.33 \\
\hline 112 & 5 & 3 & 2 & 5 & 0.29 & 21.72 & 17.33 \\
\hline 112 & 5 & 3 & 2 & 5 & 0.29 & 10.83 & 17.33 \\
\hline 112 & 5 & 3 & 2 & 5 & 0.29 & 14.44 & 17.33 \\
\hline 112 & 5 & 3 & 2 & 5 & 0.29 & 22.58 & 17.33 \\
\hline 113 & 5 & 3 & 2 & 10 & 0.31 & 21.32 & 22.52 \\
\hline 113 & 5 & 3 & 2 & 10 & 0.31 & 26.00 & 22.52 \\
\hline 113 & 5 & 3 & 2 & 10 & 0.31 & 15.87 & 22.52 \\
\hline 113 & 5 & 3 & 2 & 10 & 0.31 & 19.39 & 22.52 \\
\hline 113 & 5 & 3 & 2 & 10 & 0.31 & 27.04 & 22.52 \\
\hline 114 & 5 & 3 & 2 & 20 & 0.35 & 30.39 & 32.85 \\
\hline 114 & 5 & 3 & 2 & 20 & 0.35 & 35.29 & 32.85 \\
\hline 114 & 5 & 3 & 2 & 20 & 0.35 & 24.78 & 32.85 \\
\hline 114 & 5 & 3 & 2 & 20 & 0.35 & 28.42 & 32.85 \\
\hline 114 & 5 & 3 & 2 & 20 & 0.35 & 36.13 & 32.85 \\
\hline 115 & 5 & 3 & 2 & 30 & 0.40 & 39.85 & 43.06 \\
\hline 115 & 5 & 3 & 2 & 30 & 0.40 & 45.13 & 43.06 \\
\hline 115 & 5 & 3 & 2 & 30 & 0.40 & 34.42 & 43.06 \\
\hline 115 & 5 & 3 & 2 & 30 & 0.40 & 38.00 & 43.06 \\
\hline 115 & 5 & 3 & 2 & 30 & 0.40 & 44.98 & 43.06 \\
\hline 116 & 6 & 3 & 2 & 0 & 0.33 & 12.74 & 14.38 \\
\hline 116 & 6 & 3 & 2 & 0 & 0.33 & 18.37 & 14.38 \\
\hline 116 & 6 & 3 & 2 & 0 & 0.33 & 5.58 & 14.38 \\
\hline 116 & 6 & 3 & 2 & 0 & 0.33 & 9.69 & 14.38 \\
\hline 116 & 6 & 3 & 2 & 0 & 0.33 & 19.34 & 14.38 \\
\hline 116 & 6 & 3 & 2 & 0 & 0.33 & 12.39 & 14.38 \\
\hline 117 & 6 & 3 & 2 & 5 & 0.35 & 17.26 & 19.57 \\
\hline
\end{tabular}




\begin{tabular}{|c|c|c|c|c|c|c|c|}
\hline Inst. & Products & Servers & Subtasks & $\begin{array}{l}\text { Failure } \\
\text { Rate }\end{array}$ & $\begin{array}{l}\text { Net Traffic } \\
\text { Intensity }\end{array}$ & $\begin{array}{c}\text { Error } \\
\text { sim-anal }(\%)\end{array}$ & $\begin{array}{l}\text { Predicted } \\
\text { Error }(\%)\end{array}$ \\
\hline 117 & 6 & 3 & 2 & 5 & 0.35 & 22.31 & 19.57 \\
\hline 117 & 6 & 3 & 2 & 5 & 0.35 & 10.16 & 19.57 \\
\hline 117 & 6 & 3 & 2 & 5 & 0.35 & 14.55 & 19.57 \\
\hline 117 & 6 & 3 & 2 & 5 & 0.35 & 23.87 & 19.57 \\
\hline 117 & 6 & 3 & 2 & 5 & 0.35 & 17.41 & 19.57 \\
\hline 118 & 6 & 3 & 2 & 10 & 0.37 & 21.94 & 24.73 \\
\hline 118 & 6 & 3 & 2 & 10 & 0.37 & 27.93 & 24.73 \\
\hline 118 & 6 & 3 & 2 & 10 & 0.37 & 15.56 & 24.73 \\
\hline 118 & 6 & 3 & 2 & 10 & 0.37 & 19.39 & 24.73 \\
\hline 118 & 6 & 3 & 2 & 10 & 0.37 & 29.04 & 24.73 \\
\hline 118 & 6 & 3 & 2 & 10 & 0.37 & 21.96 & 24.73 \\
\hline 119 & 6 & 3 & 2 & 20 & 0.42 & 30.50 & 34.97 \\
\hline 119 & 6 & 3 & 2 & 20 & 0.42 & 36.58 & 34.97 \\
\hline 119 & 6 & 3 & 2 & 20 & 0.42 & 24.21 & 34.97 \\
\hline 119 & 6 & 3 & 2 & 20 & 0.42 & 28.30 & 34.97 \\
\hline 119 & 6 & 3 & 2 & 20 & 0.42 & 37.70 & 34.97 \\
\hline 119 & 6 & 3 & 2 & 20 & 0.42 & 31.25 & 34.97 \\
\hline 120 & 6 & 3 & 2 & 30 & 0.48 & 40.99 & 45.08 \\
\hline 120 & 6 & 3 & 2 & 30 & 0.48 & 45.84 & 45.08 \\
\hline 120 & 6 & 3 & 2 & 30 & 0.48 & 34.78 & 45.08 \\
\hline 120 & 6 & 3 & 2 & 30 & 0.48 & 38.45 & 45.08 \\
\hline 120 & 6 & 3 & 2 & 30 & 0.48 & 47.04 & 45.08 \\
\hline 120 & 6 & 3 & 2 & 30 & 0.48 & 41.09 & 45.08 \\
\hline 121 & 1 & 4 & 2 & 0 & 0.02 & 8.93 & 7.94 \\
\hline 122 & 1 & 4 & 2 & 5 & 0.02 & 12.55 & 13.31 \\
\hline 123 & 1 & 4 & 2 & 10 & 0.02 & 17.17 & 18.67 \\
\hline 124 & 1 & 4 & 2 & 20 & 0.03 & 26.64 & 29.40 \\
\hline 125 & 1 & 4 & 2 & 30 & 0.03 & 36.16 & 40.11 \\
\hline 126 & 2 & 4 & 2 & 0 & 0.04 & 8.52 & 10.62 \\
\hline 126 & 2 & 4 & 2 & 0 & 0.04 & 8.34 & 10.62 \\
\hline 127 & 2 & 4 & 2 & 5 & 0.04 & 12.44 & 15.97 \\
\hline 127 & 2 & 4 & 2 & 5 & 0.04 & 13.38 & 15.97 \\
\hline 128 & 2 & 4 & 2 & 10 & 0.04 & 17.16 & 21.33 \\
\hline 128 & 2 & 4 & 2 & 10 & 0.04 & 17.63 & 21.33 \\
\hline 129 & 2 & 4 & 2 & 20 & 0.05 & 25.83 & 32.03 \\
\hline 129 & 2 & 4 & 2 & 20 & 0.05 & 26.74 & 32.03 \\
\hline 130 & 2 & 4 & 2 & 30 & 0.05 & 35.07 & 42.71 \\
\hline 130 & 2 & 4 & 2 & 30 & 0.05 & 36.08 & 42.71 \\
\hline 131 & 3 & 4 & 2 & 0 & 0.08 & 10.07 & 12.95 \\
\hline 131 & 3 & 4 & 2 & 0 & 0.08 & 10.94 & 12.95 \\
\hline
\end{tabular}




\begin{tabular}{|c|c|c|c|c|c|c|c|}
\hline Inst. & Products & Servers & Subtasks & $\begin{array}{l}\text { Failure } \\
\text { Rate }\end{array}$ & $\begin{array}{l}\text { Net Traffic } \\
\text { Intensity }\end{array}$ & $\begin{array}{c}\text { Error } \\
\text { sim-anal }(\%)\end{array}$ & $\begin{array}{l}\text { Predicted } \\
\text { Error }(\%)\end{array}$ \\
\hline 131 & 3 & 4 & 2 & 0 & 0.08 & 8.09 & 12.95 \\
\hline 132 & 3 & 4 & 2 & 5 & 0.09 & 14.23 & 18.28 \\
\hline 132 & 3 & 4 & 2 & 5 & 0.09 & 15.12 & 18.28 \\
\hline 132 & 3 & 4 & 2 & 5 & 0.09 & 12.26 & 18.28 \\
\hline 133 & 3 & 4 & 2 & 10 & 0.09 & 18.45 & 23.60 \\
\hline 133 & 3 & 4 & 2 & 10 & 0.09 & 20.46 & 23.60 \\
\hline 133 & 3 & 4 & 2 & 10 & 0.09 & 16.85 & 23.60 \\
\hline 134 & 3 & 4 & 2 & 20 & 0.11 & 27.45 & 34.22 \\
\hline 134 & 3 & 4 & 2 & 20 & 0.11 & 29.71 & 34.22 \\
\hline 134 & 3 & 4 & 2 & 20 & 0.11 & 25.81 & 34.22 \\
\hline 135 & 3 & 4 & 2 & 30 & 0.12 & 37.39 & 44.81 \\
\hline 135 & 3 & 4 & 2 & 30 & 0.12 & 38.65 & 44.81 \\
\hline 135 & 3 & 4 & 2 & 30 & 0.12 & 35.55 & 44.81 \\
\hline 136 & 4 & 4 & 2 & 0 & 0.15 & 10.54 & 15.09 \\
\hline 136 & 4 & 4 & 2 & 0 & 0.15 & 13.07 & 15.09 \\
\hline 136 & 4 & 4 & 2 & 0 & 0.15 & 6.93 & 15.09 \\
\hline 136 & 4 & 4 & 2 & 0 & 0.15 & 8.50 & 15.09 \\
\hline 137 & 4 & 4 & 2 & 5 & 0.16 & 14.81 & 20.38 \\
\hline 137 & 4 & 4 & 2 & 5 & 0.16 & 18.25 & 20.38 \\
\hline 137 & 4 & 4 & 2 & 5 & 0.16 & 11.25 & 20.38 \\
\hline 137 & 4 & 4 & 2 & 5 & 0.16 & 13.31 & 20.38 \\
\hline 138 & 4 & 4 & 2 & 10 & 0.17 & 18.83 & 25.66 \\
\hline 138 & 4 & 4 & 2 & 10 & 0.17 & 21.86 & 25.66 \\
\hline 138 & 4 & 4 & 2 & 10 & 0.17 & 15.90 & 25.66 \\
\hline 138 & 4 & 4 & 2 & 10 & 0.17 & 17.87 & 25.66 \\
\hline 139 & 4 & 4 & 2 & 20 & 0.19 & 28.72 & 36.18 \\
\hline 139 & 4 & 4 & 2 & 20 & 0.19 & 31.75 & 36.18 \\
\hline 139 & 4 & 4 & 2 & 20 & 0.19 & 25.76 & 36.18 \\
\hline 139 & 4 & 4 & 2 & 20 & 0.19 & 27.50 & 36.18 \\
\hline 140 & 4 & 4 & 2 & 30 & 0.21 & 37.39 & 46.65 \\
\hline 140 & 4 & 4 & 2 & 30 & 0.21 & 40.73 & 46.65 \\
\hline 140 & 4 & 4 & 2 & 30 & 0.21 & 34.63 & 46.65 \\
\hline 140 & 4 & 4 & 2 & 30 & 0.21 & 36.48 & 46.65 \\
\hline 141 & 5 & 4 & 2 & 0 & 0.21 & 9.45 & 17.27 \\
\hline 141 & 5 & 4 & 2 & 0 & 0.21 & 13.66 & 17.27 \\
\hline 141 & 5 & 4 & 2 & 0 & 0.21 & 4.91 & 17.27 \\
\hline 141 & 5 & 4 & 2 & 0 & 0.21 & 7.40 & 17.27 \\
\hline 141 & 5 & 4 & 2 & 0 & 0.21 & 13.87 & 17.27 \\
\hline 142 & 5 & 4 & 2 & 5 & 0.22 & 13.61 & 22.53 \\
\hline 142 & 5 & 4 & 2 & 5 & 0.22 & 17.86 & 22.53 \\
\hline
\end{tabular}




\begin{tabular}{|c|c|c|c|c|c|c|c|}
\hline Inst. & Products & Servers & Subtasks & $\begin{array}{l}\text { Failure } \\
\text { Rate }\end{array}$ & $\begin{array}{l}\text { Net Traffic } \\
\text { Intensity }\end{array}$ & $\begin{array}{c}\text { Error } \\
\text { sim-anal }(\%)\end{array}$ & $\begin{array}{l}\text { Predicted } \\
\text { Error (\%) }\end{array}$ \\
\hline 142 & 5 & 4 & 2 & 5 & 0.22 & 9.43 & 22.53 \\
\hline 142 & 5 & 4 & 2 & 5 & 0.22 & 12.68 & 22.53 \\
\hline 142 & 5 & 4 & 2 & 5 & 0.22 & 18.89 & 22.53 \\
\hline 143 & 5 & 4 & 2 & 10 & 0.23 & 18.47 & 27.77 \\
\hline 143 & 5 & 4 & 2 & 10 & 0.23 & 22.55 & 27.77 \\
\hline 143 & 5 & 4 & 2 & 10 & 0.23 & 14.80 & 27.77 \\
\hline 143 & 5 & 4 & 2 & 10 & 0.23 & 16.82 & 27.77 \\
\hline 143 & 5 & 4 & 2 & 10 & 0.23 & 23.41 & 27.77 \\
\hline 144 & 5 & 4 & 2 & 20 & 0.26 & 27.89 & 38.21 \\
\hline 144 & 5 & 4 & 2 & 20 & 0.26 & 32.09 & 38.21 \\
\hline 144 & 5 & 4 & 2 & 20 & 0.26 & 23.84 & 38.21 \\
\hline 144 & 5 & 4 & 2 & 20 & 0.26 & 26.55 & 38.21 \\
\hline 144 & 5 & 4 & 2 & 20 & 0.26 & 32.81 & 38.21 \\
\hline 145 & 5 & 4 & 2 & 30 & 0.30 & 37.70 & 48.55 \\
\hline 145 & 5 & 4 & 2 & 30 & 0.30 & 42.05 & 48.55 \\
\hline 145 & 5 & 4 & 2 & 30 & 0.30 & 33.61 & 48.55 \\
\hline 145 & 5 & 4 & 2 & 30 & 0.30 & 36.39 & 48.55 \\
\hline 145 & 5 & 4 & 2 & 30 & 0.30 & 41.79 & 48.55 \\
\hline 146 & 6 & 4 & 2 & 0 & 0.25 & 10.11 & 19.69 \\
\hline 146 & 6 & 4 & 2 & 0 & 0.25 & 14.18 & 19.69 \\
\hline 146 & 6 & 4 & 2 & 0 & 0.25 & 4.88 & 19.69 \\
\hline 146 & 6 & 4 & 2 & 0 & 0.25 & 8.03 & 19.69 \\
\hline 146 & 6 & 4 & 2 & 0 & 0.25 & 15.77 & 19.69 \\
\hline 146 & 6 & 4 & 2 & 0 & 0.25 & 9.13 & 19.69 \\
\hline 147 & 6 & 4 & 2 & 5 & 0.26 & 14.13 & 24.92 \\
\hline 147 & 6 & 4 & 2 & 5 & 0.26 & 18.56 & 24.92 \\
\hline 147 & 6 & 4 & 2 & 5 & 0.26 & 9.39 & 24.92 \\
\hline 147 & 6 & 4 & 2 & 5 & 0.26 & 12.21 & 24.92 \\
\hline 147 & 6 & 4 & 2 & 5 & 0.26 & 19.97 & 24.92 \\
\hline 147 & 6 & 4 & 2 & 5 & 0.26 & 14.59 & 24.92 \\
\hline 148 & 6 & 4 & 2 & 10 & 0.28 & 19.50 & 30.14 \\
\hline 148 & 6 & 4 & 2 & 10 & 0.28 & 24.04 & 30.14 \\
\hline 148 & 6 & 4 & 2 & 10 & 0.28 & 13.86 & 30.14 \\
\hline 148 & 6 & 4 & 2 & 10 & 0.28 & 17.22 & 30.14 \\
\hline 148 & 6 & 4 & 2 & 10 & 0.28 & 24.25 & 30.14 \\
\hline 148 & 6 & 4 & 2 & 10 & 0.28 & 19.08 & 30.14 \\
\hline 149 & 6 & 4 & 2 & 20 & 0.31 & 28.56 & 40.51 \\
\hline 149 & 6 & 4 & 2 & 20 & 0.31 & 32.31 & 40.51 \\
\hline 149 & 6 & 4 & 2 & 20 & 0.31 & 24.40 & 40.51 \\
\hline 149 & 6 & 4 & 2 & 20 & 0.31 & 26.95 & 40.51 \\
\hline
\end{tabular}




Inst.
\begin{tabular}{|c|c|c|c|c|c|c|c|}
\hline 149 & Products & Servers & Subtasks & $\begin{array}{c}\text { Failure } \\
\text { Rate }\end{array}$ & $\begin{array}{c}\text { Net Traffic } \\
\text { Intensity }\end{array}$ & $\begin{array}{c}\text { Error } \\
\text { sim-anal }(\%)\end{array}$ & $\begin{array}{c}\text { Predicted } \\
\text { Error }(\%)\end{array}$ \\
\hline 149 & 6 & 4 & 2 & 20 & 0.31 & 34.07 & 40.51 \\
\hline 150 & 6 & 4 & 2 & 20 & 0.31 & 28.68 & 40.51 \\
\hline 150 & 6 & 4 & 2 & 30 & 0.36 & 42.16 & 50.78 \\
\hline 150 & 6 & 4 & 2 & 30 & 0.36 & 33.84 & 50.78 \\
\hline 150 & 6 & 4 & 2 & 30 & 0.36 & 35.92 & 50.78 \\
\hline 150 & 6 & 4 & 2 & 30 & 0.36 & 43.22 & 50.78 \\
\hline 150 & 6 & 4 & 2 & 30 & 0.36 & 38.36 & 50.78 \\
\hline
\end{tabular}




\section{APPENDIX D}

Flow time error using correction factors for three product instances

\begin{tabular}{|c|c|c|c|c|c|c|}
\hline Inst. & Prod & $\begin{array}{c}\text { Anal. } \\
\text { Flow T. }\end{array}$ & $\begin{array}{c}\text { Sim. } \\
\text { Flow T. }\end{array}$ & $\begin{array}{c}\text { Error } \\
(\%)\end{array}$ & $\begin{array}{c}\text { Adj. Anal. } \\
\text { Flow T. }\end{array}$ & $\begin{array}{c}\text { Adj. Error } \\
(\%)\end{array}$ \\
\hline 39 & 1 & 0.190 & 0.146 & 29.85 & 0.139 & 4.89 \\
\hline 39 & 2 & 0.168 & 0.124 & 35.67 & 0.118 & 5.08 \\
\hline 39 & 3 & 0.168 & 0.124 & 35.50 & 0.118 & 5.21 \\
\hline 40 & 1 & 0.118 & 0.105 & 12.32 & 0.097 & 7.18 \\
\hline 40 & 2 & 0.115 & 0.103 & 10.82 & 0.094 & 8.92 \\
\hline 40 & 3 & 0.114 & 0.104 & 9.57 & 0.094 & 9.16 \\
\hline 41 & 1 & 0.182 & 0.141 & 28.58 & 0.127 & 9.79 \\
\hline 41 & 2 & 0.181 & 0.152 & 19.08 & 0.132 & 12.85 \\
\hline 41 & 3 & 0.181 & 0.153 & 18.38 & 0.132 & 13.36 \\
\hline 42 & 1 & 0.417 & 0.388 & 7.53 & 0.365 & 5.92 \\
\hline 42 & 2 & 0.172 & 0.158 & 8.79 & 0.155 & 1.91 \\
\hline 42 & 3 & 0.183 & 0.164 & 11.64 & 0.166 & 1.35 \\
\hline 43 & 1 & 0.364 & 0.349 & 4.11 & 0.323 & 7.44 \\
\hline 43 & 2 & 0.098 & 0.082 & 18.56 & 0.085 & 3.16 \\
\hline 43 & 3 & 0.100 & 0.085 & 17.76 & 0.087 & 2.86 \\
\hline 44 & 1 & 0.267 & 0.252 & 5.95 & 0.236 & 6.32 \\
\hline 44 & 2 & 0.131 & 0.119 & 10.17 & 0.121 & 1.75 \\
\hline 44 & 3 & 0.130 & 0.118 & 10.12 & 0.120 & 1.66 \\
\hline 45 & 1 & 0.277 & 0.269 & 2.84 & 0.252 & 6.55 \\
\hline 45 & 2 & 0.099 & 0.090 & 10.35 & 0.093 & 3.32 \\
\hline 45 & 3 & 0.101 & 0.091 & 10.88 & 0.095 & 3.93 \\
\hline 46 & 1 & 0.381 & 0.364 & 4.49 & 0.364 & 0.08 \\
\hline 46 & 2 & 0.208 & 0.200 & 3.90 & 0.196 & 1.86 \\
\hline 46 & 3 & 0.218 & 0.210 & 3.72 & 0.207 & 1.75 \\
\hline 47 & 1 & 0.359 & 0.339 & 5.85 & 0.317 & 6.41 \\
\hline 47 & 2 & 0.170 & 0.149 & 14.44 & 0.151 & 1.44 \\
\hline 47 & 3 & 0.174 & 0.151 & 15.59 & 0.155 & 2.78 \\
\hline 48 & 1 & 1.092 & 1.039 & 5.10 & 0.869 & 16.38 \\
\hline 48 & 2 & 0.254 & 0.229 & 11.14 & 0.227 & 0.78 \\
\hline 48 & 3 & 0.267 & 0.234 & 14.38 & 0.241 & 3.03 \\
\hline 49 & 1 & 0.583 & 0.552 & 5.67 & 0.450 & 18.58 \\
\hline 49 & 2 & 0.220 & 0.176 & 25.35 & 0.174 & 0.86 \\
\hline 49 & 3 & 0.220 & 0.176 & 25.38 & 0.174 & 0.84 \\
\hline 50 & 1 & 0.299 & 0.238 & 25.44 & 0.230 & 3.59 \\
\hline 50 & 2 & 0.218 & 0.156 & 39.43 & 0.159 & 1.56 \\
\hline 50 & 3 & 0.217 & 0.155 & 39.43 & 0.157 & 1.35 \\
\hline 51 & 1 & 0.579 & 0.550 & 5.34 & 0.445 & 19.04 \\
\hline 51 & 2 & 0.140 & 0.112 & 24.79 & 0.114 & 1.45 \\
\hline & & & & & & \\
\hline
\end{tabular}


Flow time error using correction factors for three product instances

\begin{tabular}{|c|c|c|c|c|c|c|}
\hline Inst. & Prod & $\begin{array}{c}\text { Anal. } \\
\text { Flow T. }\end{array}$ & $\begin{array}{c}\text { Sim. } \\
\text { Flow T. }\end{array}$ & $\begin{array}{c}\text { Error } \\
(\%)\end{array}$ & $\begin{array}{c}\text { Adj. Anal. } \\
\text { Flow T. }\end{array}$ & $\begin{array}{c}\text { Adj. Error } \\
(\%)\end{array}$ \\
\hline 51 & 3 & 0.141 & 0.114 & 24.26 & 0.115 & 1.24 \\
\hline
\end{tabular}

Flow time error using correction factors for four product instances

Inst. Prod Anal. Sim. Error Adj. Anal. Adj. Error

$\begin{array}{lllll}\text { Flow T. } & \text { Flow T. } & (\%) & \text { Flow T. } & (\%)\end{array}$

\begin{tabular}{|c|c|c|c|c|c|c|}
\hline 52 & 1 & 0.977 & 0.711 & 37.52 & 0.604 & 15.07 \\
\hline 52 & 2 & 0.254 & 0.206 & 23.03 & 0.200 & 3.03 \\
\hline 52 & 3 & 0.265 & 0.211 & 25.51 & 0.212 & 0.13 \\
\hline 52 & 4 & 1.020 & 0.724 & 40.88 & 0.647 & 10.67 \\
\hline 53 & 1 & 0.609 & 0.481 & 26.70 & 0.457 & 4.83 \\
\hline 53 & 2 & 0.118 & 0.092 & 29.26 & 0.095 & 3.45 \\
\hline 53 & 3 & 0.120 & 0.094 & 28.02 & 0.097 & 2.93 \\
\hline 53 & 4 & 0.609 & 0.479 & 27.05 & 0.457 & 4.57 \\
\hline 54 & 1 & 0.504 & 0.371 & 35.98 & 0.342 & 7.66 \\
\hline 54 & 2 & 0.175 & 0.142 & 23.19 & 0.147 & 2.95 \\
\hline 54 & 3 & 0.175 & 0.142 & 23.24 & 0.146 & 2.91 \\
\hline 54 & 4 & 0.511 & 0.377 & 35.73 & 0.349 & 7.23 \\
\hline 55 & 1 & 0.541 & 0.420 & 28.70 & 0.377 & 10.30 \\
\hline 55 & 2 & 0.137 & 0.112 & 22.33 & 0.117 & 4.47 \\
\hline 55 & 3 & 0.139 & 0.113 & 23.05 & 0.119 & 5.31 \\
\hline 55 & 4 & 0.542 & 0.422 & 28.46 & 0.378 & 10.42 \\
\hline 56 & 1 & 0.666 & 0.512 & 30.01 & 0.510 & 0.35 \\
\hline 56 & 2 & 0.233 & 0.212 & 9.91 & 0.210 & 0.74 \\
\hline 56 & 3 & 0.243 & 0.222 & 9.52 & 0.220 & 0.63 \\
\hline 56 & 4 & 0.477 & 0.348 & 37.06 & 0.331 & 4.86 \\
\hline 57 & 1 & 0.744 & 0.574 & 29.55 & 0.492 & 14.29 \\
\hline 57 & 2 & 0.258 & 0.197 & 30.95 & 0.199 & 0.95 \\
\hline 57 & 3 & 0.262 & 0.198 & 31.96 & 0.203 & 2.22 \\
\hline 57 & 4 & 0.765 & 0.579 & 32.24 & 0.510 & 11.92 \\
\hline 58 & 1 & 1.259 & 1.166 & 7.95 & 0.945 & 18.98 \\
\hline 58 & 2 & 0.283 & 0.252 & 12.28 & 0.247 & 2.02 \\
\hline 58 & 3 & 0.299 & 0.256 & 16.97 & 0.264 & 3.36 \\
\hline 58 & 4 & 0.379 & 0.336 & 12.99 & 0.309 & 7.82 \\
\hline 59 & 1 & 0.692 & 0.616 & 12.46 & 0.496 & 19.39 \\
\hline 59 & 2 & 0.230 & 0.186 & 23.51 & 0.180 & 3.48 \\
\hline 59 & 3 & 0.230 & 0.187 & 23.24 & 0.180 & 3.69 \\
\hline 59 & 4 & 0.267 & 0.227 & 17.40 & 0.207 & 8.89 \\
\hline 60 & 1 & 0.350 & 0.269 & 29.74 & 0.253 & 5.98 \\
\hline 60 & 2 & 0.223 & 0.162 & 37.60 & 0.158 & 2.24 \\
\hline
\end{tabular}




\begin{tabular}{|c|c|c|c|c|c|c|}
\hline Inst. & Prod & $\begin{array}{c}\text { Anal. } \\
\text { Flow T. }\end{array}$ & $\begin{array}{c}\text { Sim. } \\
\text { Flow T. }\end{array}$ & $\begin{array}{c}\text { Error } \\
(\%)\end{array}$ & $\begin{array}{c}\text { Adj. Anal. } \\
\text { Flow T. }\end{array}$ & $\begin{array}{c}\text { Adj. Error } \\
(\%)\end{array}$ \\
\hline 60 & 3 & 0.222 & 0.161 & 37.66 & 0.157 & 2.40 \\
\hline 60 & 4 & 0.161 & 0.146 & 9.82 & 0.135 & 7.68 \\
\hline 61 & 1 & 0.617 & 0.571 & 7.92 & 0.453 & 20.71 \\
\hline 61 & 2 & 0.152 & 0.121 & 25.95 & 0.120 & 0.40 \\
\hline 61 & 3 & 0.153 & 0.122 & 25.54 & 0.121 & 0.56 \\
\hline 61 & 4 & 0.143 & 0.126 & 13.15 & 0.120 & 4.62 \\
\hline
\end{tabular}

Flow time error using correction factors for five product instances Inst. Prod Anal. Sim. Error Adj. Anal. Adj. Error

Flow T. Flow T. sim-anal (\%) Flow T. $\quad(\%)$

\begin{tabular}{|c|c|c|c|c|c|c|}
\hline \hline 62 & 1 & 1.049 & 0.748 & 40.28 & 0.624 & 16.55 \\
\hline 62 & 2 & 0.341 & 0.249 & 36.82 & 0.242 & 2.80 \\
\hline 62 & 3 & 0.353 & 0.253 & 39.36 & 0.254 & 0.50 \\
\hline 62 & 4 & 1.093 & 0.761 & 43.71 & 0.670 & 12.00 \\
\hline 62 & 5 & 0.349 & 0.250 & 39.62 & 0.251 & 0.33 \\
\hline 63 & 1 & 0.685 & 0.512 & 33.93 & 0.488 & 4.51 \\
\hline 63 & 2 & 0.195 & 0.123 & 59.20 & 0.127 & 3.86 \\
\hline 63 & 3 & 0.197 & 0.125 & 57.95 & 0.129 & 3.53 \\
\hline 63 & 4 & 0.685 & 0.511 & 34.01 & 0.489 & 4.45 \\
\hline 63 & 5 & 0.197 & 0.125 & 58.07 & 0.129 & 3.65 \\
\hline 64 & 1 & 0.598 & 0.412 & 44.97 & 0.381 & 7.56 \\
\hline 64 & 2 & 0.269 & 0.184 & 45.76 & 0.193 & 4.38 \\
\hline 64 & 3 & 0.268 & 0.184 & 46.11 & 0.192 & 4.51 \\
\hline 64 & 4 & 0.605 & 0.416 & 45.24 & 0.388 & 6.77 \\
\hline 64 & 5 & 0.268 & 0.184 & 45.85 & 0.192 & 4.37 \\
\hline 65 & 1 & 0.614 & 0.455 & 35.06 & 0.409 & 10.15 \\
\hline 65 & 2 & 0.212 & 0.148 & 43.73 & 0.157 & 6.43 \\
\hline 65 & 3 & 0.214 & 0.148 & 44.13 & 0.159 & 7.04 \\
\hline 65 & 4 & 0.615 & 0.457 & 34.59 & 0.409 & 10.42 \\
\hline 65 & 5 & 0.212 & 0.148 & 43.74 & 0.157 & 6.51 \\
\hline 66 & 1 & 0.842 & 0.619 & 36.06 & 0.599 & 3.21 \\
\hline 66 & 2 & 0.412 & 0.319 & 29.18 & 0.304 & 4.62 \\
\hline 66 & 3 & 0.423 & 0.330 & 28.15 & 0.315 & 4.57 \\
\hline 66 & 4 & 0.509 & 0.361 & 40.95 & 0.340 & 5.87 \\
\hline 66 & 5 & 0.418 & 0.325 & 28.45 & 0.310 & 4.69 \\
\hline 67 & 1 & 0.897 & 0.639 & 40.46 & 0.551 & 13.70 \\
\hline 67 & 2 & 0.410 & 0.265 & 54.85 & 0.266 & 0.73 \\
\hline 67 & 3 & 0.414 & 0.265 & 55.97 & 0.271 & 2.01 \\
\hline 67 & 4 & 0.925 & 0.643 & 43.73 & 0.569 & 11.58 \\
\hline 67 & 5 & 0.420 & 0.275 & 52.92 & 0.277 & 0.84 \\
\hline
\end{tabular}




\begin{tabular}{|c|c|c|c|c|c|c|}
\hline 68 & 1 & 1.723 & 1.343 & 28.23 & 1.127 & 16.12 \\
\hline 68 & 2 & 0.767 & 0.457 & 67.88 & 0.445 & 2.58 \\
\hline 68 & 3 & 0.791 & 0.459 & 72.38 & 0.471 & 2.61 \\
\hline 68 & 4 & 0.450 & 0.387 & 16.28 & 0.356 & 8.17 \\
\hline 68 & 5 & 0.767 & 0.457 & 68.00 & 0.445 & 2.51 \\
\hline 69 & 1 & 0.776 & 0.654 & 18.74 & 0.528 & 19.24 \\
\hline 69 & 2 & 0.402 & 0.254 & 57.99 & 0.251 & 1.34 \\
\hline 69 & 3 & 0.402 & 0.254 & 58.12 & 0.251 & 1.26 \\
\hline 69 & 4 & 0.279 & 0.243 & 14.82 & 0.218 & 10.32 \\
\hline 69 & 5 & 0.402 & 0.254 & 58.05 & 0.251 & 1.31 \\
\hline 70 & 1 & 0.498 & 0.316 & 57.90 & 0.306 & 2.92 \\
\hline 70 & 2 & 0.366 & 0.207 & 77.01 & 0.211 & 1.96 \\
\hline 70 & 3 & 0.365 & 0.205 & 77.58 & 0.210 & 2.05 \\
\hline 70 & 4 & 0.165 & 0.150 & 9.89 & 0.139 & 7.24 \\
\hline 70 & 5 & 0.368 & 0.208 & 76.79 & 0.213 & 2.25 \\
\hline 71 & 1 & 0.673 & 0.594 & 13.43 & 0.471 & 20.65 \\
\hline 71 & 2 & 0.208 & 0.142 & 46.54 & 0.145 & 1.59 \\
\hline 71 & 3 & 0.210 & 0.144 & 46.07 & 0.146 & 1.40 \\
\hline 71 & 4 & 0.155 & 0.134 & 15.55 & 0.128 & 4.34 \\
\hline 71 & 5 & 0.212 & 0.143 & 48.10 & 0.149 & 3.63 \\
\hline & & & & & & \\
\hline
\end{tabular}

Flow time error using correction factors for six product instances Instance Prod Anal. Sim. Error Adj. Anal. Adj. Error Flow T. Flow T. (\%) Flow T.

\begin{tabular}{|c|c|c|c|c|c|c|}
\hline \hline 72 & 1 & 1.129 & 0.771 & 46.33 & 0.652 & 15.47 \\
\hline 72 & 2 & 0.442 & 0.286 & 54.69 & 0.285 & 0.27 \\
\hline 72 & 3 & 0.454 & 0.289 & 56.99 & 0.298 & 2.88 \\
\hline 72 & 4 & 1.175 & 0.783 & 49.10 & 0.700 & 10.68 \\
\hline 72 & 5 & 0.451 & 0.286 & 57.86 & 0.294 & 3.06 \\
\hline 72 & 6 & 0.434 & 0.287 & 51.29 & 0.276 & 3.82 \\
\hline 73 & 1 & 0.891 & 0.583 & 52.71 & 0.566 & 3.02 \\
\hline 73 & 2 & 0.272 & 0.147 & 85.25 & 0.155 & 5.29 \\
\hline 73 & 3 & 0.274 & 0.149 & 83.55 & 0.156 & 4.81 \\
\hline 73 & 4 & 0.891 & 0.583 & 52.93 & 0.566 & 2.88 \\
\hline 73 & 5 & 0.274 & 0.149 & 83.45 & 0.157 & 4.86 \\
\hline 73 & 6 & 0.457 & 0.255 & 79.50 & 0.252 & 1.15 \\
\hline 74 & 1 & 0.746 & 0.477 & 56.20 & 0.446 & 6.51 \\
\hline 74 & 2 & 0.330 & 0.217 & 52.09 & 0.229 & 5.54 \\
\hline 74 & 3 & 0.330 & 0.217 & 52.06 & 0.229 & 5.41 \\
\hline 74 & 4 & 0.753 & 0.482 & 56.14 & 0.453 & 5.96 \\
\hline 74 & 5 & 0.330 & 0.217 & 52.03 & 0.229 & 5.43 \\
\hline
\end{tabular}




\begin{tabular}{|c|c|c|c|c|c|c|}
\hline 74 & 6 & 0.372 & 0.250 & 48.36 & 0.249 & 0.53 \\
\hline 75 & 1 & 0.765 & 0.515 & 48.39 & 0.471 & 8.58 \\
\hline 75 & 2 & 0.314 & 0.188 & 67.53 & 0.204 & 8.86 \\
\hline 75 & 3 & 0.316 & 0.188 & 68.39 & 0.206 & 9.75 \\
\hline 75 & 4 & 0.765 & 0.516 & 48.18 & 0.472 & 8.67 \\
\hline 75 & 5 & 0.315 & 0.188 & 67.82 & 0.205 & 9.13 \\
\hline 75 & 6 & 0.433 & 0.261 & 65.78 & 0.273 & 4.76 \\
\hline 76 & 1 & 1.116 & 0.746 & 49.57 & 0.730 & 2.23 \\
\hline 76 & 2 & 0.514 & 0.348 & 47.78 & 0.340 & 2.30 \\
\hline 76 & 3 & 0.525 & 0.359 & 46.06 & 0.351 & 2.45 \\
\hline 76 & 4 & 0.771 & 0.488 & 57.86 & 0.454 & 6.98 \\
\hline 76 & 5 & 0.520 & 0.354 & 46.89 & 0.346 & 2.33 \\
\hline 76 & 6 & 0.512 & 0.335 & 52.58 & 0.326 & 2.80 \\
\hline 77 & 1 & 1.080 & 0.707 & 52.83 & 0.623 & 11.85 \\
\hline 77 & 2 & 0.518 & 0.308 & 68.29 & 0.315 & 2.41 \\
\hline 77 & 3 & 0.522 & 0.309 & 69.23 & 0.319 & 3.51 \\
\hline 77 & 4 & 1.112 & 0.711 & 56.38 & 0.639 & 10.22 \\
\hline 77 & 5 & 0.529 & 0.318 & 66.18 & 0.326 & 2.44 \\
\hline 77 & 6 & 0.566 & 0.351 & 61.14 & 0.347 & 1.09 \\
\hline 78 & 1 & 2.021 & 1.472 & 37.27 & 1.250 & 15.11 \\
\hline 78 & 2 & 0.827 & 0.496 & 66.79 & 0.478 & 3.52 \\
\hline 78 & 3 & 0.855 & 0.497 & 71.20 & 0.510 & 2.56 \\
\hline 78 & 4 & 0.743 & 0.520 & 43.04 & 0.481 & 7.33 \\
\hline 78 & 5 & 0.827 & 0.496 & 66.60 & 0.478 & 3.63 \\
\hline 78 & 6 & 0.753 & 0.519 & 45.18 & 0.493 & 4.98 \\
\hline 79 & 1 & 1.034 & 0.757 & 36.67 & 0.628 & 16.97 \\
\hline 79 & 2 & 0.416 & 0.270 & 54.30 & 0.265 & 1.66 \\
\hline 79 & 3 & 0.416 & 0.269 & 54.43 & 0.265 & 1.58 \\
\hline 79 & 4 & 0.535 & 0.346 & 54.62 & 0.318 & 7.87 \\
\hline 79 & 5 & 0.416 & 0.269 & 54.55 & 0.265 & 1.51 \\
\hline 79 & 6 & 0.538 & 0.348 & 54.55 & 0.322 & 7.53 \\
\hline 80 & 1 & 0.652 & 0.384 & 69.59 & 0.369 & 4.09 \\
\hline 80 & 2 & 0.377 & 0.217 & 73.41 & 0.220 & 1.42 \\
\hline 80 & 3 & 0.375 & 0.216 & 74.08 & 0.219 & 1.60 \\
\hline 80 & 4 & 0.309 & 0.217 & 42.80 & 0.201 & 7.31 \\
\hline 80 & 5 & 0.379 & 0.219 & 73.13 & 0.222 & 1.65 \\
\hline 80 & 6 & 0.308 & 0.215 & 43.42 & 0.199 & 7.18 \\
\hline 81 & 1 & 0.761 & 0.629 & 21.07 & 0.508 & 19.21 \\
\hline 81 & 2 & 0.228 & 0.153 & 49.47 & 0.157 & 2.73 \\
\hline 81 & 3 & 0.229 & 0.154 & 48.35 & 0.157 & 1.99 \\
\hline 81 & 4 & 0.242 & 0.174 & 39.27 & 0.167 & 3.52 \\
\hline
\end{tabular}




\begin{tabular}{|l|l|l|l|l|l|l|}
\hline 81 & 5 & 0.232 & 0.154 & 51.20 & 0.161 & 4.96 \\
\hline 81 & 6 & 0.243 & 0.177 & 37.43 & 0.168 & 4.74 \\
\hline
\end{tabular}

Flow time error using correction factor in fork-join nodes with less number of subtasks than servers

Inst. \# Prod Prod Anal. Sim. Error Adj. Anal. Adj. Error

Flow T. Flow T. sim-anal (\%) $\quad$ Flow T. $\quad(\%)$

\begin{tabular}{|c|c|c|c|c|c|c|c|}
\hline 82 & 3 & 1 & 0.397 & 0.374 & 6.09 & 0.346 & 7.48 \\
\hline 82 & 3 & 2 & 0.149 & 0.140 & 6.94 & 0.134 & 3.94 \\
\hline 82 & 3 & 3 & 0.159 & 0.146 & 8.74 & 0.144 & 1.57 \\
\hline 83 & 3 & 1 & 0.331 & 0.327 & 1.27 & 0.317 & 3.04 \\
\hline 83 & 3 & 2 & 0.176 & 0.172 & 2.05 & 0.165 & 4.57 \\
\hline 83 & 3 & 3 & 0.186 & 0.183 & 1.68 & 0.175 & 4.49 \\
\hline 84 & 4 & 1 & 0.483 & 0.355 & 35.81 & 0.321 & 9.57 \\
\hline 84 & 4 & 2 & 0.154 & 0.123 & 24.97 & 0.124 & 1.08 \\
\hline 84 & 4 & 3 & 0.153 & 0.123 & 24.63 & 0.124 & 0.69 \\
\hline 84 & 4 & 4 & 0.490 & 0.359 & 36.29 & 0.328 & 8.59 \\
\hline 85 & 4 & 1 & 1.215 & 1.121 & 8.39 & 0.903 & 19.44 \\
\hline 85 & 4 & 2 & 0.248 & 0.215 & 15.41 & 0.213 & 0.66 \\
\hline 85 & 4 & 3 & 0.256 & 0.220 & 16.10 & 0.223 & 1.14 \\
\hline 85 & 4 & 4 & 0.344 & 0.298 & 15.58 & 0.276 & 7.35 \\
\hline 86 & 5 & 1 & 0.566 & 0.384 & 47.15 & 0.349 & 9.25 \\
\hline 86 & 5 & 2 & 0.237 & 0.153 & 54.52 & 0.158 & 3.37 \\
\hline 86 & 5 & 3 & 0.236 & 0.153 & 54.57 & 0.158 & 3.24 \\
\hline 86 & 5 & 4 & 0.573 & 0.390 & 47.02 & 0.356 & 8.63 \\
\hline 86 & 5 & 5 & 0.236 & 0.153 & 54.65 & 0.158 & 3.36 \\
\hline 87 & 5 & 1 & 1.235 & 1.155 & 6.95 & 0.923 & 20.10 \\
\hline 87 & 5 & 2 & 0.264 & 0.240 & 10.02 & 0.229 & 4.46 \\
\hline 87 & 5 & 3 & 0.275 & 0.246 & 11.95 & 0.242 & 1.54 \\
\hline 87 & 5 & 4 & 0.360 & 0.323 & 11.31 & 0.291 & 9.87 \\
\hline 88 & 5 & 1 & 0.484 & 0.298 & 62.16 & 0.291 & 2.67 \\
\hline 88 & 5 & 2 & 0.346 & 0.190 & 81.78 & 0.191 & 0.43 \\
\hline 88 & 5 & 3 & 0.345 & 0.189 & 82.35 & 0.190 & 0.51 \\
\hline 88 & 5 & 4 & 0.142 & 0.132 & 6.99 & 0.121 & 8.72 \\
\hline 88 & 5 & 5 & 0.348 & 0.192 & 80.91 & 0.193 & 0.46 \\
\hline 89 & 6 & 1 & 0.700 & 0.431 & 62.27 & 0.398 & 7.64 \\
\hline 89 & 6 & 2 & 0.284 & 0.170 & 67.25 & 0.178 & 4.87 \\
\hline 89 & 6 & 3 & 0.284 & 0.170 & 67.08 & 0.177 & 4.60 \\
\hline 89 & 6 & 4 & 0.707 & 0.436 & 62.14 & 0.405 & 7.02 \\
\hline 89 & 6 & 5 & 0.284 & 0.170 & 67.38 & 0.178 & 4.85 \\
\hline 89 & 6 & 6 & 0.325 & 0.205 & 58.65 & 0.202 & 1.53 \\
\hline
\end{tabular}




\begin{tabular}{|c|c|c|c|c|c|c|c|}
\hline Inst. & \# Prod & Prod & $\begin{array}{l}\text { Anal. } \\
\text { Flow T. }\end{array}$ & $\begin{array}{c}\text { Sim. } \\
\text { Flow T. }\end{array}$ & $\begin{array}{c}\text { Error } \\
\text { sim-anal (\%) }\end{array}$ & $\begin{array}{c}\text { Adj. Anal. } \\
\text { Flow T. }\end{array}$ & $\begin{array}{c}\text { Adj. Error } \\
(\%)\end{array}$ \\
\hline 90 & 6 & 1 & 1.957 & 1.418 & 38.02 & 1.184 & 16.46 \\
\hline 90 & 6 & 2 & 0.775 & 0.444 & 74.62 & 0.426 & 4.08 \\
\hline 90 & 6 & 3 & 0.791 & 0.449 & 76.24 & 0.445 & 0.95 \\
\hline 90 & 6 & 4 & 0.692 & 0.467 & 48.16 & 0.429 & 8.09 \\
\hline 90 & 6 & 5 & 0.775 & 0.444 & 74.61 & 0.426 & 4.09 \\
\hline 90 & 6 & 6 & 0.696 & 0.469 & 48.63 & 0.435 & 7.16 \\
\hline
\end{tabular}




\section{APPENDIX E}

Approximate and simulated flow times for single product class with general distributions

\begin{tabular}{|c|c|c|c|}
\hline \multicolumn{1}{l|}{ Inst. } & \multicolumn{1}{c}{ Appr. } & Sim. & $\begin{array}{c}\text { Error } \\
\text { sim-anal (\%) }\end{array}$ \\
\hline 1 & 30.39 & 28.95 & 4.98 \\
\hline 7 & 30.22 & 29.81 & 1.35 \\
\hline 13 & 34.24 & 33.04 & 3.62 \\
\hline 19 & 22.44 & 21.99 & 2.04 \\
\hline 25 & 34.06 & 32.67 & 4.25 \\
\hline 31 & 32.64 & 32.54 & 0.33 \\
\hline 37 & 36.78 & 35.94 & 2.33 \\
\hline 43 & 24.09 & 23.99 & 0.42 \\
\hline 49 & 17.11 & 15.90 & 7.59 \\
\hline 55 & 19.02 & 20.15 & 5.61 \\
\hline 61 & 20.56 & 21.49 & 4.31 \\
\hline 67 & 12.70 & 13.27 & 4.28 \\
\hline 73 & 17.59 & 15.51 & 13.38 \\
\hline 79 & 16.30 & 16.03 & 1.66 \\
\hline 85 & 17.86 & 17.55 & 1.77 \\
\hline 91 & 11.64 & 11.60 & 0.32 \\
\hline 97 & 15.40 & 15.63 & 1.48 \\
\hline 103 & 18.90 & 18.71 & 1.00 \\
\hline 109 & 19.40 & 19.35 & 0.29 \\
\hline 115 & 12.39 & 13.71 & 9.68 \\
\hline & & & \\
\hline
\end{tabular}

Approximate and simulated flow times for two product classes with general distributions

\begin{tabular}{c|c|c|c|c|c|c|}
\hline Inst. & \multicolumn{2}{|c|}{ Appr. } & \multicolumn{2}{c|}{ Sim. } & \multicolumn{2}{c|}{ Error sim-anal (\%) } \\
& Prod_1 & Prod_2 & \multicolumn{2}{c|}{ Prod_1 } & Prod_2 & \multicolumn{2}{c|}{ Prod_1 } & \multicolumn{1}{c|}{ Prod_2 } \\
\hline 2 & 31.42 & 10.72 & 29.98 & 10.77 & 4.81 & 0.47 \\
\hline 8 & 31.63 & 12.41 & 30.90 & 12.26 & 2.36 & 1.16 \\
\hline 14 & 36.09 & 15.88 & 34.78 & 15.83 & 3.76 & 0.30 \\
\hline 20 & 25.63 & 15.48 & 23.96 & 14.00 & 6.98 & 10.52 \\
\hline 26 & 35.90 & 12.51 & 34.29 & 12.40 & 4.71 & 0.91 \\
\hline 32 & 34.90 & 14.07 & 34.26 & 13.81 & 1.86 & 1.84 \\
\hline 38 & 40.03 & 18.43 & 38.79 & 18.20 & 3.20 & 1.25 \\
\hline 44 & 29.20 & 18.09 & 27.11 & 15.98 & 7.70 & 13.15 \\
\hline 50 & 18.16 & 6.32 & 16.92 & 6.50 & 7.28 & 2.79 \\
\hline
\end{tabular}




\begin{tabular}{|c|c|c|c|c|c|c|}
\hline Inst. & \multicolumn{2}{|c|}{ Appr. } & \multicolumn{2}{c|}{ Sim. } & \multicolumn{2}{c|}{ Error sim-anal (\%) } \\
\multicolumn{1}{l|}{ Prod_1 } & Prod_2 & \multicolumn{1}{c|}{ Prod_1 } & Prod_2 & \multicolumn{2}{c|}{ Prod_1 } & Prod_2 \\
\hline 56 & 20.48 & 8.18 & 21.87 & 8.53 & 6.35 & 4.03 \\
\hline 62 & 23.24 & 11.27 & 24.25 & 11.65 & 4.20 & 3.25 \\
\hline 68 & 15.51 & 9.78 & 15.68 & 9.39 & 1.11 & 4.14 \\
\hline 74 & 18.50 & 6.17 & 16.11 & 5.73 & 14.84 & 7.65 \\
\hline 80 & 17.08 & 6.77 & 16.60 & 6.48 & 2.87 & 4.49 \\
\hline 86 & 19.16 & 8.75 & 18.58 & 8.54 & 3.09 & 2.39 \\
\hline 92 & 13.23 & 8.03 & 12.81 & 7.71 & 3.27 & 4.17 \\
\hline 98 & 16.39 & 6.14 & 16.48 & 6.20 & 0.55 & 0.85 \\
\hline 104 & 20.10 & 8.00 & 19.67 & 7.40 & 2.18 & 8.09 \\
\hline 110 & 21.50 & 10.24 & 21.04 & 9.82 & 2.19 & 4.23 \\
\hline 116 & 14.69 & 9.18 & 15.81 & 9.57 & 7.11 & 3.99 \\
\hline
\end{tabular}

Approximate and simulated flow times for three product classes with general distributions

\begin{tabular}{|c|c|c|c|c|c|c|c|c|c|}
\hline \multirow[t]{2}{*}{ Inst. } & \multicolumn{3}{|c|}{ Appr. } & \multicolumn{3}{|c|}{ Sim. } & \multicolumn{3}{|c|}{ Error sim-anal (\%) } \\
\hline & Prod_1 & Prod_2 & Prod_3 & Prod_1 & Prod_2 & Prod_3 & Prod_1 & Prod_2 & Prod_3 \\
\hline 3 & 33.08 & 13.04 & 12.88 & 31.35 & 12.13 & 11.98 & 5.53 & 7.50 & 7.52 \\
\hline 9 & 33.44 & 14.80 & 13.64 & 31.98 & 13.31 & 12.59 & 4.57 & 11.21 & 8.28 \\
\hline 15 & 40.55 & 21.94 & 22.25 & 37.62 & 18.77 & 18.81 & 7.81 & 16.88 & 18.25 \\
\hline 21 & 30.45 & 20.37 & 18.96 & 26.41 & 16.39 & 15.30 & 15.30 & 24.34 & 23.90 \\
\hline 27 & 38.98 & 16.22 & 16.06 & 36.59 & 14.69 & 14.51 & 6.52 & 10.45 & 10.70 \\
\hline 33 & 37.92 & 17.65 & 16.49 & 36.17 & 15.69 & 15.03 & 4.83 & 12.50 & 9.72 \\
\hline 39 & 47.57 & 27.52 & 27.82 & 43.43 & 23.06 & 23.05 & 9.53 & 19.34 & 20.68 \\
\hline 45 & 37.07 & 25.64 & 24.22 & 31.18 & 19.81 & 18.79 & 18.89 & 29.44 & 28.93 \\
\hline 51 & 19.86 & 8.00 & 7.92 & 18.40 & 7.93 & 7.86 & 7.96 & 0.84 & 0.80 \\
\hline 57 & 22.57 & 10.24 & 9.65 & 23.66 & 10.37 & 9.99 & 4.60 & 1.32 & 3.41 \\
\hline 63 & 29.66 & 18.08 & 18.23 & 28.89 & 16.36 & 16.34 & 2.64 & 10.49 & 11.56 \\
\hline 69 & 20.61 & 14.63 & 13.93 & 18.70 & 12.25 & 11.64 & 10.20 & 19.47 & 19.67 \\
\hline 75 & 19.75 & 7.21 & 7.13 & 16.92 & 6.54 & 6.46 & 16.75 & 10.28 & 10.45 \\
\hline 81 & 18.08 & 7.77 & 7.18 & 17.28 & 7.11 & 6.72 & 4.67 & 9.29 & 6.96 \\
\hline 87 & 22.08 & 11.82 & 11.97 & 20.32 & 10.34 & 10.33 & 8.68 & 14.33 & 15.83 \\
\hline 93 & 15.98 & 10.80 & 10.09 & 14.42 & 9.28 & 8.65 & 10.82 & 16.31 & 16.69 \\
\hline 99 & 17.96 & 7.56 & 7.48 & 17.65 & 7.35 & 7.26 & 1.78 & 2.94 & 3.14 \\
\hline 105 & 21.69 & 9.30 & 8.71 & 20.78 & 8.50 & 8.09 & 4.42 & 9.36 & 7.70 \\
\hline 111 & 26.25 & 14.88 & 15.03 & 23.88 & 12.79 & 12.80 & 9.89 & 16.30 & 17.42 \\
\hline 117 & 19.00 & 13.40 & 12.70 & 18.52 & 12.22 & 11.50 & 2.62 & 9.65 & 10.43 \\
\hline
\end{tabular}


Approximate and simulated flow times for four product classes with general distributions

\begin{tabular}{|c|c|c|c|c|}
\hline \multicolumn{1}{l}{ Inst. } & Prod. & Appr. & Sim. & $\begin{array}{c}\text { Error } \\
\text { sim-anal }(\%)\end{array}$ \\
\hline 4 & 1 & 44.35 & 37.65 & 17.79 \\
\hline 4 & 2 & 13.99 & 12.60 & 11.01 \\
\hline 4 & 3 & 13.83 & 12.46 & 11.06 \\
\hline 4 & 4 & 33.95 & 27.69 & 22.60 \\
\hline 10 & 1 & 47.73 & 39.19 & 21.79 \\
\hline 10 & 2 & 17.13 & 14.67 & 16.75 \\
\hline 10 & 3 & 15.96 & 14.07 & 13.43 \\
\hline 10 & 4 & 46.29 & 38.81 & 19.27 \\
\hline 16 & 1 & 46.75 & 40.57 & 15.23 \\
\hline 16 & 2 & 23.10 & 19.82 & 16.59 \\
\hline 16 & 3 & 23.41 & 19.84 & 18.01 \\
\hline 16 & 4 & 21.07 & 19.28 & 9.29 \\
\hline 22 & 1 & 35.37 & 30.25 & 16.90 \\
\hline 22 & 2 & 20.91 & 17.11 & 22.26 \\
\hline 22 & 3 & 19.50 & 16.00 & 21.88 \\
\hline 22 & 4 & 19.23 & 18.45 & 4.22 \\
\hline 28 & 1 & 57.26 & 47.20 & 21.31 \\
\hline 28 & 2 & 17.60 & 15.54 & 13.26 \\
\hline 28 & 3 & 17.44 & 15.42 & 13.12 \\
\hline 28 & 4 & 43.19 & 34.89 & 23.77 \\
\hline 34 & 1 & 59.76 & 48.72 & 22.65 \\
\hline 34 & 2 & 21.88 & 18.31 & 19.49 \\
\hline 34 & 3 & 20.71 & 17.77 & 16.57 \\
\hline 34 & 4 & 58.32 & 48.45 & 20.37 \\
\hline 40 & 1 & 57.01 & 48.04 & 18.67 \\
\hline 40 & 2 & 30.03 & 24.77 & 21.26 \\
\hline 40 & 3 & 30.33 & 24.78 & 22.41 \\
\hline 40 & 4 & 26.54 & 23.25 & 14.18 \\
\hline 46 & 1 & 46.14 & 37.20 & 24.03 \\
\hline 46 & 2 & 27.02 & 21.03 & 28.49 \\
\hline 46 & 3 & 25.61 & 20.00 & 28.04 \\
\hline 46 & 4 & 24.30 & 22.22 & 9.36 \\
\hline 52 & 1 & 31.89 & 25.86 & 23.29 \\
\hline 52 & 2 & 9.12 & 8.60 & 6.05 \\
\hline 52 & 3 & 9.04 & 8.52 & 6.07 \\
\hline 52 & 4 & 25.63 & 19.84 & 29.15 \\
\hline 58 & 1 & 40.31 & 36.32 & 10.96 \\
\hline & & 13.48 & 12.92 & 4.36 \\
\hline
\end{tabular}




\begin{tabular}{|c|c|c|c|c|}
\hline Inst. & Prod. & Appr. & Sim. & $\begin{array}{c}\text { Error } \\
\text { sim-anal }(\%)\end{array}$ \\
\hline 58 & 3 & 12.90 & 12.59 & 2.44 \\
\hline 58 & 4 & 39.58 & 36.05 & 9.79 \\
\hline 64 & 1 & 35.11 & 33.24 & 5.61 \\
\hline 64 & 2 & 19.63 & 17.83 & 10.10 \\
\hline 64 & 3 & 19.78 & 17.82 & 10.98 \\
\hline 64 & 4 & 16.15 & 15.85 & 1.89 \\
\hline 70 & 1 & 26.24 & 23.08 & 13.70 \\
\hline 70 & 2 & 14.89 & 12.81 & 16.20 \\
\hline 70 & 3 & 14.18 & 12.20 & 16.24 \\
\hline 71 & 4 & 13.80 & 13.36 & 3.27 \\
\hline 76 & 1 & 26.27 & 20.96 & 25.36 \\
\hline 76 & 2 & 7.97 & 6.82 & 16.94 \\
\hline 76 & 3 & 7.89 & 6.73 & 17.25 \\
\hline 76 & 4 & 20.12 & 15.68 & 28.33 \\
\hline 82 & 1 & 25.61 & 21.89 & 17.01 \\
\hline 82 & 2 & 9.17 & 7.88 & 16.36 \\
\hline 82 & 3 & 8.59 & 7.55 & 13.74 \\
\hline 82 & 4 & 24.89 & 21.57 & 15.42 \\
\hline 88 & 1 & 24.27 & 22.06 & 10.03 \\
\hline 88 & 2 & 12.51 & 10.92 & 14.58 \\
\hline 88 & 3 & 12.66 & 10.95 & 15.64 \\
\hline 88 & 4 & 11.22 & 10.58 & 6.04 \\
\hline 94 & 1 & 18.72 & 16.84 & 11.14 \\
\hline 94 & 2 & 11.17 & 9.68 & 15.32 \\
\hline 94 & 3 & 10.46 & 9.06 & 15.51 \\
\hline 94 & 4 & 11.24 & 10.37 & 8.35 \\
\hline 100 & 1 & 27.75 & 24.22 & 14.56 \\
\hline 100 & 2 & 8.61 & 7.84 & 9.88 \\
\hline 100 & 3 & 8.53 & 7.77 & 9.84 \\
\hline 100 & 4 & 22.55 & 18.89 & 19.34 \\
\hline 106 & 1 & 34.19 & 29.56 & 15.65 \\
\hline 106 & 2 & 11.78 & 9.94 & 18.55 \\
\hline 106 & 3 & 11.20 & 9.56 & 17.13 \\
\hline 106 & 4 & 33.46 & 29.18 & 14.68 \\
\hline 112 & 1 & 29.13 & 26.59 & 9.55 \\
\hline 112 & 2 & 16.00 & 13.59 & 17.76 \\
\hline 112 & 3 & 16.15 & 13.57 & 19.05 \\
\hline 112 & 4 & 13.67 & 12.53 & 9.08 \\
\hline 118 & 1 & 23.57 & 22.59 & 4.35 \\
\hline 118 & 2 & 13.86 & 12.94 & 7.11 \\
\hline
\end{tabular}




\begin{tabular}{l|c|c|c|c|}
\hline Inst. & Prod. & Appr. & Sim. & $\begin{array}{c}\text { Error } \\
\text { sim-anal (\%) }\end{array}$ \\
\hline 118 & 3 & 13.15 & 12.23 & 7.52 \\
\hline 118 & 4 & 13.86 & 13.67 & 1.38 \\
\hline
\end{tabular}

Approximate and simulated flow times for five product classes with general distributions

\begin{tabular}{l|l|l|l|c|}
\hline Inst. & Prod. & Appr. & Sim. & $\begin{array}{c}\text { Error } \\
\text { sim-anal (\%) }\end{array}$ \\
\hline 5 & 1 & 48.30 & 39.95 & 20.90 \\
\hline 5 & 2 & 18.46 & 14.90 & 23.91 \\
\hline 5 & 3 & 18.31 & 14.76 & 24.00 \\
\hline 5 & 4 & 36.00 & 28.55 & 26.12 \\
\hline 5 & 5 & 18.17 & 14.65 & 23.99 \\
\hline 11 & 1 & 53.32 & 41.97 & 27.04 \\
\hline 11 & 2 & 22.63 & 17.36 & 30.31 \\
\hline 11 & 3 & 21.46 & 16.90 & 26.98 \\
\hline 11 & 4 & 51.87 & 41.69 & 24.42 \\
\hline 11 & 5 & 22.45 & 17.10 & 31.28 \\
\hline 17 & 1 & 61.57 & 46.59 & 32.15 \\
\hline 17 & 2 & 37.58 & 26.32 & 42.81 \\
\hline 17 & 3 & 37.88 & 26.32 & 43.95 \\
\hline 17 & 4 & 23.10 & 20.50 & 12.66 \\
\hline 17 & 5 & 37.58 & 26.33 & 42.75 \\
\hline 23 & 1 & 47.17 & 35.36 & 33.39 \\
\hline 23 & 2 & 33.30 & 22.01 & 51.29 \\
\hline 23 & 3 & 31.89 & 20.99 & 51.93 \\
\hline 23 & 4 & 20.18 & 19.37 & 4.19 \\
\hline 23 & 5 & 31.86 & 21.06 & 51.29 \\
\hline 29 & 1 & 64.95 & 51.11 & 27.09 \\
\hline 29 & 2 & 25.10 & 19.58 & 28.20 \\
\hline 29 & 3 & 24.95 & 19.43 & 28.39 \\
\hline 29 & 4 & 46.79 & 36.12 & 29.55 \\
\hline 29 & 5 & 24.81 & 19.33 & 28.33 \\
\hline 35 & 1 & 70.00 & 53.49 & 30.85 \\
\hline 35 & 2 & 31.86 & 23.09 & 38.00 \\
\hline 35 & 3 & 30.69 & 22.69 & 35.26 \\
\hline 35 & 4 & 68.55 & 53.25 & 28.73 \\
\hline 35 & 5 & 31.68 & 22.87 & 38.54 \\
\hline 41 & 1 & 81.32 & 58.16 & 39.83 \\
\hline 41 & 2 & 54.85 & 35.40 & 54.96 \\
\hline
\end{tabular}




\begin{tabular}{|c|c|c|c|c|}
\hline Inst. & Prod. & Appr. & \multicolumn{1}{l|}{ Sim. } & $\begin{array}{c}\text { Error } \\
\text { sim-anal (\%) }\end{array}$ \\
\hline 41 & 3 & 55.16 & 35.47 & 55.52 \\
\hline 41 & 4 & 30.80 & 25.79 & 19.40 \\
\hline 41 & 5 & 54.85 & 35.39 & 55.00 \\
\hline 47 & 1 & 66.93 & 45.61 & 46.73 \\
\hline 47 & 2 & 46.99 & 29.03 & 61.84 \\
\hline 47 & 3 & 45.57 & 28.15 & 61.87 \\
\hline 47 & 4 & 26.51 & 23.91 & 10.90 \\
\hline 47 & 5 & 45.54 & 28.21 & 61.43 \\
\hline 53 & 1 & 36.43 & 28.32 & 28.66 \\
\hline 53 & 2 & 13.37 & 11.07 & 20.82 \\
\hline 53 & 3 & 13.30 & 10.98 & 21.10 \\
\hline 53 & 4 & 28.28 & 20.91 & 35.26 \\
\hline 53 & 5 & 13.23 & 10.91 & 21.19 \\
\hline 59 & 1 & 48.46 & 40.75 & 18.91 \\
\hline 59 & 2 & 21.37 & 17.42 & 22.71 \\
\hline 59 & 3 & 20.79 & 17.18 & 21.04 \\
\hline 59 & 4 & 47.74 & 40.58 & 17.64 \\
\hline 59 & 5 & 21.29 & 17.26 & 23.32 \\
\hline 65 & 1 & 55.01 & 42.92 & 28.15 \\
\hline 65 & 2 & 41.24 & 27.99 & 47.34 \\
\hline 65 & 3 & 41.39 & 27.96 & 48.04 \\
\hline 65 & 4 & 18.76 & 18.01 & 4.12 \\
\hline 65 & 5 & 41.24 & 27.99 & 47.33 \\
\hline 71 & 1 & 40.49 & 29.28 & 38.30 \\
\hline 71 & 2 & 28.33 & 18.72 & 51.32 \\
\hline 71 & 3 & 27.62 & 18.14 & 52.24 \\
\hline 71 & 4 & 14.05 & 14.16 & 0.79 \\
\hline 71 & 5 & 27.60 & 18.17 & 51.89 \\
\hline 77 & 1 & 28.76 & 22.40 & 28.36 \\
\hline 77 & 2 & 10.78 & 8.26 & 30.62 \\
\hline 77 & 3 & 10.71 & 8.17 & 30.99 \\
\hline 77 & 4 & 21.38 & 16.31 & 31.09 \\
\hline 77 & 5 & 10.64 & 8.11 & 31.21 \\
\hline 83 & 1 & 28.58 & 23.50 & 21.59 \\
\hline 83 & 2 & 12.19 & 9.49 & 28.52 \\
\hline 83 & 3 & 11.61 & 9.22 & 25.83 \\
\hline 83 & 4 & 27.86 & 23.26 & 19.78 \\
\hline 83 & 5 & 12.10 & 9.35 & 29.48 \\
\hline 89 & 1 & 32.28 & 25.94 & 24.42 \\
\hline & 2 & 20.80 & 15.07 & 38.04 \\
\hline
\end{tabular}




\begin{tabular}{|c|c|c|c|c|}
\hline Inst. & Prod. & Appr. & Sim. & $\begin{array}{c}\text { Error } \\
\text { sim-anal }(\%)\end{array}$ \\
\hline 89 & 3 & 20.95 & 15.09 & 38.90 \\
\hline 89 & 4 & 12.42 & 11.31 & 9.80 \\
\hline 89 & 5 & 20.80 & 15.06 & 38.14 \\
\hline 95 & 1 & 25.82 & 20.21 & 27.72 \\
\hline 95 & 2 & 18.51 & 12.97 & 42.65 \\
\hline 95 & 3 & 17.80 & 12.39 & 43.69 \\
\hline 95 & 4 & 11.85 & 10.99 & 7.79 \\
\hline 95 & 5 & 17.79 & 12.42 & 43.15 \\
\hline 101 & 1 & 31.36 & 26.11 & 20.08 \\
\hline 101 & 2 & 12.35 & 9.81 & 25.97 \\
\hline 101 & 3 & 12.27 & 9.70 & 26.50 \\
\hline 101 & 4 & 24.59 & 19.80 & 24.16 \\
\hline 101 & 5 & 12.20 & 9.64 & 26.58 \\
\hline 107 & 1 & 39.26 & 32.45 & 20.98 \\
\hline 107 & 2 & 17.01 & 12.79 & 32.98 \\
\hline 107 & 3 & 16.42 & 12.50 & 31.41 \\
\hline 107 & 4 & 38.54 & 32.10 & 20.07 \\
\hline 107 & 5 & 16.92 & 12.60 & 34.23 \\
\hline 113 & 1 & 41.82 & 33.03 & 26.63 \\
\hline 113 & 2 & 29.93 & 20.34 & 47.17 \\
\hline 113 & 3 & 30.08 & 20.32 & 48.01 \\
\hline 113 & 4 & 15.55 & 13.66 & 13.82 \\
\hline 113 & 5 & 29.93 & 20.31 & 47.34 \\
\hline 119 & 1 & 35.50 & 28.35 & 25.21 \\
\hline 119 & 2 & 25.54 & 18.52 & 37.89 \\
\hline 119 & 3 & 24.83 & 17.88 & 38.89 \\
\hline 119 & 4 & 14.48 & 14.70 & 1.48 \\
\hline 119 & 5 & 24.82 & 17.91 & 38.61 \\
\hline
\end{tabular}


Approximate and simulated flow times for six product classes with general distributions

\begin{tabular}{|c|c|c|c|c|}
\hline Inst. & Prod. & Appr. & \multicolumn{1}{c}{ Sim. } & $\begin{array}{c}\text { Error } \\
\text { sim-anal (\%) }\end{array}$ \\
\hline 6 & 1 & 56.27 & 44.58 & 26.21 \\
\hline 6 & 2 & 20.02 & 15.36 & 30.30 \\
\hline 6 & 3 & 19.86 & 15.24 & 30.29 \\
\hline 6 & 4 & 43.84 & 33.54 & 30.71 \\
\hline 6 & 5 & 19.72 & 15.09 & 30.65 \\
\hline 6 & 6 & 30.65 & 25.21 & 21.61 \\
\hline 12 & 1 & 59.28 & 45.07 & 31.53 \\
\hline 12 & 2 & 27.57 & 20.11 & 37.06 \\
\hline 12 & 3 & 26.40 & 19.70 & 34.01 \\
\hline 12 & 4 & 57.83 & 44.83 & 29.01 \\
\hline 12 & 5 & 27.39 & 19.84 & 38.03 \\
\hline 12 & 6 & 22.82 & 19.97 & 14.27 \\
\hline 18 & 1 & 73.34 & 52.76 & 39.00 \\
\hline 18 & 2 & 41.58 & 28.93 & 43.72 \\
\hline 18 & 3 & 41.89 & 28.95 & 44.68 \\
\hline 18 & 4 & 34.50 & 26.59 & 29.74 \\
\hline 18 & 5 & 41.58 & 28.95 & 43.64 \\
\hline 18 & 6 & 35.22 & 26.53 & 32.76 \\
\hline 24 & 1 & 55.93 & 41.20 & 35.76 \\
\hline 24 & 2 & 34.81 & 23.87 & 45.82 \\
\hline 24 & 3 & 33.39 & 22.93 & 45.62 \\
\hline 24 & 4 & 29.94 & 24.71 & 21.16 \\
\hline 24 & 5 & 33.36 & 23.00 & 45.03 \\
\hline 24 & 6 & 27.51 & 23.57 & 16.71 \\
\hline 30 & 1 & 79.38 & 58.71 & 35.20 \\
\hline 30 & 2 & 27.38 & 20.36 & 34.45 \\
\hline 30 & 3 & 27.22 & 20.22 & 34.59 \\
\hline 30 & 4 & 60.76 & 44.14 & 37.64 \\
\hline 30 & 5 & 27.08 & 20.12 & 34.58 \\
\hline 30 & 6 & 44.08 & 33.27 & 32.51 \\
\hline 36 & 1 & 81.50 & 59.14 & 37.80 \\
\hline 36 & 2 & 41.64 & 28.10 & 48.17 \\
\hline 36 & 3 & 40.47 & 27.72 & 46.00 \\
\hline 36 & 4 & 80.05 & 58.97 & 35.76 \\
\hline 36 & 5 & 41.46 & 27.84 & 48.94 \\
\hline 36 & 6 & 34.05 & 26.73 & 27.39 \\
\hline 42 & 1 & 103.95 & 68.56 & 51.63 \\
\hline 42 & 2 & 63.78 & 40.24 & 58.53 \\
\hline
\end{tabular}




\begin{tabular}{|c|c|c|c|c|}
\hline Inst. & Prod. & Appr. & Sim. & $\begin{array}{c}\text { Error } \\
\text { sim-anal }(\%)\end{array}$ \\
\hline 42 & 3 & 64.09 & 40.26 & 59.19 \\
\hline 42 & 4 & 52.61 & 36.09 & 45.80 \\
\hline 42 & 5 & 63.78 & 40.33 & 58.14 \\
\hline 42 & 6 & 54.04 & 35.99 & 50.16 \\
\hline 48 & 1 & 84.44 & 55.63 & 51.79 \\
\hline 48 & 2 & 50.83 & 32.48 & 56.51 \\
\hline 48 & 3 & 49.42 & 31.58 & 56.49 \\
\hline 48 & 4 & 43.10 & 32.94 & 30.85 \\
\hline 48 & 5 & 49.39 & 31.63 & 56.15 \\
\hline 48 & 6 & 40.67 & 31.87 & 27.63 \\
\hline 54 & 1 & 47.32 & 32.29 & 46.54 \\
\hline 54 & 2 & 15.20 & 11.68 & 30.16 \\
\hline 54 & 3 & 15.12 & 11.62 & 30.21 \\
\hline 54 & 4 & 39.12 & 24.91 & 57.05 \\
\hline 54 & 5 & 15.05 & 11.52 & 30.64 \\
\hline 54 & 6 & 25.67 & 16.28 & 57.67 \\
\hline 60 & 1 & 57.13 & 45.42 & 25.78 \\
\hline 60 & 2 & 28.89 & 21.75 & 32.85 \\
\hline 60 & 3 & 28.31 & 21.52 & 31.54 \\
\hline 60 & 4 & 56.40 & 45.32 & 24.45 \\
\hline 60 & 5 & 28.80 & 21.58 & 33.48 \\
\hline 60 & 6 & 23.50 & 19.72 & 19.20 \\
\hline 66 & 1 & 72.88 & 51.66 & 41.09 \\
\hline 66 & 2 & 46.84 & 31.11 & 50.57 \\
\hline 66 & 3 & 46.99 & 31.16 & 50.80 \\
\hline 66 & 4 & 36.11 & 26.74 & 35.04 \\
\hline 66 & 5 & 46.84 & 31.14 & 50.40 \\
\hline 66 & 6 & 35.96 & 26.67 & 34.83 \\
\hline 72 & 1 & 50.49 & 35.23 & 43.33 \\
\hline 72 & 2 & 28.66 & 19.76 & 45.01 \\
\hline 72 & 3 & 27.95 & 19.19 & 45.68 \\
\hline 72 & 4 & 22.98 & 19.92 & 15.35 \\
\hline 72 & 5 & 27.94 & 19.24 & 45.17 \\
\hline 72 & 6 & 21.76 & 19.23 & 13.17 \\
\hline 78 & 1 & 34.57 & 25.31 & 36.59 \\
\hline 78 & 2 & 11.98 & 8.53 & 40.51 \\
\hline 78 & 3 & 11.91 & 8.44 & 41.05 \\
\hline 78 & 4 & 27.15 & 19.37 & 40.13 \\
\hline 78 & 5 & 11.84 & 8.38 & 41.24 \\
\hline 78 & 6 & 21.00 & 14.26 & 47.26 \\
\hline
\end{tabular}




\begin{tabular}{|c|c|c|c|c|}
\hline Inst. & Prod. & Appr. & Sim. & $\begin{array}{c}\text { Error } \\
\text { sim-anal }(\%)\end{array}$ \\
\hline 84 & 1 & 31.85 & 25.45 & 25.12 \\
\hline 84 & 2 & 15.27 & 11.11 & 37.44 \\
\hline 84 & 3 & 14.68 & 10.88 & 34.99 \\
\hline 84 & 4 & 31.12 & 25.22 & 23.41 \\
\hline 84 & 5 & 15.18 & 10.98 & 38.28 \\
\hline 84 & 6 & 14.15 & 11.08 & 27.62 \\
\hline 90 & 1 & 38.50 & 29.86 & 28.95 \\
\hline 90 & 2 & 23.06 & 16.75 & 37.67 \\
\hline 90 & 3 & 23.21 & 16.71 & 38.91 \\
\hline 90 & 4 & 18.71 & 15.19 & 23.20 \\
\hline 90 & 5 & 23.06 & 16.73 & 37.86 \\
\hline 90 & 6 & 19.16 & 15.17 & 26.28 \\
\hline 96 & 1 & 30.68 & 24.09 & 27.35 \\
\hline 96 & 2 & 19.52 & 14.21 & 37.41 \\
\hline 96 & 3 & 18.81 & 13.62 & 38.14 \\
\hline 96 & 4 & 17.20 & 14.50 & 18.63 \\
\hline 96 & 5 & 18.80 & 13.66 & 37.62 \\
\hline 96 & 6 & 15.99 & 13.80 & 15.85 \\
\hline 102 & 1 & 52.65 & 38.07 & 38.30 \\
\hline 102 & 2 & 16.60 & 10.98 & 51.08 \\
\hline 102 & 3 & 16.52 & 10.89 & 51.61 \\
\hline 102 & 4 & 45.91 & 31.79 & 44.40 \\
\hline 102 & 5 & 16.45 & 10.83 & 51.83 \\
\hline 102 & 6 & 37.35 & 22.96 & 62.71 \\
\hline 108 & 1 & 44.83 & 35.64 & 25.79 \\
\hline 108 & 2 & 22.61 & 15.48 & 46.10 \\
\hline 108 & 3 & 22.03 & 15.19 & 45.05 \\
\hline 108 & 4 & 44.11 & 35.30 & 24.93 \\
\hline 108 & 5 & 22.53 & 15.31 & 47.15 \\
\hline 108 & 6 & 21.53 & 14.65 & 47.02 \\
\hline 114 & 1 & 52.18 & 38.72 & 34.78 \\
\hline 114 & 2 & 33.45 & 22.15 & 51.05 \\
\hline 114 & 3 & 33.60 & 22.17 & 51.55 \\
\hline 114 & 4 & 26.18 & 19.38 & 35.09 \\
\hline 114 & 5 & 33.45 & 22.19 & 50.75 \\
\hline 114 & 6 & 26.03 & 19.33 & 34.66 \\
\hline 120 & 1 & 43.42 & 34.78 & 24.87 \\
\hline 120 & 2 & 26.50 & 20.54 & 29.00 \\
\hline 120 & 3 & 25.79 & 19.91 & 29.57 \\
\hline 120 & 4 & 22.34 & 20.74 & 7.74 \\
\hline
\end{tabular}




\begin{tabular}{l|c|c|c|c|}
\hline Inst. & Prod. & Appr. & Sim. & $\begin{array}{c}\text { Error } \\
\text { sim-anal }(\%)\end{array}$ \\
\hline 120 & 5 & 25.78 & 19.98 & 28.99 \\
\hline 120 & 6 & 21.13 & 19.91 & 6.10 \\
\hline
\end{tabular}




\section{APPENDIX F}

Approximate and simulated flow times for the $E_{2}-E_{2}$ case

Prod. Server Subtasks Net Traffic Anal. Sim $\quad$ Error $\quad$ Predicted

Intensity Flow T. Flow T. sim-anal (\%) Error (\%)

\begin{tabular}{|c|c|c|c|c|c|c|c|}
\hline 1 & 2 & 2 & 0.10 & 2.79 & 2.90 & 4.15 & 1.59 \\
\hline 1 & 2 & 2 & 0.11 & 2.94 & 2.91 & 1.04 & 6.90 \\
\hline 1 & 2 & 2 & 0.12 & 3.31 & 2.93 & 11.48 & 17.52 \\
\hline 2 & 2 & 2 & 0.19 & 2.97 & 3.07 & 3.64 & 3.45 \\
\hline 2 & 2 & 2 & 0.19 & 2.74 & 2.79 & 1.99 & 3.45 \\
\hline 2 & 2 & 2 & 0.20 & 3.15 & 3.09 & 1.80 & 8.71 \\
\hline 2 & 2 & 2 & 0.20 & 2.91 & 2.81 & 3.43 & 8.71 \\
\hline 2 & 2 & 2 & 0.22 & 3.58 & 3.15 & 12.20 & 19.21 \\
\hline 2 & 2 & 2 & 0.22 & 3.32 & 2.86 & 13.93 & 19.21 \\
\hline 3 & 2 & 2 & 0.31 & 3.39 & 3.37 & 0.65 & 4.99 \\
\hline 3 & 2 & 2 & 0.31 & 3.17 & 3.06 & 3.45 & 4.99 \\
\hline 3 & 2 & 2 & 0.31 & 3.98 & 4.21 & 5.80 & 4.99 \\
\hline 3 & 2 & 2 & 0.33 & 3.63 & 3.42 & 5.96 & 10.19 \\
\hline 3 & 2 & 2 & 0.33 & 3.41 & 3.11 & 8.85 & 10.19 \\
\hline 3 & 2 & 2 & 0.33 & 4.27 & 4.27 & 0.10 & 10.19 \\
\hline 3 & 2 & 2 & 0.37 & 4.28 & 3.54 & 17.33 & 20.52 \\
\hline 3 & 2 & 2 & 0.37 & 4.02 & 3.22 & 19.94 & 20.52 \\
\hline 3 & 2 & 2 & 0.37 & 4.98 & 4.43 & 11.17 & 20.52 \\
\hline 4 & 2 & 2 & 0.44 & 4.17 & 3.85 & 7.73 & 6.37 \\
\hline 4 & 2 & 2 & 0.44 & 3.97 & 3.50 & 11.74 & 6.37 \\
\hline 4 & 2 & 2 & 0.44 & 4.73 & 4.81 & 1.83 & 6.37 \\
\hline 4 & 2 & 2 & 0.44 & 5.13 & 5.50 & 7.19 & 6.37 \\
\hline 4 & 2 & 2 & 0.47 & 4.57 & 3.96 & 13.42 & 11.49 \\
\hline 4 & 2 & 2 & 0.47 & 4.37 & 3.60 & 17.59 & 11.49 \\
\hline 4 & 2 & 2 & 0.47 & 5.17 & 4.95 & 4.32 & 11.49 \\
\hline 4 & 2 & 2 & 0.47 & 5.60 & 5.66 & 0.93 & 11.49 \\
\hline 4 & 2 & 2 & 0.52 & 5.69 & 4.26 & 25.15 & 21.64 \\
\hline 4 & 2 & 2 & 0.52 & 5.43 & 3.87 & 28.75 & 21.64 \\
\hline 4 & 2 & 2 & 0.52 & 6.37 & 5.32 & 16.46 & 21.64 \\
\hline 4 & 2 & 2 & 0.52 & 6.86 & 6.08 & 11.42 & 21.64 \\
\hline 5 & 2 & 2 & 0.51 & 4.60 & 4.18 & 9.17 & 8.50 \\
\hline 5 & 2 & 2 & 0.51 & 4.40 & 3.80 & 13.50 & 8.50 \\
\hline 5 & 2 & 2 & 0.51 & 5.15 & 5.23 & 1.42 & 8.50 \\
\hline 5 & 2 & 2 & 0.51 & 5.53 & 5.97 & 8.02 & 8.50 \\
\hline 5 & 2 & 2 & 0.51 & 4.11 & 3.22 & 21.73 & 8.50 \\
\hline 5 & 2 & 2 & 0.54 & 5.13 & 4.35 & 15.23 & 13.59 \\
\hline
\end{tabular}




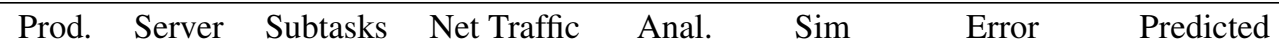
Intensity Flow T. Flow T. sim-anal (\%) Error (\%)

\begin{tabular}{|c|c|c|c|c|c|c|c|}
\hline 5 & 2 & 2 & 0.54 & 4.91 & 3.95 & 19.63 & 13.59 \\
\hline 5 & 2 & 2 & 0.54 & 5.71 & 5.43 & 4.81 & 13.59 \\
\hline 5 & 2 & 2 & 0.54 & 6.11 & 6.21 & 1.55 & 13.59 \\
\hline 5 & 2 & 2 & 0.54 & 4.58 & 3.34 & 27.03 & 13.59 \\
\hline 5 & 2 & 2 & 0.60 & 6.63 & 4.81 & 27.43 & 23.65 \\
\hline 5 & 2 & 2 & 0.60 & 6.39 & 4.38 & 31.53 & 23.65 \\
\hline 5 & 2 & 2 & 0.60 & 7.30 & 6.02 & 17.59 & 23.65 \\
\hline 5 & 2 & 2 & 0.60 & 7.79 & 6.88 & 11.70 & 23.65 \\
\hline 5 & 2 & 2 & 0.60 & 6.04 & 3.70 & 38.72 & 23.65 \\
\hline 6 & 2 & 2 & 0.59 & 5.28 & 4.73 & 10.35 & 10.48 \\
\hline 6 & 2 & 2 & 0.59 & 5.07 & 4.30 & 15.21 & 10.48 \\
\hline 6 & 2 & 2 & 0.59 & 5.79 & 5.91 & 2.19 & 10.48 \\
\hline 6 & 2 & 2 & 0.59 & 6.19 & 6.76 & 9.16 & 10.48 \\
\hline 6 & 2 & 2 & 0.59 & 4.79 & 3.64 & 23.97 & 10.48 \\
\hline 6 & 2 & 2 & 0.59 & 4.91 & 3.94 & 19.77 & 10.48 \\
\hline 6 & 2 & 2 & 0.62 & 5.99 & 5.01 & 16.38 & 15.52 \\
\hline 6 & 2 & 2 & 0.62 & 5.77 & 4.55 & 21.12 & 15.52 \\
\hline 6 & 2 & 2 & 0.62 & 6.55 & 6.26 & 4.52 & 15.52 \\
\hline 6 & 2 & 2 & 0.62 & 6.96 & 7.15 & 2.76 & 15.52 \\
\hline 6 & 2 & 2 & 0.62 & 5.48 & 3.85 & 29.68 & 15.52 \\
\hline 6 & 2 & 2 & 0.62 & 5.62 & 4.17 & 25.73 & 15.52 \\
\hline 6 & 2 & 2 & 0.69 & 8.41 & 5.87 & 30.13 & 25.47 \\
\hline 6 & 2 & 2 & 0.69 & 8.18 & 5.34 & 34.74 & 25.47 \\
\hline 6 & 2 & 2 & 0.69 & 9.04 & 7.34 & 18.80 & 25.47 \\
\hline 6 & 2 & 2 & 0.69 & 9.51 & 8.39 & 11.81 & 25.47 \\
\hline 6 & 2 & 2 & 0.69 & 7.80 & 4.52 & 42.09 & 25.47 \\
\hline 6 & 2 & 2 & 0.69 & 7.99 & 4.89 & 38.75 & 25.47 \\
\hline 1 & 3 & 3 & 0.10 & 3.26 & 3.39 & 3.98 & 3.36 \\
\hline 1 & 3 & 3 & 0.11 & 3.44 & 3.40 & 1.14 & 8.68 \\
\hline 1 & 3 & 3 & 0.12 & 3.87 & 3.43 & 11.57 & 19.29 \\
\hline 2 & 3 & 3 & 0.19 & 3.45 & 3.59 & 3.86 & 5.22 \\
\hline 2 & 3 & 3 & 0.19 & 3.20 & 3.26 & 1.99 & 5.22 \\
\hline 2 & 3 & 3 & 0.20 & 3.67 & 3.61 & 1.49 & 10.49 \\
\hline 2 & 3 & 3 & 0.20 & 3.40 & 3.28 & 3.37 & 10.49 \\
\hline 2 & 3 & 3 & 0.22 & 4.18 & 3.67 & 12.17 & 20.98 \\
\hline 2 & 3 & 3 & 0.22 & 3.88 & 3.34 & 13.90 & 20.98 \\
\hline 3 & 3 & 3 & 0.31 & 3.93 & 3.93 & 0.10 & 6.76 \\
\hline 3 & 3 & 3 & 0.31 & 3.70 & 3.57 & 3.40 & 6.76 \\
\hline 3 & 3 & 3 & 0.31 & 4.61 & 4.91 & 6.50 & 6.76 \\
\hline 3 & 3 & 3 & 0.33 & 4.24 & 3.99 & 5.88 & 11.96 \\
\hline
\end{tabular}


$\begin{array}{lllllll}\text { Prod. } & \text { Server } & \text { Subtasks Net Traffic } & \text { Anal. } & \text { Sim } & \text { Error } & \text { Predicted }\end{array}$ Intensity Flow T. Flow T. sim-anal (\%) Error (\%)

\begin{tabular}{|c|c|c|c|c|c|c|c|}
\hline 3 & 3 & 3 & 0.33 & 3.97 & 3.62 & 8.76 & 11.96 \\
\hline 3 & 3 & 3 & 0.33 & 4.95 & 4.98 & 0.59 & 11.96 \\
\hline 3 & 3 & 3 & 0.37 & 4.97 & 4.13 & 16.92 & 22.29 \\
\hline 3 & 3 & 3 & 0.37 & 4.68 & 3.75 & 19.86 & 22.29 \\
\hline 3 & 3 & 3 & 0.37 & 5.79 & 5.16 & 10.79 & 22.29 \\
\hline 4 & 3 & 3 & 0.44 & 4.85 & 4.49 & 7.47 & 8.14 \\
\hline 4 & 3 & 3 & 0.44 & 4.63 & 4.08 & 11.78 & 8.14 \\
\hline 4 & 3 & 3 & 0.44 & 5.48 & 5.61 & 2.32 & 8.14 \\
\hline 4 & 3 & 3 & 0.44 & 5.94 & 6.41 & 7.98 & 8.14 \\
\hline 4 & 3 & 3 & 0.47 & 5.32 & 4.61 & 13.27 & 13.26 \\
\hline 4 & 3 & 3 & 0.47 & 5.07 & 4.19 & 17.29 & 13.26 \\
\hline 4 & 3 & 3 & 0.47 & 5.99 & 5.77 & 3.73 & 13.26 \\
\hline 4 & 3 & 3 & 0.47 & 6.48 & 6.59 & 1.80 & 13.26 \\
\hline 4 & 3 & 3 & 0.52 & 6.62 & 4.96 & 25.03 & 23.41 \\
\hline 4 & 3 & 3 & 0.52 & 6.34 & 4.51 & 28.92 & 23.41 \\
\hline 4 & 3 & 3 & 0.52 & 7.37 & 6.20 & 15.89 & 23.41 \\
\hline 4 & 3 & 3 & 0.52 & 7.91 & 7.09 & 10.45 & 23.41 \\
\hline 5 & 3 & 3 & 0.51 & 5.33 & 4.87 & 8.58 & 10.28 \\
\hline 5 & 3 & 3 & 0.51 & 5.11 & 4.43 & 13.26 & 10.28 \\
\hline 5 & 3 & 3 & 0.51 & 5.94 & 6.09 & 2.49 & 10.28 \\
\hline 5 & 3 & 3 & 0.51 & 6.38 & 6.96 & 9.15 & 10.28 \\
\hline 5 & 3 & 3 & 0.51 & 4.78 & 3.75 & 21.50 & 10.28 \\
\hline 5 & 3 & 3 & 0.54 & 5.93 & 5.06 & 14.57 & 15.36 \\
\hline 5 & 3 & 3 & 0.54 & 5.69 & 4.60 & 19.10 & 15.36 \\
\hline 5 & 3 & 3 & 0.54 & 6.57 & 6.33 & 3.71 & 15.36 \\
\hline 5 & 3 & 3 & 0.54 & 7.06 & 7.23 & 2.45 & 15.36 \\
\hline 5 & 3 & 3 & 0.54 & 5.34 & 3.89 & 27.07 & 15.36 \\
\hline 5 & 3 & 3 & 0.60 & 7.71 & 5.61 & 27.27 & 25.42 \\
\hline 5 & 3 & 3 & 0.60 & 7.45 & 5.10 & 31.60 & 25.42 \\
\hline 5 & 3 & 3 & 0.60 & 8.44 & 7.01 & 17.00 & 25.42 \\
\hline 5 & 3 & 3 & 0.60 & 8.97 & 8.01 & 10.75 & 25.42 \\
\hline 5 & 3 & 3 & 0.60 & 7.05 & 4.31 & 38.80 & 25.42 \\
\hline 6 & 3 & 3 & 0.59 & 6.09 & 5.51 & 9.53 & 12.25 \\
\hline 6 & 3 & 3 & 0.59 & 5.88 & 5.01 & 14.91 & 12.25 \\
\hline 6 & 3 & 3 & 0.59 & 6.68 & 6.89 & 3.05 & 12.25 \\
\hline 6 & 3 & 3 & 0.59 & 7.10 & 7.87 & 10.77 & 12.25 \\
\hline 6 & 3 & 3 & 0.59 & 5.57 & 4.24 & 23.96 & 12.25 \\
\hline 6 & 3 & 3 & 0.59 & 5.73 & 4.59 & 19.85 & 12.25 \\
\hline 6 & 3 & 3 & 0.62 & 6.98 & 5.83 & 16.51 & 17.29 \\
\hline 6 & 3 & 3 & 0.62 & 6.74 & 5.30 & 21.36 & 17.29 \\
\hline
\end{tabular}




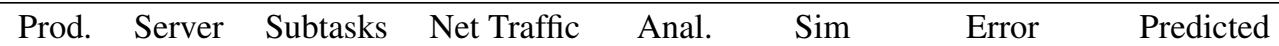
Intensity Flow T. Flow T. sim-anal (\%) Error (\%)

\begin{tabular}{|c|c|c|c|c|c|c|c|}
\hline 6 & 3 & 3 & 0.62 & 7.58 & 7.28 & 3.91 & 17.29 \\
\hline 6 & 3 & 3 & 0.62 & 8.04 & 8.32 & 3.59 & 17.29 \\
\hline 6 & 3 & 3 & 0.62 & 6.40 & 4.48 & 29.94 & 17.29 \\
\hline 6 & 3 & 3 & 0.62 & 6.56 & 4.86 & 26.02 & 17.29 \\
\hline 6 & 3 & 3 & 0.69 & 9.79 & 6.83 & 30.23 & 27.24 \\
\hline 6 & 3 & 3 & 0.69 & 9.52 & 6.21 & 34.75 & 27.24 \\
\hline 6 & 3 & 3 & 0.69 & 10.47 & 8.54 & 18.44 & 27.24 \\
\hline 6 & 3 & 3 & 0.69 & 10.99 & 9.76 & 11.15 & 27.24 \\
\hline 6 & 3 & 3 & 0.69 & 9.14 & 5.26 & 42.46 & 27.24 \\
\hline 6 & 3 & 3 & 0.69 & 9.30 & 5.69 & 38.78 & 27.24 \\
\hline 1 & 3 & 2 & 0.07 & 3.21 & 2.85 & 11.27 & 6.35 \\
\hline 1 & 3 & 2 & 0.07 & 3.38 & 2.85 & 15.64 & 11.69 \\
\hline 1 & 3 & 2 & 0.08 & 3.80 & 2.87 & 24.55 & 22.35 \\
\hline 2 & 3 & 2 & 0.13 & 3.35 & 2.95 & 11.91 & 8.54 \\
\hline 2 & 3 & 2 & 0.13 & 3.08 & 2.68 & 12.92 & 8.54 \\
\hline 2 & 3 & 2 & 0.13 & 3.54 & 2.96 & 16.40 & 13.84 \\
\hline 2 & 3 & 2 & 0.13 & 3.26 & 2.69 & 17.38 & 13.84 \\
\hline 2 & 3 & 2 & 0.15 & 4.02 & 2.99 & 25.52 & 24.42 \\
\hline 2 & 3 & 2 & 0.15 & 3.70 & 2.72 & 26.42 & 24.42 \\
\hline 3 & 3 & 2 & 0.21 & 3.67 & 3.11 & 15.21 & 10.52 \\
\hline 3 & 3 & 2 & 0.21 & 3.41 & 2.83 & 17.19 & 10.52 \\
\hline 3 & 3 & 2 & 0.21 & 4.39 & 3.89 & 11.41 & 10.52 \\
\hline 3 & 3 & 2 & 0.22 & 3.91 & 3.13 & 19.89 & 15.77 \\
\hline 3 & 3 & 2 & 0.22 & 4.02 & 2.85 & 29.08 & 15.77 \\
\hline 3 & 3 & 2 & 0.22 & 4.74 & 3.92 & 17.37 & 15.77 \\
\hline 3 & 3 & 2 & 0.24 & 4.48 & 3.19 & 28.72 & 26.25 \\
\hline 3 & 3 & 2 & 0.24 & 4.19 & 2.90 & 30.66 & 26.25 \\
\hline 3 & 3 & 2 & 0.24 & 5.33 & 3.99 & 25.14 & 26.25 \\
\hline 4 & 3 & 2 & 0.30 & 4.18 & 3.33 & 20.31 & 12.39 \\
\hline 4 & 3 & 2 & 0.30 & 3.92 & 3.03 & 22.82 & 12.39 \\
\hline 4 & 3 & 2 & 0.30 & 4.87 & 4.16 & 14.59 & 12.39 \\
\hline 4 & 3 & 2 & 0.30 & 5.38 & 4.76 & 11.55 & 12.39 \\
\hline 4 & 3 & 2 & 0.31 & 4.50 & 3.37 & 25.04 & 17.60 \\
\hline 4 & 3 & 2 & 0.31 & 4.22 & 3.07 & 27.39 & 17.60 \\
\hline 4 & 3 & 2 & 0.31 & 5.24 & 4.22 & 19.51 & 17.60 \\
\hline 4 & 3 & 2 & 0.31 & 5.75 & 4.82 & 16.24 & 17.60 \\
\hline 4 & 3 & 2 & 0.35 & 5.29 & 3.49 & 34.13 & 27.95 \\
\hline 4 & 3 & 2 & 0.35 & 4.99 & 3.17 & 36.53 & 27.95 \\
\hline 4 & 3 & 2 & 0.35 & 6.13 & 4.36 & 28.95 & 27.95 \\
\hline 4 & 3 & 2 & 0.35 & 6.72 & 4.98 & 25.92 & 27.95 \\
\hline
\end{tabular}




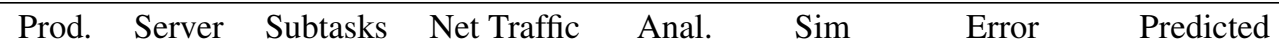
Intensity Flow T. Flow T. sim-anal (\%) Error (\%)

\begin{tabular}{|c|c|c|c|c|c|c|c|}
\hline 5 & 3 & 2 & 0.34 & 4.39 & 3.46 & 21.25 & 14.76 \\
\hline 5 & 3 & 2 & 0.34 & 4.14 & 3.14 & 24.05 & 14.76 \\
\hline 5 & 3 & 2 & 0.34 & 5.09 & 4.32 & 15.03 & 14.76 \\
\hline 5 & 3 & 2 & 0.34 & 5.58 & 4.94 & 11.42 & 14.76 \\
\hline 5 & 3 & 2 & 0.34 & 3.75 & 2.66 & 29.05 & 14.76 \\
\hline 5 & 3 & 2 & 0.36 & 4.75 & 3.52 & 25.96 & 19.94 \\
\hline 5 & 3 & 2 & 0.36 & 4.48 & 3.20 & 28.59 & 19.94 \\
\hline 5 & 3 & 2 & 0.36 & 5.48 & 4.40 & 19.80 & 19.94 \\
\hline 5 & 3 & 2 & 0.36 & 6.00 & 5.02 & 16.29 & 19.94 \\
\hline 5 & 3 & 2 & 0.36 & 4.06 & 2.71 & 33.40 & 19.94 \\
\hline 5 & 3 & 2 & 0.40 & 5.67 & 3.67 & 35.32 & 30.24 \\
\hline 5 & 3 & 2 & 0.40 & 5.36 & 3.33 & 37.84 & 30.24 \\
\hline 5 & 3 & 2 & 0.40 & 6.50 & 4.58 & 29.42 & 30.24 \\
\hline 5 & 3 & 2 & 0.40 & 7.08 & 5.24 & 26.00 & 30.24 \\
\hline 5 & 3 & 2 & 0.40 & 4.89 & 2.82 & 42.36 & 30.24 \\
\hline 6 & 3 & 2 & 0.39 & 4.67 & 3.64 & 22.03 & 17.03 \\
\hline 6 & 3 & 2 & 0.39 & 4.42 & 3.31 & 25.06 & 17.03 \\
\hline 6 & 3 & 2 & 0.39 & 5.36 & 4.55 & 15.08 & 17.03 \\
\hline 6 & 3 & 2 & 0.39 & 5.85 & 5.20 & 11.05 & 17.03 \\
\hline 6 & 3 & 2 & 0.39 & 4.02 & 2.80 & 30.40 & 17.03 \\
\hline 6 & 3 & 2 & 0.39 & 4.21 & 3.03 & 27.89 & 17.03 \\
\hline 6 & 3 & 2 & 0.41 & 5.84 & 3.72 & 36.32 & 22.18 \\
\hline 6 & 3 & 2 & 0.41 & 5.56 & 3.38 & 39.10 & 22.18 \\
\hline 6 & 3 & 2 & 0.41 & 6.59 & 4.65 & 29.37 & 22.18 \\
\hline 6 & 3 & 2 & 0.41 & 7.12 & 5.32 & 25.37 & 22.18 \\
\hline 6 & 3 & 2 & 0.41 & 5.12 & 2.86 & 44.10 & 22.18 \\
\hline 6 & 3 & 2 & 0.41 & 5.32 & 3.10 & 41.73 & 22.18 \\
\hline 6 & 3 & 2 & 0.46 & 6.17 & 3.93 & 36.24 & 32.40 \\
\hline 6 & 3 & 2 & 0.46 & 5.87 & 3.58 & 39.11 & 32.40 \\
\hline 6 & 3 & 2 & 0.46 & 7.01 & 4.92 & 29.86 & 32.40 \\
\hline 6 & 3 & 2 & 0.46 & 7.58 & 5.62 & 25.88 & 32.40 \\
\hline 6 & 3 & 2 & 0.46 & 5.44 & 3.03 & 44.41 & 32.40 \\
\hline 6 & 3 & 2 & 0.46 & 5.64 & 3.28 & 41.83 & 32.40 \\
\hline 1 & 4 & 4 & 0.10 & 3.60 & 3.74 & 4.04 & 5.13 \\
\hline 1 & 4 & 4 & 0.11 & 3.80 & 3.75 & 1.22 & 10.45 \\
\hline 1 & 4 & 4 & 0.12 & 4.27 & 3.78 & 11.55 & 21.07 \\
\hline 2 & 4 & 4 & 0.19 & 3.81 & 3.96 & 4.01 & 6.99 \\
\hline 2 & 4 & 4 & 0.19 & 3.52 & 3.60 & 2.08 & 6.99 \\
\hline 2 & 4 & 4 & 0.20 & 4.04 & 3.98 & 1.43 & 12.26 \\
\hline 2 & 4 & 4 & 0.20 & 3.75 & 3.62 & 3.38 & 12.26 \\
\hline
\end{tabular}


$\begin{array}{lllllll}\text { Prod. } & \text { Server } & \text { Subtasks Net Traffic } & \text { Anal. } & \text { Sim } & \text { Error } & \text { Predicted }\end{array}$ Intensity Flow T. Flow T. sim-anal (\%) Error (\%)

\begin{tabular}{|c|c|c|c|c|c|c|c|}
\hline 2 & 4 & 4 & 0.22 & 4.61 & 4.05 & 12.10 & 22.75 \\
\hline 2 & 4 & 4 & 0.22 & 4.29 & 3.68 & 14.09 & 22.75 \\
\hline 3 & 4 & 4 & 0.31 & 4.34 & 4.33 & 0.26 & 8.53 \\
\hline 3 & 4 & 4 & 0.31 & 4.07 & 3.94 & 3.15 & 8.53 \\
\hline 3 & 4 & 4 & 0.31 & 5.08 & 5.42 & 6.55 & 8.53 \\
\hline 3 & 4 & 4 & 0.33 & 4.66 & 4.39 & 5.69 & 13.73 \\
\hline 3 & 4 & 4 & 0.33 & 4.37 & 3.99 & 8.66 & 13.73 \\
\hline 3 & 4 & 4 & 0.33 & 5.45 & 5.49 & 0.87 & 13.73 \\
\hline 3 & 4 & 4 & 0.37 & 5.48 & 4.55 & 16.88 & 24.06 \\
\hline 3 & 4 & 4 & 0.37 & 5.16 & 4.14 & 19.81 & 24.06 \\
\hline 3 & 4 & 4 & 0.37 & 6.34 & 5.69 & 10.22 & 24.06 \\
\hline 4 & 4 & 4 & 0.44 & 5.35 & 4.94 & 7.57 & 9.92 \\
\hline 4 & 4 & 4 & 0.44 & 5.10 & 4.49 & 11.85 & 9.92 \\
\hline 4 & 4 & 4 & 0.44 & 6.03 & 6.18 & 2.42 & 9.92 \\
\hline 4 & 4 & 4 & 0.44 & 6.53 & 7.06 & 8.17 & 9.92 \\
\hline 4 & 4 & 4 & 0.47 & 5.85 & 5.08 & 13.14 & 15.04 \\
\hline 4 & 4 & 4 & 0.47 & 5.57 & 4.62 & 17.09 & 15.04 \\
\hline 4 & 4 & 4 & 0.47 & 6.58 & 6.35 & 3.44 & 15.04 \\
\hline 4 & 4 & 4 & 0.47 & 7.08 & 7.26 & 2.47 & 15.04 \\
\hline 4 & 4 & 4 & 0.52 & 7.25 & 5.46 & 24.65 & 25.19 \\
\hline 4 & 4 & 4 & 0.52 & 6.97 & 4.96 & 28.77 & 25.19 \\
\hline 4 & 4 & 4 & 0.52 & 8.06 & 6.83 & 15.37 & 25.19 \\
\hline 4 & 4 & 4 & 0.52 & 8.65 & 7.80 & 9.82 & 25.19 \\
\hline 5 & 4 & 4 & 0.51 & 5.86 & 5.37 & 8.46 & 12.05 \\
\hline 5 & 4 & 4 & 0.51 & 5.64 & 4.88 & 13.44 & 12.05 \\
\hline 5 & 4 & 4 & 0.51 & 6.53 & 6.71 & 2.69 & 12.05 \\
\hline 5 & 4 & 4 & 0.51 & 7.00 & 7.67 & 9.48 & 12.05 \\
\hline 5 & 4 & 4 & 0.51 & 5.27 & 4.13 & 21.68 & 12.05 \\
\hline 5 & 4 & 4 & 0.54 & 6.53 & 5.57 & 14.67 & 17.13 \\
\hline 5 & 4 & 4 & 0.54 & 6.27 & 5.07 & 19.17 & 17.13 \\
\hline 5 & 4 & 4 & 0.54 & 7.21 & 6.97 & 3.45 & 17.13 \\
\hline 5 & 4 & 4 & 0.54 & 7.73 & 7.96 & 3.05 & 17.13 \\
\hline 5 & 4 & 4 & 0.54 & 5.90 & 4.29 & 27.31 & 17.13 \\
\hline 5 & 4 & 4 & 0.60 & 8.43 & 6.17 & 26.83 & 27.19 \\
\hline 5 & 4 & 4 & 0.60 & 8.16 & 5.61 & 31.25 & 27.19 \\
\hline 5 & 4 & 4 & 0.60 & 9.20 & 7.71 & 16.19 & 27.19 \\
\hline 5 & 4 & 4 & 0.60 & 9.78 & 8.81 & 9.88 & 27.19 \\
\hline 5 & 4 & 4 & 0.60 & 7.73 & 4.74 & 38.64 & 27.19 \\
\hline 6 & 4 & 4 & 0.59 & 6.69 & 6.06 & 9.43 & 14.03 \\
\hline 6 & 4 & 4 & 0.59 & 6.47 & 5.51 & 14.80 & 14.03 \\
\hline
\end{tabular}




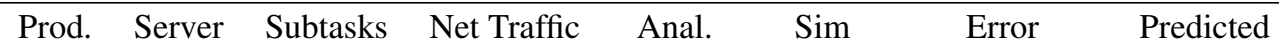
Intensity Flow T. Flow T. sim-anal (\%) Error (\%)

\begin{tabular}{|c|c|c|c|c|c|c|c|}
\hline 6 & 4 & 4 & 0.59 & 7.31 & 7.58 & 3.64 & 14.03 \\
\hline 6 & 4 & 4 & 0.59 & 7.78 & 8.66 & 11.36 & 14.03 \\
\hline 6 & 4 & 4 & 0.59 & 6.13 & 4.66 & 23.94 & 14.03 \\
\hline 6 & 4 & 4 & 0.59 & 6.27 & 5.05 & 19.46 & 14.03 \\
\hline 6 & 4 & 4 & 0.62 & 7.62 & 6.41 & 15.92 & 19.06 \\
\hline 6 & 4 & 4 & 0.62 & 7.39 & 5.83 & 21.11 & 19.06 \\
\hline 6 & 4 & 4 & 0.62 & 8.29 & 8.01 & 3.33 & 19.06 \\
\hline 6 & 4 & 4 & 0.62 & 8.77 & 9.16 & 4.37 & 19.06 \\
\hline 6 & 4 & 4 & 0.62 & 7.03 & 4.93 & 29.86 & 19.06 \\
\hline 6 & 4 & 4 & 0.62 & 7.20 & 5.34 & 25.77 & 19.06 \\
\hline 6 & 4 & 4 & 0.69 & 10.71 & 7.51 & 29.88 & 29.02 \\
\hline 6 & 4 & 4 & 0.69 & 10.48 & 6.83 & 34.84 & 29.02 \\
\hline 6 & 4 & 4 & 0.69 & 11.47 & 9.39 & 18.12 & 29.02 \\
\hline 6 & 4 & 4 & 0.69 & 11.99 & 10.73 & 10.52 & 29.02 \\
\hline 6 & 4 & 4 & 0.69 & 10.09 & 5.78 & 42.71 & 29.02 \\
\hline 6 & 4 & 4 & 0.69 & 10.28 & 6.26 & 39.08 & 29.02 \\
\hline 1 & 4 & 2 & 0.05 & 3.09 & 2.82 & 8.80 & 10.93 \\
\hline 1 & 4 & 2 & 0.05 & 3.26 & 2.83 & 13.21 & 16.28 \\
\hline 1 & 4 & 2 & 0.06 & 3.66 & 2.84 & 22.43 & 26.96 \\
\hline 2 & 4 & 2 & 0.10 & 3.19 & 2.89 & 9.14 & 13.28 \\
\hline 2 & 4 & 2 & 0.10 & 2.93 & 2.63 & 10.04 & 13.28 \\
\hline 2 & 4 & 2 & 0.10 & 3.38 & 2.90 & 13.99 & 18.61 \\
\hline 2 & 4 & 2 & 0.10 & 3.09 & 2.64 & 14.67 & 18.61 \\
\hline 2 & 4 & 2 & 0.11 & 3.80 & 2.92 & 23.09 & 29.23 \\
\hline 2 & 4 & 2 & 0.11 & 3.49 & 2.66 & 23.91 & 29.23 \\
\hline 3 & 4 & 2 & 0.16 & 3.41 & 3.00 & 12.02 & 15.48 \\
\hline 3 & 4 & 2 & 0.16 & 3.15 & 2.73 & 13.37 & 15.48 \\
\hline 3 & 4 & 2 & 0.16 & 4.12 & 3.75 & 8.79 & 15.48 \\
\hline 3 & 4 & 2 & 0.16 & 3.63 & 3.02 & 16.87 & 20.77 \\
\hline 3 & 4 & 2 & 0.16 & 3.36 & 2.74 & 18.24 & 20.77 \\
\hline 3 & 4 & 2 & 0.16 & 4.37 & 3.77 & 13.57 & 20.77 \\
\hline 3 & 4 & 2 & 0.18 & 4.12 & 3.06 & 25.88 & 31.31 \\
\hline 3 & 4 & 2 & 0.18 & 3.82 & 2.78 & 27.32 & 31.31 \\
\hline 3 & 4 & 2 & 0.18 & 4.97 & 3.82 & 23.08 & 31.31 \\
\hline 4 & 4 & 2 & 0.22 & 3.75 & 3.14 & 16.26 & 17.60 \\
\hline 4 & 4 & 2 & 0.22 & 3.50 & 2.86 & 18.31 & 17.60 \\
\hline 4 & 4 & 2 & 0.22 & 4.44 & 3.93 & 11.49 & 17.60 \\
\hline 4 & 4 & 2 & 0.22 & 4.95 & 4.49 & 9.23 & 17.60 \\
\hline 4 & 4 & 2 & 0.23 & 4.01 & 3.17 & 20.90 & 22.85 \\
\hline 4 & 4 & 2 & 0.23 & 3.74 & 2.88 & 22.87 & 22.85 \\
\hline
\end{tabular}




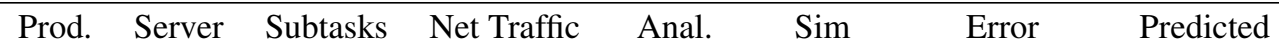
Intensity Flow T. Flow T. sim-anal (\%) $\quad$ Error (\%)

\begin{tabular}{|c|c|c|c|c|c|c|c|}
\hline 4 & 4 & 2 & 0.23 & 4.74 & 3.96 & 16.41 & 22.85 \\
\hline 4 & 4 & 2 & 0.23 & 5.27 & 4.53 & 14.07 & 22.85 \\
\hline 4 & 4 & 2 & 0.26 & 4.64 & 3.24 & 30.23 & 33.30 \\
\hline 4 & 4 & 2 & 0.26 & 4.34 & 2.94 & 32.20 & 33.30 \\
\hline 4 & 4 & 2 & 0.26 & 5.47 & 4.05 & 26.03 & 33.30 \\
\hline 4 & 4 & 2 & 0.26 & 6.05 & 4.62 & 23.57 & 33.30 \\
\hline 5 & 4 & 2 & 0.26 & 3.87 & 3.22 & 16.74 & 20.09 \\
\hline 5 & 4 & 2 & 0.26 & 3.62 & 2.93 & 19.10 & 20.09 \\
\hline 5 & 4 & 2 & 0.26 & 4.57 & 4.03 & 11.94 & 20.09 \\
\hline 5 & 4 & 2 & 0.26 & 5.07 & 4.60 & 9.23 & 20.09 \\
\hline 5 & 4 & 2 & 0.26 & 3.23 & 2.48 & 23.35 & 20.09 \\
\hline 5 & 4 & 2 & 0.27 & 4.13 & 3.25 & 21.24 & 25.32 \\
\hline 5 & 4 & 2 & 0.27 & 3.87 & 2.96 & 23.47 & 25.32 \\
\hline 5 & 4 & 2 & 0.27 & 4.87 & 4.07 & 16.53 & 25.32 \\
\hline 5 & 4 & 2 & 0.27 & 5.40 & 4.65 & 13.97 & 25.32 \\
\hline 5 & 4 & 2 & 0.27 & 3.47 & 2.50 & 27.91 & 25.32 \\
\hline 5 & 4 & 2 & 0.30 & 4.85 & 3.34 & 31.16 & 35.73 \\
\hline 5 & 4 & 2 & 0.30 & 4.53 & 3.04 & 33.01 & 35.73 \\
\hline 5 & 4 & 2 & 0.30 & 5.66 & 4.17 & 26.24 & 35.73 \\
\hline 5 & 4 & 2 & 0.30 & 6.26 & 4.77 & 23.80 & 35.73 \\
\hline 5 & 4 & 2 & 0.30 & 4.08 & 2.57 & 37.10 & 35.73 \\
\hline 6 & 4 & 2 & 0.30 & 4.03 & 3.33 & 17.45 & 22.51 \\
\hline 6 & 4 & 2 & 0.30 & 3.78 & 3.02 & 20.12 & 22.51 \\
\hline 6 & 4 & 2 & 0.30 & 4.73 & 4.16 & 12.08 & 22.51 \\
\hline 6 & 4 & 2 & 0.30 & 5.22 & 4.75 & 8.93 & 22.51 \\
\hline 6 & 4 & 2 & 0.30 & 3.39 & 2.56 & 24.61 & 22.51 \\
\hline 6 & 4 & 2 & 0.30 & 3.57 & 2.77 & 22.39 & 22.51 \\
\hline 6 & 4 & 2 & 0.31 & 4.33 & 3.37 & 22.15 & 27.71 \\
\hline 6 & 4 & 2 & 0.31 & 4.06 & 3.06 & 24.63 & 27.71 \\
\hline 6 & 4 & 2 & 0.31 & 5.05 & 4.21 & 16.68 & 27.71 \\
\hline 6 & 4 & 2 & 0.31 & 5.57 & 4.81 & 13.58 & 27.71 \\
\hline 6 & 4 & 2 & 0.31 & 3.66 & 2.59 & 29.21 & 27.71 \\
\hline 6 & 4 & 2 & 0.31 & 3.84 & 2.81 & 26.85 & 27.71 \\
\hline 6 & 4 & 2 & 0.35 & 5.10 & 3.48 & 31.81 & 38.07 \\
\hline 6 & 4 & 2 & 0.35 & 4.82 & 3.16 & 34.37 & 38.07 \\
\hline 6 & 4 & 2 & 0.35 & 5.93 & 4.35 & 26.61 & 38.07 \\
\hline 6 & 4 & 2 & 0.35 & 6.50 & 4.97 & 23.53 & 38.07 \\
\hline 6 & 4 & 2 & 0.35 & 4.35 & 2.68 & 38.45 & 38.07 \\
\hline 6 & 4 & 2 & 0.35 & 4.57 & 2.90 & 36.49 & 38.07 \\
\hline 1 & 4 & 3 & 0.08 & 4.03 & 3.34 & 17.05 & 8.03 \\
\hline
\end{tabular}




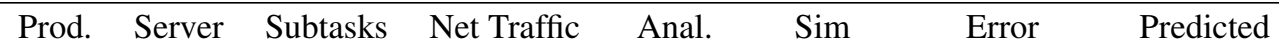
Intensity Flow T. Flow T. sim-anal (\%) Error (\%)

\begin{tabular}{|c|c|c|c|c|c|c|c|}
\hline 1 & 4 & 3 & 0.08 & 4.25 & 3.35 & 21.25 & 13.36 \\
\hline 1 & 4 & 3 & 0.09 & 4.78 & 3.37 & 29.58 & 24.01 \\
\hline 2 & 4 & 3 & 0.14 & 4.22 & 3.48 & 17.64 & 10.14 \\
\hline 2 & 4 & 3 & 0.14 & 3.90 & 3.16 & 19.01 & 10.14 \\
\hline 2 & 4 & 3 & 0.15 & 4.48 & 3.50 & 21.99 & 15.43 \\
\hline 2 & 4 & 3 & 0.15 & 4.13 & 3.18 & 23.08 & 15.43 \\
\hline 2 & 4 & 3 & 0.17 & 5.08 & 3.54 & 30.43 & 25.99 \\
\hline 2 & 4 & 3 & 0.17 & 4.71 & 3.21 & 31.80 & 25.99 \\
\hline 3 & 4 & 3 & 0.23 & 4.69 & 3.70 & 21.25 & 12.01 \\
\hline 3 & 4 & 3 & 0.23 & 4.38 & 3.36 & 23.34 & 12.01 \\
\hline 3 & 4 & 3 & 0.23 & 5.57 & 4.62 & 17.10 & 12.01 \\
\hline 3 & 4 & 3 & 0.25 & 5.01 & 3.73 & 25.52 & 17.25 \\
\hline 3 & 4 & 3 & 0.25 & 4.67 & 3.39 & 27.36 & 17.25 \\
\hline 3 & 4 & 3 & 0.25 & 5.93 & 4.66 & 21.33 & 17.25 \\
\hline 3 & 4 & 3 & 0.27 & 5.81 & 3.81 & 34.32 & 27.69 \\
\hline 3 & 4 & 3 & 0.27 & 5.42 & 3.47 & 36.08 & 27.69 \\
\hline 3 & 4 & 3 & 0.27 & 6.84 & 4.77 & 30.31 & 27.69 \\
\hline 4 & 4 & 3 & 0.33 & 5.49 & 4.01 & 26.92 & 13.76 \\
\hline 4 & 4 & 3 & 0.33 & 5.19 & 3.65 & 29.75 & 13.76 \\
\hline 4 & 4 & 3 & 0.33 & 6.33 & 5.01 & 20.80 & 13.76 \\
\hline 4 & 4 & 3 & 0.33 & 6.93 & 5.73 & 17.32 & 13.76 \\
\hline 4 & 4 & 3 & 0.35 & 5.91 & 4.07 & 31.09 & 18.94 \\
\hline 4 & 4 & 3 & 0.35 & 5.59 & 3.70 & 33.77 & 18.94 \\
\hline 4 & 4 & 3 & 0.35 & 6.81 & 5.09 & 25.20 & 18.94 \\
\hline 4 & 4 & 3 & 0.35 & 7.45 & 5.82 & 21.82 & 18.94 \\
\hline 4 & 4 & 3 & 0.39 & 7.08 & 4.24 & 40.12 & 29.24 \\
\hline 4 & 4 & 3 & 0.39 & 6.71 & 3.86 & 42.56 & 29.24 \\
\hline 4 & 4 & 3 & 0.39 & 8.07 & 5.30 & 34.28 & 29.24 \\
\hline 4 & 4 & 3 & 0.39 & 8.78 & 6.06 & 30.97 & 29.24 \\
\hline 5 & 4 & 3 & 0.38 & 5.80 & 4.20 & 27.60 & 16.07 \\
\hline 5 & 4 & 3 & 0.38 & 5.51 & 3.82 & 30.71 & 16.07 \\
\hline 5 & 4 & 3 & 0.38 & 6.67 & 5.25 & 21.24 & 16.07 \\
\hline 5 & 4 & 3 & 0.38 & 7.25 & 6.00 & 17.23 & 16.07 \\
\hline 5 & 4 & 3 & 0.38 & 5.06 & 3.23 & 36.18 & 16.07 \\
\hline 5 & 4 & 3 & 0.40 & 6.33 & 4.29 & 32.29 & 21.23 \\
\hline 5 & 4 & 3 & 0.40 & 6.01 & 3.90 & 35.15 & 21.23 \\
\hline 5 & 4 & 3 & 0.40 & 7.21 & 5.36 & 25.62 & 21.23 \\
\hline 5 & 4 & 3 & 0.40 & 7.84 & 6.13 & 21.90 & 21.23 \\
\hline 5 & 4 & 3 & 0.40 & 5.52 & 3.30 & 40.28 & 21.23 \\
\hline 5 & 4 & 3 & 0.45 & 7.73 & 4.52 & 41.53 & 31.46 \\
\hline
\end{tabular}




\begin{tabular}{|c|c|c|c|c|c|c|c|}
\hline Prod. & Server & Subtasks & $\begin{array}{l}\text { Net Traffic } \\
\text { Intensity }\end{array}$ & $\begin{array}{l}\text { Anal. } \\
\text { Flow T. }\end{array}$ & $\begin{array}{c}\text { Sim } \\
\text { Flow T. }\end{array}$ & $\begin{array}{c}\text { Error } \\
\text { sim-anal }(\%)\end{array}$ & $\begin{array}{l}\text { Predicted } \\
\text { Error }(\%)\end{array}$ \\
\hline 5 & 4 & 3 & 0.45 & 7.36 & 4.11 & 44.18 & 31.46 \\
\hline 5 & 4 & 3 & 0.45 & 8.69 & 5.65 & 35.03 & 31.46 \\
\hline 5 & 4 & 3 & 0.45 & 9.39 & 6.45 & 31.28 & 31.46 \\
\hline 5 & 4 & 3 & 0.45 & 6.81 & 3.48 & 48.97 & 31.46 \\
\hline 6 & 4 & 3 & 0.44 & 6.29 & 4.48 & 28.76 & 18.27 \\
\hline 6 & 4 & 3 & 0.44 & 6.00 & 4.07 & 32.09 & 18.27 \\
\hline 6 & 4 & 3 & 0.44 & 7.10 & 5.60 & 21.15 & 18.27 \\
\hline 6 & 4 & 3 & 0.44 & 7.69 & 6.40 & 16.78 & 18.27 \\
\hline 6 & 4 & 3 & 0.44 & 5.54 & 3.45 & 37.86 & 18.27 \\
\hline 6 & 4 & 3 & 0.44 & 5.74 & 3.73 & 35.02 & 18.27 \\
\hline 6 & 4 & 3 & 0.47 & 6.91 & 4.60 & 33.36 & 23.39 \\
\hline 6 & 4 & 3 & 0.47 & 6.62 & 4.19 & 36.74 & 23.39 \\
\hline 6 & 4 & 3 & 0.47 & 7.76 & 5.75 & 25.87 & 23.39 \\
\hline 6 & 4 & 3 & 0.47 & 8.41 & 6.58 & 21.77 & 23.39 \\
\hline 6 & 4 & 3 & 0.47 & 6.12 & 3.54 & 42.11 & 23.39 \\
\hline 6 & 4 & 3 & 0.47 & 6.35 & 3.84 & 39.54 & 23.39 \\
\hline 6 & 4 & 3 & 0.52 & 8.64 & 4.95 & 42.75 & 33.54 \\
\hline 6 & 4 & 3 & 0.52 & 8.30 & 4.50 & 45.85 & 33.54 \\
\hline 6 & 4 & 3 & 0.52 & 9.61 & 6.18 & 35.66 & 33.54 \\
\hline 6 & 4 & 3 & 0.52 & 10.29 & 7.06 & 31.38 & 33.54 \\
\hline 6 & 4 & 3 & 0.52 & 7.77 & 3.80 & 51.03 & 33.54 \\
\hline 6 & 4 & 3 & 0.52 & 8.00 & 4.12 & 48.46 & 33.54 \\
\hline
\end{tabular}

Approximate and simulated flow times for the $E_{2}-\operatorname{Exp}$ case

\begin{tabular}{|c|c|c|c|c|c|c|c|}
\hline Prod. & Server & Subtasks & $\begin{array}{c}\text { Net Traffic } \\
\text { Intensity }\end{array}$ & \multicolumn{1}{c}{$\begin{array}{c}\text { Anal. } \\
\text { Flow T. }\end{array}$} & \multicolumn{2}{c}{$\begin{array}{c}\text { Sim. } \\
\text { Flow }\end{array}$} & \multicolumn{2}{c|}{$\begin{array}{c}\text { Error } \\
\text { sim-anal (\%) }\end{array}$} & $\begin{array}{c}\text { Predicted } \\
\text { Error (\%) }\end{array}$ \\
\hline 1 & 2 & 2 & 0.10 & 3.08 & 3.24 & 5.23 & 0.79 \\
\hline 1 & 2 & 2 & 0.11 & 3.25 & 3.25 & 0.03 & 6.10 \\
\hline 1 & 2 & 2 & 0.12 & 3.67 & 3.29 & 10.40 & 16.72 \\
\hline 2 & 2 & 2 & 0.19 & 3.34 & 3.51 & 4.98 & 2.65 \\
\hline 2 & 2 & 2 & 0.19 & 3.10 & 3.19 & 2.78 & 2.65 \\
\hline 2 & 2 & 2 & 0.20 & 3.57 & 3.54 & 0.62 & 7.91 \\
\hline 2 & 2 & 2 & 0.20 & 3.30 & 3.22 & 2.47 & 7.91 \\
\hline 2 & 2 & 2 & 0.22 & 4.06 & 3.63 & 10.62 & 18.41 \\
\hline 2 & 2 & 2 & 0.22 & 3.78 & 3.30 & 12.80 & 18.41 \\
\hline 3 & 2 & 2 & 0.31 & 3.98 & 3.98 & 0.04 & 4.19 \\
\hline 3 & 2 & 2 & 0.31 & 3.75 & 3.61 & 3.67 & 4.19 \\
\hline 3 & 2 & 2 & 0.31 & 4.60 & 4.97 & 8.06 & 4.19 \\
\hline 3 & 2 & 2 & 0.33 & 4.28 & 4.05 & 5.40 & 9.39 \\
\hline
\end{tabular}




\begin{tabular}{|c|c|c|c|c|c|c|c|}
\hline Prod. & Server & Subtasks & $\begin{array}{c}\text { Net Traffic } \\
\text { Intensity }\end{array}$ & $\begin{array}{c}\text { Anal. } \\
\text { Flow T. }\end{array}$ & $\begin{array}{l}\text { Sim. } \\
\text { Flow }\end{array}$ & $\begin{array}{c}\text { Error } \\
\text { sim-anal }(\%)\end{array}$ & $\begin{array}{l}\text { Predicted } \\
\text { Error }(\%)\end{array}$ \\
\hline 3 & 2 & 2 & 0.33 & 4.04 & 3.68 & 8.83 & 9.39 \\
\hline 3 & 2 & 2 & 0.33 & 4.95 & 5.06 & 2.27 & 9.39 \\
\hline 3 & 2 & 2 & 0.37 & 5.06 & 4.25 & 16.04 & 19.72 \\
\hline 3 & 2 & 2 & 0.37 & 4.77 & 3.86 & 19.02 & 19.72 \\
\hline 3 & 2 & 2 & 0.37 & 5.79 & 5.31 & 8.38 & 19.72 \\
\hline 4 & 2 & 2 & 0.44 & 5.16 & 4.73 & 8.21 & 5.57 \\
\hline 4 & 2 & 2 & 0.44 & 4.95 & 4.30 & 13.02 & 5.57 \\
\hline 4 & 2 & 2 & 0.44 & 5.75 & 5.92 & 2.88 & 5.57 \\
\hline 4 & 2 & 2 & 0.44 & 6.16 & 6.76 & 9.69 & 5.57 \\
\hline 4 & 2 & 2 & 0.47 & 5.67 & 4.90 & 13.43 & 10.69 \\
\hline 4 & 2 & 2 & 0.47 & 5.45 & 4.46 & 18.16 & 10.69 \\
\hline 4 & 2 & 2 & 0.47 & 6.30 & 6.13 & 2.68 & 10.69 \\
\hline 4 & 2 & 2 & 0.47 & 6.74 & 7.01 & 4.02 & 10.69 \\
\hline 4 & 2 & 2 & 0.52 & 7.05 & 5.37 & 23.77 & 20.84 \\
\hline 4 & 2 & 2 & 0.52 & 6.80 & 4.89 & 28.17 & 20.84 \\
\hline 4 & 2 & 2 & 0.52 & 7.76 & 6.72 & 13.42 & 20.84 \\
\hline 4 & 2 & 2 & 0.52 & 8.26 & 7.68 & 7.08 & 20.84 \\
\hline 5 & 2 & 2 & 0.51 & 5.79 & 5.26 & 9.18 & 7.70 \\
\hline 5 & 2 & 2 & 0.51 & 5.59 & 4.78 & 14.52 & 7.70 \\
\hline 5 & 2 & 2 & 0.51 & 6.36 & 6.57 & 3.40 & 7.70 \\
\hline 5 & 2 & 2 & 0.51 & 6.75 & 7.51 & 11.19 & 7.70 \\
\hline 5 & 2 & 2 & 0.51 & 5.27 & 4.04 & 23.28 & 7.70 \\
\hline 5 & 2 & 2 & 0.54 & 6.48 & 5.51 & 14.97 & 12.79 \\
\hline 5 & 2 & 2 & 0.54 & 6.25 & 5.01 & 19.86 & 12.79 \\
\hline 5 & 2 & 2 & 0.54 & 7.07 & 6.89 & 2.53 & 12.79 \\
\hline 5 & 2 & 2 & 0.54 & 7.54 & 7.88 & 4.51 & 12.79 \\
\hline 5 & 2 & 2 & 0.54 & 5.92 & 4.24 & 28.34 & 12.79 \\
\hline 5 & 2 & 2 & 0.60 & 8.50 & 6.25 & 26.42 & 22.85 \\
\hline 5 & 2 & 2 & 0.60 & 8.25 & 5.68 & 31.08 & 22.85 \\
\hline 5 & 2 & 2 & 0.60 & 9.17 & 7.82 & 14.76 & 22.85 \\
\hline 5 & 2 & 2 & 0.60 & 9.67 & 8.93 & 7.66 & 22.85 \\
\hline 5 & 2 & 2 & 0.60 & 7.83 & 4.81 & 38.59 & 22.85 \\
\hline 6 & 2 & 2 & 0.59 & 6.80 & 6.12 & 9.94 & 9.68 \\
\hline 6 & 2 & 2 & 0.59 & 6.58 & 5.56 & 15.40 & 9.68 \\
\hline 6 & 2 & 2 & 0.59 & 7.33 & 7.65 & 4.34 & 9.68 \\
\hline 6 & 2 & 2 & 0.59 & 7.73 & 8.74 & 13.14 & 9.68 \\
\hline 6 & 2 & 2 & 0.59 & 6.29 & 4.71 & 25.19 & 9.68 \\
\hline 6 & 2 & 2 & 0.59 & 6.42 & 5.10 & 20.61 & 9.68 \\
\hline 6 & 2 & 2 & 0.62 & 7.81 & 6.55 & 16.06 & 14.72 \\
\hline 6 & 2 & 2 & 0.62 & 7.59 & 5.96 & 21.52 & 14.72 \\
\hline
\end{tabular}


$\begin{array}{lllllll}\text { Prod. Server Subtasks Net Traffic Anal. Sim. } & \text { Error } & \text { Predicted }\end{array}$ Intensity Flow T. Flow sim-anal (\%) Error (\%)

\begin{tabular}{|c|c|c|c|c|c|c|c|}
\hline 6 & 2 & 2 & 0.62 & 8.42 & 8.19 & 2.71 & 14.72 \\
\hline 6 & 2 & 2 & 0.62 & 8.83 & 9.36 & 6.07 & 14.72 \\
\hline 6 & 2 & 2 & 0.62 & 7.29 & 5.04 & 30.80 & 14.72 \\
\hline 6 & 2 & 2 & 0.62 & 7.43 & 5.46 & 26.51 & 14.72 \\
\hline 6 & 2 & 2 & 0.69 & 11.00 & 7.92 & 27.99 & 24.67 \\
\hline 6 & 2 & 2 & 0.69 & 10.79 & 7.20 & 33.27 & 24.67 \\
\hline 6 & 2 & 2 & 0.69 & 11.68 & 9.90 & 15.22 & 24.67 \\
\hline 6 & 2 & 2 & 0.69 & 12.16 & 11.32 & 6.91 & 24.67 \\
\hline 6 & 2 & 2 & 0.69 & 10.40 & 6.09 & 41.38 & 24.67 \\
\hline 6 & 2 & 2 & 0.69 & 10.57 & 6.60 & 37.56 & 24.67 \\
\hline 1 & 3 & 3 & 0.10 & 3.77 & 3.95 & 4.87 & 2.56 \\
\hline 1 & 3 & 3 & 0.11 & 3.97 & 3.97 & 0.13 & 7.88 \\
\hline 1 & 3 & 3 & 0.12 & 4.49 & 4.01 & 10.75 & 18.49 \\
\hline 2 & 3 & 3 & 0.19 & 4.06 & 4.27 & 5.22 & 4.42 \\
\hline 2 & 3 & 3 & 0.19 & 3.78 & 3.88 & 2.72 & 4.42 \\
\hline 2 & 3 & 3 & 0.20 & 4.32 & 4.31 & 0.25 & 9.69 \\
\hline 2 & 3 & 3 & 0.20 & 4.02 & 3.92 & 2.60 & 9.69 \\
\hline 2 & 3 & 3 & 0.22 & 4.95 & 4.41 & 11.02 & 20.18 \\
\hline 2 & 3 & 3 & 0.22 & 4.62 & 4.01 & 13.15 & 20.18 \\
\hline 3 & 3 & 3 & 0.31 & 4.82 & 4.82 & 0.09 & 5.96 \\
\hline 3 & 3 & 3 & 0.31 & 4.56 & 4.38 & 3.82 & 5.96 \\
\hline 3 & 3 & 3 & 0.31 & 5.56 & 6.03 & 8.46 & 5.96 \\
\hline 3 & 3 & 3 & 0.33 & 5.17 & 4.91 & 5.01 & 11.16 \\
\hline 3 & 3 & 3 & 0.33 & 4.89 & 4.47 & 8.67 & 11.16 \\
\hline 3 & 3 & 3 & 0.33 & 5.96 & 6.14 & 3.08 & 11.16 \\
\hline 3 & 3 & 3 & 0.37 & 6.09 & 5.14 & 15.49 & 21.49 \\
\hline 3 & 3 & 3 & 0.37 & 5.80 & 4.68 & 19.41 & 21.49 \\
\hline 3 & 3 & 3 & 0.37 & 6.97 & 6.43 & 7.75 & 21.49 \\
\hline 4 & 3 & 3 & 0.44 & 6.24 & 5.72 & 8.25 & 7.34 \\
\hline 4 & 3 & 3 & 0.44 & 5.98 & 5.20 & 12.97 & 7.34 \\
\hline 4 & 3 & 3 & 0.44 & 6.91 & 7.15 & 3.51 & 7.34 \\
\hline 4 & 3 & 3 & 0.44 & 7.43 & 8.18 & 10.08 & 7.34 \\
\hline 4 & 3 & 3 & 0.47 & 6.83 & 5.93 & 13.29 & 12.46 \\
\hline 4 & 3 & 3 & 0.47 & 6.59 & 5.39 & 18.20 & 12.46 \\
\hline 4 & 3 & 3 & 0.47 & 7.56 & 7.41 & 2.02 & 12.46 \\
\hline 4 & 3 & 3 & 0.47 & 8.12 & 8.47 & 4.32 & 12.46 \\
\hline 4 & 3 & 3 & 0.52 & 8.58 & 6.48 & 24.44 & 22.62 \\
\hline 4 & 3 & 3 & 0.52 & 8.26 & 5.89 & 28.63 & 22.62 \\
\hline 4 & 3 & 3 & 0.52 & 9.39 & 8.10 & 13.70 & 22.62 \\
\hline 4 & 3 & 3 & 0.52 & 9.99 & 9.26 & 7.33 & 22.62 \\
\hline
\end{tabular}


\begin{tabular}{lllllll}
\hline Prod. Server Subtasks Net Traffic Anal. Sim. & Error & Predicted
\end{tabular} Intensity Flow T. Flow sim-anal (\%) Error (\%)

\begin{tabular}{|c|c|c|c|c|c|c|c|}
\hline 5 & 3 & 3 & 0.51 & 6.97 & 6.34 & 9.00 & 9.48 \\
\hline 5 & 3 & 3 & 0.51 & 6.75 & 5.77 & 14.50 & 9.48 \\
\hline 5 & 3 & 3 & 0.51 & 7.64 & 7.93 & 3.77 & 9.48 \\
\hline 5 & 3 & 3 & 0.51 & 8.11 & 9.06 & 11.73 & 9.48 \\
\hline 5 & 3 & 3 & 0.51 & 6.39 & 4.88 & 23.61 & 9.48 \\
\hline 5 & 3 & 3 & 0.54 & 7.80 & 6.65 & 14.79 & 14.56 \\
\hline 5 & 3 & 3 & 0.54 & 7.55 & 6.04 & 19.99 & 14.56 \\
\hline 5 & 3 & 3 & 0.54 & 8.50 & 8.31 & 2.20 & 14.56 \\
\hline 5 & 3 & 3 & 0.54 & 9.01 & 9.50 & 5.44 & 14.56 \\
\hline 5 & 3 & 3 & 0.54 & 7.17 & 5.11 & 28.67 & 14.56 \\
\hline 5 & 3 & 3 & 0.60 & 10.22 & 7.53 & 26.37 & 24.62 \\
\hline 5 & 3 & 3 & 0.60 & 9.95 & 6.84 & 31.26 & 24.62 \\
\hline 5 & 3 & 3 & 0.60 & 11.01 & 9.41 & 14.57 & 24.62 \\
\hline 5 & 3 & 3 & 0.60 & 11.57 & 10.75 & 7.11 & 24.62 \\
\hline 5 & 3 & 3 & 0.60 & 9.52 & 5.79 & 39.21 & 24.62 \\
\hline 6 & 3 & 3 & 0.59 & 8.19 & 7.37 & 10.06 & 11.45 \\
\hline 6 & 3 & 3 & 0.59 & 7.96 & 6.70 & 15.88 & 11.45 \\
\hline 6 & 3 & 3 & 0.59 & 8.82 & 9.21 & 4.42 & 11.45 \\
\hline 6 & 3 & 3 & 0.59 & 9.27 & 10.52 & 13.55 & 11.45 \\
\hline 6 & 3 & 3 & 0.59 & 7.63 & 5.67 & 25.71 & 11.45 \\
\hline 6 & 3 & 3 & 0.59 & 7.79 & 6.14 & 21.17 & 11.45 \\
\hline 6 & 3 & 3 & 0.62 & 9.36 & 7.88 & 15.82 & 16.49 \\
\hline 6 & 3 & 3 & 0.62 & 9.14 & 7.17 & 21.64 & 16.49 \\
\hline 6 & 3 & 3 & 0.62 & 10.03 & 9.85 & 1.74 & 16.49 \\
\hline 6 & 3 & 3 & 0.62 & 10.53 & 11.26 & 6.93 & 16.49 \\
\hline 6 & 3 & 3 & 0.62 & 8.80 & 6.06 & 31.07 & 16.49 \\
\hline 6 & 3 & 3 & 0.62 & 8.94 & 6.57 & 26.56 & 16.49 \\
\hline 6 & 3 & 3 & 0.69 & 13.25 & 9.50 & 28.27 & 26.44 \\
\hline 6 & 3 & 3 & 0.69 & 12.97 & 8.64 & 33.37 & 26.44 \\
\hline 6 & 3 & 3 & 0.69 & 14.00 & 11.88 & 15.13 & 26.44 \\
\hline 6 & 3 & 3 & 0.69 & 14.51 & 13.58 & 6.44 & 26.44 \\
\hline 6 & 3 & 3 & 0.69 & 12.58 & 7.31 & 41.91 & 26.44 \\
\hline 6 & 3 & 3 & 0.69 & 12.78 & 7.92 & 38.03 & 26.44 \\
\hline 1 & 3 & 2 & 0.07 & 3.41 & 3.15 & 7.40 & 5.55 \\
\hline 1 & 3 & 2 & 0.07 & 3.59 & 3.16 & 11.88 & 10.89 \\
\hline 1 & 3 & 2 & 0.08 & 4.03 & 3.18 & 21.05 & 21.55 \\
\hline 2 & 3 & 2 & 0.13 & 3.59 & 3.32 & $\begin{array}{l}7.59 \\
\end{array}$ & 7.74 \\
\hline 2 & 3 & 2 & 0.13 & 3.31 & 3.01 & 8.92 & 7.74 \\
\hline 2 & 3 & 2 & 0.13 & 3.80 & 3.33 & 12.33 & 13.04 \\
\hline 2 & 3 & 2 & 0.13 & 3.51 & 3.03 & 13.66 & 13.04 \\
\hline
\end{tabular}




\begin{tabular}{|c|c|c|c|c|c|c|c|}
\hline Prod. & Server & Subtasks & $\begin{array}{c}\text { Net Traffic } \\
\text { Intensity }\end{array}$ & $\begin{array}{c}\text { Anal. } \\
\text { Flow T. }\end{array}$ & $\begin{array}{l}\text { Sim. } \\
\text { Flow }\end{array}$ & $\begin{array}{c}\text { Error } \\
\text { sim-anal }(\%)\end{array}$ & $\begin{array}{l}\text { Predicted } \\
\text { Error }(\%)\end{array}$ \\
\hline 2 & 3 & 2 & 0.15 & 4.30 & 3.38 & 21.35 & 23.62 \\
\hline 2 & 3 & 2 & 0.15 & 3.98 & 3.07 & 22.76 & 23.62 \\
\hline 3 & 3 & 2 & 0.21 & 4.01 & 3.57 & 11.11 & 9.72 \\
\hline 3 & 3 & 2 & 0.21 & 3.73 & 3.24 & 13.13 & 9.72 \\
\hline 3 & 3 & 2 & 0.21 & 4.75 & 4.46 & 6.18 & 9.72 \\
\hline 3 & 3 & 2 & 0.22 & 4.27 & 3.60 & 15.66 & 14.98 \\
\hline 3 & 3 & 2 & 0.22 & 3.98 & 3.28 & 17.79 & 14.98 \\
\hline 3 & 3 & 2 & 0.22 & 5.05 & 4.50 & 10.89 & 14.98 \\
\hline 3 & 3 & 2 & 0.24 & 4.91 & 3.70 & 24.67 & 25.45 \\
\hline 3 & 3 & 2 & 0.24 & 4.58 & 3.36 & 26.67 & 25.45 \\
\hline 3 & 3 & 2 & 0.24 & 5.79 & 4.62 & 20.15 & 25.45 \\
\hline 4 & 3 & 2 & 0.30 & 4.69 & 3.91 & 16.53 & 11.59 \\
\hline 4 & 3 & 2 & 0.30 & 4.42 & 3.56 & 19.51 & 11.59 \\
\hline 4 & 3 & 2 & 0.30 & 5.42 & 4.89 & 9.72 & 11.59 \\
\hline 4 & 3 & 2 & 0.30 & 5.94 & 5.59 & 5.93 & 11.59 \\
\hline 4 & 3 & 2 & 0.31 & 5.04 & 3.98 & 21.07 & 16.80 \\
\hline 4 & 3 & 2 & 0.31 & 4.76 & 3.62 & 24.02 & 16.80 \\
\hline 4 & 3 & 2 & 0.31 & 5.81 & 4.98 & 14.34 & 16.80 \\
\hline 4 & 3 & 2 & 0.31 & 6.37 & 5.69 & 10.65 & 16.80 \\
\hline 4 & 3 & 2 & 0.35 & 5.97 & 4.16 & 30.29 & 27.15 \\
\hline 4 & 3 & 2 & 0.35 & 5.65 & 3.78 & 33.07 & 27.15 \\
\hline 4 & 3 & 2 & 0.35 & 6.82 & 5.20 & 23.77 & 27.15 \\
\hline 4 & 3 & 2 & 0.35 & 7.44 & 5.94 & 20.11 & 27.15 \\
\hline 5 & 3 & 2 & 0.34 & 4.96 & 4.12 & 16.94 & 13.96 \\
\hline 5 & 3 & 2 & 0.34 & 4.69 & 3.74 & 20.19 & 13.96 \\
\hline 5 & 3 & 2 & 0.34 & 5.68 & 5.15 & 9.42 & 13.96 \\
\hline 5 & 3 & 2 & 0.34 & 6.19 & 5.88 & 5.04 & 13.96 \\
\hline 5 & 3 & 2 & 0.34 & 4.29 & 3.17 & 26.14 & 13.96 \\
\hline 5 & 3 & 2 & 0.36 & 5.38 & 4.21 & 21.74 & 19.15 \\
\hline 5 & 3 & 2 & 0.36 & 5.11 & 3.83 & 25.12 & 19.15 \\
\hline 5 & 3 & 2 & 0.36 & 6.14 & 5.26 & 14.35 & 19.15 \\
\hline 5 & 3 & 2 & 0.36 & 6.68 & 6.01 & 10.03 & 19.15 \\
\hline 5 & 3 & 2 & 0.36 & 4.67 & 3.24 & 30.63 & 19.15 \\
\hline 5 & 3 & 2 & 0.40 & 6.46 & 4.45 & 31.15 & 29.44 \\
\hline 5 & 3 & 2 & 0.40 & 6.12 & 4.04 & 34.02 & 29.44 \\
\hline 5 & 3 & 2 & 0.40 & 7.30 & 5.56 & 23.92 & 29.44 \\
\hline 5 & 3 & 2 & 0.40 & 7.94 & 6.35 & 20.05 & 29.44 \\
\hline 5 & 3 & 2 & 0.40 & 5.66 & 3.42 & 39.58 & 29.44 \\
\hline 6 & 3 & 2 & 0.39 & 5.34 & 4.41 & 17.52 & 16.23 \\
\hline 6 & 3 & 2 & 0.39 & 5.08 & 4.00 & 21.17 & 16.23 \\
\hline
\end{tabular}


$\begin{array}{lllllll}\text { Prod. Server Subtasks Net Traffic Anal. Sim. } & \text { Error } & \text { Predicted }\end{array}$ Intensity Flow T. Flow sim-anal (\%) Error (\%)

\begin{tabular}{|c|c|c|c|c|c|c|c|}
\hline 6 & 3 & 2 & 0.39 & 6.06 & 5.51 & 9.11 & 16.23 \\
\hline 6 & 3 & 2 & 0.39 & 6.57 & 6.29 & 4.15 & 16.23 \\
\hline 6 & 3 & 2 & 0.39 & 4.68 & 3.39 & 27.63 & 16.23 \\
\hline 6 & 3 & 2 & 0.39 & 4.87 & 3.67 & 24.69 & 16.23 \\
\hline 6 & 3 & 2 & 0.41 & 5.81 & 4.53 & 21.99 & 21.38 \\
\hline 6 & 3 & 2 & 0.41 & 5.55 & 4.12 & 25.75 & 21.38 \\
\hline 6 & 3 & 2 & 0.41 & 6.56 & 5.66 & 13.68 & 21.38 \\
\hline 6 & 3 & 2 & 0.41 & 7.11 & 6.47 & 8.90 & 21.38 \\
\hline 6 & 3 & 2 & 0.41 & 5.10 & 3.49 & 31.61 & 21.38 \\
\hline 6 & 3 & 2 & 0.41 & 5.32 & 3.78 & 29.06 & 21.38 \\
\hline 6 & 3 & 2 & 0.46 & 14.84 & 4.87 & 67.21 & 31.60 \\
\hline 6 & 3 & 2 & 0.46 & 14.53 & 4.42 & 69.56 & 31.60 \\
\hline 6 & 3 & 2 & 0.46 & 15.58 & 6.08 & 60.95 & 31.60 \\
\hline 6 & 3 & 2 & 0.46 & 16.19 & 6.95 & 57.05 & 31.60 \\
\hline 6 & 3 & 2 & 0.46 & 14.18 & 3.74 & 73.60 & 31.60 \\
\hline 6 & 3 & 2 & 0.46 & 14.32 & 4.06 & 71.68 & 31.60 \\
\hline 1 & 4 & 4 & 0.10 & 4.28 & 4.48 & 4.86 & 4.33 \\
\hline 1 & 4 & 4 & 0.11 & 4.51 & 4.50 & 0.23 & 9.65 \\
\hline 1 & 4 & 4 & 0.12 & 5.09 & 4.55 & 10.71 & 20.27 \\
\hline 2 & 4 & 4 & 0.19 & 4.60 & 4.84 & 5.25 & 6.19 \\
\hline 2 & 4 & 4 & 0.19 & 4.28 & 4.40 & 2.79 & 6.19 \\
\hline 2 & 4 & 4 & 0.20 & 4.90 & 4.88 & 0.30 & 11.46 \\
\hline 2 & 4 & 4 & 0.20 & 4.56 & 4.44 & 2.64 & 11.46 \\
\hline 2 & 4 & 4 & 0.22 & 5.60 & 4.99 & 10.83 & 21.95 \\
\hline 2 & 4 & 4 & 0.22 & 5.22 & 4.54 & 13.02 & 21.95 \\
\hline 3 & 4 & 4 & 0.31 & 5.44 & 5.45 & 0.23 & 7.73 \\
\hline 3 & 4 & 4 & 0.31 & 5.14 & 4.96 & 3.61 & 7.73 \\
\hline 3 & 4 & 4 & 0.31 & 6.26 & 6.81 & 8.91 & 7.73 \\
\hline 3 & 4 & 4 & 0.33 & 5.86 & 5.55 & 5.22 & 12.93 \\
\hline 3 & 4 & 4 & 0.33 & 5.54 & 5.05 & 8.92 & 12.93 \\
\hline 3 & 4 & 4 & 0.33 & 6.73 & 6.94 & 3.15 & 12.93 \\
\hline 3 & 4 & 4 & 0.37 & 6.93 & 5.81 & 16.22 & 23.26 \\
\hline 3 & 4 & 4 & 0.37 & 6.57 & 5.28 & 19.58 & 23.26 \\
\hline 3 & 4 & 4 & 0.37 & 7.89 & 7.26 & 8.00 & 23.26 \\
\hline 4 & 4 & 4 & 0.44 & 7.04 & 6.45 & 8.33 & 9.12 \\
\hline 4 & 4 & 4 & 0.44 & 6.77 & 5.86 & 13.38 & 9.12 \\
\hline 4 & 4 & 4 & 0.44 & 7.81 & 8.06 & 3.24 & 9.12 \\
\hline 4 & 4 & 4 & 0.44 & 8.35 & 9.22 & 10.36 & 9.12 \\
\hline 4 & 4 & 4 & 0.47 & 7.73 & 6.68 & 13.58 & 14.24 \\
\hline 4 & 4 & 4 & 0.47 & 7.42 & 6.07 & 18.21 & 14.24 \\
\hline
\end{tabular}




\begin{tabular}{|c|c|c|c|c|c|c|c|}
\hline Prod. & Server & Subtasks & $\begin{array}{c}\text { Net Traffic } \\
\text { Intensity }\end{array}$ & $\begin{array}{c}\text { Anal. } \\
\text { Flow T. }\end{array}$ & $\begin{array}{l}\text { Sim. } \\
\text { Flow }\end{array}$ & $\begin{array}{c}\text { Error } \\
\text { sim-anal }(\%)\end{array}$ & $\begin{array}{l}\text { Predicted } \\
\text { Error }(\%)\end{array}$ \\
\hline 4 & 4 & 4 & 0.47 & 8.52 & 8.35 & 2.03 & 14.24 \\
\hline 4 & 4 & 4 & 0.47 & 9.09 & 9.54 & 4.90 & 14.24 \\
\hline 4 & 4 & 4 & 0.52 & 9.66 & 7.30 & 24.51 & 24.39 \\
\hline 4 & 4 & 4 & 0.52 & 9.33 & 6.63 & 28.93 & 24.39 \\
\hline 4 & 4 & 4 & 0.52 & 10.53 & 9.12 & 13.43 & 24.39 \\
\hline 4 & 4 & 4 & 0.52 & 11.22 & 10.42 & 7.07 & 24.39 \\
\hline 5 & 4 & 4 & 0.51 & 7.85 & 7.14 & 9.06 & 11.25 \\
\hline 5 & 4 & 4 & 0.51 & 7.61 & 6.49 & 14.70 & 11.25 \\
\hline 5 & 4 & 4 & 0.51 & 8.58 & 8.93 & 4.04 & 11.25 \\
\hline 5 & 4 & 4 & 0.51 & 9.11 & 10.20 & 11.96 & 11.25 \\
\hline 5 & 4 & 4 & 0.51 & 7.20 & 5.49 & 23.70 & 11.25 \\
\hline 5 & 4 & 4 & 0.54 & 8.77 & 7.48 & 14.69 & 16.33 \\
\hline 5 & 4 & 4 & 0.54 & 8.50 & 6.80 & 20.00 & 16.33 \\
\hline 5 & 4 & 4 & 0.54 & 9.53 & 9.35 & 1.90 & 16.33 \\
\hline 5 & 4 & 4 & 0.54 & 10.09 & 10.68 & 5.89 & 16.33 \\
\hline 5 & 4 & 4 & 0.54 & 8.10 & 5.75 & 28.96 & 16.33 \\
\hline 5 & 4 & 4 & 0.60 & 11.56 & 8.45 & 26.88 & 26.39 \\
\hline 5 & 4 & 4 & 0.60 & 11.25 & 7.69 & 31.71 & 26.39 \\
\hline 5 & 4 & 4 & 0.60 & 12.43 & 10.57 & 14.97 & 26.39 \\
\hline 5 & 4 & 4 & 0.60 & 13.01 & 12.08 & 7.17 & 26.39 \\
\hline 5 & 4 & 4 & 0.60 & 10.79 & 6.50 & 39.75 & 26.39 \\
\hline 6 & 4 & 4 & 0.59 & 9.19 & 8.28 & 9.96 & 13.23 \\
\hline 6 & 4 & 4 & 0.59 & 8.94 & 7.53 & 15.82 & 13.23 \\
\hline 6 & 4 & 4 & 0.59 & 9.87 & 10.35 & 4.82 & 13.23 \\
\hline 6 & 4 & 4 & 0.59 & 10.35 & 11.83 & 14.21 & 13.23 \\
\hline 6 & 4 & 4 & 0.59 & 8.58 & 6.37 & 25.81 & 13.23 \\
\hline 6 & 4 & 4 & 0.59 & 8.75 & 6.90 & 21.12 & 13.23 \\
\hline 6 & 4 & 4 & 0.62 & 10.57 & 8.85 & 16.29 & 18.26 \\
\hline 6 & 4 & 4 & 0.62 & 10.07 & 8.05 & 20.10 & 18.26 \\
\hline 6 & 4 & 4 & 0.62 & 10.43 & 11.06 & 6.05 & 18.26 \\
\hline 6 & 4 & 4 & 0.62 & 10.62 & 12.64 & 19.00 & 18.26 \\
\hline 6 & 4 & 4 & 0.62 & 9.94 & 6.81 & 31.51 & 18.26 \\
\hline 6 & 4 & 4 & 0.62 & 10.01 & 7.37 & 26.30 & 18.26 \\
\hline 6 & 4 & 4 & 0.69 & 14.84 & 10.65 & 28.23 & 28.22 \\
\hline 6 & 4 & 4 & 0.69 & 14.53 & 9.68 & 33.37 & 28.22 \\
\hline 6 & 4 & 4 & 0.69 & 15.58 & 13.32 & 14.54 & 28.22 \\
\hline 6 & 4 & 4 & 0.69 & 16.19 & 15.22 & 6.00 & 28.22 \\
\hline 6 & 4 & 4 & 0.69 & 14.18 & 8.19 & 42.22 & 28.22 \\
\hline 6 & 4 & 4 & 0.69 & 14.32 & 8.88 & 38.03 & 28.22 \\
\hline 1 & 4 & 2 & 0.10 & 3.30 & 3.11 & 5.76 & 9.58 \\
\hline
\end{tabular}


\begin{tabular}{lllllll}
\hline Prod. Server Subtasks Net Traffic Anal. Sim. & Error & Predicted
\end{tabular} Intensity Flow T. Flow sim-anal (\%) Error (\%)

\begin{tabular}{|c|c|c|c|c|c|c|c|}
\hline 1 & 4 & 2 & 0.11 & 3.48 & 3.12 & 10.22 & 14.90 \\
\hline 1 & 4 & 2 & 0.12 & 3.91 & 3.14 & 19.73 & 25.52 \\
\hline 2 & 4 & 2 & 0.19 & 3.43 & 3.23 & 5.78 & 11.44 \\
\hline 2 & 4 & 2 & 0.19 & 3.15 & 2.93 & 6.81 & 11.44 \\
\hline 2 & 4 & 2 & 0.20 & 3.62 & 3.24 & 10.48 & 16.71 \\
\hline 2 & 4 & 2 & 0.20 & 3.33 & 2.95 & 11.58 & 16.71 \\
\hline 2 & 4 & 2 & 0.22 & 4.10 & 3.27 & 20.15 & 27.20 \\
\hline 2 & 4 & 2 & 0.22 & 3.77 & 2.98 & 21.13 & 27.20 \\
\hline 3 & 4 & 2 & 0.31 & 3.73 & 3.40 & 8.91 & 12.98 \\
\hline 3 & 4 & 2 & 0.31 & 3.45 & 3.09 & 10.52 & 12.98 \\
\hline 3 & 4 & 2 & 0.31 & 4.47 & 4.25 & 4.96 & 12.98 \\
\hline 3 & 4 & 2 & 0.33 & 3.95 & 3.42 & 13.41 & 18.18 \\
\hline 3 & 4 & 2 & 0.33 & 3.67 & 3.11 & 15.26 & 18.18 \\
\hline 3 & 4 & 2 & 0.33 & 4.74 & 4.28 & 9.82 & 18.18 \\
\hline 3 & 4 & 2 & 0.37 & 4.52 & 3.48 & 22.95 & 28.51 \\
\hline 3 & 4 & 2 & 0.37 & 4.20 & 3.17 & 24.55 & 28.51 \\
\hline 3 & 4 & 2 & 0.37 & 5.41 & 4.35 & 19.44 & 28.51 \\
\hline 4 & 4 & 2 & 0.44 & 4.18 & 3.62 & 13.35 & 14.37 \\
\hline 4 & 4 & 2 & 0.44 & 3.91 & 3.29 & 15.92 & 14.37 \\
\hline 4 & 4 & 2 & 0.44 & 4.91 & 4.52 & 7.83 & 14.37 \\
\hline 4 & 4 & 2 & 0.44 & 5.42 & 5.17 & 4.63 & 14.37 \\
\hline 4 & 4 & 2 & 0.47 & 4.47 & 3.66 & 18.05 & 19.49 \\
\hline 4 & 4 & 2 & 0.47 & 4.19 & 3.33 & 20.59 & 19.49 \\
\hline 4 & 4 & 2 & 0.47 & 5.23 & 4.58 & 12.53 & 19.49 \\
\hline 4 & 4 & 2 & 0.47 & 5.80 & 5.23 & 9.85 & 19.49 \\
\hline 4 & 4 & 2 & 0.52 & 5.18 & 3.77 & 27.33 & 29.64 \\
\hline 4 & 4 & 2 & 0.52 & 4.86 & 3.42 & 29.57 & 29.64 \\
\hline 4 & 4 & 2 & 0.52 & 6.05 & 4.71 & 22.13 & 29.64 \\
\hline 4 & 4 & 2 & 0.52 & 6.66 & 5.38 & 19.25 & 29.64 \\
\hline 5 & 4 & 2 & 0.51 & 4.34 & 3.74 & 13.70 & 16.50 \\
\hline 5 & 4 & 2 & 0.51 & 4.08 & 3.40 & 16.59 & 16.50 \\
\hline 5 & 4 & 2 & 0.51 & 5.07 & 4.68 & 7.78 & 16.50 \\
\hline 5 & 4 & 2 & 0.51 & 5.60 & 5.35 & 4.46 & 16.50 \\
\hline 5 & 4 & 2 & 0.51 & 3.66 & 2.88 & 21.43 & 16.50 \\
\hline 5 & 4 & 2 & 0.54 & 4.66 & 3.80 & 18.53 & 21.58 \\
\hline 5 & 4 & 2 & 0.54 & 4.37 & 3.45 & 20.99 & 21.58 \\
\hline 5 & 4 & 2 & 0.54 & 5.42 & 4.74 & 12.52 & 21.58 \\
\hline 5 & 4 & 2 & 0.54 & 5.96 & 5.42 & 9.04 & 21.58 \\
\hline 5 & 4 & 2 & 0.54 & 3.95 & 2.92 & 26.11 & 21.58 \\
\hline 5 & 4 & 2 & 0.60 & 5.46 & 3.93 & 27.99 & 31.64 \\
\hline
\end{tabular}




\begin{tabular}{|c|c|c|c|c|c|c|c|}
\hline Prod. & Server & Subtasks & $\begin{array}{c}\text { Net Traffic } \\
\text { Intensity }\end{array}$ & $\begin{array}{c}\text { Anal. } \\
\text { Flow T. }\end{array}$ & $\begin{array}{l}\text { Sim. } \\
\text { Flow }\end{array}$ & $\begin{array}{c}\text { Error } \\
\text { sim-anal }(\%)\end{array}$ & $\begin{array}{l}\text { Predicted } \\
\text { Error }(\%)\end{array}$ \\
\hline 5 & 4 & 2 & 0.60 & 5.12 & 3.57 & 30.29 & 31.64 \\
\hline 5 & 4 & 2 & 0.60 & 6.31 & 4.91 & 22.21 & 31.64 \\
\hline 5 & 4 & 2 & 0.60 & 6.94 & 5.61 & 19.10 & 31.64 \\
\hline 5 & 4 & 2 & 0.60 & 4.65 & 3.02 & 34.99 & 31.64 \\
\hline 6 & 4 & 2 & 0.59 & 4.55 & 3.91 & 14.10 & 18.48 \\
\hline 6 & 4 & 2 & 0.59 & 4.28 & 3.55 & 16.95 & 18.48 \\
\hline 6 & 4 & 2 & 0.59 & 5.26 & 4.88 & 7.10 & 18.48 \\
\hline 6 & 4 & 2 & 0.59 & 5.79 & 5.58 & 3.57 & 18.48 \\
\hline 6 & 4 & 2 & 0.59 & 3.88 & 3.01 & 22.54 & 18.48 \\
\hline 6 & 4 & 2 & 0.59 & 4.04 & 3.26 & 19.50 & 18.48 \\
\hline 6 & 4 & 2 & 0.62 & 4.89 & 3.98 & 18.65 & 23.51 \\
\hline 6 & 4 & 2 & 0.62 & 4.60 & 3.61 & 21.46 & 23.51 \\
\hline 6 & 4 & 2 & 0.62 & 5.64 & 4.97 & 11.93 & 23.51 \\
\hline 6 & 4 & 2 & 0.62 & 6.19 & 5.68 & 8.20 & 23.51 \\
\hline 6 & 4 & 2 & 0.62 & 4.19 & 3.06 & 27.00 & 23.51 \\
\hline 6 & 4 & 2 & 0.62 & 4.39 & 3.31 & 24.50 & 23.51 \\
\hline 6 & 4 & 2 & 0.69 & 5.79 & 4.15 & 28.33 & 33.47 \\
\hline 6 & 4 & 2 & 0.69 & 5.49 & 3.77 & 31.29 & 33.47 \\
\hline 6 & 4 & 2 & 0.69 & 6.65 & 5.19 & 22.00 & 33.47 \\
\hline 6 & 4 & 2 & 0.69 & 7.26 & 5.93 & 18.29 & 33.47 \\
\hline 6 & 4 & 2 & 0.69 & 5.01 & 3.19 & 36.24 & 33.47 \\
\hline 6 & 4 & 2 & 0.69 & 5.23 & 3.46 & 33.79 & 33.47 \\
\hline 1 & 4 & 3 & 0.08 & 4.38 & 3.88 & 11.50 & 7.23 \\
\hline 1 & 4 & 3 & 0.08 & 4.62 & 3.89 & 15.95 & 12.56 \\
\hline 1 & 4 & 3 & 0.09 & 5.20 & 3.92 & 24.76 & 23.21 \\
\hline 2 & 4 & 3 & 0.14 & 4.65 & 4.09 & 11.92 & 9.34 \\
\hline 2 & 4 & 3 & 0.14 & 4.28 & 3.72 & 13.09 & 9.34 \\
\hline 2 & 4 & 3 & 0.15 & 4.92 & 4.12 & 16.20 & 14.63 \\
\hline 2 & 4 & 3 & 0.15 & 4.55 & 3.75 & 17.62 & 14.63 \\
\hline 2 & 4 & 3 & 0.17 & 5.60 & 4.19 & 25.20 & 25.19 \\
\hline 2 & 4 & 3 & 0.17 & 5.17 & 3.81 & 26.41 & 25.19 \\
\hline 3 & 4 & 3 & 0.23 & 5.26 & 4.45 & 15.52 & 11.21 \\
\hline 3 & 4 & 3 & 0.23 & 4.91 & 4.04 & 17.74 & 11.21 \\
\hline 3 & 4 & 3 & 0.23 & 6.18 & 5.56 & 10.13 & 11.21 \\
\hline 3 & 4 & 3 & 0.25 & 5.61 & 4.50 & 19.83 & 16.45 \\
\hline 3 & 4 & 3 & 0.25 & 5.26 & 4.09 & 22.15 & 16.45 \\
\hline 3 & 4 & 3 & 0.25 & 6.59 & 5.63 & 14.69 & 16.45 \\
\hline 3 & 4 & 3 & 0.27 & 6.52 & 4.64 & 28.88 & 26.89 \\
\hline 3 & 4 & 3 & 0.27 & 6.12 & 4.21 & 31.19 & 26.89 \\
\hline 3 & 4 & 3 & 0.27 & 7.63 & 5.79 & 24.01 & 26.89 \\
\hline
\end{tabular}


\begin{tabular}{lllllll}
\hline Prod. Server Subtasks Net Traffic Anal. Sim. & Error & Predicted
\end{tabular} Intensity Flow T. Flow sim-anal (\%) Error (\%)

\begin{tabular}{|c|c|c|c|c|c|c|c|}
\hline 4 & 4 & 3 & 0.33 & 6.28 & 4.95 & 21.09 & 12.96 \\
\hline 4 & 4 & 3 & 0.33 & 5.96 & 4.50 & 24.47 & 12.96 \\
\hline 4 & 4 & 3 & 0.33 & 7.19 & 6.19 & 13.89 & 12.96 \\
\hline 4 & 4 & 3 & 0.33 & 7.82 & 7.07 & 9.52 & 12.96 \\
\hline 4 & 4 & 3 & 0.35 & 6.80 & 5.06 & 25.67 & 18.14 \\
\hline 4 & 4 & 3 & 0.35 & 6.49 & 4.60 & 29.16 & 18.14 \\
\hline 4 & 4 & 3 & 0.35 & 7.77 & 6.32 & 18.69 & 18.14 \\
\hline 4 & 4 & 3 & 0.35 & 8.45 & 7.22 & 14.57 & 18.14 \\
\hline 4 & 4 & 3 & 0.39 & 8.18 & 5.32 & 34.93 & 28.44 \\
\hline 4 & 4 & 3 & 0.39 & 7.81 & 4.84 & 37.99 & 28.44 \\
\hline 4 & 4 & 3 & 0.39 & 9.22 & 6.66 & 27.81 & 28.44 \\
\hline 4 & 4 & 3 & 0.39 & 10.01 & 7.61 & 23.97 & 28.44 \\
\hline 5 & 4 & 3 & 0.38 & 6.73 & 5.26 & 21.88 & 15.27 \\
\hline 5 & 4 & 3 & 0.38 & 6.42 & 4.78 & 25.58 & 15.27 \\
\hline 5 & 4 & 3 & 0.38 & 7.62 & 6.57 & 13.71 & 15.27 \\
\hline 5 & 4 & 3 & 0.38 & 8.26 & 7.51 & 8.98 & 15.27 \\
\hline 5 & 4 & 3 & 0.38 & 5.93 & 4.05 & 31.82 & 15.27 \\
\hline 5 & 4 & 3 & 0.40 & 7.33 & 5.40 & 26.34 & 20.43 \\
\hline 5 & 4 & 3 & 0.40 & 6.99 & 4.91 & 29.72 & 20.43 \\
\hline 5 & 4 & 3 & 0.40 & 8.27 & 6.75 & 18.35 & 20.43 \\
\hline 5 & 4 & 3 & 0.40 & 8.93 & 7.71 & 13.62 & 20.43 \\
\hline 5 & 4 & 3 & 0.40 & 6.49 & 4.15 & 36.04 & 20.43 \\
\hline 5 & 4 & 3 & 0.45 & 9.00 & 5.77 & 35.87 & 30.66 \\
\hline 5 & 4 & 3 & 0.45 & 8.60 & 5.25 & 38.99 & 30.66 \\
\hline 5 & 4 & 3 & 0.45 & 9.99 & 7.21 & 27.81 & 30.66 \\
\hline 5 & 4 & 3 & 0.45 & 10.76 & 8.24 & 23.39 & 30.66 \\
\hline 5 & 4 & 3 & 0.45 & 8.03 & 4.44 & 44.71 & 30.66 \\
\hline 6 & 4 & 3 & 0.44 & 7.37 & 5.71 & 22.58 & 17.47 \\
\hline 6 & 4 & 3 & 0.44 & 7.06 & 5.19 & 26.45 & 17.47 \\
\hline 6 & 4 & 3 & 0.44 & 8.22 & 7.13 & 13.20 & 17.47 \\
\hline 6 & 4 & 3 & 0.44 & 8.84 & 8.15 & 7.77 & 17.47 \\
\hline 6 & 4 & 3 & 0.44 & 6.57 & 4.39 & 33.16 & 17.47 \\
\hline 6 & 4 & 3 & 0.44 & 6.78 & 4.76 & 29.83 & 17.47 \\
\hline 6 & 4 & 3 & 0.47 & 8.09 & 5.91 & 27.00 & 22.59 \\
\hline 6 & 4 & 3 & 0.47 & 7.79 & 5.37 & 31.09 & 22.59 \\
\hline 6 & 4 & 3 & 0.47 & 9.00 & 7.39 & 17.96 & 22.59 \\
\hline 6 & 4 & 3 & 0.47 & 9.65 & 8.44 & 12.54 & 22.59 \\
\hline 6 & 4 & 3 & 0.47 & 7.27 & 4.55 & 37.49 & 22.59 \\
\hline 6 & 4 & 3 & 0.47 & 7.49 & 4.92 & 34.27 & 22.59 \\
\hline 6 & 4 & 3 & 0.52 & 10.18 & 6.46 & 36.53 & 32.74 \\
\hline
\end{tabular}




\begin{tabular}{|c|c|c|c|c|c|c|c|}
\hline Prod. & Server & Subtasks & $\begin{array}{c}\text { Net Traffic } \\
\text { Intensity }\end{array}$ & $\begin{array}{c}\text { Anal. } \\
\text { Flow T. }\end{array}$ & $\begin{array}{l}\text { Sim. } \\
\text { Flow }\end{array}$ & $\begin{array}{c}\text { Error } \\
\text { sim-anal }(\%)\end{array}$ & $\begin{array}{l}\text { Predicted } \\
\text { Error (\%) }\end{array}$ \\
\hline 6 & 4 & 3 & 0.52 & 9.82 & 5.87 & 40.18 & 32.74 \\
\hline 6 & 4 & 3 & 0.52 & 11.19 & 8.07 & 27.84 & 32.74 \\
\hline 6 & 4 & 3 & 0.52 & 11.91 & 9.23 & 22.50 & 32.74 \\
\hline 6 & 4 & 3 & 0.52 & 9.24 & 4.97 & 46.25 & 32.74 \\
\hline 6 & 4 & 3 & 0.52 & 9.52 & 5.38 & 43.44 & 32.74 \\
\hline
\end{tabular}

Approximate and simulated flow times for the $\operatorname{Exp}-E_{2}$ case

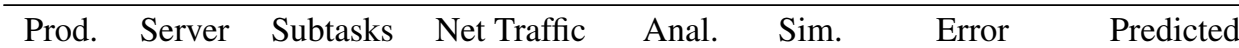
Intensity Flow T. Flow sim-anal (\%) Error (\%)

\begin{tabular}{|c|c|c|c|c|c|c|c|}
\hline 1 & 2 & 2 & 0.10 & 1.48 & 1.48 & 0.09 & 0.93 \\
\hline 1 & 2 & 2 & 0.11 & 1.57 & 1.49 & 5.26 & 4.39 \\
\hline 1 & 2 & 2 & 0.12 & 1.78 & 1.50 & 15.50 & 15.01 \\
\hline 2 & 2 & 2 & 0.19 & 1.59 & 1.60 & 0.46 & 0.94 \\
\hline 2 & 2 & 2 & 0.19 & 1.47 & 1.45 & 0.97 & 0.94 \\
\hline 2 & 2 & 2 & 0.20 & 1.69 & 1.62 & 4.67 & 6.20 \\
\hline 2 & 2 & 2 & 0.20 & 1.57 & 1.47 & 6.30 & 6.20 \\
\hline 2 & 2 & 2 & 0.22 & 1.95 & 1.65 & 15.43 & 16.70 \\
\hline 2 & 2 & 2 & 0.22 & 1.80 & 1.50 & 16.73 & 16.70 \\
\hline 3 & 2 & 2 & 0.31 & 1.80 & 1.81 & 0.60 & 2.48 \\
\hline 3 & 2 & 2 & 0.31 & 1.68 & 1.64 & 1.98 & 2.48 \\
\hline 3 & 2 & 2 & 0.31 & 1.93 & 2.26 & 17.16 & 2.48 \\
\hline 3 & 2 & 2 & 0.33 & 1.94 & 1.84 & 5.02 & 7.67 \\
\hline 3 & 2 & 2 & 0.33 & 1.81 & 1.67 & 7.58 & 7.67 \\
\hline 3 & 2 & 2 & 0.33 & 2.08 & 2.30 & 10.67 & 7.67 \\
\hline 3 & 2 & 2 & 0.37 & 2.29 & 1.93 & 15.78 & 18.01 \\
\hline 3 & 2 & 2 & 0.37 & 2.13 & 1.75 & 17.97 & 18.01 \\
\hline 3 & 2 & 2 & 0.37 & 2.45 & 2.41 & 1.55 & 18.01 \\
\hline 4 & 2 & 2 & 0.44 & 2.27 & 2.14 & 5.58 & 3.86 \\
\hline 4 & 2 & 2 & 0.44 & 2.15 & 1.95 & 9.58 & 3.86 \\
\hline 4 & 2 & 2 & 0.44 & 2.40 & 2.68 & 11.74 & 3.86 \\
\hline 4 & 2 & 2 & 0.44 & 2.82 & 3.06 & 8.35 & 3.86 \\
\hline 4 & 2 & 2 & 0.47 & 2.50 & 2.22 & 11.31 & 8.98 \\
\hline 4 & 2 & 2 & 0.47 & 2.38 & 2.02 & 15.29 & 8.98 \\
\hline 4 & 2 & 2 & 0.47 & 2.64 & 2.77 & 4.92 & 8.98 \\
\hline 4 & 2 & 2 & 0.47 & 3.10 & 3.17 & 2.03 & 8.98 \\
\hline 4 & 2 & 2 & 0.52 & 3.14 & 2.43 & 22.82 & 19.13 \\
\hline 4 & 2 & 2 & 0.52 & 2.99 & 2.20 & 26.30 & 19.13 \\
\hline 4 & 2 & 2 & 0.52 & 3.30 & 3.03 & 8.15 & 19.13 \\
\hline 4 & 2 & 2 & 0.52 & 3.83 & 3.46 & 9.45 & 19.13 \\
\hline
\end{tabular}




\begin{tabular}{|c|c|c|c|c|c|c|c|}
\hline Prod. & Server & Subtasks & $\begin{array}{c}\text { Net Traffic } \\
\text { Intensity }\end{array}$ & $\begin{array}{c}\text { Anal. } \\
\text { Flow T. }\end{array}$ & $\begin{array}{l}\text { Sim. } \\
\text { Flow }\end{array}$ & $\begin{array}{c}\text { Error } \\
\text { sim-anal (\%) }\end{array}$ & $\begin{array}{l}\text { Predicted } \\
\text { Error }(\%)\end{array}$ \\
\hline 5 & 2 & 2 & 0.51 & 2.54 & 2.37 & 6.52 & 5.99 \\
\hline 5 & 2 & 2 & 0.51 & 2.42 & 2.16 & 10.80 & 5.99 \\
\hline 5 & 2 & 2 & 0.51 & 2.66 & 2.97 & 11.34 & 5.99 \\
\hline 5 & 2 & 2 & 0.51 & 3.09 & 3.39 & 9.75 & 5.99 \\
\hline 5 & 2 & 2 & 0.51 & 2.25 & 1.83 & 18.95 & 5.99 \\
\hline 5 & 2 & 2 & 0.54 & 2.83 & 2.49 & 12.22 & 11.08 \\
\hline 5 & 2 & 2 & 0.54 & 2.71 & 2.26 & 16.59 & 11.08 \\
\hline 5 & 2 & 2 & 0.54 & 2.97 & 3.11 & 4.63 & 11.08 \\
\hline 5 & 2 & 2 & 0.54 & 3.42 & 3.55 & 3.87 & 11.08 \\
\hline 5 & 2 & 2 & 0.54 & 2.52 & 1.91 & 24.22 & 11.08 \\
\hline 5 & 2 & 2 & 0.60 & 3.71 & 2.81 & 24.09 & 21.14 \\
\hline 5 & 2 & 2 & 0.60 & 3.57 & 2.56 & 28.27 & 21.14 \\
\hline 5 & 2 & 2 & 0.60 & 3.86 & 3.52 & 8.96 & 21.14 \\
\hline 5 & 2 & 2 & 0.60 & 4.39 & 4.02 & 8.38 & 21.14 \\
\hline 5 & 2 & 2 & 0.60 & 3.34 & 2.16 & 35.23 & 21.14 \\
\hline 6 & 2 & 2 & 0.59 & 2.72 & 2.55 & 6.44 & 8.41 \\
\hline 6 & 2 & 2 & 0.59 & 2.61 & 2.31 & 11.22 & 8.41 \\
\hline 6 & 2 & 2 & 0.59 & 2.85 & 3.18 & 11.73 & 8.41 \\
\hline 6 & 2 & 2 & 0.59 & 3.27 & 3.64 & 11.23 & 8.41 \\
\hline 6 & 2 & 2 & 0.59 & 2.43 & 1.96 & 19.55 & 8.41 \\
\hline 6 & 2 & 2 & 0.59 & 2.52 & 2.12 & 15.70 & 8.41 \\
\hline 6 & 2 & 2 & 0.62 & 3.08 & 2.69 & 12.69 & 13.47 \\
\hline 6 & 2 & 2 & 0.62 & 2.96 & 2.45 & 17.27 & 13.47 \\
\hline 6 & 2 & 2 & 0.62 & 3.22 & 3.37 & 4.55 & 13.47 \\
\hline 6 & 2 & 2 & 0.62 & 3.67 & 3.85 & 4.75 & 13.47 \\
\hline 6 & 2 & 2 & 0.62 & 2.77 & 2.07 & 25.28 & 13.47 \\
\hline 6 & 2 & 2 & 0.62 & 2.86 & 2.24 & 21.57 & 13.47 \\
\hline 6 & 2 & 2 & 0.69 & 4.18 & 3.13 & 25.01 & 23.48 \\
\hline 6 & 2 & 2 & 0.69 & 4.04 & 2.85 & 29.52 & 23.48 \\
\hline 6 & 2 & 2 & 0.69 & 4.35 & 3.91 & 10.00 & 23.48 \\
\hline 6 & 2 & 2 & 0.69 & 4.86 & 4.47 & 8.02 & 23.48 \\
\hline 6 & 2 & 2 & 0.69 & 3.83 & 2.41 & 37.13 & 23.48 \\
\hline 6 & 2 & 2 & 0.69 & 3.93 & 2.61 & 33.57 & 23.48 \\
\hline 1 & 3 & 3 & 0.10 & 1.73 & 1.73 & 0.02 & 0.85 \\
\hline 1 & 3 & 3 & 0.11 & 1.83 & 1.73 & 5.21 & 6.17 \\
\hline 1 & 3 & 3 & 0.12 & 2.08 & 1.75 & 15.69 & 16.78 \\
\hline 2 & 3 & 3 & 0.19 & 1.85 & 1.86 & 0.62 & 2.71 \\
\hline 2 & 3 & 3 & 0.19 & 1.71 & 1.69 & 1.02 & 2.71 \\
\hline 2 & 3 & 3 & 0.20 & 1.97 & 1.88 & 4.83 & 7.98 \\
\hline 2 & 3 & 3 & 0.20 & 1.82 & 1.71 & 6.32 & 7.98 \\
\hline
\end{tabular}




\begin{tabular}{|c|c|c|c|c|c|c|c|}
\hline Prod. & Server & Subtasks & $\begin{array}{c}\text { Net Traffic } \\
\text { Intensity }\end{array}$ & $\begin{array}{c}\text { Anal. } \\
\text { Flow T. }\end{array}$ & $\begin{array}{l}\text { Sim. } \\
\text { Flow }\end{array}$ & $\begin{array}{c}\text { Error } \\
\text { sim-anal }(\%)\end{array}$ & $\begin{array}{l}\text { Predicted } \\
\text { Error }(\%)\end{array}$ \\
\hline 2 & 3 & 3 & 0.22 & 2.27 & 1.92 & 15.43 & 18.47 \\
\hline 2 & 3 & 3 & 0.22 & 2.09 & 1.74 & 16.76 & 18.47 \\
\hline 3 & 3 & 3 & 0.31 & 2.15 & 2.09 & 2.62 & 4.25 \\
\hline 3 & 3 & 3 & 0.31 & 2.01 & 1.90 & 5.63 & 4.25 \\
\hline 3 & 3 & 3 & 0.31 & 2.53 & 2.61 & 3.24 & 4.25 \\
\hline 3 & 3 & 3 & 0.33 & 2.32 & 2.13 & 8.06 & 9.45 \\
\hline 3 & 3 & 3 & 0.33 & 2.17 & 1.94 & 10.96 & 9.45 \\
\hline 3 & 3 & 3 & 0.33 & 2.72 & 2.66 & 2.20 & 9.45 \\
\hline 3 & 3 & 3 & 0.37 & 2.75 & 2.23 & 19.18 & 19.78 \\
\hline 3 & 3 & 3 & 0.37 & 2.59 & 2.02 & 21.83 & 19.78 \\
\hline 3 & 3 & 3 & 0.37 & 3.21 & 2.78 & 13.24 & 19.78 \\
\hline 4 & 3 & 3 & 0.44 & 2.73 & 2.47 & 9.60 & 5.63 \\
\hline 4 & 3 & 3 & 0.44 & 2.60 & 2.24 & 13.67 & 5.63 \\
\hline 4 & 3 & 3 & 0.44 & 3.09 & 3.08 & 0.33 & 5.63 \\
\hline 4 & 3 & 3 & 0.44 & 3.36 & 3.53 & 5.07 & 5.63 \\
\hline 4 & 3 & 3 & 0.47 & 3.02 & 2.55 & 15.43 & 10.75 \\
\hline 4 & 3 & 3 & 0.47 & 2.87 & 2.32 & 19.21 & 10.75 \\
\hline 4 & 3 & 3 & 0.47 & 3.40 & 3.19 & 6.23 & 10.75 \\
\hline 4 & 3 & 3 & 0.47 & 3.68 & 3.65 & 0.98 & 10.75 \\
\hline 4 & 3 & 3 & 0.52 & 3.79 & 2.79 & 26.54 & 20.90 \\
\hline 4 & 3 & 3 & 0.52 & 3.64 & 2.53 & 30.42 & 20.90 \\
\hline 4 & 3 & 3 & 0.52 & 4.24 & 3.48 & 17.84 & 20.90 \\
\hline 4 & 3 & 3 & 0.52 & 4.57 & 3.98 & 12.83 & 20.90 \\
\hline 5 & 3 & 3 & 0.51 & 3.06 & 2.73 & 10.91 & 7.77 \\
\hline 5 & 3 & 3 & 0.51 & 2.93 & 2.48 & 15.41 & 7.77 \\
\hline 5 & 3 & 3 & 0.51 & 3.42 & 3.41 & 0.20 & 7.77 \\
\hline 5 & 3 & 3 & 0.51 & 3.67 & 3.90 & 6.26 & 7.77 \\
\hline 5 & 3 & 3 & 0.51 & 2.74 & 2.10 & 23.41 & 7.77 \\
\hline 5 & 3 & 3 & 0.54 & 3.43 & 2.86 & 16.63 & 12.85 \\
\hline 5 & 3 & 3 & 0.54 & 3.29 & 2.60 & 21.06 & 12.85 \\
\hline 5 & 3 & 3 & 0.54 & 3.81 & 3.57 & 6.32 & 12.85 \\
\hline 5 & 3 & 3 & 0.54 & 4.08 & 4.08 & 0.14 & 12.85 \\
\hline 5 & 3 & 3 & 0.54 & 3.09 & 2.20 & 28.85 & 12.85 \\
\hline 5 & 3 & 3 & 0.60 & 4.53 & 3.22 & 28.85 & 22.91 \\
\hline 5 & 3 & 3 & 0.60 & 4.37 & 2.93 & 32.96 & 22.91 \\
\hline 5 & 3 & 3 & 0.60 & 4.95 & 4.03 & 18.66 & 22.91 \\
\hline 5 & 3 & 3 & 0.60 & 5.28 & 4.60 & 12.80 & 22.91 \\
\hline 5 & 3 & 3 & 0.60 & 4.13 & 2.48 & 39.92 & 22.91 \\
\hline 6 & 3 & 3 & 0.59 & 3.29 & 2.92 & 11.15 & 10.18 \\
\hline 6 & 3 & 3 & 0.59 & 3.16 & 2.66 & 15.90 & 10.18 \\
\hline
\end{tabular}




\begin{tabular}{|c|c|c|c|c|c|c|c|}
\hline Prod. & Server & Subtasks & $\begin{array}{c}\text { Net Traffic } \\
\text { Intensity }\end{array}$ & $\begin{array}{c}\text { Anal. } \\
\text { Flow T. }\end{array}$ & $\begin{array}{l}\text { Sim. } \\
\text { Flow }\end{array}$ & $\begin{array}{c}\text { Error } \\
\text { sim-anal }(\%)\end{array}$ & $\begin{array}{l}\text { Predicted } \\
\text { Error }(\%)\end{array}$ \\
\hline 6 & 3 & 3 & 0.59 & 3.64 & 3.65 & 0.49 & 10.18 \\
\hline 6 & 3 & 3 & 0.59 & 3.90 & 4.17 & 7.12 & 10.18 \\
\hline 6 & 3 & 3 & 0.59 & 2.98 & 2.25 & 24.53 & 10.18 \\
\hline 6 & 3 & 3 & 0.59 & 3.06 & 2.44 & 20.38 & 10.18 \\
\hline 6 & 3 & 3 & 0.62 & 3.73 & 3.09 & 17.27 & 15.24 \\
\hline 6 & 3 & 3 & 0.62 & 3.61 & 2.81 & 22.21 & 15.24 \\
\hline 6 & 3 & 3 & 0.62 & 4.11 & 3.86 & 6.07 & 15.24 \\
\hline 6 & 3 & 3 & 0.62 & 4.39 & 4.41 & 0.47 & 15.24 \\
\hline 6 & 3 & 3 & 0.62 & 3.39 & 2.37 & 30.04 & 15.24 \\
\hline 6 & 3 & 3 & 0.62 & 3.49 & 2.57 & 26.29 & 15.24 \\
\hline 6 & 3 & 3 & 0.69 & 5.11 & 3.58 & 29.93 & 25.25 \\
\hline 6 & 3 & 3 & 0.69 & 4.97 & 3.25 & 34.53 & 25.25 \\
\hline 6 & 3 & 3 & 0.69 & 5.54 & 4.48 & 19.27 & 25.25 \\
\hline 6 & 3 & 3 & 0.69 & 5.86 & 5.11 & 12.68 & 25.25 \\
\hline 6 & 3 & 3 & 0.69 & 4.73 & 2.75 & 41.76 & 25.25 \\
\hline 6 & 3 & 3 & 0.69 & 4.85 & 2.98 & 38.43 & 25.25 \\
\hline 1 & 3 & 2 & 0.07 & 1.67 & 1.44 & 13.50 & 3.84 \\
\hline 1 & 3 & 2 & 0.07 & 1.77 & 1.45 & 18.04 & 9.17 \\
\hline 1 & 3 & 2 & 0.08 & 1.99 & 1.46 & 26.71 & 19.84 \\
\hline 2 & 3 & 2 & 0.13 & 1.75 & 1.51 & 13.44 & 6.03 \\
\hline 2 & 3 & 2 & 0.13 & 1.61 & 1.38 & 14.48 & 6.03 \\
\hline 2 & 3 & 2 & 0.13 & 1.86 & 1.52 & 18.07 & 11.33 \\
\hline 2 & 3 & 2 & 0.13 & 1.71 & 1.38 & 18.87 & 11.33 \\
\hline 2 & 3 & 2 & 0.15 & 2.11 & 1.54 & 26.73 & 21.91 \\
\hline 2 & 3 & 2 & 0.15 & 1.94 & 1.40 & 27.75 & 21.91 \\
\hline 3 & 3 & 2 & 0.21 & 1.93 & 1.63 & 15.64 & 8.01 \\
\hline 3 & 3 & 2 & 0.21 & 1.79 & 1.48 & 17.41 & 8.01 \\
\hline 3 & 3 & 2 & 0.21 & 2.32 & 2.03 & 12.31 & 8.01 \\
\hline 3 & 3 & 2 & 0.22 & 2.06 & 1.64 & 20.28 & 13.26 \\
\hline 3 & 3 & 2 & 0.22 & 1.92 & 1.49 & 22.21 & 13.26 \\
\hline 3 & 3 & 2 & 0.22 & 2.47 & 2.05 & 17.01 & 13.26 \\
\hline 3 & 3 & 2 & 0.24 & 2.38 & 1.68 & 29.31 & 23.74 \\
\hline 3 & 3 & 2 & 0.24 & 2.22 & 1.53 & 30.95 & 23.74 \\
\hline 3 & 3 & 2 & 0.24 & 2.84 & 2.10 & 26.03 & 23.74 \\
\hline 4 & 3 & 2 & 0.30 & 2.22 & 1.78 & 19.96 & 9.88 \\
\hline 4 & 3 & 2 & 0.30 & 2.08 & 1.62 & 22.40 & 9.88 \\
\hline 4 & 3 & 2 & 0.30 & 2.60 & 2.22 & 14.61 & 9.88 \\
\hline 4 & 3 & 2 & 0.30 & 2.88 & 2.54 & 11.74 & 9.88 \\
\hline 4 & 3 & 2 & 0.31 & 2.40 & 1.81 & 24.63 & 15.09 \\
\hline 4 & 3 & 2 & 0.31 & 2.25 & 1.64 & 26.94 & 15.09 \\
\hline
\end{tabular}




\begin{tabular}{|c|c|c|c|c|c|c|c|}
\hline Prod. & Server & Subtasks & $\begin{array}{c}\text { Net Traffic } \\
\text { Intensity }\end{array}$ & $\begin{array}{c}\text { Anal. } \\
\text { Flow T. }\end{array}$ & $\begin{array}{l}\text { Sim. } \\
\text { Flow }\end{array}$ & $\begin{array}{c}\text { Error } \\
\text { sim-anal }(\%)\end{array}$ & $\begin{array}{l}\text { Predicted } \\
\text { Error }(\%)\end{array}$ \\
\hline 4 & 3 & 2 & 0.31 & 2.80 & 2.26 & 19.17 & 15.09 \\
\hline 4 & 3 & 2 & 0.31 & 3.10 & 2.58 & 16.52 & 15.09 \\
\hline 4 & 3 & 2 & 0.35 & 2.85 & 1.89 & 33.65 & 25.44 \\
\hline 4 & 3 & 2 & 0.35 & 2.67 & 1.72 & 35.75 & 25.44 \\
\hline 4 & 3 & 2 & 0.35 & 3.30 & 2.36 & 28.49 & 25.44 \\
\hline 4 & 3 & 2 & 0.35 & 3.63 & 2.70 & 25.70 & 25.44 \\
\hline 5 & 3 & 2 & 0.34 & 2.35 & 1.87 & 20.34 & 12.25 \\
\hline 5 & 3 & 2 & 0.34 & 2.21 & 1.70 & 23.05 & 12.25 \\
\hline 5 & 3 & 2 & 0.34 & 2.72 & 2.34 & 14.26 & 12.25 \\
\hline 5 & 3 & 2 & 0.34 & 3.00 & 2.67 & 10.87 & 12.25 \\
\hline 5 & 3 & 2 & 0.34 & 2.00 & 1.44 & 28.03 & 12.25 \\
\hline 5 & 3 & 2 & 0.36 & 2.54 & 1.91 & 24.74 & 17.43 \\
\hline 5 & 3 & 2 & 0.36 & 2.39 & 1.74 & 27.41 & 17.43 \\
\hline 5 & 3 & 2 & 0.36 & 2.94 & 2.39 & 18.93 & 17.43 \\
\hline 5 & 3 & 2 & 0.36 & 3.24 & 2.73 & 15.72 & 17.43 \\
\hline 5 & 3 & 2 & 0.36 & 2.17 & 1.47 & 32.39 & 17.43 \\
\hline 5 & 3 & 2 & 0.40 & 3.05 & 2.01 & 33.94 & 27.73 \\
\hline 5 & 3 & 2 & 0.40 & 2.89 & 1.83 & 36.60 & 27.73 \\
\hline 5 & 3 & 2 & 0.40 & 3.51 & 2.52 & 28.29 & 27.73 \\
\hline 5 & 3 & 2 & 0.40 & 3.84 & 2.88 & 24.98 & 27.73 \\
\hline 5 & 3 & 2 & 0.40 & 2.63 & 1.55 & 41.17 & 27.73 \\
\hline 6 & 3 & 2 & 0.39 & 2.43 & 1.93 & 20.46 & 14.82 \\
\hline 6 & 3 & 2 & 0.39 & 2.29 & 1.75 & 23.25 & 14.82 \\
\hline 6 & 3 & 2 & 0.39 & 2.80 & 2.41 & 14.00 & 14.82 \\
\hline 6 & 3 & 2 & 0.39 & 3.08 & 2.76 & 10.41 & 14.82 \\
\hline 6 & 3 & 2 & 0.39 & 2.08 & 1.48 & 28.50 & 14.82 \\
\hline 6 & 3 & 2 & 0.39 & 2.17 & 1.61 & 25.99 & 14.82 \\
\hline 6 & 3 & 2 & 0.41 & 2.64 & 1.98 & 25.00 & 19.98 \\
\hline 6 & 3 & 2 & 0.41 & 2.49 & 1.80 & 27.70 & 19.98 \\
\hline 6 & 3 & 2 & 0.41 & 3.04 & 2.47 & 18.66 & 19.98 \\
\hline 6 & 3 & 2 & 0.41 & 3.32 & 2.82 & 15.03 & 19.98 \\
\hline 6 & 3 & 2 & 0.41 & 2.27 & 1.52 & 32.91 & 19.98 \\
\hline 6 & 3 & 2 & 0.41 & 2.37 & 1.65 & 30.49 & 19.98 \\
\hline 6 & 3 & 2 & 0.46 & 3.20 & 2.10 & 34.26 & 30.24 \\
\hline 6 & 3 & 2 & 0.46 & 3.04 & 1.91 & 37.16 & 30.24 \\
\hline 6 & 3 & 2 & 0.46 & 3.66 & 2.63 & 28.29 & 30.24 \\
\hline 6 & 3 & 2 & 0.46 & 3.99 & 3.00 & 24.69 & 30.24 \\
\hline 6 & 3 & 2 & 0.46 & 2.78 & 1.62 & 41.91 & 30.24 \\
\hline 6 & 3 & 2 & 0.46 & 2.90 & 1.75 & 39.51 & 30.24 \\
\hline 1 & 4 & 4 & 0.10 & 1.90 & 1.90 & 0.15 & 2.62 \\
\hline
\end{tabular}




\begin{tabular}{|c|c|c|c|c|c|c|c|}
\hline Prod. & Server & Subtasks & $\begin{array}{c}\text { Net Traffic } \\
\text { Intensity }\end{array}$ & $\begin{array}{c}\text { Anal. } \\
\text { Flow T. }\end{array}$ & $\begin{array}{l}\text { Sim. } \\
\text { Flow }\end{array}$ & $\begin{array}{c}\text { Error } \\
\text { sim-anal (\%) }\end{array}$ & $\begin{array}{l}\text { Predicted } \\
\text { Error }(\%)\end{array}$ \\
\hline 1 & 4 & 4 & 0.11 & 2.01 & 1.91 & 5.19 & 7.94 \\
\hline 1 & 4 & 4 & 0.12 & 2.28 & 1.93 & 15.39 & 18.56 \\
\hline 2 & 4 & 4 & 0.19 & 2.04 & 2.05 & 0.46 & 4.48 \\
\hline 2 & 4 & 4 & 0.19 & 1.88 & 1.86 & 1.08 & 4.48 \\
\hline 2 & 4 & 4 & 0.20 & 2.16 & 2.06 & 4.62 & 9.75 \\
\hline 2 & 4 & 4 & 0.20 & 2.00 & 1.88 & 6.22 & 9.75 \\
\hline 2 & 4 & 4 & 0.22 & 2.49 & 2.11 & 15.18 & 20.24 \\
\hline 2 & 4 & 4 & 0.22 & 2.30 & 1.92 & 16.78 & 20.24 \\
\hline 3 & 4 & 4 & 0.31 & 2.36 & 2.29 & 2.79 & 6.02 \\
\hline 3 & 4 & 4 & 0.31 & 2.21 & 2.09 & 5.74 & 6.02 \\
\hline 3 & 4 & 4 & 0.31 & 2.77 & 2.87 & 3.38 & 6.02 \\
\hline 3 & 4 & 4 & 0.33 & 2.55 & 2.33 & 8.34 & 11.22 \\
\hline 3 & 4 & 4 & 0.33 & 2.39 & 2.12 & 11.08 & 11.22 \\
\hline 3 & 4 & 4 & 0.33 & 2.99 & 2.92 & 2.44 & 11.22 \\
\hline 3 & 4 & 4 & 0.37 & 3.03 & 2.44 & 19.42 & 21.55 \\
\hline 3 & 4 & 4 & 0.37 & 2.85 & 2.22 & 22.10 & 21.55 \\
\hline 3 & 4 & 4 & 0.37 & 3.52 & 3.05 & 13.48 & 21.55 \\
\hline 4 & 4 & 4 & 0.44 & 3.00 & 2.70 & 10.12 & 7.40 \\
\hline 4 & 4 & 4 & 0.44 & 2.86 & 2.45 & 14.22 & 7.40 \\
\hline 4 & 4 & 4 & 0.44 & 3.39 & 3.37 & 0.57 & 7.40 \\
\hline 4 & 4 & 4 & 0.44 & 3.68 & 3.86 & 4.88 & 7.40 \\
\hline 4 & 4 & 4 & 0.47 & 3.31 & 2.79 & 15.65 & 12.53 \\
\hline 4 & 4 & 4 & 0.47 & 3.16 & 2.54 & 19.60 & 12.53 \\
\hline 4 & 4 & 4 & 0.47 & 3.72 & 3.49 & 6.32 & 12.53 \\
\hline 4 & 4 & 4 & 0.47 & 4.03 & 3.99 & 1.03 & 12.53 \\
\hline 4 & 4 & 4 & 0.52 & 4.17 & 3.04 & 27.04 & 22.68 \\
\hline 4 & 4 & 4 & 0.52 & 4.00 & 2.76 & 30.81 & 22.68 \\
\hline 4 & 4 & 4 & 0.52 & 4.63 & 3.80 & 17.84 & 22.68 \\
\hline 4 & 4 & 4 & 0.52 & 4.98 & 4.34 & 12.82 & 22.68 \\
\hline 5 & 4 & 4 & 0.51 & 3.35 & 2.98 & 11.18 & 9.54 \\
\hline 5 & 4 & 4 & 0.51 & 3.22 & 2.71 & 15.80 & 9.54 \\
\hline 5 & 4 & 4 & 0.51 & 3.74 & 3.72 & 0.51 & 9.54 \\
\hline 5 & 4 & 4 & 0.51 & 4.01 & 4.26 & 6.00 & 9.54 \\
\hline 5 & 4 & 4 & 0.51 & 3.01 & 2.29 & 23.98 & 9.54 \\
\hline 5 & 4 & 4 & 0.54 & 3.75 & 3.12 & 17.02 & 14.62 \\
\hline 5 & 4 & 4 & 0.54 & 3.61 & 2.83 & 21.51 & 14.62 \\
\hline 5 & 4 & 4 & 0.54 & 4.15 & 3.89 & 6.23 & 14.62 \\
\hline 5 & 4 & 4 & 0.54 & 4.45 & 4.45 & 0.02 & 14.62 \\
\hline 5 & 4 & 4 & 0.54 & 3.38 & 2.40 & 29.14 & 14.62 \\
\hline 5 & 4 & 4 & 0.60 & 4.96 & 3.51 & 29.18 & 24.68 \\
\hline
\end{tabular}




\begin{tabular}{|c|c|c|c|c|c|c|c|}
\hline Prod. & Server & Subtasks & $\begin{array}{c}\text { Net Traffic } \\
\text { Intensity }\end{array}$ & $\begin{array}{c}\text { Anal. } \\
\text { Flow T. }\end{array}$ & $\begin{array}{l}\text { Sim. } \\
\text { Flow }\end{array}$ & $\begin{array}{c}\text { Error } \\
\text { sim-anal (\%) }\end{array}$ & $\begin{array}{l}\text { Predicted } \\
\text { Error }(\%)\end{array}$ \\
\hline 5 & 4 & 4 & 0.60 & 4.80 & 3.19 & 33.46 & 24.68 \\
\hline 5 & 4 & 4 & 0.60 & 5.42 & 4.39 & 18.99 & 24.68 \\
\hline 5 & 4 & 4 & 0.60 & 5.76 & 5.02 & 13.00 & 24.68 \\
\hline 5 & 4 & 4 & 0.60 & 4.55 & 2.70 & 40.61 & 24.68 \\
\hline 6 & 4 & 4 & 0.59 & 3.59 & 3.19 & 11.21 & 11.95 \\
\hline 6 & 4 & 4 & 0.59 & 3.46 & 2.90 & 16.18 & 11.95 \\
\hline 6 & 4 & 4 & 0.59 & 3.97 & 3.98 & 0.36 & 11.95 \\
\hline 6 & 4 & 4 & 0.59 & 4.24 & 4.55 & 7.42 & 11.95 \\
\hline 6 & 4 & 4 & 0.59 & 3.26 & 2.45 & 24.70 & 11.95 \\
\hline 6 & 4 & 4 & 0.59 & 3.35 & 2.66 & 20.77 & 11.95 \\
\hline 6 & 4 & 4 & 0.62 & 4.08 & 3.36 & 17.53 & 17.01 \\
\hline 6 & 4 & 4 & 0.62 & 3.94 & 3.06 & 22.36 & 17.01 \\
\hline 6 & 4 & 4 & 0.62 & 4.48 & 4.21 & 6.06 & 17.01 \\
\hline 6 & 4 & 4 & 0.62 & 4.78 & 4.81 & 0.61 & 17.01 \\
\hline 6 & 4 & 4 & 0.62 & 3.73 & 2.59 & 30.53 & 17.01 \\
\hline 6 & 4 & 4 & 0.62 & 3.83 & 2.80 & 26.73 & 17.01 \\
\hline 6 & 4 & 4 & 0.69 & 5.59 & 3.89 & 30.31 & 27.02 \\
\hline 6 & 4 & 4 & 0.69 & 5.43 & 3.54 & 34.81 & 27.02 \\
\hline 6 & 4 & 4 & 0.69 & 6.04 & 4.87 & 19.39 & 27.02 \\
\hline 6 & 4 & 4 & 0.69 & 6.38 & 5.56 & 12.80 & 27.02 \\
\hline 6 & 4 & 4 & 0.69 & 5.19 & 3.00 & 42.23 & 27.02 \\
\hline 6 & 4 & 4 & 0.69 & 5.31 & 3.25 & 38.83 & 27.02 \\
\hline 1 & 4 & 2 & 0.05 & 1.59 & 1.43 & 10.56 & 8.42 \\
\hline 1 & 4 & 2 & 0.05 & 1.68 & 1.43 & 15.23 & 13.76 \\
\hline 1 & 4 & 2 & 0.06 & 1.89 & 1.43 & 24.02 & 24.45 \\
\hline 2 & 4 & 2 & 0.10 & 1.65 & 1.48 & 10.57 & 10.77 \\
\hline 2 & 4 & 2 & 0.10 & 1.51 & 1.34 & 11.25 & 10.77 \\
\hline 2 & 4 & 2 & 0.10 & 1.75 & 1.48 & 15.19 & 16.10 \\
\hline 2 & 4 & 2 & 0.10 & 1.60 & 1.35 & 15.96 & 16.10 \\
\hline 2 & 4 & 2 & 0.11 & 1.97 & 1.50 & 24.10 & 26.72 \\
\hline 2 & 4 & 2 & 0.11 & 1.81 & 1.36 & 24.88 & 26.72 \\
\hline 3 & 4 & 2 & 0.16 & 1.77 & 1.55 & 12.41 & 12.97 \\
\hline 3 & 4 & 2 & 0.16 & 1.63 & 1.41 & 13.70 & 12.97 \\
\hline 3 & 4 & 2 & 0.16 & 2.15 & 1.94 & 9.67 & 12.97 \\
\hline 3 & 4 & 2 & 0.16 & 1.88 & 1.56 & 17.07 & 18.26 \\
\hline 3 & 4 & 2 & 0.16 & 1.74 & 1.42 & 18.40 & 18.26 \\
\hline 3 & 4 & 2 & 0.16 & 2.28 & 1.95 & 14.19 & 18.26 \\
\hline 3 & 4 & 2 & 0.18 & 2.15 & 1.59 & 26.20 & 28.80 \\
\hline 3 & 4 & 2 & 0.18 & 1.99 & 1.44 & 27.30 & 28.80 \\
\hline 3 & 4 & 2 & 0.18 & 2.60 & 1.99 & 23.67 & 28.80 \\
\hline
\end{tabular}




\begin{tabular}{|c|c|c|c|c|c|c|c|}
\hline Prod. & Server & Subtasks & $\begin{array}{c}\text { Net Traffic } \\
\text { Intensity }\end{array}$ & $\begin{array}{c}\text { Anal. } \\
\text { Flow T. }\end{array}$ & $\begin{array}{l}\text { Sim. } \\
\text { Flow }\end{array}$ & $\begin{array}{c}\text { Error } \\
\text { sim-anal (\%) }\end{array}$ & $\begin{array}{l}\text { Predicted } \\
\text { Error }(\%)\end{array}$ \\
\hline 4 & 4 & 2 & 0.22 & 1.95 & 1.65 & 15.63 & 15.09 \\
\hline 4 & 4 & 2 & 0.22 & 1.82 & 1.50 & 17.73 & 15.09 \\
\hline 4 & 4 & 2 & 0.22 & 2.33 & 2.06 & 11.45 & 15.09 \\
\hline 4 & 4 & 2 & 0.22 & 2.60 & 2.36 & 9.30 & 15.09 \\
\hline 4 & 4 & 2 & 0.23 & 2.10 & 1.67 & 20.41 & 20.34 \\
\hline 4 & 4 & 2 & 0.23 & 1.95 & 1.52 & 22.25 & 20.34 \\
\hline 4 & 4 & 2 & 0.23 & 2.49 & 2.08 & 16.26 & 20.34 \\
\hline 4 & 4 & 2 & 0.23 & 2.77 & 2.38 & 13.96 & 20.34 \\
\hline 4 & 4 & 2 & 0.26 & 2.44 & 1.71 & 29.65 & 30.79 \\
\hline 4 & 4 & 2 & 0.26 & 2.28 & 1.56 & 31.57 & 30.79 \\
\hline 4 & 4 & 2 & 0.26 & 2.88 & 2.14 & 25.51 & 30.79 \\
\hline 4 & 4 & 2 & 0.26 & 3.20 & 2.45 & 23.37 & 30.79 \\
\hline 5 & 4 & 2 & 0.26 & 2.03 & 1.70 & 16.00 & 17.58 \\
\hline 5 & 4 & 2 & 0.26 & 1.89 & 1.55 & 18.14 & 17.58 \\
\hline 5 & 4 & 2 & 0.26 & 2.39 & 2.13 & 11.10 & 17.58 \\
\hline 5 & 4 & 2 & 0.26 & 2.66 & 2.43 & 8.46 & 17.58 \\
\hline 5 & 4 & 2 & 0.26 & 1.68 & 1.31 & 22.11 & 17.58 \\
\hline 5 & 4 & 2 & 0.27 & 2.17 & 1.73 & 20.59 & 22.81 \\
\hline 5 & 4 & 2 & 0.27 & 2.03 & 1.57 & 22.60 & 22.81 \\
\hline 5 & 4 & 2 & 0.27 & 2.57 & 2.16 & 15.85 & 22.81 \\
\hline 5 & 4 & 2 & 0.27 & 2.85 & 2.47 & 13.35 & 22.81 \\
\hline 5 & 4 & 2 & 0.27 & 1.81 & 1.33 & 26.77 & 22.81 \\
\hline 5 & 4 & 2 & 0.30 & 2.54 & 1.79 & 29.82 & 33.22 \\
\hline 5 & 4 & 2 & 0.30 & 2.38 & 1.62 & 31.89 & 33.22 \\
\hline 5 & 4 & 2 & 0.30 & 2.99 & 2.23 & 25.25 & 33.22 \\
\hline 5 & 4 & 2 & 0.30 & 3.31 & 2.55 & 22.84 & 33.22 \\
\hline 5 & 4 & 2 & 0.30 & 2.14 & 1.37 & 35.82 & 33.22 \\
\hline 6 & 4 & 2 & 0.30 & 2.07 & 1.74 & 15.87 & 20.22 \\
\hline 6 & 4 & 2 & 0.30 & 1.93 & 1.58 & 18.18 & 20.22 \\
\hline 6 & 4 & 2 & 0.30 & 2.44 & 2.17 & 10.85 & 20.22 \\
\hline 6 & 4 & 2 & 0.30 & 2.70 & 2.48 & 8.04 & 20.22 \\
\hline 6 & 4 & 2 & 0.30 & 1.73 & 1.34 & 22.78 & 20.22 \\
\hline 6 & 4 & 2 & 0.30 & 1.82 & 1.45 & 20.43 & 20.22 \\
\hline 6 & 4 & 2 & 0.31 & 2.22 & 1.77 & 20.56 & 25.43 \\
\hline 6 & 4 & 2 & 0.31 & 2.08 & 1.60 & 22.80 & 25.43 \\
\hline 6 & 4 & 2 & 0.31 & 2.61 & 2.21 & 15.57 & 25.43 \\
\hline 6 & 4 & 2 & 0.31 & 2.89 & 2.52 & 12.78 & 25.43 \\
\hline 6 & 4 & 2 & 0.31 & 1.86 & 1.36 & 27.11 & 25.43 \\
\hline 6 & 4 & 2 & 0.31 & 1.96 & 1.47 & 25.00 & 25.43 \\
\hline 6 & 4 & 2 & 0.35 & 2.62 & 1.83 & 30.03 & 35.81 \\
\hline
\end{tabular}




\begin{tabular}{|c|c|c|c|c|c|c|c|}
\hline Prod. & Server & Subtasks & $\begin{array}{c}\text { Net Traffic } \\
\text { Intensity }\end{array}$ & $\begin{array}{c}\text { Anal. } \\
\text { Flow T. }\end{array}$ & $\begin{array}{l}\text { Sim. } \\
\text { Flow }\end{array}$ & $\begin{array}{c}\text { Error } \\
\text { sim-anal }(\%)\end{array}$ & $\begin{array}{l}\text { Predicted } \\
\text { Error }(\%)\end{array}$ \\
\hline 6 & 4 & 2 & 0.35 & 2.45 & 1.67 & 32.06 & 35.81 \\
\hline 6 & 4 & 2 & 0.35 & 3.06 & 2.29 & 25.05 & 35.81 \\
\hline 6 & 4 & 2 & 0.35 & 3.37 & 2.62 & 22.30 & 35.81 \\
\hline 6 & 4 & 2 & 0.35 & 2.21 & 1.41 & 36.19 & 35.81 \\
\hline 6 & 4 & 2 & 0.35 & 2.33 & 1.53 & 34.34 & 35.81 \\
\hline 1 & 4 & 3 & 0.08 & 2.11 & 1.69 & 19.57 & 5.52 \\
\hline 1 & 4 & 3 & 0.08 & 2.23 & 1.70 & 23.65 & 10.85 \\
\hline 1 & 4 & 3 & 0.09 & 2.51 & 1.71 & 31.83 & 21.50 \\
\hline 2 & 4 & 3 & 0.14 & 2.22 & 1.79 & 19.65 & 7.63 \\
\hline 2 & 4 & 3 & 0.14 & 2.04 & 1.62 & 20.59 & 7.63 \\
\hline 2 & 4 & 3 & 0.15 & 2.35 & 1.80 & 23.66 & 12.92 \\
\hline 2 & 4 & 3 & 0.15 & 2.17 & 1.63 & 24.70 & 12.92 \\
\hline 2 & 4 & 3 & 0.17 & 2.68 & 1.82 & 32.07 & 23.48 \\
\hline 2 & 4 & 3 & 0.17 & 2.47 & 1.66 & 32.96 & 23.48 \\
\hline 3 & 4 & 3 & 0.23 & 2.49 & 1.93 & 22.31 & 9.50 \\
\hline 3 & 4 & 3 & 0.23 & 2.32 & 1.76 & 24.20 & 9.50 \\
\hline 3 & 4 & 3 & 0.23 & 2.97 & 2.42 & 18.57 & 9.50 \\
\hline 3 & 4 & 3 & 0.25 & 2.66 & 1.96 & 26.53 & 14.74 \\
\hline 3 & 4 & 3 & 0.25 & 2.48 & 1.78 & 28.34 & 14.74 \\
\hline 3 & 4 & 3 & 0.25 & 3.17 & 2.44 & 22.82 & 14.74 \\
\hline 3 & 4 & 3 & 0.27 & 3.09 & 2.01 & 34.93 & 25.18 \\
\hline 3 & 4 & 3 & 0.27 & 2.89 & 1.83 & 36.76 & 25.18 \\
\hline 3 & 4 & 3 & 0.27 & 3.66 & 2.52 & 31.27 & 25.18 \\
\hline 4 & 4 & 3 & 0.33 & 2.94 & 2.14 & 27.14 & 11.25 \\
\hline 4 & 4 & 3 & 0.33 & 2.77 & 1.95 & 29.69 & 11.25 \\
\hline 4 & 4 & 3 & 0.33 & 3.41 & 2.68 & 21.38 & 11.25 \\
\hline 4 & 4 & 3 & 0.33 & 3.74 & 3.06 & 18.05 & 11.25 \\
\hline 4 & 4 & 3 & 0.35 & 3.19 & 2.19 & 31.36 & 16.43 \\
\hline 4 & 4 & 3 & 0.35 & 3.01 & 1.99 & 33.99 & 16.43 \\
\hline 4 & 4 & 3 & 0.35 & 3.68 & 2.74 & 25.75 & 16.43 \\
\hline 4 & 4 & 3 & 0.35 & 4.04 & 3.13 & 22.55 & 16.43 \\
\hline 4 & 4 & 3 & 0.39 & 3.83 & 2.30 & 39.89 & 26.73 \\
\hline 4 & 4 & 3 & 0.39 & 3.63 & 2.09 & 42.40 & 26.73 \\
\hline 4 & 4 & 3 & 0.39 & 4.37 & 2.88 & 34.24 & 26.73 \\
\hline 4 & 4 & 3 & 0.39 & 4.78 & 3.29 & 31.25 & 26.73 \\
\hline 5 & 4 & 3 & 0.38 & 3.14 & 2.27 & 27.59 & 13.56 \\
\hline 5 & 4 & 3 & 0.38 & 2.98 & 2.07 & 30.56 & 13.56 \\
\hline 5 & 4 & 3 & 0.38 & 3.60 & 2.84 & 20.96 & 13.56 \\
\hline 5 & 4 & 3 & 0.38 & 3.93 & 3.25 & 17.27 & 13.56 \\
\hline 5 & 4 & 3 & 0.38 & 2.73 & 1.75 & 35.83 & 13.56 \\
\hline
\end{tabular}




\begin{tabular}{|c|c|c|c|c|c|c|c|}
\hline Prod. & Server & Subtasks & $\begin{array}{c}\text { Net Traffic } \\
\text { Intensity }\end{array}$ & $\begin{array}{l}\text { Anal. } \\
\text { Flow T. }\end{array}$ & $\begin{array}{l}\text { Sim. } \\
\text { Flow }\end{array}$ & $\begin{array}{c}\text { Error } \\
\text { sim-anal }(\%)\end{array}$ & $\begin{array}{l}\text { Predicted } \\
\text { Error }(\%)\end{array}$ \\
\hline 5 & 4 & 3 & 0.40 & 3.43 & 2.33 & 31.94 & 18.72 \\
\hline 5 & 4 & 3 & 0.40 & 3.25 & 2.12 & 34.85 & 18.72 \\
\hline 5 & 4 & 3 & 0.40 & 3.91 & 2.92 & 25.45 & 18.72 \\
\hline 5 & 4 & 3 & 0.40 & 4.26 & 3.33 & 21.86 & 18.72 \\
\hline 5 & 4 & 3 & 0.40 & 2.99 & 1.79 & 39.93 & 18.72 \\
\hline 5 & 4 & 3 & 0.45 & 4.20 & 2.49 & 40.71 & 28.95 \\
\hline 5 & 4 & 3 & 0.45 & 4.00 & 2.26 & 43.47 & 28.95 \\
\hline 5 & 4 & 3 & 0.45 & 4.74 & 3.11 & 34.42 & 28.95 \\
\hline 5 & 4 & 3 & 0.45 & 5.14 & 3.55 & 30.90 & 28.95 \\
\hline 5 & 4 & 3 & 0.45 & 3.69 & 1.91 & 48.16 & 28.95 \\
\hline 6 & 4 & 3 & 0.44 & 3.27 & 2.36 & 27.84 & 16.09 \\
\hline 6 & 4 & 3 & 0.44 & 3.11 & 2.15 & 30.85 & 16.09 \\
\hline 6 & 4 & 3 & 0.44 & 3.72 & 2.95 & 20.72 & 16.09 \\
\hline 6 & 4 & 3 & 0.44 & 4.05 & 3.37 & 16.69 & 16.09 \\
\hline 6 & 4 & 3 & 0.44 & 2.86 & 1.82 & 36.41 & 16.09 \\
\hline 6 & 4 & 3 & 0.44 & 2.98 & 1.97 & 33.83 & 16.09 \\
\hline 6 & 4 & 3 & 0.47 & 3.59 & 2.43 & 32.23 & 21.22 \\
\hline 6 & 4 & 3 & 0.47 & 3.42 & 2.21 & 35.31 & 21.22 \\
\hline 6 & 4 & 3 & 0.47 & 4.07 & 3.04 & 25.35 & 21.22 \\
\hline 6 & 4 & 3 & 0.47 & 4.41 & 3.48 & 21.28 & 21.22 \\
\hline 6 & 4 & 3 & 0.47 & 3.15 & 1.87 & 40.66 & 21.22 \\
\hline 6 & 4 & 3 & 0.47 & 3.27 & 2.03 & 38.01 & 21.22 \\
\hline 6 & 4 & 3 & 0.52 & 4.44 & 2.62 & 40.94 & 31.42 \\
\hline 6 & 4 & 3 & 0.52 & 4.25 & 2.38 & 43.90 & 31.42 \\
\hline 6 & 4 & 3 & 0.52 & 4.99 & 3.28 & 34.32 & 31.42 \\
\hline 6 & 4 & 3 & 0.52 & 5.39 & 3.75 & 30.47 & 31.42 \\
\hline 6 & 4 & 3 & 0.52 & 3.94 & 2.02 & 48.84 & 31.42 \\
\hline 6 & 4 & 3 & 0.52 & 4.08 & 2.18 & 46.49 & 31.42 \\
\hline
\end{tabular}

Approximate and simulated flow times for the $H_{2}-\operatorname{Exp}$ case

\begin{tabular}{|c|c|c|c|c|c|c|c|}
\hline Prod. & Server & Subtasks & $\begin{array}{c}\text { Net Traffic } \\
\text { Intensity }\end{array}$ & $\begin{array}{l}\text { Anal. } \\
\text { Flow T. }\end{array}$ & Sim. & $\begin{array}{c}\text { Error } \\
\text { sim-anal }(\%)\end{array}$ & $\begin{array}{l}\text { Predicted } \\
\text { Error (\%) }\end{array}$ \\
\hline 1 & 2 & 2 & 0.10 & 1.73 & 1.68 & 2.64 & 4.24 \\
\hline 1 & 2 & 2 & 0.11 & 1.84 & 1.69 & 7.98 & 1.08 \\
\hline 1 & 2 & 2 & 0.12 & 2.10 & 1.72 & 18.09 & 11.70 \\
\hline 2 & 2 & 2 & 0.19 & 1.95 & 1.91 & 1.90 & 2.48 \\
\hline 2 & 2 & 2 & 0.19 & 1.95 & 1.91 & 1.69 & 2.48 \\
\hline 2 & 2 & 2 & 0.20 & 2.09 & 1.94 & 7.01 & 2.78 \\
\hline 2 & 2 & 2 & 0.20 & 2.09 & 1.94 & 7.03 & 2.78 \\
\hline
\end{tabular}




\begin{tabular}{|c|c|c|c|c|c|c|c|}
\hline Prod. & Server & Subtasks & $\begin{array}{l}\text { Net Traffic } \\
\text { Intensity }\end{array}$ & $\begin{array}{c}\text { Anal. } \\
\text { Flow T. }\end{array}$ & Sim. & $\begin{array}{c}\text { Error } \\
\text { sim-anal (\%) }\end{array}$ & $\begin{array}{l}\text { Predicted } \\
\text { Error (\%) }\end{array}$ \\
\hline 2 & 2 & 2 & 0.22 & 2.42 & 2.01 & 17.18 & 13.26 \\
\hline 2 & 2 & 2 & 0.22 & 2.42 & 2.01 & 17.07 & 13.26 \\
\hline 3 & 2 & 2 & 0.31 & 2.22 & 2.21 & 0.69 & 0.72 \\
\hline 3 & 2 & 2 & 0.31 & 2.22 & 2.21 & 0.64 & 0.72 \\
\hline 3 & 2 & 2 & 0.31 & 2.23 & 2.21 & 0.88 & 0.72 \\
\hline 3 & 2 & 2 & 0.33 & 2.41 & 2.26 & 6.27 & 4.48 \\
\hline 3 & 2 & 2 & 0.33 & 2.42 & 2.26 & 6.35 & 4.48 \\
\hline 3 & 2 & 2 & 0.33 & 2.41 & 2.26 & 6.31 & 4.48 \\
\hline 3 & 2 & 2 & 0.37 & 2.88 & 2.40 & 16.72 & 14.83 \\
\hline 3 & 2 & 2 & 0.37 & 2.88 & 2.40 & 16.53 & 14.83 \\
\hline 3 & 2 & 2 & 0.37 & 2.88 & 2.40 & 16.60 & 14.83 \\
\hline 4 & 2 & 2 & 0.44 & 2.61 & 2.60 & 0.25 & 1.04 \\
\hline 4 & 2 & 2 & 0.44 & 2.61 & 2.60 & 0.28 & 1.04 \\
\hline 4 & 2 & 2 & 0.44 & 2.61 & 2.60 & 0.46 & 1.04 \\
\hline 4 & 2 & 2 & 0.44 & 2.61 & 2.60 & 0.48 & 1.04 \\
\hline 4 & 2 & 2 & 0.47 & 2.87 & 2.70 & 5.83 & 6.18 \\
\hline 4 & 2 & 2 & 0.47 & 2.86 & 2.70 & 5.76 & 6.18 \\
\hline 4 & 2 & 2 & 0.47 & 2.86 & 2.70 & 5.56 & 6.18 \\
\hline 4 & 2 & 2 & 0.47 & 2.87 & 2.70 & 5.76 & 6.18 \\
\hline 4 & 2 & 2 & 0.52 & 3.56 & 2.97 & 16.55 & 16.39 \\
\hline 4 & 2 & 2 & 0.52 & 3.55 & 2.97 & 16.54 & 16.39 \\
\hline 4 & 2 & 2 & 0.52 & 3.56 & 2.97 & 16.61 & 16.39 \\
\hline 4 & 2 & 2 & 0.52 & 3.55 & 2.97 & 16.40 & 16.39 \\
\hline 5 & 2 & 2 & 0.51 & 2.86 & 2.85 & 0.35 & 3.34 \\
\hline 5 & 2 & 2 & 0.51 & 2.87 & 2.85 & 0.72 & 3.34 \\
\hline 5 & 2 & 2 & 0.51 & 2.87 & 2.85 & 0.58 & 3.34 \\
\hline 5 & 2 & 2 & 0.51 & 2.86 & 2.85 & 0.30 & 3.34 \\
\hline 5 & 2 & 2 & 0.51 & 2.94 & 3.07 & 4.50 & 0.83 \\
\hline 5 & 2 & 2 & 0.54 & 3.16 & 2.98 & 5.61 & 8.46 \\
\hline 5 & 2 & 2 & 0.54 & 3.18 & 2.98 & 6.08 & 8.46 \\
\hline 5 & 2 & 2 & 0.54 & 3.17 & 2.98 & 5.75 & 8.46 \\
\hline 5 & 2 & 2 & 0.54 & 3.17 & 2.98 & 5.79 & 8.46 \\
\hline 5 & 2 & 2 & 0.54 & 3.24 & 3.23 & 0.50 & 5.95 \\
\hline 5 & 2 & 2 & 0.60 & 4.00 & 3.36 & 16.16 & 18.60 \\
\hline 5 & 2 & 2 & 0.60 & 4.01 & 3.36 & 16.23 & 18.60 \\
\hline 5 & 2 & 2 & 0.60 & 4.01 & 3.36 & 16.40 & 18.60 \\
\hline 5 & 2 & 2 & 0.60 & 4.00 & 3.36 & 16.05 & 18.60 \\
\hline 5 & 2 & 2 & 0.60 & 4.12 & 3.66 & 11.19 & 16.09 \\
\hline 6 & 2 & 2 & 0.59 & 3.17 & 3.15 & 0.55 & 5.65 \\
\hline 6 & 2 & 2 & 0.59 & 3.16 & 3.15 & 0.47 & 5.65 \\
\hline
\end{tabular}




\begin{tabular}{|c|c|c|c|c|c|c|c|}
\hline Prod. & Server & Subtasks & $\begin{array}{l}\text { Net Traffic } \\
\text { Intensity }\end{array}$ & $\begin{array}{l}\text { Anal. } \\
\text { Flow T. }\end{array}$ & Sim. & $\begin{array}{c}\text { Error } \\
\text { sim-anal }(\%)\end{array}$ & $\begin{array}{l}\text { Predicted } \\
\text { Error }(\%)\end{array}$ \\
\hline 6 & 2 & 2 & 0.59 & 3.17 & 3.15 & 0.65 & 5.65 \\
\hline 6 & 2 & 2 & 0.59 & 3.16 & 3.15 & 0.45 & 5.65 \\
\hline 6 & 2 & 2 & 0.59 & 3.23 & 3.42 & 5.78 & 3.14 \\
\hline 6 & 2 & 2 & 0.59 & 3.23 & 3.42 & $\begin{array}{l}5.82 \\
\end{array}$ & 3.14 \\
\hline 6 & 2 & 2 & 0.62 & 3.55 & 3.33 & 6.12 & 10.74 \\
\hline 6 & 2 & 2 & 0.62 & 3.56 & 3.33 & 6.29 & 10.74 \\
\hline 6 & 2 & 2 & 0.62 & 3.56 & 3.33 & 6.34 & 10.74 \\
\hline 6 & 2 & 2 & 0.62 & 3.56 & 3.33 & 6.24 & 10.74 \\
\hline 6 & 2 & 2 & 0.62 & 3.64 & 3.63 & 0.26 & 8.22 \\
\hline 6 & 2 & 2 & 0.62 & 3.65 & 3.63 & 0.45 & 8.22 \\
\hline 6 & 2 & 2 & 0.69 & 4.66 & 3.86 & 17.22 & 20.81 \\
\hline 6 & 2 & 2 & 0.69 & 4.65 & 3.86 & 17.09 & 20.81 \\
\hline 6 & 2 & 2 & 0.69 & 4.66 & 3.86 & 17.23 & 20.81 \\
\hline 6 & 2 & 2 & 0.69 & 4.66 & 3.86 & 17.23 & 20.81 \\
\hline 6 & 2 & 2 & 0.69 & 4.78 & 4.24 & 11.25 & 18.30 \\
\hline 6 & 2 & 2 & 0.69 & 4.79 & 4.24 & 11.57 & 18.30 \\
\hline 1 & 3 & 3 & 0.10 & 2.10 & 2.04 & 2.83 & 2.46 \\
\hline 1 & 3 & 3 & 0.11 & 2.23 & 2.05 & 7.83 & 2.85 \\
\hline 1 & 3 & 3 & 0.12 & 2.54 & 2.08 & 17.96 & 13.47 \\
\hline 2 & 3 & 3 & 0.19 & 2.36 & 2.30 & 2.23 & 0.71 \\
\hline 2 & 3 & 3 & 0.19 & 2.36 & 2.30 & 2.25 & 0.71 \\
\hline 2 & 3 & 3 & 0.20 & 2.52 & 2.33 & 7.30 & 4.55 \\
\hline 2 & 3 & 3 & 0.20 & 2.52 & 2.33 & 7.29 & 4.55 \\
\hline 2 & 3 & 3 & 0.22 & 2.92 & 2.41 & 17.55 & 15.04 \\
\hline 2 & 3 & 3 & 0.22 & 2.92 & 2.41 & 17.48 & 15.04 \\
\hline 3 & 3 & 3 & 0.31 & 2.68 & 2.64 & 1.58 & 1.05 \\
\hline 3 & 3 & 3 & 0.31 & 2.69 & 2.64 & 1.76 & 1.05 \\
\hline 3 & 3 & 3 & 0.31 & 2.69 & 2.64 & 1.77 & 1.05 \\
\hline 3 & 3 & 3 & 0.33 & 2.89 & 2.70 & 6.64 & 6.25 \\
\hline 3 & 3 & 3 & 0.33 & 2.90 & 2.70 & 6.74 & 6.25 \\
\hline 3 & 3 & 3 & 0.33 & 2.89 & 2.70 & 6.59 & 6.25 \\
\hline 3 & 3 & 3 & 0.37 & 3.44 & 2.86 & 16.97 & 16.60 \\
\hline 3 & 3 & 3 & 0.37 & 3.45 & 2.86 & 17.12 & 16.60 \\
\hline 3 & 3 & 3 & 0.37 & 3.46 & 2.86 & 17.28 & 16.60 \\
\hline 4 & 3 & 3 & 0.44 & 3.13 & 3.09 & 1.27 & 2.81 \\
\hline 4 & 3 & 3 & 0.44 & 3.12 & 3.09 & 1.16 & 2.81 \\
\hline 4 & 3 & 3 & 0.44 & 3.13 & 3.09 & 1.24 & 2.81 \\
\hline 4 & 3 & 3 & 0.44 & 3.12 & 3.09 & 1.17 & 2.81 \\
\hline 4 & 3 & 3 & 0.47 & 3.43 & 3.20 & 6.62 & 7.95 \\
\hline 4 & 3 & 3 & 0.47 & 3.43 & 3.20 & 6.75 & 7.95 \\
\hline
\end{tabular}




\begin{tabular}{|c|c|c|c|c|c|c|c|}
\hline Prod. & Server & Subtasks & $\begin{array}{l}\text { Net Traffic } \\
\text { Intensity }\end{array}$ & $\begin{array}{l}\text { Anal. } \\
\text { Flow T. }\end{array}$ & Sim. & $\begin{array}{c}\text { Error } \\
\text { sim-anal }(\%)\end{array}$ & $\begin{array}{l}\text { Predicted } \\
\text { Error }(\%)\end{array}$ \\
\hline 4 & 3 & 3 & 0.47 & 3.43 & 3.20 & 6.70 & 7.95 \\
\hline 4 & 3 & 3 & 0.47 & 3.43 & 3.20 & 6.59 & 7.95 \\
\hline 4 & 3 & 3 & 0.52 & 4.22 & 3.50 & 16.88 & 18.17 \\
\hline 4 & 3 & 3 & 0.52 & 4.22 & 3.50 & 16.99 & 18.17 \\
\hline 4 & 3 & 3 & 0.52 & 4.22 & 3.50 & 17.01 & 18.17 \\
\hline 4 & 3 & 3 & 0.52 & 4.22 & 3.50 & 17.03 & 18.17 \\
\hline 5 & 3 & 3 & 0.51 & 3.42 & 3.37 & 1.41 & 5.11 \\
\hline 5 & 3 & 3 & 0.51 & 3.41 & 3.37 & 1.26 & 5.11 \\
\hline 5 & 3 & 3 & 0.51 & 3.42 & 3.37 & 1.31 & 5.11 \\
\hline 5 & 3 & 3 & 0.51 & 3.42 & 3.37 & 1.44 & 5.11 \\
\hline 5 & 3 & 3 & 0.51 & 3.48 & 3.60 & 3.32 & 2.60 \\
\hline 5 & 3 & 3 & 0.54 & 3.77 & 3.52 & 6.54 & 10.23 \\
\hline 5 & 3 & 3 & 0.54 & 3.77 & 3.52 & 6.41 & 10.23 \\
\hline 5 & 3 & 3 & 0.54 & 3.77 & 3.52 & 6.41 & 10.23 \\
\hline 5 & 3 & 3 & 0.54 & 3.77 & 3.52 & 6.51 & 10.23 \\
\hline 5 & 3 & 3 & 0.54 & 3.85 & 3.77 & 2.05 & 7.72 \\
\hline 5 & 3 & 3 & 0.60 & 4.77 & 3.95 & 17.19 & 20.37 \\
\hline 5 & 3 & 3 & 0.60 & 4.76 & 3.95 & 17.12 & 20.37 \\
\hline 5 & 3 & 3 & 0.60 & 4.77 & 3.95 & 17.28 & 20.37 \\
\hline 5 & 3 & 3 & 0.60 & 4.77 & 3.95 & 17.18 & 20.37 \\
\hline 5 & 3 & 3 & 0.60 & 4.88 & 4.26 & 12.73 & 17.86 \\
\hline 6 & 3 & 3 & 0.59 & 3.77 & 3.71 & 1.56 & 7.42 \\
\hline 6 & 3 & 3 & 0.59 & 3.77 & 3.71 & 1.61 & 7.42 \\
\hline 6 & 3 & 3 & 0.59 & 3.77 & 3.71 & 1.45 & 7.42 \\
\hline 6 & 3 & 3 & 0.59 & 3.77 & 3.71 & 1.40 & 7.42 \\
\hline 6 & 3 & 3 & 0.59 & 3.85 & 3.99 & 3.44 & 4.91 \\
\hline 6 & 3 & 3 & 0.59 & 3.86 & 3.99 & 3.21 & 4.91 \\
\hline 6 & 3 & 3 & 0.62 & 4.20 & 3.92 & 6.68 & 12.51 \\
\hline 6 & 3 & 3 & 0.62 & 4.20 & 3.92 & 6.66 & 12.51 \\
\hline 6 & 3 & 3 & 0.62 & 4.20 & 3.92 & 6.64 & 12.51 \\
\hline 6 & 3 & 3 & 0.62 & 4.20 & 3.92 & 6.57 & 12.51 \\
\hline 6 & 3 & 3 & 0.62 & 4.30 & 4.23 & 1.73 & 10.00 \\
\hline 6 & 3 & 3 & 0.62 & 4.30 & 4.23 & 1.65 & 10.00 \\
\hline 6 & 3 & 3 & 0.69 & 5.47 & 4.52 & 17.34 & 22.58 \\
\hline 6 & 3 & 3 & 0.69 & 5.47 & 4.52 & 17.37 & 22.58 \\
\hline 6 & 3 & 3 & 0.69 & 5.47 & 4.52 & 17.35 & 22.58 \\
\hline 6 & 3 & 3 & 0.69 & 5.47 & 4.52 & 17.45 & 22.58 \\
\hline 6 & 3 & 3 & 0.69 & 5.62 & 4.91 & 12.68 & 20.07 \\
\hline 6 & 3 & 3 & 0.69 & 5.61 & 4.91 & 12.55 & 20.07 \\
\hline 1 & 3 & 2 & 0.07 & 1.83 & 1.62 & 11.81 & 0.53 \\
\hline
\end{tabular}




\begin{tabular}{|c|c|c|c|c|c|c|c|}
\hline Prod. & Server & Subtasks & $\begin{array}{l}\text { Net Traffic } \\
\text { Intensity }\end{array}$ & $\begin{array}{l}\text { Anal. } \\
\text { Flow T. }\end{array}$ & Sim. & $\begin{array}{c}\text { Error } \\
\text { sim-anal }(\%)\end{array}$ & $\begin{array}{l}\text { Predicted } \\
\text { Error }(\%)\end{array}$ \\
\hline 1 & 3 & 2 & 0.07 & 1.94 & 1.62 & 16.12 & 5.86 \\
\hline 1 & 3 & 2 & 0.08 & 2.20 & 1.64 & 25.31 & 16.53 \\
\hline 2 & 3 & 2 & 0.13 & 1.97 & 1.75 & 11.09 & 2.65 \\
\hline 2 & 3 & 2 & 0.13 & 1.97 & 1.75 & 11.15 & 2.65 \\
\hline 2 & 3 & 2 & 0.13 & 2.09 & 1.77 & 15.47 & 7.95 \\
\hline 2 & 3 & 2 & 0.13 & 2.10 & 1.77 & 15.59 & 7.95 \\
\hline 2 & 3 & 2 & 0.15 & 2.40 & 1.81 & 24.78 & 18.52 \\
\hline 2 & 3 & 2 & 0.15 & 2.40 & 1.81 & 24.56 & 18.52 \\
\hline 3 & 3 & 2 & 0.21 & 2.13 & 1.91 & 10.23 & 4.77 \\
\hline 3 & 3 & 2 & 0.21 & 2.13 & 1.91 & 10.25 & 4.77 \\
\hline 3 & 3 & 2 & 0.21 & 2.13 & 1.91 & 10.38 & 4.77 \\
\hline 3 & 3 & 2 & 0.22 & 2.28 & 1.94 & 14.74 & 10.03 \\
\hline 3 & 3 & 2 & 0.22 & 2.28 & 1.94 & 14.88 & 10.03 \\
\hline 3 & 3 & 2 & 0.22 & 2.27 & 1.94 & 14.69 & 10.03 \\
\hline 3 & 3 & 2 & 0.24 & 2.64 & 2.01 & 24.09 & 20.51 \\
\hline 3 & 3 & 2 & 0.24 & 2.65 & 2.01 & 24.14 & 20.51 \\
\hline 3 & 3 & 2 & 0.24 & 2.64 & 2.01 & 23.97 & 20.51 \\
\hline 4 & 3 & 2 & 0.30 & 2.32 & 2.10 & 9.61 & 6.89 \\
\hline 4 & 3 & 2 & 0.30 & 2.32 & 2.10 & 9.61 & 6.89 \\
\hline 4 & 3 & 2 & 0.30 & 2.32 & 2.10 & 9.54 & 6.89 \\
\hline 4 & 3 & 2 & 0.30 & 2.32 & 2.10 & 9.48 & 6.89 \\
\hline 4 & 3 & 2 & 0.31 & 2.50 & 2.14 & 14.11 & 12.12 \\
\hline 4 & 3 & 2 & 0.31 & 2.50 & 2.14 & 14.15 & 12.12 \\
\hline 4 & 3 & 2 & 0.31 & 2.50 & 2.14 & 14.19 & 12.12 \\
\hline 4 & 3 & 2 & 0.31 & 2.49 & 2.14 & 14.03 & 12.12 \\
\hline 4 & 3 & 2 & 0.35 & 2.95 & 2.25 & 23.48 & 22.51 \\
\hline 4 & 3 & 2 & 0.35 & 2.94 & 2.25 & 23.43 & 22.51 \\
\hline 4 & 3 & 2 & 0.35 & 2.94 & 2.25 & 23.34 & 22.51 \\
\hline 4 & 3 & 2 & 0.35 & 2.94 & 2.25 & 23.30 & 22.51 \\
\hline 5 & 3 & 2 & 0.34 & 2.44 & 2.21 & 9.41 & 9.38 \\
\hline 5 & 3 & 2 & 0.34 & 2.43 & 2.21 & 9.28 & 9.38 \\
\hline 5 & 3 & 2 & 0.34 & 2.44 & 2.21 & 9.52 & 9.38 \\
\hline 5 & 3 & 2 & 0.34 & 2.43 & 2.21 & 9.32 & 9.38 \\
\hline 5 & 3 & 2 & 0.34 & 2.45 & 2.32 & 5.29 & 6.87 \\
\hline 5 & 3 & 2 & 0.36 & 2.63 & 2.26 & 13.88 & 14.59 \\
\hline 5 & 3 & 2 & 0.36 & 2.63 & 2.26 & 13.89 & 14.59 \\
\hline 5 & 3 & 2 & 0.36 & 2.63 & 2.26 & 13.92 & 14.59 \\
\hline 5 & 3 & 2 & 0.36 & 2.63 & 2.26 & 14.01 & 14.59 \\
\hline 5 & 3 & 2 & 0.36 & 2.66 & 2.38 & 10.21 & 12.07 \\
\hline 5 & 3 & 2 & 0.40 & 3.12 & 2.40 & 23.11 & 24.93 \\
\hline
\end{tabular}




\begin{tabular}{|c|c|c|c|c|c|c|c|}
\hline Prod. & Server & Subtasks & $\begin{array}{l}\text { Net Traffic } \\
\text { Intensity }\end{array}$ & $\begin{array}{c}\text { Anal. } \\
\text { Flow T. }\end{array}$ & Sim. & $\begin{array}{c}\text { Error } \\
\text { sim-anal }(\%)\end{array}$ & $\begin{array}{l}\text { Predicted } \\
\text { Error }(\%)\end{array}$ \\
\hline 5 & 3 & 2 & 0.40 & 3.12 & 2.40 & 23.19 & 24.93 \\
\hline 5 & 3 & 2 & 0.40 & 3.13 & 2.40 & 23.21 & 24.93 \\
\hline 5 & 3 & 2 & 0.40 & 3.12 & 2.40 & 23.01 & 24.93 \\
\hline 5 & 3 & 2 & 0.40 & 3.16 & 2.55 & 19.39 & 22.42 \\
\hline 6 & 3 & 2 & 0.39 & 2.56 & 2.32 & 9.16 & 11.87 \\
\hline 6 & 3 & 2 & 0.39 & 2.56 & 2.32 & 9.02 & 11.87 \\
\hline 6 & 3 & 2 & 0.39 & 2.56 & 2.32 & 9.08 & 11.87 \\
\hline 6 & 3 & 2 & 0.39 & 2.55 & 2.32 & 8.93 & 11.87 \\
\hline 6 & 3 & 2 & 0.39 & 2.59 & 2.46 & 4.96 & 9.36 \\
\hline 6 & 3 & 2 & 0.39 & 2.59 & 2.46 & 5.07 & 9.36 \\
\hline 6 & 3 & 2 & 0.41 & 2.77 & 2.39 & 13.80 & 17.05 \\
\hline 6 & 3 & 2 & 0.41 & 2.78 & 2.39 & 13.93 & 17.05 \\
\hline 6 & 3 & 2 & 0.41 & 2.78 & 2.39 & 13.98 & 17.05 \\
\hline 6 & 3 & 2 & 0.41 & 2.78 & 2.39 & 14.03 & 17.05 \\
\hline 6 & 3 & 2 & 0.41 & 2.81 & 2.54 & 9.86 & 14.54 \\
\hline 6 & 3 & 2 & 0.41 & 2.82 & 2.54 & 10.04 & 14.54 \\
\hline 6 & 3 & 2 & 0.46 & 3.33 & 2.56 & 22.97 & 27.35 \\
\hline 6 & 3 & 2 & 0.46 & 3.33 & 2.56 & 23.05 & 27.35 \\
\hline 6 & 3 & 2 & 0.46 & 3.33 & 2.56 & 22.89 & 27.35 \\
\hline 6 & 3 & 2 & 0.46 & 3.33 & 2.56 & 22.93 & 27.35 \\
\hline 6 & 3 & 2 & 0.46 & 3.38 & 2.74 & 18.96 & 24.84 \\
\hline 6 & 3 & 2 & 0.46 & 3.38 & 2.74 & 19.11 & 24.84 \\
\hline 1 & 4 & 4 & 0.10 & 2.38 & 2.31 & 3.02 & 0.69 \\
\hline 1 & 4 & 4 & 0.11 & 2.52 & 2.32 & 7.96 & 4.63 \\
\hline 1 & 4 & 4 & 0.12 & 2.87 & 2.35 & 17.97 & 15.24 \\
\hline 2 & 4 & 4 & 0.19 & 2.65 & 2.59 & 2.30 & 1.07 \\
\hline 2 & 4 & 4 & 0.19 & 2.66 & 2.59 & 2.40 & 1.07 \\
\hline 2 & 4 & 4 & 0.20 & 2.84 & 2.63 & 7.46 & 6.33 \\
\hline 2 & 4 & 4 & 0.20 & 2.84 & 2.63 & 7.43 & 6.33 \\
\hline 2 & 4 & 4 & 0.22 & 3.28 & 2.71 & 17.47 & 16.81 \\
\hline 2 & 4 & 4 & 0.22 & 3.29 & 2.71 & 17.63 & 16.81 \\
\hline 3 & 4 & 4 & 0.31 & 3.01 & 2.96 & 1.91 & 2.82 \\
\hline 3 & 4 & 4 & 0.31 & 3.01 & 2.96 & 1.66 & 2.82 \\
\hline 3 & 4 & 4 & 0.31 & 3.01 & 2.96 & 1.74 & 2.82 \\
\hline 3 & 4 & 4 & 0.33 & 3.25 & 3.02 & 6.98 & 8.03 \\
\hline 3 & 4 & 4 & 0.33 & 3.26 & 3.02 & 7.17 & 8.03 \\
\hline 3 & 4 & 4 & 0.33 & 3.25 & 3.02 & 6.97 & 8.03 \\
\hline 3 & 4 & 4 & 0.37 & 3.87 & 3.19 & 17.51 & 18.37 \\
\hline 3 & 4 & 4 & 0.37 & 3.87 & 3.19 & 17.40 & 18.37 \\
\hline 3 & 4 & 4 & 0.37 & 3.88 & 3.19 & 17.58 & 18.37 \\
\hline
\end{tabular}




\begin{tabular}{|c|c|c|c|c|c|c|c|}
\hline Prod. & Server & Subtasks & $\begin{array}{l}\text { Net Traffic } \\
\text { Intensity }\end{array}$ & $\begin{array}{l}\text { Anal. } \\
\text { Flow T. }\end{array}$ & Sim. & $\begin{array}{c}\text { Error } \\
\text { sim-anal }(\%)\end{array}$ & $\begin{array}{l}\text { Predicted } \\
\text { Error }(\%)\end{array}$ \\
\hline 4 & 4 & 4 & 0.44 & 3.50 & 3.44 & 1.62 & 4.58 \\
\hline 4 & 4 & 4 & 0.44 & 3.50 & 3.44 & 1.57 & 4.58 \\
\hline 4 & 4 & 4 & 0.44 & 3.50 & 3.44 & 1.81 & 4.58 \\
\hline 4 & 4 & 4 & 0.44 & 3.50 & 3.44 & 1.55 & 4.58 \\
\hline 4 & 4 & 4 & 0.47 & 3.83 & 3.56 & 6.90 & 9.73 \\
\hline 4 & 4 & 4 & 0.47 & 3.83 & 3.56 & 6.92 & 9.73 \\
\hline 4 & 4 & 4 & 0.47 & 3.83 & 3.56 & 6.86 & 9.73 \\
\hline 4 & 4 & 4 & 0.47 & 3.83 & 3.56 & 6.95 & 9.73 \\
\hline 4 & 4 & 4 & 0.52 & 4.71 & 3.89 & 17.35 & 19.94 \\
\hline 4 & 4 & 4 & 0.52 & 4.71 & 3.89 & 17.34 & 19.94 \\
\hline 4 & 4 & 4 & 0.52 & 4.71 & 3.89 & 17.35 & 19.94 \\
\hline 4 & 4 & 4 & 0.52 & 4.72 & 3.89 & 17.43 & 19.94 \\
\hline 5 & 4 & 4 & 0.51 & 3.82 & 3.75 & 1.77 & 6.89 \\
\hline 5 & 4 & 4 & 0.51 & 3.81 & 3.75 & 1.61 & 6.89 \\
\hline 5 & 4 & 4 & 0.51 & 3.81 & 3.75 & 1.65 & 6.89 \\
\hline 5 & 4 & 4 & 0.51 & 3.81 & 3.75 & 1.63 & 6.89 \\
\hline 5 & 4 & 4 & 0.51 & 3.89 & 3.98 & 2.27 & 4.37 \\
\hline 5 & 4 & 4 & 0.54 & 4.21 & 3.92 & 7.03 & 12.00 \\
\hline 5 & 4 & 4 & 0.54 & 4.22 & 3.92 & 7.11 & 12.00 \\
\hline 5 & 4 & 4 & 0.54 & 4.22 & 3.92 & 7.15 & 12.00 \\
\hline 5 & 4 & 4 & 0.54 & 4.22 & 3.92 & 7.11 & 12.00 \\
\hline 5 & 4 & 4 & 0.54 & 4.31 & 4.17 & 3.26 & 9.49 \\
\hline 5 & 4 & 4 & 0.60 & 5.33 & 4.37 & 17.94 & 22.15 \\
\hline 5 & 4 & 4 & 0.60 & 5.32 & 4.37 & 17.79 & 22.15 \\
\hline 5 & 4 & 4 & 0.60 & 5.32 & 4.37 & 17.83 & 22.15 \\
\hline 5 & 4 & 4 & 0.60 & 5.34 & 4.37 & 18.06 & 22.15 \\
\hline 5 & 4 & 4 & 0.60 & 5.48 & 4.69 & 14.41 & 19.63 \\
\hline 6 & 4 & 4 & 0.59 & 4.20 & 4.12 & 1.92 & 9.19 \\
\hline 6 & 4 & 4 & 0.59 & 4.20 & 4.12 & 1.98 & 9.19 \\
\hline 6 & 4 & 4 & 0.59 & 4.20 & 4.12 & 1.82 & 9.19 \\
\hline 6 & 4 & 4 & 0.59 & 4.21 & 4.12 & 2.13 & 9.19 \\
\hline 6 & 4 & 4 & 0.59 & 4.30 & 4.40 & 2.24 & 6.68 \\
\hline 6 & 4 & 4 & 0.59 & 4.29 & 4.40 & 2.60 & 6.68 \\
\hline 6 & 4 & 4 & 0.62 & 4.68 & 4.35 & 7.14 & 14.28 \\
\hline 6 & 4 & 4 & 0.62 & 4.68 & 4.35 & 7.12 & 14.28 \\
\hline 6 & 4 & 4 & 0.62 & 4.67 & 4.35 & 7.00 & 14.28 \\
\hline 6 & 4 & 4 & 0.62 & 4.69 & 4.35 & 7.30 & 14.28 \\
\hline 6 & 4 & 4 & 0.62 & 4.78 & 4.65 & 2.61 & 11.77 \\
\hline 6 & 4 & 4 & 0.62 & 4.78 & 4.65 & 2.63 & 11.77 \\
\hline 6 & 4 & 4 & 0.69 & 6.09 & 4.99 & 18.04 & 24.35 \\
\hline
\end{tabular}




\begin{tabular}{|c|c|c|c|c|c|c|c|}
\hline Prod. & Server & Subtasks & $\begin{array}{l}\text { Net Traffic } \\
\text { Intensity }\end{array}$ & $\begin{array}{l}\text { Anal. } \\
\text { Flow T. }\end{array}$ & Sim. & $\begin{array}{c}\text { Error } \\
\text { sim-anal }(\%)\end{array}$ & $\begin{array}{l}\text { Predicted } \\
\text { Error }(\%)\end{array}$ \\
\hline 6 & 4 & 4 & 0.69 & 6.09 & 4.99 & 18.06 & 24.35 \\
\hline 6 & 4 & 4 & 0.69 & 6.08 & 4.99 & 17.91 & 24.35 \\
\hline 6 & 4 & 4 & 0.69 & 6.09 & 4.99 & 17.99 & 24.35 \\
\hline 6 & 4 & 4 & 0.69 & 6.23 & 5.39 & 13.53 & 21.84 \\
\hline 6 & 4 & 4 & 0.69 & 6.23 & 5.39 & 13.45 & 21.84 \\
\hline 1 & 4 & 2 & 0.05 & 1.75 & 1.59 & 9.15 & 5.11 \\
\hline 1 & 4 & 2 & 0.05 & 1.85 & 1.59 & 13.77 & 10.45 \\
\hline 1 & 4 & 2 & 0.06 & 2.08 & 1.60 & 22.97 & 21.14 \\
\hline 2 & 4 & 2 & 0.10 & 1.84 & 1.68 & 8.62 & 7.41 \\
\hline 2 & 4 & 2 & 0.10 & 1.84 & 1.68 & 8.65 & 7.41 \\
\hline 2 & 4 & 2 & 0.10 & 1.95 & 1.69 & 13.26 & 12.73 \\
\hline 2 & 4 & 2 & 0.10 & 1.95 & 1.69 & 13.22 & 12.73 \\
\hline 2 & 4 & 2 & 0.11 & 2.22 & 1.72 & 22.54 & 23.35 \\
\hline 2 & 4 & 2 & 0.11 & 2.22 & 1.72 & 22.38 & 23.35 \\
\hline 3 & 4 & 2 & 0.16 & 1.95 & 1.79 & 7.92 & 9.72 \\
\hline 3 & 4 & 2 & 0.16 & 1.95 & 1.79 & 7.96 & 9.72 \\
\hline 3 & 4 & 2 & 0.16 & 1.95 & 1.79 & 7.96 & 9.72 \\
\hline 3 & 4 & 2 & 0.16 & 2.07 & 1.81 & 12.68 & 15.01 \\
\hline 3 & 4 & 2 & 0.16 & 2.07 & 1.81 & 12.59 & 15.01 \\
\hline 3 & 4 & 2 & 0.16 & 2.08 & 1.81 & 12.93 & 15.01 \\
\hline 3 & 4 & 2 & 0.18 & 2.38 & 1.85 & 22.05 & 25.56 \\
\hline 3 & 4 & 2 & 0.18 & 2.38 & 1.85 & 22.02 & 25.56 \\
\hline 3 & 4 & 2 & 0.18 & 2.38 & 1.85 & 22.02 & 25.56 \\
\hline 4 & 4 & 2 & 0.22 & 2.07 & 1.91 & 7.47 & 12.02 \\
\hline 4 & 4 & 2 & 0.22 & 2.07 & 1.91 & 7.59 & 12.02 \\
\hline 4 & 4 & 2 & 0.22 & 2.07 & 1.91 & 7.45 & 12.02 \\
\hline 4 & 4 & 2 & 0.22 & 2.07 & 1.91 & 7.70 & 12.02 \\
\hline 4 & 4 & 2 & 0.23 & 2.21 & 1.94 & 12.27 & 17.28 \\
\hline 4 & 4 & 2 & 0.23 & 2.21 & 1.94 & 12.22 & 17.28 \\
\hline 4 & 4 & 2 & 0.23 & 2.21 & 1.94 & 12.23 & 17.28 \\
\hline 4 & 4 & 2 & 0.23 & 2.21 & 1.94 & 12.14 & 17.28 \\
\hline 4 & 4 & 2 & 0.26 & 2.56 & 2.01 & 21.53 & 27.76 \\
\hline 4 & 4 & 2 & 0.26 & 2.55 & 2.01 & 21.30 & 27.76 \\
\hline 4 & 4 & 2 & 0.26 & 2.56 & 2.01 & 21.65 & 27.76 \\
\hline 4 & 4 & 2 & 0.26 & 2.56 & 2.01 & 21.59 & 27.76 \\
\hline 5 & 4 & 2 & 0.26 & 2.14 & 1.98 & 7.47 & 14.60 \\
\hline 5 & 4 & 2 & 0.26 & 2.14 & 1.98 & 7.32 & 14.60 \\
\hline 5 & 4 & 2 & 0.26 & 2.13 & 1.98 & 7.27 & 14.60 \\
\hline 5 & 4 & 2 & 0.26 & 2.14 & 1.98 & 7.43 & 14.60 \\
\hline 5 & 4 & 2 & 0.26 & 2.15 & 2.05 & 4.73 & 12.09 \\
\hline
\end{tabular}




\begin{tabular}{|c|c|c|c|c|c|c|c|}
\hline Prod. & Server & Subtasks & $\begin{array}{l}\text { Net Traffic } \\
\text { Intensity }\end{array}$ & $\begin{array}{c}\text { Anal. } \\
\text { Flow T. }\end{array}$ & Sim. & $\begin{array}{c}\text { Error } \\
\text { sim-anal }(\%)\end{array}$ & $\begin{array}{l}\text { Predicted } \\
\text { Error }(\%)\end{array}$ \\
\hline 5 & 4 & 2 & 0.27 & 2.28 & 2.01 & 11.90 & 19.85 \\
\hline 5 & 4 & 2 & 0.27 & 2.29 & 2.01 & 12.03 & 19.85 \\
\hline 5 & 4 & 2 & 0.27 & 2.29 & 2.01 & 11.98 & 19.85 \\
\hline 5 & 4 & 2 & 0.27 & 2.29 & 2.01 & 11.98 & 19.85 \\
\hline 5 & 4 & 2 & 0.27 & 2.30 & 2.09 & 9.11 & 17.34 \\
\hline 5 & 4 & 2 & 0.30 & 2.66 & 2.09 & 21.19 & 30.30 \\
\hline 5 & 4 & 2 & 0.30 & 2.66 & 2.09 & 21.13 & 30.30 \\
\hline 5 & 4 & 2 & 0.30 & 2.66 & 2.09 & 21.26 & 30.30 \\
\hline 5 & 4 & 2 & 0.30 & 2.66 & 2.09 & 21.18 & 30.30 \\
\hline 5 & 4 & 2 & 0.30 & 2.68 & 2.06 & 23.40 & 27.78 \\
\hline 6 & 4 & 2 & 0.30 & 2.21 & 2.05 & 7.18 & 17.18 \\
\hline 6 & 4 & 2 & 0.30 & 2.21 & 2.05 & 7.15 & 17.18 \\
\hline 6 & 4 & 2 & 0.30 & 2.21 & 2.05 & 7.19 & 17.18 \\
\hline 6 & 4 & 2 & 0.30 & 2.21 & 2.05 & 7.28 & 17.18 \\
\hline 6 & 4 & 2 & 0.30 & 2.22 & 2.09 & 5.74 & 14.67 \\
\hline 6 & 4 & 2 & 0.30 & 2.22 & 2.14 & 3.65 & 14.67 \\
\hline 6 & 4 & 2 & 0.31 & 2.37 & 2.09 & 11.99 & 22.41 \\
\hline 6 & 4 & 2 & 0.31 & 2.37 & 2.09 & 11.88 & 22.41 \\
\hline 6 & 4 & 2 & 0.31 & 2.37 & 2.09 & 11.77 & 22.41 \\
\hline 6 & 4 & 2 & 0.31 & 2.37 & 2.09 & 11.75 & 22.41 \\
\hline 6 & 4 & 2 & 0.31 & 2.39 & 2.19 & 8.19 & 19.90 \\
\hline 6 & 4 & 2 & 0.31 & 2.39 & 2.19 & 8.40 & 19.90 \\
\hline 6 & 4 & 2 & 0.35 & 2.78 & 2.19 & 21.21 & 32.83 \\
\hline 6 & 4 & 2 & 0.35 & 2.77 & 2.19 & 21.03 & 32.83 \\
\hline 6 & 4 & 2 & 0.35 & 2.78 & 2.19 & 21.19 & 32.83 \\
\hline 6 & 4 & 2 & 0.35 & 2.76 & 2.19 & 20.77 & 32.83 \\
\hline 6 & 4 & 2 & 0.35 & 2.79 & 2.14 & 23.45 & 30.31 \\
\hline 6 & 4 & 2 & 0.35 & 2.80 & 2.14 & 23.50 & 30.31 \\
\hline 1 & 4 & 3 & 0.08 & 2.37 & 1.99 & 16.20 & 2.21 \\
\hline 1 & 4 & 3 & 0.08 & 2.51 & 1.99 & 20.59 & 7.54 \\
\hline 1 & 4 & 3 & 0.09 & 2.84 & 2.02 & 29.12 & 18.19 \\
\hline 2 & 4 & 3 & 0.14 & 2.57 & 2.17 & 15.66 & 4.24 \\
\hline 2 & 4 & 3 & 0.14 & 2.57 & 2.17 & 15.63 & 4.24 \\
\hline 2 & 4 & 3 & 0.15 & 2.73 & 2.19 & 19.88 & 9.53 \\
\hline 2 & 4 & 3 & 0.15 & 2.74 & 2.19 & 20.10 & 9.53 \\
\hline 2 & 4 & 3 & 0.17 & 3.13 & 2.24 & 28.66 & 20.08 \\
\hline 2 & 4 & 3 & 0.17 & 3.13 & 2.24 & 28.60 & 20.08 \\
\hline 3 & 4 & 3 & 0.23 & 2.80 & 2.38 & 15.12 & 6.27 \\
\hline 3 & 4 & 3 & 0.23 & 2.81 & 2.38 & 15.23 & 6.27 \\
\hline 3 & 4 & 3 & 0.23 & 2.80 & 2.38 & 15.13 & 6.27 \\
\hline
\end{tabular}




\begin{tabular}{|c|c|c|c|c|c|c|c|}
\hline Prod. & Server & Subtasks & $\begin{array}{l}\text { Net Traffic } \\
\text { Intensity }\end{array}$ & $\begin{array}{l}\text { Anal. } \\
\text { Flow T. }\end{array}$ & Sim. & $\begin{array}{c}\text { Error } \\
\text { sim-anal }(\%)\end{array}$ & $\begin{array}{l}\text { Predicted } \\
\text { Error }(\%)\end{array}$ \\
\hline 3 & 4 & 3 & 0.25 & 3.00 & 2.42 & 19.53 & 11.52 \\
\hline 3 & 4 & 3 & 0.25 & 3.00 & 2.42 & 19.52 & 11.52 \\
\hline 3 & 4 & 3 & 0.25 & 3.01 & 2.42 & 19.62 & 11.52 \\
\hline 3 & 4 & 3 & 0.27 & 3.50 & 2.51 & 28.22 & 21.96 \\
\hline 3 & 4 & 3 & 0.27 & 3.50 & 2.51 & 28.36 & 21.96 \\
\hline 3 & 4 & 3 & 0.27 & 3.50 & 2.51 & 28.28 & 21.96 \\
\hline 4 & 4 & 3 & 0.33 & 3.10 & 2.64 & 14.91 & 8.30 \\
\hline 4 & 4 & 3 & 0.33 & 3.10 & 2.64 & 14.76 & 8.30 \\
\hline 4 & 4 & 3 & 0.33 & 3.09 & 2.64 & 14.71 & 8.30 \\
\hline 4 & 4 & 3 & 0.33 & 3.10 & 2.64 & 14.84 & 8.30 \\
\hline 4 & 4 & 3 & 0.35 & 3.35 & 2.70 & 19.35 & 13.50 \\
\hline 4 & 4 & 3 & 0.35 & 3.35 & 2.70 & 19.27 & 13.50 \\
\hline 4 & 4 & 3 & 0.35 & 3.34 & 2.70 & 19.21 & 13.50 \\
\hline 4 & 4 & 3 & 0.35 & 3.35 & 2.70 & 19.34 & 13.50 \\
\hline 4 & 4 & 3 & 0.39 & 3.97 & 2.86 & 27.93 & 23.85 \\
\hline 4 & 4 & 3 & 0.39 & 3.97 & 2.86 & 27.95 & 23.85 \\
\hline 4 & 4 & 3 & 0.39 & 3.97 & 2.86 & 28.08 & 23.85 \\
\hline 4 & 4 & 3 & 0.39 & 3.98 & 2.86 & 28.09 & 23.85 \\
\hline 5 & 4 & 3 & 0.38 & 3.27 & 2.79 & 14.73 & 10.74 \\
\hline 5 & 4 & 3 & 0.38 & 3.27 & 2.79 & 14.61 & 10.74 \\
\hline 5 & 4 & 3 & 0.38 & 3.27 & 2.79 & 14.52 & 10.74 \\
\hline 5 & 4 & 3 & 0.38 & 3.27 & 2.79 & 14.54 & 10.74 \\
\hline 5 & 4 & 3 & 0.38 & 3.31 & 2.79 & 15.61 & 8.23 \\
\hline 5 & 4 & 3 & 0.40 & 3.54 & 2.87 & 19.01 & 15.93 \\
\hline 5 & 4 & 3 & 0.40 & 3.55 & 2.87 & 19.12 & 15.93 \\
\hline 5 & 4 & 3 & 0.40 & 3.54 & 2.87 & 18.99 & 15.93 \\
\hline 5 & 4 & 3 & 0.40 & 3.55 & 2.87 & 19.14 & 15.93 \\
\hline 5 & 4 & 3 & 0.40 & 3.60 & 2.87 & 20.23 & 13.41 \\
\hline 5 & 4 & 3 & 0.45 & 4.25 & 3.07 & 27.80 & 26.22 \\
\hline 5 & 4 & 3 & 0.45 & 4.26 & 3.07 & 27.83 & 26.22 \\
\hline 5 & 4 & 3 & 0.45 & 4.26 & 3.07 & 27.92 & 26.22 \\
\hline 5 & 4 & 3 & 0.45 & 4.26 & 3.07 & 27.94 & 26.22 \\
\hline 5 & 4 & 3 & 0.45 & 4.31 & 3.07 & 28.72 & 23.71 \\
\hline 6 & 4 & 3 & 0.44 & 3.47 & 2.96 & 14.64 & 13.19 \\
\hline 6 & 4 & 3 & 0.44 & 3.47 & 2.96 & 14.57 & 13.19 \\
\hline 6 & 4 & 3 & 0.44 & 3.47 & 2.96 & 14.68 & 13.19 \\
\hline 6 & 4 & 3 & 0.44 & 3.47 & 2.96 & 14.64 & 13.19 \\
\hline 6 & 4 & 3 & 0.44 & 3.51 & 2.96 & 15.68 & 10.67 \\
\hline 6 & 4 & 3 & 0.44 & 3.51 & 2.96 & 15.67 & 10.67 \\
\hline 6 & 4 & 3 & 0.47 & 3.78 & 3.06 & 19.00 & 18.35 \\
\hline
\end{tabular}




\begin{tabular}{|c|c|c|c|c|c|c|c|}
\hline Prod. & Server & Subtasks & $\begin{array}{l}\text { Net Traffic } \\
\text { Intensity }\end{array}$ & $\begin{array}{c}\text { Anal. } \\
\text { Flow T. }\end{array}$ & Sim. & $\begin{array}{c}\text { Error } \\
\text { sim-anal }(\%)\end{array}$ & $\begin{array}{l}\text { Predicted } \\
\text { Error }(\%)\end{array}$ \\
\hline 6 & 4 & 3 & 0.47 & 3.78 & 3.06 & 19.03 & 18.35 \\
\hline 6 & 4 & 3 & 0.47 & 3.79 & 3.06 & 19.23 & 18.35 \\
\hline 6 & 4 & 3 & 0.47 & 3.78 & 3.06 & 19.04 & 18.35 \\
\hline 6 & 4 & 3 & 0.47 & 3.83 & 3.06 & 20.13 & 15.83 \\
\hline 6 & 4 & 3 & 0.47 & 3.84 & 3.06 & 20.26 & 15.83 \\
\hline 6 & 4 & 3 & 0.52 & 4.58 & 3.32 & 27.65 & 28.59 \\
\hline 6 & 4 & 3 & 0.52 & 4.59 & 3.32 & 27.78 & 28.59 \\
\hline 6 & 4 & 3 & 0.52 & 4.60 & 3.32 & 27.94 & 28.59 \\
\hline 6 & 4 & 3 & 0.52 & 4.60 & 3.32 & 27.93 & 28.59 \\
\hline 6 & 4 & 3 & 0.52 & 4.68 & 3.32 & 29.10 & 26.08 \\
\hline 6 & 4 & 3 & 0.52 & 4.67 & 3.32 & 29.01 & 26.08 \\
\hline
\end{tabular}

Approximate and simulated flow times for the $\operatorname{Exp}-\mathrm{H}_{2}$ case

Prod. Server Subtasks Net Traffic Anal. Sim. Error $\quad$ Predicted Intensity Flow T. Flow T. sim-anal (\%) $\quad$ Error (\%)

\begin{tabular}{|c|c|c|c|c|c|c|c|}
\hline 1 & 2 & 2 & 0.10 & 1.76 & 1.76 & 0.23 & 2.52 \\
\hline 1 & 2 & 2 & 0.11 & 1.86 & 1.77 & 5.01 & 2.79 \\
\hline 1 & 2 & 2 & 0.12 & 2.11 & 1.80 & 14.87 & 13.41 \\
\hline 2 & 2 & 2 & 0.19 & 2.01 & 1.97 & 1.83 & 0.77 \\
\hline 2 & 2 & 2 & 0.19 & 2.02 & 1.97 & 2.34 & 0.77 \\
\hline 2 & 2 & 2 & 0.20 & 2.14 & 2.00 & 6.71 & 4.49 \\
\hline 2 & 2 & 2 & 0.20 & 2.14 & 2.00 & 6.69 & 4.49 \\
\hline 2 & 2 & 2 & 0.22 & 2.48 & 2.07 & 16.54 & 14.98 \\
\hline 2 & 2 & 2 & 0.22 & 2.48 & 2.07 & 16.73 & 14.98 \\
\hline 3 & 2 & 2 & 0.31 & 2.34 & 2.25 & 3.84 & 0.99 \\
\hline 3 & 2 & 2 & 0.31 & 2.34 & 2.25 & 3.76 & 0.99 \\
\hline 3 & 2 & 2 & 0.31 & 2.34 & 2.25 & 3.86 & 0.99 \\
\hline 3 & 2 & 2 & 0.33 & 2.51 & 2.30 & 8.49 & 6.19 \\
\hline 3 & 2 & 2 & 0.33 & 2.52 & 2.30 & 8.80 & 6.19 \\
\hline 3 & 2 & 2 & 0.33 & 2.52 & 2.30 & 8.57 & 6.19 \\
\hline 3 & 2 & 2 & 0.37 & 2.98 & 2.44 & 18.30 & 16.54 \\
\hline 3 & 2 & 2 & 0.37 & 2.98 & 2.44 & 18.40 & 16.54 \\
\hline 3 & 2 & 2 & 0.37 & 2.98 & 2.44 & 18.27 & 16.54 \\
\hline 4 & 2 & 2 & 0.44 & 2.77 & 2.61 & 5.70 & 2.75 \\
\hline 4 & 2 & 2 & 0.44 & 2.78 & 2.61 & 6.03 & 2.75 \\
\hline 4 & 2 & 2 & 0.44 & 2.78 & 2.61 & 5.89 & 2.75 \\
\hline 4 & 2 & 2 & 0.44 & 2.77 & 2.61 & 5.83 & 2.75 \\
\hline 4 & 2 & 2 & 0.47 & 3.02 & 2.71 & 10.40 & 7.89 \\
\hline 4 & 2 & 2 & 0.47 & 3.02 & 2.71 & 10.38 & 7.89 \\
\hline
\end{tabular}


$\begin{array}{lllllll}\text { Prod. } & \text { Server } & \text { Subtasks Net Traffic } & \text { Anal. Sim. } & \text { Error } & \text { Predicted }\end{array}$ Intensity Flow T. Flow T. sim-anal (\%) Error (\%)

\begin{tabular}{|c|c|c|c|c|c|c|c|}
\hline 4 & 2 & 2 & 0.47 & 3.02 & 2.71 & 10.37 & 7.89 \\
\hline 4 & 2 & 2 & 0.47 & 3.02 & 2.71 & 10.32 & 7.89 \\
\hline 4 & 2 & 2 & 0.52 & 3.70 & 2.96 & 20.00 & 18.10 \\
\hline 4 & 2 & 2 & 0.52 & 3.71 & 2.96 & 20.10 & 18.10 \\
\hline 4 & 2 & 2 & 0.52 & 3.70 & 2.96 & 20.01 & 18.10 \\
\hline 4 & 2 & 2 & 0.52 & 3.71 & 2.96 & 20.08 & 18.10 \\
\hline 5 & 2 & 2 & 0.51 & 3.38 & 3.12 & 7.61 & 4.51 \\
\hline 5 & 2 & 2 & 0.51 & 3.37 & 3.12 & 7.26 & 4.51 \\
\hline 5 & 2 & 2 & 0.51 & 3.37 & 3.12 & 7.44 & 4.51 \\
\hline 5 & 2 & 2 & 0.51 & 3.36 & 3.12 & 7.24 & 4.51 \\
\hline 5 & 2 & 2 & 0.51 & 3.37 & 3.12 & 7.34 & 4.51 \\
\hline 5 & 2 & 2 & 0.54 & 3.76 & 3.29 & 12.40 & 9.59 \\
\hline 5 & 2 & 2 & 0.54 & 3.75 & 3.29 & 12.27 & 9.59 \\
\hline 5 & 2 & 2 & 0.54 & 3.77 & 3.29 & 12.61 & 9.59 \\
\hline 5 & 2 & 2 & 0.54 & 3.75 & 3.29 & 12.14 & 9.59 \\
\hline 5 & 2 & 2 & 0.54 & 3.76 & 3.29 & 12.53 & 9.59 \\
\hline 5 & 2 & 2 & 0.60 & 4.83 & 3.78 & 21.62 & 19.67 \\
\hline 5 & 2 & 2 & 0.60 & 4.82 & 3.78 & 21.52 & 19.67 \\
\hline 5 & 2 & 2 & 0.60 & 4.82 & 3.78 & 21.56 & 19.67 \\
\hline 5 & 2 & 2 & 0.60 & 4.82 & 3.78 & 21.49 & 19.67 \\
\hline 5 & 2 & 2 & 0.60 & 4.82 & 3.78 & 21.53 & 19.67 \\
\hline 6 & 2 & 2 & 0.59 & 3.76 & 3.46 & 8.00 & 6.81 \\
\hline 6 & 2 & 2 & 0.59 & 3.76 & 3.46 & 8.02 & 6.81 \\
\hline 6 & 2 & 2 & 0.59 & 3.77 & 3.46 & 8.36 & 6.81 \\
\hline 6 & 2 & 2 & 0.59 & 3.77 & 3.46 & 8.26 & 6.81 \\
\hline 6 & 2 & 2 & 0.59 & 3.77 & 3.46 & 8.23 & 6.81 \\
\hline 6 & 2 & 2 & 0.59 & 3.77 & 3.42 & 9.06 & 6.81 \\
\hline 6 & 2 & 2 & 0.62 & 4.25 & 3.69 & 13.09 & 11.87 \\
\hline 6 & 2 & 2 & 0.62 & 4.26 & 3.69 & 13.24 & 11.87 \\
\hline 6 & 2 & 2 & 0.62 & 4.26 & 3.69 & 13.27 & 11.87 \\
\hline 6 & 2 & 2 & 0.62 & 4.25 & 3.69 & 13.01 & 11.87 \\
\hline 6 & 2 & 2 & 0.62 & 4.25 & 3.69 & 13.06 & 11.87 \\
\hline 6 & 2 & 2 & 0.62 & 4.25 & 3.66 & 14.05 & 11.87 \\
\hline 6 & 2 & 2 & 0.69 & 5.70 & 4.40 & 22.88 & 21.88 \\
\hline 6 & 2 & 2 & 0.69 & 5.71 & 4.40 & 23.01 & 21.88 \\
\hline 6 & 2 & 2 & 0.69 & 5.70 & 4.40 & 22.91 & 21.88 \\
\hline 6 & 2 & 2 & 0.69 & 5.69 & 4.40 & 22.78 & 21.88 \\
\hline 6 & 2 & 2 & 0.69 & 5.70 & 4.40 & 22.89 & 21.88 \\
\hline 6 & 2 & 2 & 0.69 & 5.70 & 4.35 & 23.80 & 21.88 \\
\hline 1 & 3 & 3 & 0.10 & 2.19 & 2.27 & 3.54 & 0.75 \\
\hline
\end{tabular}


\begin{tabular}{lllllll}
\hline Prod. & Server & Subtasks Net Traffic & Anal. & Sim. & Error & Predicted
\end{tabular} Intensity Flow T. Flow T. sim-anal (\%) Error (\%)

\begin{tabular}{|c|c|c|c|c|c|c|c|}
\hline 1 & 3 & 3 & 0.11 & 2.32 & 2.29 & 1.35 & 4.57 \\
\hline 1 & 3 & 3 & 0.12 & 2.63 & 2.33 & 11.37 & 15.18 \\
\hline 2 & 3 & 3 & 0.19 & 2.50 & 2.52 & 0.63 & 1.00 \\
\hline 2 & 3 & 3 & 0.19 & 2.50 & 2.52 & 0.61 & 1.00 \\
\hline 2 & 3 & 3 & 0.20 & 2.67 & 2.55 & 4.23 & 0.89 \\
\hline 2 & 3 & 3 & 0.20 & 2.67 & 2.55 & 4.35 & 0.89 \\
\hline 2 & 3 & 3 & 0.22 & 3.08 & 2.64 & 14.28 & 0.62 \\
\hline 2 & 3 & 3 & 0.22 & 3.08 & 2.64 & 14.41 & 0.62 \\
\hline 3 & 3 & 3 & 0.31 & 2.89 & 2.81 & 2.95 & 2.76 \\
\hline 3 & 3 & 3 & 0.31 & 2.89 & 2.81 & 2.87 & 2.76 \\
\hline 3 & 3 & 3 & 0.31 & 2.89 & 2.81 & 2.87 & 2.76 \\
\hline 3 & 3 & 3 & 0.33 & 3.12 & 2.87 & 8.10 & 7.97 \\
\hline 3 & 3 & 3 & 0.33 & 3.12 & 2.87 & 7.99 & 7.97 \\
\hline 3 & 3 & 3 & 0.33 & 3.12 & 2.87 & 8.08 & 7.97 \\
\hline 3 & 3 & 3 & 0.37 & 3.69 & 3.02 & 18.30 & 18.31 \\
\hline 3 & 3 & 3 & 0.37 & 3.69 & 3.02 & 18.25 & 18.31 \\
\hline 3 & 3 & 3 & 0.37 & 3.69 & 3.02 & 18.29 & 18.31 \\
\hline 4 & 3 & 3 & 0.44 & 3.41 & 3.16 & 7.31 & 4.52 \\
\hline 4 & 3 & 3 & 0.44 & 3.42 & 3.16 & 7.40 & 4.52 \\
\hline 4 & 3 & 3 & 0.44 & 3.42 & 3.16 & 7.44 & 4.52 \\
\hline 4 & 3 & 3 & 0.44 & 3.42 & 3.16 & 7.38 & 4.52 \\
\hline 4 & 3 & 3 & 0.47 & 3.72 & 3.26 & 12.47 & 9.67 \\
\hline 4 & 3 & 3 & 0.47 & 3.73 & 3.26 & 12.63 & 9.67 \\
\hline 4 & 3 & 3 & 0.47 & 3.73 & 3.26 & 12.52 & 9.67 \\
\hline 4 & 3 & 3 & 0.47 & 3.73 & 3.26 & 12.69 & 9.67 \\
\hline 4 & 3 & 3 & 0.52 & 4.55 & 3.51 & 22.85 & 19.88 \\
\hline 4 & 3 & 3 & 0.52 & 4.55 & 3.51 & 22.92 & 19.88 \\
\hline 4 & 3 & 3 & 0.52 & 4.55 & 3.51 & 22.86 & 19.88 \\
\hline 4 & 3 & 3 & 0.52 & 4.55 & 3.51 & 22.84 & 19.88 \\
\hline 5 & 3 & 3 & 0.51 & 4.13 & 3.62 & 12.28 & 6.28 \\
\hline 5 & 3 & 3 & 0.51 & 4.14 & 3.62 & 12.40 & 6.28 \\
\hline 5 & 3 & 3 & 0.51 & 4.14 & 3.62 & 12.52 & 6.28 \\
\hline 5 & 3 & 3 & 0.51 & 4.13 & 3.62 & 12.23 & 6.28 \\
\hline 5 & 3 & 3 & 0.51 & 4.14 & 3.62 & 12.39 & 6.28 \\
\hline 5 & 3 & 3 & 0.54 & 4.60 & 3.78 & 17.73 & 11.37 \\
\hline 5 & 3 & 3 & 0.54 & 4.61 & 3.78 & 18.00 & 11.37 \\
\hline 5 & 3 & 3 & 0.54 & 4.60 & 3.78 & 17.86 & 11.37 \\
\hline 5 & 3 & 3 & 0.54 & 4.60 & 3.78 & 17.75 & 11.37 \\
\hline 5 & 3 & 3 & 0.54 & 4.60 & 3.78 & 17.85 & 11.37 \\
\hline 5 & 3 & 3 & 0.60 & 5.93 & 4.22 & 28.74 & 21.44 \\
\hline
\end{tabular}


\begin{tabular}{lllllll}
\hline Prod. & Server & Subtasks Net Traffic & Anal. & Sim. & Error & Predicted
\end{tabular} Intensity Flow T. Flow T. sim-anal (\%) Error (\%)

\begin{tabular}{|c|c|c|c|c|c|c|c|}
\hline 5 & 3 & 3 & 0.60 & 5.92 & 4.22 & 28.71 & 21.44 \\
\hline 5 & 3 & 3 & 0.60 & 5.93 & 4.22 & 28.76 & 21.44 \\
\hline 5 & 3 & 3 & 0.60 & 5.93 & 4.22 & 28.79 & 21.44 \\
\hline 5 & 3 & 3 & 0.60 & 5.94 & 4.22 & 28.90 & 21.44 \\
\hline 6 & 3 & 3 & 0.59 & 4.60 & 3.91 & 14.86 & 8.58 \\
\hline 6 & 3 & 3 & 0.59 & 4.60 & 3.91 & 14.92 & 8.58 \\
\hline 6 & 3 & 3 & 0.59 & 4.60 & 3.91 & 14.87 & 8.58 \\
\hline 6 & 3 & 3 & 0.59 & 4.61 & 3.91 & 15.03 & 8.58 \\
\hline 6 & 3 & 3 & 0.59 & 4.61 & 3.91 & 15.02 & 8.58 \\
\hline 6 & 3 & 3 & 0.59 & 4.61 & 3.79 & 17.79 & 8.58 \\
\hline 6 & 3 & 3 & 0.62 & 5.19 & 4.12 & 20.62 & 13.64 \\
\hline 6 & 3 & 3 & 0.62 & 5.19 & 4.12 & 20.56 & 13.64 \\
\hline 6 & 3 & 3 & 0.62 & 5.19 & 4.12 & 20.52 & 13.64 \\
\hline 6 & 3 & 3 & 0.62 & 5.17 & 4.12 & 20.34 & 13.64 \\
\hline 6 & 3 & 3 & 0.62 & 5.19 & 4.12 & 20.60 & 13.64 \\
\hline 6 & 3 & 3 & 0.62 & 5.19 & 3.98 & 23.32 & 13.64 \\
\hline 6 & 3 & 3 & 0.69 & 6.94 & 4.73 & 31.89 & 23.65 \\
\hline 6 & 3 & 3 & 0.69 & 6.94 & 4.73 & 31.87 & 23.65 \\
\hline 6 & 3 & 3 & 0.69 & 6.93 & 4.73 & 31.76 & 23.65 \\
\hline 6 & 3 & 3 & 0.69 & 6.95 & 4.73 & 31.96 & 23.65 \\
\hline 6 & 3 & 3 & 0.69 & 6.94 & 4.73 & 31.90 & 23.65 \\
\hline 6 & 3 & 3 & 0.69 & 6.94 & 4.54 & 34.55 & 23.65 \\
\hline 1 & 3 & 2 & 0.07 & 1.84 & 1.69 & 7.89 & 2.24 \\
\hline 1 & 3 & 2 & 0.07 & 1.94 & 1.70 & 12.37 & 7.58 \\
\hline 1 & 3 & 2 & 0.08 & 2.19 & 1.72 & 21.53 & 18.24 \\
\hline 2 & 3 & 2 & 0.13 & 1.99 & 1.82 & 8.66 & 4.36 \\
\hline 2 & 3 & 2 & 0.13 & 2.00 & 1.82 & 8.75 & 4.36 \\
\hline 2 & 3 & 2 & 0.13 & 2.13 & 1.84 & 13.55 & 4.28 \\
\hline 2 & 3 & 2 & 0.13 & 2.12 & 1.84 & 13.40 & 4.28 \\
\hline 2 & 3 & 2 & 0.15 & 2.42 & 1.88 & 22.41 & 4.10 \\
\hline 2 & 3 & 2 & 0.15 & 2.42 & 1.88 & 22.48 & 4.10 \\
\hline 3 & 3 & 2 & 0.21 & 2.19 & 1.97 & 9.79 & 6.48 \\
\hline 3 & 3 & 2 & 0.21 & 2.19 & 1.97 & 9.82 & 6.48 \\
\hline 3 & 3 & 2 & 0.21 & 2.19 & 1.97 & 10.08 & 6.48 \\
\hline 3 & 3 & 2 & 0.22 & 2.34 & 2.00 & 14.39 & 11.74 \\
\hline 3 & 3 & 2 & 0.22 & 2.34 & 2.00 & 14.40 & 11.74 \\
\hline 3 & 3 & 2 & 0.22 & 2.33 & 2.00 & 14.32 & 11.74 \\
\hline 3 & 3 & 2 & 0.24 & 2.69 & 2.07 & 23.22 & 22.23 \\
\hline 3 & 3 & 2 & 0.24 & 2.70 & 2.07 & 23.37 & 22.23 \\
\hline 3 & 3 & 2 & 0.24 & 2.69 & 2.07 & 23.20 & 22.23 \\
\hline
\end{tabular}


\begin{tabular}{lllllll}
\hline Prod. & Server & Subtasks Net Traffic & Anal. & Sim. & Error & Predicted
\end{tabular} Intensity Flow T. Flow T. sim-anal (\%) $\quad$ Error (\%)

\begin{tabular}{|c|c|c|c|c|c|c|c|}
\hline 4 & 3 & 2 & 0.30 & 2.40 & 2.15 & 10.70 & 8.61 \\
\hline 4 & 3 & 2 & 0.30 & 2.41 & 2.15 & 10.81 & 8.61 \\
\hline 4 & 3 & 2 & 0.30 & 2.41 & 2.15 & 10.91 & 8.61 \\
\hline 4 & 3 & 2 & 0.30 & 2.41 & 2.15 & 10.88 & 8.61 \\
\hline 4 & 3 & 2 & 0.31 & 2.58 & 2.19 & 15.25 & 13.83 \\
\hline 4 & 3 & 2 & 0.31 & 2.59 & 2.19 & 15.54 & 13.83 \\
\hline 4 & 3 & 2 & 0.31 & 2.58 & 2.19 & 15.21 & 13.83 \\
\hline 4 & 3 & 2 & 0.31 & 2.58 & 2.19 & 15.11 & 13.83 \\
\hline 4 & 3 & 2 & 0.35 & 3.04 & 2.30 & 24.28 & 24.22 \\
\hline 4 & 3 & 2 & 0.35 & 3.04 & 2.30 & 24.28 & 24.22 \\
\hline 4 & 3 & 2 & 0.35 & 3.04 & 2.30 & 24.24 & 24.22 \\
\hline 4 & 3 & 2 & 0.35 & 3.03 & 2.30 & 24.12 & 24.22 \\
\hline 5 & 3 & 2 & 0.34 & 2.66 & 2.36 & 11.47 & 10.73 \\
\hline 5 & 3 & 2 & 0.34 & 2.66 & 2.36 & 11.46 & 10.73 \\
\hline 5 & 3 & 2 & 0.34 & 2.66 & 2.36 & 11.48 & 10.73 \\
\hline 5 & 3 & 2 & 0.34 & 2.67 & 2.36 & 11.61 & 10.73 \\
\hline 5 & 3 & 2 & 0.34 & 2.66 & 2.36 & 11.53 & 10.73 \\
\hline 5 & 3 & 2 & 0.36 & 2.89 & 2.42 & 16.13 & 15.91 \\
\hline 5 & 3 & 2 & 0.36 & 2.90 & 2.42 & 16.35 & 15.91 \\
\hline 5 & 3 & 2 & 0.36 & 2.89 & 2.42 & 16.05 & 15.91 \\
\hline 5 & 3 & 2 & 0.36 & 2.89 & 2.42 & 16.32 & 15.91 \\
\hline 5 & 3 & 2 & 0.36 & 2.89 & 2.42 & 16.17 & 15.91 \\
\hline 5 & 3 & 2 & 0.40 & 3.45 & 2.59 & 24.98 & 26.21 \\
\hline 5 & 3 & 2 & 0.40 & 3.47 & 2.59 & 25.30 & 26.21 \\
\hline 5 & 3 & 2 & 0.40 & 3.46 & 2.59 & 25.12 & 26.21 \\
\hline 5 & 3 & 2 & 0.40 & 3.47 & 2.59 & 25.30 & 26.21 \\
\hline 5 & 3 & 2 & 0.40 & 3.47 & 2.59 & 25.37 & 26.21 \\
\hline 6 & 3 & 2 & 0.39 & 2.81 & 2.48 & 11.78 & 13.22 \\
\hline 6 & 3 & 2 & 0.39 & 2.82 & 2.48 & 12.05 & 13.22 \\
\hline 6 & 3 & 2 & 0.39 & 2.82 & 2.48 & 12.00 & 13.22 \\
\hline 6 & 3 & 2 & 0.39 & 2.82 & 2.48 & 12.16 & 13.22 \\
\hline 6 & 3 & 2 & 0.39 & 2.82 & 2.48 & 12.02 & 13.22 \\
\hline 6 & 3 & 2 & 0.39 & 2.82 & 2.45 & 12.90 & 13.22 \\
\hline 6 & 3 & 2 & 0.41 & 3.07 & 2.56 & 16.72 & 18.38 \\
\hline 6 & 3 & 2 & 0.41 & 3.07 & 2.56 & 16.72 & 18.38 \\
\hline 6 & 3 & 2 & 0.41 & 3.07 & 2.56 & 16.59 & 18.38 \\
\hline 6 & 3 & 2 & 0.41 & 3.07 & 2.56 & 16.61 & 18.38 \\
\hline 6 & 3 & 2 & 0.41 & 3.07 & 2.56 & 16.73 & 18.38 \\
\hline 6 & 3 & 2 & 0.41 & 3.08 & 2.53 & 17.81 & 18.38 \\
\hline 6 & 3 & 2 & 0.46 & 3.72 & 2.76 & 25.81 & 28.64 \\
\hline
\end{tabular}




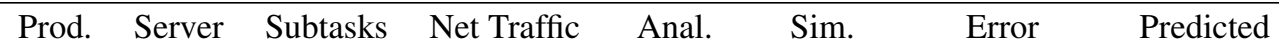
Intensity Flow T. Flow T. sim-anal (\%) Error (\%)

\begin{tabular}{|c|c|c|c|c|c|c|c|}
\hline 6 & 3 & 2 & 0.46 & 3.72 & 2.76 & 25.76 & 28.64 \\
\hline 6 & 3 & 2 & 0.46 & 3.72 & 2.76 & 25.68 & 28.64 \\
\hline 6 & 3 & 2 & 0.46 & 3.73 & 2.76 & 25.92 & 28.64 \\
\hline 6 & 3 & 2 & 0.46 & 3.72 & 2.76 & 25.75 & 28.64 \\
\hline 6 & 3 & 2 & 0.46 & 3.73 & 2.73 & 26.69 & 28.64 \\
\hline 1 & 4 & 4 & 0.10 & 2.53 & 2.63 & 4.24 & 1.02 \\
\hline 1 & 4 & 4 & 0.11 & 2.68 & 2.66 & 1.01 & 6.34 \\
\hline 1 & 4 & 4 & 0.12 & 3.04 & 2.71 & 10.94 & 16.96 \\
\hline 2 & 4 & 4 & 0.19 & 2.88 & 2.92 & 1.33 & 2.78 \\
\hline 2 & 4 & 4 & 0.19 & 2.88 & 2.92 & 1.09 & 2.78 \\
\hline 2 & 4 & 4 & 0.20 & 3.07 & 2.96 & 3.77 & 2.66 \\
\hline 2 & 4 & 4 & 0.20 & 3.08 & 2.96 & 3.91 & 2.66 \\
\hline 2 & 4 & 4 & 0.22 & 3.56 & 3.05 & 14.07 & 2.39 \\
\hline 2 & 4 & 4 & 0.22 & 3.55 & 3.05 & 14.05 & 2.39 \\
\hline 3 & 4 & 4 & 0.31 & 3.32 & 3.24 & 2.47 & 4.53 \\
\hline 3 & 4 & 4 & 0.31 & 3.32 & 3.24 & 2.36 & 4.53 \\
\hline 3 & 4 & 4 & 0.31 & 3.33 & 3.24 & 2.68 & 4.53 \\
\hline 3 & 4 & 4 & 0.33 & 3.58 & 3.31 & 7.75 & 9.74 \\
\hline 3 & 4 & 4 & 0.33 & 3.58 & 3.31 & 7.62 & 9.74 \\
\hline 3 & 4 & 4 & 0.33 & 3.57 & 3.31 & 7.53 & 9.74 \\
\hline 3 & 4 & 4 & 0.37 & 4.25 & 3.47 & 18.31 & 20.08 \\
\hline 3 & 4 & 4 & 0.37 & 4.24 & 3.47 & 18.20 & 20.08 \\
\hline 3 & 4 & 4 & 0.37 & 4.25 & 3.47 & 18.35 & 20.08 \\
\hline 4 & 4 & 4 & 0.44 & 3.90 & 3.63 & 7.10 & 6.29 \\
\hline 4 & 4 & 4 & 0.44 & 3.91 & 3.63 & 7.18 & 6.29 \\
\hline 4 & 4 & 4 & 0.44 & 3.91 & 3.63 & 7.18 & 6.29 \\
\hline 4 & 4 & 4 & 0.44 & 3.91 & 3.63 & 7.18 & 6.29 \\
\hline 4 & 4 & 4 & 0.47 & 4.27 & 3.73 & 12.51 & 11.44 \\
\hline 4 & 4 & 4 & 0.47 & 4.27 & 3.73 & 12.59 & 11.44 \\
\hline 4 & 4 & 4 & 0.47 & 4.27 & 3.73 & 12.56 & 11.44 \\
\hline 4 & 4 & 4 & 0.47 & 4.27 & 3.73 & 12.59 & 11.44 \\
\hline 4 & 4 & 4 & 0.52 & 5.21 & 4.01 & 23.15 & 21.65 \\
\hline 4 & 4 & 4 & 0.52 & 5.22 & 4.01 & 23.26 & 21.65 \\
\hline 4 & 4 & 4 & 0.52 & 5.22 & 4.01 & 23.28 & 21.65 \\
\hline 4 & 4 & 4 & 0.52 & 5.23 & 4.01 & 23.48 & 21.65 \\
\hline 5 & 4 & 4 & 0.51 & 4.72 & 4.12 & 12.74 & 8.05 \\
\hline 5 & 4 & 4 & 0.51 & 4.72 & 4.12 & 12.76 & 8.05 \\
\hline 5 & 4 & 4 & 0.51 & 4.72 & 4.12 & 12.81 & 8.05 \\
\hline 5 & 4 & 4 & 0.51 & 4.72 & 4.12 & 12.72 & 8.05 \\
\hline 5 & 4 & 4 & 0.51 & 4.73 & 4.12 & 12.84 & 8.05 \\
\hline
\end{tabular}


$\begin{array}{lllllll}\text { Prod. } & \text { Server } & \text { Subtasks Net Traffic } & \text { Anal. Sim. } & \text { Error } & \text { Predicted }\end{array}$ Intensity Flow T. Flow T. sim-anal (\%) Error (\%)

\begin{tabular}{|c|c|c|c|c|c|c|c|}
\hline 5 & 4 & 4 & 0.54 & 5.26 & 4.29 & 18.40 & 13.14 \\
\hline 5 & 4 & 4 & 0.54 & 5.26 & 4.29 & 18.39 & 13.14 \\
\hline 5 & 4 & 4 & 0.54 & 5.26 & 4.29 & 18.44 & 13.14 \\
\hline 5 & 4 & 4 & 0.54 & 5.26 & 4.29 & 18.39 & 13.14 \\
\hline 5 & 4 & 4 & 0.54 & 5.26 & 4.29 & 18.46 & 13.14 \\
\hline 5 & 4 & 4 & 0.60 & 6.73 & 4.76 & 29.30 & 23.21 \\
\hline 5 & 4 & 4 & 0.60 & 6.73 & 4.76 & 29.31 & 23.21 \\
\hline 5 & 4 & 4 & 0.60 & 6.74 & 4.76 & 29.41 & 23.21 \\
\hline 5 & 4 & 4 & 0.60 & 6.74 & 4.76 & 29.36 & 23.21 \\
\hline 5 & 4 & 4 & 0.60 & 6.73 & 4.76 & 29.29 & 23.21 \\
\hline 6 & 4 & 4 & 0.59 & 5.25 & 4.43 & 15.74 & 10.36 \\
\hline 6 & 4 & 4 & 0.59 & 5.25 & 4.43 & 15.78 & 10.36 \\
\hline 6 & 4 & 4 & 0.59 & 5.26 & 4.43 & 15.83 & 10.36 \\
\hline 6 & 4 & 4 & 0.59 & 5.26 & 4.43 & 15.93 & 10.36 \\
\hline 6 & 4 & 4 & 0.59 & 5.24 & 4.43 & 15.58 & 10.36 \\
\hline 6 & 4 & 4 & 0.59 & 5.25 & 4.27 & 18.50 & 10.36 \\
\hline 6 & 4 & 4 & 0.62 & 5.89 & 4.65 & 21.09 & 15.41 \\
\hline 6 & 4 & 4 & 0.62 & 5.89 & 4.65 & 21.11 & 15.41 \\
\hline 6 & 4 & 4 & 0.62 & 5.90 & 4.65 & 21.19 & 15.41 \\
\hline 6 & 4 & 4 & 0.62 & 5.90 & 4.65 & 21.22 & 15.41 \\
\hline 6 & 4 & 4 & 0.62 & 5.90 & 4.65 & 21.19 & 15.41 \\
\hline 6 & 4 & 4 & 0.62 & 5.91 & 4.48 & 24.23 & 15.41 \\
\hline 6 & 4 & 4 & 0.69 & 7.89 & 5.28 & 33.06 & 25.42 \\
\hline 6 & 4 & 4 & 0.69 & 7.87 & 5.28 & 32.88 & 25.42 \\
\hline 6 & 4 & 4 & 0.69 & 7.89 & 5.28 & 33.04 & 25.42 \\
\hline 6 & 4 & 4 & 0.69 & 7.88 & 5.28 & 32.93 & 25.42 \\
\hline 6 & 4 & 4 & 0.69 & 7.89 & 5.28 & 33.06 & 25.42 \\
\hline 6 & 4 & 4 & 0.69 & 7.86 & 5.06 & 35.69 & 25.42 \\
\hline 1 & 4 & 2 & 0.05 & 1.77 & 1.66 & 5.79 & 6.82 \\
\hline 1 & 4 & 2 & 0.05 & 1.86 & 1.67 & 10.43 & 12.16 \\
\hline 1 & 4 & 2 & 0.06 & 2.10 & 1.68 & 19.70 & 22.85 \\
\hline 2 & 4 & 2 & 0.10 & 1.88 & 1.76 & 6.56 & 9.12 \\
\hline 2 & 4 & 2 & 0.10 & 1.88 & 1.76 & 6.70 & 9.12 \\
\hline 2 & 4 & 2 & 0.10 & 1.99 & 1.77 & 11.32 & 9.06 \\
\hline 2 & 4 & 2 & 0.10 & 2.00 & 1.77 & 11.54 & 9.06 \\
\hline 2 & 4 & 2 & 0.11 & 2.26 & 1.80 & 20.56 & 8.93 \\
\hline 2 & 4 & 2 & 0.11 & 2.26 & 1.80 & 20.55 & 8.93 \\
\hline 3 & 4 & 2 & 0.16 & 2.01 & 1.86 & 7.72 & 11.43 \\
\hline 3 & 4 & 2 & 0.16 & 2.01 & 1.86 & 7.51 & 11.43 \\
\hline 3 & 4 & 2 & 0.16 & 2.01 & 1.86 & 7.50 & 11.43 \\
\hline
\end{tabular}




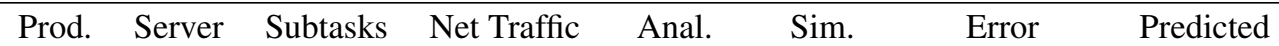
Intensity Flow T. Flow T. sim-anal (\%) Error (\%)

\begin{tabular}{|c|c|c|c|c|c|c|c|}
\hline 3 & 4 & 2 & 0.16 & 2.13 & 1.88 & 12.09 & 16.72 \\
\hline 3 & 4 & 2 & 0.16 & 2.13 & 1.88 & 11.83 & 16.72 \\
\hline 3 & 4 & 2 & 0.16 & 2.13 & 1.88 & 11.89 & 16.72 \\
\hline 3 & 4 & 2 & 0.18 & 2.44 & 1.92 & 21.34 & 27.27 \\
\hline 3 & 4 & 2 & 0.18 & 2.44 & 1.92 & 21.22 & 27.27 \\
\hline 3 & 4 & 2 & 0.18 & 2.45 & 1.92 & 21.36 & 27.27 \\
\hline 4 & 4 & 2 & 0.22 & 2.15 & 1.97 & 8.37 & 13.73 \\
\hline 4 & 4 & 2 & 0.22 & 2.15 & 1.97 & 8.40 & 13.73 \\
\hline 4 & 4 & 2 & 0.22 & 2.15 & 1.97 & 8.49 & 13.73 \\
\hline 4 & 4 & 2 & 0.22 & 2.15 & 1.97 & 8.46 & 13.73 \\
\hline 4 & 4 & 2 & 0.23 & 2.29 & 2.00 & 12.86 & 18.99 \\
\hline 4 & 4 & 2 & 0.23 & 2.30 & 2.00 & 12.92 & 18.99 \\
\hline 4 & 4 & 2 & 0.23 & 2.29 & 2.00 & 12.81 & 18.99 \\
\hline 4 & 4 & 2 & 0.23 & 2.30 & 2.00 & 12.98 & 18.99 \\
\hline 4 & 4 & 2 & 0.26 & 2.66 & 2.07 & 22.16 & 29.48 \\
\hline 4 & 4 & 2 & 0.26 & 2.66 & 2.07 & 22.32 & 29.48 \\
\hline 4 & 4 & 2 & 0.26 & 2.66 & 2.07 & 22.11 & 29.48 \\
\hline 4 & 4 & 2 & 0.26 & 2.66 & 2.07 & 22.19 & 29.48 \\
\hline 5 & 4 & 2 & 0.26 & 2.32 & 2.10 & 9.31 & 16.04 \\
\hline 5 & 4 & 2 & 0.26 & 2.31 & 2.10 & 9.21 & 16.04 \\
\hline 5 & 4 & 2 & 0.26 & 2.32 & 2.10 & 9.32 & 16.04 \\
\hline 5 & 4 & 2 & 0.26 & 2.32 & 2.10 & 9.33 & 16.04 \\
\hline 5 & 4 & 2 & 0.26 & 2.32 & 2.10 & 9.30 & 16.04 \\
\hline 5 & 4 & 2 & 0.27 & 2.48 & 2.14 & 13.65 & 21.27 \\
\hline 5 & 4 & 2 & 0.27 & 2.47 & 2.14 & 13.49 & 21.27 \\
\hline 5 & 4 & 2 & 0.27 & 2.47 & 2.14 & 13.47 & 21.27 \\
\hline 5 & 4 & 2 & 0.27 & 2.48 & 2.14 & 13.65 & 21.27 \\
\hline 5 & 4 & 2 & 0.27 & 2.47 & 2.14 & 13.51 & 21.27 \\
\hline 5 & 4 & 2 & 0.30 & 2.91 & 2.24 & 23.12 & 31.68 \\
\hline 5 & 4 & 2 & 0.30 & 2.91 & 2.24 & 23.01 & 31.68 \\
\hline 5 & 4 & 2 & 0.30 & 2.90 & 2.24 & 22.98 & 31.68 \\
\hline 5 & 4 & 2 & 0.30 & 2.90 & 2.24 & 22.92 & 31.68 \\
\hline 5 & 4 & 2 & 0.30 & 2.90 & 2.24 & 22.93 & 31.68 \\
\hline 6 & 4 & 2 & 0.30 & 2.40 & 2.17 & 9.49 & 18.62 \\
\hline 6 & 4 & 2 & 0.30 & 2.41 & 2.17 & 9.71 & 18.62 \\
\hline 6 & 4 & 2 & 0.30 & 2.40 & 2.17 & 9.51 & 18.62 \\
\hline 6 & 4 & 2 & 0.30 & 2.40 & 2.17 & 9.40 & 18.62 \\
\hline 6 & 4 & 2 & 0.30 & 2.41 & 2.17 & 9.73 & 18.62 \\
\hline 6 & 4 & 2 & 0.30 & 2.40 & 2.15 & 10.42 & 18.62 \\
\hline 6 & 4 & 2 & 0.31 & 2.59 & 2.22 & 14.30 & 23.84 \\
\hline
\end{tabular}


\begin{tabular}{lllllll}
\hline Prod. & Server & Subtasks Net Traffic & Anal. & Sim. & Error & Predicted
\end{tabular} Intensity Flow T. Flow T. sim-anal (\%) Error (\%)

\begin{tabular}{|c|c|c|c|c|c|c|c|}
\hline 6 & 4 & 2 & 0.31 & 2.58 & 2.22 & 14.19 & 23.84 \\
\hline 6 & 4 & 2 & 0.31 & 2.58 & 2.22 & 14.12 & 23.84 \\
\hline 6 & 4 & 2 & 0.31 & 2.58 & 2.22 & 14.22 & 23.84 \\
\hline 6 & 4 & 2 & 0.31 & 2.58 & 2.22 & 14.20 & 23.84 \\
\hline 6 & 4 & 2 & 0.31 & 2.58 & 2.19 & 14.99 & 23.84 \\
\hline 6 & 4 & 2 & 0.35 & 3.05 & 2.33 & 23.63 & 34.22 \\
\hline 6 & 4 & 2 & 0.35 & 3.05 & 2.33 & 23.48 & 34.22 \\
\hline 6 & 4 & 2 & 0.35 & 3.04 & 2.33 & 23.28 & 34.22 \\
\hline 6 & 4 & 2 & 0.35 & 3.05 & 2.33 & 23.59 & 34.22 \\
\hline 6 & 4 & 2 & 0.35 & 3.05 & 2.33 & 23.56 & 34.22 \\
\hline 6 & 4 & 2 & 0.35 & 3.05 & 2.31 & 24.41 & 34.22 \\
\hline 1 & 4 & 3 & 0.08 & 2.41 & 2.21 & 8.17 & 3.92 \\
\hline 1 & 4 & 3 & 0.08 & 2.56 & 2.23 & 12.95 & 9.25 \\
\hline 1 & 4 & 3 & 0.09 & 2.88 & 2.26 & 21.37 & 19.90 \\
\hline 2 & 4 & 3 & 0.14 & 2.64 & 2.39 & 9.45 & 5.95 \\
\hline 2 & 4 & 3 & 0.14 & 2.64 & 2.39 & 9.69 & 5.95 \\
\hline 2 & 4 & 3 & 0.15 & 2.81 & 2.41 & 14.17 & 5.86 \\
\hline 2 & 4 & 3 & 0.15 & 2.80 & 2.41 & 13.82 & 5.86 \\
\hline 2 & 4 & 3 & 0.17 & 3.22 & 2.48 & 23.09 & 5.66 \\
\hline 2 & 4 & 3 & 0.17 & 3.21 & 2.48 & 22.83 & 5.66 \\
\hline 3 & 4 & 3 & 0.23 & 2.91 & 2.58 & 11.21 & 7.98 \\
\hline 3 & 4 & 3 & 0.23 & 2.92 & 2.58 & 11.43 & 7.98 \\
\hline 3 & 4 & 3 & 0.23 & 2.92 & 2.58 & 11.41 & 7.98 \\
\hline 3 & 4 & 3 & 0.25 & 3.12 & 2.63 & 15.92 & 13.23 \\
\hline 3 & 4 & 3 & 0.25 & 3.12 & 2.63 & 15.98 & 13.23 \\
\hline 3 & 4 & 3 & 0.25 & 3.13 & 2.63 & 15.99 & 13.23 \\
\hline 3 & 4 & 3 & 0.27 & 3.63 & 2.73 & 24.86 & 23.68 \\
\hline 3 & 4 & 3 & 0.27 & 3.63 & 2.73 & 24.98 & 23.68 \\
\hline 3 & 4 & 3 & 0.27 & 3.63 & 2.73 & 25.02 & 23.68 \\
\hline 4 & 4 & 3 & 0.33 & 3.25 & 2.81 & 13.54 & 10.01 \\
\hline 4 & 4 & 3 & 0.33 & 3.24 & 2.81 & 13.46 & 10.01 \\
\hline 4 & 4 & 3 & 0.33 & 3.25 & 2.81 & 13.62 & 10.01 \\
\hline 4 & 4 & 3 & 0.33 & 3.25 & 2.81 & 13.69 & 10.01 \\
\hline 4 & 4 & 3 & 0.35 & 3.50 & 2.87 & 18.06 & 15.22 \\
\hline 4 & 4 & 3 & 0.35 & 3.51 & 2.87 & 18.41 & 15.22 \\
\hline 4 & 4 & 3 & 0.35 & 3.50 & 2.87 & 18.10 & 15.22 \\
\hline 4 & 4 & 3 & 0.35 & 3.50 & 2.87 & 18.14 & 15.22 \\
\hline 4 & 4 & 3 & 0.39 & 4.16 & 3.02 & 27.49 & 25.56 \\
\hline 4 & 4 & 3 & 0.39 & 4.16 & 3.02 & 27.44 & 25.56 \\
\hline 4 & 4 & 3 & 0.39 & 4.16 & 3.02 & 27.56 & 25.56 \\
\hline
\end{tabular}


\begin{tabular}{lllllll}
\hline Prod. & Server & Subtasks Net Traffic & Anal. & Sim. & Error & Predicted
\end{tabular} Intensity Flow T. Flow T. sim-anal (\%) Error (\%)

\begin{tabular}{|c|c|c|c|c|c|c|c|}
\hline 4 & 4 & 3 & 0.39 & 4.16 & 3.02 & 27.51 & 25.56 \\
\hline 5 & 4 & 3 & 0.38 & 3.66 & 3.07 & 16.14 & 12.04 \\
\hline 5 & 4 & 3 & 0.38 & 3.66 & 3.07 & 16.15 & 12.04 \\
\hline 5 & 4 & 3 & 0.38 & 3.67 & 3.07 & 16.38 & 12.04 \\
\hline 5 & 4 & 3 & 0.38 & 3.66 & 3.07 & 16.32 & 12.04 \\
\hline 5 & 4 & 3 & 0.38 & 3.66 & 3.07 & 16.15 & 12.04 \\
\hline 5 & 4 & 3 & 0.40 & 3.98 & 3.15 & 20.84 & 17.20 \\
\hline 5 & 4 & 3 & 0.40 & 3.98 & 3.15 & 20.86 & 17.20 \\
\hline 5 & 4 & 3 & 0.40 & 3.98 & 3.15 & 20.88 & 17.20 \\
\hline 5 & 4 & 3 & 0.40 & 3.98 & 3.15 & 20.87 & 17.20 \\
\hline 5 & 4 & 3 & 0.40 & 3.98 & 3.15 & 20.82 & 17.20 \\
\hline 5 & 4 & 3 & 0.45 & 4.85 & 3.37 & 30.47 & 27.45 \\
\hline 5 & 4 & 3 & 0.45 & 4.85 & 3.37 & 30.41 & 27.45 \\
\hline 5 & 4 & 3 & 0.45 & 4.84 & 3.37 & 30.31 & 27.45 \\
\hline 5 & 4 & 3 & 0.45 & 4.85 & 3.37 & 30.43 & 27.45 \\
\hline 5 & 4 & 3 & 0.45 & 4.85 & 3.37 & 30.51 & 27.45 \\
\hline 6 & 4 & 3 & 0.44 & 3.90 & 3.21 & 17.56 & 14.49 \\
\hline 6 & 4 & 3 & 0.44 & 3.89 & 3.21 & 17.42 & 14.49 \\
\hline 6 & 4 & 3 & 0.44 & 3.91 & 3.21 & 17.70 & 14.49 \\
\hline 6 & 4 & 3 & 0.44 & 3.90 & 3.21 & 17.65 & 14.49 \\
\hline 6 & 4 & 3 & 0.44 & 3.90 & 3.21 & 17.66 & 14.49 \\
\hline 6 & 4 & 3 & 0.44 & 3.91 & 3.12 & 20.15 & 14.49 \\
\hline 6 & 4 & 3 & 0.47 & 4.27 & 3.32 & 22.39 & 19.63 \\
\hline 6 & 4 & 3 & 0.47 & 4.27 & 3.32 & 22.37 & 19.63 \\
\hline 6 & 4 & 3 & 0.47 & 4.26 & 3.32 & 22.24 & 19.63 \\
\hline 6 & 4 & 3 & 0.47 & 4.27 & 3.32 & 22.29 & 19.63 \\
\hline 6 & 4 & 3 & 0.47 & 4.27 & 3.32 & 22.34 & 19.63 \\
\hline 6 & 4 & 3 & 0.47 & 4.27 & 3.21 & 24.77 & 19.63 \\
\hline 6 & 4 & 3 & 0.52 & 5.27 & 3.58 & 32.00 & 29.82 \\
\hline 6 & 4 & 3 & 0.52 & 5.26 & 3.58 & 31.84 & 29.82 \\
\hline 6 & 4 & 3 & 0.52 & 5.27 & 3.58 & 32.00 & 29.82 \\
\hline 6 & 4 & 3 & 0.52 & 5.27 & 3.58 & 31.94 & 29.82 \\
\hline 6 & 4 & 3 & 0.52 & 5.27 & 3.58 & 31.96 & 29.82 \\
\hline 6 & 4 & 3 & 0.52 & 5.26 & 3.46 & 34.29 & 29.82 \\
\hline
\end{tabular}




\section{APPENDIX G}

Flow time error using correction factors for three product instances

\begin{tabular}{|c|c|c|c|c|c|c|c|}
\hline Prod \# & Ca & Cs & Analitical & Sim. & Error & $\begin{array}{c}\text { Adj. } \\
\text { Anali. }\end{array}$ & Adj Error \\
\hline 1 & & & Flow T. & $(\%)$ \\
\hline 2 & 0.5 & 0.5 & 33.08 & 31.35 & 5.53 & 33.44 & 6.67 \\
\hline 3 & 0.5 & 0.5 & 12.88 & 11.98 & 7.52 & 13.11 & 9.43 \\
\hline 1 & 0.5 & 0.5 & 33.44 & 31.98 & 4.57 & 32.65 & 2.11 \\
\hline 2 & 0.5 & 0.5 & 14.80 & 13.31 & 11.21 & 14.46 & 8.66 \\
\hline 3 & 0.5 & 0.5 & 13.64 & 12.59 & 8.28 & 13.30 & 5.59 \\
\hline 1 & 0.5 & 0.5 & 40.55 & 37.62 & 7.81 & 38.52 & 2.42 \\
\hline 2 & 0.5 & 0.5 & 21.94 & 18.77 & 16.88 & 19.89 & 5.96 \\
\hline 3 & 0.5 & 0.5 & 22.25 & 18.81 & 18.25 & 20.20 & 7.35 \\
\hline 1 & 0.5 & 0.5 & 30.45 & 26.41 & 15.30 & 27.83 & 5.39 \\
\hline 2 & 0.5 & 0.5 & 20.37 & 16.39 & 24.34 & 17.92 & 9.35 \\
\hline 3 & 0.5 & 0.5 & 18.96 & 15.30 & 23.90 & 16.50 & 7.85 \\
\hline 1 & 0.5 & 1 & 38.98 & 36.59 & 6.52 & 39.14 & 6.97 \\
\hline 2 & 0.5 & 1 & 16.22 & 14.69 & 10.45 & 16.43 & 11.88 \\
\hline 3 & 0.5 & 1 & 16.06 & 14.51 & 10.70 & 16.27 & 12.15 \\
\hline 1 & 0.5 & 1 & 37.92 & 36.17 & 4.83 & 36.56 & 1.08 \\
\hline 2 & 0.5 & 1 & 17.65 & 15.69 & 12.50 & 17.06 & 8.75 \\
\hline 3 & 0.5 & 1 & 16.49 & 15.03 & 9.72 & 15.90 & 5.80 \\
\hline 1 & 0.5 & 1 & 47.57 & 43.43 & 9.53 & 44.30 & 1.99 \\
\hline 2 & 0.5 & 1 & 27.52 & 23.06 & 19.34 & 24.40 & 5.83 \\
\hline 3 & 0.5 & 1 & 27.82 & 23.05 & 20.68 & 24.70 & 7.16 \\
\hline 1 & 0.5 & 1 & 37.07 & 31.18 & 18.89 & 33.07 & 6.08 \\
\hline 2 & 0.5 & 1 & 25.64 & 19.81 & 29.44 & 22.02 & 11.15 \\
\hline 3 & 0.5 & 1 & 24.22 & 18.79 & 28.93 & 20.60 & 9.66 \\
\hline 1 & 1 & 1.5 & 19.86 & 18.40 & 7.96 & 18.88 & 2.62 \\
\hline 2 & 1 & 1.5 & 8.00 & 7.93 & 0.84 & 7.72 & 2.65 \\
\hline 3 & 1 & 1.5 & 7.92 & 7.86 & 0.80 & 7.64 & 2.73 \\
\hline 1 & 1 & 2 & 22.57 & 23.66 & 4.60 & 21.05 & 11.01 \\
\hline 2 & 1 & 2 & 10.24 & 10.37 & 1.32 & 9.52 & 8.19 \\
\hline 3 & 1 & 2 & 9.65 & 9.99 & 3.41 & 8.94 & 10.54 \\
\hline 1 & 1 & 2 & 29.66 & 28.89 & 2.64 & 26.28 & 9.04 \\
\hline 2 & 1 & 2 & 18.08 & 16.36 & 10.49 & 15.08 & 7.86 \\
\hline 3 & 1 & 2 & 18.23 & 16.34 & 11.56 & 15.23 & 6.82 \\
\hline 1 & 1 & 1.5 & 20.61 & 18.70 & 10.20 & 17.62 & 5.80 \\
\hline 3 & 1 & 1.5 & 14.63 & 12.25 & 19.47 & 11.98 & 2.19 \\
\hline 1 & 1 & 0.5 & 19.75 & 16.92 & 16.75 & 19.26 & 13.81 \\
\hline & & & & & & & \\
\hline 1 & & & & & \\
\hline
\end{tabular}


Flow time error using correction factors for three product instances

\begin{tabular}{|c|c|c|c|c|c|c|c|}
\hline Prod \# & Ca & Cs & Analitical & Sim. & Error & $\begin{array}{c}\text { Adj. } \\
\text { Anali. } \\
\text { Flow T. }\end{array}$ & $\begin{array}{c}\text { Adj Error } \\
(\%)\end{array}$ \\
\hline 2 & 1 & 0.5 & 7.21 & 6.54 & 10.28 & 7.16 & 9.48 \\
\hline 3 & 1 & 0.5 & 7.13 & 6.46 & 10.45 & 7.08 & 9.64 \\
\hline 1 & 1 & 0.5 & 18.08 & 17.28 & 4.67 & 17.39 & 0.69 \\
\hline 2 & 1 & 0.5 & 7.77 & 7.11 & 9.29 & 7.50 & 5.46 \\
\hline 3 & 1 & 0.5 & 7.18 & 6.72 & 6.96 & 6.91 & 2.92 \\
\hline 1 & 1 & 0.5 & 22.08 & 20.32 & 8.68 & 20.56 & 1.19 \\
\hline 2 & 1 & 0.5 & 11.82 & 10.34 & 14.33 & 10.52 & 1.76 \\
\hline 3 & 1 & 0.5 & 11.97 & 10.33 & 15.83 & 10.67 & 3.25 \\
\hline 1 & 1 & 0.5 & 15.98 & 14.42 & 10.82 & 14.43 & 0.07 \\
\hline 2 & 1 & 0.5 & 10.80 & 9.28 & 16.31 & 9.36 & 0.84 \\
\hline 3 & 1 & 0.5 & 10.09 & 8.65 & 16.69 & 8.65 & 0.08 \\
\hline 1 & 1.5 & 1 & 17.96 & 17.65 & 1.78 & 17.13 & 2.90 \\
\hline 2 & 1.5 & 1 & 7.56 & 7.35 & 2.94 & 7.28 & 0.92 \\
\hline 3 & 1.5 & 1 & 7.48 & 7.26 & 3.14 & 7.20 & 0.77 \\
\hline 1 & 2 & 1 & 21.69 & 20.78 & 4.42 & 20.28 & 2.38 \\
\hline 2 & 2 & 1 & 9.30 & 8.50 & 9.36 & 8.70 & 2.29 \\
\hline 3 & 2 & 1 & 8.71 & 8.09 & 7.70 & 8.11 & 0.27 \\
\hline 1 & 1.5 & 1 & 26.25 & 23.88 & 9.89 & 23.57 & 1.30 \\
\hline 2 & 1.5 & 1 & 14.88 & 12.79 & 16.30 & 12.65 & 1.15 \\
\hline 3 & 1.5 & 1 & 15.03 & 12.80 & 17.42 & 12.80 & 0.03 \\
\hline 1 & 1.5 & 1 & 19.00 & 18.52 & 2.62 & 16.42 & 11.32 \\
\hline 2 & 1.5 & 1 & 13.40 & 12.22 & 9.65 & 11.09 & 9.29 \\
\hline 3 & 1.5 & 1 & 12.70 & 11.50 & 10.43 & 10.38 & 9.72 \\
\hline
\end{tabular}

Flow time error using correction factors for four product instances

\begin{tabular}{|c|c|c|c|c|c|c|c|}
\hline Prod \# & $C a$ & $C s$ & $\begin{array}{c}\text { Anal. } \\
\text { Flow T. }\end{array}$ & $\begin{array}{c}\text { Sim. } \\
\text { Flow T. }\end{array}$ & $\begin{array}{c}\text { Error } \\
(\%)\end{array}$ & $\begin{array}{c}\text { Adj. Anal. } \\
\text { Flow T. }\end{array}$ & $\begin{array}{c}\text { Adj Error } \\
(\%)\end{array}$ \\
\hline 1 & 0.5 & 0.5 & 44.35 & 37.65 & 17.79 & 40.27 & 6.97 \\
\hline 2 & 0.5 & 0.5 & 13.99 & 12.60 & 11.01 & 13.90 & 10.31 \\
\hline 3 & 0.5 & 0.5 & 13.83 & 12.46 & 11.06 & 13.75 & 10.35 \\
\hline 4 & 0.5 & 0.5 & 33.95 & 27.69 & 22.60 & 29.27 & 5.69 \\
\hline 1 & 0.5 & 0.5 & 47.73 & 39.19 & 21.79 & 40.90 & 4.36 \\
\hline 2 & 0.5 & 0.5 & 17.13 & 14.67 & 16.75 & 16.03 & 9.24 \\
\hline 3 & 0.5 & 0.5 & 15.96 & 14.07 & 13.43 & 14.86 & 5.60 \\
\hline 4 & 0.5 & 0.5 & 46.29 & 38.81 & 19.27 & 39.45 & 1.66 \\
\hline 1 & 0.5 & 0.5 & 46.75 & 40.57 & 15.23 & 42.66 & 5.15 \\
\hline 2 & 0.5 & 0.5 & 23.10 & 19.82 & 16.59 & 20.64 & 4.14 \\
\hline 3 & 0.5 & 0.5 & 23.41 & 19.84 & 18.01 & 20.94 & 5.58 \\
\hline
\end{tabular}


Flow time error using correction factors for four product instances

\begin{tabular}{|c|c|c|c|c|c|c|c|}
\hline Prod \# & $\mathrm{Ca}$ & $C s$ & $\begin{array}{c}\text { Anal. } \\
\text { Flow T. }\end{array}$ & $\begin{array}{l}\text { Sim. } \\
\text { Flow T. }\end{array}$ & $\begin{array}{c}\text { Error } \\
(\%)\end{array}$ & $\begin{array}{c}\text { Adj. Anal. } \\
\text { Flow T. }\end{array}$ & $\begin{array}{c}\text { Adj Error } \\
(\%)\end{array}$ \\
\hline 4 & 0.5 & 0.5 & 21.07 & 19.28 & 9.29 & 20.12 & 4.36 \\
\hline 1 & 0.5 & 0.5 & 35.37 & 30.25 & 16.90 & 30.88 & 2.08 \\
\hline 2 & 0.5 & 0.5 & 20.91 & 17.11 & 22.26 & 18.25 & 6.71 \\
\hline 3 & 0.5 & 0.5 & 19.50 & 16.00 & 21.88 & 16.84 & 5.25 \\
\hline 4 & 0.5 & 0.5 & 19.23 & 18.45 & 4.22 & 18.31 & 0.77 \\
\hline 1 & 0.5 & 1 & 57.26 & 47.20 & 21.31 & 50.66 & 7.33 \\
\hline 2 & 0.5 & 1 & 17.60 & 15.54 & 13.26 & 17.35 & 11.66 \\
\hline 3 & 0.5 & 1 & 17.44 & 15.42 & 13.12 & 17.19 & 11.51 \\
\hline 4 & 0.5 & 1 & 43.19 & 34.89 & 23.77 & 36.03 & 3.26 \\
\hline 1 & 0.5 & 1 & 59.76 & 48.72 & 22.65 & 49.25 & 1.09 \\
\hline 2 & 0.5 & 1 & 21.88 & 18.31 & 19.49 & 20.02 & 9.35 \\
\hline 3 & 0.5 & 1 & 20.71 & 17.77 & 16.57 & 18.86 & 6.12 \\
\hline 4 & 0.5 & 1 & 58.32 & 48.45 & 20.37 & 47.81 & 1.32 \\
\hline 1 & 0.5 & 1 & 57.01 & 48.04 & 18.67 & 50.72 & 5.58 \\
\hline 2 & 0.5 & 1 & 30.03 & 24.77 & 21.26 & 26.18 & 5.71 \\
\hline 3 & 0.5 & 1 & 30.33 & 24.78 & 22.41 & 26.48 & 6.88 \\
\hline 4 & 0.5 & 1 & 26.54 & 23.25 & 14.18 & 24.92 & 7.19 \\
\hline 1 & 0.5 & 1 & 46.14 & 37.20 & 24.03 & 38.92 & 4.62 \\
\hline 2 & 0.5 & 1 & 27.02 & 21.03 & 28.49 & 23.06 & 9.66 \\
\hline 3 & 0.5 & 1 & 25.61 & 20.00 & 28.04 & 21.65 & 8.24 \\
\hline 4 & 0.5 & 1 & 24.30 & 22.22 & 9.36 & 22.69 & 2.08 \\
\hline 1 & 1 & 1.5 & 31.89 & 25.86 & 23.29 & 25.35 & 1.96 \\
\hline 2 & 1 & 1.5 & 9.12 & 8.60 & 6.05 & 8.37 & 2.65 \\
\hline 3 & 1 & 1.5 & 9.04 & 8.52 & 6.07 & 8.29 & 2.70 \\
\hline 4 & 1 & 1.5 & 25.63 & 19.84 & 29.15 & 19.25 & 3.00 \\
\hline 1 & 1 & 2 & 40.31 & 36.32 & 10.96 & 30.74 & 15.38 \\
\hline 2 & 1 & 2 & 13.48 & 12.92 & 4.36 & 11.51 & 10.92 \\
\hline 3 & 1 & 2 & 12.90 & 12.59 & 2.44 & 10.92 & 13.24 \\
\hline 4 & 1 & 2 & 39.58 & 36.05 & 9.79 & 30.01 & 16.75 \\
\hline 1 & 1 & 2 & 35.11 & 33.24 & 5.61 & 29.53 & 11.18 \\
\hline 2 & 1 & 2 & 19.63 & 17.83 & 10.10 & 15.81 & 11.31 \\
\hline 3 & 1 & 2 & 19.78 & 17.82 & 10.98 & 15.96 & 10.44 \\
\hline 4 & 1 & 2 & 16.15 & 15.85 & 1.89 & 14.27 & 9.95 \\
\hline 1 & 1 & 1.5 & 26.24 & 23.08 & 13.70 & 20.84 & 9.72 \\
\hline 2 & 1 & 1.5 & 14.89 & 12.81 & 16.20 & 11.92 & 7.01 \\
\hline 3 & 1 & 1.5 & 14.18 & 12.20 & 16.24 & 11.21 & 8.13 \\
\hline 4 & 1 & 1.5 & 13.80 & 13.36 & 3.27 & 11.92 & 10.76 \\
\hline 1 & 1 & 0.5 & 26.27 & 20.96 & 25.36 & 22.81 & 8.84 \\
\hline 2 & 1 & 0.5 & 7.97 & 6.82 & 16.94 & 7.63 & 11.92 \\
\hline
\end{tabular}


Flow time error using correction factors for four product instances

\begin{tabular}{|c|c|c|c|c|c|c|c|}
\hline Prod \# & $C a$ & $C s$ & $\begin{array}{c}\text { Anal. } \\
\text { Flow T. }\end{array}$ & $\begin{array}{c}\text { Sim. } \\
\text { Flow T. }\end{array}$ & $\begin{array}{c}\text { Error } \\
(\%)\end{array}$ & $\begin{array}{c}\text { Adj. Anal. } \\
\text { Flow T. }\end{array}$ & $\begin{array}{c}\text { Adj Error } \\
(\%)\end{array}$ \\
\hline 3 & 1 & 0.5 & 7.89 & 6.73 & 17.25 & 7.55 & 12.17 \\
\hline 4 & 1 & 0.5 & 20.12 & 15.68 & 28.33 & 16.48 & 5.10 \\
\hline 1 & 1 & 0.5 & 25.61 & 21.89 & 17.01 & 21.52 & 1.67 \\
\hline 2 & 1 & 0.5 & 9.17 & 7.88 & 16.36 & 8.45 & 7.19 \\
\hline 3 & 1 & 0.5 & 8.59 & 7.55 & 13.74 & 7.86 & 4.17 \\
\hline 4 & 1 & 0.5 & 24.89 & 21.57 & 15.42 & 20.80 & 3.54 \\
\hline 1 & 1 & 0.5 & 24.27 & 22.06 & 10.03 & 21.96 & 0.45 \\
\hline 2 & 1 & 0.5 & 12.51 & 10.92 & 14.58 & 10.96 & 0.40 \\
\hline 3 & 1 & 0.5 & 12.66 & 10.95 & 15.64 & 11.12 & 1.50 \\
\hline 4 & 1 & 0.5 & 11.22 & 10.58 & 6.04 & 10.56 & 0.23 \\
\hline 1 & 1 & 0.5 & 18.72 & 16.84 & 11.14 & 16.14 & 4.16 \\
\hline 2 & 1 & 0.5 & 11.17 & 9.68 & 15.32 & 9.61 & 0.75 \\
\hline 3 & 1 & 0.5 & 10.46 & 9.06 & 15.51 & 8.90 & 1.68 \\
\hline 4 & 1 & 0.5 & 11.24 & 10.37 & 8.35 & 10.27 & 1.03 \\
\hline 1 & 1.5 & 1 & 27.75 & 24.22 & 14.56 & 22.38 & 7.59 \\
\hline 2 & 1.5 & 1 & 8.61 & 7.84 & 9.88 & 7.89 & 0.65 \\
\hline 3 & 1.5 & 1 & 8.53 & 7.77 & 9.84 & 7.81 & 0.54 \\
\hline 4 & 1.5 & 1 & 22.55 & 18.89 & 19.34 & 17.29 & 8.47 \\
\hline 1 & 2 & 1 & 34.19 & 29.56 & 15.65 & 26.86 & 9.12 \\
\hline 2 & 2 & 1 & 11.78 & 9.94 & 18.55 & 10.30 & 3.67 \\
\hline 3 & 2 & 1 & 11.20 & 9.56 & 17.13 & 9.72 & 1.66 \\
\hline 4 & 2 & 1 & 33.46 & 29.18 & 14.68 & 26.14 & 10.42 \\
\hline 1 & 1.5 & 1 & 29.13 & 26.59 & 9.55 & 25.25 & 5.04 \\
\hline 2 & 1.5 & 1 & 16.00 & 13.59 & 17.76 & 13.26 & 2.40 \\
\hline 3 & 1.5 & 1 & 16.15 & 13.57 & 19.05 & 13.41 & 1.13 \\
\hline 4 & 1.5 & 1 & 13.67 & 12.53 & 9.08 & 12.32 & 1.66 \\
\hline 1 & 1.5 & 1 & 23.57 & 22.59 & 4.35 & 19.12 & 15.35 \\
\hline 2 & 1.5 & 1 & 13.86 & 12.94 & 7.11 & 11.29 & 12.73 \\
\hline 3 & 1.5 & 1 & 13.15 & 12.23 & 7.52 & 10.59 & 13.46 \\
\hline 4 & 1.5 & 1 & 13.86 & 13.67 & 1.38 & 11.96 & 12.49 \\
\hline & & & & & & & \\
\hline
\end{tabular}

Flow time error using correction factors for five product instances

\begin{tabular}{|c|c|c|c|c|c|c|c|}
\hline Prod \# & $C a$ & $C s$ & $\begin{array}{c}\text { Anal. } \\
\text { Flow T. }\end{array}$ & $\begin{array}{c}\text { Sim. } \\
\text { Flow T. }\end{array}$ & $\begin{array}{c}\text { Error } \\
(\%)\end{array}$ & $\begin{array}{c}\text { Adj. Anal. } \\
\text { Flow T. }\end{array}$ & $\begin{array}{c}\text { Adj Error } \\
(\%)\end{array}$ \\
\hline 1 & 0.5 & 0.5 & 48.30 & 39.95 & 20.90 & 42.88 & 7.31 \\
\hline 2 & 0.5 & 0.5 & 18.46 & 14.90 & 23.91 & 16.74 & 12.35 \\
\hline 3 & 0.5 & 0.5 & 18.31 & 14.76 & 24.00 & 16.58 & 12.33 \\
\hline 4 & 0.5 & 0.5 & 36.00 & 28.55 & 26.12 & 30.15 & 5.61 \\
\hline 5 & 0.5 & 0.5 & 18.17 & 14.65 & 23.99 & 16.44 & 12.24 \\
\hline
\end{tabular}


Flow time error using correction factors for five product instances

\begin{tabular}{|c|c|c|c|c|c|c|c|}
\hline Prod \# & $\mathrm{Ca}$ & $C s$ & $\begin{array}{c}\text { Anal. } \\
\text { Flow T. }\end{array}$ & $\begin{array}{l}\text { Sim. } \\
\text { Flow T. }\end{array}$ & $\begin{array}{c}\text { Error } \\
(\%)\end{array}$ & $\begin{array}{c}\text { Adj. Anal. } \\
\text { Flow T. }\end{array}$ & $\begin{array}{c}\text { Adj Error } \\
(\%)\end{array}$ \\
\hline 1 & 0.5 & 0.5 & 53.32 & 41.97 & 27.04 & 43.68 & 4.09 \\
\hline 2 & 0.5 & 0.5 & 22.63 & 17.36 & 30.31 & 19.13 & 10.15 \\
\hline 3 & 0.5 & 0.5 & 21.46 & 16.90 & 26.98 & 17.96 & 6.27 \\
\hline 4 & 0.5 & 0.5 & 51.87 & 41.69 & 24.42 & 42.24 & 1.32 \\
\hline 5 & 0.5 & 0.5 & 22.45 & 17.10 & 31.28 & 18.95 & 10.81 \\
\hline 1 & 0.5 & 0.5 & 61.57 & 46.59 & 32.15 & 48.94 & 5.03 \\
\hline 2 & 0.5 & 0.5 & 37.58 & 26.32 & 42.81 & 26.91 & 2.25 \\
\hline 3 & 0.5 & 0.5 & 37.88 & 26.32 & 43.95 & 27.21 & 3.39 \\
\hline 4 & 0.5 & 0.5 & 23.10 & 20.50 & 12.66 & 21.67 & 5.70 \\
\hline 5 & 0.5 & 0.5 & 37.58 & 26.33 & 42.75 & 26.91 & 2.20 \\
\hline 1 & 0.5 & 0.5 & 47.17 & 35.36 & 33.39 & 35.72 & 1.00 \\
\hline 2 & 0.5 & 0.5 & 33.30 & 22.01 & 51.29 & 23.47 & 6.63 \\
\hline 3 & 0.5 & 0.5 & 31.89 & 20.99 & 51.93 & 22.06 & 5.09 \\
\hline 4 & 0.5 & 0.5 & 20.18 & 19.37 & 4.19 & 19.39 & 0.14 \\
\hline 5 & 0.5 & 0.5 & 31.86 & 21.06 & 51.29 & 22.03 & 4.60 \\
\hline 1 & 0.5 & 1 & 64.95 & 51.11 & 27.09 & 55.75 & 9.08 \\
\hline 2 & 0.5 & 1 & 25.10 & 19.58 & 28.20 & 22.48 & 14.80 \\
\hline 3 & 0.5 & 1 & 24.95 & 19.43 & 28.39 & 22.32 & 14.88 \\
\hline 4 & 0.5 & 1 & 46.79 & 36.12 & 29.55 & 37.53 & 3.92 \\
\hline 5 & 0.5 & 1 & 24.81 & 19.33 & 28.33 & 22.18 & 14.75 \\
\hline 1 & 0.5 & 1 & 70.00 & 53.49 & 30.85 & 54.65 & 2.16 \\
\hline 2 & 0.5 & 1 & 31.86 & 23.09 & 38.00 & 25.83 & 11.89 \\
\hline 3 & 0.5 & 1 & 30.69 & 22.69 & 35.26 & 24.66 & 8.70 \\
\hline 4 & 0.5 & 1 & 68.55 & 53.25 & 28.73 & 53.20 & 0.10 \\
\hline 5 & 0.5 & 1 & 31.68 & 22.87 & 38.54 & 25.65 & 12.18 \\
\hline 1 & 0.5 & 1 & 81.32 & 58.16 & 39.83 & 61.74 & 6.16 \\
\hline 2 & 0.5 & 1 & 54.85 & 35.40 & 54.96 & 37.44 & 5.76 \\
\hline 3 & 0.5 & 1 & 55.16 & 35.47 & 55.52 & 37.74 & 6.42 \\
\hline 4 & 0.5 & 1 & 30.80 & 25.79 & 19.40 & 28.27 & 9.60 \\
\hline 5 & 0.5 & 1 & 54.85 & 35.39 & 55.00 & 37.44 & 5.79 \\
\hline 1 & 0.5 & 1 & 66.93 & 45.61 & 46.73 & 48.21 & 5.70 \\
\hline 2 & 0.5 & 1 & 46.99 & 29.03 & 61.84 & 32.00 & 10.21 \\
\hline 3 & 0.5 & 1 & 45.57 & 28.15 & 61.87 & 30.58 & 8.62 \\
\hline 4 & 0.5 & 1 & 26.51 & 23.91 & 10.90 & 25.08 & 4.92 \\
\hline 5 & 0.5 & 1 & 45.54 & 28.21 & 61.43 & 30.55 & 8.29 \\
\hline 1 & 1 & 1.5 & 36.43 & 28.32 & 28.66 & 27.52 & 2.80 \\
\hline 2 & 1 & 1.5 & 13.37 & 11.07 & 20.82 & 10.67 & 3.59 \\
\hline 3 & 1 & 1.5 & 13.30 & 10.98 & 21.10 & 10.59 & 3.51 \\
\hline 4 & 1 & 1.5 & 28.28 & 20.91 & 35.26 & 20.16 & 3.57 \\
\hline
\end{tabular}


Flow time error using correction factors for five product instances

\begin{tabular}{|c|c|c|c|c|c|c|c|}
\hline Prod \# & $\mathrm{Ca}$ & $C s$ & $\begin{array}{c}\text { Anal. } \\
\text { Flow T. }\end{array}$ & $\begin{array}{l}\text { Sim. } \\
\text { Flow T. }\end{array}$ & $\begin{array}{c}\text { Error } \\
(\%)\end{array}$ & $\begin{array}{c}\text { Adj. Anal. } \\
\text { Flow T. }\end{array}$ & $\begin{array}{c}\text { Adj Error } \\
(\%)\end{array}$ \\
\hline 5 & 1 & 1.5 & 13.23 & 10.91 & 21.19 & 10.52 & 3.57 \\
\hline 1 & 1 & 2 & 48.46 & 40.75 & 18.91 & 34.41 & 15.55 \\
\hline 2 & 1 & 2 & 21.37 & 17.42 & 22.71 & 15.47 & 11.21 \\
\hline 3 & 1 & 2 & 20.79 & 17.18 & 21.04 & 14.88 & 13.35 \\
\hline 4 & 1 & 2 & 47.74 & 40.58 & 17.64 & 33.69 & 16.98 \\
\hline 5 & 1 & 2 & 21.29 & 17.26 & 23.32 & 15.38 & 10.90 \\
\hline 1 & 1 & 2 & 55.01 & 42.92 & 28.15 & 37.78 & 11.98 \\
\hline 2 & 1 & 2 & 41.24 & 27.99 & 47.34 & 24.73 & 11.63 \\
\hline 3 & 1 & 2 & 41.39 & 27.96 & 48.04 & 24.89 & 10.99 \\
\hline 4 & 1 & 2 & 18.76 & 18.01 & 4.12 & 15.81 & 12.24 \\
\hline 5 & 1 & 2 & 41.24 & 27.99 & 47.33 & 24.73 & 11.63 \\
\hline 1 & 1 & 1.5 & 40.49 & 29.28 & 38.30 & 26.35 & 10.01 \\
\hline 2 & 1 & 1.5 & 28.33 & 18.72 & 51.32 & 17.16 & 8.33 \\
\hline 3 & 1 & 1.5 & 27.62 & 18.14 & 52.24 & 16.45 & 9.31 \\
\hline 4 & 1 & 1.5 & 14.05 & 14.16 & 0.79 & 12.12 & 14.43 \\
\hline 5 & 1 & 1.5 & 27.60 & 18.17 & 51.89 & 16.44 & 9.55 \\
\hline 1 & 1 & 0.5 & 28.76 & 22.40 & 28.36 & 24.37 & 8.79 \\
\hline 2 & 1 & 0.5 & 10.78 & 8.26 & 30.62 & 9.37 & 13.52 \\
\hline 3 & 1 & 0.5 & 10.71 & 8.17 & 30.99 & 9.29 & 13.71 \\
\hline 4 & 1 & 0.5 & 21.38 & 16.31 & 31.09 & 16.96 & 3.96 \\
\hline 5 & 1 & 0.5 & 10.64 & 8.11 & 31.21 & 9.22 & 13.79 \\
\hline 1 & 1 & 0.5 & 28.58 & 23.50 & 21.59 & 23.04 & 1.97 \\
\hline 2 & 1 & 0.5 & 12.19 & 9.49 & 28.52 & 10.18 & 7.27 \\
\hline 3 & 1 & 0.5 & 11.61 & 9.22 & 25.83 & 9.59 & 3.98 \\
\hline 4 & 1 & 0.5 & 27.86 & 23.26 & 19.78 & 22.32 & 4.03 \\
\hline 5 & 1 & 0.5 & 12.10 & 9.35 & 29.48 & 10.09 & 7.92 \\
\hline 1 & 1 & 0.5 & 32.28 & 25.94 & 24.42 & 25.40 & 2.10 \\
\hline 2 & 1 & 0.5 & 20.80 & 15.07 & 38.04 & 14.58 & 3.28 \\
\hline 3 & 1 & 0.5 & 20.95 & 15.09 & 38.90 & 14.73 & 2.37 \\
\hline 4 & 1 & 0.5 & 12.42 & 11.31 & 9.80 & 11.48 & 1.52 \\
\hline 5 & 1 & 0.5 & 20.80 & 15.06 & 38.14 & 14.58 & 3.21 \\
\hline 1 & 1 & 0.5 & 25.82 & 20.21 & 27.72 & 19.13 & 5.36 \\
\hline 2 & 1 & 0.5 & 18.51 & 12.97 & 42.65 & 12.76 & 1.63 \\
\hline 3 & 1 & 0.5 & 17.80 & 12.39 & 43.69 & 12.05 & 2.69 \\
\hline 4 & 1 & 0.5 & 11.85 & 10.99 & 7.79 & 10.93 & 0.57 \\
\hline 5 & 1 & 0.5 & 17.79 & 12.42 & 43.15 & 12.04 & 3.09 \\
\hline 1 & 1.5 & 1 & 31.36 & 26.11 & 20.08 & 24.22 & 7.27 \\
\hline 2 & 1.5 & 1 & 12.35 & 9.81 & 25.97 & 9.98 & 1.75 \\
\hline 3 & 1.5 & 1 & 12.27 & 9.70 & 26.50 & 9.90 & 2.02 \\
\hline
\end{tabular}


Flow time error using correction factors for five product instances

\begin{tabular}{|c|c|c|c|c|c|c|c|}
\hline Prod \# & $C a$ & $C s$ & $\begin{array}{c}\text { Anal. } \\
\text { Flow T. }\end{array}$ & $\begin{array}{c}\text { Sim. } \\
\text { Flow T. }\end{array}$ & $\begin{array}{c}\text { Error } \\
(\%)\end{array}$ & $\begin{array}{c}\text { Adj. Anal. } \\
\text { Flow T. }\end{array}$ & $\begin{array}{c}\text { Adj Error } \\
(\%)\end{array}$ \\
\hline 4 & 1.5 & 1 & 24.59 & 19.80 & 24.16 & 17.98 & 9.19 \\
\hline 5 & 1.5 & 1 & 12.20 & 9.64 & 26.58 & 9.83 & 1.94 \\
\hline 1 & 2 & 1 & 39.26 & 32.45 & 20.98 & 29.31 & 9.69 \\
\hline 2 & 2 & 1 & 17.01 & 12.79 & 32.98 & 13.11 & 2.49 \\
\hline 3 & 2 & 1 & 16.42 & 12.50 & 31.41 & 12.52 & 0.21 \\
\hline 4 & 2 & 1 & 38.54 & 32.10 & 20.07 & 28.59 & 10.94 \\
\hline 5 & 2 & 1 & 16.92 & 12.60 & 34.23 & 13.02 & 3.30 \\
\hline 1 & 1.5 & 1 & 41.82 & 33.03 & 26.63 & 30.58 & 7.42 \\
\hline 2 & 1.5 & 1 & 29.93 & 20.34 & 47.17 & 19.14 & 5.89 \\
\hline 3 & 1.5 & 1 & 30.08 & 20.32 & 48.01 & 19.29 & 5.09 \\
\hline 4 & 1.5 & 1 & 15.55 & 13.66 & 13.82 & 13.60 & 0.47 \\
\hline 5 & 1.5 & 1 & 29.93 & 20.31 & 47.34 & 19.14 & 5.79 \\
\hline 1 & 1.5 & 1 & 35.50 & 28.35 & 25.21 & 23.94 & 15.56 \\
\hline 2 & 1.5 & 1 & 25.54 & 18.52 & 37.89 & 16.12 & 12.99 \\
\hline 3 & 1.5 & 1 & 24.83 & 17.88 & 38.89 & 15.41 & 13.82 \\
\hline 4 & 1.5 & 1 & 14.48 & 14.70 & 1.48 & 12.57 & 14.49 \\
\hline 5 & 1.5 & 1 & 24.82 & 17.91 & 38.61 & 15.40 & 14.02 \\
\hline
\end{tabular}

Flow time error using correction factors for six product instances

\begin{tabular}{|c|c|c|c|c|c|c|c|}
\hline Prod \# & $C a$ & $C s$ & $\begin{array}{c}\text { Anal. } \\
\text { Flow T. }\end{array}$ & $\begin{array}{c}\text { Sim. } \\
\text { Flow T. }\end{array}$ & $\begin{array}{c}\text { Error } \\
(\%)\end{array}$ & $\begin{array}{c}\text { Adj. Anal. } \\
\text { Flow T. }\end{array}$ & $\begin{array}{c}\text { Adj Error } \\
(\%)\end{array}$ \\
\hline 1 & 0.5 & 0.5 & 56.27 & 44.58 & 26.21 & 47.37 & 6.24 \\
\hline 2 & 0.5 & 0.5 & 20.02 & 15.36 & 30.30 & 17.53 & 14.09 \\
\hline 3 & 0.5 & 0.5 & 19.86 & 15.24 & 30.29 & 17.37 & 13.96 \\
\hline 4 & 0.5 & 0.5 & 43.84 & 33.54 & 30.71 & 34.18 & 1.91 \\
\hline 5 & 0.5 & 0.5 & 19.72 & 15.09 & 30.65 & 17.23 & 14.15 \\
\hline 6 & 0.5 & 0.5 & 30.65 & 25.21 & 21.61 & 26.89 & 6.66 \\
\hline 1 & 0.5 & 0.5 & 59.28 & 45.07 & 31.53 & 47.19 & 4.71 \\
\hline 2 & 0.5 & 0.5 & 27.57 & 20.11 & 37.06 & 22.10 & 9.88 \\
\hline 3 & 0.5 & 0.5 & 26.40 & 19.70 & 34.01 & 20.93 & 6.25 \\
\hline 4 & 0.5 & 0.5 & 57.83 & 44.83 & 29.01 & 45.74 & 2.04 \\
\hline 5 & 0.5 & 0.5 & 27.39 & 19.84 & 38.03 & 21.92 & 10.48 \\
\hline 6 & 0.5 & 0.5 & 22.82 & 19.97 & 14.27 & 20.20 & 1.13 \\
\hline 1 & 0.5 & 0.5 & 73.34 & 52.76 & 39.00 & 55.31 & 4.83 \\
\hline 2 & 0.5 & 0.5 & 41.58 & 28.93 & 43.72 & 29.70 & 2.64 \\
\hline 3 & 0.5 & 0.5 & 41.89 & 28.95 & 44.68 & 30.00 & 3.62 \\
\hline 4 & 0.5 & 0.5 & 34.50 & 26.59 & 29.74 & 27.98 & 5.20 \\
\hline 5 & 0.5 & 0.5 & 41.58 & 28.95 & 43.64 & 29.70 & 2.58 \\
\hline 6 & 0.5 & 0.5 & 35.22 & 26.53 & 32.76 & 28.85 & 8.73 \\
\hline
\end{tabular}


Flow time error using correction factors for six product instances

\begin{tabular}{|c|c|c|c|c|c|c|c|}
\hline Prod \# & $\mathrm{Ca}$ & $C s$ & $\begin{array}{c}\text { Anal. } \\
\text { Flow T. }\end{array}$ & $\begin{array}{l}\text { Sim. } \\
\text { Flow T. }\end{array}$ & $\begin{array}{c}\text { Error } \\
(\%)\end{array}$ & $\begin{array}{c}\text { Adj. Anal. } \\
\text { Flow T. }\end{array}$ & $\begin{array}{c}\text { Adj Error } \\
(\%)\end{array}$ \\
\hline 1 & 0.5 & 0.5 & 55.93 & 41.20 & 35.76 & 40.75 & 1.09 \\
\hline 2 & 0.5 & 0.5 & 34.81 & 23.87 & 45.82 & 25.23 & 5.69 \\
\hline 3 & 0.5 & 0.5 & 33.39 & 22.93 & 45.62 & 23.81 & 3.84 \\
\hline 4 & 0.5 & 0.5 & 29.94 & 24.71 & 21.16 & 25.16 & 1.82 \\
\hline 5 & 0.5 & 0.5 & 33.36 & 23.00 & 45.03 & 23.78 & 3.39 \\
\hline 6 & 0.5 & 0.5 & 27.51 & 23.57 & 16.71 & 22.74 & 3.56 \\
\hline 1 & 0.5 & 1 & 79.38 & 58.71 & 35.20 & 64.04 & 9.07 \\
\hline 2 & 0.5 & 1 & 27.38 & 20.36 & 34.45 & 23.67 & 16.22 \\
\hline 3 & 0.5 & 1 & 27.22 & 20.22 & 34.59 & 23.51 & 16.24 \\
\hline 4 & 0.5 & 1 & 60.76 & 44.14 & 37.64 & 44.92 & 1.76 \\
\hline 5 & 0.5 & 1 & 27.08 & 20.12 & 34.58 & 23.37 & 16.13 \\
\hline 6 & 0.5 & 1 & 44.08 & 33.27 & 32.51 & 36.90 & 10.92 \\
\hline 1 & 0.5 & 1 & 81.50 & 59.14 & 37.80 & 61.66 & 4.25 \\
\hline 2 & 0.5 & 1 & 41.64 & 28.10 & 48.17 & 31.98 & 13.78 \\
\hline 3 & 0.5 & 1 & 40.47 & 27.72 & 46.00 & 30.81 & 11.13 \\
\hline 4 & 0.5 & 1 & 80.05 & 58.97 & 35.76 & 60.21 & 2.10 \\
\hline 5 & 0.5 & 1 & 41.46 & 27.84 & 48.94 & 31.80 & 14.22 \\
\hline 6 & 0.5 & 1 & 34.05 & 26.73 & 27.39 & 28.76 & 7.60 \\
\hline 1 & 0.5 & 1 & 103.95 & 68.56 & 51.63 & 74.30 & 8.37 \\
\hline 2 & 0.5 & 1 & 63.78 & 40.24 & 58.53 & 43.65 & 8.48 \\
\hline 3 & 0.5 & 1 & 64.09 & 40.26 & 59.19 & 43.95 & 9.17 \\
\hline 4 & 0.5 & 1 & 52.61 & 36.09 & 45.80 & 40.63 & 12.60 \\
\hline 5 & 0.5 & 1 & 63.78 & 40.33 & 58.14 & 43.65 & 8.22 \\
\hline 6 & 0.5 & 1 & 54.04 & 35.99 & 50.16 & 42.30 & 17.53 \\
\hline 1 & 0.5 & 1 & 84.44 & 55.63 & 51.79 & 58.46 & 5.09 \\
\hline 2 & 0.5 & 1 & 50.83 & 32.48 & 56.51 & 36.08 & 11.10 \\
\hline 3 & 0.5 & 1 & 49.42 & 31.58 & 56.49 & 34.67 & 9.79 \\
\hline 4 & 0.5 & 1 & 43.10 & 32.94 & 30.85 & 35.37 & 7.38 \\
\hline 5 & 0.5 & 1 & 49.39 & 31.63 & 56.15 & 34.64 & 9.52 \\
\hline 6 & 0.5 & 1 & 40.67 & 31.87 & 27.63 & 32.94 & 3.37 \\
\hline 1 & 1 & 1.5 & 47.32 & 32.29 & 46.54 & 32.04 & 0.79 \\
\hline 2 & 1 & 1.5 & 15.20 & 11.68 & 30.16 & 11.46 & 1.85 \\
\hline 3 & 1 & 1.5 & 15.12 & 11.62 & 30.21 & 11.38 & 1.98 \\
\hline 4 & 1 & 1.5 & 39.12 & 24.91 & 57.05 & 24.53 & 1.53 \\
\hline 5 & 1 & 1.5 & 15.05 & 11.52 & 30.64 & 11.31 & 1.80 \\
\hline 6 & 1 & 1.5 & 25.67 & 16.28 & 57.67 & 16.04 & 1.51 \\
\hline 1 & 1 & 2 & 57.13 & 45.42 & 25.78 & 38.62 & 14.96 \\
\hline 2 & 1 & 2 & 28.89 & 21.75 & 32.85 & 19.07 & 12.29 \\
\hline 3 & 1 & 2 & 28.31 & 21.52 & 31.54 & 18.49 & 14.08 \\
\hline
\end{tabular}


Flow time error using correction factors for six product instances

\begin{tabular}{|c|c|c|c|c|c|c|c|}
\hline Prod \# & $C a$ & $C s$ & $\begin{array}{c}\text { Anal. } \\
\text { Flow T. }\end{array}$ & $\begin{array}{l}\text { Sim. } \\
\text { Flow T. }\end{array}$ & $\begin{array}{c}\text { Error } \\
(\%)\end{array}$ & $\begin{array}{c}\text { Adj. Anal. } \\
\text { Flow T. }\end{array}$ & $\begin{array}{c}\text { Adj Error } \\
(\%)\end{array}$ \\
\hline 4 & 1 & 2 & 56.40 & 45.32 & 24.45 & 37.90 & 16.38 \\
\hline 5 & 1 & 2 & 28.80 & 21.58 & 33.48 & 18.99 & 12.01 \\
\hline 6 & 1 & 2 & 23.50 & 19.72 & 19.20 & 16.89 & 14.34 \\
\hline 1 & 1 & 2 & 72.88 & 51.66 & 41.09 & 45.79 & 11.36 \\
\hline 2 & 1 & 2 & 46.84 & 31.11 & 50.57 & 27.17 & 12.67 \\
\hline 3 & 1 & 2 & 46.99 & 31.16 & 50.80 & 27.32 & 12.33 \\
\hline 4 & 1 & 2 & 36.11 & 26.74 & 35.04 & 23.87 & 10.73 \\
\hline 5 & 1 & 2 & 46.84 & 31.14 & 50.40 & 27.17 & 12.77 \\
\hline 6 & 1 & 2 & 35.96 & 26.67 & 34.83 & 23.72 & 11.06 \\
\hline 1 & 1 & 1.5 & 50.49 & 35.23 & 43.33 & 30.60 & 13.15 \\
\hline 2 & 1 & 1.5 & 28.66 & 19.76 & 45.01 & 17.31 & 12.42 \\
\hline 3 & 1 & 1.5 & 27.95 & 19.19 & 45.68 & 16.60 & 13.47 \\
\hline 4 & 1 & 1.5 & 22.98 & 19.92 & 15.35 & 16.17 & 18.79 \\
\hline 5 & 1 & 1.5 & 27.94 & 19.24 & 45.17 & 16.59 & 13.81 \\
\hline 6 & 1 & 1.5 & 21.76 & 19.23 & 13.17 & 14.96 & 22.20 \\
\hline 1 & 1 & 0.5 & 34.57 & 25.31 & 36.59 & 27.35 & 8.05 \\
\hline 2 & 1 & 0.5 & 11.98 & 8.53 & 40.51 & 9.94 & 16.53 \\
\hline 3 & 1 & 0.5 & 11.91 & 8.44 & 41.05 & 9.86 & 16.81 \\
\hline 4 & 1 & 0.5 & 27.15 & 19.37 & 40.13 & 19.69 & 1.63 \\
\hline 5 & 1 & 0.5 & 11.84 & 8.38 & 41.24 & 9.79 & 16.83 \\
\hline 6 & 1 & 0.5 & 21.00 & 14.26 & 47.26 & 16.29 & 14.20 \\
\hline 1 & 1 & 0.5 & 31.85 & 25.45 & 25.12 & 25.05 & 1.57 \\
\hline 2 & 1 & 0.5 & 15.27 & 11.11 & 37.44 & 12.06 & 8.58 \\
\hline 3 & 1 & 0.5 & 14.68 & 10.88 & 34.99 & 11.48 & 5.51 \\
\hline 4 & 1 & 0.5 & 31.12 & 25.22 & 23.41 & 24.33 & 3.53 \\
\hline 5 & 1 & 0.5 & 15.18 & 10.98 & 38.28 & 11.97 & 9.07 \\
\hline 6 & 1 & 0.5 & 14.15 & 11.08 & 27.62 & 11.86 & 7.05 \\
\hline 1 & 1 & 0.5 & 38.50 & 29.86 & 28.95 & 28.90 & 3.23 \\
\hline 2 & 1 & 0.5 & 23.06 & 16.75 & 37.67 & 16.23 & 3.15 \\
\hline 3 & 1 & 0.5 & 23.21 & 16.71 & 38.91 & 16.38 & 2.01 \\
\hline 4 & 1 & 0.5 & 18.71 & 15.19 & 23.20 & 15.04 & 0.99 \\
\hline 5 & 1 & 0.5 & 23.06 & 16.73 & 37.86 & 16.23 & 3.01 \\
\hline 6 & 1 & 0.5 & 19.16 & 15.17 & 26.28 & 15.56 & 2.52 \\
\hline 1 & 1 & 0.5 & 30.68 & 24.09 & 27.35 & 22.02 & 8.61 \\
\hline 2 & 1 & 0.5 & 19.52 & 14.21 & 37.41 & 13.91 & 2.06 \\
\hline 3 & 1 & 0.5 & 18.81 & 13.62 & 38.14 & 13.21 & 3.03 \\
\hline 4 & 1 & 0.5 & 17.20 & 14.50 & 18.63 & 14.06 & 3.05 \\
\hline 5 & 1 & 0.5 & 18.80 & 13.66 & 37.62 & 13.19 & 3.43 \\
\hline 6 & 1 & 0.5 & 15.99 & 13.80 & 15.85 & 12.84 & 6.93 \\
\hline
\end{tabular}


Flow time error using correction factors for six product instances

\begin{tabular}{|c|c|c|c|c|c|c|c|}
\hline Prod \# & $C a$ & $C s$ & $\begin{array}{c}\text { Anal. } \\
\text { Flow T. }\end{array}$ & $\begin{array}{c}\text { Sim. } \\
\text { Flow T. }\end{array}$ & $\begin{array}{c}\text { Error } \\
(\%)\end{array}$ & $\begin{array}{c}\text { Adj. Anal. } \\
\text { Flow T. }\end{array}$ & $\begin{array}{c}\text { Adj Error } \\
(\%)\end{array}$ \\
\hline 1 & 1.5 & 1 & 52.65 & 38.07 & 38.30 & 31.77 & 16.55 \\
\hline 2 & 1.5 & 1 & 16.60 & 10.98 & 51.08 & 11.58 & 5.44 \\
\hline 3 & 1.5 & 1 & 16.52 & 10.89 & 51.61 & 11.50 & 5.59 \\
\hline 4 & 1.5 & 1 & 45.91 & 31.79 & 44.40 & 25.44 & 19.98 \\
\hline 5 & 1.5 & 1 & 16.45 & 10.83 & 51.83 & 11.43 & 5.54 \\
\hline 6 & 1.5 & 1 & 37.35 & 22.96 & 62.71 & 19.19 & 16.39 \\
\hline 1 & 2 & 1 & 44.83 & 35.64 & 25.79 & 32.41 & 9.05 \\
\hline 2 & 2 & 1 & 22.61 & 15.48 & 46.10 & 16.17 & 4.45 \\
\hline 3 & 2 & 1 & 22.03 & 15.19 & 45.05 & 15.58 & 2.60 \\
\hline 4 & 2 & 1 & 44.11 & 35.30 & 24.93 & 31.69 & 10.23 \\
\hline 5 & 2 & 1 & 22.53 & 15.31 & 47.15 & 16.08 & 5.03 \\
\hline 6 & 2 & 1 & 21.53 & 14.65 & 47.02 & 16.23 & 10.79 \\
\hline 1 & 1.5 & 1 & 52.18 & 38.72 & 34.78 & 35.62 & 7.99 \\
\hline 2 & 1.5 & 1 & 33.45 & 22.15 & 51.05 & 21.09 & 4.77 \\
\hline 3 & 1.5 & 1 & 33.60 & 22.17 & 51.55 & 21.24 & 4.20 \\
\hline 4 & 1.5 & 1 & 26.18 & 19.38 & 35.09 & 18.91 & 2.42 \\
\hline 5 & 1.5 & 1 & 33.45 & 22.19 & 50.75 & 21.09 & 4.96 \\
\hline 6 & 1.5 & 1 & 26.03 & 19.33 & 34.66 & 18.76 & 2.95 \\
\hline 1 & 1.5 & 1 & 43.42 & 34.78 & 24.87 & 27.80 & 20.06 \\
\hline 2 & 1.5 & 1 & 26.50 & 20.54 & 29.00 & 17.05 & 17.01 \\
\hline 3 & 1.5 & 1 & 25.79 & 19.91 & 29.57 & 16.34 & 17.91 \\
\hline 4 & 1.5 & 1 & 22.34 & 20.74 & 7.74 & 16.52 & 20.35 \\
\hline 5 & 1.5 & 1 & 25.78 & 19.98 & 28.99 & 16.33 & 18.30 \\
\hline 6 & 1.5 & 1 & 21.13 & 19.91 & 6.10 & 15.30 & 23.15 \\
\hline & & & & & & & \\
\hline
\end{tabular}


VITA

MARIA DE LOURDES HULETT

May 17, 1972

Born, Caracas, Venezuela

B.S., Industrial Engineering

University of Tachira

Tachira, Venezuela

1996-2001

Planner Engineer

Planning Council. University of Tachira

Tachira, Venezuela

2001

M. Sc., Business Administration

University of Tachira

Tachira, Venezuela

2001-2004

Assistant Professor

Department of Industrial Engineering

University of Tachira

Tachira, Venezuela

2004-2006

Aggregate Professor

Department of Industrial Engineering

University of Tachira

Tachira, Venezuela

2007

M. Sc., Engineering Management

Florida International University

Miami, Florida

2009

Doctoral Candidate in Industrial and Systems Engineering

Florida International University

Miami, Florida

\section{PUBLICATIONS AND PRESENTATIONS}

Hulett, M., Damodaran, P. Analytical Approximations to Predict Performance Measures of an Assembly Line. Proceedings of the 2009 Industrial Engineering Research Conference, Miami 2009.

Hulett, M., Damodaran, P. Queuing Network Approximations for Server Assembly. INFORMS Annual Meeting, Washington, 2008. 
Hulett, M., Damodaran, P., Mestry, S. Minimizing the Makespan of Identical Batch Processing Machines at an Electronics Manufacturing Facility, INFORMS Annual Meeting, Seattle, 2007.

Hulett, M. Key factors for the implementation of a business incubator in a local context. Case UNET. XI Seminario Latino-Iberoamericano de Gestión Tecnológica Brasil, 2005.

Hulett, M. The Business Incubator: An alternative for transferring technology. International Conference on CAD/CAM, Robotics and Factories of the Future (CARS \& FOF 2004), Venezuela, 2004.

Hulett, M. Business Incubation Model for Innovative Entrepreneurs, World Conference on Business Incubation, Brasil, 2001.

Hulett, M. A Strategic Model of a Business Incubator for the UNET, IX Seminario LatinoIberoamericano de Gestión Tecnológica, Costa Rica, 2001.

Hulett, M. (2001). A strategic model for the UNET Business Incubator. Revista de Ciencia e Tecnología, Política e Gestao para a Periferia. ISSN 1415-3262. Recife, Brasil, v.5, n.2, p.252-265. 